\title{
New Tricks for an Old Dog: Grubbs Catalysts Enable Efficient Hydrogen Production from Aqueous-Phase Methanol Reforming
}

Qian Wang, ${ }^{a, b, *}$ Jialing Lan, ${ }^{a, b, *}$ Rong Liang, ${ }^{b}$ Yihao Xia, ${ }^{b}$ Lei Qin,,$^{b, *}$ Lung Wa

Chung, ${ }^{b, c, *} \&$ Zhiping Zheng, ${ }^{b, c, *}$

[a] Q. Wang, J. Lan

School of Chemistry and Chemical Engineering

Harbin Institute of Technology

Harbin, 150001, China.

[b] Q. Wang, J. Lan, R. Liang, Y. Xia, L. Qin, L. W. Chung, Z. Zheng

Department of Chemistry

Southern University of Science and Technology

Shenzhen, 518055, China.

[c] L. W. Chung, Z. Zheng

Shenzhen Grubbs Institute and Guangdong Provincial Key Laboratory of Catalysis

Southern University of Science and Technology

Shenzhen, 518055, China.

E-mail: qinl@ sustech.edu.cn; oscarchung@ sustech.edu.cn; zhengzp@ sustech.edu.cn

*Q. Wang and J. Lan contributed equally. 


\section{Supporting Information}

\section{Contents}

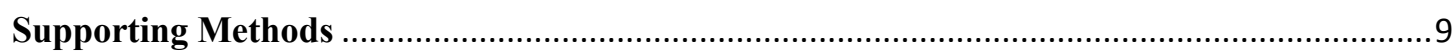

Supplementary Tables...............................................................................................11

Table S1. The set temperature and corresponding actual inner reaction temperature in $\mathrm{CH}_{3} \mathrm{OH} / \mathrm{H}_{2} \mathrm{O}(9: 1 v / v, 10 \mathrm{~mL})$ and $8 \mathrm{M} \mathrm{KOH}$ solutions.

Table S2. The quantitative relationship between $\mathrm{H}_{2}$ and HCOOK with various catalyst loadings

Supplementary Figures

Figure S1. GC result for the reaction using G-III. Reaction condition: $\mathrm{MeOH} / \mathrm{H}_{2} \mathrm{O}=9: 1,8 \mathrm{M}$

$\mathrm{KOH}, T_{\text {set }}=100{ }^{\circ} \mathrm{C}, 3$ hours, the unit for $\mathrm{CO}$ is ppm. The trace $\mathrm{CO}_{2}$ accounted for only $0.017 \%$ of the gas mixture produced

Figure S2. The ${ }^{1} \mathrm{H}-\mathrm{NMR}$ of G-III in basic $(8 \mathrm{M} \mathrm{KOH}), \mathrm{MeOH} / \mathrm{H}_{2} \mathrm{O}(9: 1,10 \mathrm{~mL})$ solutions after methanol reforming reaction for 1.5 hours. Inserts are the partially magnified picture at indicated ranges

Figure S3. The ${ }^{1} \mathrm{H}$ NMR of G-III in basic $(8 \mathrm{M} \mathrm{KOH}), \mathrm{MeOH} / \mathrm{H}_{2} \mathrm{O}(9: 1,10 \mathrm{~mL})$ solutions after methanol reforming reaction for 6 hours. Inserts are the partially magnified picture at indicated ranges. Notably, this measurement took hours to complete, and our result cannot accurately reflect the realistic state when the reaction is ceased.

Figure S4. The ${ }^{13} \mathrm{C}-\mathrm{NMR}$ of G-III in basic $(8 \mathrm{M} \mathrm{KOH}), \mathrm{MeOH} / \mathrm{H}_{2} \mathrm{O}(9: 1,10 \mathrm{~mL})$ solutions after methanol reforming reaction for 1.5 hours

Figure S5. a HRESI-MS of the reaction mixture using G-III as catalyst. Insert: the key species structures of F-1 (left), F-2 (right, top), and F-3 (right, bottom). b Proposed reaction intermediates based on the HRESI-MS data

Scheme S1. Catalytic performance comparison of (top) the alkylidene moiety-containing and (bottom) carbene-free species based on G-III.

Figure S6. The ${ }^{1} \mathrm{H}$ NMR of the decomposition complex from G-I applied for APMR reaction.

Figure S7. The ${ }^{1} \mathrm{H}$ NMR of the decomposition complex from G-II applied for APMR reaction. 
Scheme S2. The equations of dehydrogenation reactions using other substrates or the so-called liquid organic hydrogen carriers. ${ }^{2-4}$

Figure S8. The ${ }^{13} \mathrm{C}$ NMR spectrum of the product solution from the reaction of G-III ( $\left.5 \mu \mathrm{mol}\right)$ and $8 \mathrm{M} \mathrm{KOH}$ in $\mathrm{EtOH} / \mathrm{H}_{2} \mathrm{O}(v / v$ 9:1, $5 \mathrm{~mL})$ mixture; the thermostat was set at $100{ }^{\circ} \mathrm{C} . \ldots . . .18$

Figure S9. Free energy profile for the most favorable pathway (1) of the $\mathrm{Ru}(\mathrm{II})$-catalyzed aqueous methanol reforming reaction in solution by the PCM M06-L//M06-L method. This pathway with less favorable conformations is given in Figure S16.

Figure S10. Free energy profile for the pathway (2) involving the formation of $R u(I I)-$ dihydride species in solution by the PCM M06-L//M06-L method. TS $_{8-9}$ was computed to be higher in electronic energy (without ZPE correction) than 9 by $0.7 \mathrm{kcal} / \mathrm{mol}$ in gas phase, but becomes lower in energy with inclusion of the ZPE correction.

Figure S11. Free energy profile for the anionic pathway (3) of the $\mathrm{Ru}(\mathrm{II})$-catalyzed aqueous methanol reforming reaction in solution by the PCM M06-L//M06-L method.

Figure S12. Free energy profile for pathway 4 of the $\mathrm{Ru}(\mathrm{II})$-catalyzed aqueous methanol reforming reaction via the Ru-alkyl species in solution by the PCM M06-L//M06-L method.

Figure S13. Free energy profile for the higher-energy $\beta-\mathrm{H}$ elimination pathways in gas phase by the M06-L method.

Figure S14. Free energy profile for the pathway (2) involving the formation of $\mathrm{Ru}(\mathrm{II})-$ dihydride species in solution using the 3-Br-pyridine as the ligand (instead of pyridine) by the PCM M06-L//M06-L method

Figure S15. Free energy profile for the hydrogen evolution processes from $\mathrm{MeOH}, \mathrm{CH}_{2}(\mathrm{OH})_{2}$ and $\mathrm{HCO}_{2} \mathrm{H}$ in solution using the 3-Br-pyridine as the ligand (instead of pyridine) by the PCM M06-L//M06-L method.

Figure S16. Calculated key structural parameters of the intermediates and transition states for the pathway 1 by the M06-L method with the key bond lengths (Ångstroms). Unimportant H atoms are omitted for clarity. Their relative free energies in solution by PCM M06-L//M06-L method are also given.

Figure S17. Calculated key structural parameters of the intermediates and transition states for the pathway 2 by the M06-L method with the key bond lengths (Angstroms). Unimportant H atoms are omitted for clarity. Their relative free energies in solution by PCM M06-L//M06-L method are also given. 
Figure S18. Calculated key structural parameters of the intermediates and transition states for the anionic pathway 3 by the M06-L method with the key bond lengths (Ångstroms). Unimportant $\mathrm{H}$ atoms are omitted for clarity. Their relative free energies in solution by PCM M06-L//M06-L method are also given.

Figure S19. Calculated key structural parameters of the intermediates and transition states for the pathway 4 by the M06-L method with the key bond lengths (Ångstroms). Unimportant H atoms are omitted for clarity. Their relative free energies in solution by PCM M06-L//M06-L method are also given.

Figure S20. Calculated key structural parameters of the intermediates and transition states for the higher-energy $\beta-\mathrm{H}$ elimination pathways by the M06-L method with the key bond lengths (Ångstroms). Unimportant $\mathrm{H}$ atoms are omitted for clarity. Their relative free energies in solution by M06-L method are also given.

Figure S21. Calculated key structural parameters of the Ru-dihydride-dihydrogen and Ruhydride-dihydrogen intermediates by the M06-L method. Unimportant $\mathrm{H}$ atoms are omitted for clarity. Their relative free energies in solution by PCM M06-L/M06-L method are also given.

Figure S22. Calculated key structural parameters of the key geometries with 3-Br-pyridine as the ligand by the M06-L method. Unimportant $\mathrm{H}$ atoms are omitted for clarity. Their relative free energies in solution by PCM M06-L//M06-L method are also given.

Figure S23. | Calculated key structural parameters of the geometries with 3-Br-pyridine as the ligand for dehydrogenation of $\mathrm{HOCH}_{2} \mathrm{OH}$ to $\mathrm{HCOOH}$ by the M06-L method. Unimportant $\mathrm{H}$ atoms are omitted for clarity. Their relative free energies in solution by PCM M06-L//M06-L method are also given.

Figure S24. | Calculated key structural parameters of the geometries with 3-Br-pyridine as the ligand for dehydrogenation of $\mathrm{HCOOH}$ to $\mathrm{CO}_{2}$ by the M06-L method. Unimportant $\mathrm{H}$ atoms are omitted for clarity. Their relative free energies in solution by PCM M06-L//M06-L method are also given.

Table S3. The absolute energies (in Hartree) for the pathway 1 of the Ru-catalyzed lowtemperature aqueous methanol reforming by the M06-L method.

Table S4. The relative energies (in $\mathrm{kcal} / \mathrm{mol}$ ) for the pathway 1 of the Ru-catalyzed lowtemperature aqueous methanol reforming by the M06-L method. The relative free energies in solution with the standard state correction $\left(\Delta \mathrm{G}_{\text {soln-pcm-cor }}\right)$ are also given.

Table S5. The absolute energies (in Hartree) for the pathways $\mathbf{2}$ and $\mathbf{3}$ of the Ru-catalyzed lowtemperature aqueous methanol reforming by the M06-L method. .51 
Table S6. The relative energies (in $\mathrm{kcal} / \mathrm{mol}$ ) for the pathways $\mathbf{2}$ and $\mathbf{3}$ of the Ru-catalyzed lowtemperature aqueous methanol reforming by the M06-L method. The relative free energies in solution with the standard state correction $\left(\Delta \mathrm{G}_{\text {soln-pcm-cor }}\right)$ are also given. .52

Table S7. The absolute energies (in Hartree) for the pathway 4 of the Ru-catalyzed lowtemperature aqueous methanol reforming by the M06-L method. 53

Table S8. The relative energies (in $\mathrm{kcal} / \mathrm{mol}$ ) for the pathway 4 of the Ru-catalyzed lowtemperature aqueous methanol reforming by the M06-L method. The relative free energies in solution with the standard state correction $\left(\Delta \mathrm{G}_{\text {soln-pcm-cor }}\right)$ are also given

Table S9. The absolute electronic energies (in Hartree) for the key geometries of the Rucatalyzed low-temperature aqueous methanol reforming in solution by different DFT methods.

Table S10.The relative electronic energies (in $\mathrm{kcal} / \mathrm{mol}$ ) for the key geometries of the $\mathrm{Ru}$ catalyzed low-temperature aqueous methanol reforming in solution by different DFT methods.

Table S11.The relative estimated Gibbs free energies (in $\mathrm{kcal} / \mathrm{mol}$ ) for the key geometries of the Ru-catalyzed low-temperature aqueous methanol reforming in solution by different DFT methods.

Table S12. The corrected relative estimated Gibbs free energies (in $\mathrm{kcal} / \mathrm{mol}$ ) for the key geometries of the Ru-catalyzed low-temperature aqueous methanol reforming in solution by different DFT methods. The relative free energies were corrected with the standard state correction.

Table S13. The absolute (in Hartree) and relative energies (in $\mathrm{kcal} / \mathrm{mol}$ ) for the higher-energy $\beta-\mathrm{H}$ elimination pathways in gas phase by the M06-L method

Table S14. The absolute energies (in Hartree) for the Ru-dihydride-dihydrogen and Ruhydride-didydrogen intermediates by the M06-L method.

Table S15. The relative energies (in $\mathrm{kcal} / \mathrm{mol}$ ) for the Ru-dihydride-dihydrogen and Ruhydride-didydrogen intermediates by the M06-L method. The relative free energies with the standard state correction $\left(\Delta \mathrm{G}_{\text {soln-pcm-cor }}\right)$ are also given.

Table S16. The absolute energies (in Hartree) for the key geometries with 3-Br-pyridine as the ligand in the main text by the M06-L method.

Table S17. The relative energies (in $\mathrm{kcal} / \mathrm{mol}$ ) for the key geometries with using the 3-Brpyridine as the ligand (instead of pyridine) by the M06-L method. The relative free energies in 
solution with the standard state correction $\left(\Delta \mathrm{G}_{\text {soln-pcm-cor }}\right)$ are also given

Table S18. The metal-ligand bond distances (in $\AA$ ) for the key geometries of the Ru-catalyzed low-temperature aqueous methanol reforming in solution by the M06-L method

Table S19. The absolute energies (in Hartree) for for the geometries with 3-Br-pyridine as the ligand for dehydrogenation of $\mathrm{HOCH}_{2} \mathrm{OH}$ to $\mathrm{HCOOH}$ by the M06-L method.

Table S20. The relative energies (in $\mathrm{kcal} / \mathrm{mol}$ ) for the geometries with 3 -Br-pyridine as the ligand for dehydrogenation of $\mathrm{HOCH}_{2} \mathrm{OH}$ to $\mathrm{HCOOH}$ by the $\mathrm{M} 06-\mathrm{L}$ method. The relative free energies in solution with the standard state correction $\left(\Delta \mathrm{G}_{\text {soln-pcm-cor }}\right)$ are also given.

Table S21. The absolute energies (in Hartree) for for the geometries with 3-Br-pyridine as the ligand for dehydrogenation of $\mathrm{HCOOH}$ to $\mathrm{CO}_{2}$ by the M06-L method.

Table S22. The relative energies (in $\mathrm{kcal} / \mathrm{mol}$ ) for the geometries with 3 -Br-pyridine as the ligand for dehydrogenation of $\mathrm{HCOOH}$ to $\mathrm{CO}_{2}$ by the $\mathrm{M} 06-\mathrm{L}$ method. The relative free energies in solution with the standard state correction $\left(\Delta \mathrm{G}_{\text {soln-pcm-cor }}\right)$ are also given. 70

Table S23. The absolute electronic energies (in Hartree) for the key geometries with 3-Brpyridine as the ligand for dehydrogenation of $\mathrm{HOCH}_{2} \mathrm{OH}$ to $\mathrm{HCOOH}$ in solution by different DFT methods .71

Table S24. The relative electronic and estimated Gibbs free energies (in $\mathrm{kcal} / \mathrm{mol}$ ) for the key geometries with 3-Br-pyridine as the ligand for dehydrogenation of $\mathrm{HOCH}_{2} \mathrm{OH}$ to $\mathrm{HCOOH}$ in solution by different DFT methods. .72

Table S25. The corrected relative estimated Gibbs free energies (in $\mathrm{kcal} / \mathrm{mol}$ ) for the key geometries with 3-Br-pyridine as the ligand for dehydrogenation of $\mathrm{HOCH}_{2} \mathrm{OH}$ to $\mathrm{HCOOH}$ in solution by different DFT methods. The relative free energies were corrected with the standard state correction .73

Table S26. The absolute electronic energies (in Hartree) for the key geometries with 3-Brpyridine as the ligand for dehydrogenation of $\mathrm{HCOOH}$ to $\mathrm{CO}_{2}$ in solution by different DFT methods

Table S27. The relative electronic and estimated Gibbs free energies (in $\mathrm{kcal} / \mathrm{mol}$ ) for the key geometries with 3-Br-pyridine as the ligand for dehydrogenation of $\mathrm{HCOOH}$ to $\mathrm{CO}_{2}$ in solution by different DFT methods. .75

Table S28. The corrected relative estimated Gibbs free energies (in kcal/mol) for the key geometries with 3-Br-pyridine as the ligand for dehydrogenation of $\mathrm{HCOOH}$ to $\mathrm{CO}_{2}$ in solution by different DFT methods. The relative free energies were corrected with the standard state 
correction.

Table S29. The absolute electronic energies (in Hartree) for the key geometries with 3-Brpyridine as the ligand for dehydrogenation of $\mathrm{MeOH}$ in solution by PCM M06-L/6-

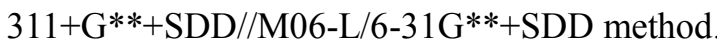

Table S30. The relative energies (in $\mathrm{kcal} / \mathrm{mol}$ ) for the key geometries with 3-Br-pyridine as the ligand for dehydrogenation of $\mathrm{MeOH}$ in solution by PCM M06-L/6-311+G**+SDD//M06$\mathrm{L} / 6-31 \mathrm{G}^{* *+}+\mathrm{SDD}$ method. The relative free energies in solution with the standard state correction $\left(\Delta \mathbf{G}_{\text {soln-cor-B }}\right)$ are also given.

Table S31. The absolute electronic energies (in Hartree) for the key geometries with pyridine as the ligand for dehydrogenation of $\mathrm{MeOH}$ in solution by PCM M06-L/6$311+\mathrm{G}^{* *+} \mathrm{SDD} / / \mathrm{M} 06-\mathrm{L} / 6-31 \mathrm{G}^{* *+} \mathrm{SDD}$ method

Table S32. The relative energies (in $\mathrm{kcal} / \mathrm{mol}$ ) for the key geometries with pyridine as the ligand for dehydrogenation of $\mathrm{MeOH}$ in solution by $\mathrm{PCM}$ M06-L/6-311+ $\mathrm{G}^{* *+\mathrm{SDD} / / \mathrm{M} 06-}$ $\mathrm{L} / 6-31 \mathrm{G}^{* *+}+\mathrm{SDD}$ method. The relative free energies in solution with the standard state correction $\left(\Delta \mathbf{G}_{\text {soln-cor-B }}\right)$ are also given.

Table S33. The driving force (in $\mathrm{kcal} / \mathrm{mol}$ ) for the overall catalyzed reaction by PCM M06L/6-31G**+SDD//M06-L/6-31G**+SDD (method A), PCM M06-L/6-311+G**+SDD//M06$\mathrm{L} / 6-31 \mathrm{G}^{* *+} \mathrm{SDD}(\operatorname{method} \mathrm{B})$ and experimental values (Table S33).

Table S34. Experimental values of standard molar Gibbs free energy (in $\mathrm{kJ} / \mathrm{mol}$ ) for the overall catalyzed reaction taken from ref. 20

Table S35. The absolute enthalpy energies (in Hartree) for $\mathbf{G}-\mathbf{I}, \mathbf{G}-\mathbf{I}_{\mathbf{D}}, \mathbf{G}-\mathbf{I I}, \mathbf{G}-\mathbf{I I}_{\mathbf{D}}$ and $\mathbf{P C \mathbf { y } _ { 3 }}$ in gas phase by the M06-L method.

Table S36. Bond dissociation enthalpies (in $\mathrm{kcal} / \mathrm{mol}$ ) for the phosphine ligand in G-I and GII in gas phase by the M06-L method $\left(\Delta \mathbf{H}_{\text {DFT }}\right)$ and experiments $\left(\Delta \mathbf{H}_{\text {expt }}\right)^{[21]}$

Table S37. The absolute (in Hartree) and relative (in $\mathrm{kcal} / \mathrm{mol}$ ) energies for the direct hydride transfer from the methanolate intermediate $\mathbf{2 5}$ to the Ru metal optimized in solvent by the PCM M06-L method. The relative free energies in solution with the standard state correction $\left(\Delta \mathbf{G}_{\text {soln- }}\right.$ cor) are also given. 82

Table S38. The absolute (in Hartree) and relative (in $\mathrm{kcal} / \mathrm{mol}$ ) electronic energies for potential energy scan with various Ru-H bond distance ( $\mathrm{R}$, in $\AA$ ) in gas and solvent phases by the M06L and PCM M06-L methods, respectively.

Table S39. The absolute (in Hartree) energies for the 8-m-r pathway in the gas phase optimized 
by M06-L/6-31G**+SDD method, and corresponding single-point energies in solvent phase by PCM M06-L/6-31G**+SDD//M06-L/6-31G**+SDD and PCM M06-L/6-

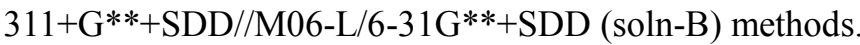

Table S40. The relative (in $\mathrm{kcal} / \mathrm{mol}$ ) energies for the 8-m-r pathway in the gas phase by M06$\mathrm{L} / 6-31 \mathrm{G}^{* *+}$ SDD method, and the relative energies in solvent phase by PCM M06-L/6-

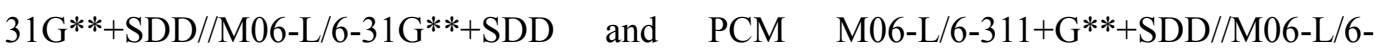
$31 \mathrm{G}^{* *+\mathrm{SDD}}$ (soln-B) methods. The relative free energies in solution with the standard state correction ( $\boldsymbol{\Delta} \mathbf{G}_{\text {soln-cor }}$ and $\left.\boldsymbol{\Delta} \mathbf{G}_{\text {soln-B-cor }}\right)$ are also given.

Table S41. The calculated H/D KIE value for different key transition states in gas phase at $373.15 \mathrm{~K}$ by the M06-L method 


\section{Supporting Methods}

NMR experiments. ${ }^{1} \mathrm{H}$ and ${ }^{13} \mathrm{C}$ NMR spectra were recorded on a Bruker Ascend ${ }^{\mathrm{TM}} 400$ spectrometer. Chemical shifts are referenced to internal solvent resonances and reported in parts per million. All NMR measurements were performed with cooled reaction mixtures.

High-resolution electrospray ionization mass spectrometry. Mass spectrometry was recorded on a MAT 95XP ThermoFisher Mass Spectrometer using Electron Impact Ionization (EI, $70 \mathrm{eV}$ ) mode. The reaction mixture using $12 \mathrm{mg}$ of G-III as catalyst was transferred into Ar-protected glove box after cooling down to room temperature (Reaction condition: $\left.\mathrm{MeOH} / \mathrm{H}_{2} \mathrm{O}(v / v)=9: 1(10 \mathrm{~mL}), 8 \mathrm{M} \mathrm{KOH}, T_{\text {set }}=100{ }^{\circ} \mathrm{C}\right)$. Then, $100-\mu \mathrm{L}$ mixture was diluted in $5 \mathrm{~mL}$ of toluene, and $1 \mathrm{~mL}$ of the resulting solution was taken for the HRESI-MS measurement immediately.

Preparation of alkylidene moiety-free species by in situ decomposition of G-I and G-II. The carbene-free complexes resulting from the decomposition of G-I and G-II were synthesized by using reported methods. ${ }^{1}$

Preparation of alkylidene moiety-free species by in situ decomposition of G-III. To a degassed $\mathrm{MeOH} / \mathrm{H}_{2} \mathrm{O}(9 \mathrm{~mL} / 1 \mathrm{~mL})$ solution containing $8 \mathrm{M} \mathrm{KOH}, 5 \mu \mathrm{mol}$ of G-III was added. The mixture was stirred at room temperature for a week until no carbene signal could be detected (monitored by ${ }^{1} \mathrm{H}$ NMR). Then, the mixture was degassed and heated to $100{ }^{\circ} \mathrm{C}$. No obvious gas evolution was observed after a day. 
The schematic presentation of the reaction apparatus.

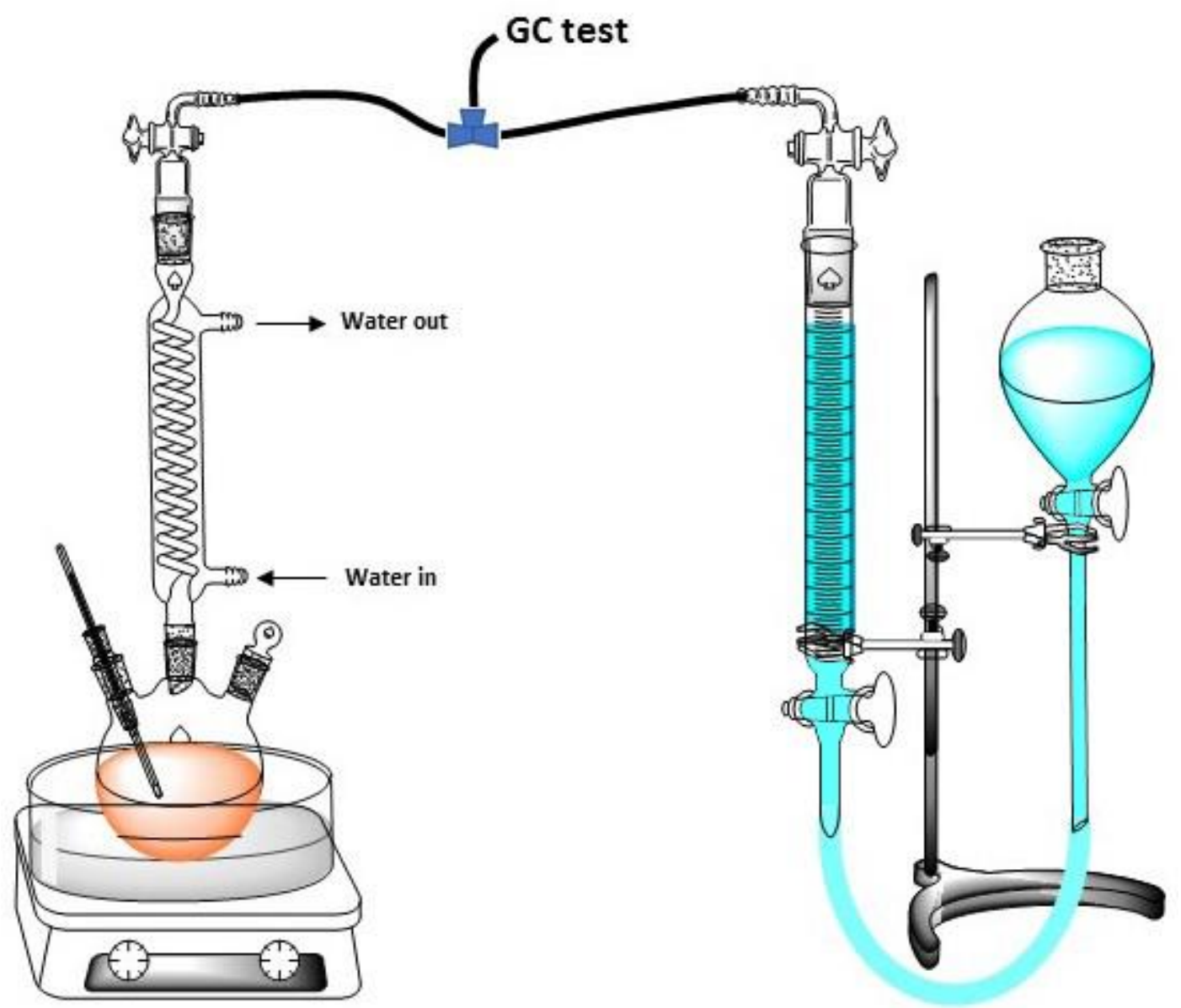




\section{Supplementary Tables}

Table S1. The set temperature and corresponding actual inner reaction temperature in $\mathrm{CH}_{3} \mathrm{OH} / \mathrm{H}_{2} \mathrm{O}(9: 1 v / v, 10 \mathrm{~mL})$ and $8 \mathrm{M} \mathrm{KOH}$ solutions

\begin{tabular}{ccc}
\hline Entry & $T_{\text {set }}{ }^{\circ} \mathrm{C}$ & $T_{\text {inner }} /{ }^{\circ} \mathrm{C}$ \\
\hline 1 & 80 & 75 \\
2 & 90 & 85 \\
3 & 100 & 88 \\
4 & 110 & 97 \\
5 & 120 & 102 \\
6 & 130 & 104 \\
\hline
\end{tabular}

Table S2. The quantitative relationship between $\mathrm{H}_{2}$ and $\mathrm{HCOOK}$ with various catalyst loadings $^{a}$

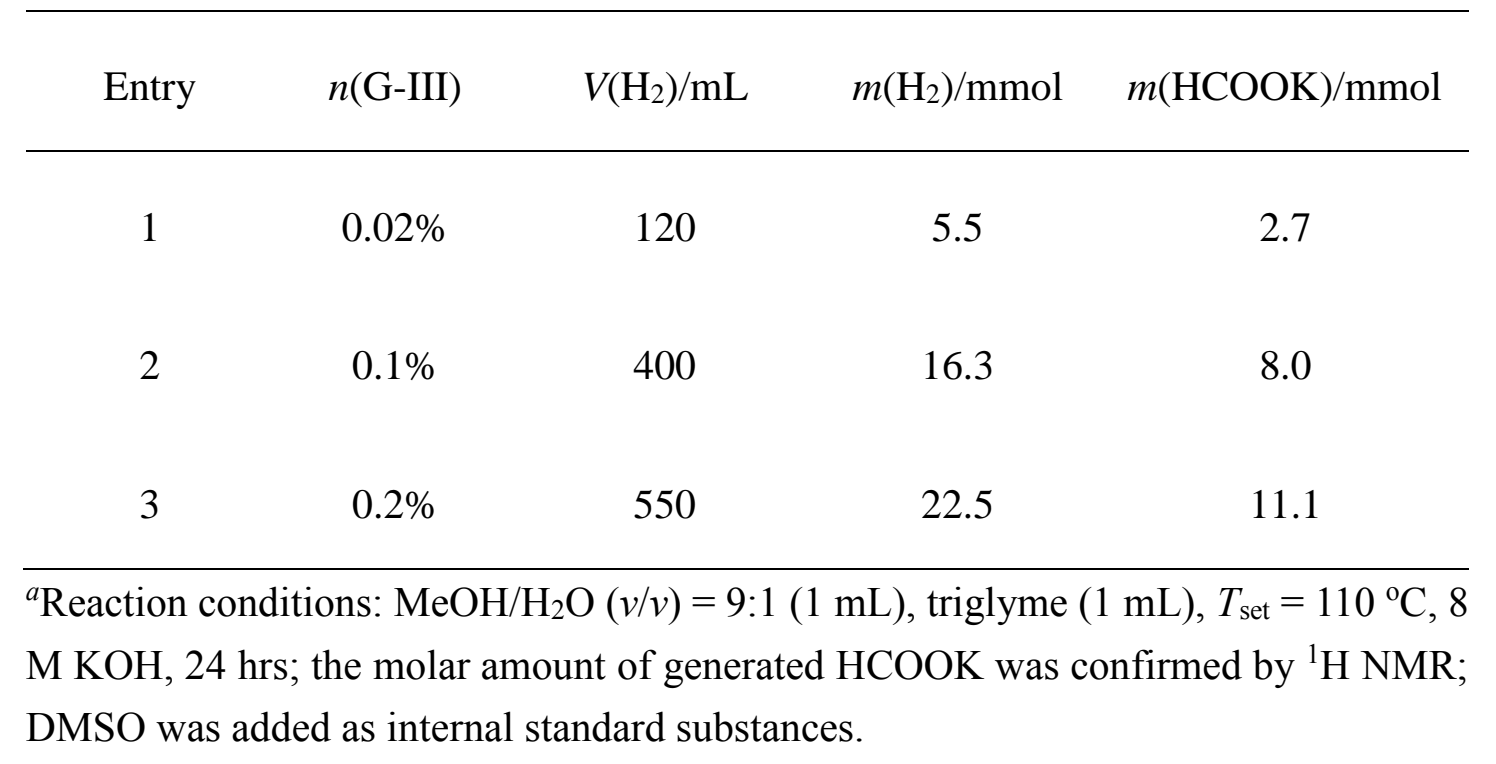




\section{Supplementary Figures}

\section{Report}

Analysis Method: $\quad$ D: $\backslash$ Data $\backslash$ Method $\backslash$ Test-20181030-1. 50.met

Data File: D: Data \Result \QinLei \Grubbs Catalyst \Grubbs-3\q1-3-27-grubbs-3-8M

KOH-5ummolCat-9bi1. rs1t \q1-3-27-grubbs-3-8M-KOH-5ummolCat-9 bi1. dat

User: $\quad$ Lei Qin

Acquisition time: 2018/11/30 16:52:38 (GMT +08:00)

Print time: $\quad 2018 / 11 / 3017: 26: 09$ (GMT +08:00)
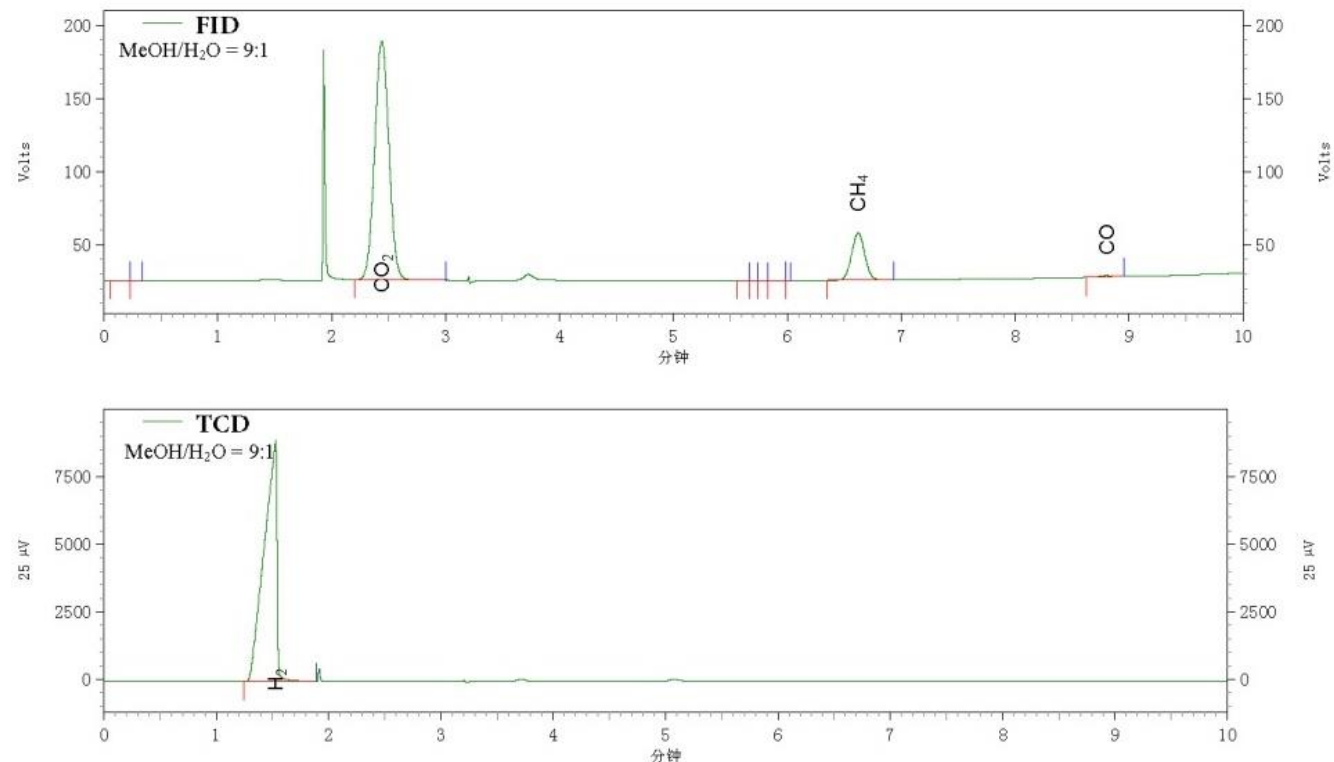

FID result

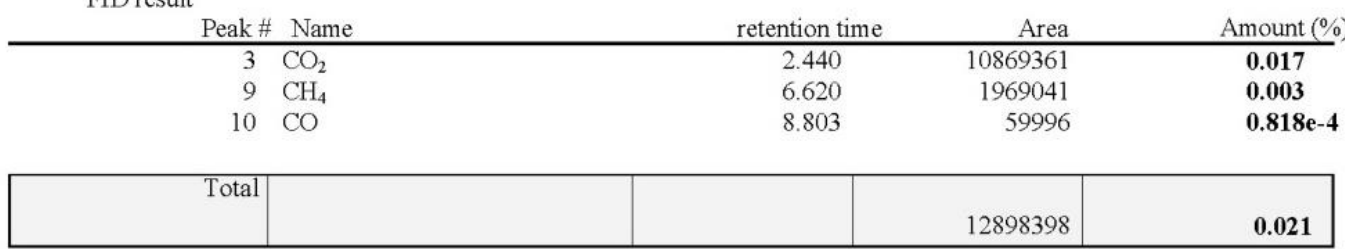

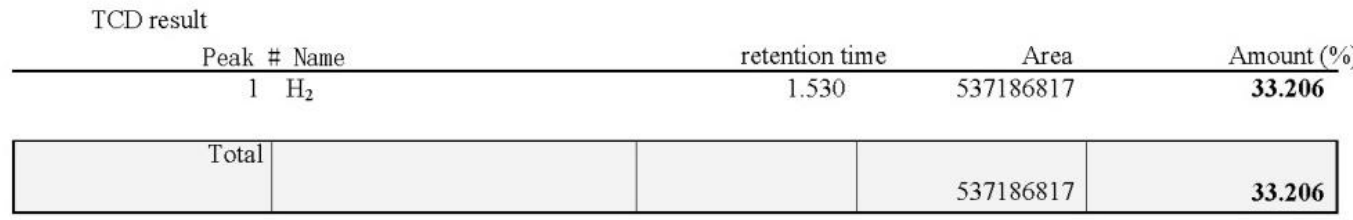

Figure S1. GC result for the reaction using G-III. Reaction condition: $\mathrm{MeOH} / \mathrm{H}_{2} \mathrm{O}=$ 9:1, $8 \mathrm{M} \mathrm{KOH}, T_{\text {set }}=100{ }^{\circ} \mathrm{C}, 3$ hours, the unit for $\mathrm{CO}$ is ppm. The trace $\mathrm{CO}_{2}$ accounted for only $0.017 \%$ of the gas mixture produced. 


\section{NMR and HRESI-MS experiments}

For this work of using G-III as catalyst, distinct patterns of $\mathrm{m} / \mathrm{z}$ peaks in the HRESI-MS spectra were produced (Supplementary Fig. 5) as a result of the seven characteristic isotopes of Ru. Based on the molecular structure of G-III, a combined analysis of the NMR and mass spectra, coupled with careful formula simulations, led to the identification of three prominent fragments in the reaction mixtures (Supplementary Fig. 5a). These mononuclear alkylidene species can be formulated as $\left[\left(\mathrm{H}_{2} \mathrm{Imes}\right)(\mathrm{H}) \mathrm{Ru}=\mathrm{CH}-\mathrm{Ph}\right]^{+}(\mathbf{F}-\mathbf{1}, m / z=499.1679),\left[\left(\mathrm{H}_{2} \mathrm{Imes}\right)\left(\mathrm{CH}_{3} \mathrm{O}\right) \mathrm{Ru}=\mathrm{CH}-\mathrm{Ph}\right]^{+}(\mathbf{F}-$ 2, $m / z=529.1800)$, and $\left[\left(\mathrm{H}_{2} \mathrm{Imes}\right)\left(\mathrm{HOCH}_{2} \mathrm{O}\right) \mathrm{Ru}=\mathrm{CH}-\mathrm{Ph}\right]^{+}$or $\left[\left(\mathrm{H}_{2} \mathrm{Imes}\right)(\mathrm{H})(\mathrm{HCOO}) \mathrm{Ru}=\mathrm{CH}-\mathrm{Ph}+\right.$ $\mathrm{H}]^{+}(\mathbf{F}-3, m / z=545.1736)$ (Supplementary Fig. 5a). In addition to these three fragments, simulated plots for the other peaks in the HRESI-MS spectrum matched well with the experimental results, all within 5 ppm between the experimental and the calculated formula masses and with perfectly matched isotope distribution patterns. With this information of the prominent fragments, we propose three corresponding intermediates, $\left[\left(\mathrm{H}_{2} \mathrm{Imes}\right)(\mathrm{H})(\mathrm{R}) \mathrm{Ru}=\mathrm{CH}-\mathrm{Ph}\right]\left[\right.$ Supplementary Fig. $5 \mathrm{~b}, \mathrm{R}=\mathrm{MeO}^{-}$ (I-1), $\mathrm{HOCH}_{2} \mathrm{O}^{-}(\mathbf{I}-2)$, and $\left.\mathrm{HCOO}^{-}(\mathbf{I}-3)\right]$ and involved sequentially in the three $\mathrm{H}_{2}$-producing steps of the AMR process (Fig. 1A). 


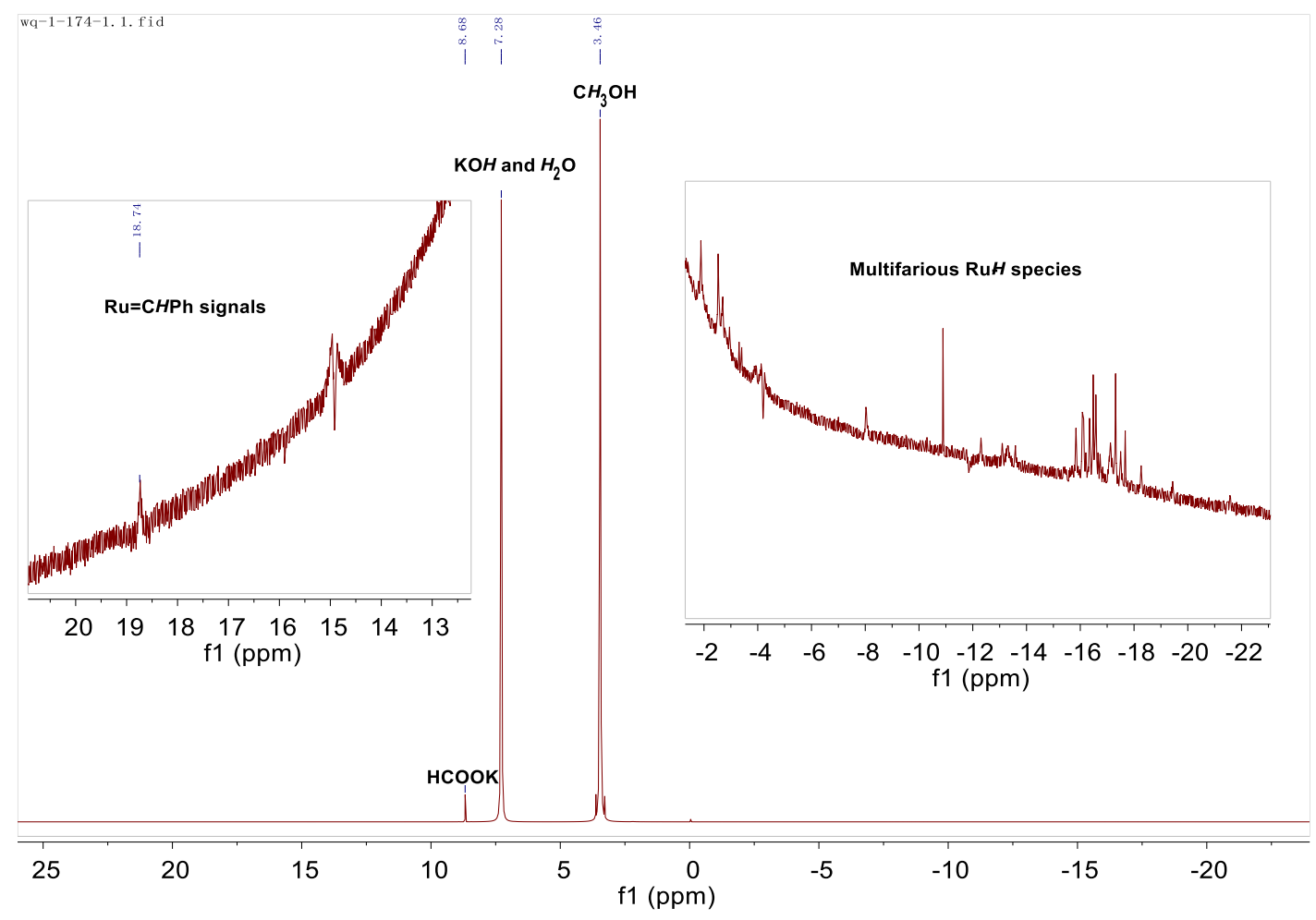

Figure S2. The ${ }^{1} \mathrm{H}-\mathrm{NMR}$ of G-III in basic $(8 \mathrm{M} \mathrm{KOH}), \mathrm{MeOH} / \mathrm{H}_{2} \mathrm{O}(9: 1,10 \mathrm{~mL})$ solutions after methanol reforming reaction for 1.5 hours. Inserts are the partially magnified picture at indicated ranges.

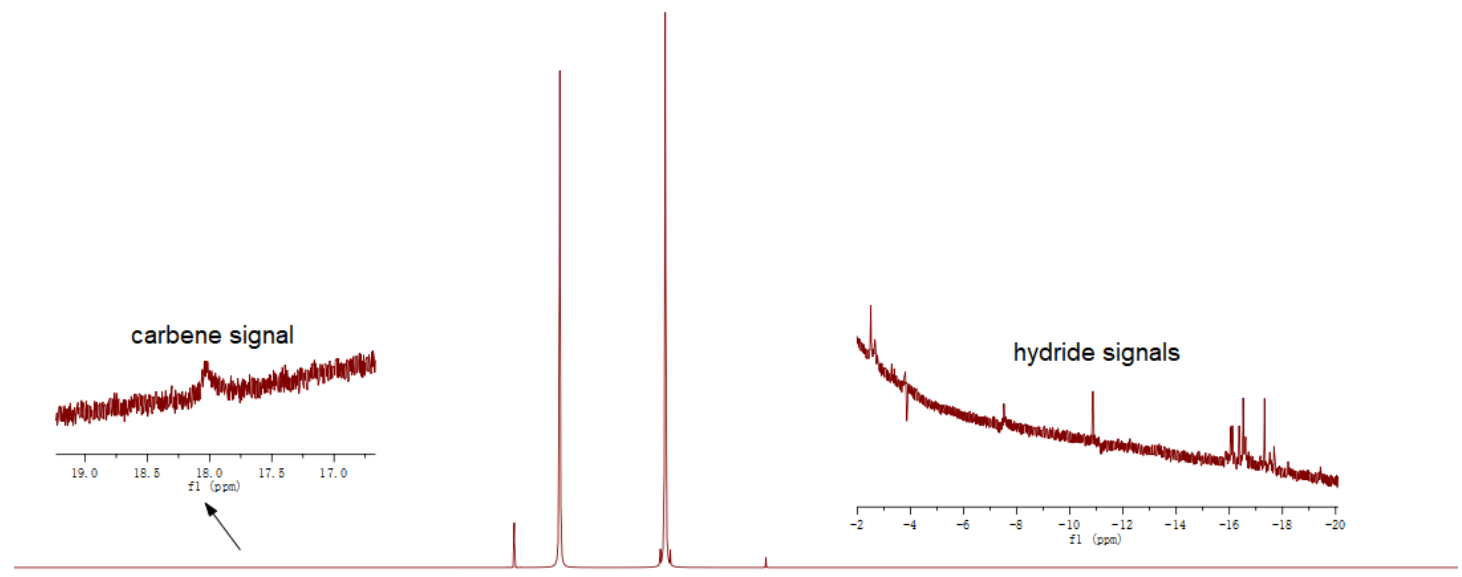

Figure S3. The ${ }^{1} \mathrm{H}$ NMR of G-III in basic $(8 \mathrm{M} \mathrm{KOH}), \mathrm{MeOH} / \mathrm{H}_{2} \mathrm{O}(9: 1,10 \mathrm{~mL})$ solutions after methanol reforming reaction for 6 hours. Inserts are the partially magnified picture at indicated ranges. Notably, this measurement took hours to complete, and our result cannot accurately reflect the realistic state when the reaction is ceased. 


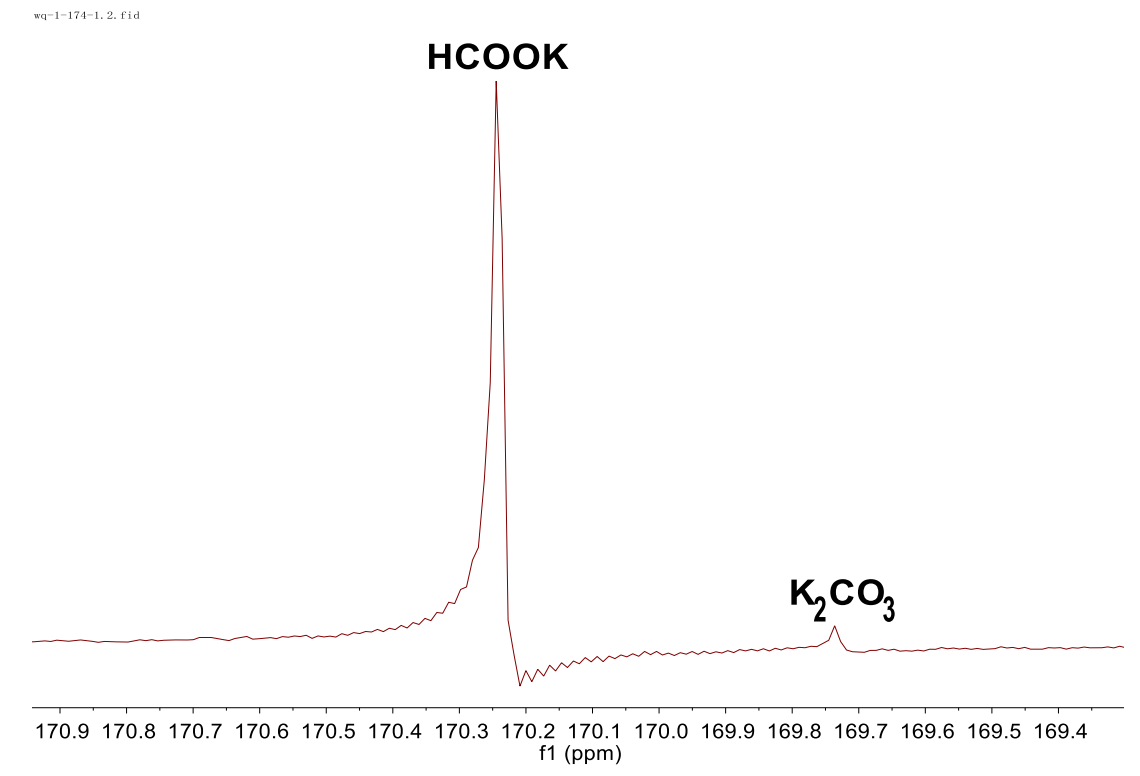

Figure S4. The ${ }^{13} \mathrm{C}-\mathrm{NMR}$ of G-III in basic $(8 \mathrm{M} \mathrm{KOH}), \mathrm{MeOH} / \mathrm{H}_{2} \mathrm{O}(9: 1,10 \mathrm{~mL})$ solutions after methanol reforming reaction for 1.5 hours.

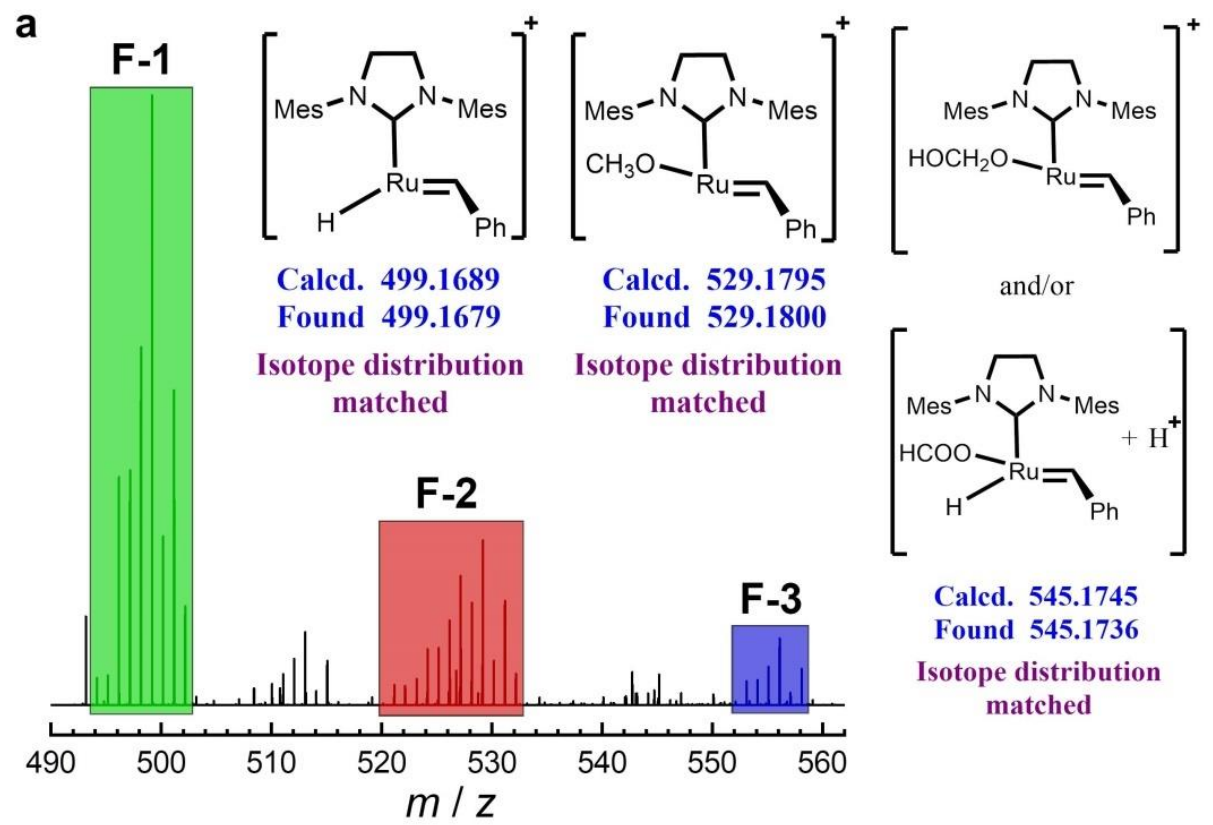

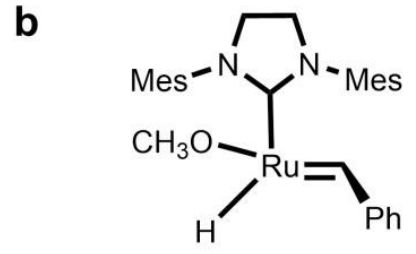

I-1

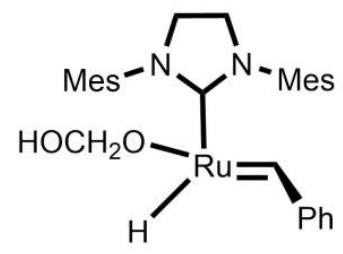

I-2<smiles>[H]/[R](=C\Pc1ccccc1)C1N(C)CCN1C</smiles>

I-3

Figure S5. a HRESI-MS of the reaction mixture using G-III as catalyst. Insert: the key species structures of F-1 (left), F-2 (right, top), and F-3 (right, bottom). b Proposed reaction intermediates based on the HRESI-MS data. 


\section{Control experiment}

The common presence of the alkylidene moiety in the proposed complex intermediates suggests its essentiality for the observed catalysis. To verify this conjecture, a control experiment was carried out at room temperature for 7 days (Supplementary Scheme 1) by using an identical reaction mixture to the one used for entry 3 (Table 1). The NMR spectra of this reaction mixture indicate the absence of alkylidene-containing species, thus suggesting the decomposition of G-III under the conditions used. As anticipated, no evolution of $\mathrm{H}_{2}$ was observed with heating of this mixture at $100{ }^{\circ} \mathrm{C}$. In stark contrast and as already observed, quickly heating the same reaction mixture led to the instant production of $\mathrm{H}_{2}$. These observations clearly indicate the vital role of the alkylidene ligand in the catalytically active species; once entering the catalytic cycle, such species, whose key component fragments have been identified in the HRESI-MS spectra, can survive the decomposition observed in the control experiment. Other decomposed species from G-I and G-II were prepared according to the literature. ${ }^{1}$

Scheme S1. Catalytic performance comparison of (top) the alkylidene moietycontaining and (bottom) carbene-free species based on G-III.

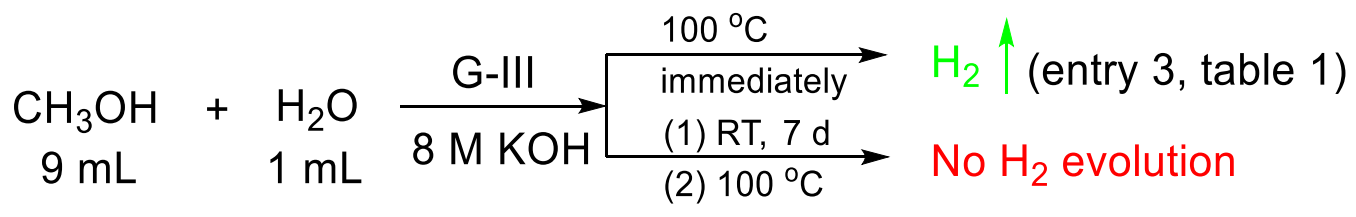




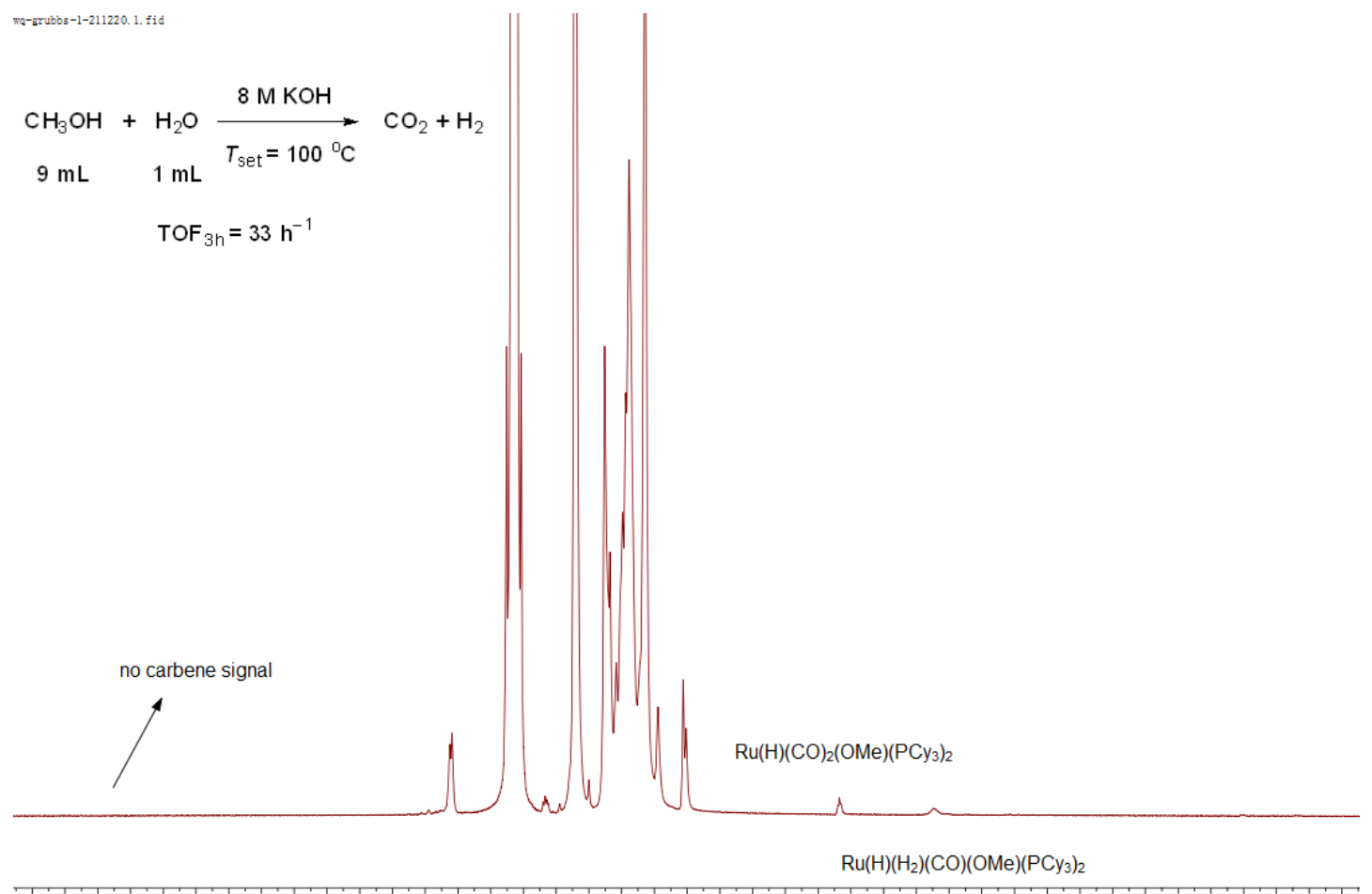

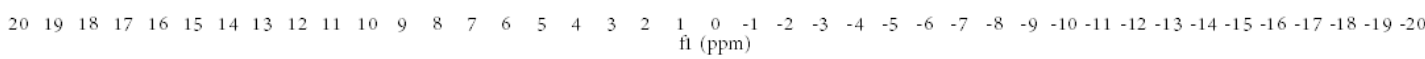

Figure S6. The ${ }^{1} \mathrm{H}$ NMR of the decomposition complex from G-I applied for APMR reaction.

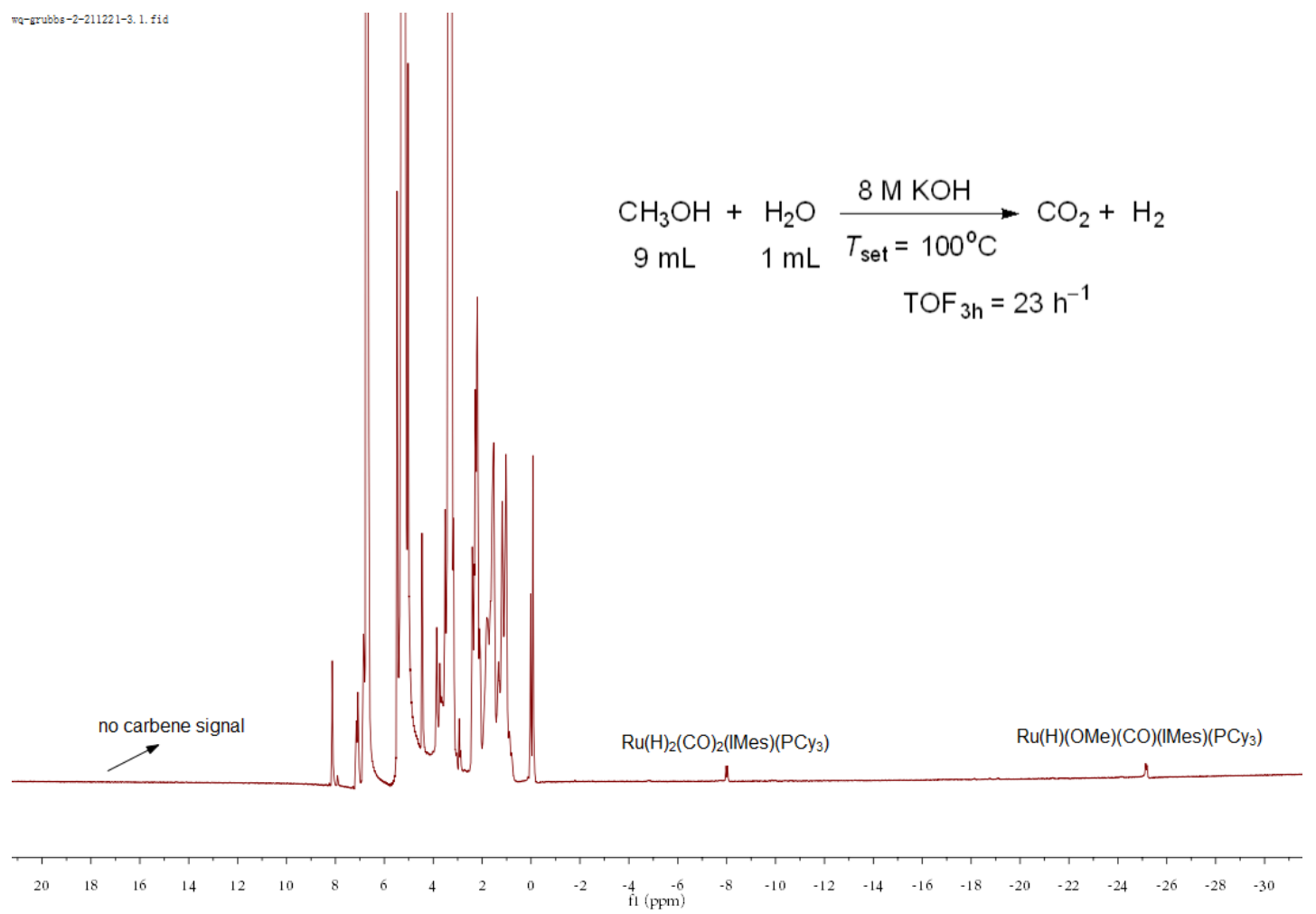

Figure S7. The ${ }^{1} \mathrm{H}$ NMR of the decomposition complex from G-II applied for APMR reaction. 
Scheme S2. The equations of dehydrogenation reactions using other substrates or the so-called liquid organic hydrogen carriers. ${ }^{2-4}$

$$
\begin{gathered}
\widehat{\mathrm{OH}}+\mathrm{H}_{2} \mathrm{O} \stackrel{\mathrm{G}-\text { III, } \mathrm{KOH}, 100^{\circ} \mathrm{C}}{\longrightarrow} \mathrm{CH}_{3} \mathrm{COOK}+\mathrm{H}_{2} \\
\mathrm{HCOOH} \stackrel{\mathrm{G}-I I I, \mathrm{HCOOK}, 100^{\circ} \mathrm{C}}{\longrightarrow} \mathrm{CO}_{2}+\mathrm{H}_{2}
\end{gathered}
$$

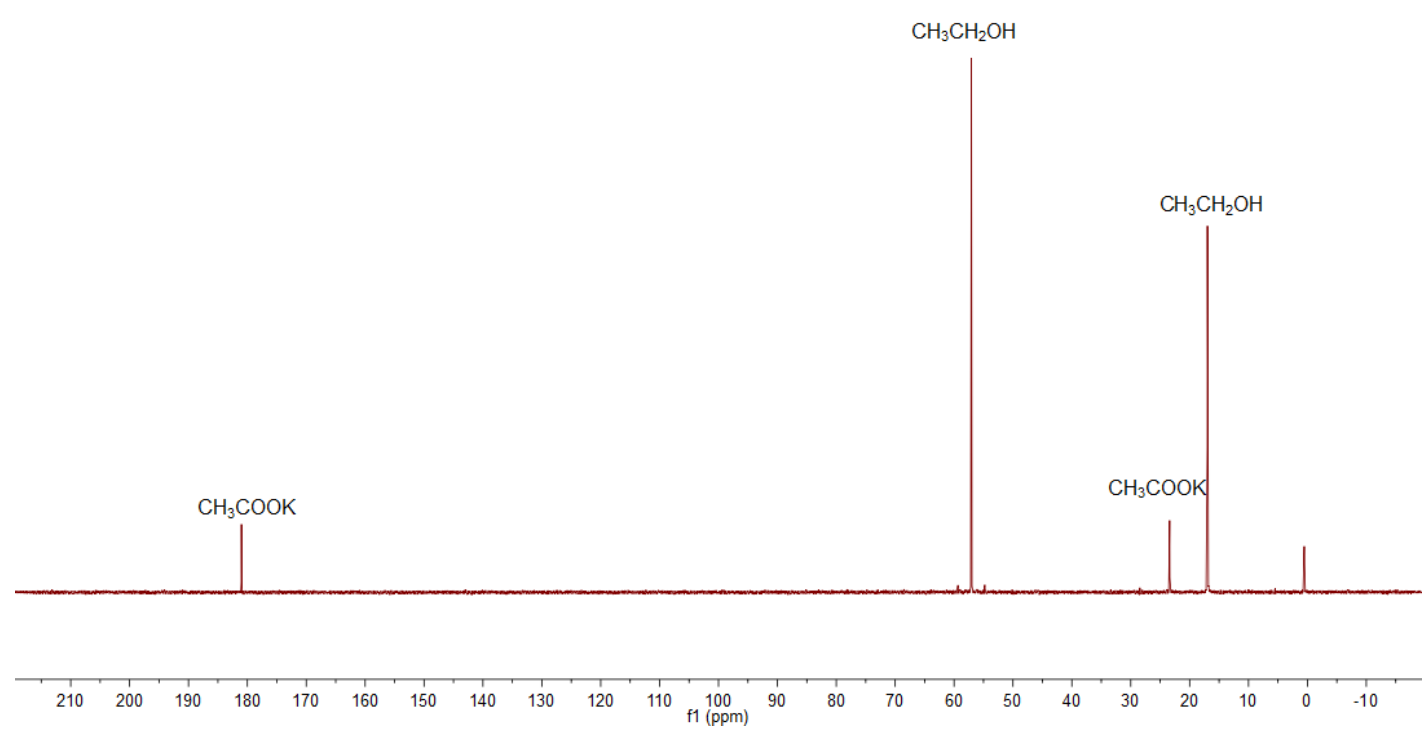

Figure S8. The ${ }^{13} \mathrm{C}$ NMR spectrum of the product solution from the reaction of G-III $(5 \mu \mathrm{mol})$ and $8 \mathrm{M} \mathrm{KOH}$ in $\mathrm{EtOH} / \mathrm{H}_{2} \mathrm{O}(v / v 9: 1,5 \mathrm{~mL})$ mixture; the thermostat was set at $100{ }^{\circ} \mathrm{C}$. 


\section{DFT results}

\section{Computational details}

The reaction mechanism of the dehydrogenation of methanol, which were reported to be likely the slowest step for the methanol reforming process and undergo the same type of the mechanism for the subsequent two dehydrogenation steps, ${ }^{5}$ was systematically carried out using the third-generation Grubbs catalyst (giving the highest TOF) and DFT methods (mainly the M06-L method) in this study. All DFT calculations were performed using the Gaussian 09 program (except for the single-point solvent calculations by implicit CPCM solvent model performed by the Gaussian 16 program). ${ }^{6-7}$ 3-bromopyridine ligand was generally replaced by pyridine in our calculations, while 3-bromopyridine ligand was also used for the most critical steps. 3bromopyridine ligand gave slightly higher relative free energies only. M06-L method ${ }^{11}$ was employed to fully optimize the reactants, intermediates, products and transition states (TSs) in gas phase. The $6-31 \mathrm{G}^{* *}$ basis set was used for $\mathrm{H}, \mathrm{C}, \mathrm{N}$, and $\mathrm{O}$ atoms, while the SDD basis set and its effective-core potential were used for the $\mathrm{Ru}$ and $\mathrm{Br}$ atoms. Frequency calculations were then carried out to confirm transition states with one imaginary frequency and minima without imaginary frequency. Solvent effects (methanol) were also included through implicit PCM and CPCM solvent models ${ }^{8-10}$ by carrying single-point solvent calculations on the optimized geometries in gas phase. Both implicit solvent models gave almost same results (in the absence of the below standard state correction) and, thus, the PCM results are only discussed. Single-point calculations in solution by several DFT functionals (B3LYP-D3, M06, PBE0-D3 and $\varpi B 97 X D)^{11-16}$ were also performed to examine effect of the functionals on the energetic profiles. These DFT methods all supported the key mechanistic pathways and conclusion. All the structural Figures were generated using CLYview. ${ }^{17}$ The standard state correction was applied for the computed relative free energy of all species (1.9 $\mathrm{kcal} / \mathrm{mol}$ per molecule) except methanol solvent $(24 \mathrm{M}$, which contributes to 3.8 $\mathrm{kcal} / \mathrm{mol}$ correction per methanol molecule) and $\mathrm{H}_{2} \mathrm{O}$ molecules (55 M, which contributes to $4.3 \mathrm{kcal} / \mathrm{mol}$ correction per $\mathrm{H}_{2} \mathrm{O}$ molecule) in solution by the abovementioned PCM DFT methods. ${ }^{18-19}$ These relative free energies with such free energy correction are given in Figs. S9-S24 as well as used for our discussion unless stated otherwise.

Our DFT calculations suggest that the Ru(II) monohydride monomethoxide species with coordination of two methanol solvent molecules (3) is proposed mainly responsible for the $\mathrm{H}_{2}$ formation via 6-m-r solvent-assisted metathesis TS, TS 3-4. While, the $\mathrm{Ru}$ (II) dihydride species require much higher barriers for the $\mathrm{H}_{2}$ formation. Since TS3-4 (the most favorable $\mathrm{H}_{2}$ formation pathway) and TS5-6 (the formation of $\mathrm{Ru}(\mathrm{II}$ ) dihydride species pathway) are computed to have comparable energies and the latter ones cannot form $\mathrm{H}_{2}$, these pathways could compete, as well as the $\mathrm{Ru}$ (II) dihydride species could undergo a reversible reaction to re-generate the active $\mathrm{Ru}(\mathrm{II})$ monohydride monomethoxide species or further undergo the observed dimerization degradation process. In addition, attempts to locate reductive elimination products 
$(\mathrm{Ru}(0)$-dihydrogen) and transition states from the $\mathrm{Ru}(\mathrm{II})$ dihydride species essentially failed and the optimizations gave $\mathrm{Ru}(\mathrm{II})$ dihydride species only except with coordination of $\mathrm{H}_{2} \mathrm{CO}$ (leading to very unstable formal $\mathrm{Ru}(0)$-dihydrogen intermediates 22a, 22b and 22c, 39.0-69.5 kcal/mol in gas phase, see Figure S13).

Moreover, intermediate $\mathbf{6}$ (coordination with one $\mathrm{H}_{2} \mathrm{CO}$ ) is computed to be the most stable form of the $\mathrm{Ru}$ (II) dihydride species. Whereas, intermediates 7-A, 7-B and 7-C (coordination with one or two methanol) are less stable. As the observed Ru(II) dihydride species with coordination one methanol and one $\mathrm{K}$ cation by our ESI-MS study, low boiling-point $\mathrm{H}_{2} \mathrm{CO}$ (about $-19{ }^{\circ} \mathrm{C}$ ) is proposed to dissociate and evaporate from intermediate 6 and, then, the resultant species could coordinate with $\mathrm{MeOH}$ (boiling point: $\sim 65^{\circ} \mathrm{C}$ ) solvent molecule and $\mathrm{K}$ cation under the gas-phase ESI-MS conditions. In addition, our calculations suggest that another observed intermediate by the ESI-MS should be Ru(II) hydride dihydrogen alkyl intermediate $\mathbf{2 4}$ (Figure S21), which is much stable than the related $\mathrm{Ru}(\mathrm{II})$ dihydride dihydrogen carbene intermediates (23a-23d).

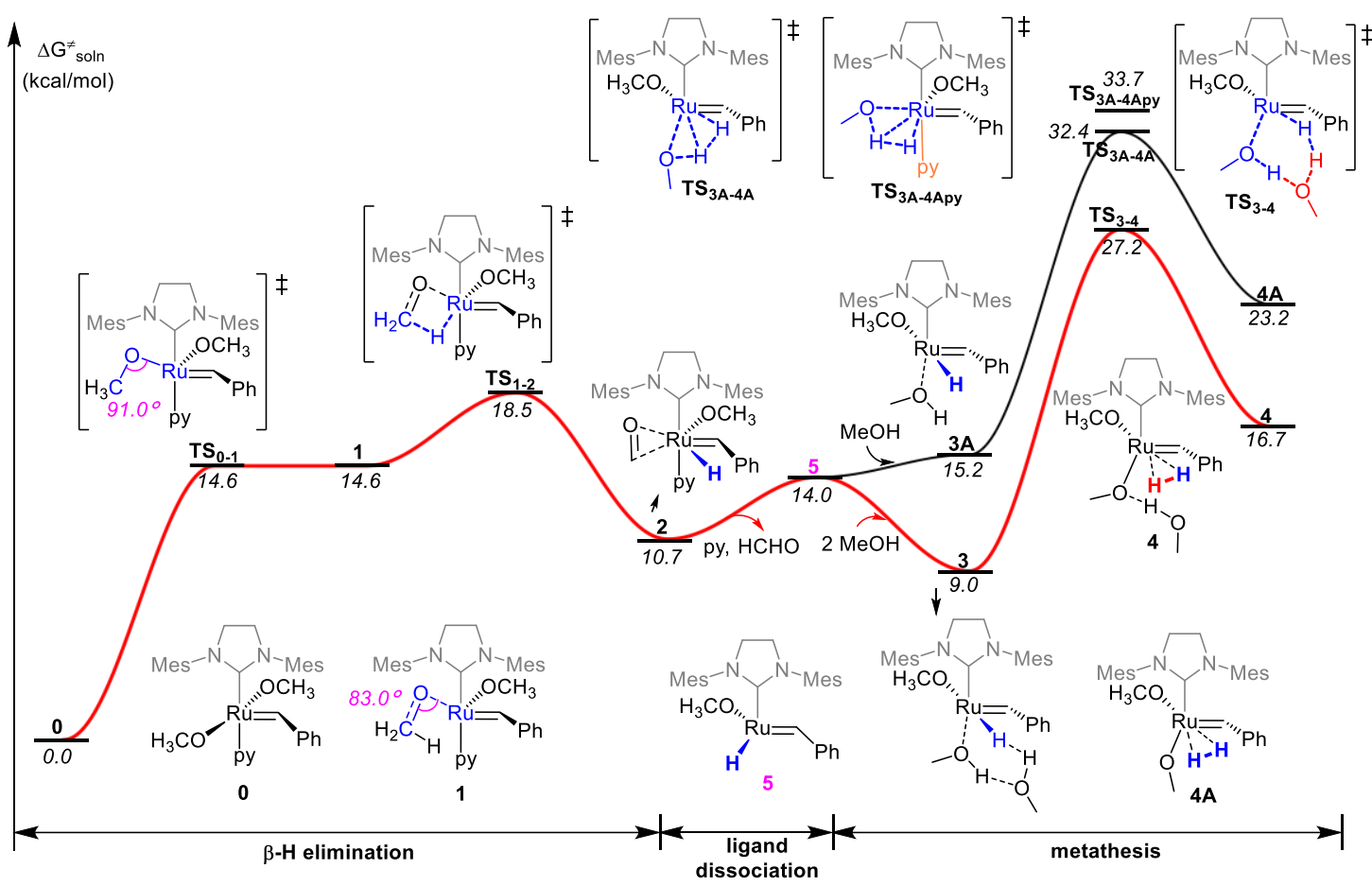

Figure S9. Free energy profile for the most favorable pathway (1) of the $\mathrm{Ru}(\mathrm{II})$ catalyzed aqueous methanol reforming reaction in solution by the PCM M06-L//M06L method. This pathway with less favorable conformations is given in Figure S16. 


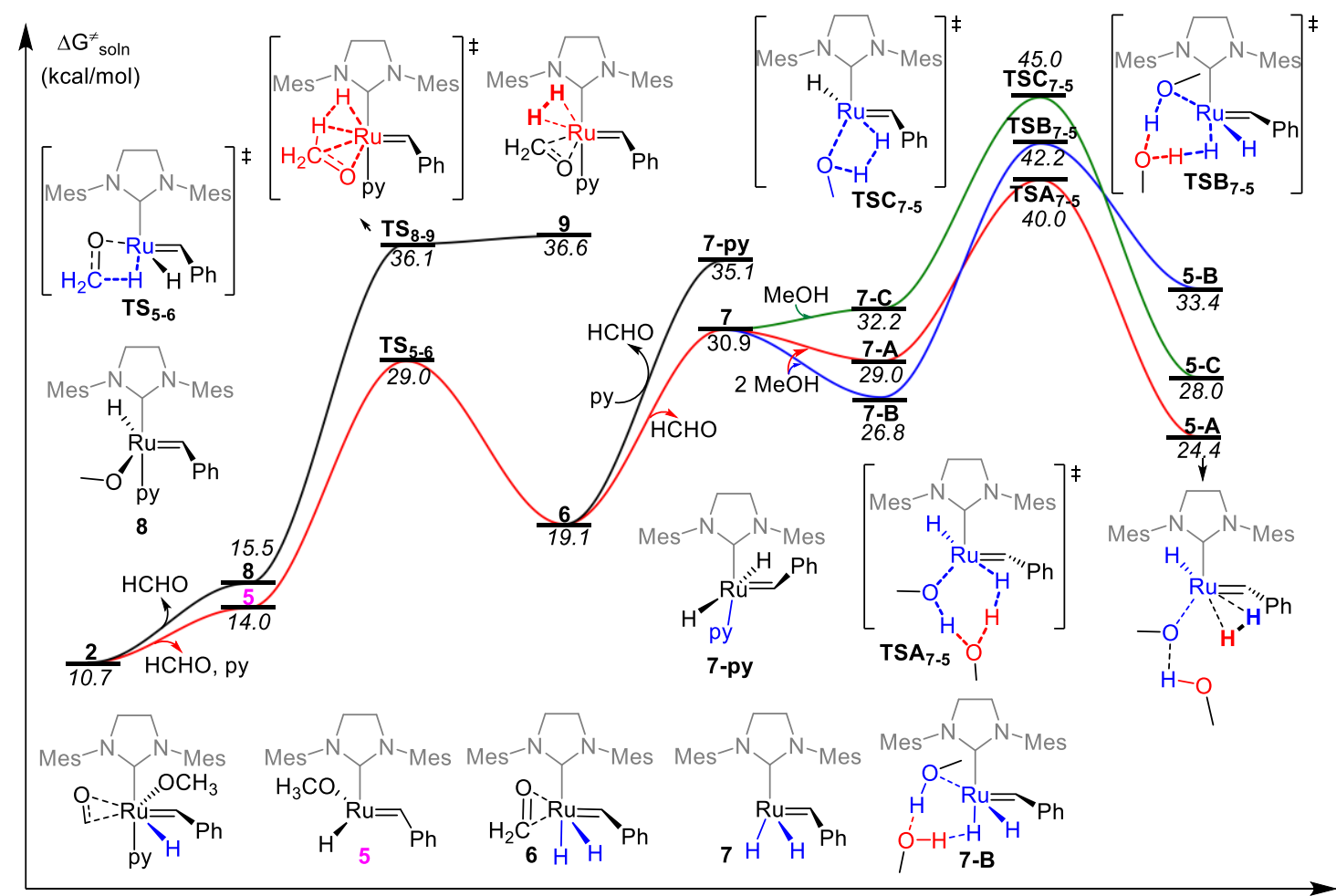

Figure S10. Free energy profile for the pathway (2) involving the formation of $\mathrm{Ru}(\mathrm{II})-$ dihydride species in solution by the PCM M06-L//M06-L method. TS8-9 was computed to be higher in electronic energy (without ZPE correction) than 9 by $0.7 \mathrm{kcal} / \mathrm{mol}$ in gas phase, but becomes lower in energy with inclusion of the ZPE correction. 


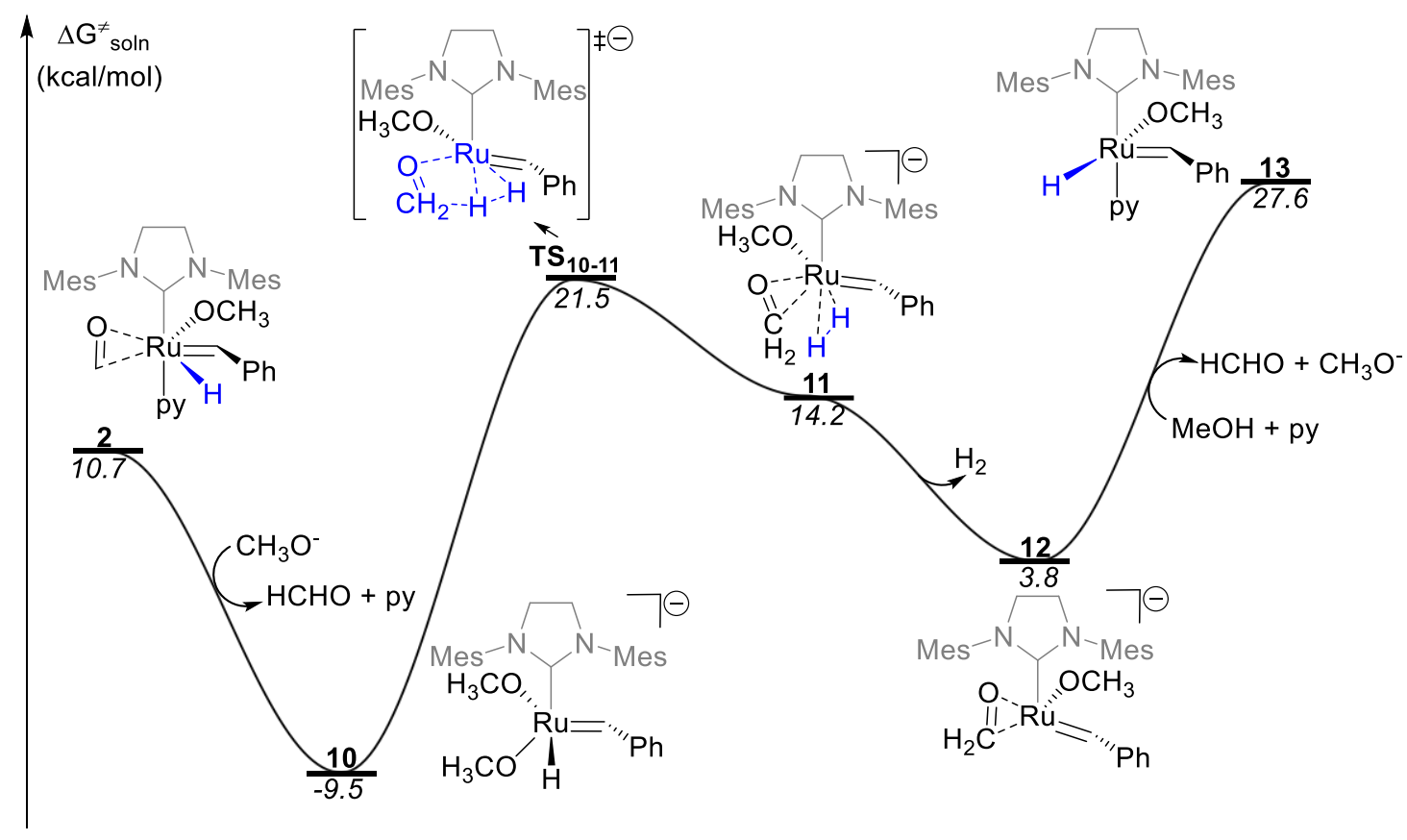

Figure S11. Free energy profile for the anionic pathway (3) of the $\mathrm{Ru}(\mathrm{II})$-catalyzed aqueous methanol reforming reaction in solution by the PCM M06-L//M06-L method. 


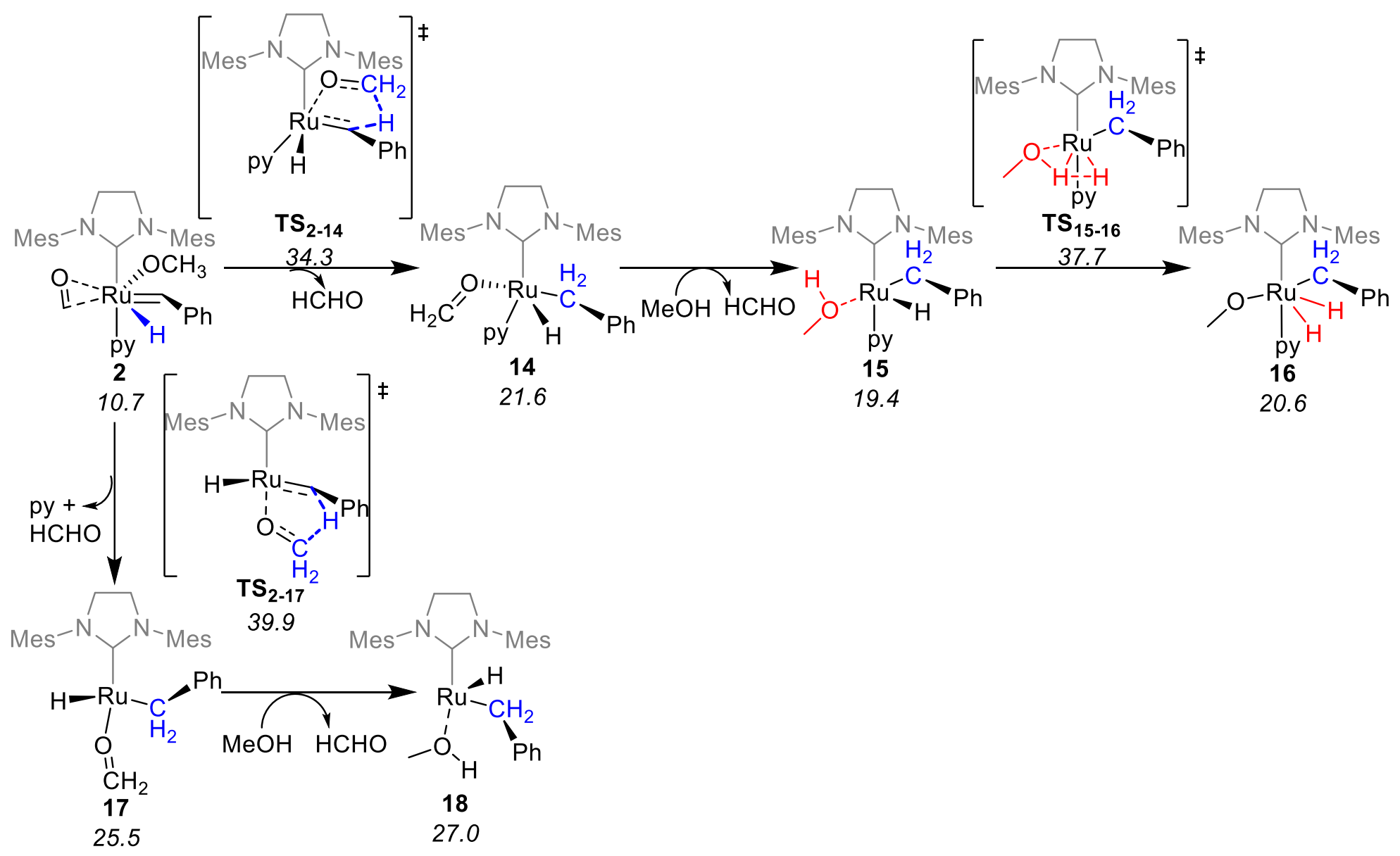

Figure S12. Free energy profile for pathway 4 of the Ru(II)-catalyzed aqueous methanol reforming reaction via the Ru-alkyl species in solution by the PCM M06-L//M06-L method. 


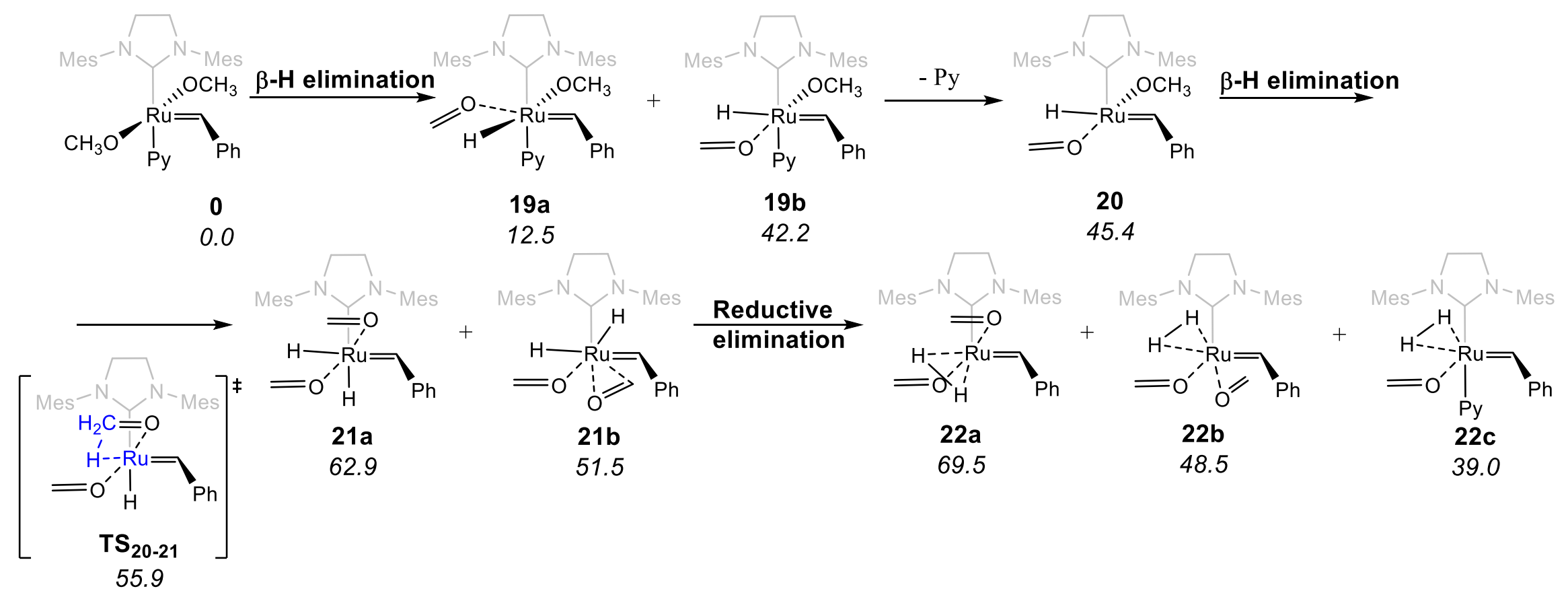

Figure S13. Free energy profile for the higher-energy $\beta-\mathrm{H}$ elimination pathways in gas phase by the M06-L method. 


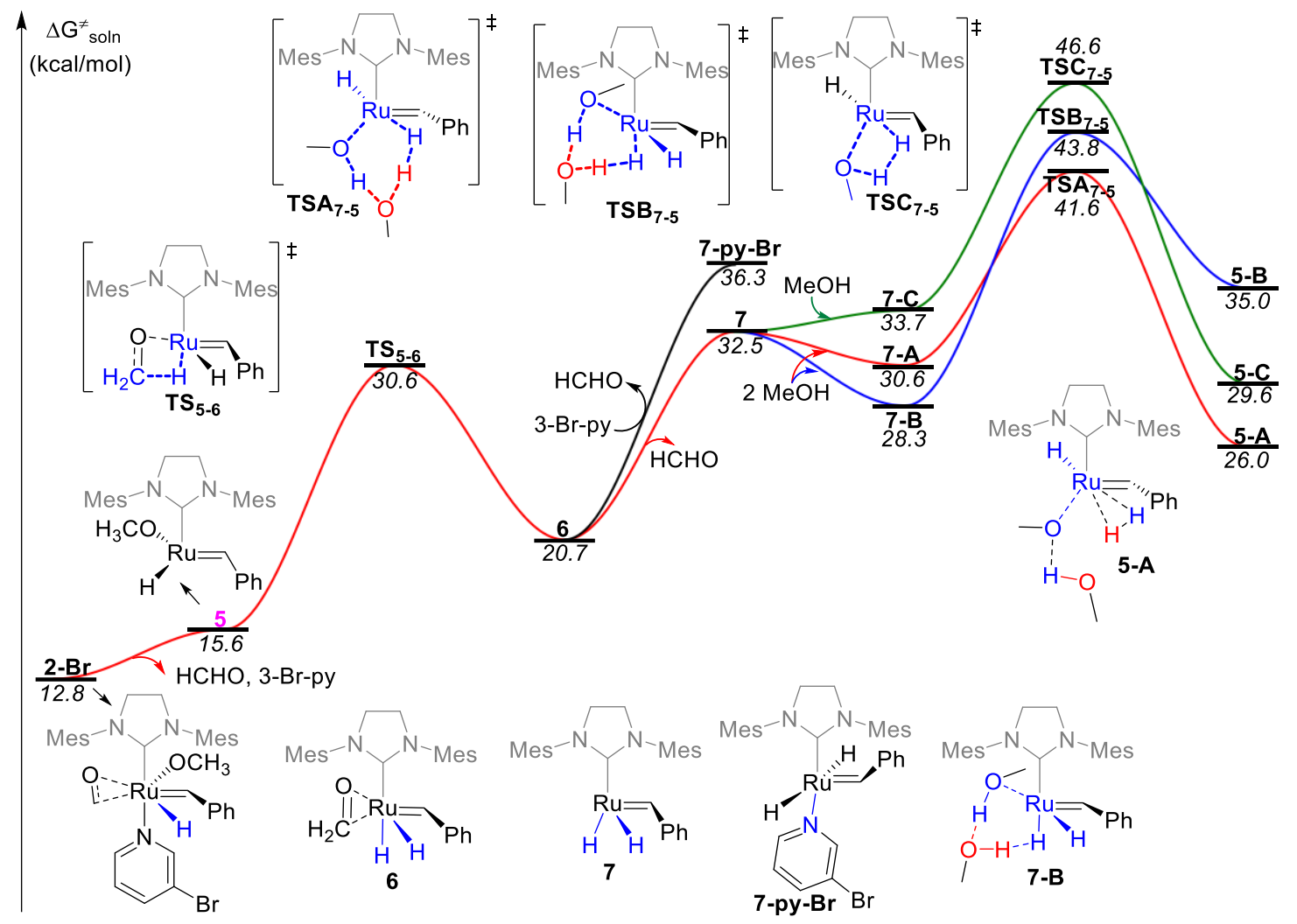

Figure S14. Free energy profile for the pathway (2) involving the formation of $\mathrm{Ru}(\mathrm{II})$ dihydride species in solution using the 3-Br-pyridine as the ligand (instead of pyridine) by the PCM M06-L//M06-L method. 
(a) The most favorable hydrogen evolution process from $\mathrm{MeOH}$
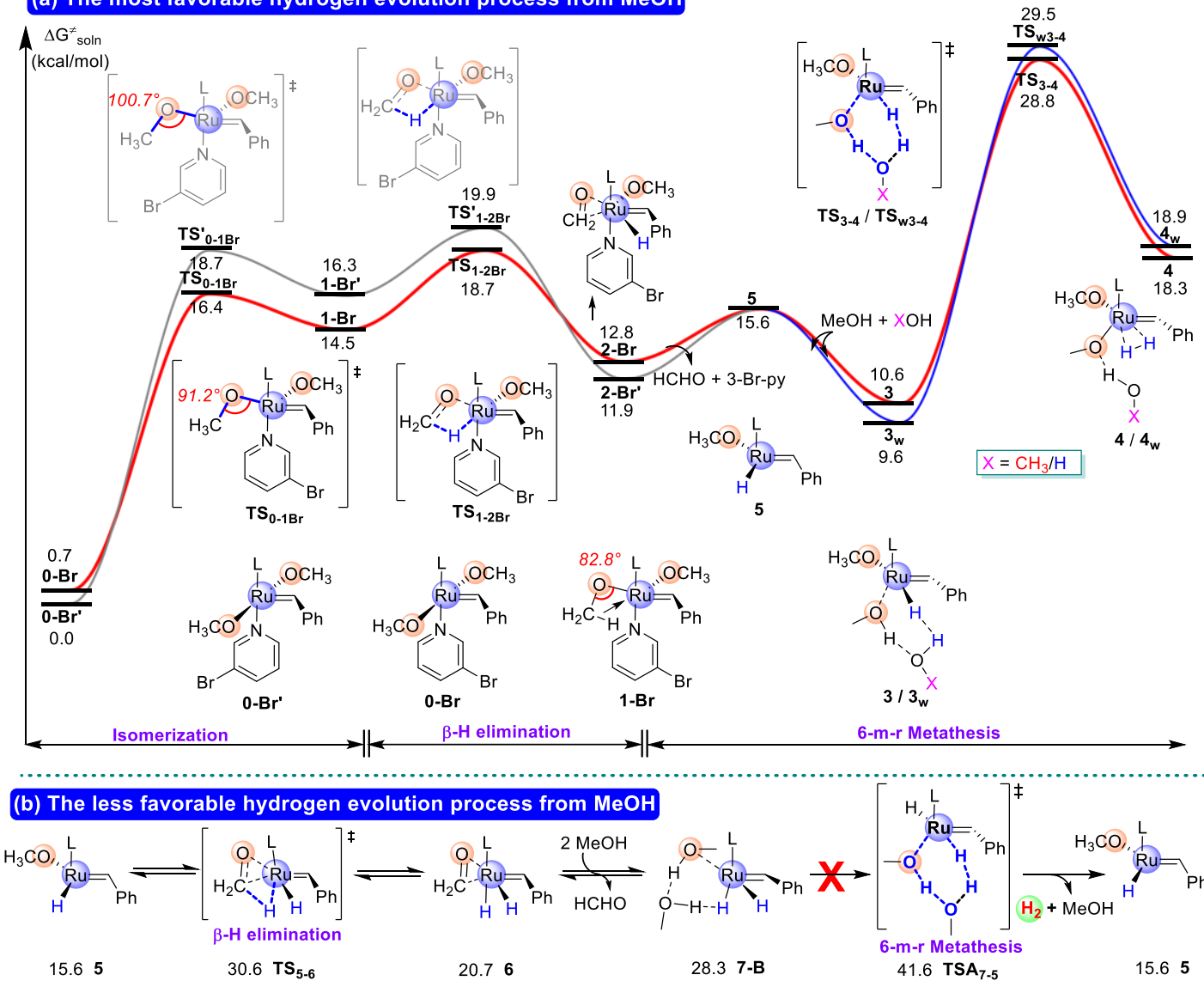

\section{(c) Hydrogen evolution processes from $\mathrm{CH}_{2}(\mathrm{OH})_{2}$ and $\mathrm{HCO}_{2} \mathrm{H}$}

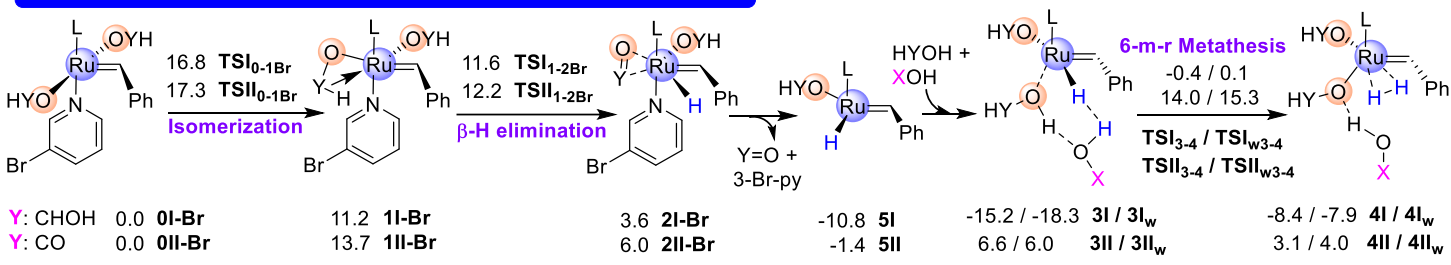

Figure S15. Free energy profile for the hydrogen evolution processes from $\mathrm{MeOH}$, $\mathrm{CH}_{2}(\mathrm{OH})_{2}$ and $\mathrm{HCO}_{2} \mathrm{H}$ in solution using the 3-Br-pyridine as the ligand (instead of pyridine) by the PCM M06-L//M06-L method. 

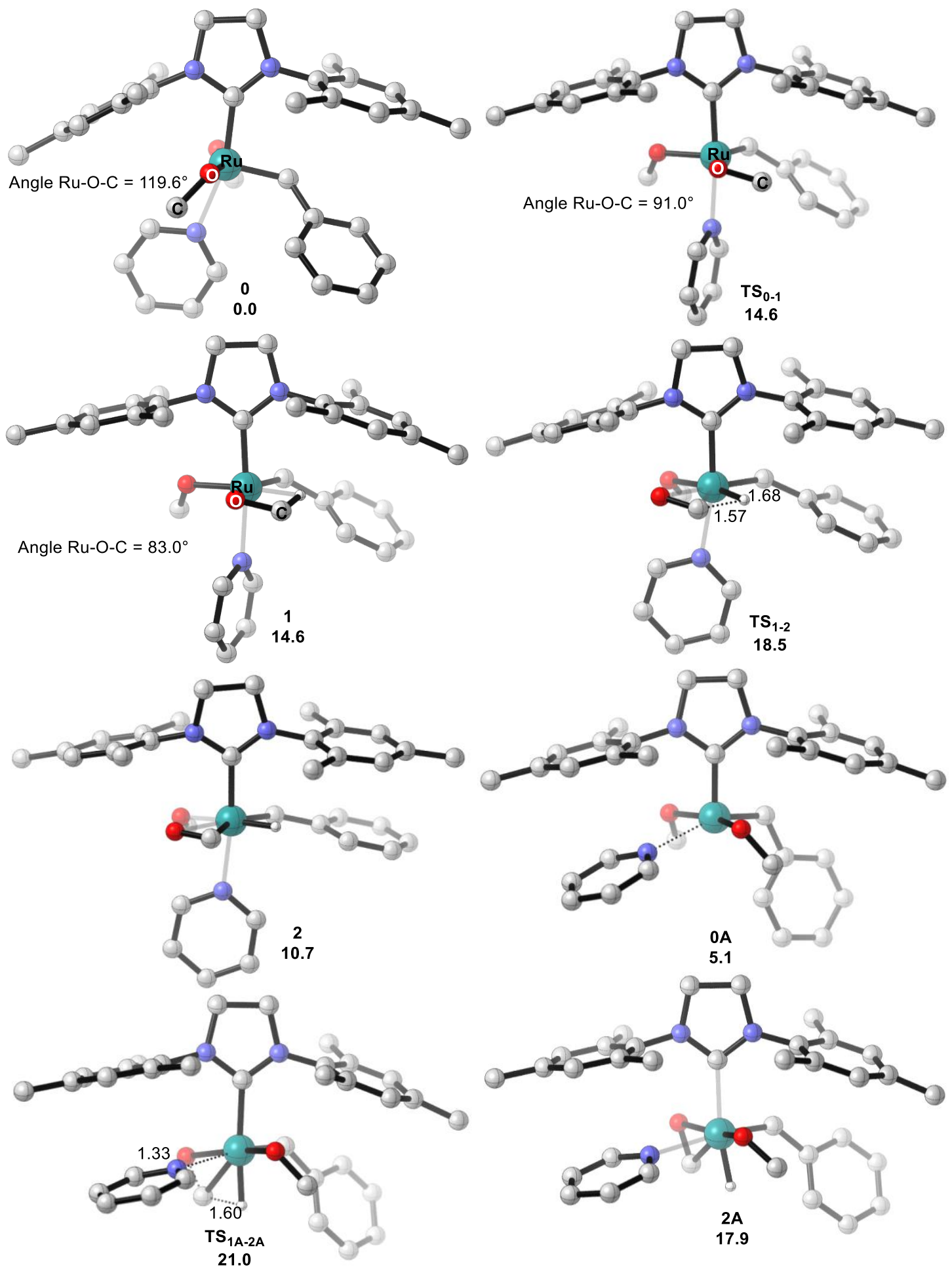

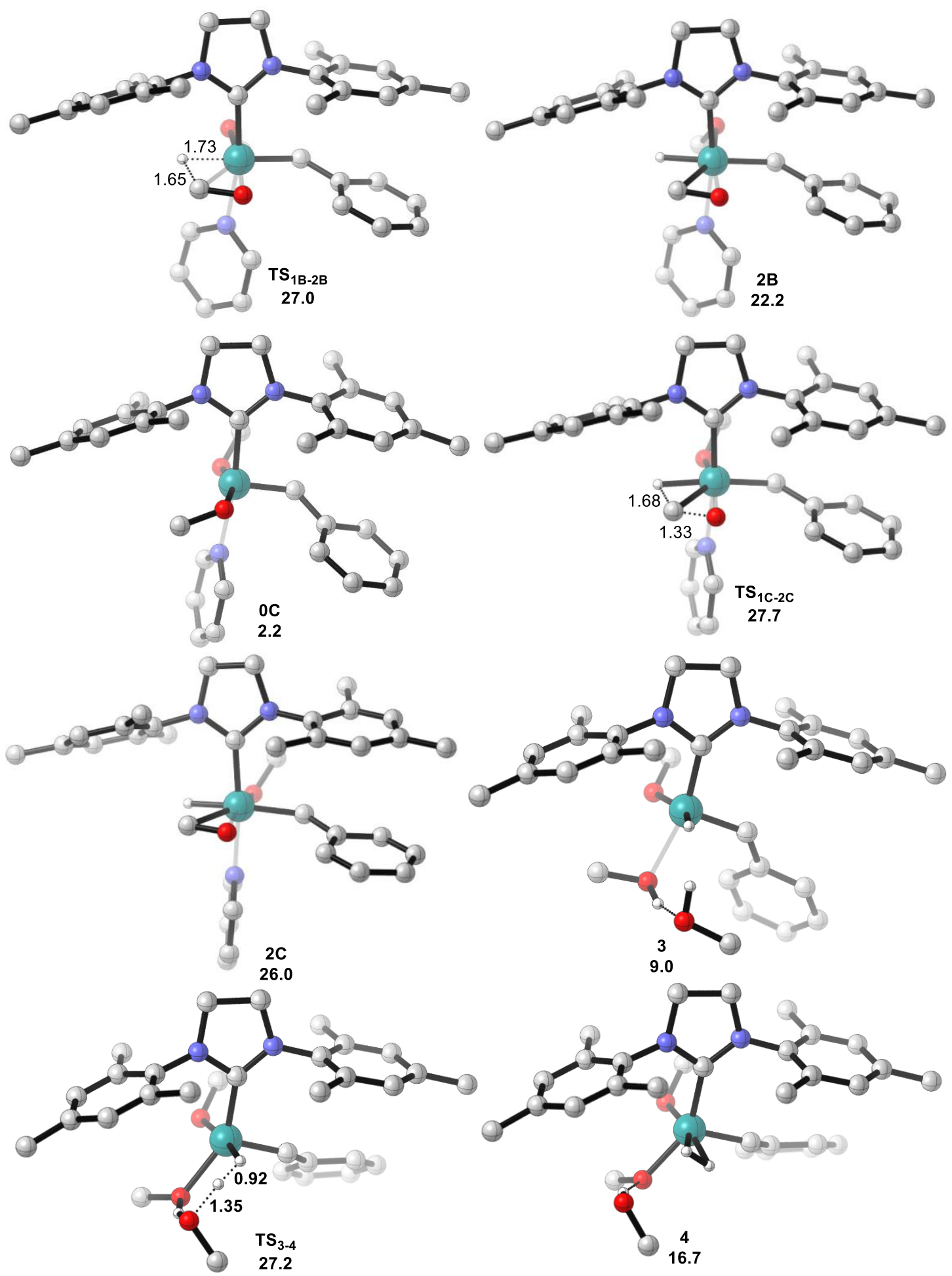

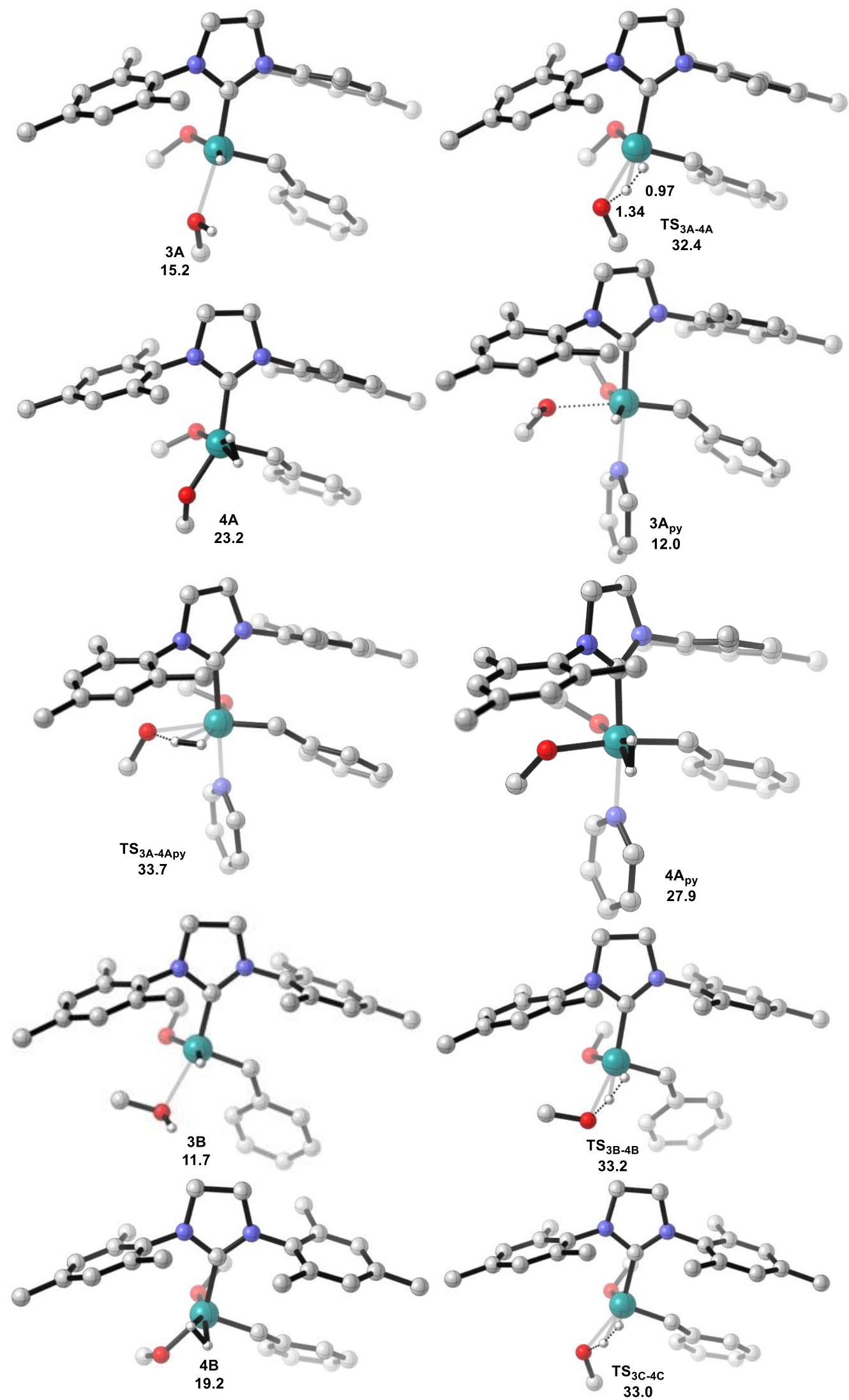

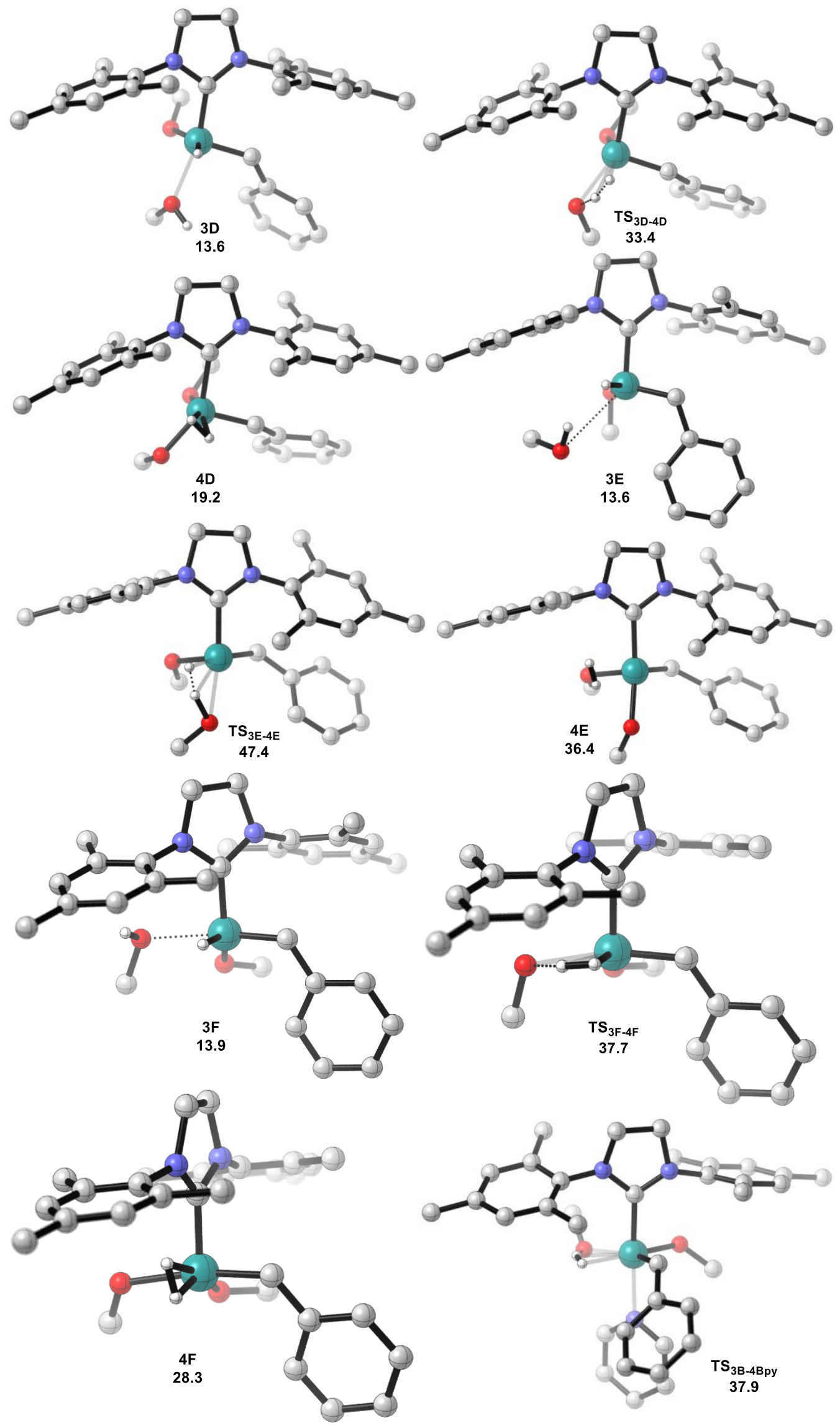

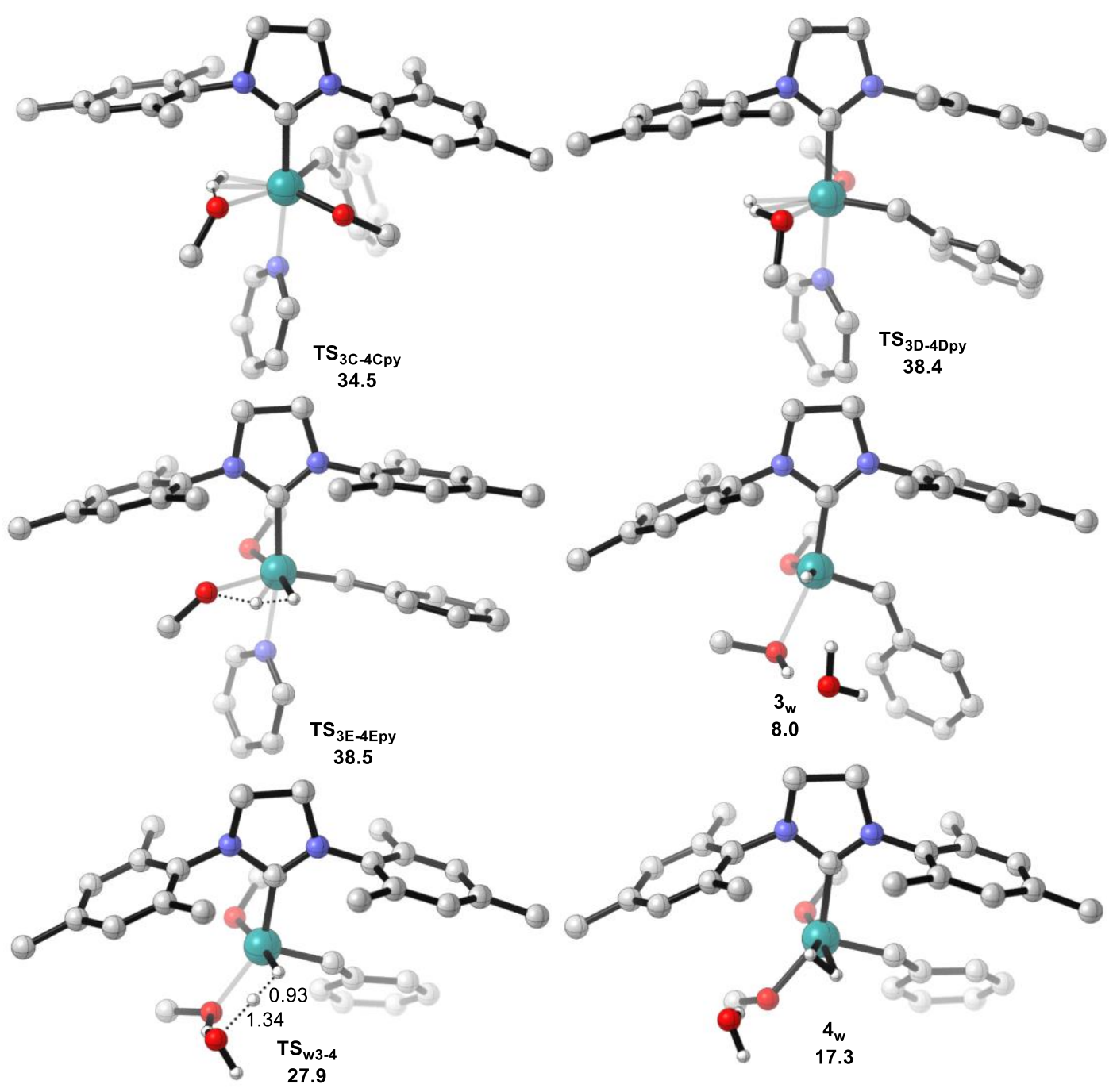

Figure S16. Calculated key structural parameters of the intermediates and transition states for the pathway 1 by the M06-L method with the key bond lengths (Ångstroms). Unimportant $\mathrm{H}$ atoms are omitted for clarity. Their relative free energies in solution by PCM M06-L//M06-L method are also given. 

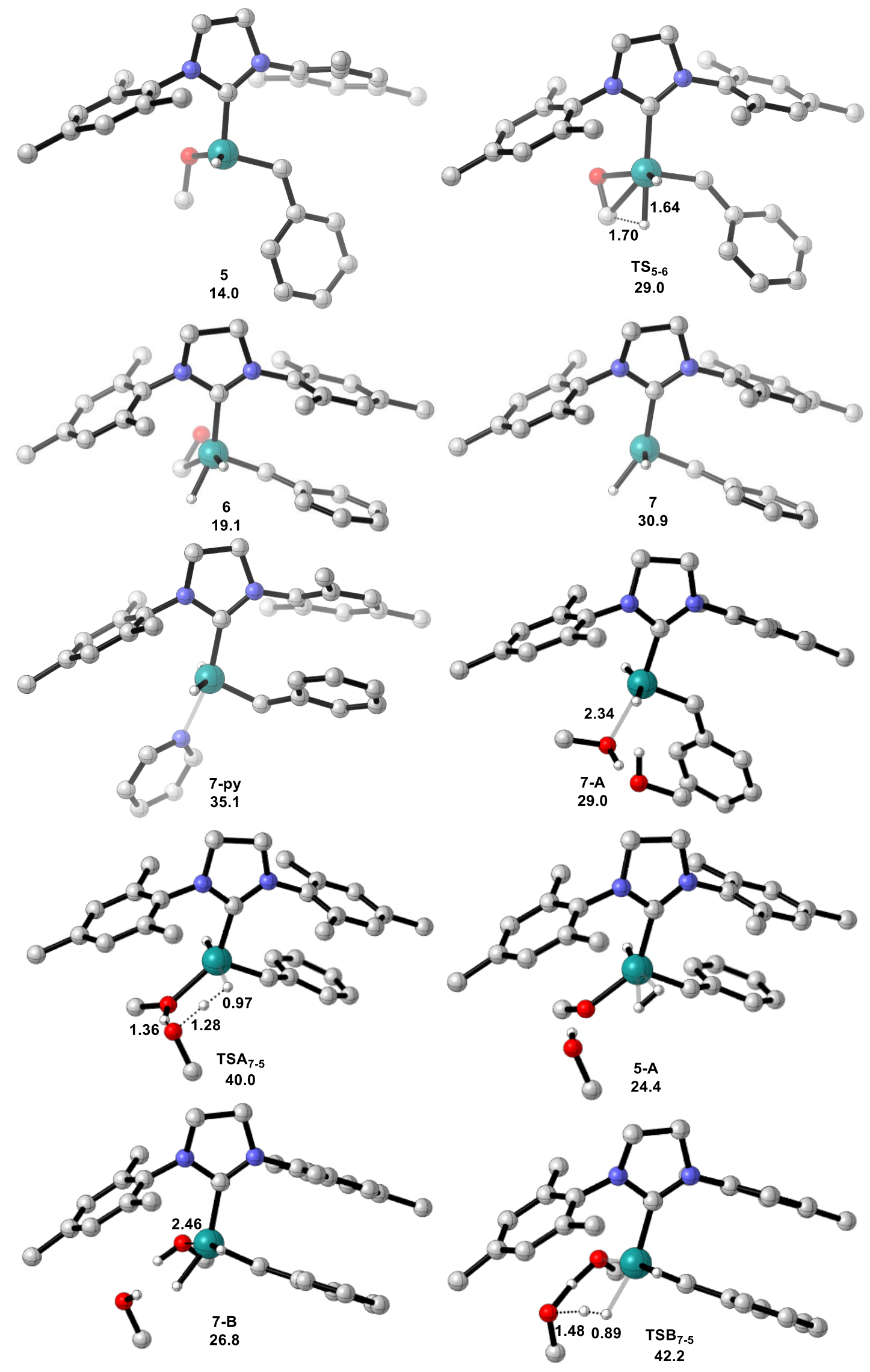

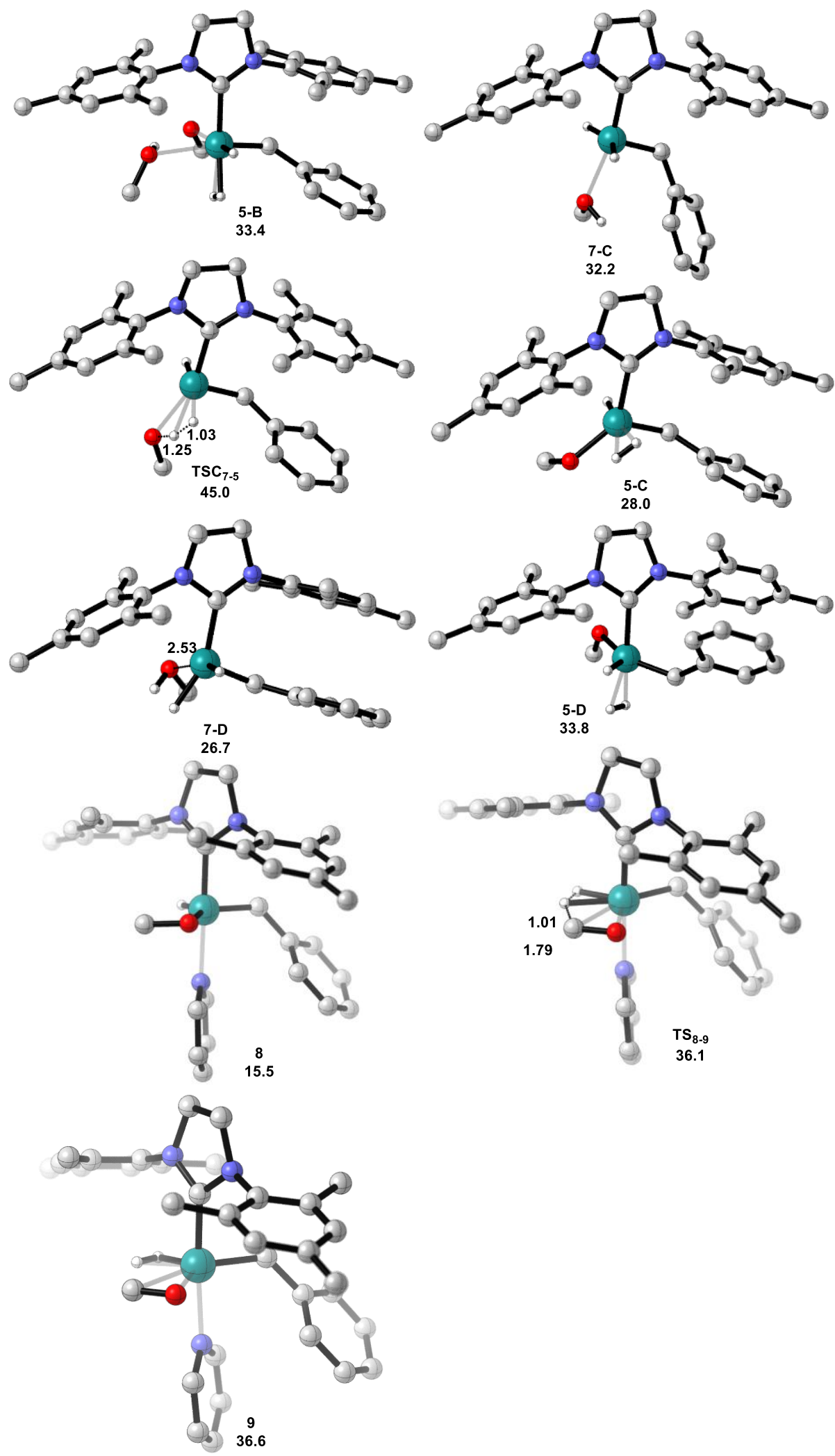

Figure S17. Calculated key structural parameters of the intermediates and transition states for the pathway 2 by the M06-L method with the key bond lengths (Ångstroms). Unimportant $\mathrm{H}$ atoms are omitted for clarity. Their relative free energies in solution by PCM M06-L//M06-L method are also given. 

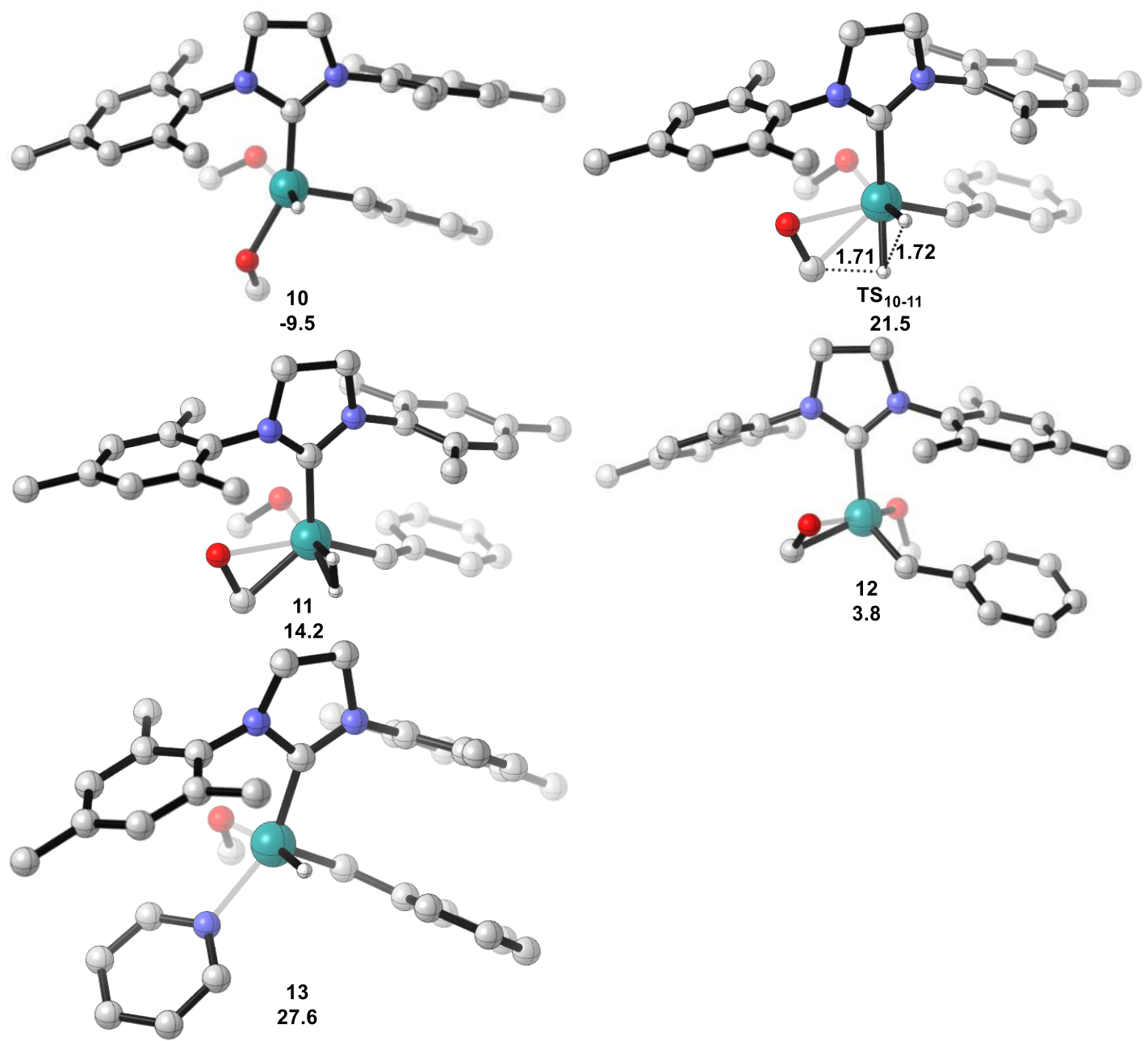

Figure S18. Calculated key structural parameters of the intermediates and transition states for the anionic pathway 3 by the M06-L method with the key bond lengths (Ångstroms). Unimportant $\mathrm{H}$ atoms are omitted for clarity. Their relative free energies in solution by PCM M06-L//M06-L method are also given. 

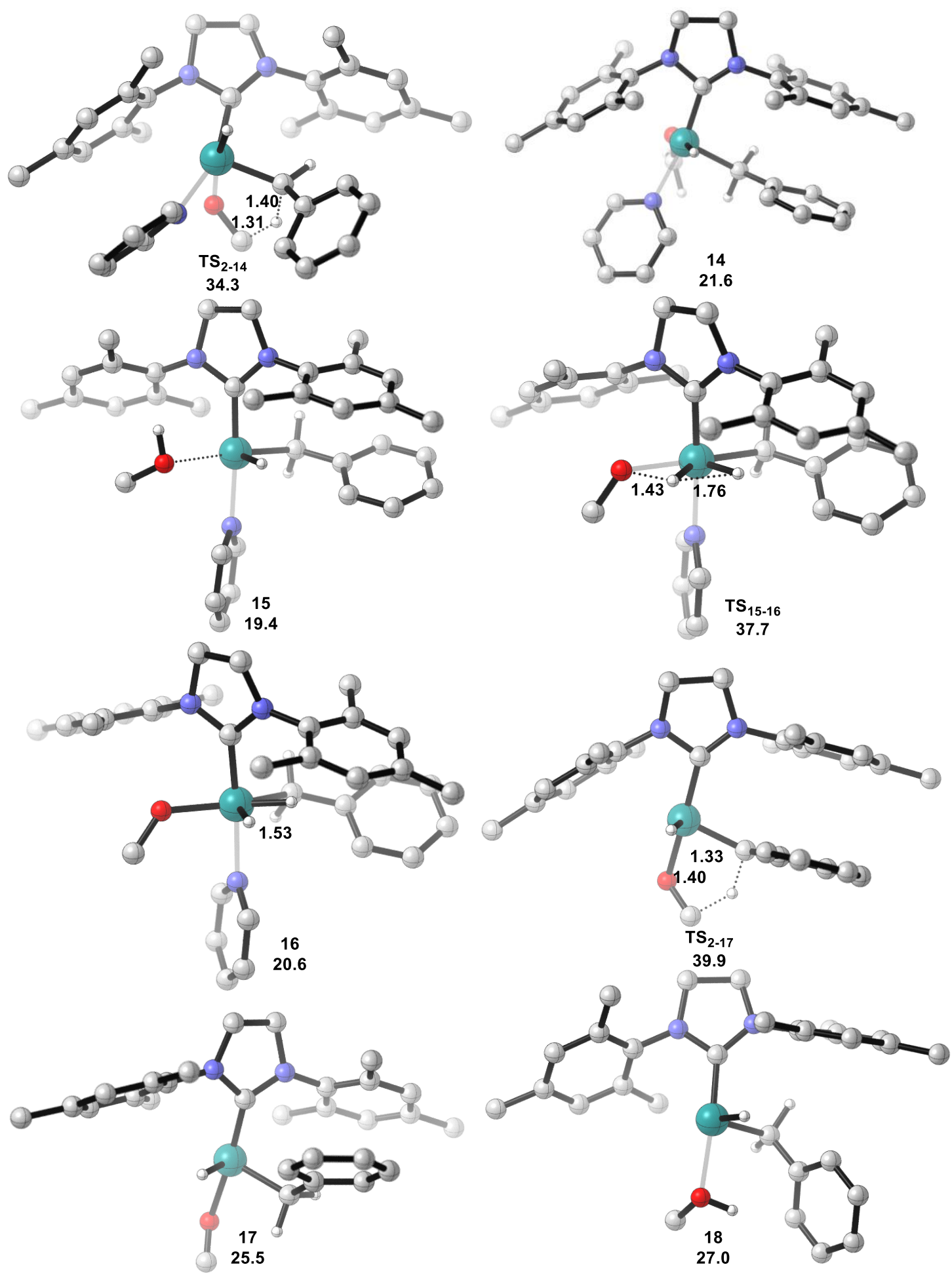

Figure S19. Calculated key structural parameters of the intermediates and transition states for the pathway 4 by the M06-L method with the key bond lengths (Ångstroms). Unimportant $\mathrm{H}$ atoms are omitted for clarity. Their relative free energies in solution by PCM M06-L//M06-L method are also given. 

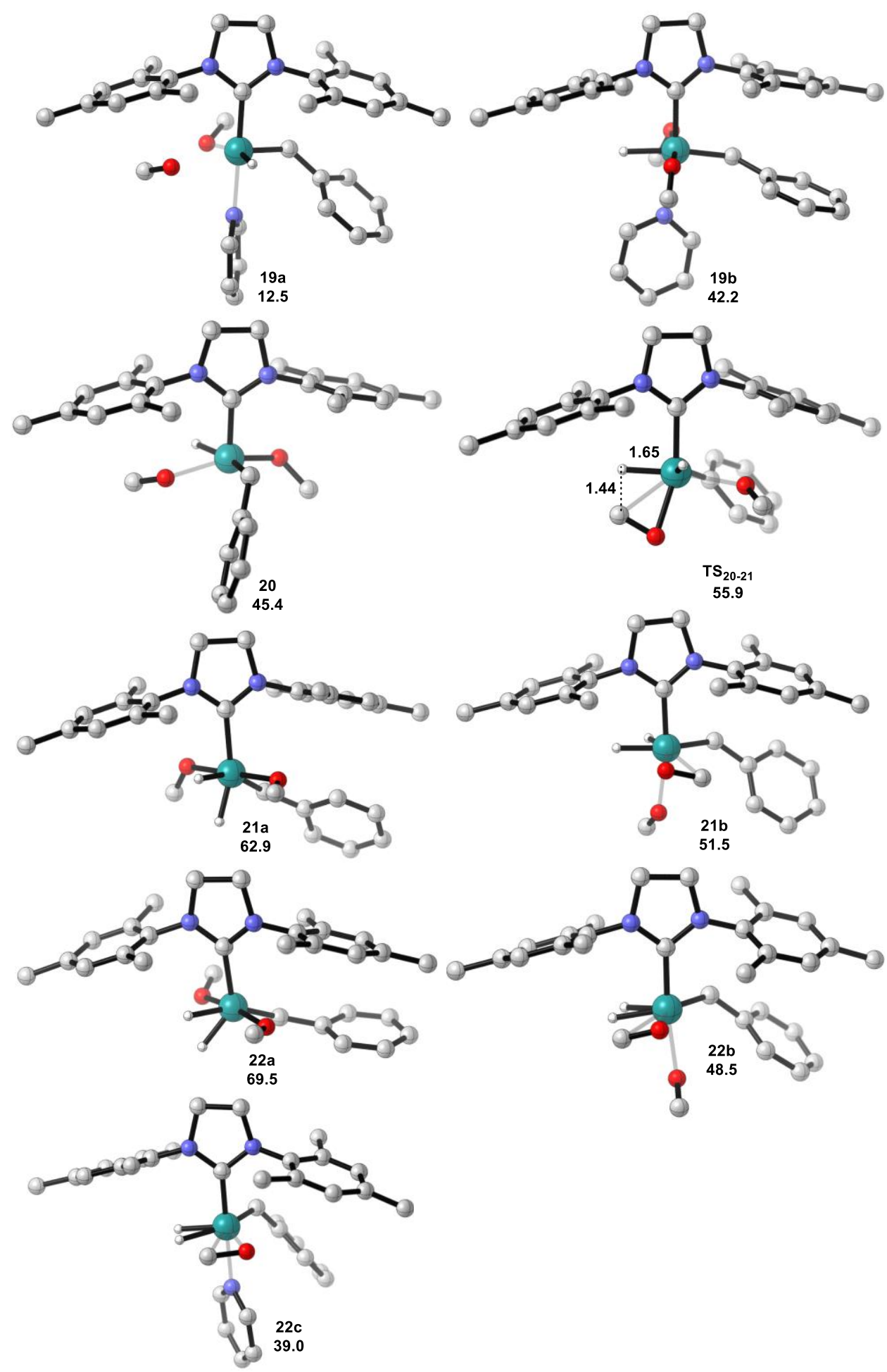

Figure S20. Calculated key structural parameters of the intermediates and transition states for the higher-energy $\beta-\mathrm{H}$ elimination pathways by the M06-L method with the key bond lengths (Ångstroms). Unimportant $\mathrm{H}$ atoms are omitted for clarity. Their relative free energies in solution by M06-L method are also given. 

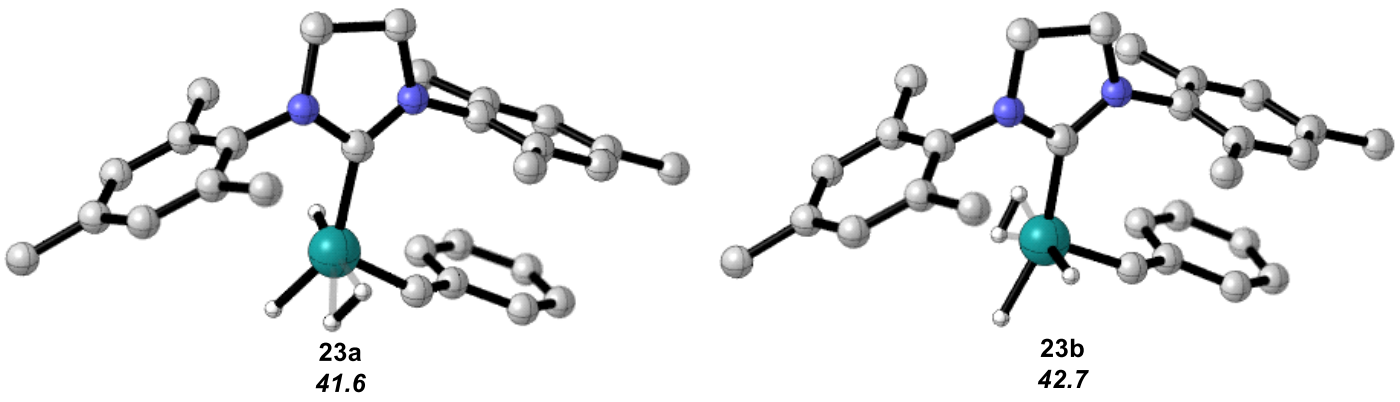

23b
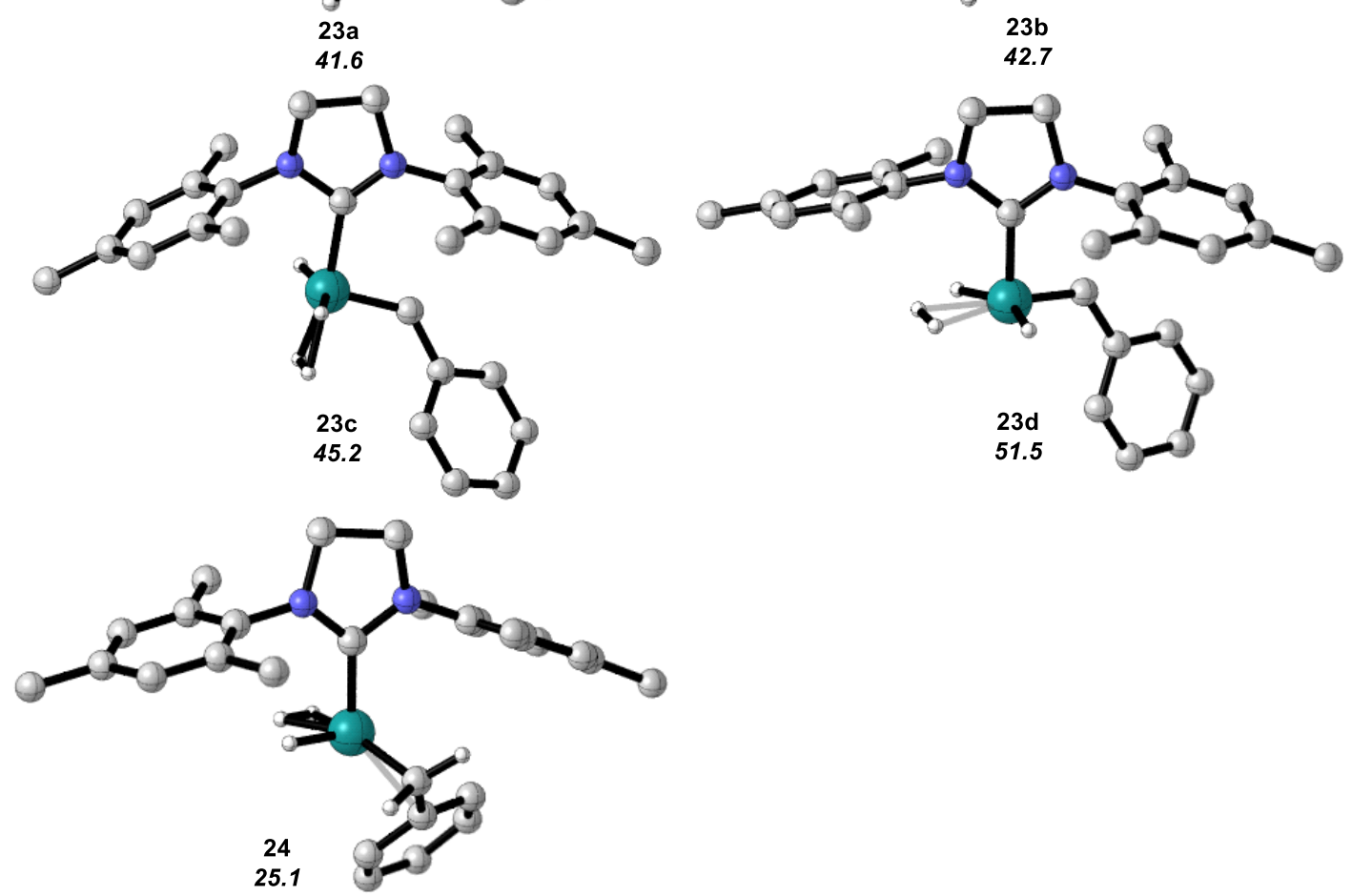

Figure S21. Calculated key structural parameters of the Ru-dihydride-dihydrogen and Ru-hydride-dihydrogen intermediates by the M06-L method. Unimportant $\mathrm{H}$ atoms are omitted for clarity. Their relative free energies in solution by PCM M06-L//M06-L method are also given. 

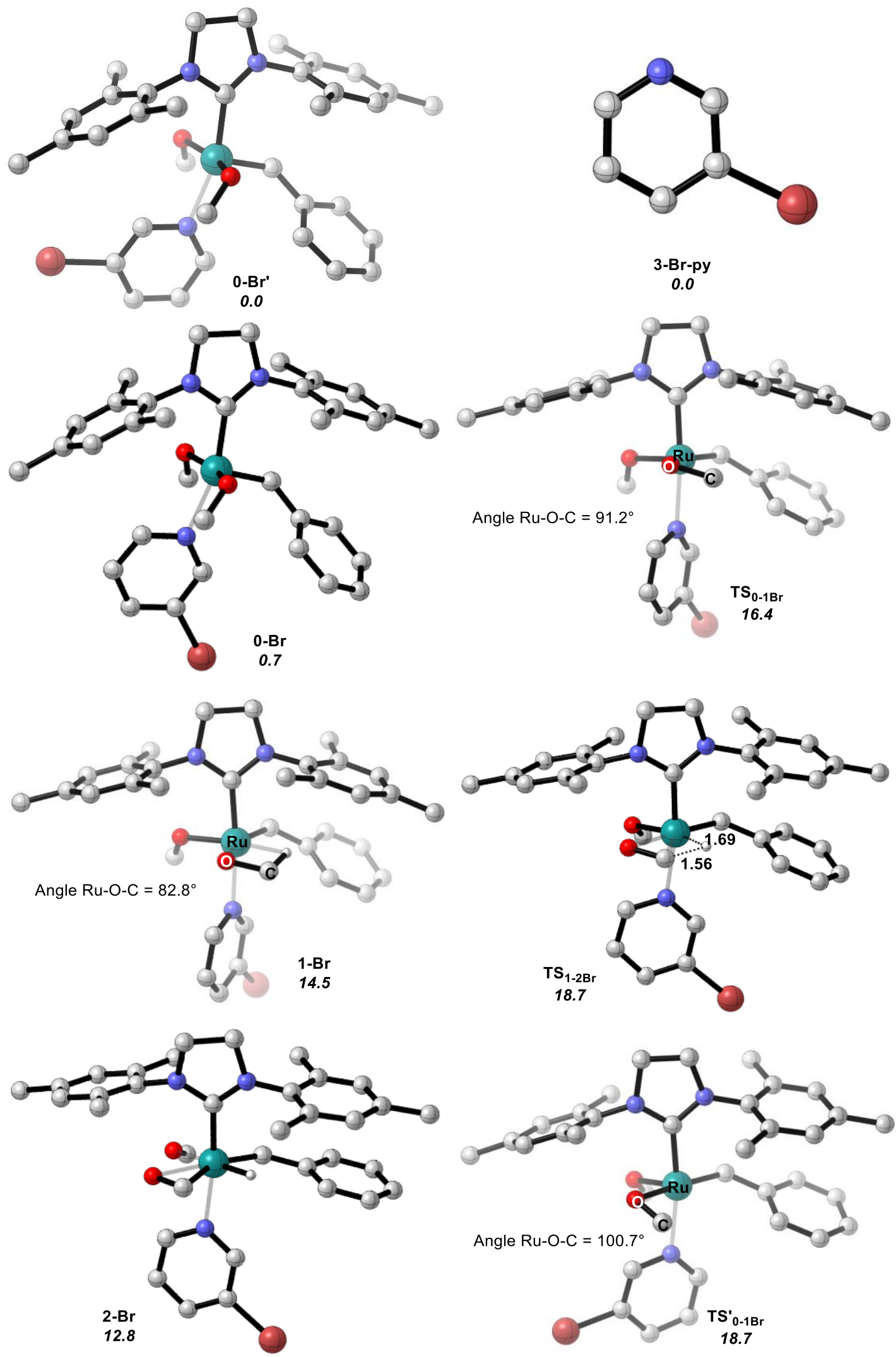

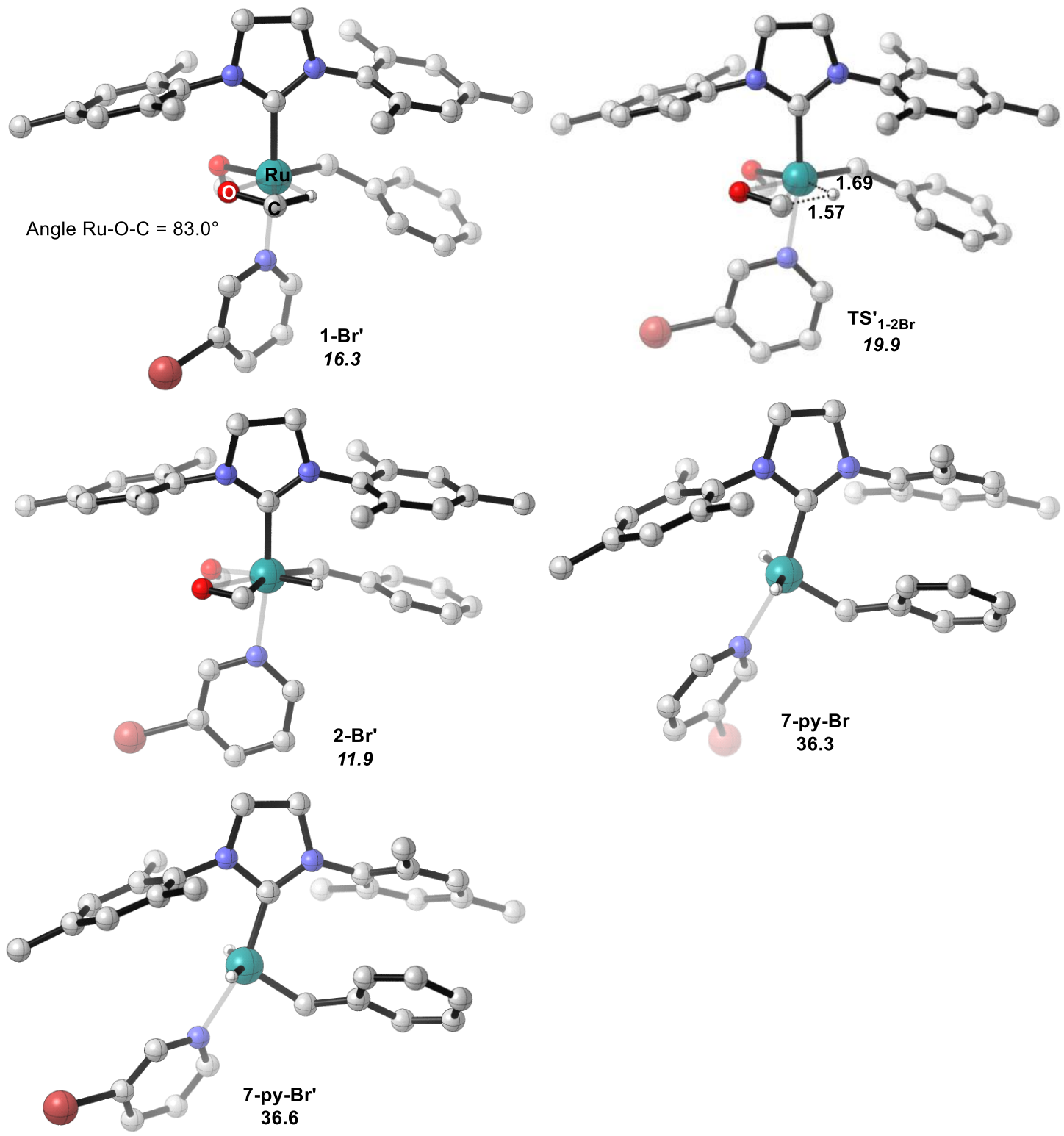

Figure S22. Calculated key structural parameters of the key geometries with 3-Brpyridine as the ligand by the M06-L method. Unimportant $\mathrm{H}$ atoms are omitted for clarity. Their relative free energies in solution by PCM M06-L//M06-L method are also given. 

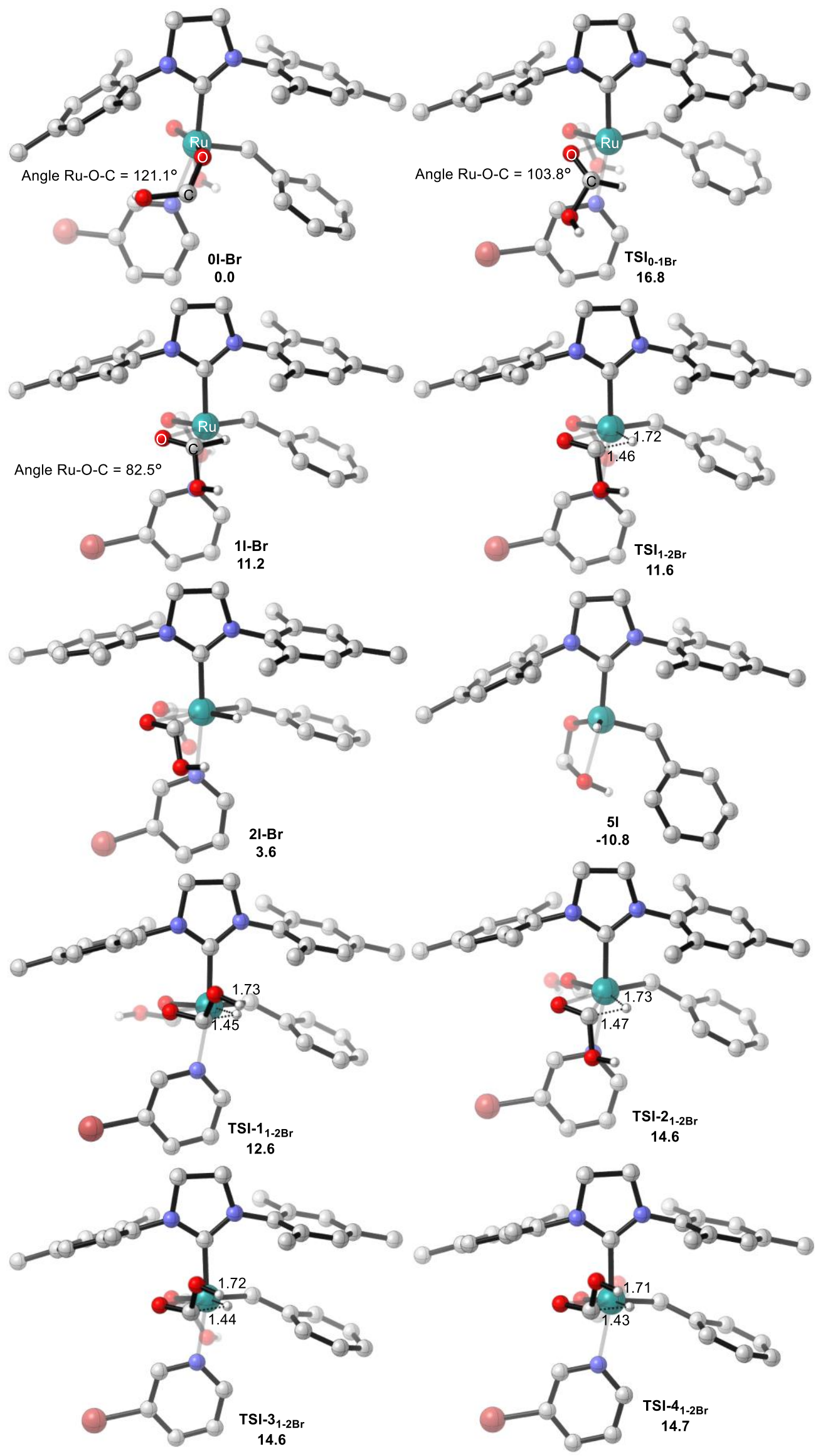

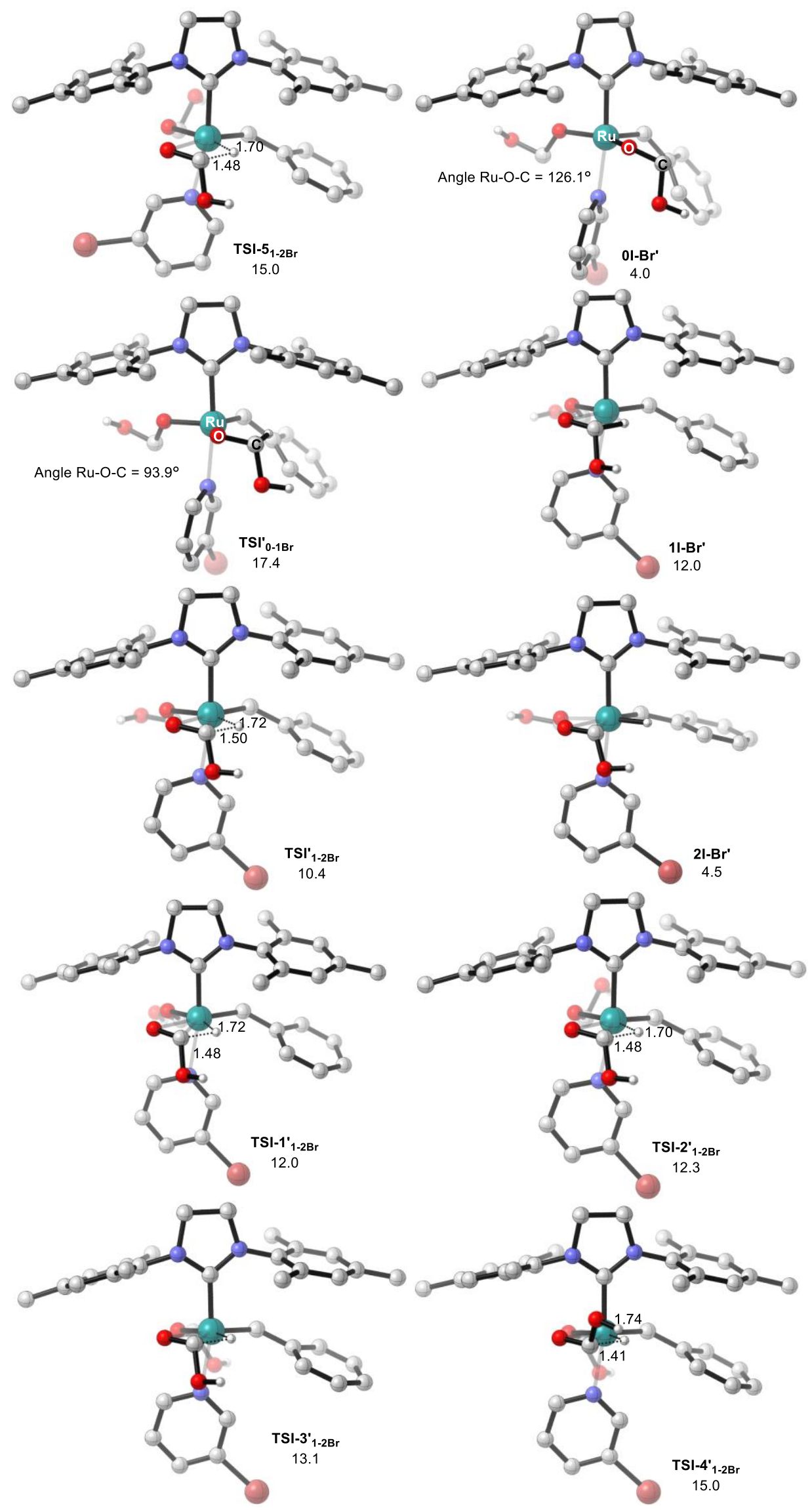

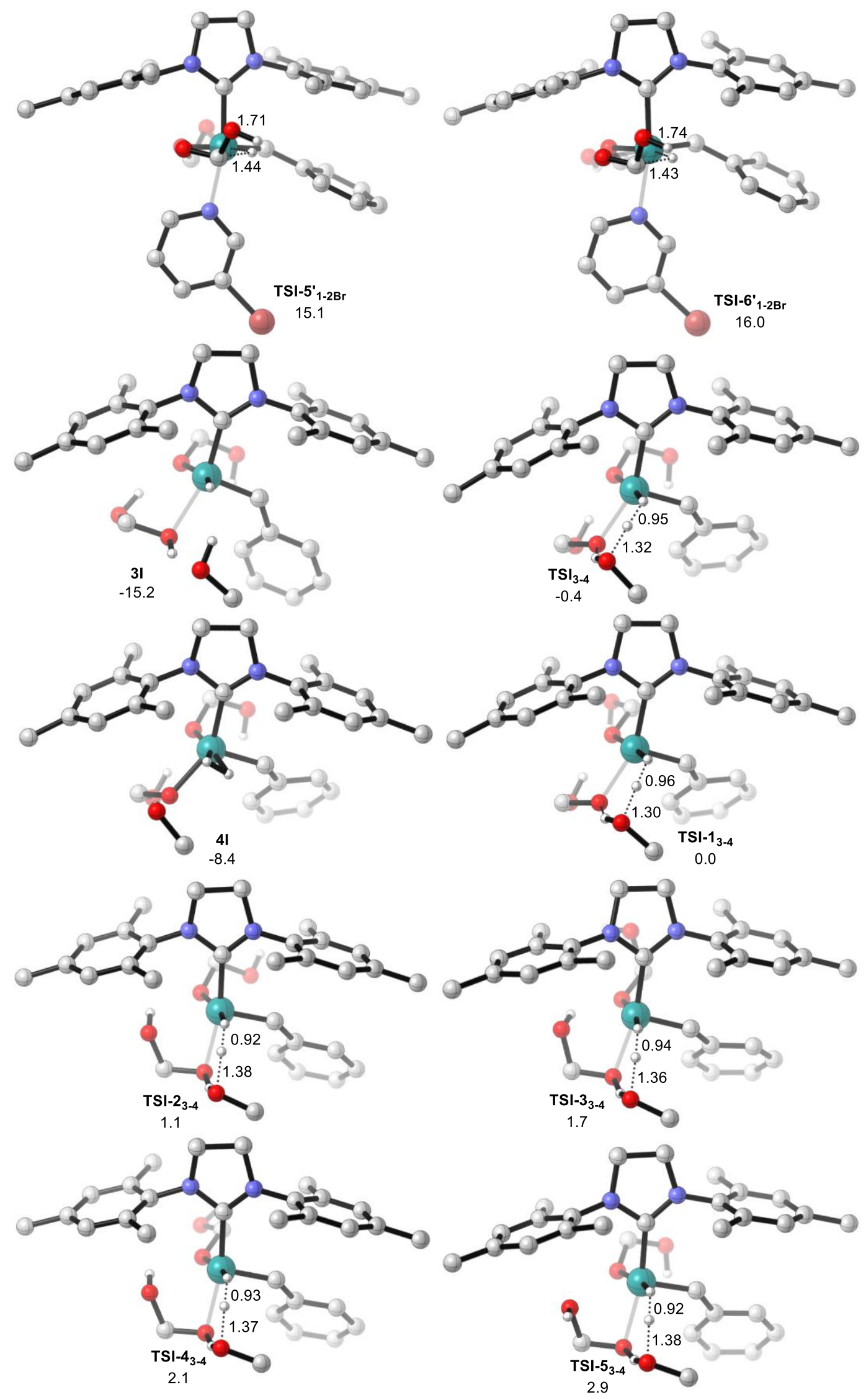

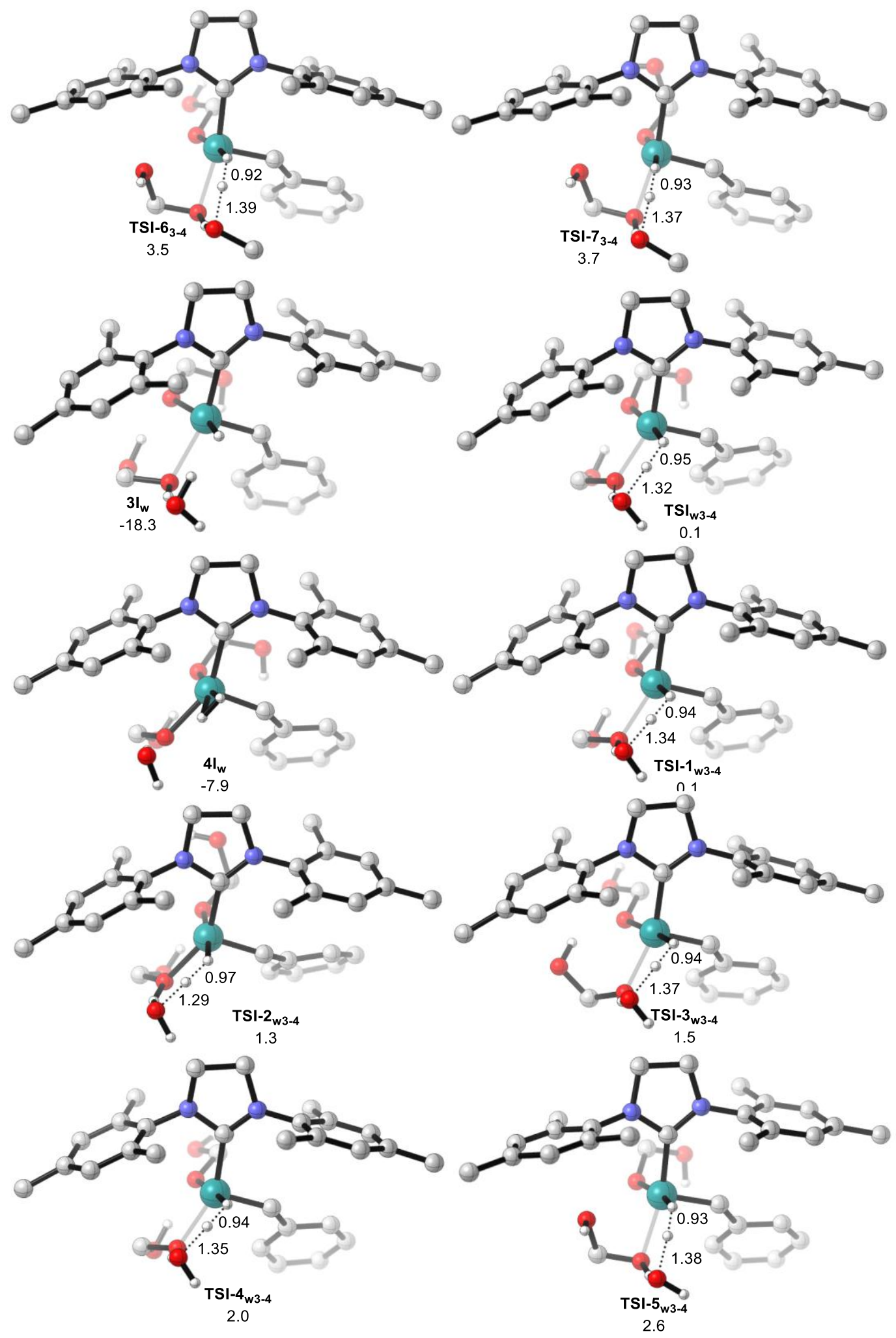

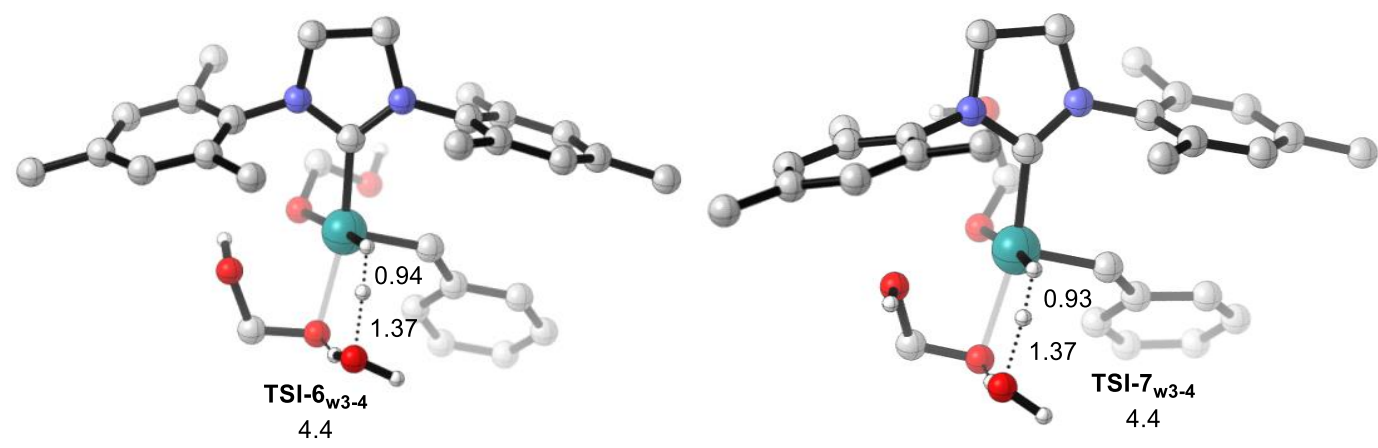

Figure S23. | Calculated key structural parameters of the geometries with 3-Br-pyridine as the ligand for dehydrogenation of $\mathrm{HOCH}_{2} \mathrm{OH}$ to $\mathrm{HCOOH}$ by the M06-L method. Unimportant $\mathrm{H}$ atoms are omitted for clarity. Their relative free energies in solution by PCM M06-L//M06-L method are also given. 

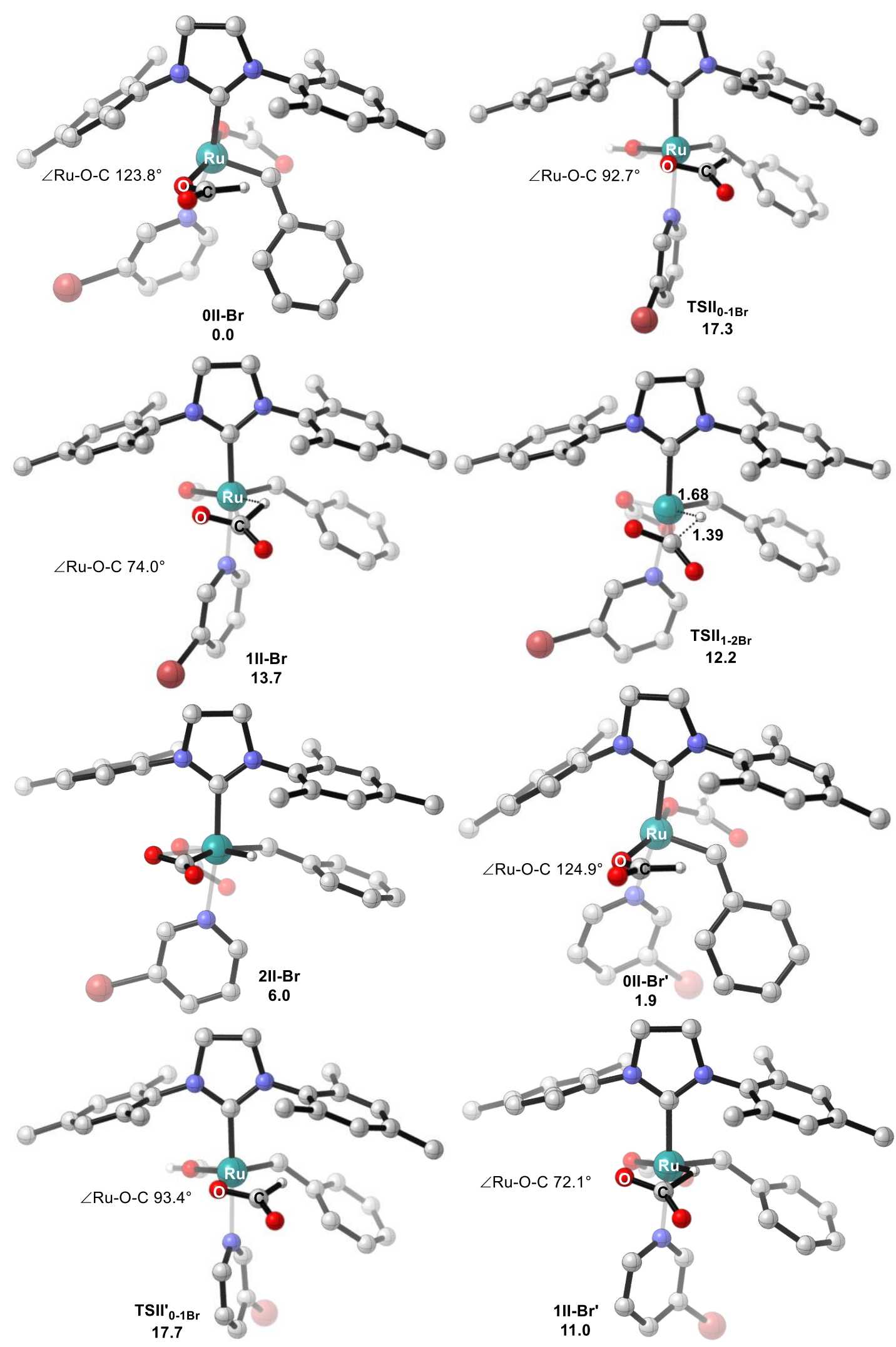

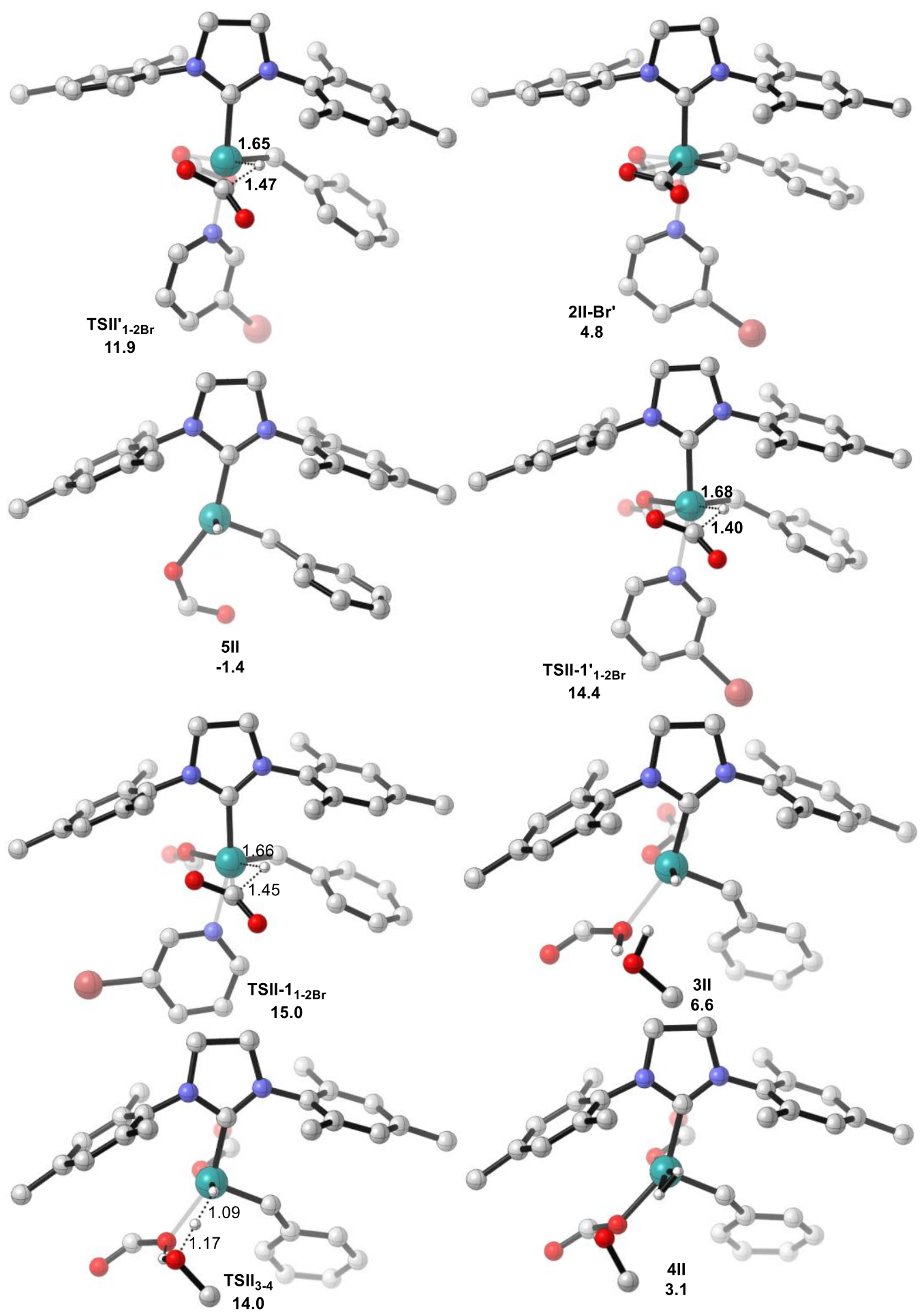

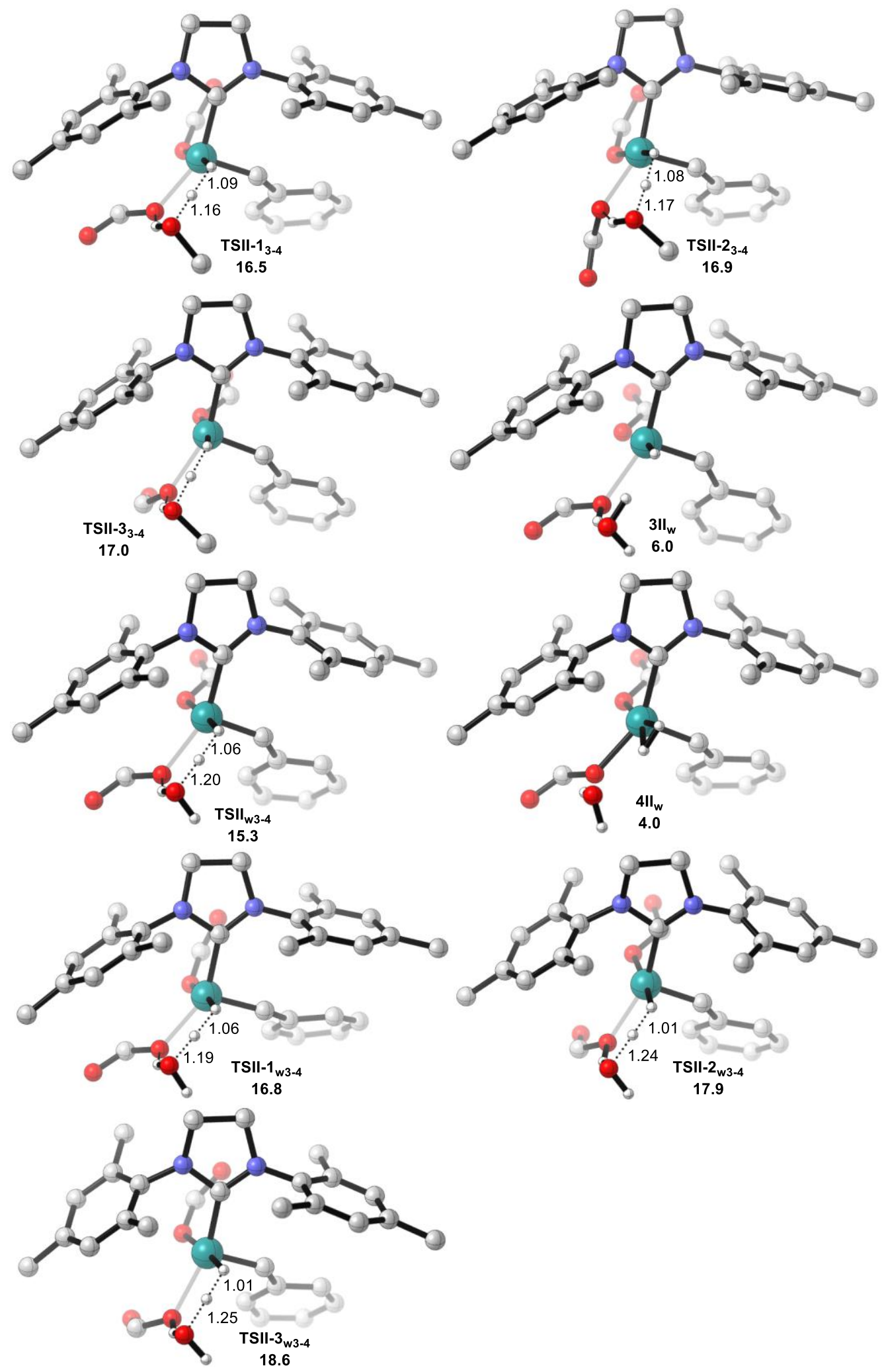

Figure S24. | Calculated key structural parameters of the geometries with 3-Br-pyridine as the ligand for dehydrogenation of $\mathrm{HCOOH}$ to $\mathrm{CO}_{2}$ by the M06-L method. Unimportant $\mathrm{H}$ atoms are omitted for clarity. Their relative free energies in solution by PCM M06-L//M06-L method are also given. 
Table S3. The absolute energies (in Hartree) for the pathway 1 of the Ru-catalyzed lowtemperature aqueous methanol reforming by the M06-L method.

\begin{tabular}{|c|c|c|c|c|c|}
\hline & $\mathbf{E}$ & $\mathbf{E}+\mathrm{ZPE}$ & G & Esoln-pem $_{\text {s. }}$ & Esoln-cpcm \\
\hline $\mathrm{CH}_{2} \mathrm{O}$ & -114.486683 & -114.460213 & -114.481875 & -114.491726 & -114.491775 \\
\hline py & -248.255369 & -248.166575 & -248.193990 & -248.261309 & -248.261384 \\
\hline $\mathbf{H}_{2}$ & -1.170077 & -1.160085 & -1.171574 & -1.170201 & -1.170203 \\
\hline МеОН & -115.702581 & -115.650986 & -115.673762 & -115.708311 & -115.708388 \\
\hline $\mathrm{H}_{2} \mathrm{O}$ & -76.411023 & -76.389158 & -76.406799 & -76.418119 & -76.418225 \\
\hline $\mathbf{O H}^{-}$ & -75.717922 & -75.709626 & -75.725893 & -75.851732 & -75.851820 \\
\hline $\mathrm{CH}_{3} \mathrm{O}^{-}$ & -115.063911 & -115.028938 & -115.050116 & -115.171079 & -115.171151 \\
\hline 0 & -1769.142512 & -1768.436906 & -1768.512350 & -1769.155459 & -1769.155928 \\
\hline $\mathbf{T S}_{0-1}$ & -1769.119270 & -1768.413978 & -1768.487924 & -1769.133335 & -1769.133979 \\
\hline 1 & -1769.120286 & -1768.413770 & -1768.487075 & -1769.135265 & -1769.135946 \\
\hline $\mathbf{T S}_{1-2}$ & -1769.109304 & -1768.408078 & -1768.481461 & -1769.123729 & -176 \\
\hline 2 & -1769.119491 & -1768.417202 & -1768.491593 & -1769.136125 & -1769.136833 \\
\hline $\mathbf{0 A}$ & -1769.137467 & -1768.431448 & -1768.506156 & -1769.148440 & -1769.148874 \\
\hline TS $_{1 \mathrm{~A}-2 \mathrm{~A}}$ & -1769.110412 & -1768.407450 & -1768.479897 & -1769.122419 & -1769.122874 \\
\hline $2 A$ & -1769.116295 & -1768.411916 & -1768.484371 & -1769.128644 & -1769.129077 \\
\hline $\mathbf{T S}_{1 \mathrm{~B}-2 \mathrm{~B}}$ & -1769.095068 & -1768.393727 & -1768.468119 & -1769.109169 & -1769.109586 \\
\hline 2B & -1769.102609 & -1768.401147 & -1768.476269 & -1769.116190 & -1769.116634 \\
\hline $\mathbf{0 C}$ & -1769.140507 & -1768.434497 & -1768.507983 & -1769.154345 & -1769.154840 \\
\hline TS $_{1 \mathrm{C}-2 \mathrm{C}}$ & -1769.09 & -1768.396681 & -1768.468548 & -1769.111972 & -1769.112370 \\
\hline 2C & -1769.102269 & -1768.398709 & -1768.470704 & -1769.115452 & -1769.115895 \\
\hline 3 & -1637.776355 & -1637.087552 & -1637.164655 & -1637.788720 & -1637.789131 \\
\hline $\mathbf{T S}_{3-4}$ & -1637.743185 & -1637.061000 & -1637.135033 & -1637.756174 & -1637.756611 \\
\hline 4 & -1637.761898 & -1637.074998 & -1637.149858 & -1637.776820 & -1637.777374 \\
\hline $\mathbf{3 A}$ & -1522.044302 & -1521.409300 & -1521.479878 & -1522.058209 & -1522.058706 \\
\hline $\mathbf{T S}_{3 \mathrm{~A}-4 \mathrm{~A}}$ & -1522.013306 & -1521.384089 & -1521.453527 & -1522.026054 & -1522.026502 \\
\hline $4 \mathrm{~A}$ & -1522.030896 & -1521.397567 & -1521.465369 & -1522.046490 & -1522.046984 \\
\hline $3 A_{p y}$ & -1770.334176 & -1769.608313 & -1769.684077 & -1770.345872 & -1770.346268 \\
\hline TS 3A-4Apy & -1770.296723 & -1769.576090 & -1769.649889 & -1770.307895 & -1770.308301 \\
\hline $4 A_{p y}$ & -1770.307714 & -1769.583745 & -1769.656695 & -1770.321467 & -1770.321994 \\
\hline 3B & -1522.047178 & -1521.413429 & -1521.485562 & -1522.060834 & -1522.061257 \\
\hline $\mathbf{T S}_{3 \mathrm{~B}-4 \mathrm{~B}}$ & -1522.010765 & -1521.381341 & -1521.450160 & -1522.025668 & -1522.026090 \\
\hline 4B & -1522.035233 & -1521.401603 & -1521.469285 & -1522.053342 & -1522.053936 \\
\hline $\mathbf{T S}_{3 \mathrm{C}-4 \mathrm{C}}$ & -1522.008093 & -1521.379402 & -1521.449178 & -1522.024237 & -1522.024843 \\
\hline 3D & -1522.046300 & -1521.411234 & -1521.481778 & -1522.060838 & -1522.061376 \\
\hline $\mathbf{T S}_{3 \mathrm{D}-4 \mathrm{D}}$ & -1522.007875 & -1521.378359 & -1521.448410 & -1522.024248 & -1522.024888 \\
\hline 4D & -1522.035233 & -1521.401599 & -1521.469259 & -1522.053345 & -1522.053940 \\
\hline $3 \mathbf{E}$ & -1522.044446 & -1521.410667 & -1521.483161 & -1522.057504 & -1522.057988 \\
\hline $\mathbf{T S}_{3 \mathrm{E}-4 \mathrm{E}}$ & -1521.988116 & -1521.360184 & -1521.428834 & -1522.001749 & -1522.002255 \\
\hline $4 E$ & -1522.008359 & -1521.377506 & -1521.445410 & -1522.022850 & -1522.023401 \\
\hline $3 \mathbf{F}$ & -1522.046954 & -1521.411932 & -1521.483098 & -1522.059703 & -1522.060203 \\
\hline
\end{tabular}




\begin{tabular}{|c|c|c|c|c|c|}
\hline $\mathbf{T S}_{3 \mathrm{~F}-4 \mathrm{~F}}$ & -1522.004157 & -1521.373390 & -1521.444113 & -1522.017953 & -1522.018461 \\
\hline $4 F$ & -1522.018398 & -1521.386727 & -1521.458031 & -1522.033231 & -1522.033722 \\
\hline TS 3 B-4Bрy & -1770.286911 & -1769.565110 & -1769.639911 & -1770.301429 & -1770.301968 \\
\hline TS 3 C-4Cpy & -1770.294991 & -1769.571992 & -1769.646755 & -1770.308093 & -1770.308584 \\
\hline TS $_{\text {3D-4Dpy }}$ & -1770.286013 & -1769.566300 & -1769.641683 & -1770.297981 & -1770.298439 \\
\hline TS 3 E-4Epy & -1770.290408 & -1769.569415 & -1769.642127 & -1770.301765 & -1770.302159 \\
\hline $3 w$ & -1598.485057 & -1597.825229 & -1597.899253 & -1598.498013 & -1598.498388 \\
\hline $\mathbf{T S}_{\mathbf{w} 3-4}$ & -1598.451676 & -1597.796633 & -1597.866982 & -1598.465145 & -1598.465527 \\
\hline $4_{w}$ & -1598.469917 & -1597.810850 & -1597.881775 & -1598.485488 & -1598.485924 \\
\hline
\end{tabular}


Table S4. The relative energies (in $\mathrm{kcal} / \mathrm{mol}$ ) for the pathway 1 of the Ru-catalyzed low-temperature aqueous methanol reforming by the M06-L method. The relative free energies in solution with the standard state correction $\left(\Delta \mathrm{G}_{\text {soln-pcm-cor }}\right)$ are also given.

\begin{tabular}{|c|c|c|c|c|c|c|c|c|}
\hline & $\Delta \mathbf{E}$ & $\Delta \mathbf{E}+\mathbf{Z P E}$ & $\Delta \mathbf{G}$ & $\Delta \mathbf{E}_{\text {soln-pcm }}$ & $\Delta \mathbf{E}_{\text {soln-cpcm }}$ & $\begin{array}{c}\Delta \mathbf{G}_{\text {soln- }} \\
\text { pcm }\end{array}$ & $\Delta \mathbf{G}_{\text {soln-cpcm }}$ & $\Delta \mathbf{G}_{\text {soln-pcm-cor }}$ \\
\hline $\mathbf{0}$ & 0.0 & 0.0 & 0.0 & 0.0 & 0.0 & 0.0 & 0.0 & 0.0 \\
\hline $\mathbf{T S}_{0-1}$ & 14.6 & 14.4 & 15.3 & 13.9 & 13.8 & 14.6 & 14.5 & 14.6 \\
\hline 1 & 13.9 & 14.5 & 15.9 & 12.7 & 12.5 & 14.6 & 14.5 & 14.6 \\
\hline $\mathbf{T S}_{1-2}$ & 20.8 & 18.1 & 19.4 & 19.9 & 19.8 & 18.5 & 18.3 & 18.5 \\
\hline 2 & 14.4 & 12.4 & 13.0 & 12.1 & 12.0 & 10.7 & 10.6 & 10.7 \\
\hline $\mathbf{O A}$ & 3.2 & 3.4 & 3.9 & 4.4 & 4.4 & 5.1 & 5.1 & 5.1 \\
\hline $\mathbf{T S}_{1 \mathrm{~A}-2 \mathrm{~A}}$ & 20.1 & 18.5 & 20.4 & 20.7 & 20.7 & 21.0 & 21.0 & 21.0 \\
\hline $2 A$ & 16.5 & 15.7 & 17.6 & 16.8 & 16.8 & 17.9 & 18.0 & 17.9 \\
\hline TS 1 1B-2B & 29.8 & 27.1 & 27.8 & 29.0 & 29.1 & 27.0 & 27.1 & 27.0 \\
\hline $2 B$ & 25.0 & 22.4 & 22.6 & 24.6 & 24.7 & 22.2 & 22.3 & 22.2 \\
\hline $\mathrm{OC}$ & 1.3 & 1.5 & 2.7 & 0.7 & 0.7 & 2.2 & 2.2 & 2.2 \\
\hline TS $1 \mathrm{C}-2 \mathrm{C}$ & 27.1 & 25.2 & 27.5 & 27.3 & 27.3 & 27.7 & 27.7 & 27.7 \\
\hline 2C & 25.3 & 24.0 & 26.1 & 25.1 & 25.1 & 26.0 & 26.0 & 26.0 \\
\hline 3 & 18.4 & 15.4 & 12.1 & 19.0 & 19.1 & 12.8 & 12.9 & 9.0 \\
\hline $\mathbf{T S}_{3-4}$ & 39.2 & 32.1 & 30.7 & 39.5 & 39.5 & 31.0 & 31.0 & 27.2 \\
\hline 4 & 27.4 & 23.3 & 21.4 & 26.5 & 26.5 & 20.5 & 20.5 & 16.7 \\
\hline $\mathbf{3 A}$ & 36.9 & 32.5 & 19.1 & 33.0 & 32.9 & 15.2 & 15.1 & 15.2 \\
\hline $\mathbf{T S}_{3 \mathrm{~A}-4 \mathrm{~A}}$ & 56.3 & 48.3 & 35.6 & 53.1 & 53.1 & 32.4 & 32.4 & 32.4 \\
\hline $4 \mathrm{~A}$ & 45.3 & 39.9 & 28.2 & 40.3 & 40.3 & 23.2 & 23.2 & 23.2 \\
\hline $\mathbf{3} \mathbf{A}_{\mathrm{py}}$ & 15.2 & 12.2 & 12.7 & 16.4 & 16.5 & 13.9 & 13.9 & 12.0 \\
\hline $\mathbf{T S}_{3 \mathrm{~A}-}$ & & & & & & & & 33.7 \\
\hline 4Apy & 38.7 & 32.4 & 34.1 & 40.3 & 40.3 & 35.6 & 35.7 & \\
\hline $4 A_{p y}$ & 31.8 & 27.6 & 29.8 & 31.7 & 31.7 & 29.8 & 29.7 & 27.9 \\
\hline 3B & 35.1 & 29.9 & 15.5 & 31.3 & 31.3 & 11.7 & 11.7 & 11.7 \\
\hline $\mathbf{T S}_{3 \mathrm{~B}-4 \mathrm{~B}}$ & 57.9 & 50.1 & 37.7 & 53.4 & 53.4 & 33.2 & 33.2 & 33.2 \\
\hline $4 B$ & 42.6 & 37.3 & 25.7 & 36.0 & 35.9 & 19.2 & 19.1 & 19.2 \\
\hline $\mathrm{TS}_{3 \mathrm{C}-4 \mathrm{C}}$ & 59.6 & 51.3 & 38.3 & 54.3 & 54.2 & 33.0 & 32.9 & 33.0 \\
\hline 3D & 35.6 & 31.3 & 17.9 & 31.3 & 31.2 & 13.6 & 13.5 & 13.6 \\
\hline TS $_{3 D-4 D}$ & 59.7 & 51.9 & 38.8 & 54.3 & 54.1 & 33.4 & 33.2 & 33.4 \\
\hline 4D & 42.6 & 37.3 & 25.7 & 36.0 & 35.9 & 19.2 & 19.1 & 19.2 \\
\hline $3 \mathbf{E}$ & 36.8 & 31.6 & 17.0 & 33.4 & 33.4 & 13.6 & 13.6 & 13.6 \\
\hline $\mathbf{T S}_{3 \mathrm{E}-4 \mathrm{E}}$ & 72.1 & 63.3 & 51.1 & 68.4 & 68.3 & 47.4 & 47.3 & 47.4 \\
\hline $4 E$ & 59.4 & 52.5 & 40.7 & 55.1 & 55.1 & 36.4 & 36.3 & 36.4 \\
\hline $3 F$ & 35.2 & 30.9 & 17.0 & 32.0 & 32.0 & 13.9 & 13.8 & 13.9 \\
\hline $\mathrm{TS}_{3 \mathrm{~F}-4 \mathrm{~F}}$ & 62.1 & 55.0 & 41.5 & 58.2 & 58.2 & 37.7 & 37.6 & 37.7 \\
\hline $4 F$ & 53.1 & 46.7 & 32.8 & 48.6 & 48.6 & 28.3 & 28.2 & 28.3 \\
\hline $\begin{array}{c}\text { TS } \\
\text { 4B- } \\
\text { 4Bpy }\end{array}$ & 44.9 & 39.3 & 40.4 & 44.3 & 44.3 & 39.8 & 39.8 & 37.9 \\
\hline
\end{tabular}




\begin{tabular}{|c|c|c|c|c|c|c|c|c|}
\hline $\begin{array}{c}\text { TS 3C- } \\
\text { 4Cpy }\end{array}$ & 39.8 & 34.9 & 36.1 & 40.1 & 40.1 & 36.4 & 36.4 & 34.5 \\
\hline $\begin{array}{c}\text { TS }_{3 D-} \\
\text { 4Dpy }\end{array}$ & 45.4 & 38.5 & 39.3 & 46.5 & 46.5 & 40.3 & 40.3 & 38.4 \\
\hline $\begin{array}{c}\text { TS3E- } \\
\text { 4Epy }\end{array}$ & 42.7 & 36.6 & 39.0 & 44.1 & 44.2 & 40.4 & 40.5 & 38.5 \\
\hline $\mathbf{3}_{w}$ & 18.2 & 15.7 & 11.2 & 19.4 & 19.4 & 12.3 & 12.4 & 8.0 \\
\hline $\mathbf{T S}_{\mathrm{w} 3-4}$ & 39.1 & 33.7 & 31.4 & 40.0 & 40.1 & 32.2 & 32.3 & 27.9 \\
\hline $4_{w}$ & 27.7 & 24.7 & 22.1 & 27.2 & 27.3 & 21.6 & 21.7 & 17.3 \\
\hline
\end{tabular}

Table S5. The absolute energies (in Hartree) for the pathways $\mathbf{2}$ and $\mathbf{3}$ of the Rucatalyzed low-temperature aqueous methanol reforming by the M06-L method.

\begin{tabular}{|c|c|c|c|c|c|}
\hline & $\mathbf{E}$ & $\mathrm{E}+\mathrm{ZPE}$ & $\mathbf{G}$ & Esoln-pcm $_{\text {s. }}$ & Esoln-cpcm \\
\hline 5 & -1406.321961 & -1405.742159 & -1405.809244 & -1406.334844 & -1406.335297 \\
\hline TS5-6 & -1406.294979 & -1405.718677 & -1405.785001 & -1406.308275 & -1406.308727 \\
\hline 6 & -1406.312368 & -1405.735046 & -1405.799036 & -1406.327320 & -1406.327770 \\
\hline 7 & -1291.773122 & -1291.227625 & -1291.289726 & -1291.794716 & -1291.795533 \\
\hline 7-py & -1540.056033 & -1539.419553 & -1539.487021 & -1540.070609 & -1540.071154 \\
\hline 7-A & -1523.228187 & -1522.575221 & -1522.648174 & -1523.241285 & -1523.241812 \\
\hline $\mathbf{T S A}_{7-5}$ & -1523.208558 & -1522.561332 & -1522.631619 & -1523.220625 & -1523.221034 \\
\hline 5-A & -1523.237087 & -1522.585726 & -1522.657183 & -1523.248463 & -1523.248846 \\
\hline 7-B & -1523.233339 & -1522.579065 & -1522.650309 & -1523.247854 & -1523.248449 \\
\hline $\mathrm{TSB}_{7-5}$ & -1523.205877 & -1522.558628 & -1522.628641 & -1523.217416 & -1523.217826 \\
\hline 5-B & -1523.227226 & -1522.575511 & -1522.644457 & -1523.237040 & -1523.237362 \\
\hline 7-C & -1407.498282 & -1406.898962 & -1406.967111 & -1407.513974 & -1407.514615 \\
\hline $\mathrm{TSC}_{7-5}$ & -1407.474127 & -1406.879752 & -1406.947773 & -1407.488682 & -1407.489229 \\
\hline 5-C & -1407.505163 & -1406.907504 & -1406.974553 & -1407.519977 & -1407.520457 \\
\hline 7-D & -1407.502698 & -1406.903878 & -1406.973690 & -1407.520439 & -1407.521094 \\
\hline 5-D & -1407.501647 & -1406.904552 & -1406.970032 & -1407.511766 & -1407.512152 \\
\hline 8 & -1654.603898 & -1653.932011 & -1654.003245 & -1654.617400 & -1654.618031 \\
\hline TS8-9 & -1654.564209 & -1653.899350 & -1653.971290 & -1654.576857 & -1654.577469 \\
\hline 9 & -1654.565378 & -1653.897959 & -1653.970661 & -1654.577815 & -1654.578435 \\
\hline 10 & -1521.482546 & -1520.863557 & -1520.934192 & -1521.562276 & -1521.563462 \\
\hline $\mathbf{T S}_{10-11}$ & -1521.437636 & -1520.823813 & -1520.890430 & -1521.511634 & -1521.512577 \\
\hline 11 & -1521.451638 & -1520.834436 & -1520.901709 & -1521.526105 & -1521.527079 \\
\hline 12 & -1520.280766 & -1519.681576 & -1519.750466 & -1520.354349 & -1520.355348 \\
\hline 13 & -1654.606091 & -1653.934561 & -1654.005406 & -1654.618944 & -1654.619366 \\
\hline
\end{tabular}


Table S6. The relative energies (in $\mathrm{kcal} / \mathrm{mol}$ ) for the pathways $\mathbf{2}$ and $\mathbf{3}$ of the $\mathrm{Ru}-$ catalyzed low-temperature aqueous methanol reforming by the M06-L method. The relative free energies in solution with the standard state correction $\left(\Delta \mathrm{G}_{\text {soln-pcm-cor }}\right)$ are also given.

\begin{tabular}{|c|c|c|c|c|c|c|c|c|}
\hline & $\Delta \mathbf{E}$ & $\Delta \mathrm{E}+\mathrm{ZPE}$ & $\Delta \mathbf{G}$ & $\Delta \mathbf{E}_{\text {soln-pcm }}$ & $\Delta \mathbf{E}_{\text {soln-cpcm }}$ & $\begin{array}{c}\Delta \mathbf{G}_{\text {soln- }} \\
\text { pcm }\end{array}$ & $\Delta \mathbf{G}_{\text {soln-cpem }}$ & $\Delta \mathbf{G}_{\text {soln-pcm-cor }}$ \\
\hline 5 & 49.3 & 42.6 & 17.1 & 42.4 & 42.3 & 10.2 & 10.2 & 14.0 \\
\hline $\mathbf{T S}_{5-6}$ & 66.2 & 57.4 & 32.3 & 59.1 & 59.0 & 25.2 & 25.1 & 29.0 \\
\hline 6 & 55.3 & 47.1 & 23.5 & 47.1 & 47.1 & 15.3 & 15.3 & 19.1 \\
\hline 7 & 88.3 & 76.7 & 40.7 & 72.8 & 72.5 & 25.2 & 24.9 & 30.9 \\
\hline 7-py & 71.0 & 60.8 & 38.6 & 63.6 & 63.5 & 31.3 & 31.2 & 35.1 \\
\hline $7-A$ & 56.9 & 48.1 & 33.9 & 54.0 & 53.9 & 30.9 & 30.9 & 29.0 \\
\hline TSA $_{7-5}$ & 69.3 & 56.8 & 44.2 & 67.0 & 67.0 & 41.9 & 42.0 & 40.0 \\
\hline 5-A & 51.4 & 41.5 & 28.2 & 49.5 & 49.5 & 26.3 & 26.4 & 24.4 \\
\hline 7-B & 53.7 & 45.7 & 32.5 & 49.9 & 49.8 & 28.7 & 28.6 & 26.8 \\
\hline $\mathrm{TSB}_{7-5}$ & 70.9 & 58.5 & 46.1 & 69.0 & 69.0 & 44.1 & 44.2 & 42.2 \\
\hline 5-B & 57.6 & 47.9 & 36.2 & 56.7 & 56.7 & 35.3 & 35.4 & 33.4 \\
\hline 7-C & 74.1 & 64.0 & 38.4 & 65.9 & 65.7 & 30.3 & 30.1 & 32.2 \\
\hline $\mathrm{TSC}_{7.5}$ & 89.3 & 76.0 & 50.6 & 81.8 & 81.7 & 43.1 & 43.0 & 45.0 \\
\hline 5-C & 69.8 & 58.6 & 33.8 & 62.1 & 62.1 & 26.1 & 26.1 & 28.0 \\
\hline 7-D & 71.3 & 60.9 & 34.3 & 61.9 & 61.7 & 24.8 & 24.7 & 26.7 \\
\hline 5-D & 72.0 & 60.5 & 36.6 & 67.3 & 67.3 & 31.9 & 31.9 & 33.8 \\
\hline 8 & 32.6 & 28.0 & 17.1 & 29.1 & 28.9 & 13.6 & 13.4 & 15.5 \\
\hline TS8-9 & 57.5 & 48.5 & 37.1 & 54.5 & 54.4 & 34.2 & 34.0 & 36.1 \\
\hline 9 & 56.8 & 49.4 & 37.5 & 53.9 & 53.8 & 34.7 & 34.6 & 36.6 \\
\hline 10 & -11.4 & -15.4 & -29.9 & 7.0 & 6.6 & -11.4 & -11.9 & -9.5 \\
\hline $\mathbf{T S}_{10-11}$ & 16.8 & 9.6 & -2.4 & 38.8 & 38.5 & 19.6 & 19.3 & 21.5 \\
\hline 11 & 8.0 & 2.9 & -9.5 & 29.7 & 29.4 & 12.3 & 11.9 & 14.2 \\
\hline 12 & 8.5 & -1.6 & -22.2 & 30.7 & 30.4 & 0.0 & -0.4 & 3.8 \\
\hline 13 & 60.0 & 45.7 & 28.5 & 57.2 & 57.2 & 25.7 & 25.7 & 27.6 \\
\hline
\end{tabular}


Table S7. The absolute energies (in Hartree) for the pathway 4 of the Ru-catalyzed lowtemperature aqueous methanol reforming by the M06-L method.

\begin{tabular}{cccccc}
\hline & $\mathbf{E}$ & $\mathbf{E}+\mathbf{Z P E}$ & $\mathbf{G}$ & $\mathbf{E}_{\text {soln-pcm }}$ & Esoln-cpcm \\
\hline $\mathbf{T S}_{\mathbf{2}-14}$ & -1654.570500 & -1653.903285 & -1653.974350 & -1654.582928 & -1654.583396 \\
$\mathbf{1 4}$ & -1654.596493 & -1653.926221 & -1653.996630 & -1654.606804 & -1654.607155 \\
$\mathbf{1 5}$ & -1655.805980 & -1655.112530 & -1655.187905 & -1655.818176 & -1655.818563 \\
$\mathbf{T S}_{\mathbf{1 5}-16}$ & -1655.773423 & -1655.084200 & -1655.156780 & -1655.787566 & -1655.788145 \\
$\mathbf{1 6}$ & -1655.800275 & -1655.108707 & -1655.182676 & -1655.815663 & -1655.816360 \\
$\mathbf{T S}_{\mathbf{2}-17}$ & -1406.278792 & -1405.702479 & -1405.766333 & -1406.293319 & -1406.293899 \\
$\mathbf{1 7}$ & -1406.307402 & -1405.728097 & -1405.792676 & -1406.318526 & -1406.318897 \\
$\mathbf{1 8}$ & -1407.515113 & -1406.910319 & -1406.976373 & -1407.529808 & -1407.530285 \\
\hline
\end{tabular}

Table S8. The relative energies (in $\mathrm{kcal} / \mathrm{mol}$ ) for the pathway 4 of the Ru-catalyzed low-temperature aqueous methanol reforming by the M06-L method. The relative free energies in solution with the standard state correction $\left(\Delta \mathrm{G}_{\text {soln-pcm-cor }}\right)$ are also given.

\begin{tabular}{ccccccccc}
\hline & $\boldsymbol{\Delta} \mathbf{E}$ & $\Delta \mathbf{E}+\mathbf{Z P E}$ & $\boldsymbol{\Delta} \mathbf{G}$ & $\Delta \mathbf{E}_{\text {soln-pcm }}$ & $\Delta \mathbf{E}_{\text {soln-cpcm }}$ & $\Delta \mathbf{G}_{\text {soln-pcm }}$ & $\Delta \mathbf{G}_{\text {soln-cpcm }}$ & $\Delta \mathbf{G}_{\text {soln-pcm-cor }}$ \\
\hline $\mathbf{T S}_{\mathbf{2 - 1 4}}$ & 53.5 & 46.1 & 35.2 & 50.7 & 50.7 & 32.4 & 32.4 & 34.3 \\
$\mathbf{1 4}$ & 37.2 & 31.7 & 21.2 & 35.7 & 35.8 & 19.7 & 19.8 & 21.6 \\
$\mathbf{1 5}$ & 41.3 & 34.5 & 21.6 & 39.0 & 39.0 & 19.4 & 19.4 & 19.4 \\
$\mathbf{T S}_{\mathbf{1 5 - 1 6}}$ & 61.7 & 52.3 & 41.2 & 58.2 & 58.1 & 37.7 & 37.6 & 37.7 \\
$\mathbf{1 6}$ & 44.8 & 36.9 & 24.9 & 40.6 & 40.4 & 20.6 & 20.5 & 20.6 \\
$\mathbf{T S}_{\mathbf{2 - 1 7}}$ & 76.3 & 67.5 & 44.0 & 68.5 & 68.3 & 36.1 & 36.0 & 39.9 \\
$\mathbf{1 7}$ & 58.4 & 51.5 & 27.5 & 52.6 & 52.6 & 21.7 & 21.7 & 25.5 \\
$\mathbf{1 8}$ & 63.5 & 56.8 & 32.6 & 56.0 & 55.9 & 25.1 & 25.0 & 27.0 \\
\hline
\end{tabular}


Table S9. The absolute electronic energies (in Hartree) for the key geometries of the Ru-catalyzed low-temperature aqueous methanol reforming in solution by different DFT methods.

\begin{tabular}{|c|c|c|c|c|}
\hline & Eв3LYP & Ем06 & Epbe0 & $\mathbf{E}_{\omega \mathrm{B} 97 \mathrm{XD}}$ \\
\hline $\mathrm{CH}_{2} \mathrm{O}$ & -114.507750 & -114.441885 & -114.375246 & -114.467524 \\
\hline py & -248.301643 & -248.105429 & -248.003899 & -248.207679 \\
\hline $\mathbf{H}_{2}$ & -1.178670 & -1.169425 & -1.167179 & -1.175186 \\
\hline МeOH & -115.729568 & -115.657646 & -115.594213 & -115.691338 \\
\hline $\mathrm{H}_{2} \mathrm{O}$ & -76.426457 & -76.393011 & -76.343954 & -76.405037 \\
\hline $\mathbf{O H}^{-}$ & -75.859481 & -75.827133 & -75.776768 & -75.837487 \\
\hline $\mathrm{CH}_{3} \mathrm{O}^{-}$ & -115.184988 & -115.117835 & -115.050195 & -115.144590 \\
\hline $\mathbf{0}$ & -1769.425319 & -1768.012531 & -1767.379526 & -1768.797597 \\
\hline $\mathbf{T S}_{0-1}$ & -1769.390423 & -1767.984582 & -1767.348935 & -1768.768154 \\
\hline 1 & -1769.391456 & -1767.986986 & -1767.351285 & -1768.770213 \\
\hline $\mathbf{T S}_{1-2}$ & -1769.382244 & -1767.977428 & -1767.343055 & -1768.760924 \\
\hline 2 & -1769.391994 & -1767.987671 & -1767.351700 & -1768.770186 \\
\hline $\mathbf{0 A}$ & -1769.410808 & -1767.999366 & -1767.365830 & -1768.783984 \\
\hline $\mathbf{T S}_{1 \mathrm{~A}-2 \mathrm{~A}}$ & -1769.379560 & -1767.975658 & -1767.339649 & -1768.758958 \\
\hline $2 A$ & -1769.384740 & -1767.980127 & -1767.342246 & -1768.762379 \\
\hline 3 & -1638.049477 & -1636.758258 & -1636.159490 & -1637.478470 \\
\hline $\mathbf{T S}_{3-4}$ & -1638.021005 & -1636.724233 & -1636.129366 & -1637.443702 \\
\hline 4 & -1638.030602 & -1636.740990 & -1636.138698 & -1637.459018 \\
\hline $\mathbf{3 A}$ & -1522.293486 & -1521.079275 & -1520.539534 & -1521.763505 \\
\hline $\mathbf{T S}_{3 \mathrm{~A}-4 \mathrm{~A}}$ & -1522.257513 & -1521.045204 & -1520.503833 & -1521.727228 \\
\hline $4 \mathrm{~A}$ & -1522.275230 & -1521.062571 & -1520.519613 & -1521.744829 \\
\hline $\mathbf{3} \mathbf{A}_{\mathrm{py}}$ & -1770.617148 & -1769.201561 & -1768.567423 & -1769.994867 \\
\hline TS 3A-4Apy & -1770.575590 & -1769.159880 & -1768.525611 & -1769.952074 \\
\hline $4 A_{p y}$ & -1770.586717 & -1769.172844 & -1768.535231 & -1769.962786 \\
\hline 5 & -1406.544948 & -1405.406532 & -1404.928406 & -1406.053505 \\
\hline TS $_{5-6}$ & -1406.514712 & -1405.382107 & -1404.902186 & -1406.028679 \\
\hline 6 & -1406.533697 & -1405.399414 & -1404.918367 & -1406.046902 \\
\hline 7 & -1291.991496 & -1290.919751 & -1290.500293 & -1291.541430 \\
\hline 7-py & -1540.305746 & -1539.033216 & -1538.522481 & -1539.763439 \\
\hline 7-A & -1523.485878 & -1522.263552 & -1521.726715 & -1522.956365 \\
\hline $\mathbf{T S A}_{7-5}$ & -1523.469230 & -1522.240716 & -1521.708102 & -1522.934455 \\
\hline 5-A & -1523.491487 & -1522.266819 & -1521.727663 & -1522.958453 \\
\hline 7-B & -1523.492912 & -1522.270835 & -1521.731584 & -1522.963621 \\
\hline $\mathrm{TSB}_{7-5}$ & -1523.463128 & -1522.237206 & -1521.701324 & -1522.928166 \\
\hline 5-B & -1523.475539 & -1522.256605 & -1521.715019 & -1522.947051 \\
\hline $7-\mathrm{C}$ & -1407.734247 & -1406.587145 & -1406.111056 & -1407.245209 \\
\hline $\mathrm{TSC}_{7.5}$ & -1407.706761 & -1406.560006 & -1406.082598 & -1407.214739 \\
\hline 5-C & -1407.737133 & -1406.589719 & -1406.109487 & -1407.244208 \\
\hline 7-D & -1407.739134 & -1406.593782 & -1406.113576 & -1407.250288 \\
\hline
\end{tabular}




\begin{tabular}{ccccc}
\hline $\mathbf{5 - D}$ & -1407.724421 & -1406.579559 & -1406.098745 & -1407.235158 \\
$\mathbf{8}$ & -1654.861565 & -1653.526698 & -1652.952424 & -1654.283415 \\
$\mathbf{T S} 8-9$ & -1654.819628 & -1653.485564 & -1652.914206 & -1654.241216 \\
$\mathbf{9}$ & -1654.819168 & -1653.486973 & -1652.911940 & -1654.239973 \\
$\mathbf{1 0}$ & -1521.788533 & -1520.581420 & -1520.036149 & -1521.256572 \\
$\mathbf{T S}$ & -1521.735955 & -1520.533401 & -1519.987707 & -1521.207598 \\
$\mathbf{1 1}$ & -1521.748544 & -1520.546836 & -1519.999622 & -1521.219201 \\
$\mathbf{1 2}$ & -1520.572665 & -1519.374758 & -1518.828452 & -1520.038630 \\
$\mathbf{2 3 a}$ & -1293.180949 & -1292.105645 & -1291.685649 & -1292.733676 \\
$\mathbf{2 3 b}$ & -1293.179297 & -1292.103294 & -1291.683724 & -1292.731743 \\
$\mathbf{2 3 c}$ & -1293.173959 & -1292.098785 & -1291.680880 & -1292.725865 \\
$\mathbf{2 3 d}$ & -1293.161316 & -1292.083207 & -1291.666764 & -1292.709428 \\
$\mathbf{2 4}$ & -1293.206762 & -1292.139939 & -1291.721730 & -1292.767382 \\
\hline
\end{tabular}


Table S10.The relative electronic energies (in $\mathrm{kcal} / \mathrm{mol}$ ) for the key geometries of the Ru-catalyzed low-temperature aqueous methanol reforming in solution by different DFT methods.

\begin{tabular}{|c|c|c|c|c|}
\hline & $\Delta \mathbf{E}_{\text {B3LYP }}$ & $\Delta \mathbf{E}_{M 06}$ & $\Delta \mathbf{E}_{\text {PBE0 }}$ & $\Delta \mathbf{E}_{\omega \mathrm{B} 97 \mathrm{XD}}$ \\
\hline 0 & 0.0 & 0.0 & 0.0 & 0.0 \\
\hline $\mathbf{T S}_{0-1}$ & 21.9 & 17.5 & 19.2 & 18.5 \\
\hline 1 & 21.2 & 16.0 & 17.7 & 17.2 \\
\hline $\mathrm{TS}_{1-2}$ & 27.0 & 22.0 & 22.9 & 23.0 \\
\hline 2 & 20.9 & 15.6 & 17.5 & 17.2 \\
\hline $\mathbf{O A}$ & 9.1 & 8.3 & 8.6 & 8.5 \\
\hline TS $_{1 \mathrm{~A}-2 \mathrm{~A}}$ & 28.7 & 23.1 & 25.0 & 24.2 \\
\hline $2 A$ & 25.5 & 20.3 & 23.4 & 22.1 \\
\hline 3 & 16.1 & 14.0 & 18.4 & 16.7 \\
\hline TS $_{3-4}$ & 33.9 & 35.3 & 37.3 & 38.5 \\
\hline 4 & 27.9 & 24.8 & 31.4 & 28.9 \\
\hline $\mathbf{3 A}$ & 32.6 & 27.4 & 34.6 & 31.5 \\
\hline $\mathbf{T S}_{3 \mathrm{~A}-4 \mathrm{~A}}$ & 55.2 & 48.7 & 57.0 & 54.3 \\
\hline $4 \mathrm{~A}$ & 44.1 & 37.8 & 47.1 & 43.2 \\
\hline $\mathbf{3} \mathbf{A}_{\mathrm{py}}$ & 18.8 & 16.8 & 19.5 & 16.7 \\
\hline $\mathbf{T S}_{\mathbf{3 A - 4 A p y}}$ & 44.9 & 42.9 & 45.7 & 43.5 \\
\hline $4 A_{p y}$ & 37.9 & 34.8 & 39.7 & 36.8 \\
\hline 5 & 44.5 & 36.8 & 45.2 & 43.2 \\
\hline TS $_{5-6}$ & 63.5 & 52.2 & 61.6 & 58.8 \\
\hline 6 & 51.6 & 41.3 & 51.5 & 47.4 \\
\hline 7 & 73.2 & 65.0 & 78.3 & 71.2 \\
\hline 7-py & 65.3 & 60.0 & 66.9 & 62.2 \\
\hline 7-A & 51.1 & 47.1 & 54.5 & 50.9 \\
\hline TSA $7-5$ & 61.5 & 61.4 & 66.2 & 64.7 \\
\hline 5-A & 47.6 & 45.1 & 53.9 & 49.6 \\
\hline 7-B & 46.7 & 42.5 & 51.4 & 46.4 \\
\hline $\mathrm{TSB}_{7-5}$ & 65.4 & 63.6 & 70.4 & 68.6 \\
\hline 5-B & 57.6 & 51.5 & 61.8 & 56.8 \\
\hline 7-C & 64.9 & 58.9 & 68.0 & 63.4 \\
\hline $\mathrm{TSC}_{7-5}$ & 82.2 & 75.9 & 85.8 & 82.5 \\
\hline 5-C & 63.1 & 57.3 & 68.9 & 64.0 \\
\hline 7-D & 61.9 & 54.7 & 66.4 & 60.2 \\
\hline 5-D & 71.1 & 63.6 & 75.7 & 69.7 \\
\hline 8 & 35.1 & 27.6 & 32.5 & 29.3 \\
\hline TS8-9 & 61.5 & 53.4 & 56.5 & 55.8 \\
\hline 9 & 61.7 & 52.5 & 57.9 & 56.5 \\
\hline 10 & 7.8 & 1.0 & 9.1 & 6.5 \\
\hline $\mathbf{T S}_{10-11}$ & 40.8 & 31.2 & 39.5 & 37.3 \\
\hline 11 & 32.9 & 22.7 & 32.0 & 30.0 \\
\hline 12 & 31.1 & 24.4 & 34.5 & 33.4 \\
\hline
\end{tabular}




\begin{tabular}{ccccc}
\hline 23a & 93.5 & 83.7 & 99.4 & 91.0 \\
23b & 94.6 & 85.2 & 100.6 & 92.2 \\
$\mathbf{2 3 c}$ & 97.9 & 88.0 & 102.4 & 95.9 \\
$\mathbf{2 3 d}$ & 105.8 & 97.8 & 111.3 & 106.2 \\
$\mathbf{2 4}$ & 77.3 & 62.2 & 76.8 & 69.8 \\
\hline
\end{tabular}


Table S11.The relative estimated Gibbs free energies (in $\mathrm{kcal} / \mathrm{mol}$ ) for the key geometries of the Ru-catalyzed low-temperature aqueous methanol reforming in solution by different DFT methods.

\begin{tabular}{|c|c|c|c|c|}
\hline & $\Delta \mathbf{G}_{\text {BзLYP }}$ & $\Delta \mathbf{G}_{\text {M06 }}$ & $\Delta \mathbf{G}_{\text {PBE0 }}$ & $\Delta \mathbf{G}_{\omega \mathrm{B} 97 \mathrm{XD}}$ \\
\hline 0 & 0.0 & 0.0 & 0.0 & 0.0 \\
\hline $\mathbf{T S}_{0-1}$ & 22.6 & 18.3 & 19.9 & 19.2 \\
\hline 1 & 23.2 & 17.9 & 19.6 & 19.1 \\
\hline $\mathbf{T S}_{1-2}$ & 25.6 & 20.6 & 21.4 & 21.6 \\
\hline 2 & 19.5 & 14.2 & 16.0 & 15.8 \\
\hline $\mathbf{0 A}$ & 9.8 & 9.0 & 9.3 & 9.3 \\
\hline $\mathbf{T S}_{1 \mathrm{~A}-2 \mathrm{~A}}$ & 28.9 & 23.4 & 25.2 & 24.5 \\
\hline $2 A$ & 26.6 & 21.4 & 24.5 & 23.2 \\
\hline 3 & 9.8 & 7.7 & 12.2 & 10.5 \\
\hline TS3-4 & 25.5 & 26.9 & 28.9 & 30.1 \\
\hline 4 & 21.9 & 18.8 & 25.4 & 22.9 \\
\hline $\mathbf{3 A}$ & 14.8 & 9.5 & 16.7 & 13.7 \\
\hline $\mathbf{T S}_{3 \mathrm{~A}-4 \mathrm{~A}}$ & 34.5 & 28.0 & 36.2 & 33.6 \\
\hline $4 \mathrm{~A}$ & 27.0 & 20.7 & 29.9 & 26.1 \\
\hline $3 A_{p y}$ & 16.3 & 14.2 & 16.9 & 14.1 \\
\hline TS $_{3 A-4 A p y}$ & 40.3 & 38.3 & 41.1 & 38.9 \\
\hline $4 A_{p y}$ & 35.9 & 32.8 & 37.7 & 34.8 \\
\hline 5 & 12.4 & 4.7 & 13.0 & 11.1 \\
\hline TS $_{5-6}$ & 29.6 & 18.3 & 27.7 & 24.9 \\
\hline 6 & 19.8 & 9.5 & 19.7 & 15.6 \\
\hline 7 & 25.7 & 17.5 & 30.8 & 23.6 \\
\hline 7-py & 33.0 & 27.6 & 34.5 & 29.9 \\
\hline 7-A & 28.0 & 24.0 & 31.4 & 27.9 \\
\hline TSA7-5 $_{7}$ & 36.5 & 36.4 & 41.2 & 39.7 \\
\hline 5-A & 24.4 & 21.9 & 30.7 & 26.5 \\
\hline 7-B & 25.5 & 21.3 & 30.2 & 25.2 \\
\hline $\mathrm{TSB}_{7-5}$ & 40.5 & 38.8 & 45.6 & 43.8 \\
\hline 5-B & 36.2 & 30.1 & 40.5 & 35.4 \\
\hline 7-C & 29.3 & 23.2 & 32.3 & 27.7 \\
\hline $\mathrm{TSC}_{7-5}$ & 43.5 & 37.2 & 47.1 & 43.8 \\
\hline 5-C & 27.1 & 21.3 & 32.9 & 28.0 \\
\hline 7-D & 24.9 & 17.7 & 29.4 & 23.2 \\
\hline 5-D & 35.7 & 28.3 & 40.3 & 34.3 \\
\hline 8 & 19.6 & 12.1 & 17.0 & 13.8 \\
\hline TS8-9 & 41.1 & 33.0 & 36.2 & 35.4 \\
\hline 9 & 42.5 & 33.3 & 38.7 & 37.3 \\
\hline 10 & -10.7 & -17.4 & -9.4 & -11.9 \\
\hline $\mathbf{T S}_{10-11}$ & 21.6 & 12.0 & 20.3 & 18.1 \\
\hline 11 & 15.4 & 5.3 & 14.5 & 12.5 \\
\hline 12 & 0.4 & -6.3 & 3.8 & 2.6 \\
\hline
\end{tabular}




\begin{tabular}{ccccc}
\hline 23a & 40.3 & 30.5 & 46.2 & 37.7 \\
23b & 41.0 & 31.6 & 47.1 & 38.6 \\
23c & 43.6 & 33.7 & 48.1 & 41.5 \\
23d & 50.4 & 42.3 & 55.8 & 50.7 \\
$\mathbf{2 4}$ & 26.6 & 11.5 & 26.1 & 19.1 \\
\hline
\end{tabular}


Table S12. The corrected relative estimated Gibbs free energies (in $\mathrm{kcal} / \mathrm{mol}$ ) for the key geometries of the Ru-catalyzed low-temperature aqueous methanol reforming in solution by different DFT methods. The relative free energies were corrected with the standard state correction.

\begin{tabular}{|c|c|c|c|c|}
\hline & $\Delta \mathbf{G}_{\text {B3LYP-cor }}$ & $\Delta \mathbf{G}_{M 06-c o r}$ & $\Delta \mathbf{G P B E 0}$-cor & $\Delta \mathbf{G}_{\omega \mathrm{B}}{ } \mathbf{3 7 \mathrm { XD } - \mathrm { cor }}$ \\
\hline 0 & 0.0 & 0.0 & 0.0 & 0.0 \\
\hline $\mathbf{T S}_{0-1}$ & 22.6 & 18.3 & 19.9 & 19.2 \\
\hline 1 & 23.2 & 17.9 & 19.6 & 19.1 \\
\hline $\mathbf{T S}_{1-2}$ & 25.6 & 20.6 & 21.4 & 21.6 \\
\hline 2 & 19.5 & 14.2 & 16.0 & 15.8 \\
\hline $\mathbf{O A}$ & 9.8 & 9.0 & 9.3 & 9.3 \\
\hline $\mathbf{T S}_{1 \mathrm{~A}-2 \mathrm{~A}}$ & 28.9 & 23.4 & 25.2 & 24.5 \\
\hline $2 A$ & 26.6 & 21.4 & 24.5 & 23.2 \\
\hline 3 & 6.0 & 3.9 & 8.4 & 6.7 \\
\hline TS3-4 & 21.7 & 23.1 & 25.1 & 26.3 \\
\hline 4 & 18.1 & 15.0 & 21.6 & 19.1 \\
\hline $\mathbf{3 A}$ & 14.8 & 9.5 & 16.7 & 13.7 \\
\hline $\mathbf{T S}_{3 \mathrm{~A}-\mathbf{4 A}}$ & 34.5 & 28 & 36.2 & 33.6 \\
\hline $4 \mathrm{~A}$ & 27 & 20.7 & 29.9 & 26.1 \\
\hline $\mathbf{3} \mathbf{A}_{\mathrm{py}}$ & 14.4 & 12.3 & 15.0 & 12.2 \\
\hline TS $_{3 A-4 A p y}$ & 38.4 & 36.4 & 39.2 & 37.0 \\
\hline $4 A_{p y}$ & 34.0 & 30.9 & 35.8 & 32.9 \\
\hline 5 & 16.2 & 8.5 & 16.8 & 14.9 \\
\hline TS5-6 & 33.4 & 22.1 & 31.5 & 28.7 \\
\hline 6 & 23.6 & 13.3 & 23.5 & 19.4 \\
\hline 7 & 31.4 & 23.2 & 36.5 & 29.3 \\
\hline 7-py & 36.8 & 31.4 & 38.3 & 33.7 \\
\hline 7-A & 26.1 & 22.1 & 29.5 & 26.0 \\
\hline $\mathrm{TSA}_{7-5}$ & 34.6 & 34.5 & 39.3 & 37.8 \\
\hline 5-A & 22.5 & 20.0 & 28.8 & 24.6 \\
\hline 7-B & 23.6 & 19.4 & 28.3 & 23.3 \\
\hline $\mathrm{TSB}_{7-5}$ & 38.6 & 36.9 & 43.7 & 41.9 \\
\hline 5-B & 34.3 & 28.2 & 38.6 & 33.5 \\
\hline 7-C & 31.2 & 25.1 & 34.2 & 29.6 \\
\hline $\mathbf{T S C}_{7-5}$ & 45.4 & 39.1 & 49.0 & 45.7 \\
\hline 5-C & 29.0 & 23.2 & 34.8 & 29.9 \\
\hline 7-D & 26.8 & 19.6 & 31.3 & 25.1 \\
\hline 5-D & 37.6 & 30.2 & 42.2 & 36.2 \\
\hline 8 & 21.5 & 14.0 & 18.9 & 15.7 \\
\hline TS8-9 & 43.0 & 34.9 & 38.1 & 37.3 \\
\hline
\end{tabular}




\begin{tabular}{ccccc}
\hline $\mathbf{9}$ & 44.4 & 35.2 & 40.6 & 39.2 \\
$\mathbf{1 0}$ & -8.8 & -15.5 & -7.5 & -10.0 \\
$\mathbf{T S} \mathbf{1 0 - 1 1}$ & 23.5 & 13.9 & 22.2 & 20.0 \\
$\mathbf{1 1}$ & 17.3 & 7.2 & 16.4 & 14.4 \\
$\mathbf{1 2}$ & 4.2 & -2.5 & 7.6 & 6.4 \\
$\mathbf{2 3 a}$ & 44.1 & 34.3 & 50.0 & 41.5 \\
$\mathbf{2 3 b}$ & 44.8 & 35.4 & 50.9 & 42.4 \\
$\mathbf{2 3 c}$ & 47.4 & 37.5 & 51.9 & 45.3 \\
$\mathbf{2 3 d}$ & 54.2 & 46.1 & 59.6 & 54.5 \\
$\mathbf{2 4}$ & 30.4 & 15.3 & 29.9 & 22.9 \\
\hline
\end{tabular}

Table S13. The absolute (in Hartree) and relative energies (in $\mathrm{kcal} / \mathrm{mol}$ ) for the higherenergy $\beta$-H elimination pathways in gas phase by the M06-L method.

\begin{tabular}{cccccccc}
\hline & $\mathbf{E}$ & $\mathbf{E}+\mathbf{Z P E}$ & $\mathbf{G}$ & $\Delta \mathbf{E}$ & $\Delta \mathbf{E}+\mathbf{Z P E}$ & $\Delta \mathbf{G}$ & $\Delta \mathbf{G}$ cor \\
\hline $\mathbf{1 9 a}$ & -1769.116534 & -1768.416771 & -1768.492399 & 16.3 & 12.6 & 12.5 & 12.5 \\
$\mathbf{1 9 b}$ & -1769.069883 & -1768.369433 & -1768.445039 & 45.6 & 42.3 & 42.2 & 42.2 \\
$\mathbf{2 0}$ & -1520.786838 & -1520.178302 & -1520.249007 & 62.9 & 57.7 & 43.5 & 45.4 \\
$\mathbf{T S} \mathbf{S}_{\mathbf{2 0 - 2 1}}$ & -1520.768857 & -1520.164471 & -1520.232272 & 74.2 & 66.4 & 54.0 & 55.9 \\
$\mathbf{2 1 a}$ & -1520.755977 & -1520.151423 & -1520.221131 & 82.3 & 74.6 & 61.0 & 62.9 \\
$\mathbf{2 1 b}$ & -1520.777093 & -1520.170524 & -1520.239268 & 69.1 & 62.6 & 49.6 & 51.5 \\
$\mathbf{2 2 a}$ & -1520.746379 & -1520.143259 & -1520.210576 & 88.3 & 79.7 & 67.6 & 69.5 \\
$\mathbf{2 2 b}$ & -1520.780823 & -1520.176653 & -1520.244041 & 66.7 & 58.8 & 46.6 & 48.5 \\
$\mathbf{2 2 c}$ & -1654.564209 & -1653.89935 & -1653.97129 & 57.5 & 48.5 & 37.1 & 39.0 \\
\hline
\end{tabular}

Table S14. The absolute energies (in Hartree) for the Ru-dihydride-dihydrogen and Ruhydride-didydrogen intermediates by the M06-L method.

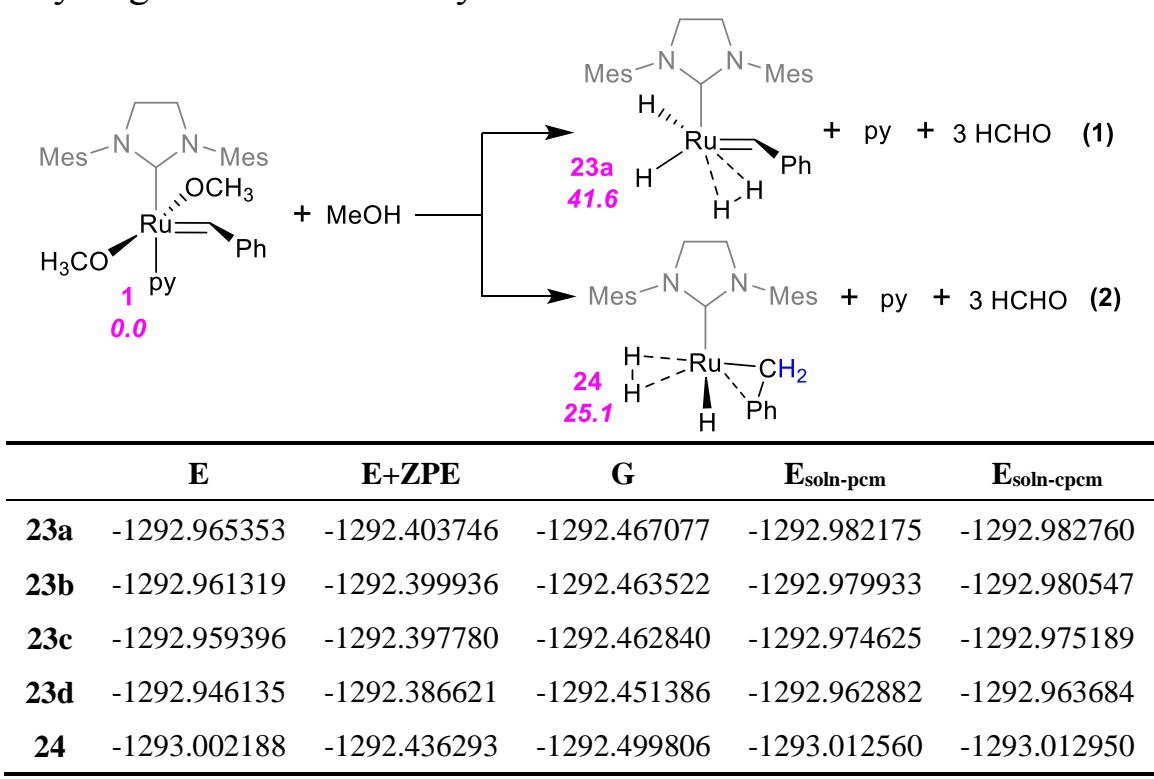


Table S15. The relative energies (in $\mathrm{kcal} / \mathrm{mol}$ ) for the Ru-dihydride-dihydrogen and Ru-hydride-didydrogen intermediates by the M06-L method. The relative free energies with the standard state correction $\left(\Delta \mathrm{G}_{\text {soln-pcm-cor }}\right)$ are also given.

\begin{tabular}{ccccccccc}
\hline & $\boldsymbol{\Delta} \mathbf{E}$ & $\boldsymbol{\Delta} \mathbf{E}+\mathbf{Z P E}$ & $\boldsymbol{\Delta} \mathbf{G}$ & $\boldsymbol{\Delta} \mathbf{E}_{\text {soln-pcm }}$ & $\Delta \mathbf{E}_{\text {soln-cpcm }}$ & $\Delta \mathbf{G}_{\text {soln-pcm }}$ & $\Delta \mathbf{G}_{\text {soln-cpcm }}$ & $\Delta \mathbf{G}_{\text {soln-pcm-cor }}$ \\
\hline 23a & 103.1 & 85.9 & 49.8 & 91.1 & 90.9 & 37.8 & 37.6 & 41.6 \\
$\mathbf{2 3 b}$ & 105.6 & 88.3 & 52.1 & 92.5 & 92.3 & 38.9 & 38.7 & 42.7 \\
$\mathbf{2 3 c}$ & 106.9 & 89.7 & 52.5 & 95.8 & 95.6 & 41.4 & 41.3 & 45.2 \\
$\mathbf{2 3 d}$ & 115.2 & 96.7 & 59.7 & 103.2 & 102.9 & 47.7 & 47.4 & 51.5 \\
$\mathbf{2 4}$ & 80.0 & 65.5 & 29.3 & 72.0 & 71.9 & 21.3 & 21.2 & 25.1 \\
\hline
\end{tabular}

Table S16. The absolute energies (in Hartree) for the key geometries with 3-Br-pyridine as the ligand in the main text by the M06-L method.

\begin{tabular}{|c|c|c|c|c|}
\hline & $\mathbf{E}$ & $\mathbf{E}+\mathrm{ZPE}$ & G & Esoln-pcm $_{\text {s. }}$ \\
\hline 0-Br' & -1781.907858 & -1781.213017 & -1781.292580 & -1781.920677 \\
\hline 3-Br-py & -261.020806 & -260.942271 & -260.973176 & -261.025152 \\
\hline 0-Br & -1781.906539 & -1781.212212 & -1781.291528 & -1781.919237 \\
\hline $\mathrm{TS}_{0-1 \mathrm{Br}}$ & -1781.883959 & -1781.188355 & -1781.265244 & -1781.898026 \\
\hline 1-Br & -1781.885047 & -1781.189752 & -1781.267143 & -1781.900173 \\
\hline $\mathbf{T S}_{1-2 \mathrm{Br}}$ & -1781.873995 & -1781.183498 & -1781.260594 & -1781.889029 \\
\hline $2-\mathrm{Br}$ & -1781.883865 & -1781.191855 & -1781.268666 & -1781.900239 \\
\hline $\mathbf{T S}_{0-1 \mathrm{Br}}^{\prime}$ & -1781.877197 & -1781.183922 & -1781.260682 & -1781.892045 \\
\hline 1-Br' & -1781.881590 & -1781.187357 & -1781.264042 & -1781.896964 \\
\hline $\mathbf{T S}^{\prime}{ }_{1-2 \mathrm{Br}}$ & -1781.873723 & -1781.182678 & -1781.258763 & -1781.888671 \\
\hline 2-Br' & -1781.883759 & -1781.192139 & -1781.269460 & -1781.900776 \\
\hline 7-py-Br & -1552.820199 & -1552.194735 & -1552.266085 & -1552.833961 \\
\hline 7-py-Br' & -1552.820744 & -1552.195272 & -1552.265679 & -1552.834406 \\
\hline
\end{tabular}


Table S17. The relative energies (in $\mathrm{kcal} / \mathrm{mol}$ ) for the key geometries with using the 3 Br-pyridine as the ligand (instead of pyridine) by the M06-L method. The relative free energies in solution with the standard state correction $\left(\Delta \mathrm{G}_{\text {soln-pcm-cor }}\right)$ are also given.

\begin{tabular}{|c|c|c|c|c|c|c|}
\hline & $\Delta \mathbf{E}$ & $\Delta \mathrm{E}+\mathrm{ZPE}$ & $\Delta \mathbf{G}$ & $\Delta \mathbf{E}_{\text {soln-pcm }}$ & $\Delta \mathbf{G}_{\text {soln-pcm }}$ & $\Delta \mathbf{G}_{\text {soln-pcm-cor }}$ \\
\hline O-Br' & 0.0 & 0.0 & 0.0 & 0.0 & 0.0 & 0.0 \\
\hline 0-Br & 0.8 & 0.5 & 0.7 & 0.9 & 0.7 & 0.7 \\
\hline $\mathbf{T S}_{\mathbf{0 - 1 B r}}$ & 15.0 & 15.5 & 17.2 & 14.2 & 16.4 & 16.4 \\
\hline $1-\mathrm{Br}$ & 14.3 & 14.6 & 16.0 & 12.9 & 14.5 & 14.5 \\
\hline $\mathbf{T S}_{1-2 \mathrm{Br}}$ & 21.2 & 18.5 & 20.1 & 19.9 & 18.7 & 18.7 \\
\hline $2-\mathrm{Br}$ & 15.1 & 13.3 & 15.0 & 12.8 & 12.8 & 12.8 \\
\hline$T^{\prime}{ }_{0-1 B r}$ & 19.2 & 18.3 & 20.0 & 18.0 & 18.7 & 18.7 \\
\hline 1-Br' & 16.5 & 16.1 & 17.9 & 14.9 & 16.3 & 16.3 \\
\hline $\mathrm{TS}^{\prime}{ }_{1-2 \mathrm{Br}}$ & 21.4 & 19.0 & 21.2 & 20.1 & 19.9 & 19.9 \\
\hline $2-\mathbf{B r}^{\prime}$ & 15.1 & 13.1 & 14.5 & 12.5 & 11.9 & 11.9 \\
\hline 3 & 18.3 & 15.7 & 12.8 & 19.9 & 14.4 & 10.6 \\
\hline TS3-4 & 39.1 & 32.3 & 31.4 & 40.3 & 32.6 & 28.8 \\
\hline 4 & 27.4 & 23.5 & 22.1 & 27.4 & 22.1 & 18.3 \\
\hline 5 & 49.2 & 42.9 & 17.7 & 43.3 & 11.8 & 15.6 \\
\hline $\mathbf{T S}_{5-6}$ & 66.1 & 57.6 & 33.0 & 59.9 & 26.8 & 30.6 \\
\hline 6 & 55.2 & 47.4 & 24.2 & 48.0 & 16.9 & 20.7 \\
\hline 7-A & 56.9 & 48.4 & 34.5 & 54.9 & 32.5 & 30.6 \\
\hline TSA $7-5$ & 69.2 & 57.1 & 44.9 & 67.8 & 43.5 & 41.6 \\
\hline 5-A & 51.3 & 41.8 & 28.9 & 50.3 & 27.9 & 26.0 \\
\hline 7-B & 53.7 & 46.0 & 33.2 & 50.7 & 30.2 & 28.3 \\
\hline $\mathbf{T S B}_{7-5}$ & 70.9 & 58.8 & 46.8 & 69.8 & 45.7 & 43.8 \\
\hline 5-B & 57.5 & 48.2 & 36.8 & 57.5 & 36.9 & 35.0 \\
\hline 7-C & 74.0 & 64.2 & 39.1 & 66.8 & 31.8 & 33.7 \\
\hline TSC $_{7-5}$ & 89.2 & 76.3 & 51.2 & 82.6 & 44.7 & 46.6 \\
\hline 5-C & 69.7 & 58.9 & 34.4 & 63.0 & 27.7 & 29.6 \\
\hline 7 & 88.2 & 77.0 & 41.4 & 73.6 & 26.8 & 32.5 \\
\hline 7-py-Br & 71.7 & 61.4 & 39.4 & 64.8 & 32.5 & 36.3 \\
\hline 7-py-Br' & 71.4 & 61.1 & 39.6 & 64.5 & 32.8 & 36.6 \\
\hline 24 & 79.9 & 65.8 & 30.0 & 72.9 & 22.9 & 26.7 \\
\hline $3 w$ & 18.1 & 16.0 & 11.8 & 20.2 & 13.9 & 9.6 \\
\hline $\mathbf{T S}_{\mathrm{w} 3-4}$ & 39.1 & 33.9 & 32.1 & 40.8 & 33.8 & 29.5 \\
\hline $4_{w}$ & 27.6 & 25.0 & 22.8 & 28.1 & 23.2 & 18.9 \\
\hline $\mathbf{T S}_{\mathbf{3 A - 4 A}}$ & 56.3 & 48.6 & 36.2 & 54.0 & 34.0 & 34.0 \\
\hline
\end{tabular}


Table S18. The metal-ligand bond distances (in $\AA$ ) for the key geometries of the Rucatalyzed low-temperature aqueous methanol reforming in solution by the M06-L method.
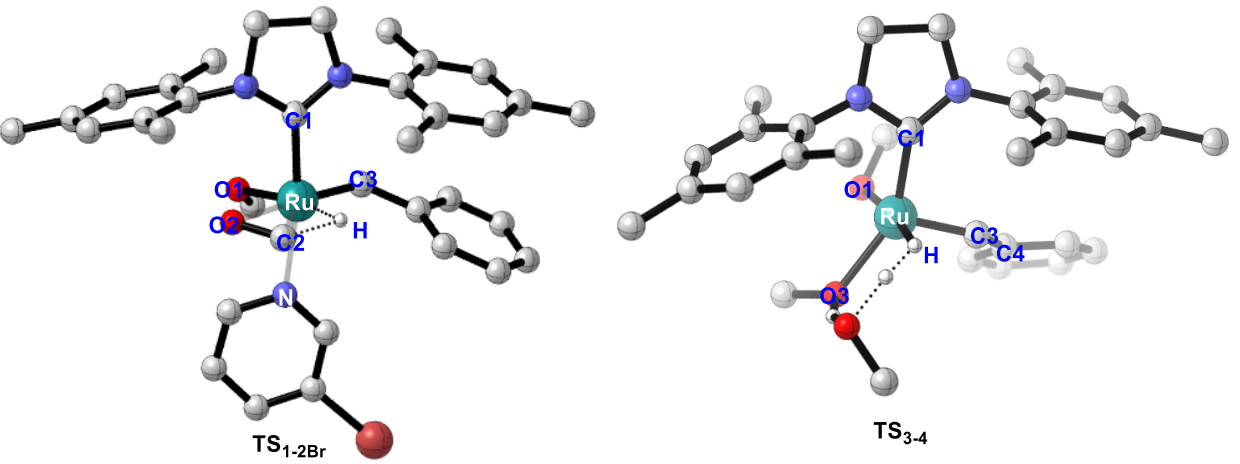

\begin{tabular}{|c|c|c|c|c|c|c|c|c|c|}
\hline & Ru-C1 & Ru-O1 & Ru-O2 & Ru-O3 & Ru-C2 & Ru-H & $\mathrm{Ru}-\mathrm{C3}$ & $\mathbf{R u}-\mathbf{N}$ & Ru-C4 \\
\hline $\mathbf{0}$ & 1.99 & 2.05 & 2.07 & - & - & - & 1.85 & 2.23 & - \\
\hline 0-Br' & 1.99 & 2.04 & 2.06 & - & - & - & 1.85 & 2.23 & - \\
\hline $\mathbf{T S}_{1-2}$ & 2.03 & 2.08 & 2.20 & - & 2.24 & 1.68 & 1.89 & 2.22 & - \\
\hline $\mathbf{T S}_{1-2 \mathrm{Br}}$ & 2.03 & 2.08 & 2.20 & - & 2.24 & 1.69 & 1.89 & 2.22 & - \\
\hline 2 & 2.04 & 2.16 & 2.15 & - & 2.15 & 1.61 & 1.90 & 2.21 & - \\
\hline $2-\mathrm{Br}$ & 2.03 & 2.16 & 2.15 & - & 2.15 & 1.61 & 1.90 & 2.22 & - \\
\hline 3 & 1.94 & 2.02 & - & 2.32 & - & 1.68 & 1.85 & - & - \\
\hline $\mathbf{T S}_{3-4}$ & 1.97 & 2.02 & - & 2.13 & - & 1.79 & 1.85 & - & - \\
\hline 4 & 2.02 & 2.01 & - & 2.02 & - & 1.77 & 1.88 & - & - \\
\hline 5 & 1.92 & 2.01 & - & - & - & 1.62 & 1.85 & - & - \\
\hline $\mathbf{T S}_{5-6}$ & 2.12 & 2.08 & - & - & 2.14 & $1.61,1.64$ & 1.85 & - & - \\
\hline 6 & 2.08 & 2.20 & - & - & 2.14 & $1.60,1.63$ & 1.83 & - & - \\
\hline 7-B & 2.01 & - & - & 2.46 & - & $1.58,1.70$ & 1.83 & - & - \\
\hline TSA $_{7-5}$ & 1.96 & - & - & 2.12 & - & $1.62,1.90$ & 1.85 & - & - \\
\hline 24 & 1.94 & - & - & - & - & $1.57,1.74,1.77$ & -2.15 & - & 2.2 \\
\hline
\end{tabular}


Table S19. The absolute energies (in Hartree) for for the geometries with 3-Br-pyridine as the ligand for dehydrogenation of $\mathrm{HOCH}_{2} \mathrm{OH}$ to $\mathrm{HCOOH}$ by the M06-L method.

\begin{tabular}{|c|c|c|c|c|}
\hline & $\mathbf{E}$ & $\mathrm{E}+\mathrm{ZPE}$ & G & $E_{\text {soln-pcm }}$ \\
\hline 0I-Br & -1932.351199 & -1931.644465 & -1931.723824 & -1932.369415 \\
\hline $\mathrm{CO}_{2}$ & -188.576690 & -188.564836 & -188.585546 & -188.579002 \\
\hline НСООН & -189.743494 & -189.709396 & -189.733473 & -189.749773 \\
\hline $\mathrm{HOCH}_{2} \mathrm{OH}$ & -190.917015 & -190.859958 & -190.885138 & -190.925268 \\
\hline TSI $_{0-1 \mathrm{Br}}$ & -1932.319961 & -1931.615245 & -1931.693812 & -1932.341355 \\
\hline 1I-Br & -1932.321802 & -1931.619739 & -1931.700702 & -1932.345358 \\
\hline TSI $_{1-2 \mathrm{Br}}$ & -1932.320648 & -1931.620899 & -1931.700283 & -1932.343904 \\
\hline 2I-Br & -1932.335065 & -1931.632885 & -1931.712043 & -1932.359253 \\
\hline 5 I & -1481.552776 & -1480.966738 & -1481.033891 & -1481.566980 \\
\hline TSI-1 1-2Br & -1932.322757 & -1931.622311 & -1931.701996 & -1932.342778 \\
\hline TSI-21-2Br & -1932.316803 & -1931.616186 & -1931.695002 & -1932.340589 \\
\hline TSI-31-2Br & -1932.321234 & -1931.619698 & -1931.697569 & -1932.342440 \\
\hline TSI-41-2Br & -1932.320803 & -1931.619449 & -1931.697816 & -1932.341579 \\
\hline TSI-51-2Br & -1932.321160 & -1931.619731 & -1931.697044 & -1932.342210 \\
\hline OI-Br' & -1932.341034 & -1931.634117 & -1931.715867 & .360803 \\
\hline TSI' $_{0-1 \mathrm{Br}}$ & -1932.322553 & -1931.616858 & -1931.695664 & -1932.341219 \\
\hline 1I-Br' & -1932.324954 & -1931.621665 & -1931.701933 & -1932.345942 \\
\hline TSI' $_{1-2 \mathrm{Br}}$ & -1932.323278 & -1931.623675 & -1931.704699 & -1932.344069 \\
\hline 2I-Br' & -1932.336135 & -1931.633517 & -1931.712847 & -1932.358226 \\
\hline TSI-1'1-2Br & -1932.317177 & -1931.617698 & -1931.699873 & -1932.340281 \\
\hline TSI-2' ${ }_{1-2 \mathrm{Br}}$ & -1932.321390 & -1931.621334 & -1931.702078 & -1932.341829 \\
\hline TSI-3' $_{1-2 \mathrm{Br}}$ & -1932.321497 & -1931.620794 & -1931.698396 & -1932.344262 \\
\hline TSI-4' ${ }_{1-2 \mathrm{Br}}$ & -1932.321835 & -1931.619507 & -1931.697071 & -1932.342842 \\
\hline TSI-5' ${ }_{1-2 \mathrm{Br}}$ & -1932.320750 & -1931.619621 & -1931.698784 & -1932.339999 \\
\hline TSI-6' ${ }_{1-2 \mathrm{Br}}$ & -1932.316387 & -1931.615438 & -1931.694861 & -1932.338109 \\
\hline 3I & -1788.228803 & -1787.527564 & -1787.603771 & -1788.243812 \\
\hline TSI3-4 & -1788.195445 & -1787.502431 & -1787.578116 & -1788.212554 \\
\hline $4 I$ & -1788.212693 & -1787.513928 & -1787.589682 & -1788.231070 \\
\hline TSI-13-4 & -1788.192222 & -1787.499295 & -1787.575941 & -1788.210966 \\
\hline TSI-23-4 & -1788.191055 & -1787.498170 & -1787.575335 & -1788.208651 \\
\hline TSI-33-4 & -1788.195756 & -1787.501976 & -1787.576661 & -1788.211002 \\
\hline TSI-43-4 & -1788.191836 & -1787.498285 & -1787.573172 & -1788.209871 \\
\hline TSI-53-4 & -1788.195260 & -1787.501082 & -1787.574783 & -1788.210502 \\
\hline TSI-63-4 & -1788.186156 & -1787.493362 & -1787.569530 & -1788.205678 \\
\hline TSI-73-4 & -1788.195995 & -1787.501243 & -1787.574362 & -1788.210404 \\
\hline $3 \mathbf{I}_{\mathbf{w}}$ & -1748.934965 & -1748.263079 & -1748.338756 & -1748.953676 \\
\hline TSI $_{w 3-4}$ & -1748.901328 & -1748.235863 & -1748.309126 & -1748.920247 \\
\hline $4 I_{w}$ & -1748.918390 & -1748.247364 & -1748.321113 & -1748.938084 \\
\hline TSI-1 ${ }_{\mathrm{w} 3-4}$ & -1748.901000 & -1748.235413 & -1748.308727 & -1748.920361 \\
\hline TSI-2 ${ }_{\mathrm{w} 3-4}$ & -1748.901069 & -1748.236116 & -1748.308904 & -1748.918346 \\
\hline TSI-3 ${ }_{\mathrm{w} 3-4}$ & -1748.895881 & -1748.231112 & -1748.305055 & -1748.916711 \\
\hline
\end{tabular}


$\begin{array}{lllll}\text { TSI-4 }_{\text {w3-4 }} & -1748.895779 & -1748.230978 & -1748.304103 & -1748.916734\end{array}$

$\begin{array}{llllll}\text { TSI-5 }_{\text {w3-4 }} & -1748.900552 & -1748.235192 & -1748.308108 & -1748.916518\end{array}$

$\begin{array}{llllll}\text { TSI-6 }_{w 3-4} & -1748.896555 & -1748.230428 & -1748.302575 & -1748.915217\end{array}$

$\begin{array}{llllll}\text { TSI-7 }_{\text {w3-4 }} & -1748.901902 & -1748.234582 & -1748.304965 & -1748.916988\end{array}$ 
Table S20. The relative energies (in $\mathrm{kcal} / \mathrm{mol}$ ) for the geometries with 3-Br-pyridine as the ligand for dehydrogenation of $\mathrm{HOCH}_{2} \mathrm{OH}$ to $\mathrm{HCOOH}$ by the M06-L method. The relative free energies in solution with the standard state correction $\left(\Delta \mathrm{G}_{\text {soln-pcm-cor }}\right)$ are also given.

\begin{tabular}{|c|c|c|c|c|c|c|}
\hline & $\Delta \mathbf{E}$ & $\Delta \mathbf{E}+\mathrm{ZPE}$ & $\Delta \mathbf{G}$ & $\Delta \mathbf{E}_{\text {soln-pcm }}$ & $\Delta \mathbf{G}_{\text {soln-pem }}$ & $\Delta \mathbf{G}_{\text {soln-pcm-cor }}$ \\
\hline OI-Br & 0.0 & 0.0 & 0.0 & 0.0 & 0.0 & 0.0 \\
\hline 3-Br-py & 0.0 & 0.0 & 0.0 & 0.0 & 0.0 & 0.0 \\
\hline $\mathbf{H}_{2}$ & 0.0 & 0.0 & 0.0 & 0.0 & 0.0 & 0.0 \\
\hline МeOH & 0.0 & 0.0 & 0.0 & 0.0 & 0.0 & 0.0 \\
\hline $\mathrm{CO}_{2}$ & 0.0 & 0.0 & 0.0 & 0.0 & 0.0 & 0.0 \\
\hline НСООН & 0.0 & 0.0 & 0.0 & 0.0 & 0.0 & 0.0 \\
\hline $\mathrm{HOCH}_{2} \mathrm{OH}$ & 0.0 & 0.0 & 0.0 & 0.0 & 0.0 & 0.0 \\
\hline $\mathrm{H}_{2} \mathrm{O}$ & 0.0 & 0.0 & 0.0 & 0.0 & 0.0 & 0.0 \\
\hline TSI $_{0-1 \mathrm{Br}}$ & 19.6 & 18.3 & 18.8 & 17.6 & 16.8 & 16.8 \\
\hline 1I-Br & 18.4 & 15.5 & 14.5 & 15.1 & 11.2 & 11.2 \\
\hline TSI $_{1-2 \mathrm{Br}}$ & 19.2 & 14.8 & 14.8 & 16.0 & 11.6 & 11.6 \\
\hline 2I-Br & 10.1 & 7.3 & 7.4 & 6.4 & 3.6 & 3.6 \\
\hline $5 I$ & 21.4 & 16.4 & -10.5 & 17.3 & -14.6 & -10.8 \\
\hline TSI-1 1-2Br $_{1}$ & 17.8 & 13.9 & 13.7 & 16.7 & 12.6 & 12.6 \\
\hline TSI-21-2Br & 21.6 & 17.7 & 18.1 & 18.1 & 14.6 & 14.6 \\
\hline TSI-31-2Br & 18.8 & 15.5 & 16.5 & 16.9 & 14.6 & 14.6 \\
\hline TSI-41-2Br & 19.1 & 15.7 & 16.3 & 17.5 & 14.7 & 14.7 \\
\hline TSI-51-2Br & 18.8 & 15.5 & 16.8 & 17.1 & 15.0 & 15.0 \\
\hline 0I-Br' & 6.4 & 6.5 & 5.0 & 5.4 & 4.0 & 4.0 \\
\hline $\mathrm{TSI}_{0-1 \mathrm{Br}}$ & 18.0 & 17.3 & 17.7 & 17.7 & 17.4 & 17.4 \\
\hline 1I-Br' & 16.5 & 14.3 & 13.7 & 14.7 & 12.0 & 12.0 \\
\hline $\mathrm{TSI}_{1-2 \mathrm{Br}}$ & 17.5 & 13.0 & 12.0 & 15.9 & 10.4 & 10.4 \\
\hline 2I-Br' & 9.5 & 6.9 & 6.9 & 7.0 & 4.5 & 4.5 \\
\hline TSI-1'1-2Br & 21.3 & 16.8 & 15.0 & 18.3 & 12.0 & 12.0 \\
\hline TSI-2' ${ }_{1-2 \mathrm{Br}}$ & 18.7 & 14.5 & 13.6 & 17.3 & 12.3 & 12.3 \\
\hline TSI-3' $_{1-2 \mathrm{Br}}$ & 18.6 & 14.9 & 16.0 & 15.8 & 13.1 & 13.1 \\
\hline TSI-4' ${ }_{1-2 \mathrm{Br}}$ & 18.4 & 15.7 & 16.8 & 16.7 & 15.0 & 15.0 \\
\hline TSI-5' ${ }_{1-2 \mathrm{Br}}$ & 19.1 & 15.6 & 15.7 & 18.5 & 15.1 & 15.1 \\
\hline TSI-6' ${ }_{1-2 \mathrm{Br}}$ & 21.8 & 18.2 & 18.2 & 19.6 & 16.0 & 16.0 \\
\hline $3 I$ & -14.0 & -14.9 & -17.4 & -9.9 & -13.3 & -15.2 \\
\hline TSI $_{3-4}$ & 6.9 & 0.8 & -1.3 & 9.7 & 1.5 & -0.4 \\
\hline $4 I$ & -3.9 & -6.4 & -8.5 & -1.9 & -6.5 & -8.4 \\
\hline TSI-13-4 & 9.0 & 2.8 & 0.1 & 10.7 & 1.9 & 0.0 \\
\hline TSI-23-4 & 9.7 & 3.5 & 0.5 & 12.2 & 3.0 & 1.1 \\
\hline TSI-33-4 & 6.7 & 1.1 & -0.4 & 10.7 & 3.6 & 1.7 \\
\hline TSI-43-4 & 9.2 & 3.4 & 1.8 & 11.4 & 4.0 & 2.1 \\
\hline TSI-53-4 & 7.1 & 1.7 & 0.8 & 11.0 & 4.8 & 2.9 \\
\hline TSI-63-4 & 12.8 & 6.5 & 4.1 & 14.1 & 5.4 & 3.5 \\
\hline TSI-73-4 & 6.6 & 1.6 & 1.1 & 11.1 & 5.6 & 3.7 \\
\hline
\end{tabular}




\begin{tabular}{ccccccc} 
3I $_{\mathbf{w}}$ & -12.6 & -13.3 & -18.6 & -9.9 & -15.9 & -18.3 \\
TSI $_{\mathbf{w} 3-4}$ & 8.5 & 3.8 & 0.0 & 11.1 & 2.5 & 0.1 \\
4I $_{\mathbf{w}}$ & -2.2 & -3.4 & -7.5 & -0.1 & -5.5 & -7.9 \\
TSI-1 $_{\mathbf{w} 3-4}$ & 8.7 & 4.1 & 0.2 & 11.0 & 2.5 & 0.1 \\
TSI-2 $_{\mathbf{w} 3-4}$ & 8.7 & 3.6 & 0.1 & 12.3 & 3.7 & 1.3 \\
TSI-3 $_{\mathbf{w 3}-4}$ & 12.0 & 6.8 & 2.5 & 13.3 & 3.9 & 1.5 \\
TSI-4 $_{\mathbf{w 3}-4}$ & 12.0 & 6.9 & 3.1 & 13.3 & 4.4 & 2.0 \\
TSI-5 $_{\mathbf{w} 3-4}$ & 9.0 & 4.2 & 0.6 & 13.4 & 5.0 & 2.6 \\
TSI-6 $_{\mathbf{w 3}-4}$ & 11.5 & 7.2 & 4.1 & 14.2 & 6.8 & 4.4 \\
TSI-7 $_{\mathbf{w 3}-4}$ & 8.2 & 4.6 & 2.6 & 13.1 & 7.5 & 5.1 \\
\hline
\end{tabular}


Table S21. The absolute energies (in Hartree) for for the geometries with 3-Br-pyridine as the ligand for dehydrogenation of $\mathrm{HCOOH}$ to $\mathrm{CO}_{2}$ by the M06-L method.

\begin{tabular}{|c|c|c|c|c|}
\hline & $\mathbf{E}$ & E+ZPE & G & $\mathbf{E}_{\text {soln-pcm }}$ \\
\hline 0II-Br & -1930.021943 & -1929.361105 & -1929.440717 & -1930.044185 \\
\hline TSII $_{0-1 \mathrm{Br}}$ & -1929.992438 & -1929.332276 & -1929.411549 & -1930.016277 \\
\hline 1II-Br & -1930.002143 & -1929.341776 & -1929.420329 & -1930.022882 \\
\hline TSII1-2Br & -1930.000642 & -1929.343733 & -1929.422680 & -1930.021527 \\
\hline 2II-Br & -1930.008677 & -1929.350846 & -1929.431762 & -1930.030331 \\
\hline 0II-Br' & -1930.020750 & -1929.358980 & -1929.437315 & -1930.043309 \\
\hline TSII' $_{0-1 \mathrm{Br}}$ & -1929.993349 & -1929.332628 & -1929.411212 & -1930.016926 \\
\hline 1II-Br' & -1930.002587 & -1929.343712 & -1929.423901 & -1930.024124 \\
\hline TSII' ${ }_{1-2 B r}$ & -1930.001789 & -1929.344364 & -1929.422893 & -1930.022909 \\
\hline 2II-Br' & -1930.008823 & -1929.351959 & -1929.433259 & 30830 \\
\hline 5 II & -1480.375057 & -1479.812745 & -1479.881310 & -1480.399619 \\
\hline TSII-1 1-2Br & -1929.992616 & -1929.336126 & -1929.414354 & -1930.017355 \\
\hline TSII-1'1-2Br & -1929.993034 & -1929.336498 & -1929.415490 & -1930.017525 \\
\hline 3II & -1785.858033 & -1785.205331 & -1785.282982 & -1785.878282 \\
\hline TSII3-4 & -1785.839209 & -1785.192370 & -1785.267612 & -1785.863086 \\
\hline $4 I I$ & -1785.857016 & -1785.206215 & -1785.283079 & -1785.882835 \\
\hline TSII-13-4 & -1785.843108 & -1785.195644 & -1785.270403 & -1785.860235 \\
\hline TSII-23-4 & -1785.839533 & -1785.192350 & -1785.267955 & -1785.858411 \\
\hline TSII-33-4 & -1785.829668 & -1785.183983 & -1785.259397 & -1785.857019 \\
\hline $3 I_{w}$ & -1746.565844 & -1745.940816 & -1746.015039 & -1746.588570 \\
\hline TSII $_{w 3-4}$ & -1746.544539 & -1745.925517 & -1745.997591 & -1746.570014 \\
\hline $4 I_{w}$ & -1746.564767 & -1745.941745 & -1746.014114 & -1746.591754 \\
\hline TSII-1 $_{\mathrm{w} 3-4}$ & -1746.548538 & -1745.930084 & -1746.001926 & -1746.567203 \\
\hline TSII-2 ${ }_{\mathrm{w} 3-4}$ & -1746.535724 & -1745.917824 & -1745.990721 & -1746.563953 \\
\hline TSII-3 $_{\text {w3-4 }}$ & -1746.542639 & -1745.924534 & -1745.996543 & -1746.563853 \\
\hline
\end{tabular}


Table S22. The relative energies (in $\mathrm{kcal} / \mathrm{mol}$ ) for the geometries with 3-Br-pyridine as the ligand for dehydrogenation of $\mathrm{HCOOH}$ to $\mathrm{CO}_{2}$ by the M06-L method. The relative free energies in solution with the standard state correction $\left(\Delta \mathrm{G}_{\text {soln-pcm-cor }}\right)$ are also given.

\begin{tabular}{|c|c|c|c|c|c|c|}
\hline & $\Delta \mathbf{E}$ & $\Delta \mathrm{E}+\mathrm{ZPE}$ & $\Delta \mathbf{G}$ & $\Delta \mathbf{E}_{\text {soln-pcm }}$ & $\Delta \mathbf{G}_{\text {soln-pcm }}$ & $\Delta \mathbf{G}_{\text {soln-pcm-cor }}$ \\
\hline OII-Br & 0.0 & 0.0 & 0.0 & 0.0 & 0.0 & 0.0 \\
\hline 3-Br-py & 0.0 & 0.0 & 0.0 & 0.0 & 0.0 & 0.0 \\
\hline $\mathbf{H}_{2}$ & 0.0 & 0.0 & 0.0 & 0.0 & 0.0 & 0.0 \\
\hline МeOH & 0.0 & 0.0 & 0.0 & 0.0 & 0.0 & 0.0 \\
\hline $\mathrm{CO}_{2}$ & 0.0 & 0.0 & 0.0 & 0.0 & 0.0 & 0.0 \\
\hline НСООН & 0.0 & 0.0 & 0.0 & 0.0 & 0.0 & 0.0 \\
\hline $\mathrm{HOCH}_{2} \mathrm{OH}$ & 0.0 & 0.0 & 0.0 & 0.0 & 0.0 & 0.0 \\
\hline $\mathrm{H}_{2} \mathrm{O}$ & 0.0 & 0.0 & 0.0 & 0.0 & 0.0 & 0.0 \\
\hline TSII $_{0-1 \mathrm{Br}}$ & 18.5 & 18.1 & 18.3 & 17.5 & 17.3 & 17.3 \\
\hline 1II-Br & 12.4 & 12.1 & 12.8 & 13.4 & 13.7 & 13.7 \\
\hline TSII $_{1-2 \mathrm{Br}}$ & 13.4 & 10.9 & 11.3 & 14.2 & 12.2 & 12.2 \\
\hline 2II-Br & 8.3 & 6.4 & 5.6 & 8.7 & 6.0 & 6.0 \\
\hline 0II-Br' & 0.7 & 1.3 & 2.1 & 0.5 & 1.9 & 1.9 \\
\hline TSII' $_{0-1 \mathrm{Br}}$ & 17.9 & 17.9 & 18.5 & 17.1 & 17.7 & 17.7 \\
\hline 1II-Br' & 12.1 & 10.9 & 10.6 & 12.6 & 11.0 & 11.0 \\
\hline TSII' $_{1-2 \mathrm{Br}}$ & 12.6 & 10.5 & 11.2 & 13.4 & 11.9 & 11.9 \\
\hline 2II-Br' & 8.2 & 5.7 & 4.7 & 8.4 & 4.8 & 4.8 \\
\hline 5 II & 31.0 & 25.9 & 0.4 & 25.4 & -5.2 & -1.4 \\
\hline TSII-11-2Br & 18.4 & 15.7 & 16.5 & 16.8 & 15.0 & 15.0 \\
\hline TSII-1'1-2Br & 18.1 & 15.4 & 15.8 & 16.7 & 14.4 & 14.4 \\
\hline $3 I I$ & 7.8 & 5.7 & 3.9 & 12.4 & 8.5 & 6.6 \\
\hline TSII $3-4$ & 19.6 & 13.8 & 13.6 & 22.0 & 15.9 & 14.0 \\
\hline 4 II & 8.5 & 5.1 & 3.9 & 9.6 & 5.0 & 3.1 \\
\hline TSII-13-4 & 17.2 & 11.8 & 11.8 & 23.8 & 18.4 & 16.5 \\
\hline TSII-23-4 & 19.4 & 13.8 & 13.4 & 24.9 & 18.8 & 16.9 \\
\hline TSII-33-4 & 25.6 & 19.1 & 18.7 & 25.8 & 18.9 & 17.0 \\
\hline $3 I_{w}$ & 8.2 & 7.4 & 4.5 & 12.1 & 8.4 & 6.0 \\
\hline TSII $_{w 3-4}$ & 21.6 & 17.0 & 15.5 & 23.8 & 17.7 & 15.3 \\
\hline $4 I_{w}$ & 8.9 & 6.8 & 5.1 & 10.1 & 6.4 & 4.0 \\
\hline TSII-1 $_{\text {w3-4 }}$ & 19.1 & 14.1 & 12.8 & 25.6 & 19.2 & 16.8 \\
\hline TSII-2 w3-4 & 27.1 & 21.8 & 19.8 & 27.6 & 20.3 & 17.9 \\
\hline TSII-3w3-4 & 22.8 & 17.6 & 16.1 & 27.7 & 21.0 & 18.6 \\
\hline
\end{tabular}


Table S23. The absolute electronic energies (in Hartree) for the key geometries with 3Br-pyridine as the ligand for dehydrogenation of $\mathrm{HOCH}_{2} \mathrm{OH}$ to $\mathrm{HCOOH}$ in solution by different DFT methods.

\begin{tabular}{|c|c|c|c|c|}
\hline & Eв3LYP & EM06 & Epbe0 & $\mathbf{E}_{\text {wB97XD }}$ \\
\hline OI-Br & -1932.667817 & -1931.170917 & -1930.475015 & -1931.999843 \\
\hline 3-Br-py & -261.068369 & -260.845212 & -260.774067 & -260.979860 \\
\hline $\mathrm{CO}_{2}$ & -188.583466 & -188.504535 & -188.384981 & -188.522431 \\
\hline НСОOH & -189.769420 & -189.678147 & -189.565583 & -189.708844 \\
\hline $\mathrm{HOCH}_{2} \mathrm{OH}$ & -190.953534 & -190.855296 & -190.745334 & -190.894349 \\
\hline TSI $_{0-1 \mathrm{Br}}$ & -1932.625539 & -1931.135140 & -1930.435240 & -1931.959183 \\
\hline 1I-Br & -1932.625571 & -1931.142041 & -1930.439174 & -1931.965569 \\
\hline TSI $_{1-2 \mathrm{Br}}$ & -1932.624527 & -1931.140857 & -1930.439058 & -1931.964822 \\
\hline $2 \mathrm{I}-\mathrm{Br}$ & -1932.636512 & -1931.152560 & -1930.450238 & -1931.974853 \\
\hline $5 \mathbf{I}$ & -1481.785440 & -1480.618846 & -1480.094317 & -1481.272108 \\
\hline OI-Br' & -1932.655338 & -1931.158946 & -1930.464215 & -1931.988595 \\
\hline TSI' $_{0-1 \mathrm{Br}}$ & -1932.627990 & -1931.135523 & -1930.438882 & -1931.961417 \\
\hline 1I-Br' & -1932.627825 & -1931.143493 & -1930.441982 & -1931.967355 \\
\hline TSI' $_{1-2 \mathrm{Br}}$ & -1932.626714 & -1931.142325 & -1930.441681 & -1931.966418 \\
\hline 2I-Br' & -1932.638435 & -1931.153621 & -1930.452101 & -1931.977041 \\
\hline $3 I$ & -1788.524255 & -1787.175287 & -1786.487995 & -1787.910155 \\
\hline TSI3-4 & -1788.498501 & -1787.143381 & -1786.460791 & -1787.877923 \\
\hline $4 I$ & -1788.505712 & -1787.158305 & -1786.467899 & -1787.891677 \\
\hline $3 \mathrm{I}_{\mathrm{w}}$ & -1749.223122 & -1747.912597 & -1747.238821 & -1748.624007 \\
\hline TSI $_{w 3-4}$ & -1749.193267 & -1747.877868 & -1747.208178 & -1748.588905 \\
\hline $4 I_{w}$ & -1749.199952 & -1747.892617 & -1747.214650 & -1748.601990 \\
\hline
\end{tabular}


Table S24. The relative electronic and estimated Gibbs free energies (in $\mathrm{kcal} / \mathrm{mol}$ ) for the key geometries with 3-Br-pyridine as the ligand for dehydrogenation of $\mathrm{HOCH}_{2} \mathrm{OH}$ to $\mathrm{HCOOH}$ in solution by different DFT methods.

\begin{tabular}{|c|c|c|c|c|c|c|c|c|}
\hline & $\Delta \mathbf{E}_{\text {B3LYP }}$ & $\Delta \mathbf{E}_{M 06}$ & $\Delta \mathbf{E}_{\text {PBE0 }}$ & $\Delta \mathbf{E}_{\mathrm{wB}} \mathrm{97XD}$ & $\Delta \mathbf{G}_{\text {B3LYP }}$ & $\Delta \mathbf{G}_{\text {M06 }}$ & $\Delta \mathbf{G}_{\text {PBE0 }}$ & $\Delta \mathbf{G}_{\mathrm{wB}}$ \\
\hline 0I-Br & 0.0 & 0.0 & 0.0 & 0.0 & 0.0 & 0.0 & 0.0 & 0.0 \\
\hline 3-Br-py & 0.0 & 0.0 & 0.0 & 0.0 & 0.0 & 0.0 & 0.0 & 0.0 \\
\hline $\mathbf{H}_{2}$ & 0.0 & 0.0 & 0.0 & 0.0 & 0.0 & 0.0 & 0.0 & 0.0 \\
\hline $\mathrm{MeOH}$ & 0.0 & 0.0 & 0.0 & 0.0 & 0.0 & 0.0 & 0.0 & 0.0 \\
\hline $\mathrm{CO}_{2}$ & 0.0 & 0.0 & 0.0 & 0.0 & 0.0 & 0.0 & 0.0 & 0.0 \\
\hline НСOOH & 0.0 & 0.0 & 0.0 & 0.0 & 0.0 & 0.0 & 0.0 & 0.0 \\
\hline $\mathrm{HOCH}_{2} \mathrm{OH}$ & 0.0 & 0.0 & 0.0 & 0.0 & 0.0 & 0.0 & 0.0 & 0.0 \\
\hline $\mathrm{H}_{2} \mathrm{O}$ & 0.0 & 0.0 & 0.0 & 0.0 & 0.0 & 0.0 & 0.0 & 0.0 \\
\hline TSI $_{0-1 \mathrm{Br}}$ & 26.5 & 22.5 & 25.0 & 25.5 & 25.8 & 21.7 & 24.2 & 24.7 \\
\hline 1I-Br & 26.5 & 18.1 & 22.5 & 21.5 & 22.6 & 14.2 & 18.6 & 17.6 \\
\hline TSI $_{1-2 \mathrm{Br}}$ & 27.2 & 18.9 & 22.6 & 22.0 & 22.8 & 14.5 & 18.2 & 17.6 \\
\hline 2I-Br & 19.6 & 11.5 & 15.5 & 15.7 & 16.9 & 8.8 & 12.8 & 12.9 \\
\hline $5 I$ & 28.0 & 18.0 & 25.8 & 24.5 & -3.9 & -13.9 & -6.1 & -7.4 \\
\hline OI-Br' & 7.8 & 7.5 & 6.8 & 7.1 & 6.4 & 6.1 & 5.4 & 5.7 \\
\hline $\mathrm{TSI}_{0-1 \mathrm{Br}}$ & 25.0 & 22.2 & 22.7 & 24.1 & 24.7 & 21.9 & 22.4 & 23.8 \\
\hline 1I-Br' & 25.1 & 17.2 & 20.7 & 20.4 & 22.4 & 14.5 & 18.0 & 17.7 \\
\hline $\mathrm{TSI}_{1-2 \mathrm{Br}}$ & 25.8 & 17.9 & 20.9 & 21.0 & 20.3 & 12.4 & 15.4 & 15.5 \\
\hline 2I-Br' & 18.4 & 10.9 & 14.4 & 14.3 & 15.9 & 8.3 & 11.8 & 11.7 \\
\hline $3 I$ & -7.0 & -9.3 & -8.2 & -8.4 & -10.4 & -12.7 & -11.6 & -11.7 \\
\hline TSI $_{3-4}$ & 9.2 & 10.7 & 8.9 & 11.9 & 1.0 & 2.5 & 0.6 & 3.6 \\
\hline $4 I$ & 4.7 & 1.4 & 4.4 & 3.2 & 0.0 & -3.3 & -0.2 & -1.4 \\
\hline $3 \mathbf{I}_{w}$ & -8.2 & -10.5 & -8.9 & -8.5 & -14.3 & -16.5 & -14.9 & -14.5 \\
\hline TSI $_{w 3-4}$ & 10.5 & 11.3 & 10.3 & 13.6 & 2.0 & 2.7 & 1.8 & 5.0 \\
\hline $4 I_{w}$ & 6.3 & 2.0 & 6.3 & 5.4 & 1.0 & -3.3 & 0.9 & 0.0 \\
\hline
\end{tabular}


Table S25. The corrected relative estimated Gibbs free energies (in $\mathrm{kcal} / \mathrm{mol}$ ) for the key geometries with 3-Br-pyridine as the ligand for dehydrogenation of $\mathrm{HOCH}_{2} \mathrm{OH}$ to $\mathrm{HCOOH}$ in solution by different DFT methods. The relative free energies were corrected with the standard state correction.

\begin{tabular}{|c|c|c|c|c|}
\hline & $\Delta \mathbf{G}_{\text {B3LYP-cor }}$ & $\Delta \mathbf{G}_{\mathbf{M 0 6} \text {-cor }}$ & $\Delta \mathbf{G P B E 0}$-cor & $\Delta \mathbf{G}_{\mathrm{wB}}$ 97XD-cor \\
\hline OI-Br & 0.0 & 0.0 & 0.0 & 0.0 \\
\hline 3-Br-py & 0.0 & 0.0 & 0.0 & 0.0 \\
\hline $\mathbf{H}_{2}$ & 0.0 & 0.0 & 0.0 & 0.0 \\
\hline МeOH & 0.0 & 0.0 & 0.0 & 0.0 \\
\hline $\mathrm{CO}_{2}$ & 0.0 & 0.0 & 0.0 & 0.0 \\
\hline НСООН & 0.0 & 0.0 & 0.0 & 0.0 \\
\hline $\mathrm{HOCH}_{2} \mathrm{OH}$ & 0.0 & 0.0 & 0.0 & 0.0 \\
\hline $\mathrm{H}_{2} \mathrm{O}$ & 0.0 & 0.0 & 0.0 & 0.0 \\
\hline TSI $_{0-1 \mathrm{Br}}$ & 25.8 & 21.7 & 24.2 & 24.7 \\
\hline $1 \mathrm{I}-\mathrm{Br}$ & 22.6 & 14.2 & 18.6 & 17.6 \\
\hline TSI $_{1-2 \mathrm{Br}}$ & 22.8 & 14.5 & 18.2 & 17.6 \\
\hline 2I-Br & 16.9 & 8.8 & 12.8 & 12.9 \\
\hline $5 I$ & -0.1 & -10.1 & -2.3 & -3.6 \\
\hline OI-Br' & 6.4 & 6.1 & 5.4 & 5.7 \\
\hline $\mathrm{TSI}_{0-1 \mathrm{Br}}$ & 24.7 & 21.9 & 22.4 & 23.8 \\
\hline 1I-Br' & 22.4 & 14.5 & 18.0 & 17.7 \\
\hline TSI' $_{1-2 \mathrm{Br}}$ & 20.3 & 12.4 & 15.4 & 15.5 \\
\hline 2I-Br' & 15.9 & 8.3 & 11.8 & 11.7 \\
\hline 3I & -12.3 & -14.6 & -13.5 & -13.6 \\
\hline TSI3-4 & -0.9 & 0.6 & -1.3 & 1.7 \\
\hline $4 \mathrm{I}$ & -1.9 & -5.2 & -2.1 & -3.3 \\
\hline $3 \mathbf{I}_{w}$ & -16.7 & -18.9 & -17.3 & -16.9 \\
\hline TSI $_{w 3-4}$ & -0.4 & 0.3 & -0.6 & 2.6 \\
\hline $4 I_{w}$ & -1.4 & -5.7 & -1.5 & -2.4 \\
\hline
\end{tabular}


Table S26. The absolute electronic energies (in Hartree) for the key geometries with 3Br-pyridine as the ligand for dehydrogenation of $\mathrm{HCOOH}$ to $\mathrm{CO}_{2}$ in solution by different DFT methods.

\begin{tabular}{|c|c|c|c|c|}
\hline & ЕвзLYP & Ем06 & EpвE0 & $\mathbf{E}_{w B 97 X D}$ \\
\hline OII-Br & 930.322497 & -1928.843665 & -1928.140774 & -1929.654327 \\
\hline TSII $_{0-1 \mathrm{Br}}$ & -1930.286151 & -1928.809819 & -1928.105861 & -1929.619088 \\
\hline 1II-Br & -1930.287642 & -1928.816933 & -1928.111245 & -1929.625515 \\
\hline TSII $_{1-2 B r}$ & -1930.287633 & -1928.817637 & -1928.111988 & -1929.625531 \\
\hline 2II-Br & -1930.292329 & -1928.822438 & -1928.115705 & -1929.627085 \\
\hline 0II-Br' & -1930.321126 & -1928.842418 & -1928.139738 & -1929.652804 \\
\hline TSII' $_{0-1 \mathrm{Br}}$ & -1930.286575 & -1928.810358 & -1928.106212 & -1929.619501 \\
\hline 1II-Br' & -1930.289834 & -1928.819674 & -1928.113311 & -1929.627275 \\
\hline TSII' ${ }_{1-2 B r}$ & -1930.288057 & -1928.818326 & -1928.112675 & -1929.625214 \\
\hline 2II-Br' & -1930.292899 & -1928.822775 & -1928.116224 & -1929.627716 \\
\hline $5 \mathrm{II}$ & -1480.611290 & -1479.451851 & -1478.923085 & -1480.098412 \\
\hline $3 I I$ & -1786.138429 & -1784.806492 & -1784.111354 & -1785.522222 \\
\hline TSII $_{3-4}$ & -1786.127369 & -1784.790227 & -1784.100407 & -1785.507401 \\
\hline 4 II & -1786.137110 & -1784.806591 & -1784.109288 & -1785.522946 \\
\hline $3 I_{w}$ & -1746.8 & -1745.543024 & -1744.860868 & -1746.235245 \\
\hline TSII $_{w 3-4}$ & -1746.820089 & -1745.522549 & -1744.846110 & -1746.215971 \\
\hline $4 I I_{w}$ & -1746.833115 & -1745.542356 & -1744.858002 & -1746.235106 \\
\hline
\end{tabular}


Table S27. The relative electronic and estimated Gibbs free energies (in $\mathrm{kcal} / \mathrm{mol}$ ) for the key geometries with 3-Br-pyridine as the ligand for dehydrogenation of $\mathrm{HCOOH}$ to $\mathrm{CO}_{2}$ in solution by different DFT methods.

\begin{tabular}{|c|c|c|c|c|c|c|c|c|}
\hline & $\Delta \mathbf{E}_{B 3 L Y P}$ & $\Delta \mathbf{E}_{\mathbf{M 0 6}}$ & $\Delta \mathrm{E}_{\text {PBE0 }}$ & $\Delta \mathbf{E}_{\mathrm{wB} 97 \mathrm{XD}}$ & $\Delta \mathbf{G}_{\text {B3LYP }}$ & $\Delta \mathbf{G}_{\mathbf{M 0 6}}$ & $\Delta$ GPBE0 & $\Delta \mathbf{G}_{\mathrm{wB}} \mathbf{3 7 \mathrm { XD }}$ \\
\hline 0II-Br & 0.0 & 0.0 & 0.0 & 0.0 & 0.0 & 0.0 & 0.0 & 0.0 \\
\hline 3-Br-py & 0.0 & 0.0 & 0.0 & 0.0 & 0.0 & 0.0 & 0.0 & 0.0 \\
\hline $\mathbf{H}_{2}$ & 0.0 & 0.0 & 0.0 & 0.0 & 0.0 & 0.0 & 0.0 & 0.0 \\
\hline МeOH & 0.0 & 0.0 & 0.0 & 0.0 & 0.0 & 0.0 & 0.0 & 0.0 \\
\hline $\mathrm{CO}_{2}$ & 0.0 & 0.0 & 0.0 & 0.0 & 0.0 & 0.0 & 0.0 & 0.0 \\
\hline НСООН & 0.0 & 0.0 & 0.0 & 0.0 & 0.0 & 0.0 & 0.0 & 0.0 \\
\hline $\mathrm{HOCH}_{2} \mathrm{OH}$ & 0.0 & 0.0 & 0.0 & 0.0 & 0.0 & 0.0 & 0.0 & 0.0 \\
\hline $\mathrm{H}_{2} \mathrm{O}$ & 0.0 & 0.0 & 0.0 & 0.0 & 0.0 & 0.0 & 0.0 & 0.0 \\
\hline TSII $_{0-1 \mathrm{Br}}$ & 22.8 & 21.2 & 21.9 & 22.1 & 22.6 & 21.0 & 21.7 & 21.9 \\
\hline 1II-Br & 21.9 & 16.8 & 18.5 & 18.1 & 22.2 & 17.1 & 18.9 & 18.4 \\
\hline TSII $_{1-2 \mathrm{Br}}$ & 21.9 & 16.3 & 18.1 & 18.1 & 19.8 & 14.3 & 16.0 & 16.0 \\
\hline 2II-Br & 18.9 & 13.3 & 15.7 & 17.1 & 16.2 & 10.6 & 13.0 & 14.4 \\
\hline 0II-Br' & 0.9 & 0.8 & 0.7 & 1.0 & 2.2 & 2.2 & 2.0 & 2.3 \\
\hline TSIII'0-1Br $^{\prime}$ & 22.5 & 20.9 & 21.7 & 21.9 & 23.1 & 21.5 & 22.3 & 22.4 \\
\hline 1II-Br' & 20.5 & 15.1 & 17.2 & 17.0 & 18.9 & 13.5 & 15.6 & 15.4 \\
\hline TSII' $_{1-2 \mathrm{Br}}$ & 21.6 & 15.9 & 17.6 & 18.3 & 20.1 & 14.4 & 16.2 & 16.8 \\
\hline 2III-Br' & 18.6 & 13.1 & 15.4 & 16.7 & 15.0 & 9.6 & 11.9 & 13.1 \\
\hline $5 \mathrm{II}$ & 37.3 & 26.4 & 36.8 & 33.6 & 6.7 & -4.2 & 6.2 & 3.1 \\
\hline 3II & 19.6 & 14.6 & 18.9 & 18.8 & 15.7 & 10.7 & 15.0 & 14.9 \\
\hline TSII $_{3-4}$ & 26.5 & 24.8 & 25.8 & 28.1 & 20.4 & 18.7 & 19.7 & 22.0 \\
\hline $4 I I$ & 20.4 & 14.5 & 20.2 & 18.4 & 15.8 & 9.9 & 15.6 & 13.8 \\
\hline $3 I_{w}$ & 19.8 & 13.8 & 19.1 & 19.2 & 16.1 & 10.1 & 15.4 & 15.5 \\
\hline TSII $_{w 3-4}$ & 29.1 & 26.7 & 28.3 & 31.3 & 23.0 & 20.6 & 22.2 & 25.2 \\
\hline $4 I I_{w}$ & 21.0 & 14.3 & 20.9 & 19.3 & 17.2 & 10.5 & 17.1 & 15.5 \\
\hline
\end{tabular}


Table S28. The corrected relative estimated Gibbs free energies (in $\mathrm{kcal} / \mathrm{mol}$ ) for the key geometries with 3-Br-pyridine as the ligand for dehydrogenation of $\mathrm{HCOOH}$ to $\mathrm{CO}_{2}$ in solution by different DFT methods. The relative free energies were corrected with the standard state correction.

\begin{tabular}{|c|c|c|c|c|}
\hline & $\Delta \mathbf{G}_{\text {B3LYP-cor }}$ & $\Delta \mathbf{G}_{\text {M06-cor }}$ & $\Delta \mathbf{G}_{\mathrm{PBE} 0 \text {-cor }}$ & $\Delta \mathbf{G}_{\mathrm{wB}}$ 97XD-cor \\
\hline OII-Br & 0.0 & 0.0 & 0.0 & 0.0 \\
\hline 3-Br-py & 0.0 & 0.0 & 0.0 & 0.0 \\
\hline $\mathbf{H}_{2}$ & 0.0 & 0.0 & 0.0 & 0.0 \\
\hline МeOH & 0.0 & 0.0 & 0.0 & 0.0 \\
\hline $\mathrm{CO}_{2}$ & 0.0 & 0.0 & 0.0 & 0.0 \\
\hline НСОOH & 0.0 & 0.0 & 0.0 & 0.0 \\
\hline $\mathrm{HOCH}_{2} \mathrm{OH}$ & 0.0 & 0.0 & 0.0 & 0.0 \\
\hline $\mathrm{H}_{2} \mathrm{O}$ & 0.0 & 0.0 & 0.0 & 0.0 \\
\hline TSII $_{0-1 \mathrm{Br}}$ & 22.6 & 21.0 & 21.7 & 21.9 \\
\hline 1II-Br & 22.2 & 17.1 & 18.9 & 18.4 \\
\hline TSII $_{1-2 \mathrm{Br}}$ & 19.8 & 14.3 & 16.0 & 16.0 \\
\hline 2II-Br & 16.2 & 10.6 & 13.0 & 14.4 \\
\hline 0II-Br' & 2.2 & 2.2 & 2.0 & 2.3 \\
\hline TSII' $_{0-1 \mathrm{Br}}$ & 23.1 & 21.5 & 22.3 & 22.4 \\
\hline 1II-Br' & 18.9 & 13.5 & 15.6 & 15.4 \\
\hline TSII' $_{1-2 \mathrm{Br}}$ & 20.1 & 14.4 & 16.2 & 16.8 \\
\hline 2II-Br' & 15.0 & 9.6 & 11.9 & 13.1 \\
\hline 5 II & 10.5 & -0.4 & 10.0 & 6.9 \\
\hline $3 I I$ & 13.8 & 8.8 & 13.1 & 13.0 \\
\hline TSII3-4 & 18.5 & 16.8 & 17.8 & 20.1 \\
\hline $4 I I$ & 13.9 & 8.0 & 13.7 & 11.9 \\
\hline $3 I_{w}$ & 13.7 & 7.7 & 13.0 & 13.1 \\
\hline TSII $_{w 3-4}$ & 20.6 & 18.2 & 19.8 & 22.8 \\
\hline $4 \mathrm{II}_{\mathrm{w}}$ & 14.8 & 8.1 & 14.7 & 13.1 \\
\hline
\end{tabular}


Table S29. The absolute electronic energies (in Hartree) for the key geometries with 3Br-pyridine as the ligand for dehydrogenation of $\mathrm{MeOH}$ in solution by PCM M06-L/6$311+\mathrm{G}^{* *+\mathrm{SDD}} / / \mathrm{M} 06-\mathrm{L} / 6-31 \mathrm{G}^{* *+}+\mathrm{SDD}$ method.

\begin{tabular}{|c|c|}
\hline & $E_{\text {soln-pcm-B }}$ \\
\hline $\mathrm{CH}_{2} \mathrm{O}$ & -114.526284 \\
\hline 3-Br-py & -261.079385 \\
\hline $\mathbf{H}_{2}$ & -1.171641 \\
\hline МeOH & -115.744392 \\
\hline $\mathrm{H}_{2} \mathrm{O}$ & -76.448404 \\
\hline $\mathbf{O H}^{-}$ & -75.931338 \\
\hline $\mathrm{CH}_{3} \mathrm{O}^{-}$ & -115.225941 \\
\hline $\mathrm{CO}_{2}$ & -188.633647 \\
\hline НСOOH & -189.805081 \\
\hline $\mathrm{HOCH}_{2} \mathrm{OH}$ & -190.985422 \\
\hline 0-Br' & -1782.278077 \\
\hline $\mathrm{TS}_{1-2 \mathrm{Br}}$ & -1782.247193 \\
\hline $\mathrm{TS}_{3-4}$ & -1638.093602 \\
\hline $\mathbf{T S}_{\mathrm{w} 3-4}$ & -1598.795767 \\
\hline TS5-6 & -1406.582401 \\
\hline TSA $_{7-5}$ & -1523.528798 \\
\hline $\mathrm{TSB}_{7-5}$ & -1523.526237 \\
\hline $\mathrm{TSC}_{7-5}$ & -1407.763911 \\
\hline 0I-Br & -1932.773109 \\
\hline TSI $_{0-1 B r}$ & -1932.745201 \\
\hline TSI $_{1-2 B r}$ & -1932.749276 \\
\hline 5I & -1481.861932 \\
\hline 3I & -1788.628121 \\
\hline $\mathrm{TSI}_{3-4}$ & -1788.596191 \\
\hline $3 \mathbf{I}_{\mathrm{w}}$ & -1749.330553 \\
\hline TSI $_{\text {w3-4 }}$ & -1749.296288 \\
\hline 0II-Br & -1930.446305 \\
\hline TSII0-1Br & -1930.417210 \\
\hline TSII $_{1-2 B r}$ & -1930.422829 \\
\hline $5 \mathrm{II}$ & -1480.697744 \\
\hline 3II & -1786.262103 \\
\hline TSII $_{3-4}$ & -1786.246879 \\
\hline $3 I_{w}$ & -1746.964893 \\
\hline TSII $_{\mathrm{w} 3-4}$ & -1746.945128 \\
\hline
\end{tabular}


Table S30. The relative energies (in $\mathrm{kcal} / \mathrm{mol}$ ) for the key geometries with 3-Brpyridine as the ligand for dehydrogenation of $\mathrm{MeOH}$ in solution by PCM M06-L/6-

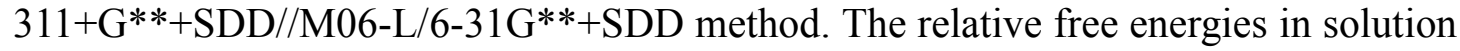
with the standard state correction $\left(\Delta \mathbf{G}_{\text {soln-cor-B }}\right)$ are also given.

\begin{tabular}{cccc}
\hline & $\Delta \mathbf{E}_{\text {soln-pcm-B }}$ & $\Delta \mathbf{G}_{\text {soln-pcm-B }}$ & $\Delta \mathbf{G}_{\text {soln-cor-B }}$ \\
\hline $\mathrm{CH}_{2} \mathbf{O}$ & 0.0 & 0.0 & 0.0 \\
3-Br-py & 0.0 & 0.0 & 0.0 \\
$\mathbf{H}_{2}$ & 0.0 & 0.0 & 0.0 \\
$\mathrm{MeOH}$ & 0.0 & 0.0 & 0.0 \\
$\mathbf{H}_{2} \mathbf{O}$ & 0.0 & 0.0 & 0.0 \\
$\mathbf{O H}^{-}$ & 0.0 & 0.0 & 0.0 \\
$\mathbf{C H}_{3} \mathbf{O}^{-}$ & 0.0 & 0.0 & 0.0 \\
$\mathbf{C O}_{2}$ & 0.0 & 0.0 & 0.0 \\
$\mathrm{HCOOH}^{-0}$ & 0.0 & 0.0 & 0.0 \\
$\mathbf{H O C H}_{2} \mathbf{O H}$ & 0.0 & 0.0 & 0.0 \\
\hline
\end{tabular}

\section{Dehydrogenation of $\mathrm{MeOH}$ to $\mathrm{HCHO}$ and $\mathrm{H}_{2}$}

\begin{tabular}{|c|c|c|c|}
\hline 0-Br' & 0.0 & 0.0 & 0.0 \\
\hline $\mathrm{TS}_{1-2 \mathrm{Br}}$ & 19.4 & 18.2 & 18.2 \\
\hline TS $_{3-4}$ & 42.4 & 34.7 & 30.9 \\
\hline $\mathbf{T S}_{\mathrm{w3-4}}$ & 43.6 & 36.6 & 32.3 \\
\hline $\mathbf{T S}_{3 \mathrm{~A}-4 \mathrm{~A}}$ & 53.0 & 33.0 & 33.0 \\
\hline $\mathbf{T S}_{5-6}$ & 56.5 & 23.3 & 27.1 \\
\hline TSA $7-5$ & 66.6 & 42.3 & 40.4 \\
\hline $\mathbf{T S B}_{7-5}$ & 68.2 & 44.1 & 42.2 \\
\hline $\mathrm{TSC}_{7-5}$ & 79.4 & 41.5 & 43.4 \\
\hline \multicolumn{4}{|c|}{ Dehydrogenation of $\mathrm{HOCH}_{2} \mathrm{OH}$ to $\mathrm{HCOOH}$ and $\mathrm{H}_{2}$} \\
\hline 0I-Br & 0.0 & 0.0 & 0.0 \\
\hline TSI $_{0-1 B r}$ & 17.5 & 16.7 & 16.7 \\
\hline TSI 1-2Br & 15.0 & 10.6 & 10.6 \\
\hline $5 I$ & 16.8 & -15.1 & -11.3 \\
\hline 3I & -6.1 & -9.4 & -11.3 \\
\hline TSI3-4 & 14.0 & 5.8 & 3.9 \\
\hline $3 \mathbf{I}_{w}$ & -5.1 & -11.1 & -13.5 \\
\hline TSI $_{\text {w3-4 }}$ & 16.4 & 7.9 & 5.5 \\
\hline \multicolumn{4}{|c|}{ Dehydrogenation of $\mathrm{HCOOH}$ to $\mathrm{CO}_{2}$ and $\mathrm{H}_{2}$} \\
\hline OII-Br & 0.0 & 0.0 & 0.0 \\
\hline TSII $_{0-1 \mathrm{Br}}$ & 18.3 & 18.0 & 18.0 \\
\hline TSII $_{1-2 B r}$ & 14.7 & 12.7 & 12.7 \\
\hline 5 II & 22.3 & -8.3 & -4.5 \\
\hline 3II & 13.0 & 9.0 & 7.1 \\
\hline TSII3-4 & 22.5 & 16.4 & 14.5 \\
\hline
\end{tabular}




\begin{tabular}{cccc}
\hline 3II $_{\mathbf{w}}$ & 13.7 & 10.0 & 7.6 \\
TSII $_{\mathbf{w} 3-4}$ & 26.1 & 20.0 & 17.6 \\
\hline
\end{tabular}

Table S31. The absolute electronic energies (in Hartree) for the key geometries with pyridine as the ligand for dehydrogenation of $\mathrm{MeOH}$ in solution by PCM M06-L/6-

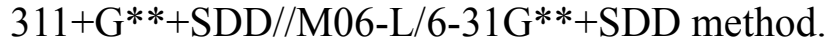

\begin{tabular}{cc} 
& Esoln-pcm-B \\
\hline py & -248.314724 \\
$\mathbf{0}$ & -1769.512740 \\
TS $_{34-4 A}$ & -1522.332404 \\
TS $_{\text {3Apy-4Apy }}$ & -1770.668073 \\
TS8-9 & -1654.905050 \\
$\mathbf{7 - p y}$ & -1540.367030 \\
$\mathbf{1 0}$ & -1521.873023 \\
TS $_{10-11}$ & -1521.824590 \\
$\mathbf{1 3}$ & -1654.946718 \\
TS $_{2-14}$ & -1654.909675 \\
TS $_{15-16}$ & -1656.118103 \\
TS2-17 $_{2}$ & -1406.568057 \\
TS $_{20-21}$ & -1521.091578 \\
\hline
\end{tabular}


Table S32. The relative energies (in $\mathrm{kcal} / \mathrm{mol}$ ) for the key geometries with pyridine as the ligand for dehydrogenation of $\mathrm{MeOH}$ in solution by PCM M06-L/6-

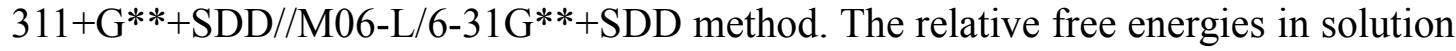
with the standard state correction $\left(\Delta \mathbf{G}_{\text {soln-cor-B }}\right)$ are also given.

\begin{tabular}{|c|c|c|c|}
\hline & $\Delta \mathbf{E}_{\text {soln-pcm-B }}$ & $\Delta \mathbf{G}_{\text {soln-pcm-B }}$ & $\Delta \mathbf{G}_{\text {soln-cor-B }}$ \\
\hline $\mathrm{CH}_{2} \mathrm{O}$ & 0.0 & 0.0 & 0.0 \\
\hline py & 0.0 & 0.0 & 0.0 \\
\hline $\mathbf{H}_{2}$ & 0.0 & 0.0 & 0.0 \\
\hline МeOH & 0.0 & 0.0 & 0.0 \\
\hline $\mathrm{H}_{2} \mathrm{O}$ & 0.0 & 0.0 & 0.0 \\
\hline $\mathbf{O H}^{-}$ & 0.0 & 0.0 & 0.0 \\
\hline $\mathrm{CH}_{3} \mathrm{O}^{-}$ & 0.0 & 0.0 & 0.0 \\
\hline 0 & 0.0 & 0.0 & 0.0 \\
\hline TS $_{3-4}$ & 42.0 & 33.5 & 29.7 \\
\hline $\mathbf{T S}_{3 \mathrm{~A}-4 \mathrm{~A}}$ & 52.5 & 31.8 & 31.8 \\
\hline TS3Apy-4Apy & 39.4 & 34.8 & 32.9 \\
\hline TS5-6 & 56.1 & 22.2 & 26.0 \\
\hline TS8-9 & 51.1 & 30.7 & 32.6 \\
\hline 7-py & 58.4 & 26.1 & 29.9 \\
\hline TSA $_{7-5}$ & 66.2 & 41.1 & 39.2 \\
\hline TSB $_{7-5}$ & 67.8 & 42.9 & 41.0 \\
\hline $\mathrm{TSC}_{7-5}$ & 79.0 & 40.3 & 42.2 \\
\hline 10 & 15.5 & -3.0 & -1.1 \\
\hline $\mathrm{TS}_{10-11}$ & 45.9 & 26.7 & 28.6 \\
\hline 13 & 54.1 & 22.6 & 24.5 \\
\hline $\mathbf{T S}_{2-14}$ & 48.2 & 29.9 & 31.8 \\
\hline $\mathrm{TS}_{15-16}$ & 54.3 & 33.7 & 33.7 \\
\hline $\mathbf{T S}_{2-17}$ & 65.1 & 32.7 & 36.5 \\
\hline $\mathbf{T S}_{20-21}$ & 66.8 & 46.6 & 48.5 \\
\hline
\end{tabular}


Table S33. The driving force (in $\mathrm{kcal} / \mathrm{mol}$ ) for the overall catalyzed reaction by PCM M06-L/6-31G**+SDD//M06-L/6-31G**+SDD (method A), PCM M06-L/6-

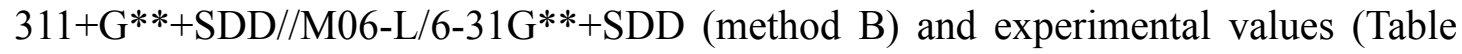
S33).

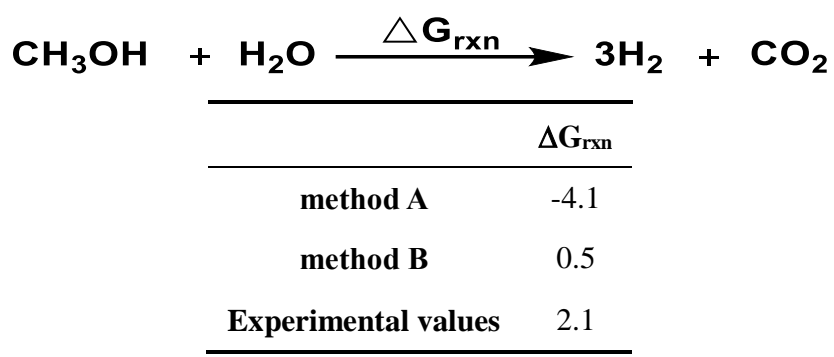

Table S34. Experimental values of standard molar Gibbs free energy (in $\mathrm{kJ} / \mathrm{mol}$ ) for the overall catalyzed reaction taken from ref. 20.

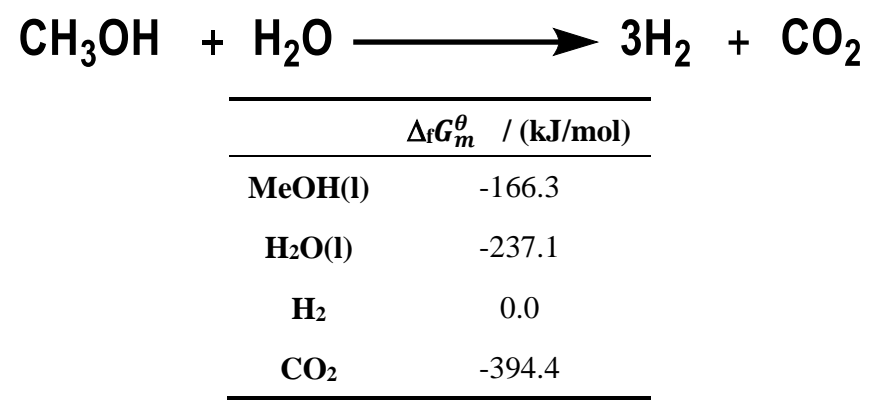

Table S35. The absolute enthalpy energies (in Hartree) for G-I, G-ID, G-II, G-IID and $\mathbf{P C} \mathbf{y}_{3}$ in gas phase by the M06-L method.

\begin{tabular}{cc}
\hline & $\mathbf{H}$ \\
\hline $\mathbf{P C y}_{\mathbf{3}}$ & -1046.589602 \\
$\mathbf{G}-\mathbf{I}$ & -3378.865343 \\
$\mathbf{G}-\mathbf{I}_{\mathbf{D}}$ & -2332.218576 \\
$\mathbf{G}-\mathbf{I I}$ & -3257.146031 \\
$\mathbf{G - I I}$ & -2210.495255 \\
\hline
\end{tabular}


Table S36. Bond dissociation enthalpies (in $\mathrm{kcal} / \mathrm{mol}$ ) for the phosphine ligand in G-I and $\mathbf{G}-\mathbf{I I}$ in gas phase by the M06-L method $\left(\Delta \mathbf{H}_{\mathbf{D F T}}\right)$ and experiments $\left(\Delta \mathbf{H}_{\text {expt }}\right)^{[21]}$

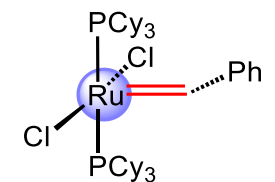

G-I<smiles>CN1CCN(C)C1/C(Cl)=C/P</smiles>

G-II

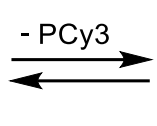

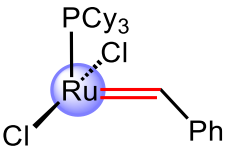

G-I $\mathbf{D}$

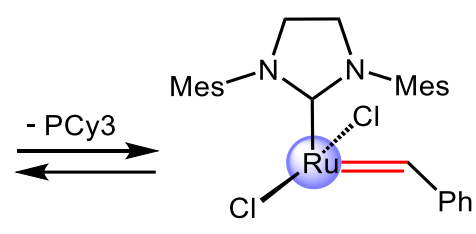

G-IID

\begin{tabular}{ccc}
\hline & $\Delta \mathbf{H}_{\text {DFT }}$ & $\Delta \mathbf{H}_{\text {expt }}$ \\
\hline G-ID & 35.9 & 33.4 \\
\hline G-IID & 38.4 & 36.9
\end{tabular}

Table S37. The absolute (in Hartree) and relative (in $\mathrm{kcal} / \mathrm{mol}$ ) energies for the direct hydride transfer from the methanolate intermediate $\mathbf{2 5}$ to the $\mathrm{Ru}$ metal optimized in solvent by the PCM M06-L method. The relative free energies in solution with the standard state correction ( $\left.\Delta \mathbf{G}_{\text {soln-cor }}\right)$ are also given.

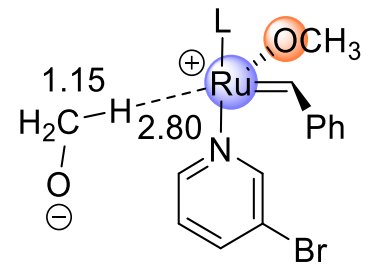

25

32.2

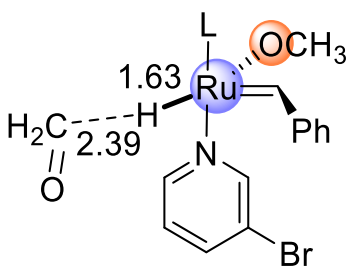

26

13.0

\begin{tabular}{cccccccc}
\hline & $\mathbf{E}_{\text {soln }}$ & $\mathbf{E}+\mathbf{Z P E}$ soln & $\mathbf{G}_{\text {soln }}$ & $\square \Delta \mathbf{E}_{\text {soln }}$ & $\square \Delta \mathbf{E}+\mathbf{Z P E} \mathbf{E}_{\text {soln }}$ & $\square \Delta \mathbf{G}_{\text {soln }}$ & $\Delta \mathbf{G}_{\text {soln-cor }}$ \\
\hline $\mathbf{0 - B \mathbf { r } ^ { \prime } \text { sol }}$ & -1781.921538 & -1781.226460 & -1781.304215 & 0.0 & 0.0 & 0.0 & 0.0 \\
$\mathbf{2 5}$ & -1781.859927 & -1781.169429 & -1781.252905 & 38.7 & 35.8 & 32.2 & 32.2 \\
$\mathbf{2 6}$ & -1781.888530 & -1781.200818 & -1781.283564 & 20.7 & 16.1 & 13.0 & 13.0 \\
\hline
\end{tabular}


Table S38. The absolute (in Hartree) and relative (in $\mathrm{kcal} / \mathrm{mol}$ ) electronic energies for potential energy scan with various $\mathrm{Ru}-\mathrm{H}$ bond distance ( $\mathrm{R}$, in $\AA$ ) in gas and solvent phases by the M06-L and PCM M06-L methods, respectively.

\begin{tabular}{cccccc}
\hline & $\mathbf{R}$ & $\mathbf{E}$ & $\Delta \mathbf{E}$ & $\mathbf{E}_{\text {soln }}$ & $\Delta \mathbf{E}_{\text {soln }}$ \\
\hline $\mathbf{0 - B r ^ { \prime }}$ & 3.3 & -1781.907858 & 0.0 & -1781.921538 & 0.0 \\
$\mathbf{2 5}$ & 2.8 & - & - & -1781.859927 & 38.7 \\
$\mathbf{2 6 - a}$ & 2.8 & -1781.836258 & 44.9 & -1781.859897 & 38.7 \\
$\mathbf{2 6 - b}$ & 2.6 & -1781.841133 & 41.9 & -1781.864924 & 35.5 \\
$\mathbf{2 6 - c}$ & 2.4 & -1781.846327 & 38.6 & -1781.871375 & 31.5 \\
$\mathbf{2 6 - d}$ & 2.2 & -1781.852311 & 34.9 & -1781.877169 & 27.8 \\
$\mathbf{2 6 - e}$ & 2.1 & -1781.855312 & 33.0 & -1781.879989 & 26.1 \\
$\mathbf{2 6 - f}$ & 2.0 & -1781.855722 & 32.7 & -1781.872664 & 30.7 \\
$\mathbf{2 6 - g}$ & 1.9 & -1781.861425 & 29.1 & -1781.878734 & 26.9 \\
$\mathbf{2 6 - h}$ & 1.8 & -1781.867716 & 25.2 & -1781.883993 & 23.6 \\
$\mathbf{2 6}$ & 1.6 & -1781.873483 & 21.6 & -1781.88853 & 20.7 \\
\hline
\end{tabular}

Table S39. The absolute (in Hartree) energies for the 8-m-r pathway in the gas phase optimized by M06-L/6-31G**+SDD method, and corresponding single-point energies in solvent phase by PCM M06-L/6-31G**+SDD//M06-L/6-31G**+SDD and PCM M06-L/6-311+G**+SDD//M06-L/6-31G**+SDD (soln-B) methods.

\begin{tabular}{cccccc}
\hline & $\mathbf{E}$ & $\mathbf{E}+\mathbf{Z P E}$ & $\mathbf{G}$ & $\mathbf{E}_{\text {soln }}$ & $\mathbf{E}_{\text {soln-B }}$ \\
\hline 38-m-r & -1753.503705 & -1752.759738 & -1752.839498 & -1753.517126 & -1753.888023 \\
$\mathbf{T S} 8-m-r$ & -1753.467108 & -1752.731292 & -1752.808154 & -1753.485001 & -1753.855297 \\
48-m-r & -1753.490350 & -1752.747484 & -1752.825142 & -1753.505916 & -1753.878610 \\
4sw8-m-r & -1753.467628 & - & - & -1753.482771 & -1753.855151 \\
\hline
\end{tabular}

Table S40. The relative (in $\mathrm{kcal} / \mathrm{mol}$ ) energies for the $8-\mathrm{m}-\mathrm{r}$ pathway in the gas phase by M06-L/6-31G**+SDD method, and the relative energies in solvent phase by PCM M06-L/6-31G**+SDD//M06-L/6-31G**+SDD and PCM M06-L/6-

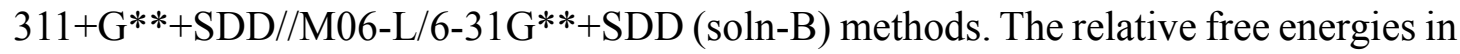
solution with the standard state correction $\left(\boldsymbol{\Delta} \mathbf{G}_{\text {soln-cor }}\right.$ and $\left.\boldsymbol{\Delta} \mathbf{G}_{\text {soln-B-cor }}\right)$ are also given.

\begin{tabular}{|c|c|c|c|c|c|c|c|c|c|}
\hline & $\Delta \mathbf{E}$ & $\Delta \mathbf{E}+\mathrm{ZPE}$ & $\Delta \mathbf{G}$ & $\Delta \mathbf{E}_{\text {soln }}$ & $\Delta \mathbf{E}_{\text {soln-B }}$ & $\Delta \mathbf{G}_{\text {soln }}$ & $\Delta \mathbf{G}_{\text {soln-B }}$ & $\Delta \mathbf{G}_{\text {soln-cor }}$ & $\Delta \mathbf{G}_{\text {soln-B-cor }}$ \\
\hline 38-m-r & 2.8 & 2.4 & 12.1 & 7.3 & 11.0 & 16.6 & 20.4 & 9.0 & 12.8 \\
\hline TS8-m-r & 25.7 & 20.2 & 31.8 & 27.4 & 31.6 & 33.5 & 37.6 & 25.9 & 30.0 \\
\hline 48-m-r & 11.1 & 10.0 & 21.1 & 14.3 & 16.9 & 24.3 & 26.9 & 16.7 & 19.3 \\
\hline 4sw8-m-r & 25.4 & - & - & 28.8 & 31.6 & - & - & - & - \\
\hline
\end{tabular}


Table S41. The calculated H/D KIE value for different key transition states in gas phase at $373.15 \mathrm{~K}$ by the M06-L method.

\begin{tabular}{cc}
\hline & KIE \\
\hline TS $_{\text {1-2Br }}$ & 3.68 \\
TS $_{3-4}$ & 7.94 \\
TS $_{5-6}$ & 2.73 \\
TS $_{\text {3A-4A }}$ & 5.26 \\
TSC $_{7-5}$ & 4.17 \\
TSA $_{7-5}$ & 6.79 \\
\hline
\end{tabular}




\section{Cartesian coordinates of all optimized structures}

$\begin{array}{cccc}\mathrm{CH}_{2} \mathrm{O} & & & \\ \mathrm{C} & 0.000000 & 0.526024 & 0.000000 \\ \mathrm{H} & 0.937868 & 1.128440 & 0.000000 \\ \mathrm{H} & -0.937869 & 1.128433 & 0.000000 \\ \mathrm{O} & 0.000000 & -0.676627 & 0.000000\end{array}$

$\begin{array}{cccc}\text { py } & & & \\ \mathrm{C} & -1.138981 & -0.718169 & -0.000213 \\ \mathrm{C} & -1.194513 & 0.673114 & -0.000124 \\ \mathrm{C} & 0.001542 & 1.382853 & 0.000104 \\ \mathrm{C} & 1.196028 & 0.670514 & 0.000216 \\ \mathrm{C} & 1.137372 & -0.720657 & 0.000113 \\ \mathrm{~N} & -0.001589 & -1.421109 & -0.000092 \\ \mathrm{H} & 0.002697 & 2.469500 & 0.000166 \\ \mathrm{H} & -2.058021 & -1.303228 & -0.000370 \\ \mathrm{H} & -2.152714 & 1.184046 & -0.000251 \\ \mathrm{H} & 2.155367 & 1.179305 & 0.000353 \\ \mathrm{H} & 2.055108 & -1.307798 & 0.000178\end{array}$

$\mathbf{H}_{2}$

$\begin{array}{llll}\mathrm{H} & 0.000000 & 0.000000 & 0.371711\end{array}$

$\begin{array}{llll}\mathrm{H} & 0.000000 & 0.000000 & -0.371711\end{array}$

\section{$\mathrm{MeOH}$}

$\begin{array}{cccc}\text { C } & 0.654739 & -0.019142 & 0.000000 \\ \mathrm{H} & 1.076622 & 0.988324 & -0.000001 \\ \mathrm{H} & 1.039008 & -0.540305 & -0.890336 \\ \mathrm{H} & 1.039008 & -0.540304 & 0.890336 \\ \mathrm{O} & -0.744956 & 0.121087 & 0.000000 \\ \mathrm{H} & -1.123420 & -0.761558 & 0.000000\end{array}$

\section{$\mathrm{H}_{2} \mathrm{O}$}

$\begin{array}{llll}\mathrm{O} & 0.000000 & 0.000000 & 0.118733\end{array}$

$\begin{array}{llll}\mathrm{H} & 0.000000 & 0.754011 & -0.474931\end{array}$

$\begin{array}{llll}\mathrm{H} & 0.000000 & -0.754011 & -0.474931\end{array}$

$\mathrm{OH}^{-}$

$\begin{array}{llll}\text { O } & 0.000000 & 0.000000 & 0.108039\end{array}$

$\begin{array}{llll}\mathrm{H} & 0.000000 & 0.000000 & -0.864312\end{array}$

\section{$\mathrm{CH}_{3} \mathrm{O}^{-}$}

$\begin{array}{llll}\text { O } & 0.000000 & 0.000000 & 0.783910\end{array}$

$$
\begin{array}{rrrr}
\mathrm{C} & 0.000000 & 0.000000 & -0.514727 \\
\mathrm{H} & 0.000000 & 1.026798 & -1.060971 \\
\mathrm{H} & 0.889233 & -0.513399 & -1.060971 \\
\mathrm{H} & -0.889233 & -0.513399 & -1.060971
\end{array}
$$

\section{0}

$\begin{array}{llll}\mathrm{Ru} & -0.331511 & 0.283419 & 0.059150\end{array}$

$\begin{array}{llll}\text { C } & -2.612574 & 2.166303 & 0.287921\end{array}$

$\begin{array}{llll}\text { C } & -0.755975 & 3.463350 & 0.716753\end{array}$

$\begin{array}{llll}\text { C } & -3.473699 & 3.203070 & 0.614171\end{array}$

$\begin{array}{llll}\mathrm{H} & -2.994932 & 1.188728 & -0.013099\end{array}$

$\begin{array}{llll}\text { C } & -1.547519 & 4.556361 & 1.043129\end{array}$

$\begin{array}{llll}\mathrm{H} & 0.328531 & 3.511717 & 0.763805\end{array}$

$\begin{array}{llll}\mathrm{H} & -4.546772 & 3.044665 & 0.569014\end{array}$

$\begin{array}{llll}\mathrm{H} & -1.078522 & 5.488536 & 1.341114\end{array}$

$\begin{array}{llll}\mathrm{N} & -1.272464 & 2.283930 & 0.335062\end{array}$

$\begin{array}{llll}\text { C } & -2.932258 & 4.427090 & 0.995084\end{array}$

$\begin{array}{llll}\mathrm{H} & -3.576191 & 5.262513 & 1.254243\end{array}$

$\begin{array}{llll}\text { C } & -0.035781 & -1.678148 & -0.072562\end{array}$

$\begin{array}{llll}\text { C } & 0.673015 & -3.916393 & -0.190716\end{array}$

$\begin{array}{llll}\text { C } & -0.844595 & -3.895510 & -0.068161\end{array}$

$\begin{array}{llll}\mathrm{H} & 1.008467 & -4.225232 & -1.192393\end{array}$

$\begin{array}{llll}\mathrm{H} & 1.159623 & -4.571068 & 0.539791\end{array}$

$\begin{array}{llll}\mathrm{H} & -1.348633 & -4.494728 & -0.833455\end{array}$

$\begin{array}{llll}\mathrm{H} & -1.186047 & -4.244531 & 0.917835\end{array}$

$\begin{array}{llll}\mathrm{N} & 1.028989 & -2.511374 & 0.043706\end{array}$

$\begin{array}{llll}\mathrm{N} & -1.135389 & -2.472164 & -0.234717\end{array}$

$\begin{array}{llll}\text { C } & 2.395533 & -2.109021 & 0.030743\end{array}$

$\begin{array}{llll}\text { C } & 3.110811 & -2.161945 & 1.233229\end{array}$

$\begin{array}{llll}\text { C } & 2.971613 & -1.636779 & -1.157186\end{array}$

$\begin{array}{llll}\text { C } & 4.434946 & -1.723498 & 1.232073\end{array}$

$\begin{array}{llll}\text { C } & 4.296934 & -1.204188 & -1.107973\end{array}$

$\begin{array}{llll}\text { C } & 5.039867 & -1.230359 & 0.073993\end{array}$

$\begin{array}{llll}\mathrm{H} & 4.999254 & -1.742600 & 2.163863\end{array}$

H $\quad 4.755673 \quad-0.819778 \quad-2.018363$

$\begin{array}{llll}\text { C } & -2.461081 & -1.936637 & -0.253671\end{array}$

$\begin{array}{llll}\text { C } & -3.199322 & -1.819304 & 0.936882\end{array}$

$\begin{array}{llll}\text { C } & -3.004193 & -1.554443 & -1.496056\end{array}$

$\begin{array}{llll}\text { C } & -4.450436 & -1.200749 & 0.870375\end{array}$

$\begin{array}{llll}\text { C } & -4.253831 & -0.933593 & -1.504935\end{array}$

$\begin{array}{llll}\text { C } & -4.982251 & -0.729269 & -0.329887\end{array}$

$\begin{array}{llll}\mathrm{H} & -5.020214 & -1.077559 & 1.790740\end{array}$ 


\begin{tabular}{|c|c|c|c|}
\hline $\mathrm{H}$ & .670444 & 05 & -2.458981 \\
\hline C & 2.168690 & -1.542438 & - 2415005 \\
\hline & .438062 & -0.723454 & -2.362345 \\
\hline & 2.813993 & -1.369679 & -3.279980 \\
\hline & .588563 & -2.454079 & -2.602081 \\
\hline & 2.4 & & 7238 \\
\hline 11 & 2.006799 & 5896 & 2006 \\
\hline $\mathrm{H}$ & 3.103673 & 94 & 0127 \\
\hline $\mathrm{H}$ & 3 & 9 & 2.707886 \\
\hline ( & 6.436383 & -0.689616 & 43 \\
\hline $\mathrm{H}$ & & & 628 \\
\hline $\mathrm{H}$ & 8 & 4 & 92 \\
\hline $\mathrm{H}$ & 7 & 38 & 6056 \\
\hline C & -2.694824 & 359 & 1839 \\
\hline $\mathrm{H}$ & 1 & 1 & 59 \\
\hline $\mathrm{H}$ & -2.928839 & -3442300 & 2.312628 \\
\hline $\mathrm{H}$ & -1.617125 & -2.228 & 2.333 \\
\hline $\mathrm{C}$ & 22 & 1 & 825 \\
\hline $\mathrm{H}$ & -1.323842 & -1.296338 & -2.793585 \\
\hline $\mathrm{H}$ & -2.050102 & 509 & 517 \\
\hline $\mathrm{H}$ & -2.870609 & -1543200 & -3.635180 \\
\hline$a_{-1}$ & -6.314606 & -0.0 & 7916 \\
\hline $\mathrm{H}$ & -6.267955 & 0.895889 & 99 \\
\hline $\mathrm{H}$ & -7.072716 & -0.66 & -0.861685 \\
\hline $\mathrm{H}$ & 66 & 7 & 13 \\
\hline$-\mathrm{cos}$ & 1.416003 & 739458 & 58309 \\
\hline $\mathrm{H}$ & 1.9 & 384 & 5930 \\
\hline $\mathrm{C}$ & 2.208601 & 1860672 & מ 00170 \\
\hline$C$ & 3.417756 & 2.166184 & 28 \\
\hline $\mathrm{C}$ & 1.8623 & 5 & 0335 \\
\hline$C$ & 5 & 0 & 0.242724 \\
\hline $\mathrm{H}$ & 3.708879 & .557023 & .519543 \\
\hline $\mathrm{C}$ & 2.678224 & 3.669177 & -1.555977 \\
\hline $\mathrm{H}$ & .957693 & 318 & 90 \\
\hline $\mathrm{C}$ & 3.854047 & 3.973973 & 9063 \\
\hline $\mathrm{H}$ & 37 & 2 & 0.775995 \\
\hline $\mathrm{H}$ & 2.400046 & 330 & 40 \\
\hline 11 & 4.4859 & 305 & + \\
\hline 0 & .395838 & (5177) & 1008200 \\
\hline $\mathrm{O}$ & -0.912569 & -0.081803 & 1.985954 \\
\hline$a_{1}$ & -1.233441 & 1.385558 & -2.581587 \\
\hline $\mathrm{H}$ & -1.191579 & 4 & 171 \\
\hline $\mathrm{H}$ & -2.301216 & .256376 & -2.301342 \\
\hline $\mathrm{H}$ & .980011 & 2.437529 & $258>-2$ \\
\hline
\end{tabular}

$\begin{array}{rrrr}\mathrm{C} & -0.638847 & 0.837937 & 2.978367 \\ \mathrm{H} & 0.446632 & 1.062004 & 3.061241 \\ \mathrm{H} & -1.143508 & 1.816269 & 2.852131 \\ \mathrm{H} & -0.965830 & 0.442497 & 3.956207\end{array}$

TS0-1

$\begin{array}{llll}\mathrm{Ru} & 0.206639 & 0.560702 & 0.186201\end{array}$

$\begin{array}{llll}\text { C } & 0.451916 & 3.311991 & 1.623484\end{array}$

$\begin{array}{llll}\text { C } & -0.660671 & 3.599786 & -0.376953\end{array}$

$\begin{array}{llll}\text { C } & 0.448285 & 4.679749 & 1.865908\end{array}$

$\begin{array}{llll}\mathrm{H} & 0.891426 & 2.586422 & 2.308899\end{array}$

$\begin{array}{llll}\text { C } & -0.712442 & 4.975022 & -0.202678\end{array}$

$\begin{array}{llll}\mathrm{H} & -1.069795 & 3.124161 & -1.264478\end{array}$

$\begin{array}{llll}\mathrm{H} & 0.906602 & 5.063502 & 2.771791\end{array}$

$\begin{array}{llll}\mathrm{H} & -1.184029 & 5.594295 & -0.958712\end{array}$

$\begin{array}{llll}\mathrm{N} & -0.095960 & 2.770707 & 0.518783\end{array}$

$\begin{array}{llll}\text { C } & -0.145527 & 5.530284 & 0.940324\end{array}$

$\mathrm{H} \quad-0.163058 \quad 6.604436 \quad 1.102710$

$\begin{array}{llll}\text { C } & 0.675978 & -1.377163 & -0.097771\end{array}$

$\begin{array}{llll}\text { C } & 0.434784 & -3.717486 & -0.393046\end{array}$

$\begin{array}{llll}\text { C } & 1.875834 & -3.329600 & -0.675607\end{array}$

$\begin{array}{llll}\mathrm{H} & 0.329707 & -4.313421 & 0.525180\end{array}$

$\begin{array}{llll}\mathrm{H} & -0.031947 & -4.281631 & -1.208019\end{array}$

$\mathrm{H} \quad 2.604284-3.886686 \quad-0.076793$

$\begin{array}{llll}\mathrm{H} & 2.146647 & -3.458308 & -1.732683\end{array}$

$\begin{array}{llll}\mathrm{N} & -0.214301 & -2.414198 & -0.228716\end{array}$

$\begin{array}{llll}\mathrm{N} & 1.907080 & -1.907379 & -0.316081\end{array}$

$\begin{array}{llll}\text { C } & -1.576550 & -2.380006 & 0.197541\end{array}$

$\begin{array}{llll}\text { C } & -2.607197 & -2.431326 & -0.753099\end{array}$

$\begin{array}{llll}\text { C } & -1.857013 & -2.381835 & 1.575047\end{array}$

$\begin{array}{llll}\text { C } & -3.923738 & -2.326747 & -0.304787\end{array}$

$\begin{array}{llll}\text { C } & -3.190076 & -2.288728 & 1.975488\end{array}$

$\begin{array}{llll}\text { C } & -4.234894 & -2.224607 & 1.051321\end{array}$

$\mathrm{H} \quad-4.727258 \quad-2.315825 \quad-1.040915$

$\begin{array}{llll}\mathrm{H} & -3.416592 & -2.263672 & 3.041158\end{array}$

$\begin{array}{llll}\text { C } & 3.181284 & -1.263442 & -0.278386\end{array}$

$\begin{array}{llll}\text { C } & 3.812404 & -0.923751 & -1.483713\end{array}$

$\begin{array}{llll}\text { C } & 3.799262 & -1.062917 & 0.959864\end{array}$

$\begin{array}{llll}\text { C } & 5.077929 & -0.348959 & -1.424279\end{array}$

$\begin{array}{llll}\text { C } & 5.067858 & -0.475789 & 0.968784\end{array}$

$\begin{array}{llll}\text { C } & 5.720465 & -0.109303 & -0.206176\end{array}$

$\begin{array}{llll}\mathrm{H} & 5.569608 & -0.062570 & -2.354067\end{array}$

$\begin{array}{llll}\mathrm{H} & 5.560249 & -0.310372 & 1.926443\end{array}$

$\begin{array}{llll}\text { C } & -0.751629 & -2.519117 & 2.577603\end{array}$ 


\begin{tabular}{|c|c|c|c|c|c|c|c|}
\hline $\mathrm{H}$ & -1.141893 & -2.467706 & 3.597318 & $\mathrm{H}$ & 1.620347 & 3.021564 & -1.417066 \\
\hline $\mathrm{H}$ & -0.244179 & -3.486495 & 2.474812 & $\mathrm{H}$ & 2.686166 & 2.004106 & -2.403993 \\
\hline $\mathrm{H}$ & 0.014759 & -1.741634 & 2.469108 & & & & \\
\hline $\mathrm{C}$ & -2.331498 & -2.642332 & -2.209781 & 1 & & & \\
\hline $\mathrm{H}$ & -2.521282 & -3.686036 & -2.489976 & $\mathrm{Ru}$ & -0.149255 & 0.576826 & -0.223732 \\
\hline $\mathrm{H}$ & -2.986889 & -2.024059 & -2.829337 & $\mathrm{C}$ & -0.269818 & 3.279423 & -1.756075 \\
\hline $\mathrm{H}$ & -1.295281 & -2.414395 & -2.470875 & $\mathrm{C}$ & 0.774901 & 3.590596 & 0.278233 \\
\hline $\mathrm{C}$ & -5.652211 & -2.039282 & 1.497343 & $\mathrm{C}$ & -0.212594 & 4.639012 & -2.035323 \\
\hline $\mathrm{H}$ & -5.775226 & -2.257793 & 2.561510 & $\mathrm{H}$ & -0.719315 & 2.550469 & -2.431539 \\
\hline $\mathrm{H}$ & -5.979071 & -1.005134 & 1.335923 & $\mathrm{C}$ & 0.875090 & 4.958147 & 0.068960 \\
\hline $\mathrm{H}$ & -6.342394 & -2.678138 & 0.937840 & $\mathrm{H}$ & 1.131204 & 3.128967 & 1.194908 \\
\hline $\mathrm{C}$ & 3.120991 & -1.125003 & -2.795981 & $\mathrm{H}$ & -0.625922 & 5.010912 & -2.967224 \\
\hline $\mathrm{H}$ & 3.535821 & -0.463538 & -3.561285 & $\mathrm{H}$ & 1.333618 & 5.584532 & 0.827146 \\
\hline $\mathrm{H}$ & 3.227259 & -2.150851 & -3.171799 & $\mathrm{~N}$ & 0.220338 & 2.753876 & -0.616875 \\
\hline $\mathrm{H}$ & 2.051446 & -0.909275 & -2.704201 & $\mathrm{C}$ & 0.371767 & 5.497186 & -1.110834 \\
\hline $\mathrm{C}$ & 3.122841 & -1.464276 & 2.232600 & $\mathrm{H}$ & 0.428606 & 6.565302 & -1.301612 \\
\hline $\mathrm{H}$ & 2.256014 & -0.827230 & 2.448697 & $\mathrm{C}$ & -0.679249 & -1.342301 & 0.170302 \\
\hline $\mathrm{H}$ & 2.747031 & -2.494018 & 2.180744 & $\mathrm{C}$ & -0.457857 & -3.636357 & 0.757390 \\
\hline $\mathrm{H}$ & 3.813658 & -1.402119 & 3.077427 & $\mathrm{C}$ & -1.934438 & -3.272441 & 0.709130 \\
\hline $\mathrm{C}$ & 7.071157 & 0.538803 & -0.171381 & $\mathrm{H}$ & -0.191505 & -4.439722 & 0.059937 \\
\hline $\mathrm{H}$ & 7.526370 & 0.470608 & 0.820209 & $\mathrm{H}$ & -0.124494 & -3.945301 & 1.757330 \\
\hline $\mathrm{H}$ & 7.759945 & 0.081342 & -0.889039 & $\mathrm{H}$ & -2.483011 & -3.796394 & -0.086112 \\
\hline $\mathrm{H}$ & 7.011178 & 1.602368 & -0.429385 & $\mathrm{H}$ & -2.459197 & -3.471087 & 1.650371 \\
\hline $\mathrm{C}$ & -1.064675 & 0.377495 & -1.202862 & $\mathrm{~N}$ & 0.194501 & -2.382819 & 0.366392 \\
\hline $\mathrm{H}$ & -0.900417 & -0.377326 & -1.990376 & $\mathrm{~N}$ & -1.917301 & -1.831205 & 0.429692 \\
\hline $\mathrm{C}$ & -2.409186 & 0.916953 & -1.296259 & $\mathrm{C}$ & 1.524342 & -2.417800 & -0.149110 \\
\hline $\mathrm{C}$ & -3.142937 & 0.738701 & -2.492853 & $\mathrm{C}$ & 2.628814 & -2.403922 & 0.715510 \\
\hline $\mathrm{C}$ & -3.074906 & 1.566692 & -0.231258 & $\mathrm{C}$ & 1.688333 & -2.522701 & -1.539909 \\
\hline $\mathrm{C}$ & -4.452359 & 1.176917 & -2.619380 & $\mathrm{C}$ & 3.902348 & -2.329055 & 0.151098 \\
\hline $\mathrm{H}$ & -2.650978 & 0.243194 & -3.328468 & $\mathrm{C}$ & 2.982275 & -2.457573 & -2.058143 \\
\hline $\mathrm{C}$ & -4.393444 & 1.983846 & -0.352653 & $\mathrm{C}$ & 4.098245 & -2.320198 & -1.230667 \\
\hline $\mathrm{H}$ & -2.545814 & 1.722448 & 0.705347 & $\mathrm{H}$ & 4.764201 & -2.262088 & 0.814704 \\
\hline $\mathrm{C}$ & -5.093171 & 1.793967 & -1.543878 & $\mathrm{H}$ & 3.119505 & -2.508616 & -3.138090 \\
\hline $\mathrm{H}$ & -4.983209 & 1.028132 & -3.556830 & $\mathrm{C}$ & -3.191833 & -1.186976 & 0.344077 \\
\hline $\mathrm{H}$ & -4.882159 & 2.464171 & 0.491761 & $\mathrm{C}$ & -3.814334 & -0.781516 & 1.533249 \\
\hline $\mathrm{H}$ & -6.124791 & 2.123023 & -1.633679 & $\mathrm{C}$ & -3.819046 & -1.062597 & -0.897671 \\
\hline $\mathrm{O}$ & 0.522846 & 0.473522 & 2.316008 & $\mathrm{C}$ & -5.083494 & -0.219470 & 1.454846 \\
\hline $\mathrm{O}$ & 1.776252 & 0.989756 & -0.915820 & $\mathrm{C}$ & -5.092768 & -0.485377 & -0.927321 \\
\hline $\mathrm{C}$ & -0.828956 & 0.607561 & 2.545977 & $\mathrm{C}$ & -5.737718 & -0.055204 & 0.229215 \\
\hline $\mathrm{H}$ & -1.196304 & -0.020293 & 3.378533 & $\mathrm{H}$ & -5.568960 & 0.115693 & 2.371350 \\
\hline $\mathrm{H}$ & -1.159576 & 1.648969 & 2.745677 & $\mathrm{H}$ & -5.593078 & -0.375500 & -1.888908 \\
\hline $\mathrm{H}$ & -1.462304 & 0.253982 & 1.677616 & $\mathrm{C}$ & 0.498898 & -2.707621 & -2.432181 \\
\hline $\mathrm{C}$ & 1.734012 & 2.012353 & -1.848630 & $\mathrm{H}$ & 0.808895 & -2.832305 & -3.473174 \\
\hline $\mathrm{H}$ & 0.911791 & 1.870091 & -2.578207 & $\mathrm{H}$ & -0.074575 & -3.600251 & -2.150438 \\
\hline
\end{tabular}




\begin{tabular}{|c|c|c|c|}
\hline 11 & -0.197479 & -1.859917 & -2.387934 \\
\hline $\mathrm{C}$ & 2.467504 & -2.517570 & 2.198910 \\
\hline $\mathrm{H}$ & 2.463727 & -3.570907 & 2.507111 \\
\hline 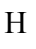 & 3.292066 & -2.030277 & 2.724867 \\
\hline $\mathrm{H}$ & 1.529446 & -2.075367 & 2.544768 \\
\hline $\mathrm{C}$ & 5.474205 & -2.166267 & -1.802315 \\
\hline $\mathrm{H}$ & 5.489308 & -2.350565 & -2.879604 \\
\hline $\mathrm{H}$ & 5.855149 & -1.151717 & -1.636226 \\
\hline $\mathrm{H}$ & 6.189385 & -2.849599 & -1.333343 \\
\hline C & -3.087740 & -0.886091 & 2.836315 \\
\hline $\mathrm{H}$ & -3.653663 & -0.415318 & 3.644292 \\
\hline $\mathrm{H}$ & -2.896856 & -1.924980 & 3.133394 \\
\hline $\mathrm{H}$ & -2.113642 & -0.390144 & 2.758392 \\
\hline$c$ & -3.147336 & -1.510695 & -2.156616 \\
\hline $\mathrm{H}$ & -2.303923 & -0.857310 & -2.413267 \\
\hline $\mathrm{H}$ & -2.739986 & -2.525144 & -2.064903 \\
\hline $\mathrm{H}$ & -3.849453 & -1.510063 & -2.994425 \\
\hline C & -7.095972 & 0.575057 & 0.168415 \\
\hline $\mathrm{H}$ & -7.529047 & 0.504136 & -0.832831 \\
\hline $\mathrm{H}$ & -7.794147 & 0.104668 & 0.868902 \\
\hline $\mathrm{H}$ & -7.056378 & 1.637749 & 0.433045 \\
\hline $\mathrm{C}$ & 1.022946 & 0.440685 & 1.262196 \\
\hline $\mathrm{H}$ & 0.759423 & -0.22 & 2.106551 \\
\hline $\mathrm{C}$ & 2.392853 & 0.905033 & 1.402498 \\
\hline $\mathrm{C}$ & 3.060561 & 0.762723 & 2.640353 \\
\hline $\mathrm{C}$ & 3.136984 & 1.458748 & 0.334470 \\
\hline $\mathrm{C}$ & 4.382459 & 1.150761 & 2.804918 \\
\hline $\mathrm{H}$ & 2.511919 & 0.327130 & 3.474333 \\
\hline $\mathrm{C}$ & 4.464541 & 1.826992 & 0.494199 \\
\hline $\mathrm{H}$ & 2.656455 & 1.576554 & -0.634343 \\
\hline C & 5.097216 & 1.678805 & 1.729386 \\
\hline $\mathrm{H}$ & 4.864497 & 1.030988 & 3.772169 \\
\hline $\mathrm{H}$ & 5.014537 & 2.233674 & -0.351196 \\
\hline $\mathrm{H}$ & 6.137343 & 1.968612 & 1.851566 \\
\hline $\mathrm{O}$ & -0.566734 & 0.428470 & -2.371108 \\
\hline ? & -1.806459 & 1.110064 & 0.716806 \\
\hline $\mathrm{C}$ & 0.788534 & 0.441738 & -2.465965 \\
\hline $\mathrm{H}$ & 1.216496 & -0.311829 & -3.154357 \\
\hline $\mathrm{H}$ & 1.237974 & 1.426730 & -2.719310 \\
\hline $\mathrm{H}$ & 1.325999 & 0.120878 & -1.484362 \\
\hline$C$ & -1.789596 & 2.164417 & 1.610828 \\
\hline $\mathrm{H}$ & -0.980774 & 2.058928 & 2.364134 \\
\hline $\mathrm{H}$ & -1.666690 & 3.158305 & 1.143667 \\
\hline $\mathrm{H}$ & -2.751791 & 2.179018 & 2.15022 \\
\hline
\end{tabular}

TS $_{1-2}$

$\begin{array}{rrrr}\mathrm{Ru} & 0.302466 & 0.597922 & 0.215000 \\ \mathrm{C} & 1.824467 & 3.203783 & 0.190046 \\ \mathrm{C} & -0.419319 & 3.727808 & 0.393727\end{array}$

$\begin{array}{llll}\text { C } & -0.419319 & 3.727808 & 0.393727\end{array}$

$\begin{array}{llll}\text { C } & 2.178304 & 4.545808 & 0.245587\end{array}$

$\begin{array}{llll}\mathrm{H} & 2.546743 & 2.403627 & 0.057405\end{array}$

$\begin{array}{llll}\text { C } & -0.143697 & 5.087315 & 0.453425\end{array}$

$\mathrm{H} \quad-1.438212 \quad 3.349636 \quad 0.439830$

$\mathrm{H} \quad 3.224503 \quad 4.826349 \quad 0.180586$

$\begin{array}{llll}\mathrm{H} & -0.957194 & 5.798614 & 0.552132\end{array}$

$\begin{array}{llll}\mathrm{N} & 0.542024 & 2.801361 & 0.265298\end{array}$

$\begin{array}{llll}\text { C } & 1.181731 & 5.505280 & 0.380860\end{array}$

$\begin{array}{llll}\mathrm{H} & 1.431671 & 6.561661 & 0.426309\end{array}$

$\begin{array}{llll}\text { C } & 0.455440 & -1.422581 & 0.236499\end{array}$

$\begin{array}{llll}\text { C } & -0.037019 & -3.724461 & 0.454281\end{array}$

$\begin{array}{llll}\text { C } & 1.459112 & -3.555748 & 0.270137\end{array}$

$\mathrm{H} \quad-0.306190 \quad-4.038463 \quad 1.474350$

$\mathrm{H} \quad-0.487302 \quad-4.440638 \quad-0.241552$

$\mathrm{H} \quad 2.056330 \quad-4.058156 \quad 1.038095$

$\mathrm{H} \quad 1.802666 \quad-3.913362-0.711511$

$\begin{array}{llll}\mathrm{N} & -0.533155 & -2.368817 & 0.205787\end{array}$

$\begin{array}{llll}\mathrm{N} & 1.630525 & -2.101040 & 0.351072\end{array}$

$\begin{array}{llll}\text { C } & -1.939986 & -2.151415 & 0.236635\end{array}$

$\begin{array}{llll}\text { C } & -2.663902 & -2.355240 & -0.950555\end{array}$

$\begin{array}{llll}\text { C } & -2.588056 & -1.799909 & 1.430908\end{array}$

$\begin{array}{llll}\text { C } & -4.041586 & -2.149061 & -0.931928\end{array}$

$\begin{array}{llll}\text { C } & -3.969006 & -1.605617 & 1.397435\end{array}$

$\begin{array}{llll}\text { C } & -4.712668 & -1.760516 & 0.227605\end{array}$

$\mathrm{H} \quad-4.604412 \quad-2.272252 \quad-1.856823$

$\mathrm{H} \quad-4.477200 \quad-1.305446 \quad 2.314292$

$\begin{array}{llll}\text { C } & 2.929510 & -1.584999 & 0.033222\end{array}$

$\begin{array}{llll}\text { C } & 3.276217 & -1.399100 & -1.314202\end{array}$

$\begin{array}{llll}\text { C } & 3.854441 & -1.382400 & 1.064481\end{array}$

$\begin{array}{llll}\text { C } & 4.554788 & -0.925239 & -1.600763\end{array}$

$\begin{array}{llll}\text { C } & 5.123090 & -0.910683 & 0.726268\end{array}$

$\begin{array}{llll}\text { C } & 5.485434 & -0.658260 & -0.596380\end{array}$

$\mathrm{H} \quad 4.826903 \quad-0.754352 \quad-2.642094$

$\mathrm{H} \quad 5.845857 \quad-0.731461 \quad 1.521721$

$\begin{array}{llll}\text { C } & -1.849431 & -1.640125 & 2.724311\end{array}$

$\begin{array}{llll}\mathrm{H} & -2.005617 & -0.640439 & 3.145600\end{array}$

$\mathrm{H} \quad-2.207273 \quad-2.358702 \quad 3.470435$

$\mathrm{H} \quad-0.771280 \quad-1.773938 \quad 2.610572$

$\begin{array}{llll}\text { C } & -1.967842 & -2.756188 & -2.214185\end{array}$ 


\begin{tabular}{|c|c|c|c|}
\hline $\mathrm{H}$ & -1.734029 & -3.828424 & -2.230773 \\
\hline $\mathrm{H}$ & -2.590540 & -2.550855 & -3.088859 \\
\hline & -1.016778 & -2.227453 & -2.334615 \\
\hline $\mathrm{C}$ & -6.184603 & -1.487236 & 0.216579 \\
\hline $\mathrm{H}$ & -6.710205 & -2.053709 & 0.992443 \\
\hline $\mathrm{H}$ & -6.384982 & -0.426214 & 0.405773 \\
\hline $\mathrm{H}$ & -6.635006 & -1.738989 & -0.747156 \\
\hline $\mathrm{C}$ & 2.347133 & -1.761454 & -2.431565 \\
\hline $\mathrm{H}$ & 2.559733 & -1.161281 & -3.319070 \\
\hline $\mathrm{H}$ & 2.462298 & -2.817026 & -2.716590 \\
\hline $\mathrm{H}$ & 1.302027 & -1.584632 & -2.168977 \\
\hline C & 3.519104 & -1.687793 & 2.491466 \\
\hline $\mathrm{H}$ & 2.439685 & -1.747564 & 2.642059 \\
\hline $\mathrm{H}$ & 3.967878 & -2.638743 & 2.807653 \\
\hline $\mathrm{H}$ & 3.902002 & -0.910456 & 3.157382 \\
\hline $\mathrm{C}$ & 6.834824 & -0.099406 & -0.931599 \\
\hline $\mathrm{H}$ & 7.570644 & -0.320674 & -0.153166 \\
\hline $\mathrm{H}$ & 7.215154 & -0.494392 & -1.878550 \\
\hline $\mathrm{H}$ & 6.797828 & 0.991234 & -1.037195 \\
\hline $\mathrm{C}$ & -1.265465 & 0.492157 & -0.835457 \\
\hline $\mathrm{H}$ & -1.180846 & -0.038334 & -1.803712 \\
\hline C & -2.609654 & 1.024385 & -0.682977 \\
\hline $\mathrm{C}$ & -3.455487 & 1.092603 & -1.810676 \\
\hline $\mathrm{C}$ & -3.127571 & 1.492362 & 0.542113 \\
\hline C & -4.732415 & 1.631015 & -1.729947 \\
\hline $\mathrm{H}$ & -3.078729 & 0.713391 & -2.759901 \\
\hline $\mathrm{C}$ & -4.408699 & 2.018097 & 0.629303 \\
\hline $\mathrm{H}$ & -2.498792 & 1.406549 & 1.427603 \\
\hline $\mathrm{C}$ & -5.214987 & 2.099013 & -0.507799 \\
\hline $\mathrm{H}$ & -5.358794 & 1.678848 & -2.616927 \\
\hline $\mathrm{H}$ & -4.788206 & 2.362268 & 1.588423 \\
\hline $\mathrm{H}$ & -6.217567 & 2.513246 & -0.438003 \\
\hline $\mathrm{O}$ & 1.895267 & 0.651015 & 1.734795 \\
\hline $\mathrm{O}$ & 1.569122 & 0.797679 & -1.428256 \\
\hline $\mathrm{C}$ & 0.788288 & 0.829336 & 2.392379 \\
\hline $\mathrm{H}$ & 0.491218 & 0.054494 & 3.126933 \\
\hline $\mathrm{H}$ & 0.544628 & 1.848549 & 2.753362 \\
\hline $\mathrm{H}$ & -0.583395 & 0.651428 & 1.647054 \\
\hline $\mathrm{C}$ & 1.117274 & 1.471971 & -2.537145 \\
\hline $\mathrm{H}$ & 0.275976 & 0.957698 & -3.056231 \\
\hline $\mathrm{H}$ & 0.753304 & 2.503217 & -2.336532 \\
\hline & 1.929561 & 1.565488 & -3.281741 \\
\hline
\end{tabular}

2

\begin{tabular}{|c|c|c|c|}
\hline & 9 & 5585 & 510 \\
\hline $\mathrm{C}$ & 710146 & 3.407620 & 0504096 \\
\hline c & 0.567693 & 661777 & .8345 \\
\hline $\mathrm{C}$ & 1.917900 & .771527 & 678140 \\
\hline $\mathrm{H}$ & -2.495795 & .695646 & 4776 \\
\hline $\mathrm{C}$ & 0 & 1016 & 583 \\
\hline $\mathrm{H}$ & 1.5 & 68 & 02 \\
\hline $\mathrm{H}$ & -2.923444 & 5.172887 & -0.604341 \\
\hline $\mathrm{H}$ & 1.318163 & 635206 & $201+1>$ \\
\hline $\mathrm{J}$ & -0.482394 & 2.866328 & 3448 \\
\hline $\mathrm{C}$ & -0.830803 & 5.597272 & \\
\hline I & -0.9 & 0 & 76 \\
\hline $\mathrm{C}$ & -0.443332 & 392 & 537 \\
\hline $\mathrm{C}$ & 291 & 0 & 36 \\
\hline $\mathrm{C}$ & -1.388429 & -3.496064 & 33 \\
\hline $\mathrm{H}$ & 0.603683 & -4.198398 & \\
\hline$\Pi$ & 0.362962 & -4.1 & 0.52409 \\
\hline $\mathrm{H}$ & -1.706334 & -3.758531 & -1 . \\
\hline $\mathrm{H}$ & -1.987399 & -4.086897 & 5571 \\
\hline $\mathrm{N}$ & 057 & 3 & 67 \\
\hline $\mathrm{N}$ & -1.602026 & -2.061763 & 917 \\
\hline $\mathrm{C}$ & 1.978463 & -2.0 & 03 \\
\hline$C_{1}$ & 2.677845 & 13 & 0.824980 \\
\hline $\mathrm{C}$ & 2.649045 & -1.8 & 044 \\
\hline$C$ & 4.065641 & -1.987391 & 60 \\
\hline C & 4.041766 & -1.762549 & 21 \\
\hline $\mathrm{C}$ & 4.766837 & -1.8 & -0. \\
\hline $\mathrm{H}$ & 4.613286 & מ 010 roe & 1.740183 \\
\hline $\mathrm{H}$ & 4.573076 & .629505 & -2 \\
\hline $\mathrm{C}$ & - & -1.630447 & 0.049827 \\
\hline $\mathrm{C}$ & -3.1 & 452143 & 94 \\
\hline $\mathrm{C}$ & -3.928380 & -1.496735 & -0.881496 \\
\hline $\mathrm{C}$ & -4 & -1.094879 & \\
\hline$C$ & -5.190867 & -1.130222 & -0.411960 \\
\hline$C$ & -5.437956 & -0.914265 & 0.94 \\
\hline$U$ & -4.565675 & 041256 & .910383 \\
\hline $\mathrm{H}$ & -6.001505 & -1.005832 & -1.129252 \\
\hline $\mathrm{C}$ & 1.897223 & -1.782234 & 0 \\
\hline 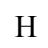 & 1.468869 & -0.780548 & 6709 \\
\hline $\mathrm{H}$ & 2.547260 & -1.984673 & -3.754929 \\
\hline $\mathrm{H}$ & 1.051548 & .477919 & 500 \\
\hline $\mathrm{C}$ & 1.941581 & -2.25 & 75 \\
\hline $\mathrm{H}$ & 1.554325 & -3.271120 & 2.258789 \\
\hline $\mathrm{H}$ & 2.59 & r 101 & \\
\hline
\end{tabular}




\begin{tabular}{|c|c|c|c|c|c|c|c|}
\hline $\mathrm{H}$ & 1.076534 & -1.582092 & 2.167054 & $\mathrm{C}$ & 2.083423 & 1.561418 & 2.274431 \\
\hline $\mathrm{C}$ & 6.257312 & -1.678210 & -0.391221 & $\mathrm{C}$ & 4.160489 & 2.417125 & 0.728266 \\
\hline $\mathrm{H}$ & 6.656245 & -1.543627 & -1.400138 & $\mathrm{H}$ & 2.890170 & 1.662342 & -0.879722 \\
\hline $\mathrm{H}$ & 6.563908 & -0.811984 & 0.207031 & $\mathrm{C}$ & 3.191978 & 2.123524 & 2.895896 \\
\hline $\mathrm{H}$ & 6.748598 & -2.553069 & 0.048011 & $\mathrm{H}$ & 1.220831 & 1.193728 & 2.827379 \\
\hline $\mathrm{C}$ & -2.005741 & -1.663443 & 2.408506 & $\mathrm{H}$ & 4.965011 & 2.737210 & 0.072379 \\
\hline $\mathrm{H}$ & -2.358299 & -1.512490 & 3.432184 & $\mathrm{H}$ & 3.217567 & 2.216690 & 3.97728 \\
\hline $\mathrm{H}$ & -1.582258 & -2.675395 & 2.344680 & $\mathrm{~N}$ & 1.995847 & 1.410101 & 0.943257 \\
\hline $\mathrm{H}$ & -1.198252 & -0.947372 & 2.221878 & $\mathrm{C}$ & 4.252331 & 2.561668 & 2.108030 \\
\hline $\mathrm{C}$ & -3.706638 & -1.757753 & -2.338147 & $\mathrm{H}$ & 5.133650 & 3.007388 & 2.562530 \\
\hline $\mathrm{H}$ & -2.665391 & -1.588341 & -2.621243 & $\mathrm{C}$ & -0.020433 & -1.312618 & -0.405111 \\
\hline $\mathrm{H}$ & -3.968047 & -2.790211 & -2.606368 & $\mathrm{C}$ & -0.581157 & -3.594857 & -0.564239 \\
\hline $\mathrm{H}$ & -4.327534 & -1.100279 & -2.951850 & $\mathrm{C}$ & 0.830244 & -3.399639 & -1.093970 \\
\hline $\mathrm{C}$ & -6.792244 & -0.487682 & 1.421688 & $\mathrm{H}$ & -0.594677 & -4.103887 & 0.412933 \\
\hline $\mathrm{H}$ & -7.558091 & -0.635667 & 0.655454 & $\mathrm{H}$ & -1.226271 & -4.159869 & -1.245175 \\
\hline $\mathrm{H}$ & -7.099104 & -1.039971 & 2.315743 & $\mathrm{H}$ & 1.560474 & -4.080279 & -0.644577 \\
\hline $\mathrm{H}$ & -6.802477 & 0.574624 & 1.690893 & $\mathrm{H}$ & 0.882932 & -3.518639 & -2.186496 \\
\hline $\mathrm{C}$ & 1.015158 & 0.825862 & 1.009526 & $\mathrm{~N}$ & -1.060380 & -2.218476 & -0.418056 \\
\hline $\mathrm{H}$ & 0.600359 & 0.816203 & 2.031811 & $\mathrm{~N}$ & 1.112015 & -2.015294 & -0.717239 \\
\hline $\mathrm{C}$ & 2.440577 & 1.075475 & 1.060648 & $\mathrm{C}$ & -2.364313 & -2.016111 & 0.137761 \\
\hline $\mathrm{C}$ & 3.059225 & 1.193215 & 2.325776 & $\mathrm{C}$ & -3.468892 & -1.954123 & -0.729612 \\
\hline $\mathrm{C}$ & 3.257216 & 1.231132 & -0.079871 & $\mathrm{C}$ & -2.540013 & -1.963453 & 1.530991 \\
\hline $\mathrm{C}$ & 4.417940 & 1.445337 & 2.449182 & $\mathrm{C}$ & -4.728870 & -1.700070 & -0.190029 \\
\hline $\mathrm{H}$ & 2.440433 & 1.078506 & 3.214358 & $\mathrm{C}$ & -3.819367 & -1.695367 & 2.021667 \\
\hline $\mathrm{C}$ & 4.612024 & 1.497549 & 0.043064 & $\mathrm{C}$ & -4.919380 & -1.530758 & 1.181984 \\
\hline $\mathrm{H}$ & 2.800970 & 1.118861 & -1.060859 & $\mathrm{H}$ & -5.582633 & -1.621671 & -0.862738 \\
\hline $\mathrm{C}$ & 5.199843 & 1.603231 & 1.305087 & $\mathrm{H}$ & -3.955432 & -1.618460 & 3.100204 \\
\hline $\mathrm{H}$ & 4.869613 & 1.528120 & 3.434047 & $\mathrm{C}$ & 2.460231 & -1.564189 & -0.595126 \\
\hline $\mathrm{H}$ & 5.223339 & 1.605663 & -0.850170 & $\mathrm{C}$ & 3.219925 & -1.295900 & -1.740970 \\
\hline $\mathrm{H}$ & 6.263859 & 1.808529 & 1.394888 & $\mathrm{C}$ & 3.025905 & -1.496310 & 0.689831 \\
\hline $\mathrm{O}$ & -2.034831 & 0.712409 & -1.632363 & $\mathrm{C}$ & 4.555594 & -0.922398 & -1.572134 \\
\hline $\mathrm{O}$ & -1.849610 & 1.072017 & 1.176258 & $\mathrm{C}$ & 4.364061 & -1.127430 & 0.807284 \\
\hline $\mathrm{C}$ & -0.973271 & 0.655554 & -2.375141 & $\mathrm{C}$ & 5.144972 & -0.830898 & -0.311548 \\
\hline $\mathrm{H}$ & -0.735178 & -0.291150 & -2.896985 & $\mathrm{H}$ & 5.149239 & -0.691484 & -2.456109 \\
\hline $\mathrm{H}$ & -0.633280 & 1.557225 & -2.915485 & $\mathrm{H}$ & 4.803341 & -1.054101 & 1.802188 \\
\hline $\mathrm{H}$ & 0.967001 & 0.527618 & -1.248698 & $\mathrm{C}$ & -1.439042 & -2.281072 & 2.493102 \\
\hline $\mathrm{C}$ & -1.606982 & 1.731953 & 2.345744 & $\mathrm{H}$ & -0.453191 & -2.160911 & 2.048708 \\
\hline $\mathrm{H}$ & -1.026980 & 1.148368 & 3.105147 & $\mathrm{H}$ & -1.473212 & -1.625985 & 3.365962 \\
\hline $\mathrm{H}$ & -1.054229 & 2.697020 & 2.237054 & $\mathrm{H}$ & -1.538086 & -3.315593 & 2.849742 \\
\hline \multirow[t]{2}{*}{$\mathrm{H}$} & -2.558120 & 1.980298 & 2.855057 & $\mathrm{C}$ & -3.319310 & -2.171702 & -2.206000 \\
\hline & & & & $\mathrm{H}$ & -3.576508 & -3.203142 & -2.477982 \\
\hline $\mathbf{0 A}$ & & & & $\mathrm{H}$ & -3.988290 & -1.520398 & -2.776693 \\
\hline $\mathrm{Ru}$ & -0.066520 & 0.578160 & -0.128848 & $\mathrm{H}$ & -2.295797 & -1.991904 & -2.545164 \\
\hline $\mathrm{C}$ & 3.019964 & 1.835311 & 0.187229 & $\mathrm{C}$ & -6.266073 & -1.179145 & 1.735116 \\
\hline
\end{tabular}




\begin{tabular}{|c|c|c|c|c|c|c|c|}
\hline $\mathrm{H}$ & -7.076290 & -1.563426 & 1.108727 & $\mathrm{H}$ & 2.986211 & 1.854067 & -1.035327 \\
\hline $\mathrm{H}$ & -6.404498 & -1.571119 & 2.746750 & $\mathrm{C}$ & 3.441954 & 1.992638 & 2.747080 \\
\hline $\mathrm{H}$ & -6.395876 & -0.092250 & 1.794223 & $\mathrm{H}$ & 1.442791 & 1.123727 & 2.688328 \\
\hline $\mathrm{C}$ & 2.621495 & -1.413459 & -3.105786 & $\mathrm{H}$ & 5.108818 & 2.799929 & -0.090342 \\
\hline $\mathrm{H}$ & 3.185997 & -0.824223 & -3.833350 & $\mathrm{H}$ & 3.509249 & 2.001559 & 3.830771 \\
\hline $\mathrm{H}$ & 2.627725 & -2.452827 & -3.459434 & $\mathrm{~N}$ & 2.150478 & 1.455440 & 0.796750 \\
\hline $\mathrm{H}$ & 1.590789 & -1.051978 & -3.099765 & $\mathrm{C}$ & 4.481158 & 2.471891 & 1.955027 \\
\hline $\mathrm{C}$ & 2.205082 & -1.793733 & 1.903790 & $\mathrm{H}$ & 5.390116 & 2.862758 & 2.405538 \\
\hline $\mathrm{H}$ & 1.370273 & -1.087500 & 2.008357 & $\mathrm{C}$ & 0.174621 & -1.270107 & -0.333676 \\
\hline $\mathrm{H}$ & 1.762378 & -2.796687 & 1.857720 & $\mathrm{C}$ & -0.302139 & -3.591086 & -0.385906 \\
\hline $\mathrm{H}$ & 2.811598 & -1.736225 & 2.811714 & $\mathrm{C}$ & 1.106870 & -3.371223 & -0.915351 \\
\hline $\mathrm{C}$ & 6.581635 & -0.435256 & -0.155015 & $\mathrm{H}$ & -0.326242 & -4.145965 & 0.562617 \\
\hline $\mathrm{H}$ & 6.717766 & 0.262841 & 0.677911 & $\mathrm{H}$ & -0.950822 & -4.118759 & -1.093726 \\
\hline $\mathrm{H}$ & 7.218271 & -1.302561 & 0.055368 & $\mathrm{H}$ & 1.872241 & -3.909568 & -0.344646 \\
\hline $\mathrm{H}$ & 6.971113 & 0.039299 & -1.060018 & $\mathrm{H}$ & 1.219778 & -3.657193 & -1.969074 \\
\hline $\mathrm{C}$ & -1.694031 & 0.731216 & -1.019307 & $\mathrm{~N}$ & -0.784396 & -2.219345 & -0.166858 \\
\hline $\mathrm{H}$ & -2.204836 & -0.026176 & -1.619915 & $\mathrm{~N}$ & 1.285662 & -1.918314 & -0.762669 \\
\hline $\mathrm{C}$ & -2.343972 & 2.025028 & -1.009173 & $\mathrm{C}$ & -2.108877 & -2.027634 & 0.335650 \\
\hline $\mathrm{C}$ & -3.656118 & 2.192987 & -1.500000 & $\mathrm{C}$ & -3.189298 & -2.088736 & -0.564275 \\
\hline $\mathrm{C}$ & -1.695718 & 3.166922 & -0.478863 & $\mathrm{C}$ & -2.323164 & -1.846486 & 1.709674 \\
\hline $\mathrm{C}$ & -4.290309 & 3.425777 & -1.447059 & $\mathrm{C}$ & -4.464378 & -1.793712 & -0.093096 \\
\hline $\mathrm{H}$ & -4.170990 & 1.324361 & -1.908080 & $\mathrm{C}$ & -3.620493 & -1.535851 & 2.130615 \\
\hline $\mathrm{C}$ & -2.329707 & 4.398810 & -0.430646 & $\mathrm{C}$ & -4.692525 & -1.463566 & 1.245335 \\
\hline $\mathrm{H}$ & -0.670995 & 3.086057 & -0.105993 & $\mathrm{H}$ & -5.297198 & -1.788768 & -0.796024 \\
\hline $\mathrm{C}$ & -3.632366 & 4.534198 & -0.911969 & $\mathrm{H}$ & -3.790048 & -1.340766 & 3.189351 \\
\hline $\mathrm{H}$ & -5.304365 & 3.528669 & -1.825591 & $\mathrm{C}$ & 2.619240 & -1.408127 & -0.717283 \\
\hline $\mathrm{H}$ & -1.808246 & 5.259946 & -0.020853 & $\mathrm{C}$ & 3.264880 & -1.019466 & -1.894548 \\
\hline $\mathrm{H}$ & -4.129311 & 5.499633 & -0.874477 & $\mathrm{C}$ & 3.279854 & -1.416253 & 0.522903 \\
\hline $\mathrm{O}$ & -0.620818 & 0.394090 & 1.810457 & $\mathrm{C}$ & 4.603337 & -0.623075 & -1.802680 \\
\hline $\mathrm{O}$ & 1.099247 & 0.901884 & -1.761789 & $\mathrm{C}$ & 4.615189 & -1.026514 & 0.562815 \\
\hline $\mathrm{C}$ & -1.529961 & 1.331839 & 2.272084 & $\mathrm{C}$ & 5.293752 & -0.624969 & -0.591519 \\
\hline $\mathrm{H}$ & -2.522042 & 1.221989 & 1.792646 & $\mathrm{H}$ & 5.118808 & -0.306198 & -2.708793 \\
\hline $\mathrm{H}$ & -1.671168 & 1.216047 & 3.361033 & $\mathrm{H}$ & 5.132449 & -1.011760 & 1.522168 \\
\hline $\mathrm{H}$ & -1.223806 & 2.386456 & 2.102220 & $\mathrm{C}$ & -1.249575 & -2.070458 & 2.728526 \\
\hline $\mathrm{C}$ & 0.754229 & 1.973167 & -2.565161 & $\mathrm{H}$ & -0.252696 & -2.041331 & 2.291293 \\
\hline $\mathrm{H}$ & -0.212800 & 1.814979 & -3.084412 & $\mathrm{H}$ & -1.278932 & -1.310563 & 3.512499 \\
\hline $\mathrm{H}$ & 0.643928 & 2.937763 & -2.023272 & $\mathrm{H}$ & -1.394223 & -3.047017 & 3.210615 \\
\hline \multirow[t]{2}{*}{$\mathrm{H}$} & 1.528175 & 2.131737 & -3.336385 & $\mathrm{C}$ & -2.988130 & -2.466771 & -2.001444 \\
\hline & & & & $\mathrm{H}$ & -2.996978 & -3.557076 & -2.129539 \\
\hline \multicolumn{2}{|c|}{$\mathbf{T S}_{1 \mathrm{~A}-2 \mathrm{~A}}$} & & & $\mathrm{H}$ & -3.789782 & -2.071989 & -2.632197 \\
\hline $\mathrm{Ru}$ & -0.018340 & 0.848395 & -0.171207 & $\mathrm{H}$ & -2.034102 & -2.106603 & -2.399515 \\
\hline $\mathrm{C}$ & 3.153195 & 1.925014 & 0.038124 & $\mathrm{C}$ & -6.050874 & -1.027155 & 1.698569 \\
\hline $\mathrm{C}$ & 2.296403 & 1.504365 & 2.130401 & $\mathrm{H}$ & -6.843580 & -1.655418 & 1.280007 \\
\hline $\mathrm{C}$ & 4.327733 & 2.441060 & 0.574020 & $\mathrm{H}$ & -6.142509 & -1.045552 & 2.787648 \\
\hline
\end{tabular}




\begin{tabular}{|c|c|c|c|c|c|c|c|}
\hline $\mathrm{H}$ & -6.254413 & -0.000944 & 1.369419 & $\mathrm{H}$ & -1.377150 & -1.552713 & 2.558325 \\
\hline $\mathrm{C}$ & 2.533930 & -1.004870 & -3.199270 & $\mathrm{H}$ & -5.074800 & -2.734871 & -0.426252 \\
\hline $\mathrm{H}$ & 3.205981 & -0.752821 & -4.023312 & $\mathrm{H}$ & -3.448537 & -2.573363 & 3.562004 \\
\hline $\mathrm{H}$ & 2.081211 & -1.977434 & -3.423219 & $\mathrm{~N}$ & -2.091713 & -1.591902 & 0.639659 \\
\hline $\mathrm{H}$ & 1.724613 & -0.267431 & -3.174853 & $\mathrm{C}$ & -4.431813 & -2.742674 & 1.640821 \\
\hline $\mathrm{C}$ & 2.538514 & -1.788441 & 1.768346 & $\mathrm{H}$ & -5.344424 & -3.185583 & 2.031601 \\
\hline $\mathrm{H}$ & 1.689198 & -1.110995 & 1.933095 & $\mathrm{C}$ & -0.243188 & 1.299008 & -0.277999 \\
\hline $\mathrm{H}$ & 2.122828 & -2.803356 & 1.718566 & $\mathrm{C}$ & 0.209928 & 3.627366 & -0.254640 \\
\hline $\mathrm{H}$ & 3.188829 & -1.738627 & 2.645569 & $\mathrm{C}$ & -1.237512 & 3.416008 & -0.670558 \\
\hline $\mathrm{C}$ & 6.726307 & -0.193565 & -0.513698 & $\mathrm{H}$ & 0.317661 & 4.203765 & 0.672652 \\
\hline $\mathrm{H}$ & 6.868587 & 0.572790 & 0.256883 & $\mathrm{H}$ & 0.809794 & 4.124655 & -1.026032 \\
\hline $\mathrm{H}$ & 7.386622 & -1.027429 & -0.250257 & $\mathrm{H}$ & -1.955405 & 3.848521 & 0.037290 \\
\hline $\mathrm{H}$ & 7.078512 & 0.217065 & -1.463560 & $\mathrm{H}$ & -1.469721 & 3.820764 & -1.662393 \\
\hline $\mathrm{C}$ & -1.717919 & 0.700889 & -0.959876 & $\mathrm{~N}$ & 0.690125 & 2.250002 & -0.046495 \\
\hline $\mathrm{H}$ & -1.937451 & -0.208258 & -1.531874 & $\mathrm{~N}$ & -1.364804 & 1.943970 & -0.674353 \\
\hline $\mathrm{C}$ & -2.849003 & 1.611975 & -1.029286 & $\mathrm{C}$ & 2.036368 & 2.045679 & 0.388197 \\
\hline $\mathrm{C}$ & -3.975700 & 1.229228 & -1.792170 & $\mathrm{C}$ & 3.076908 & 2.124807 & -0.553027 \\
\hline $\mathrm{C}$ & -2.937422 & 2.837220 & -0.332020 & $\mathrm{C}$ & 2.304227 & 1.842309 & 1.750164 \\
\hline $\mathrm{C}$ & -5.117592 & 2.016220 & -1.858948 & $\mathrm{C}$ & 4.375058 & 1.844890 & -0.132011 \\
\hline $\mathrm{H}$ & -3.933546 & 0.283930 & -2.331309 & $\mathrm{C}$ & 3.618994 & 1.547764 & 2.118588 \\
\hline $\mathrm{C}$ & -4.077019 & 3.623184 & -0.397389 & $\mathrm{C}$ & 4.659478 & 1.506869 & 1.191886 \\
\hline $\mathrm{H}$ & -2.098425 & 3.148285 & 0.285068 & $\mathrm{H}$ & 5.180081 & 1.856709 & -0.866640 \\
\hline $\mathrm{C}$ & -5.175182 & 3.220087 & -1.159988 & $\mathrm{H}$ & 3.830370 & 1.339487 & 3.166976 \\
\hline $\mathrm{H}$ & -5.965700 & 1.691000 & -2.456466 & $\mathrm{C}$ & -2.692707 & 1.418899 & -0.595628 \\
\hline $\mathrm{H}$ & -4.116222 & 4.558628 & 0.155177 & $\mathrm{C}$ & -3.419363 & 1.177597 & -1.765757 \\
\hline $\mathrm{H}$ & -6.067367 & 3.838731 & -1.205263 & $\mathrm{C}$ & -3.274007 & 1.287167 & 0.676771 \\
\hline $\mathrm{O}$ & -0.446171 & 0.473293 & 1.767290 & $\mathrm{C}$ & -4.752077 & 0.774793 & -1.635217 \\
\hline $\mathrm{O}$ & 1.027843 & 1.463019 & -1.874584 & $\mathrm{C}$ & -4.608311 & 0.897643 & 0.755851 \\
\hline $\mathrm{C}$ & -1.363906 & 1.296154 & 2.397644 & $\mathrm{C}$ & -5.363823 & 0.635226 & -0.389805 \\
\hline $\mathrm{H}$ & -2.376868 & 1.208048 & 1.961988 & $\mathrm{H}$ & -5.326802 & 0.567487 & -2.537338 \\
\hline $\mathrm{H}$ & -1.444383 & 1.017169 & 3.464987 & $\mathrm{H}$ & -5.064373 & 0.776572 & 1.738070 \\
\hline $\mathrm{H}$ & -1.090681 & 2.371136 & 2.360175 & $\mathrm{C}$ & 1.252138 & 2.021047 & 2.799473 \\
\hline $\mathrm{C}$ & 0.472137 & 2.557581 & -1.368635 & $\mathrm{H}$ & 0.276601 & 1.676022 & 2.461064 \\
\hline $\mathrm{H}$ & -0.382426 & 2.986734 & -1.915616 & $\mathrm{H}$ & 1.504151 & 1.461022 & 3.703604 \\
\hline $\mathrm{H}$ & -0.290877 & 2.480252 & 0.040798 & $\mathrm{H}$ & 1.175232 & 3.078615 & 3.086822 \\
\hline \multirow[t]{2}{*}{$\mathrm{H}$} & 1.164752 & 3.325011 & -0.978775 & $\mathrm{C}$ & 2.813622 & 2.503457 & -1.980018 \\
\hline & & & & $\mathrm{H}$ & 2.833059 & 3.592993 & -2.112571 \\
\hline $2 A$ & & & & $\mathrm{H}$ & 3.578408 & 2.095269 & -2.647190 \\
\hline $\mathrm{Ru}$ & 0.060368 & -0.949696 & -0.195306 & $\mathrm{H}$ & 1.837841 & 2.153324 & -2.331757 \\
\hline $\mathrm{C}$ & -3.104292 & -1.923108 & -0.175799 & $\mathrm{C}$ & 6.038085 & 1.082854 & 1.591685 \\
\hline $\mathrm{C}$ & -2.234096 & -1.839595 & 1.951890 & $\mathrm{H}$ & 6.810787 & 1.656946 & 1.071563 \\
\hline $\mathrm{C}$ & -4.283575 & -2.500602 & 0.280353 & $\mathrm{H}$ & 6.201246 & 1.188169 & 2.667594 \\
\hline $\mathrm{H}$ & -2.949302 & -1.678059 & -1.224808 & $\mathrm{H}$ & 6.203180 & 0.027886 & 1.339799 \\
\hline $\mathrm{C}$ & -3.383994 & -2.401572 & 2.491801 & $\mathrm{C}$ & -2.781517 & 1.329365 & -3.109683 \\
\hline
\end{tabular}




\begin{tabular}{|c|c|c|c|c|c|c|c|}
\hline $\mathrm{H}$ & -3.496332 & 1.120918 & -3.909556 & $\mathrm{H}$ & -0.924631 & 5.622314 & 1.121168 \\
\hline $\mathrm{H}$ & -2.395755 & 2.343507 & -3.265699 & $\mathrm{~N}$ & 0.437255 & 2.891898 & -0.291942 \\
\hline $\mathrm{H}$ & -1.934296 & 0.644787 & -3.209056 & $\mathrm{C}$ & 0.737641 & 5.657361 & -0.262529 \\
\hline $\mathrm{C}$ & -2.459884 & 1.519237 & 1.910480 & $\mathrm{H}$ & 0.853311 & 6.737455 & -0.253230 \\
\hline $\mathrm{H}$ & -1.626481 & 0.805436 & 1.970496 & $\mathrm{C}$ & 0.615630 & -1.307689 & -0.219188 \\
\hline $\mathrm{H}$ & -2.011801 & 2.521563 & 1.931688 & $\mathrm{C}$ & 0.203036 & -3.609990 & -0.584053 \\
\hline $\mathrm{H}$ & -3.069023 & 1.409198 & 2.811505 & $\mathrm{C}$ & 1.694761 & -3.364864 & -0.662708 \\
\hline $\mathrm{C}$ & -6.795916 & 0.210289 & -0.274612 & $\mathrm{H}$ & -0.086291 & -4.171417 & 0.318585 \\
\hline $\mathrm{H}$ & -6.926430 & -0.549956 & 0.503144 & $\mathrm{H}$ & -0.198443 & -4.145271 & -1.450567 \\
\hline $\mathrm{H}$ & -7.446186 & 1.050258 & -0.004480 & $\mathrm{H}$ & 2.282352 & -4.028987 & -0.019779 \\
\hline $\mathrm{H}$ & -7.171968 & -0.202201 & -1.214766 & $\mathrm{H}$ & 2.083669 & -3.458847 & -1.686776 \\
\hline $\mathrm{C}$ & 1.768526 & -0.733939 & -0.955899 & $\mathrm{~N}$ & -0.332859 & -2.247386 & -0.518441 \\
\hline $\mathrm{H}$ & 1.922724 & 0.170068 & -1.561715 & $\mathrm{~N}$ & 1.807131 & -1.974661 & -0.213000 \\
\hline $\mathrm{C}$ & 2.955231 & -1.569083 & -1.018602 & $\mathrm{C}$ & -1.743378 & -2.137706 & -0.363446 \\
\hline $\mathrm{C}$ & 4.062431 & -1.107159 & -1.766286 & $\mathrm{C}$ & -2.544864 & -2.144631 & -1.513885 \\
\hline $\mathrm{C}$ & 3.104671 & -2.801598 & -0.345358 & $\mathrm{C}$ & -2.300446 & -2.137130 & 0.927839 \\
\hline $\mathrm{C}$ & 5.249479 & -1.822714 & -1.835098 & $\mathrm{C}$ & -3.930263 & -2.149319 & -1.347248 \\
\hline $\mathrm{H}$ & 3.967257 & -0.158237 & -2.291680 & $\mathrm{C}$ & -3.687359 & -2.141262 & 1.042623 \\
\hline $\mathrm{C}$ & 4.289278 & -3.517566 & -0.414468 & $\mathrm{C}$ & -4.518974 & -2.152137 & -0.081282 \\
\hline $\mathrm{H}$ & 2.266660 & -3.174301 & 0.237786 & $\mathrm{H}$ & -4.567093 & -2.133342 & -2.231091 \\
\hline $\mathrm{C}$ & 5.369525 & -3.033726 & -1.155926 & $\mathrm{H}$ & -4.132462 & -2.107920 & 2.036781 \\
\hline $\mathrm{H}$ & 6.082895 & -1.438524 & -2.418294 & $\mathrm{C}$ & 3.114200 & -1.498422 & 0.102935 \\
\hline $\mathrm{H}$ & 4.377499 & -4.463293 & 0.114355 & $\mathrm{C}$ & 3.926033 & -0.933293 & -0.891931 \\
\hline $\mathrm{H}$ & 6.296624 & -3.598951 & -1.203433 & $\mathrm{C}$ & 3.581813 & -1.707551 & 1.407000 \\
\hline $\mathrm{O}$ & 0.452294 & -0.585032 & 1.741459 & $\mathrm{C}$ & 5.218752 & -0.549706 & -0.532792 \\
\hline $\mathrm{O}$ & -0.954325 & -1.075723 & -2.021740 & $\mathrm{C}$ & 4.884638 & -1.312684 & 1.716603 \\
\hline $\mathrm{C}$ & 1.439643 & -1.301016 & 2.399065 & $\mathrm{C}$ & 5.714481 & -0.725487 & 0.761544 \\
\hline $\mathrm{H}$ & 2.434985 & -1.169383 & 1.936027 & $\mathrm{H}$ & 5.859708 & -0.102342 & -1.292046 \\
\hline $\mathrm{H}$ & 1.516051 & -0.946805 & 3.442878 & $\mathrm{H}$ & 5.256135 & -1.463312 & 2.729490 \\
\hline $\mathrm{H}$ & 1.240484 & -2.390610 & 2.431071 & $\mathrm{C}$ & -1.414894 & -2.045755 & 2.128391 \\
\hline $\mathrm{C}$ & -0.479551 & -2.269845 & -1.742614 & $\mathrm{H}$ & -0.851005 & -1.101281 & 2.122557 \\
\hline $\mathrm{H}$ & 0.385526 & -2.639355 & -2.313041 & $\mathrm{H}$ & -1.994998 & -2.094841 & 3.053201 \\
\hline $\mathrm{H}$ & 0.485922 & -2.453623 & 0.199615 & $\mathrm{H}$ & -0.668391 & -2.849544 & 2.153013 \\
\hline \multirow[t]{2}{*}{$\mathrm{H}$} & -1.189211 & -3.054414 & -1.433323 & $\mathrm{C}$ & -1.908744 & -2.074527 & -2.866346 \\
\hline & & & & $\mathrm{H}$ & -1.412776 & -3.015517 & -3.136611 \\
\hline \multicolumn{2}{|c|}{$\mathrm{TS}_{1 \mathrm{~B}-2 \mathrm{~B}}$} & & & $\mathrm{H}$ & -2.651790 & -1.866170 & -3.640689 \\
\hline $\mathrm{Ru}$ & 0.332354 & 0.685556 & -0.155890 & $\mathrm{H}$ & -1.133861 & -1.294946 & -2.886119 \\
\hline $\mathrm{C}$ & 1.397817 & 3.485409 & -1.020617 & $\mathrm{C}$ & -6.007453 & -2.134305 & 0.080123 \\
\hline $\mathrm{C}$ & -0.364449 & 3.659262 & 0.462481 & $\mathrm{H}$ & -6.516859 & -2.021755 & -0.880755 \\
\hline $\mathrm{C}$ & 1.579461 & 4.860680 & -1.034145 & $\mathrm{H}$ & -6.375436 & -3.056425 & 0.543793 \\
\hline $\mathrm{H}$ & 2.025804 & 2.809169 & -1.595508 & $\mathrm{H}$ & -6.323022 & -1.307663 & 0.726565 \\
\hline $\mathrm{C}$ & -0.249477 & 5.043685 & 0.499406 & $\mathrm{C}$ & 3.409324 & -0.740050 & -2.280075 \\
\hline $\mathrm{H}$ & -1.103264 & 3.125972 & 1.055142 & $\mathrm{H}$ & 4.135203 & -0.198665 & -2.893027 \\
\hline $\mathrm{H}$ & 2.369360 & 5.293537 & -1.639051 & $\mathrm{H}$ & 3.223464 & -1.702361 & -2.773662 \\
\hline
\end{tabular}




\begin{tabular}{|c|c|c|c|c|c|c|c|}
\hline $\mathrm{H}$ & 2.450987 & -0.198745 & -2.293107 & $\mathrm{C}$ & -0.542539 & 5.696066 & 0.098511 \\
\hline $\mathrm{C}$ & 2.681389 & -2.301245 & 2.447727 & $\mathrm{H}$ & -0.604578 & 6.777729 & 0.019697 \\
\hline $\mathrm{H}$ & 1.783100 & -1.688148 & 2.592587 & $\mathrm{C}$ & -0.660951 & -1.315689 & 0.133972 \\
\hline $\mathrm{H}$ & 2.328161 & -3.301923 & 2.168711 & $\mathrm{C}$ & -0.253404 & -3.629064 & 0.388168 \\
\hline $\mathrm{H}$ & 3.192074 & -2.386389 & 3.409974 & $\mathrm{C}$ & -1.747699 & -3.391280 & 0.396994 \\
\hline $\mathrm{C}$ & 7.100881 & -0.279819 & 1.115266 & $\mathrm{H}$ & 0.088601 & -4.135491 & -0.527561 \\
\hline $\mathrm{H}$ & 7.155709 & 0.809894 & 1.218992 & $\mathrm{H}$ & 0.104056 & -4.210832 & 1.243617 \\
\hline $\mathrm{H}$ & 7.435650 & -0.712137 & 2.061878 & $\mathrm{H}$ & -2.300578 & -4.031292 & -0.298471 \\
\hline $\mathrm{H}$ & 7.825149 & -0.557539 & 0.343102 & $\mathrm{H}$ & -2.187845 & -3.519753 & 1.396358 \\
\hline $\mathrm{C}$ & -1.584081 & 0.615364 & -0.494804 & $\mathrm{~N}$ & 0.278398 & -2.261886 & 0.429982 \\
\hline $\mathrm{H}$ & -1.877561 & 0.369903 & -1.527243 & $\mathrm{~N}$ & -1.841644 & -1.983461 & -0.003276 \\
\hline $\mathrm{C}$ & -2.749250 & 0.973569 & 0.286582 & $\mathrm{C}$ & 1.694967 & -2.144882 & 0.314370 \\
\hline $\mathrm{C}$ & -4.007973 & 1.097385 & -0.341378 & $\mathrm{C}$ & 2.473185 & -2.186148 & 1.479984 \\
\hline $\mathrm{C}$ & -2.682287 & 1.191340 & 1.681690 & $\mathrm{C}$ & 2.276512 & -2.122955 & -0.965676 \\
\hline $\mathrm{C}$ & -5.145373 & 1.428935 & 0.382807 & $\mathrm{C}$ & 3.861744 & -2.186674 & 1.338636 \\
\hline $\mathrm{H}$ & -4.070644 & 0.914619 & -1.412980 & $\mathrm{C}$ & 3.665451 & -2.130189 & -1.053939 \\
\hline $\mathrm{C}$ & -3.823027 & 1.508465 & 2.404542 & $\mathrm{C}$ & 4.475425 & -2.159936 & 0.085271 \\
\hline $\mathrm{H}$ & -1.717287 & 1.093630 & 2.171686 & $\mathrm{H}$ & 4.480959 & -2.195433 & 2.235015 \\
\hline $\mathrm{C}$ & -5.056510 & 1.628547 & 1.760122 & $\mathrm{H}$ & 4.129431 & -2.080994 & -2.038743 \\
\hline $\mathrm{H}$ & -6.105050 & 1.520400 & -0.120203 & $\mathrm{C}$ & -3.153083 & -1.458847 & -0.214367 \\
\hline $\mathrm{H}$ & -3.756574 & 1.663856 & 3.478417 & $\mathrm{C}$ & -3.904881 & -0.971592 & 0.864896 \\
\hline $\mathrm{H}$ & -5.946821 & 1.879436 & 2.331334 & $\mathrm{C}$ & -3.696315 & -1.561009 & -1.502937 \\
\hline $\mathrm{O}$ & 0.369213 & 1.034219 & 1.956952 & $\mathrm{C}$ & -5.196521 & -0.515035 & 0.603520 \\
\hline $\mathrm{O}$ & 0.460521 & 0.482843 & -2.270605 & $\mathrm{C}$ & -4.993829 & -1.094004 & -1.713792 \\
\hline $\mathrm{C}$ & 1.623506 & 1.112805 & 1.542501 & $\mathrm{C}$ & -5.753929 & -0.553459 & -0.676118 \\
\hline $\mathrm{H}$ & 2.323071 & 0.346786 & 1.903278 & $\mathrm{H}$ & -5.785941 & -0.119842 & 1.430478 \\
\hline $\mathrm{H}$ & 2.053175 & 0.787686 & -0.015390 & $\mathrm{H}$ & -5.420919 & -1.157707 & -2.713909 \\
\hline $\mathrm{H}$ & 2.085150 & 2.116582 & 1.528437 & $\mathrm{C}$ & 1.417604 & -2.003402 & -2.183666 \\
\hline $\mathrm{C}$ & -0.110073 & 1.455875 & -3.058613 & $\mathrm{H}$ & 0.886694 & -1.039487 & -2.189995 \\
\hline $\mathrm{H}$ & -1.112884 & 1.794931 & -2.713677 & $\mathrm{H}$ & 2.015897 & -2.071452 & -3.095853 \\
\hline $\mathrm{H}$ & 0.488462 & 2.386711 & -3.159098 & $\mathrm{H}$ & 0.646838 & -2.783314 & -2.226917 \\
\hline \multirow[t]{2}{*}{$\mathrm{H}$} & -0.251614 & 1.076018 & -4.086867 & $\mathrm{C}$ & 1.816835 & -2.184850 & 2.823766 \\
\hline & & & & $\mathrm{H}$ & 1.278251 & -3.122294 & 3.012208 \\
\hline 2B & & & & $\mathrm{H}$ & 2.555605 & -2.068180 & 3.621017 \\
\hline $\mathrm{Ru}$ & -0.354728 & 0.680613 & 0.197520 & $\mathrm{H}$ & 1.077358 & -1.376065 & 2.890184 \\
\hline $\mathrm{C}$ & -1.380545 & 3.598818 & 0.893810 & $\mathrm{C}$ & 5.967113 & -2.145979 & -0.045148 \\
\hline $\mathrm{C}$ & 0.526702 & 3.611790 & -0.400533 & $\mathrm{H}$ & 6.454122 & -1.981747 & 0.920066 \\
\hline $\mathrm{C}$ & -1.493358 & 4.979674 & 0.818911 & $\mathrm{H}$ & 6.347228 & -3.090915 & -0.449720 \\
\hline $\mathrm{H}$ & -2.104730 & 2.989787 & 1.427625 & $\mathrm{H}$ & 6.295984 & -1.352945 & -0.725726 \\
\hline $\mathrm{C}$ & 0.483015 & 4.995090 & -0.524058 & $\mathrm{C}$ & -3.349370 & -0.967124 & 2.253246 \\
\hline $\mathrm{H}$ & 1.296191 & 3.019357 & -0.885739 & $\mathrm{H}$ & -3.889119 & -0.256877 & 2.885572 \\
\hline $\mathrm{H}$ & -2.320170 & 5.477230 & 1.314851 & $\mathrm{H}$ & -3.460380 & -1.954099 & 2.722180 \\
\hline $\mathrm{H}$ & 1.246618 & 5.504312 & -1.102870 & $\mathrm{H}$ & -2.283746 & -0.705777 & 2.270382 \\
\hline $\mathrm{N}$ & -0.381809 & 2.917196 & 0.305674 & $\mathrm{C}$ & -2.901054 & -2.160579 & -2.623472 \\
\hline
\end{tabular}




\begin{tabular}{|c|c|c|c|c|c|c|c|}
\hline $\mathrm{H}$ & -1.954436 & -1.631790 & -2.784060 & $\mathrm{C}$ & -0.460873 & -1.473649 & 0.152941 \\
\hline $\mathrm{H}$ & -2.635664 & -3.206205 & -2.423049 & $\mathrm{C}$ & -0.087662 & -3.806336 & 0.003680 \\
\hline $\mathrm{H}$ & -3.462864 & -2.137910 & -3.560353 & $\mathrm{C}$ & -1.572266 & -3.565435 & 0.227282 \\
\hline $\mathrm{C}$ & -7.131477 & -0.020442 & -0.927186 & $\mathrm{H}$ & 0.131936 & -4.203895 & -0.998527 \\
\hline $\mathrm{H}$ & -7.124465 & 1.071626 & -1.021033 & $\mathrm{H}$ & 0.348653 & -4.494704 & 0.735831 \\
\hline $\mathrm{H}$ & -7.558150 & -0.419460 & -1.851460 & $\mathrm{H}$ & -2.205519 & -4.038883 & -0.531363 \\
\hline $\mathrm{H}$ & -7.815007 & -0.261555 & -0.107521 & $\mathrm{H}$ & -1.911489 & -3.919763 & 1.212123 \\
\hline $\mathrm{C}$ & 1.613401 & 0.644752 & 0.495439 & $\mathrm{~N}$ & 0.486694 & -2.464399 & 0.144732 \\
\hline $\mathrm{H}$ & 1.799071 & 0.509914 & 1.572999 & $\mathrm{~N}$ & -1.673645 & -2.106798 & 0.151878 \\
\hline $\mathrm{C}$ & 2.841745 & 0.981960 & -0.186745 & $\mathrm{C}$ & 1.893896 & -2.304168 & -0.012955 \\
\hline $\mathrm{C}$ & 4.038746 & 1.095897 & 0.557111 & $\mathrm{C}$ & 2.743294 & -2.665515 & 1.044459 \\
\hline $\mathrm{C}$ & 2.919313 & 1.157453 & -1.588575 & $\mathrm{C}$ & 2.408354 & -1.805327 & -1.223904 \\
\hline $\mathrm{C}$ & 5.251645 & 1.369669 & -0.058835 & $\mathrm{C}$ & 4.113721 & -2.441353 & 0.898957 \\
\hline $\mathrm{H}$ & 3.990315 & 0.946065 & 1.634281 & $\mathrm{C}$ & 3.783069 & -1.596807 & -1.317236 \\
\hline $\mathrm{C}$ & 4.133792 & 1.428807 & -2.202088 & $\mathrm{C}$ & 4.649908 & -1.885365 & -0.261849 \\
\hline $\mathrm{H}$ & 2.006076 & 1.070586 & -2.168824 & $\mathrm{H}$ & 4.776576 & -2.692071 & 1.726628 \\
\hline $\mathrm{C}$ & 5.301500 & 1.533380 & -1.442833 & $\mathrm{H}$ & 4.184682 & -1.173861 & -2.237971 \\
\hline $\mathrm{H}$ & 6.160502 & 1.445974 & 0.532883 & $\mathrm{C}$ & -2.923344 & -1.489804 & -0.164588 \\
\hline $\mathrm{H}$ & 4.178176 & 1.556494 & -3.280699 & $\mathrm{C}$ & -3.823162 & -1.128372 & 0.852753 \\
\hline $\mathrm{H}$ & 6.250453 & 1.743203 & -1.930796 & $\mathrm{C}$ & -3.264829 & -1.361123 & -1.521901 \\
\hline $\mathrm{O}$ & -0.072045 & 1.066628 & -1.881506 & $\mathrm{C}$ & -5.054407 & -0.588543 & 0.477020 \\
\hline $\mathrm{O}$ & -0.335847 & 0.284797 & 2.261753 & $\mathrm{C}$ & -4.511641 & -0.825957 & -1.844469 \\
\hline $\mathrm{C}$ & -1.356356 & 1.090033 & -1.602775 & $\mathrm{C}$ & -5.416807 & -0.427684 & -0.861524 \\
\hline $\mathrm{H}$ & -1.987401 & 0.259505 & -1.943445 & $\mathrm{H}$ & -5.755682 & -0.297762 & 1.258590 \\
\hline $\mathrm{H}$ & -1.992725 & 0.874172 & 0.526915 & $\mathrm{H}$ & -4.776047 & -0.709137 & -2.895092 \\
\hline $\mathrm{H}$ & -1.872608 & 2.063704 & -1.604896 & $\mathrm{C}$ & 1.508966 & -1.479351 & -2.371488 \\
\hline $\mathrm{C}$ & -0.786863 & 1.285583 & 3.089749 & $\mathrm{H}$ & 0.845658 & -0.633435 & -2.151450 \\
\hline $\mathrm{H}$ & -0.239175 & 2.246251 & 2.985596 & $\mathrm{H}$ & 2.091925 & -1.228267 & -3.261236 \\
\hline $\mathrm{H}$ & -1.865262 & 1.523406 & 2.944459 & $\mathrm{H}$ & 0.856050 & -2.323632 & -2.623474 \\
\hline \multirow[t]{2}{*}{$\mathrm{H}$} & -0.675241 & 0.975795 & 4.144067 & $\mathrm{C}$ & 2.205206 & -3.254681 & 2.311914 \\
\hline & & & & $\mathrm{H}$ & 2.135328 & -4.348439 & 2.250651 \\
\hline OC & & & & $\mathrm{H}$ & 2.856653 & -3.028352 & 3.160120 \\
\hline $\mathrm{Ru}$ & -0.363651 & 0.500146 & 0.320482 & $\mathrm{H}$ & 1.204185 & -2.877591 & 2.540099 \\
\hline $\mathrm{C}$ & -0.921496 & 3.231893 & 1.814450 & $\mathrm{C}$ & 6.109507 & -1.569737 & -0.367719 \\
\hline $\mathrm{C}$ & -0.301827 & 3.603030 & -0.377047 & $\mathrm{H}$ & 6.688521 & -2.046533 & 0.427560 \\
\hline $\mathrm{C}$ & -1.065531 & 4.594143 & 2.044009 & $\mathrm{H}$ & 6.526744 & -1.887938 & -1.328145 \\
\hline $\mathrm{H}$ & -1.083924 & 2.477502 & 2.580961 & $\mathrm{H}$ & 6.275885 & -0.487750 & -0.295615 \\
\hline $\mathrm{C}$ & -0.415449 & 4.978063 & -0.223673 & $\mathrm{C}$ & -3.477083 & -1.293896 & 2.296502 \\
\hline $\mathrm{H}$ & -0.006171 & 3.147985 & -1.316829 & $\mathrm{H}$ & -4.370677 & -1.231851 & 2.922600 \\
\hline $\mathrm{H}$ & -1.370904 & 4.942256 & 3.025753 & $\mathrm{H}$ & -2.986415 & -2.252580 & 2.493943 \\
\hline $\mathrm{H}$ & -0.196430 & 5.631590 & -1.061839 & $\mathrm{H}$ & -2.771409 & -0.514501 & 2.607148 \\
\hline $\mathrm{N}$ & -0.551552 & 2.736557 & 0.619323 & $\mathrm{C}$ & -2.292179 & -1.749207 & -2.589530 \\
\hline $\mathrm{C}$ & -0.808118 & 5.487045 & 1.009365 & $\mathrm{H}$ & -1.413811 & -1.090828 & -2.550216 \\
\hline $\mathrm{H}$ & -0.907388 & 6.558388 & 1.161315 & $\mathrm{H}$ & -1.930682 & -2.777882 & -2.468060 \\
\hline
\end{tabular}




$\begin{array}{rrrc}\mathrm{H} & -2.743947 & -1.667414 & -3.581323 \\ \mathrm{C} & -6.759106 & 0.127470 & -1.229185 \\ \mathrm{H} & -6.795442 & 0.433545 & -2.277782 \\ \mathrm{H} & -7.552954 & -0.613336 & -1.079128 \\ \mathrm{H} & -7.019715 & 0.995185 & -0.615805 \\ \mathrm{C} & 1.470130 & 0.526223 & 0.538598 \\ \mathrm{H} & 1.876588 & -0.144001 & 1.315469 \\ \mathrm{C} & 2.500869 & 1.341317 & -0.074814 \\ \mathrm{C} & 3.761556 & 1.457697 & 0.547244 \\ \mathrm{C} & 2.333068 & 1.908306 & -1.354250 \\ \mathrm{C} & 4.798145 & 2.148064 & -0.065034 \\ \mathrm{H} & 3.913311 & 0.982827 & 1.515249 \\ \mathrm{C} & 3.382257 & 2.574431 & -1.973602 \\ \mathrm{H} & 1.380609 & 1.752733 & -1.859401 \\ \mathrm{C} & 4.612623 & 2.708266 & -1.330210 \\ \mathrm{H} & 5.758978 & 2.241337 & 0.435823 \\ \mathrm{H} & 3.245421 & 2.988769 & -2.969541 \\ \mathrm{H} & 5.428544 & 3.236974 & -1.815907 \\ \mathrm{O} & -0.636685 & 0.898493 & -1.730512 \\ \mathrm{O} & -0.768207 & 0.429236 & 2.335650 \\ \mathrm{C} & -1.867786 & 1.491130 & -1.964974 \\ \mathrm{H} & -1.788280 & 2.534670 & -2.330549 \\ \mathrm{H} & -2.454499 & 0.941847 & -2.723266 \\ \mathrm{H} & -2.517250 & 1.536826 & -1.062734 \\ \mathrm{C} & -0.278369 & -0.596444 & 3.114977 \\ \mathrm{H} & -0.603242 & -1.606720 & 2.781031 \\ \mathrm{H} & 0.830204 & -0.621046 & 3.146734 \\ \mathrm{H} & -0.621068 & -0.485976 & 4.158609\end{array}$

TS 1 1C-2C

$\begin{array}{rrrr}\mathrm{Ru} & 0.353255 & 0.751818 & -0.183472 \\ \mathrm{C} & 0.498475 & 3.600238 & -1.484300 \\ \mathrm{C} & -0.050018 & 3.734543 & 0.760824 \\ \mathrm{C} & 0.441961 & 4.981954 & -1.615873 \\ \mathrm{H} & 0.723285 & 2.920167 & -2.304995 \\ \mathrm{C} & -0.128539 & 5.119972 & 0.702949 \\ \mathrm{H} & -0.226793 & 3.169726 & 1.674678 \\ \mathrm{H} & 0.646564 & 5.436464 & -2.580090 \\ \mathrm{H} & -0.383177 & 5.681601 & 1.595892 \\ \mathrm{~N} & 0.254765 & 2.988514 & -0.313625 \\ \mathrm{C} & 0.121010 & 5.757802 & -0.507139 \\ \mathrm{H} & 0.066694 & 6.840250 & -0.584237 \\ \mathrm{C} & 0.681751 & -1.236938 & -0.249880 \\ \mathrm{C} & 0.372793 & -3.568424 & -0.541223\end{array}$

\begin{tabular}{|c|c|c|c|}
\hline & & & \\
\hline & 155933 & 34 & \\
\hline & - 037133 & & 18067 \\
\hline & 7 & & \\
\hline & 17 & & \\
\hline & 23 & -2.2 & -0.5 \\
\hline & & & \\
\hline & .64 & & \\
\hline & 2.52 & -2. & \\
\hline & -2.1 & & \\
\hline & -3.897006 & & \\
\hline & -3.497 & & \\
\hline & -4.40 & -2.2 & \\
\hline & -4.58 & & \\
\hline & -3 & & \\
\hline & 3.18 & & 0021 \\
\hline & 4.003 & -0 & 0.8 \\
\hline & 3.6 & & \\
\hline & & & \\
\hline & 4.91 & -1 & \\
\hline & 5.75 & -0 . & \\
\hline & & & \\
\hline & & & \\
\hline & -1.179 & -1.8 & 2.19 \\
\hline & -0.6 & -0.9 & \\
\hline & -1.7 & & \\
\hline & -0.40 & & \\
\hline & -2.042 & -2.75 & -2.7 \\
\hline & -2.2 & -3 & - \\
\hline & 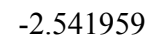 & & \\
\hline & -0.96 & -2 & -2.8 \\
\hline & -5.87 & -2 & 101 \\
\hline & -6.4502 & -2.10 & -0.4004 \\
\hline & -6.20 & & \\
\hline & -6.161 & $-1.41 \mathrm{C}$ & \\
\hline & 09 & -0.4 & 2.2 \\
\hline & 4.296877 & -0.025763 & -2.8694 \\
\hline & 1672 & & \\
\hline & 7 & 59 & 20270 \\
\hline & 124 & -2.2 & \\
\hline & 1.821415 & -1.601914 & . \\
\hline & & & \\
\hline & 3.225605 & -2 & \\
\hline & 13532 & -0.085304 & .22 \\
\hline
\end{tabular}




\begin{tabular}{|c|c|c|c|c|c|c|c|}
\hline $\mathrm{H}$ & 7.251313 & -0.024026 & 2.309954 & $\mathrm{H}$ & 0.078617 & -4.204442 & 1.207053 \\
\hline $\mathrm{H}$ & 7.887008 & -0.794272 & 0.858457 & $\mathrm{H}$ & -2.472375 & -3.906958 & -0.024438 \\
\hline $\mathrm{H}$ & 7.391976 & 0.890692 & 0.802238 & $\mathrm{H}$ & -2.138210 & -3.393539 & 1.640819 \\
\hline $\mathrm{C}$ & -1.551303 & 0.734372 & -0.615451 & $\mathrm{~N}$ & 0.246092 & -2.242590 & 0.420717 \\
\hline $\mathrm{H}$ & -1.784044 & 0.713310 & -1.694432 & $\mathrm{~N}$ & -1.895464 & -1.879348 & 0.192813 \\
\hline $\mathrm{C}$ & -2.747255 & 1.018882 & 0.145288 & $\mathrm{C}$ & 1.660406 & -2.180183 & 0.238489 \\
\hline $\mathrm{C}$ & -4.004034 & 1.039611 & -0.499698 & $\mathrm{C}$ & 2.507652 & -2.432402 & 1.329504 \\
\hline $\mathrm{C}$ & -2.717820 & 1.244918 & 1.540231 & $\mathrm{C}$ & 2.182817 & -2.019953 & -1.060477 \\
\hline $\mathrm{C}$ & -5.175826 & 1.260707 & 0.210845 & $\mathrm{C}$ & 3.888798 & -2.391383 & 1.118438 \\
\hline $\mathrm{H}$ & -4.038144 & 0.862803 & -1.573529 & $\mathrm{C}$ & 3.565092 & -2.004108 & -1.220448 \\
\hline $\mathrm{C}$ & -3.888733 & 1.480995 & 2.244616 & $\mathrm{C}$ & 4.437799 & -2.161660 & -0.140570 \\
\hline $\mathrm{H}$ & -1.756479 & 1.227699 & 2.044866 & $\mathrm{H}$ & 4.552300 & -2.556112 & 1.967314 \\
\hline $\mathrm{C}$ & -5.120283 & 1.483693 & 1.586309 & $\mathrm{H}$ & 3.973564 & -1.847997 & -2.218527 \\
\hline $\mathrm{H}$ & -6.134069 & 1.260265 & -0.303119 & $\mathrm{C}$ & -3.190837 & -1.359292 & -0.112955 \\
\hline $\mathrm{H}$ & -3.848348 & 1.657058 & 3.316627 & $\mathrm{C}$ & -3.982874 & -0.770944 & 0.884777 \\
\hline $\mathrm{H}$ & -6.036246 & 1.662813 & 2.144483 & $\mathrm{C}$ & -3.682350 & -1.572747 & -1.408733 \\
\hline $\mathrm{O}$ & 0.318088 & 1.034634 & 1.950652 & $\mathrm{C}$ & -5.268170 & -0.356960 & 0.535573 \\
\hline $\mathrm{O}$ & 0.595068 & 0.893003 & -2.275158 & $\mathrm{C}$ & -4.973436 & -1.136712 & -1.710711 \\
\hline $\mathrm{C}$ & 1.570958 & 1.193594 & 1.546027 & $\mathrm{C}$ & -5.779896 & -0.523311 & -0.752934 \\
\hline $\mathrm{H}$ & 1.978237 & 2.220064 & 1.594369 & $\mathrm{H}$ & -5.888614 & 0.111111 & 1.299198 \\
\hline $\mathrm{H}$ & 2.307387 & 0.446650 & 1.868730 & $\mathrm{H}$ & -5.357857 & -1.285427 & -2.718946 \\
\hline $\mathrm{H}$ & 2.035244 & 1.056884 & -0.063621 & $\mathrm{C}$ & 1.277259 & -1.858690 & -2.237738 \\
\hline $\mathrm{C}$ & 0.193544 & -0.128380 & -3.097982 & $\mathrm{H}$ & 0.766202 & -0.886362 & -2.226928 \\
\hline $\mathrm{H}$ & 0.772403 & -1.071932 & -2.962003 & $\mathrm{H}$ & 1.838811 & -1.938170 & -3.172426 \\
\hline $\mathrm{H}$ & -0.870818 & -0.412965 & -2.979924 & $\mathrm{H}$ & 0.495188 & -2.628229 & -2.250009 \\
\hline \multirow[t]{2}{*}{$\mathrm{H}$} & 0.323668 & 0.163960 & -4.155001 & $\mathrm{C}$ & 1.987508 & -2.813294 & 2.683060 \\
\hline & & & & $\mathrm{H}$ & 2.206688 & -3.867787 & 2.892141 \\
\hline $2 \mathrm{C}$ & & & & $\mathrm{H}$ & 2.467368 & -2.230889 & 3.475765 \\
\hline $\mathrm{Ru}$ & -0.379267 & 0.755108 & 0.151341 & $\mathrm{H}$ & 0.909964 & -2.666809 & 2.771876 \\
\hline $\mathrm{C}$ & -0.736961 & 3.573426 & 1.434316 & $\mathrm{C}$ & 5.920881 & -2.126208 & -0.345871 \\
\hline $\mathrm{C}$ & -0.030150 & 3.748950 & -0.762249 & $\mathrm{H}$ & 6.453509 & -1.959917 & 0.594895 \\
\hline $\mathrm{C}$ & -0.780675 & 4.955373 & 1.566859 & $\mathrm{H}$ & 6.291507 & -3.067085 & -0.769321 \\
\hline $\mathrm{H}$ & -0.975873 & 2.882406 & 2.240369 & $\mathrm{H}$ & 6.206735 & -1.328586 & -1.039073 \\
\hline $\mathrm{C}$ & -0.048387 & 5.136237 & -0.702424 & $\mathrm{C}$ & -3.459824 & -0.571947 & 2.268652 \\
\hline $\mathrm{H}$ & 0.256927 & 3.200759 & -1.657543 & $\mathrm{H}$ & -4.247306 & -0.212702 & 2.936260 \\
\hline $\mathrm{H}$ & -1.088607 & 5.391914 & 2.511715 & $\mathrm{H}$ & -3.062540 & -1.502436 & 2.691266 \\
\hline $\mathrm{H}$ & 0.235308 & 5.715090 & -1.575343 & $\mathrm{H}$ & -2.631486 & 0.150436 & 2.275324 \\
\hline $\mathrm{N}$ & -0.367090 & 2.981354 & 0.286930 & $\mathrm{C}$ & -2.837667 & -2.249285 & -2.446144 \\
\hline $\mathrm{C}$ & -0.431180 & 5.753861 & 0.483142 & $\mathrm{H}$ & -1.861430 & -1.763855 & -2.558996 \\
\hline $\mathrm{H}$ & -0.456734 & 6.837364 & 0.560521 & $\mathrm{H}$ & -2.630452 & -3.296552 & -2.190193 \\
\hline $\mathrm{C}$ & -0.681521 & -1.251356 & 0.193272 & $\mathrm{H}$ & -3.332309 & -2.244768 & -3.420394 \\
\hline $\mathrm{C}$ & -0.345908 & -3.585766 & 0.410058 & $\mathrm{C}$ & -7.173742 & -0.083782 & -1.083844 \\
\hline $\mathrm{C}$ & -1.815539 & -3.287089 & 0.593504 & $\mathrm{H}$ & -7.344109 & -0.058697 & -2.163284 \\
\hline $\mathrm{H}$ & -0.132009 & -4.081535 & -0.550369 & $\mathrm{H}$ & -7.920127 & -0.760717 & -0.652233 \\
\hline
\end{tabular}




\begin{tabular}{|c|c|c|c|c|c|c|c|}
\hline $\mathrm{H}$ & -7.387919 & 0.912829 & -0.686061 & $\mathrm{C}$ & 2.897755 & -1.197372 & -0.122999 \\
\hline $\mathrm{C}$ & 1.570629 & 0.792140 & 0.549931 & $\mathrm{C}$ & 3.704245 & -0.660430 & -1.137514 \\
\hline $\mathrm{H}$ & 1.750983 & 0.777621 & 1.637250 & $\mathrm{C}$ & 3.309535 & -1.155131 & 1.226933 \\
\hline $\mathrm{C}$ & 2.805147 & 1.097004 & -0.133631 & $\mathrm{C}$ & 4.889070 & -0.013903 & -0.766914 \\
\hline $\mathrm{C}$ & 4.010750 & 1.130192 & 0.605433 & $\mathrm{C}$ & 4.492079 & -0.494529 & 1.544046 \\
\hline $\mathrm{C}$ & 2.885297 & 1.298051 & -1.531910 & $\mathrm{C}$ & 5.290234 & 0.098732 & 0.560568 \\
\hline $\mathrm{C}$ & 5.234749 & 1.337972 & -0.012866 & $\mathrm{H}$ & 5.514019 & 0.414321 & -1.550227 \\
\hline $\mathrm{H}$ & 3.959864 & 0.969796 & 1.681084 & $\mathrm{H}$ & 4.805281 & -0.450342 & 2.586379 \\
\hline $\mathrm{C}$ & 4.109745 & 1.520794 & -2.144795 & $\mathrm{C}$ & -1.194278 & -2.285229 & 2.427494 \\
\hline $\mathrm{H}$ & 1.968067 & 1.267152 & -2.110366 & $\mathrm{H}$ & -0.690200 & -1.309081 & 2.434773 \\
\hline $\mathrm{C}$ & 5.286067 & 1.536221 & -1.392372 & $\mathrm{H}$ & -1.640959 & -2.448696 & 3.411133 \\
\hline $\mathrm{H}$ & 6.149657 & 1.346324 & 0.574190 & $\mathrm{H}$ & -0.408241 & -3.034698 & 2.283444 \\
\hline $\mathrm{H}$ & 4.155209 & 1.675351 & -3.219963 & $\mathrm{C}$ & -2.412038 & -2.111815 & -2.458031 \\
\hline $\mathrm{H}$ & 6.242881 & 1.703313 & -1.881493 & $\mathrm{H}$ & -3.240868 & -2.369495 & -3.121966 \\
\hline $\mathrm{O}$ & -0.080827 & 1.024024 & -1.947738 & $\mathrm{H}$ & -2.099140 & -1.088420 & -2.696222 \\
\hline $\mathrm{O}$ & -0.548084 & 0.878309 & 2.243281 & $\mathrm{H}$ & -1.559611 & -2.753833 & -2.700503 \\
\hline $\mathrm{C}$ & -1.367390 & 1.087642 & -1.672539 & $\mathrm{C}$ & -6.021549 & -2.139461 & 1.034632 \\
\hline $\mathrm{H}$ & -1.854270 & 2.075799 & -1.727404 & $\mathrm{H}$ & -6.649516 & -2.659455 & 0.305531 \\
\hline $\mathrm{H}$ & -2.018336 & 0.265156 & -1.994034 & $\mathrm{H}$ & -6.218165 & -2.570267 & 2.020033 \\
\hline $\mathrm{H}$ & -2.023765 & 0.970673 & 0.334723 & $\mathrm{H}$ & -6.365953 & -1.098912 & 1.063690 \\
\hline $\mathrm{C}$ & -0.127019 & -0.162528 & 3.032223 & $\mathrm{C}$ & 3.341442 & -0.743646 & -2.585211 \\
\hline $\mathrm{H}$ & -0.722863 & -1.095005 & 2.905163 & $\mathrm{H}$ & 2.981830 & 0.227482 & -2.940086 \\
\hline $\mathrm{H}$ & 0.928901 & -0.453999 & 2.862569 & $\mathrm{H}$ & 4.209379 & -1.029281 & -3.187442 \\
\hline \multirow[t]{2}{*}{$\mathrm{H}$} & -0.209216 & 0.116589 & 4.097173 & $\mathrm{H}$ & 2.535193 & -1.455709 & -2.770896 \\
\hline & & & & $\mathrm{C}$ & 2.494719 & -1.814146 & 2.293440 \\
\hline 3 & & & & $\mathrm{H}$ & 1.526327 & -1.314347 & 2.403834 \\
\hline $\mathrm{Ru}$ & 0.386827 & 0.632701 & -0.102657 & $\mathrm{H}$ & 2.283933 & -2.864543 & 2.058250 \\
\hline $\mathrm{C}$ & 0.433498 & -1.302224 & -0.242009 & $\mathrm{H}$ & 3.009348 & -1.783309 & 3.256776 \\
\hline $\mathrm{C}$ & 0.168909 & -3.641672 & -0.455263 & $\mathrm{C}$ & 6.534977 & 0.843804 & 0.933086 \\
\hline $\mathrm{C}$ & 1.622180 & -3.291950 & -0.754860 & $\mathrm{H}$ & 7.209933 & 0.957498 & 0.080841 \\
\hline $\mathrm{H}$ & 0.053505 & -4.204355 & 0.484581 & $\mathrm{H}$ & 6.297812 & 1.851334 & 1.294966 \\
\hline $\mathrm{H}$ & -0.303761 & -4.231089 & -1.248767 & $\mathrm{H}$ & 7.083338 & 0.343098 & 1.736484 \\
\hline $\mathrm{H}$ & 2.335415 & -3.860344 & -0.146592 & $\mathrm{C}$ & -1.419394 & 0.842240 & 0.226091 \\
\hline $\mathrm{H}$ & 1.881617 & -3.457677 & -1.811734 & $\mathrm{H}$ & -2.023640 & 0.091235 & 0.754861 \\
\hline $\mathrm{N}$ & -0.469358 & -2.326012 & -0.342607 & $\mathrm{C}$ & -2.226753 & 1.976639 & -0.212711 \\
\hline $\mathrm{N}$ & 1.672795 & -1.869335 & -0.429021 & $\mathrm{C}$ & -3.514484 & 2.155094 & 0.334310 \\
\hline $\mathrm{C}$ & -1.854310 & -2.273493 & -0.004101 & $\mathrm{C}$ & -1.789179 & 2.888292 & -1.195326 \\
\hline $\mathrm{C}$ & -2.814960 & -2.212109 & -1.022186 & $\mathrm{C}$ & -4.314699 & 3.223674 & -0.047546 \\
\hline $\mathrm{C}$ & -2.229007 & -2.316067 & 1.348132 & $\mathrm{H}$ & -3.870234 & 1.437382 & 1.073544 \\
\hline $\mathrm{C}$ & -4.162993 & -2.183411 & -0.660219 & $\mathrm{C}$ & -2.601469 & 3.943396 & -1.587112 \\
\hline $\mathrm{C}$ & -3.586879 & -2.288484 & 1.662875 & $\mathrm{H}$ & -0.813784 & 2.732784 & -1.650081 \\
\hline $\mathrm{C}$ & -4.569709 & -2.216311 & 0.673490 & $\mathrm{C}$ & -3.858742 & 4.124472 & -1.009371 \\
\hline $\mathrm{H}$ & -4.914888 & -2.126624 & -1.446386 & $\mathrm{H}$ & -5.299034 & 3.351276 & 0.395834 \\
\hline $\mathrm{H}$ & -3.883923 & -2.311641 & 2.710674 & $\mathrm{H}$ & -2.253857 & 4.633085 & -2.352146 \\
\hline
\end{tabular}




$\begin{array}{cccc}\mathrm{H} & -4.485720 & 4.957705 & -1.315893 \\ \mathrm{O} & 0.838447 & 0.970246 & -2.044237 \\ \mathrm{H} & 0.714177 & 0.459121 & 1.534024 \\ \mathrm{C} & 0.306980 & 0.205528 & -3.069220 \\ \mathrm{H} & 0.327284 & -0.883462 & -2.860466 \\ \mathrm{H} & -0.747720 & 0.465351 & -3.280209 \\ \mathrm{H} & 0.868585 & 0.362265 & -4.007147 \\ \mathrm{O} & 0.952418 & 2.875257 & 0.037394 \\ \mathrm{H} & 0.502693 & 1.860886 & 2.457892 \\ \mathrm{C} & 2.358115 & 2.941086 & -0.188439 \\ \mathrm{H} & 2.916096 & 2.332462 & 0.539842 \\ \mathrm{H} & 2.531225 & 2.543978 & -1.191182 \\ \mathrm{H} & 2.713536 & 3.976441 & -0.137271 \\ \mathrm{O} & 0.471021 & 2.806106 & 2.713121 \\ \mathrm{H} & 0.780361 & 3.110442 & 0.976517 \\ \mathrm{C} & -0.858724 & 3.090663 & 3.111797 \\ \mathrm{H} & -1.590544 & 2.834694 & 2.333067 \\ \mathrm{H} & -1.128055 & 2.561275 & 4.035142 \\ \mathrm{H} & -0.920041 & 4.163762 & 3.304003\end{array}$

TS $_{3-4}$

$\begin{array}{rrrr}\mathrm{Ru} & -0.488378 & 0.742763 & -0.230289 \\ \mathrm{C} & -0.234962 & -1.093047 & 0.439418 \\ \mathrm{C} & 0.394392 & -3.212689 & 1.240818 \\ \mathrm{C} & -1.051624 & -2.949802 & 1.646714 \\ \mathrm{H} & 0.483928 & -4.002199 & 0.478469 \\ \mathrm{H} & 1.033820 & -3.494468 & 2.083991 \\ \mathrm{H} & -1.738769 & -3.744997 & 1.338022 \\ \mathrm{H} & -1.161312 & -2.815558 & 2.733188 \\ \mathrm{~N} & 0.820197 & -1.921652 & 0.683901 \\ \mathrm{~N} & -1.348030 & -1.702904 & 0.943221 \\ \mathrm{C} & 2.145214 & -1.815967 & 0.159172 \\ \mathrm{C} & 3.207558 & -1.458121 & 1.002026 \\ \mathrm{C} & 2.374554 & -2.166150 & -1.183817 \\ \mathrm{C} & 4.499201 & -1.442585 & 0.469846 \\ \mathrm{C} & 3.679517 & -2.128196 & -1.670977 \\ \mathrm{C} & 4.757296 & -1.765229 & -0.860210 \\ \mathrm{H} & 5.324745 & -1.146281 & 1.116461 \\ \mathrm{H} & 3.858510 & -2.388017 & -2.713546 \\ \mathrm{C} & -2.679274 & -1.260037 & 0.651594 \\ \mathrm{C} & -3.423025 & -0.535565 & 1.594544 \\ \mathrm{C} & -3.230939 & -1.615308 & -0.595917 \\ \mathrm{C} & -4.710560 & -0.123459 & 1.235372 \\ \mathrm{C} & -4.508718 & -1.165758 & -0.910465\end{array}$

\begin{tabular}{|c|c|c|c|}
\hline & & & \\
\hline & -5.291188 & .450479 & 570 \\
\hline & .927415 & 10 & .8844 \\
\hline & 1.250 & & \\
\hline & 0.62 & -1.63 & -2.3 \\
\hline & 1.594 & -2.9 & 77 \\
\hline & 0. & & \\
\hline & 2.99 & -1 & 846. \\
\hline & 3.751 & & \\
\hline & 3.09 & & \\
\hline & 2.005523 & & \\
\hline & 6.14 & -1. & -1.4 \\
\hline & 6.892 & -2.04 & 0.70752 \\
\hline & 6.249243 & -2.2 & \\
\hline & 0.716 & -0.6 & \\
\hline & -2.868 & -0.1 & 202600 \\
\hline & -2.375277 & 826246 & 8412 \\
\hline & -3.660 & -0.0 & \\
\hline & -2.10 & -0.848 & \\
\hline & -2.45 & -2 & 56 \\
\hline & -1.558643 & -1.93 & -1.5 \\
\hline & -2.122 & -3.3 & -1 . \\
\hline & -3.06 & -2 & \\
\hline & -6.60 & 0.121879 & -0.39602 \\
\hline & -7.232104 & 0.320 & 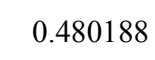 \\
\hline & -6.51 & & .9 \\
\hline & -7.1466 & -05 & -104720 \\
\hline & 1.268 & & \\
\hline & 1.6989 & 6 & 735 \\
\hline & & & \\
\hline & 3.59 & $1.71^{\circ}$ & -0.56 \\
\hline & 1.910 & 81 & 6070 \\
\hline & $4.592^{2}$ & 2.518937 & \\
\hline & & & \\
\hline & 2.907 & 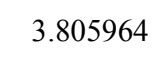 & 11200 \\
\hline & 0.8647 & 3 & .857 \\
\hline & 4.250097 & 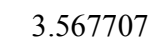 & 0.0205 \\
\hline & 5.635475 & 2.330429 & -0.26 \\
\hline & 2.640 & 4 & 17837 \\
\hline & 5.024 & & $1.24 \gamma_{2}$ \\
\hline & -0.586294 & 1.517498 & .6350 \\
\hline & -0.668500 & 0 & -1.8734 \\
\hline & & & \\
\hline & 27513 & .05202 & \\
\hline
\end{tabular}




$\begin{array}{lrrr}\mathrm{H} & 1.278935 & 1.271267 & 2.524894 \\ \mathrm{H} & -0.097141 & 1.512726 & 3.619343 \\ \mathrm{O} & -1.808038 & 2.315545 & -0.782541 \\ \mathrm{H} & -1.372616 & 0.531744 & -2.249696 \\ \mathrm{C} & -2.644697 & 3.016447 & 0.103057 \\ \mathrm{H} & -3.611917 & 2.505705 & 0.237460 \\ \mathrm{H} & -2.161889 & 3.097299 & 1.082689 \\ \mathrm{H} & -2.841289 & 4.027806 & -0.279627 \\ \mathrm{O} & -2.323524 & 1.271343 & -2.849436 \\ \mathrm{H} & -2.242510 & 1.911641 & -1.853503 \\ \mathrm{C} & -1.681069 & 1.989840 & -3.877125 \\ \mathrm{H} & -0.599778 & 2.101013 & -3.688899 \\ \mathrm{H} & -1.809121 & 1.475351 & -4.836464 \\ \mathrm{H} & -2.103745 & 2.998120 & -3.973612\end{array}$

\section{4}

$\begin{array}{cccc}\mathrm{C} & 0.205336 & -1.023677 & -0.499940 \\ \mathrm{C} & -0.476832 & -3.058269 & -1.461838 \\ \mathrm{C} & 0.972247 & -2.795822 & -1.853140 \\ \mathrm{H} & -0.583313 & -3.906352 & -0.768714 \\ \mathrm{H} & -1.129497 & -3.248214 & -2.320194 \\ \mathrm{H} & 1.646900 & -3.619919 & -1.597887 \\ \mathrm{H} & 1.086894 & -2.590120 & -2.927203 \\ \mathrm{~N} & -0.867805 & -1.810568 & -0.786771 \\ \mathrm{~N} & 1.294574 & -1.601012 & -1.071504 \\ \mathrm{C} & -2.175638 & -1.746475 & -0.214473 \\ \mathrm{C} & -3.272353 & -1.387251 & -1.010231 \\ \mathrm{C} & -2.355618 & -2.167388 & 1.116625 \\ \mathrm{C} & -4.548236 & -1.439916 & -0.443041 \\ \mathrm{C} & -3.645000 & -2.187182 & 1.643075 \\ \mathrm{C} & -4.757419 & -1.825959 & 0.878310 \\ \mathrm{H} & -5.401127 & -1.145827 & -1.053844 \\ \mathrm{H} & -3.785454 & -2.501997 & 2.676458 \\ \mathrm{C} & 2.642770 & -1.186393 & -0.811983 \\ \mathrm{C} & 3.333715 & -0.372234 & -1.718886 \\ \mathrm{C} & 3.256749 & -1.653517 & 0.367460 \\ \mathrm{C} & 4.634803 & 0.018789 & -1.385239 \\ \mathrm{C} & 4.550241 & -1.236277 & 0.651316 \\ \mathrm{C} & 5.249884 & -0.379929 & -0.202697 \\ \mathrm{H} & 5.174169 & 0.667905 & -2.074148 \\ \mathrm{H} & 5.010966 & -1.557407 & 1.583096 \\ \mathrm{C} & -1.183946 & -2.558490 & 1.958849 \\ \mathrm{H} & -0.554471 & -1.688662 & 2.181723\end{array}$

\begin{tabular}{|c|c|c|c|}
\hline & 0 & & \\
\hline & .532885 & 3.281812 & $454 \mathrm{C}$ \\
\hline & 119989 & 79 & \\
\hline & 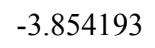 & & \\
\hline & -3.29 & 8 & \\
\hline & -2.11 & .08 & -2 \\
\hline & -6.1 & & \\
\hline & -6.899 & -2.0 & \\
\hline & -6.22 & -2.5 & \\
\hline & -6.3 & & \\
\hline & 2.719532 & & \\
\hline & & & \\
\hline & 3.47 & 0220818 & -3 \\
\hline & 1.934172 & 73 & \\
\hline & & & \\
\hline & 1.62 & $-2.1 c$ & \\
\hline & $2.21 \mathrm{C}$ & -3.4 & \\
\hline & 3.16 & -2 & \\
\hline & & & \\
\hline & 7.08 & 0. & -0.6 \\
\hline & 6.566 & & \\
\hline & 2 & & \\
\hline & -1.50 & & \\
\hline & -1.691 & 36 & 17297 \\
\hline & -2.324 & 36 & \\
\hline & -3.66 & & \\
\hline & -2.06 & 3 & -0.4064 \\
\hline & -4.705 & 2.385 & 0.2674 \\
\hline & $-3.8^{\prime}$ & & \\
\hline & & & \\
\hline & -1.03 & 3 & 0 \\
\hline & -4.42 & 9 & -05 \\
\hline & -5.732 & & \\
\hline & -2.88 & & \\
\hline & -5.23 & 4.109330 & -0.9120 \\
\hline & 0.461591 & 678973 & -1 \\
\hline & 0.525394 & 340380 & .00 \\
\hline & -0.34 & & \\
\hline & -0.1 & 8 & -2 \\
\hline & -1.4 & 10 & -2.28 \\
\hline & -0.128640 & 1.821725 & -3.4056 \\
\hline & 1. & 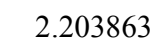 & \\
\hline & & 74 & \\
\hline & 189858 & 24033 & .0 \\
\hline
\end{tabular}




$\begin{array}{lllr}\mathrm{H} & 2.711000 & 2.879269 & -0.862542 \\ \mathrm{H} & 1.330804 & 3.830356 & -0.312440 \\ \mathrm{H} & 2.870401 & 3.916246 & 0.577159 \\ \mathrm{O} & 3.262303 & 0.757578 & 2.709870 \\ \mathrm{H} & 2.930522 & 1.298460 & 1.959747 \\ \mathrm{C} & 2.740432 & 1.349985 & 3.868749 \\ \mathrm{H} & 1.637409 & 1.308160 & 3.914380 \\ \mathrm{H} & 3.129846 & 0.806471 & 4.735352 \\ \mathrm{H} & 3.026711 & 2.407209 & 3.976160\end{array}$

$\begin{array}{rrrr}\mathrm{H} & 0.747290 & -0.157080 & 2.268820 \\ \mathrm{C} & 5.925298 & -1.398029 & 0.555040 \\ \mathrm{H} & 6.449017 & -2.229322 & 1.041858 \\ \mathrm{H} & 6.381522 & -1.263233 & -0.430085 \\ \mathrm{H} & 6.129802 & -0.499037 & 1.143740 \\ \mathrm{C} & -3.045950 & -0.702817 & 2.539337 \\ \mathrm{H} & -2.075164 & -0.191532 & 2.586124 \\ \mathrm{H} & -3.689230 & -0.327258 & 3.339527 \\ \mathrm{H} & -2.857953 & -1.766563 & 2.726559 \\ \mathrm{C} & -3.066456 & -1.473619 & -2.448264 \\ \mathrm{H} & -1.975869 & -1.525971 & -2.390224\end{array}$

3A

$\mathrm{Ru} \quad-0.440845$

$\begin{array}{llll}\text { C } & -0.619647 & -1.158139 & -0.034149\end{array}$

$\begin{array}{llll}\text { C } & -0.343104 & -3.445541 & 0.461675\end{array}$

$\begin{array}{llll}\text { C } & -1.815127 & -3.181470 & 0.197127\end{array}$

$\mathrm{H} \quad 0.054651 \quad-4.296566 \quad-0.101139$

$\mathrm{H} \quad-0.141052 \quad-3.625300 \quad 1.529364$

$\mathrm{H} \quad-2.142257 \quad-3.590091 \quad-0.772406$

$\begin{array}{llll}\mathrm{H} & -2.475764 & -3.584842 & 0.971663\end{array}$

$\begin{array}{llll}\mathrm{N} & 0.286476 & -2.191110 & 0.036622\end{array}$

$\begin{array}{llll}\mathrm{N} & -1.849543 & -1.722244 & 0.178519\end{array}$

$\begin{array}{llll}\text { C } & 1.696644 & -2.047864 & 0.213035\end{array}$

$\begin{array}{llll}\text { C } & 2.208594 & -1.488393 & 1.393352\end{array}$

$\begin{array}{llll}\text { C } & 2.541725 & -2.469456 & -0.824367\end{array}$

$\begin{array}{llll}\text { C } & 3.588821 & -1.295847 & 1.479957\end{array}$

$\begin{array}{llll}\text { C } & 3.912897 & -2.260734 & -0.689933\end{array}$

$\begin{array}{llll}\text { C } & 4.453445 & -1.654673 & 0.446483\end{array}$

$\begin{array}{llll}\mathrm{H} & 3.994684 & -0.829952 & 2.377572\end{array}$

$\begin{array}{llll}\mathrm{H} & 4.572878 & -2.549546 & -1.507564\end{array}$

$\begin{array}{llll}\text { C } & -3.070638 & -0.997437 & 0.037279\end{array}$

$\begin{array}{llll}\text { C } & -3.671401 & -0.480081 & 1.199159\end{array}$

$\begin{array}{llll}\text { C } & -3.658672 & -0.840158 & -1.226877\end{array}$

$\begin{array}{llll}\text { C } & -4.847923 & 0.253264 & 1.058096\end{array}$

$\begin{array}{llll}\text { C } & -4.838754 & -0.098353 & -1.314427\end{array}$

$\begin{array}{llll}\text { C } & -5.439945 & 0.465780 & -0.190026\end{array}$

$\begin{array}{llll}\mathrm{H} & -5.315412 & 0.669774 & 1.950055\end{array}$

$\begin{array}{llll}\mathrm{H} & -5.298396 & 0.042631 & -2.292050\end{array}$

$\begin{array}{llll}\text { C } & 1.964731 & -3.042964 & -2.081268\end{array}$

$\begin{array}{llll}\mathrm{H} & 1.197204 & -2.381579 & -2.501125\end{array}$

$\begin{array}{llll}\mathrm{H} & 2.737971 & -3.197149 & -2.837877\end{array}$

$\begin{array}{llll}\mathrm{H} & 1.475630 & -4.009656 & -1.910842\end{array}$

$\begin{array}{llll}\text { C } & 1.305045 & -1.071259 & 2.508538\end{array}$

$\begin{array}{llll}\mathrm{H} & 0.550244 & -1.835727 & 2.728028\end{array}$

H $\quad 1.875349 \quad-0.891847 \quad 3.423736$

$\begin{array}{llll}\mathrm{H} & -3.448633 & -2.492714 & -2.590409\end{array}$

$\begin{array}{llll}\mathrm{H} & -3.324920 & -0.908021 & -3.347139\end{array}$

$\begin{array}{llll}\text { C } & -6.690622 & 1.282285 & -0.311307\end{array}$

$\begin{array}{llll}\mathrm{H} & -7.123914 & 1.211900 & -1.312358\end{array}$

$\begin{array}{llll}\mathrm{H} & -7.452490 & 0.964174 & 0.407612\end{array}$

$\begin{array}{llll}\mathrm{H} & -6.496457 & 2.342235 & -0.112719\end{array}$

$\begin{array}{llll}\text { C } & 1.282334 & 0.588298 & -1.015664\end{array}$

$\begin{array}{llll}\mathrm{H} & 1.603429 & -0.262958 & -1.631732\end{array}$

$\begin{array}{llll}\text { C } & 2.394131 & 1.472225 & -0.685734\end{array}$

$\begin{array}{llll}\text { C } & 3.573459 & 1.447490 & -1.456582\end{array}$

$\begin{array}{llll}\text { C } & 2.378447 & 2.274397 & 0.474554\end{array}$

$\begin{array}{llll}\text { C } & 4.676830 & 2.213454 & -1.105006\end{array}$

$\begin{array}{llll}\mathrm{H} & 3.612300 & 0.796850 & -2.328885\end{array}$

$\begin{array}{llll}\text { C } & 3.488817 & 3.028516 & 0.830586\end{array}$

$\begin{array}{llll}\mathrm{H} & 1.489588 & 2.247232 & 1.106934\end{array}$

$\begin{array}{llll}\text { C } & 4.640083 & 3.006687 & 0.042021\end{array}$

$\begin{array}{llll}\mathrm{H} & 5.576496 & 2.180236 & -1.714638\end{array}$

$\begin{array}{llll}\mathrm{H} & 3.465915 & 3.626735 & 1.738227\end{array}$

$\begin{array}{llll}\mathrm{H} & 5.509460 & 3.592239 & 0.328657\end{array}$

$\begin{array}{llll}\mathrm{O} & -0.759537 & 1.202508 & 1.624464\end{array}$

$\begin{array}{llll}\mathrm{H} & -0.941184 & 0.437263 & -1.894334\end{array}$

$\begin{array}{llll}\text { C } & -1.470405 & 2.362707 & 1.888325\end{array}$

$\begin{array}{llll}\mathrm{H} & -0.829403 & 3.167650 & 2.300655\end{array}$

$\begin{array}{llll}\mathrm{H} & -1.983843 & 2.784496 & 1.001498\end{array}$

$\begin{array}{llll}\mathrm{H} & -2.264702 & 2.180475 & 2.636260\end{array}$

$\begin{array}{llll}\mathrm{O} & -0.692013 & 2.989202 & -1.055938\end{array}$

$\begin{array}{llll}\mathrm{H} & -0.491382 & 2.816180 & -1.983393\end{array}$

$\begin{array}{llll}\text { C } & -0.036996 & 4.191751 & -0.654593\end{array}$

$\mathrm{H} \quad 1.042826 \quad 4.147263 \quad-0.828910$

$\begin{array}{llll}\mathrm{H} & -0.466092 & 5.054309 & -1.176401\end{array}$

$\mathrm{H} \quad-0.215035 \quad 4.300924 \quad 0.416206$

TS 3A-4A 


\begin{tabular}{|c|c|c|c|c|c|c|c|}
\hline $\mathrm{Ru}$ & -0.588631 & 0.708204 & -0.521171 & $\mathrm{H}$ & -3.461439 & -2.746907 & -2.251824 \\
\hline $\mathrm{C}$ & -0.522911 & -1.184674 & 0.041391 & $\mathrm{H}$ & -3.292512 & -1.207773 & -3.092187 \\
\hline $\mathrm{C}$ & -0.070755 & -3.414944 & 0.615144 & $\mathrm{C}$ & -6.537089 & 1.210239 & -0.100935 \\
\hline $\mathrm{C}$ & -1.577415 & -3.235934 & 0.502077 & $\mathrm{H}$ & -7.247544 & 0.773216 & -0.808688 \\
\hline $\mathrm{H}$ & 0.310520 & -4.268012 & 0.044551 & $\mathrm{H}$ & -7.049136 & 1.344499 & 0.855514 \\
\hline $\mathrm{H}$ & 0.255112 & -3.536423 & 1.659108 & $\mathrm{H}$ & -6.287923 & 2.208673 & -0.477686 \\
\hline $\mathrm{H}$ & -1.984099 & -3.711271 & -0.404115 & $\mathrm{C}$ & 1.194625 & 0.778619 & -1.005646 \\
\hline $\mathrm{H}$ & -2.128290 & -3.624993 & 1.364129 & $\mathrm{H}$ & 1.538494 & 0.145993 & -1.843930 \\
\hline $\mathrm{N}$ & 0.442664 & -2.148892 & 0.073222 & $\mathrm{C}$ & 2.283978 & 1.573760 & -0.466052 \\
\hline $\mathrm{N}$ & -1.692744 & -1.781349 & 0.412609 & $\mathrm{C}$ & 3.525723 & 1.543778 & -1.133879 \\
\hline $\mathrm{C}$ & 1.845067 & -1.889011 & 0.131827 & $\mathrm{C}$ & 2.181939 & 2.325598 & 0.721947 \\
\hline $\mathrm{C}$ & 2.395959 & -1.295841 & 1.278791 & $\mathrm{C}$ & 4.620245 & 2.241829 & -0.643550 \\
\hline $\mathrm{C}$ & 2.636883 & -2.241901 & -0.969565 & $\mathrm{H}$ & 3.616583 & 0.946328 & -2.039628 \\
\hline $\mathrm{C}$ & 3.767772 & -1.043501 & 1.285884 & $\mathrm{C}$ & 3.285632 & 3.004188 & 1.219309 \\
\hline $\mathrm{C}$ & 4.003692 & -1.974322 & -0.913278 & $\mathrm{H}$ & 1.236109 & 2.311450 & 1.256290 \\
\hline $\mathrm{C}$ & 4.585526 & -1.369005 & 0.203311 & $\mathrm{C}$ & 4.504228 & 2.971027 & 0.538883 \\
\hline $\mathrm{H}$ & 4.204496 & -0.552384 & 2.154966 & $\mathrm{H}$ & 5.568857 & 2.208290 & -1.173925 \\
\hline $\mathrm{H}$ & 4.626125 & -2.225059 & -1.771558 & $\mathrm{H}$ & 3.198626 & 3.567639 & 2.144760 \\
\hline $\mathrm{C}$ & -2.943499 & -1.099829 & 0.281045 & $\mathrm{H}$ & 5.361308 & 3.513387 & 0.930241 \\
\hline $\mathrm{C}$ & -3.494573 & -0.500368 & 1.425251 & $\mathrm{O}$ & -0.663679 & 1.283796 & 1.444813 \\
\hline $\mathrm{C}$ & -3.577853 & -1.020809 & -0.971400 & $\mathrm{H}$ & -0.914583 & 0.356086 & -2.260981 \\
\hline $\mathrm{C}$ & -4.674005 & 0.229318 & 1.278561 & $\mathrm{C}$ & -1.280480 & 2.518870 & 1.637937 \\
\hline $\mathrm{C}$ & -4.750961 & -0.273394 & -1.065472 & $\mathrm{H}$ & -0.548624 & 3.346303 & 1.737004 \\
\hline $\mathrm{C}$ & -5.305493 & 0.370467 & 0.042038 & $\mathrm{H}$ & -1.967525 & 2.797101 & 0.819034 \\
\hline $\mathrm{H}$ & -5.104214 & 0.710683 & 2.155966 & $\mathrm{H}$ & -1.875884 & 2.503605 & 2.566885 \\
\hline $\mathrm{H}$ & -5.240221 & -0.186107 & -2.034682 & $\mathrm{O}$ & -1.603447 & 2.379417 & -1.515760 \\
\hline $\mathrm{C}$ & 2.007185 & -2.835976 & -2.190928 & $\mathrm{H}$ & -1.221117 & 1.277324 & -2.180324 \\
\hline $\mathrm{H}$ & 1.217379 & -2.185861 & -2.585704 & $\mathrm{C}$ & -0.821431 & 3.526985 & -1.723322 \\
\hline $\mathrm{H}$ & 2.745577 & -2.995097 & -2.980380 & $\mathrm{H}$ & 0.075502 & 3.319367 & -2.330573 \\
\hline $\mathrm{H}$ & 1.532150 & -3.801887 & -1.982627 & $\mathrm{H}$ & -1.409167 & 4.296652 & -2.243753 \\
\hline $\mathrm{C}$ & 1.530318 & -0.886834 & 2.426904 & $\mathrm{H}$ & -0.472180 & 3.956890 & -0.770309 \\
\hline $\mathrm{H}$ & 0.862951 & -1.695244 & 2.749991 & & & & \\
\hline $\mathrm{H}$ & 2.137100 & -0.590369 & 3.286041 & $4 \mathrm{~A}$ & & & \\
\hline $\mathrm{H}$ & 0.879869 & -0.042169 & 2.160664 & $\mathrm{Ru}$ & -0.460747 & 0.668659 & -0.934930 \\
\hline $\mathrm{C}$ & 6.053244 & -1.072940 & 0.237594 & $\mathrm{C}$ & -0.473047 & -1.188147 & -0.080315 \\
\hline $\mathrm{H}$ & 6.625290 & -1.915049 & 0.644733 & $\mathrm{C}$ & -0.040792 & -3.317557 & 0.811865 \\
\hline $\mathrm{H}$ & 6.446494 & -0.874086 & -0.764024 & $\mathrm{C}$ & -1.543694 & -3.142733 & 0.669394 \\
\hline $\mathrm{H}$ & 6.270965 & -0.202547 & 0.863210 & $\mathrm{H}$ & 0.335936 & -4.242132 & 0.363747 \\
\hline $\mathrm{C}$ & -2.811249 & -0.618007 & 2.749168 & $\mathrm{H}$ & 0.284598 & -3.295847 & 1.862283 \\
\hline $\mathrm{H}$ & -1.862951 & -0.067136 & 2.721204 & $\mathrm{H}$ & -1.965455 & -3.756751 & -0.140007 \\
\hline $\mathrm{H}$ & -3.434359 & -0.212761 & 3.550490 & $\mathrm{H}$ & -2.100291 & -3.368474 & 1.584374 \\
\hline $\mathrm{H}$ & -2.572620 & -1.658979 & 2.994564 & $\mathrm{~N}$ & 0.483499 & -2.139335 & 0.101603 \\
\hline $\mathrm{C}$ & -3.037439 & -1.737899 & -2.171030 & $\mathrm{~N}$ & -1.649339 & -1.722576 & 0.324132 \\
\hline $\mathrm{H}$ & -1.948960 & -1.835298 & -2.136212 & $\mathrm{C}$ & 1.886114 & -1.878811 & 0.163234 \\
\hline
\end{tabular}




\begin{tabular}{|c|c|c|c|c|c|c|c|}
\hline C & 2.408955 & -1.186300 & 1.265582 & C & 4.632082 & 2.434840 & -0.178174 \\
\hline $\mathrm{C}$ & 2.704005 & -2.344131 & -0.874326 & $\mathrm{H}$ & 3.939208 & 1.087982 & -1.706269 \\
\hline $\mathrm{C}$ & 3.785143 & -0.963829 & 1.300930 & $\mathrm{C}$ & 2.965736 & 3.157786 & 1.411196 \\
\hline $\mathrm{C}$ & 4.073448 & -2.095304 & -0.795997 & $\mathrm{H}$ & 0.967051 & 2.375976 & 1.107862 \\
\hline $\mathrm{C}$ & 4.631370 & -1.404710 & 0.283184 & $\mathrm{C}$ & 4.283364 & 3.174252 & 0.951609 \\
\hline $\mathrm{H}$ & 4.200573 & -0.390520 & 2.129071 & $\mathrm{H}$ & 5.656379 & 2.443767 & -0.543154 \\
\hline $\mathrm{H}$ & 4.718125 & -2.433952 & -1.606411 & $\mathrm{H}$ & 2.693289 & 3.737584 & 2.289362 \\
\hline $\mathrm{C}$ & -2.910386 & -1.052042 & 0.297360 & $\mathrm{H}$ & 5.034684 & 3.765977 & 1.468457 \\
\hline $\mathrm{C}$ & -3.407940 & -0.564080 & 1.519982 & $\mathrm{O}$ & -0.825713 & 1.328871 & 0.972533 \\
\hline $\mathrm{C}$ & -3.639524 & -0.926681 & -0.892581 & $\mathrm{H}$ & -0.596737 & -0.324036 & -2.384335 \\
\hline $\mathrm{C}$ & -4.646272 & 0.067056 & 1.523481 & $\mathrm{C}$ & -1.830416 & 2.269540 & 1.200075 \\
\hline $\mathrm{C}$ & -4.873807 & -0.270417 & -0.838003 & $\mathrm{H}$ & -1.444109 & 3.301080 & 1.290165 \\
\hline $\mathrm{C}$ & -5.391791 & 0.233031 & 0.351641 & $\mathrm{H}$ & -2.608094 & 2.281410 & 0.418730 \\
\hline $\mathrm{H}$ & -5.030658 & 0.461419 & 2.463350 & $\mathrm{H}$ & -2.337860 & 2.034826 & 2.152827 \\
\hline $\mathrm{H}$ & -5.445180 & -0.156403 & -1.758343 & $\mathrm{O}$ & -1.368309 & 2.238653 & -1.806164 \\
\hline $\mathrm{C}$ & 2.102921 & -3.046032 & -2.052020 & $\mathrm{H}$ & -0.214387 & 0.370212 & -2.660457 \\
\hline $\mathrm{H}$ & 1.324535 & -2.434948 & -2.523574 & $\mathrm{C}$ & -1.086505 & 3.545719 & -1.422123 \\
\hline $\mathrm{H}$ & 2.859351 & -3.276850 & -2.805705 & $\mathrm{H}$ & -0.670742 & 4.114693 & -2.271799 \\
\hline $\mathrm{H}$ & 1.620561 & -3.988468 & -1.767390 & $\mathrm{H}$ & -1.991077 & 4.080624 & -1.083763 \\
\hline $\mathrm{C}$ & 1.505955 & -0.633958 & 2.322304 & $\mathrm{H}$ & -0.347639 & 3.619393 & -0.605043 \\
\hline $\mathrm{H}$ & 0.894383 & -1.413375 & 2.794569 & & & & \\
\hline $\mathrm{H}$ & 2.082413 & -0.143384 & 3.110373 & $\mathbf{3} \mathrm{A}_{\mathrm{py}}$ & & & \\
\hline $\mathrm{H}$ & 0.802108 & 0.102924 & 1.907554 & $\mathrm{Ru}$ & -0.248333 & 0.615521 & 0.026459 \\
\hline $\mathrm{C}$ & 6.102334 & -1.127898 & 0.337801 & $\mathrm{C}$ & 0.469573 & 3.592708 & 0.862051 \\
\hline $\mathrm{H}$ & 6.663556 & -1.992868 & 0.710169 & $\mathrm{C}$ & 0.330280 & 3.333493 & -1.423995 \\
\hline $\mathrm{H}$ & 6.504023 & -0.892809 & -0.653078 & $\mathrm{C}$ & 0.826501 & 4.927496 & 0.728696 \\
\hline $\mathrm{H}$ & 6.325451 & -0.285853 & 0.998592 & $\mathrm{H}$ & 0.381464 & 3.089872 & 1.824639 \\
\hline $\mathrm{C}$ & -2.578652 & -0.659529 & 2.760183 & $\mathrm{C}$ & 0.686435 & 4.657702 & -1.641325 \\
\hline $\mathrm{H}$ & -1.641303 & -0.110917 & 2.601984 & $\mathrm{H}$ & 0.128721 & 2.648395 & -2.243352 \\
\hline $\mathrm{H}$ & -3.102229 & -0.232779 & 3.619183 & $\mathrm{H}$ & 1.022302 & 5.521862 & 1.615894 \\
\hline $\mathrm{H}$ & -2.311264 & -1.692874 & 3.012555 & $\mathrm{H}$ & 0.765667 & 5.033525 & -2.656471 \\
\hline $\mathrm{C}$ & -3.150720 & -1.465315 & -2.203351 & $\mathrm{~N}$ & 0.219184 & 2.797629 & -0.194618 \\
\hline $\mathrm{H}$ & -2.247805 & -2.072392 & -2.096151 & $\mathrm{C}$ & 0.939354 & 5.475572 & -0.545085 \\
\hline $\mathrm{H}$ & -3.918974 & -2.079494 & -2.684501 & $\mathrm{H}$ & 1.220976 & 6.516186 & -0.681425 \\
\hline $\mathrm{H}$ & -2.916560 & -0.647632 & -2.894639 & $\mathrm{C}$ & -0.708405 & -1.296495 & 0.283303 \\
\hline $\mathrm{C}$ & -6.698700 & 0.963609 & 0.377004 & $\mathrm{C}$ & -0.574799 & -3.526738 & 1.082131 \\
\hline $\mathrm{H}$ & -7.297759 & 0.754485 & -0.512921 & $\mathrm{C}$ & -2.008873 & -3.249534 & 0.681194 \\
\hline $\mathrm{H}$ & -7.293942 & 0.700876 & 1.256573 & $\mathrm{H}$ & -0.172651 & -4.450657 & 0.652799 \\
\hline $\mathrm{H}$ & -6.541804 & 2.047622 & 0.413789 & $\mathrm{H}$ & -0.453100 & -3.587668 & 2.174354 \\
\hline $\mathrm{C}$ & 1.393791 & 0.804169 & -1.100071 & $\mathrm{H}$ & -2.302330 & -3.791323 & -0.232991 \\
\hline $\mathrm{H}$ & 1.902274 & 0.271882 & -1.923202 & $\mathrm{H}$ & -2.733933 & -3.503687 & 1.462165 \\
\hline $\mathrm{C}$ & 2.332577 & 1.647043 & -0.380928 & $\mathrm{~N}$ & 0.131815 & -2.353208 & 0.565959 \\
\hline $\mathrm{C}$ & 3.669357 & 1.681420 & -0.833925 & $\mathrm{~N}$ & -1.987923 & -1.806200 & 0.430238 \\
\hline $\mathrm{C}$ & 1.995316 & 2.414380 & 0.753486 & $\mathrm{C}$ & 1.545673 & -2.462559 & 0.365073 \\
\hline
\end{tabular}




\begin{tabular}{|c|c|c|c|c|c|c|c|}
\hline $\mathrm{C}$ & 2.463913 & -2.024942 & 1.330576 & $\mathrm{C}$ & 4.859138 & 1.195789 & -1.657031 \\
\hline $\mathrm{C}$ & 1.985732 & -3.078406 & -0.819674 & $\mathrm{H}$ & 3.350242 & -0.008446 & -2.612183 \\
\hline $\mathrm{C}$ & 3.826587 & -2.164327 & 1.058142 & $\mathrm{C}$ & 4.249071 & 2.145364 & 0.474681 \\
\hline $\mathrm{C}$ & 3.355150 & -3.202115 & -1.044635 & $\mathrm{H}$ & 2.261153 & 1.676377 & 1.177935 \\
\hline $\mathrm{C}$ & 4.294355 & -2.732953 & -0.124960 & $\mathrm{C}$ & 5.188702 & 1.965145 & -0.541118 \\
\hline $\mathrm{H}$ & 4.542219 & -1.799145 & 1.794135 & $\mathrm{H}$ & 5.591931 & 1.040022 & -2.445506 \\
\hline $\mathrm{H}$ & 3.696794 & -3.657743 & -1.973616 & $\mathrm{H}$ & 4.510202 & 2.723332 & 1.358028 \\
\hline $\mathrm{C}$ & -3.170390 & -1.243679 & -0.139388 & $\mathrm{H}$ & 6.176827 & 2.410260 & -0.457436 \\
\hline $\mathrm{C}$ & -4.210476 & -0.838773 & 0.715426 & $\mathrm{O}$ & -0.023599 & 1.038661 & 2.090133 \\
\hline $\mathrm{C}$ & -3.323904 & -1.195166 & -1.534221 & $\mathrm{H}$ & -0.949017 & 0.665530 & -1.447848 \\
\hline $\mathrm{C}$ & -5.374433 & -0.318198 & 0.152345 & $\mathrm{C}$ & -0.658561 & 0.345147 & 3.096102 \\
\hline $\mathrm{C}$ & -4.502497 & -0.650082 & -2.053091 & $\mathrm{H}$ & -0.868205 & -0.716940 & 2.852134 \\
\hline $\mathrm{C}$ & -5.535777 & -0.201205 & -1.231553 & $\mathrm{H}$ & -0.057402 & 0.334348 & 4.026411 \\
\hline $\mathrm{H}$ & -6.174873 & 0.010924 & 0.814740 & $\mathrm{H}$ & -1.638802 & 0.789302 & 3.374489 \\
\hline $\mathrm{H}$ & -4.620461 & -0.596921 & -3.134750 & $\mathrm{O}$ & -2.435705 & 1.473232 & 0.667432 \\
\hline $\mathrm{C}$ & 1.005788 & -3.508396 & -1.866144 & $\mathrm{H}$ & -3.018754 & 1.429743 & -0.098374 \\
\hline $\mathrm{H}$ & 0.440063 & -2.648914 & -2.246307 & $\mathrm{C}$ & -2.642247 & 2.685201 & 1.367720 \\
\hline $\mathrm{H}$ & 1.513150 & -3.979071 & -2.711777 & $\mathrm{H}$ & -1.909259 & 2.689286 & 2.176570 \\
\hline $\mathrm{H}$ & 0.261304 & -4.219706 & -1.488416 & $\mathrm{H}$ & -2.474890 & 3.567973 & 0.735962 \\
\hline $\mathrm{C}$ & 2.009791 & -1.370918 & 2.590036 & $\mathrm{H}$ & -3.655023 & 2.726089 & 1.791842 \\
\hline $\mathrm{H}$ & 2.830522 & -1.284171 & 3.306762 & & & & \\
\hline $\mathrm{H}$ & 1.617343 & -0.367655 & 2.378745 & \multicolumn{4}{|c|}{$\mathbf{T S}_{3 \mathrm{~A}-4 \mathrm{Apy}}$} \\
\hline $\mathrm{H}$ & 1.191531 & -1.920796 & 3.066984 & $\mathrm{Ru}$ & -0.263986 & 0.634060 & 0.061381 \\
\hline $\mathrm{C}$ & 5.761009 & -2.801316 & -0.418622 & $\mathrm{C}$ & 0.774392 & 3.338109 & 1.209677 \\
\hline $\mathrm{H}$ & 6.118199 & -1.853192 & -0.838633 & $\mathrm{C}$ & 0.661168 & 3.401312 & -1.091577 \\
\hline $\mathrm{H}$ & 6.349372 & -2.988675 & 0.484422 & $\mathrm{C}$ & 1.278813 & 4.630660 & 1.258086 \\
\hline $\mathrm{H}$ & 5.994836 & -3.585004 & -1.144761 & $\mathrm{H}$ & 0.634847 & 2.730279 & 2.098758 \\
\hline $\mathrm{C}$ & -4.044813 & -0.909373 & 2.198683 & $\mathrm{C}$ & 1.171896 & 4.690119 & -1.129154 \\
\hline $\mathrm{H}$ & -4.935810 & -0.540979 & 2.713909 & $\mathrm{H}$ & 0.400907 & 2.864534 & -2.000710 \\
\hline $\mathrm{H}$ & -3.857725 & -1.931302 & 2.548373 & $\mathrm{H}$ & 1.509527 & 5.079709 & 2.218956 \\
\hline $\mathrm{H}$ & -3.184658 & -0.311128 & 2.513026 & $\mathrm{H}$ & 1.316958 & 5.183726 & -2.084468 \\
\hline $\mathrm{C}$ & -2.270632 & -1.729827 & -2.451837 & $\mathrm{~N}$ & 0.465651 & 2.725269 & 0.053788 \\
\hline $\mathrm{H}$ & -1.360070 & -1.122554 & -2.394582 & $\mathrm{C}$ & 1.484601 & 5.322918 & 0.070024 \\
\hline $\mathrm{H}$ & -1.988025 & -2.754652 & -2.184367 & $\mathrm{H}$ & 1.880414 & 6.334554 & 0.077007 \\
\hline $\mathrm{H}$ & -2.620081 & -1.739113 & -3.487229 & $\mathrm{C}$ & -0.878906 & -1.288122 & 0.242154 \\
\hline $\mathrm{C}$ & -6.798173 & 0.362211 & -1.810383 & $\mathrm{C}$ & -0.816446 & -3.566842 & 0.879108 \\
\hline $\mathrm{H}$ & -7.033502 & 1.345319 & -1.389351 & $\mathrm{C}$ & -2.239480 & -3.206502 & 0.491189 \\
\hline $\mathrm{H}$ & -6.730344 & 0.472110 & -2.895575 & $\mathrm{H}$ & -0.425508 & -4.437022 & 0.340609 \\
\hline $\mathrm{H}$ & -7.659149 & -0.281092 & -1.597648 & $\mathrm{H}$ & -0.709897 & -3.767059 & 1.955448 \\
\hline $\mathrm{C}$ & 1.332537 & 0.147833 & -0.840082 & $\mathrm{H}$ & -2.560467 & -3.686452 & -0.446310 \\
\hline $\mathrm{H}$ & 1.377031 & -0.709399 & -1.528359 & $\mathrm{H}$ & -2.978385 & -3.459285 & 1.259015 \\
\hline $\mathrm{C}$ & 2.626541 & 0.812728 & -0.757040 & $\mathrm{~N}$ & -0.071437 & -2.356431 & 0.523489 \\
\hline $\mathrm{C}$ & 3.600790 & 0.615805 & -1.755412 & $\mathrm{~N}$ & -2.160919 & -1.753425 & 0.305187 \\
\hline $\mathrm{C}$ & 2.986356 & 1.578452 & 0.371252 & $\mathrm{C}$ & 1.351041 & -2.432141 & 0.402901 \\
\hline
\end{tabular}




\begin{tabular}{|c|c|c|c|c|c|c|c|}
\hline $\mathrm{C}$ & 2.184603 & -2.244537 & 1.514342 & $\mathrm{C}$ & 4.682924 & 0.809629 & -2.218536 \\
\hline $\mathrm{C}$ & 1.886620 & -2.780382 & -0.851826 & $\mathrm{H}$ & 2.908778 & -0.050286 & -3.085626 \\
\hline $\mathrm{C}$ & 3.566092 & -2.280758 & 1.310838 & $\mathrm{C}$ & 4.469394 & 1.560078 & 0.068425 \\
\hline $\mathrm{C}$ & 3.270772 & -2.802180 & -1.003931 & $\mathrm{H}$ & 2.525362 & 1.274272 & 0.976428 \\
\hline $\mathrm{C}$ & 4.129695 & -2.521102 & 0.059386 & $\mathrm{C}$ & 5.245787 & 1.385191 & -1.078307 \\
\hline $\mathrm{H}$ & 4.220695 & -2.101067 & 2.163485 & $\mathrm{H}$ & 5.289049 & 0.658246 & -3.108403 \\
\hline $\mathrm{H}$ & 3.690216 & -3.028140 & -1.984055 & $\mathrm{H}$ & 4.916501 & 1.986089 & 0.963551 \\
\hline $\mathrm{C}$ & -3.330372 & -1.092538 & -0.183849 & $\mathrm{H}$ & 6.290915 & 1.684659 & -1.080192 \\
\hline $\mathrm{C}$ & -4.285205 & -0.626906 & 0.730522 & $\mathrm{O}$ & 0.676180 & 0.492270 & 1.889138 \\
\hline $\mathrm{C}$ & -3.528725 & -0.988608 & -1.566574 & $\mathrm{H}$ & -1.362354 & 1.005561 & -1.329614 \\
\hline $\mathrm{C}$ & -5.439581 & -0.030648 & 0.230560 & $\mathrm{C}$ & -0.259550 & 0.444538 & 2.910178 \\
\hline $\mathrm{C}$ & -4.693376 & -0.367167 & -2.021630 & $\mathrm{H}$ & -0.971021 & -0.404814 & 2.822669 \\
\hline $\mathrm{C}$ & -5.658364 & 0.119626 & -1.140774 & $\mathrm{H}$ & 0.253869 & 0.331596 & 3.881129 \\
\hline $\mathrm{H}$ & -6.180717 & 0.346102 & 0.934737 & $\mathrm{H}$ & -0.895083 & 1.352044 & 2.980919 \\
\hline $\mathrm{H}$ & -4.852909 & -0.272196 & -3.095188 & $\mathrm{O}$ & -2.248764 & 1.469933 & 0.758052 \\
\hline $\mathrm{C}$ & 0.993558 & -3.126235 & -2.004221 & $\mathrm{H}$ & -1.880448 & 1.252198 & -0.594788 \\
\hline $\mathrm{H}$ & 0.138367 & -2.448458 & -2.081605 & $\mathrm{C}$ & -2.438968 & 2.804246 & 1.080651 \\
\hline $\mathrm{H}$ & 1.541227 & -3.093912 & -2.950224 & $\mathrm{H}$ & -1.859874 & 3.114468 & 1.973290 \\
\hline $\mathrm{H}$ & 0.580649 & -4.139222 & -1.905886 & $\mathrm{H}$ & -2.151454 & 3.500662 & 0.269073 \\
\hline $\mathrm{C}$ & 1.656432 & -2.076083 & 2.903221 & $\mathrm{H}$ & -3.502689 & 2.998291 & 1.304480 \\
\hline $\mathrm{H}$ & 1.975642 & -2.916199 & 3.532418 & & & & \\
\hline $\mathrm{H}$ & 2.034353 & -1.156264 & 3.355500 & $4 A_{p y}$ & & & \\
\hline $\mathrm{H}$ & 0.568111 & -2.018312 & 2.928172 & $\mathrm{Ru}$ & -0.288101 & 0.685158 & -0.115205 \\
\hline $\mathrm{C}$ & 5.611821 & -2.455845 & -0.140933 & $\mathrm{C}$ & 0.790610 & 3.455733 & 0.896225 \\
\hline $\mathrm{H}$ & 5.951019 & -1.413251 & -0.159161 & $\mathrm{C}$ & 0.403677 & 3.507318 & -1.372417 \\
\hline $\mathrm{H}$ & 6.156794 & -2.954995 & 0.666302 & $\mathrm{C}$ & 1.186846 & 4.787263 & 0.895373 \\
\hline $\mathrm{H}$ & 5.914046 & -2.911887 & -1.087767 & $\mathrm{H}$ & 0.780560 & 2.833483 & 1.789230 \\
\hline $\mathrm{C}$ & -4.032089 & -0.694090 & 2.201003 & $\mathrm{C}$ & 0.785187 & 4.838592 & -1.458795 \\
\hline $\mathrm{H}$ & -4.945726 & -0.510200 & 2.772254 & $\mathrm{H}$ & 0.089158 & 2.953737 & -2.254067 \\
\hline $\mathrm{H}$ & -3.618893 & -1.658656 & 2.514717 & $\mathrm{H}$ & 1.493013 & 5.253949 & 1.826304 \\
\hline $\mathrm{H}$ & -3.292553 & 0.070910 & 2.464285 & $\mathrm{H}$ & 0.766568 & 5.342758 & -2.419457 \\
\hline $\mathrm{C}$ & -2.512842 & -1.510569 & -2.534956 & $\mathrm{~N}$ & 0.397351 & 2.818734 & -0.219463 \\
\hline $\mathrm{H}$ & -1.572129 & -0.949629 & -2.459927 & $\mathrm{C}$ & 1.181865 & 5.496889 & -0.299260 \\
\hline $\mathrm{H}$ & -2.266275 & -2.562828 & -2.346881 & $\mathrm{H}$ & 1.483022 & 6.540200 & -0.328467 \\
\hline $\mathrm{H}$ & -2.872396 & -1.432838 & -3.563969 & $\mathrm{C}$ & -0.849589 & -1.241818 & 0.291637 \\
\hline $\mathrm{C}$ & -6.907235 & 0.774616 & -1.647301 & $\mathrm{C}$ & -0.699878 & -3.400916 & 1.233266 \\
\hline $\mathrm{H}$ & -7.025965 & 1.783372 & -1.238149 & $\mathrm{C}$ & -2.153088 & -3.116271 & 0.881898 \\
\hline $\mathrm{H}$ & -6.906477 & 0.856637 & -2.737240 & $\mathrm{H}$ & -0.309155 & -4.307504 & 0.757254 \\
\hline $\mathrm{H}$ & -7.802689 & 0.213095 & -1.358574 & $\mathrm{H}$ & -0.536072 & -3.498666 & 2.314871 \\
\hline $\mathrm{C}$ & 1.166511 & 0.146197 & -1.061534 & $\mathrm{H}$ & -2.540709 & -3.764470 & 0.082363 \\
\hline $\mathrm{H}$ & 0.969879 & -0.502008 & -1.932660 & $\mathrm{H}$ & -2.831218 & -3.211650 & 1.737010 \\
\hline $\mathrm{C}$ & 2.540095 & 0.615786 & -1.069853 & $\mathrm{~N}$ & 0.001973 & -2.212521 & 0.729738 \\
\hline $\mathrm{C}$ & 3.350418 & 0.422291 & -2.208306 & $\mathrm{~N}$ & -2.109679 & -1.724530 & 0.417106 \\
\hline $\mathrm{C}$ & 3.132634 & 1.184241 & 0.078113 & $\mathrm{C}$ & 1.399897 & -2.325965 & 0.438402 \\
\hline
\end{tabular}




\begin{tabular}{|c|c|c|c|c|c|c|c|}
\hline $\mathrm{C}$ & 2.376882 & -2.058671 & 1.401587 & $\mathrm{C}$ & 4.987526 & 0.674356 & -1.691864 \\
\hline $\mathrm{C}$ & 1.753407 & -2.811242 & -0.838071 & $\mathrm{H}$ & 3.321695 & -0.139999 & -2.785650 \\
\hline $\mathrm{C}$ & 3.718961 & -2.194799 & 1.027033 & $\mathrm{C}$ & 4.484865 & 1.489134 & 0.524373 \\
\hline $\mathrm{C}$ & 3.098708 & -2.942423 & -1.157172 & $\mathrm{H}$ & 2.421030 & 1.314767 & 1.146307 \\
\hline $\mathrm{C}$ & 4.103627 & -2.617801 & -0.239267 & $\mathrm{C}$ & 5.410351 & 1.249911 & -0.492369 \\
\hline $\mathrm{H}$ & 4.487172 & -1.955530 & 1.762094 & $\mathrm{H}$ & 5.705298 & 0.484682 & -2.486815 \\
\hline $\mathrm{H}$ & 3.372856 & -3.292520 & -2.152320 & $\mathrm{H}$ & 4.817527 & 1.924090 & 1.464236 \\
\hline $\mathrm{C}$ & -3.324564 & -1.142212 & -0.067389 & $\mathrm{H}$ & 6.457344 & 1.506758 & -0.350737 \\
\hline $\mathrm{C}$ & -4.219650 & -0.564007 & 0.845232 & $\mathrm{O}$ & 0.504363 & 0.726200 & 1.780871 \\
\hline $\mathrm{C}$ & -3.637316 & -1.249501 & -1.427703 & $\mathrm{H}$ & -0.995124 & 1.090311 & -1.679681 \\
\hline $\mathrm{C}$ & -5.417966 & -0.052971 & 0.356348 & $\mathrm{C}$ & -0.353037 & 0.452207 & 2.832938 \\
\hline $\mathrm{C}$ & -4.845784 & -0.707462 & -1.873875 & $\mathrm{H}$ & -0.846134 & -0.539897 & 2.760055 \\
\hline $\mathrm{C}$ & -5.743705 & -0.097551 & -1.001555 & $\mathrm{H}$ & 0.224176 & 0.441895 & 3.774390 \\
\hline $\mathrm{H}$ & -6.111338 & 0.410366 & 1.057405 & $\mathrm{H}$ & -1.164884 & 1.190581 & 2.958784 \\
\hline $\mathrm{H}$ & -5.094655 & -0.781107 & -2.932053 & $\mathrm{O}$ & -2.140512 & 1.443312 & 0.565694 \\
\hline $\mathrm{C}$ & 0.693815 & -3.142549 & -1.841052 & $\mathrm{H}$ & -1.199107 & 0.277773 & -1.542331 \\
\hline $\mathrm{H}$ & 0.077365 & -2.266408 & -2.073403 & $\mathrm{C}$ & -2.302502 & 2.670503 & 1.152775 \\
\hline $\mathrm{H}$ & 1.131314 & -3.505891 & -2.774086 & $\mathrm{H}$ & -1.557303 & 2.910473 & 1.944314 \\
\hline $\mathrm{H}$ & 0.002075 & -3.913616 & -1.477345 & $\mathrm{H}$ & -2.248193 & 3.515330 & 0.431845 \\
\hline $\mathrm{C}$ & 2.052682 & -1.650533 & 2.801774 & $\mathrm{H}$ & -3.297069 & 2.747346 & 1.637384 \\
\hline $\mathrm{H}$ & 2.645622 & -2.232213 & 3.515776 & & & & \\
\hline $\mathrm{H}$ & 2.277235 & -0.591357 & 2.955771 & 3B & & & \\
\hline $\mathrm{H}$ & 0.995313 & -1.780658 & 3.035470 & $\mathrm{Ru}$ & -0.375505 & 0.758636 & -0.160365 \\
\hline $\mathrm{C}$ & 5.547292 & -2.732073 & -0.617982 & $\mathrm{C}$ & -0.405687 & -1.122737 & 0.257661 \\
\hline $\mathrm{H}$ & 5.772788 & -2.114571 & -1.494451 & $\mathrm{C}$ & -0.125761 & -3.399584 & 0.832107 \\
\hline $\mathrm{H}$ & 6.202271 & -2.402395 & 0.192629 & $\mathrm{C}$ & -1.583891 & -3.020322 & 1.064034 \\
\hline $\mathrm{H}$ & 5.820112 & -3.762057 & -0.873476 & $\mathrm{H}$ & -0.001978 & -4.088077 & -0.018914 \\
\hline $\mathrm{C}$ & -3.867183 & -0.439631 & 2.290570 & $\mathrm{H}$ & 0.339977 & -3.871855 & 1.704314 \\
\hline $\mathrm{H}$ & -4.719156 & -0.087152 & 2.877348 & $\mathrm{H}$ & -2.288864 & -3.696931 & 0.567309 \\
\hline $\mathrm{H}$ & -3.523737 & -1.384334 & 2.727881 & $\mathrm{H}$ & -1.840497 & -3.000484 & 2.135098 \\
\hline $\mathrm{H}$ & -3.044759 & 0.278037 & 2.384417 & $\mathrm{~N}$ & 0.505216 & -2.108428 & 0.545942 \\
\hline $\mathrm{C}$ & -2.725990 & -1.938197 & -2.397784 & $\mathrm{~N}$ & -1.644789 & -1.682088 & 0.490009 \\
\hline $\mathrm{H}$ & -1.862605 & -1.319662 & -2.674150 & $\mathrm{C}$ & 1.892886 & -2.087812 & 0.215240 \\
\hline $\mathrm{H}$ & -2.315052 & -2.869993 & -1.994355 & $\mathrm{C}$ & 2.835788 & -1.835644 & 1.219710 \\
\hline $\mathrm{H}$ & -3.255233 & -2.180042 & -3.323090 & $\mathrm{C}$ & 2.287344 & -2.328285 & -1.110877 \\
\hline $\mathrm{C}$ & -7.027433 & 0.492569 & -1.499746 & $\mathrm{C}$ & 4.187021 & -1.812235 & 0.869639 \\
\hline $\mathrm{H}$ & -7.015387 & 1.585914 & -1.430341 & $\mathrm{C}$ & 3.647681 & -2.297636 & -1.414350 \\
\hline $\mathrm{H}$ & -7.212781 & 0.233455 & -2.545516 & $\mathrm{C}$ & 4.612763 & -2.032631 & -0.439987 \\
\hline $\mathrm{H}$ & -7.885809 & 0.151288 & -0.912010 & $\mathrm{H}$ & 4.925695 & -1.606038 & 1.643171 \\
\hline $\mathrm{C}$ & 1.318970 & 0.169179 & -1.035492 & $\mathrm{H}$ & 3.960122 & -2.469001 & -2.443973 \\
\hline $\mathrm{H}$ & 1.239279 & -0.433346 & -1.960511 & $\mathrm{C}$ & -2.870145 & -1.056468 & 0.106506 \\
\hline $\mathrm{C}$ & 2.694267 & 0.583019 & -0.854923 & $\mathrm{C}$ & -3.669665 & -0.382982 & 1.042609 \\
\hline $\mathrm{C}$ & 3.653273 & 0.335165 & -1.862483 & $\mathrm{C}$ & -3.284389 & -1.186773 & -1.236887 \\
\hline $\mathrm{C}$ & 3.145114 & 1.170258 & 0.349098 & $\mathrm{C}$ & -4.850129 & 0.219364 & 0.592813 \\
\hline
\end{tabular}




\begin{tabular}{|c|c|c|c|}
\hline & 110 & 27 & -1.637332 \\
\hline & 251665 & 0.163359 & - 7386 \\
\hline & .465 & 0.751485 & 3146 \\
\hline & .775286 & -0.648420 & -2.676421 \\
\hline & 1.266355 & -2.494424 & -2191952 \\
\hline & & & -2.348536 \\
\hline & 1.732532 & -2.784002 & -3.136858 \\
\hline & 506668 & 582 & -1.5 \\
\hline & 770 & 03 & 31 \\
\hline $\mathrm{H}$ & 3.224332 & -1.658857 & (2) \\
\hline $\mathrm{H}$ & 2.060410 & -0.488759 & 818 \\
\hline 1 & 829 & 3 & 2.937755 \\
\hline & 6.0 & -1.9 & 0.799313 \\
\hline $\mathrm{H}$ & 6.706508 & 214 & (1) \\
\hline $\mathrm{H}$ & 9 & 2 & 34 \\
\hline $\mathrm{H}$ & 349679 & -0.920355 & -1.050927 \\
\hline C & 299649 & -0.276157 & 2.486707 \\
\hline $\mathrm{H}$ & 6 & 7 & 04 \\
\hline $\mathrm{H}$ & -4.156700 & -0.507672 & 3.126946 \\
\hline$H$ & -2.4 & 268 & 2. \\
\hline $\mathrm{C}$ & -2.476581 & -1.989799 & -2.205045 \\
\hline $\mathrm{H}$ & -1.501659 & -1.520220 & -2.3 \\
\hline $\mathrm{H}$ & $22+\frac{1}{2}$ & -3.001868 & 9 \\
\hline $\mathrm{H}$ & -2.988061 & -2.079292 & -3.166480 \\
\hline $\mathrm{C}$ & -6.488334 & 0 & 03 \\
\hline $\mathrm{H}$ & -6.247688 & 1.853217 & -1.623769 \\
\hline $\mathrm{H}$ & -7.004819 & 711 & 9144 \\
\hline $\mathrm{H}$ & -7.193658 & 1.034382 & -0.384883 \\
\hline $\mathrm{C}$ & 140 & 26288 & -0.569649 \\
\hline $\mathrm{H}$ & 2.025227 & 085670 & 4238 \\
\hline $\mathrm{C}$ & 2.2 & 2 & 7 \\
\hline C & 3.499828 & 2.178012 & -1.003055 \\
\hline $\mathrm{C}$ & .796126 & 050750 & 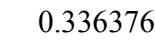 \\
\hline $\mathrm{C}$ & 4.311639 & 3.296960 & -0.876805 \\
\hline $\mathrm{H}$ & 3.844754 & 1.306159 & 1.558351 \\
\hline$C$ & 618778 & 128 & . \\
\hline $\mathrm{H}$ & 20 & 8751 & 9508 \\
\hline C & 3.872415 & 6 & 0 \\
\hline $\mathrm{H}$ & 5.293819 & 3.311371 & -1.343151 \\
\hline$U$ & 2.284824 & 5.206870 & 1.068357 \\
\hline $\mathrm{H}$ & 4.509512 & 5.269240 & -0.029543 \\
\hline $\mathrm{O}$ & ס & . & 3641 \\
\hline 20 & -0.738340 & 0.402336 & -1.742973 \\
\hline $\mathrm{C}$ & 0.317317 & 0.916337 & (2.01 \\
\hline
\end{tabular}

$\begin{array}{rrrr}\mathrm{H} & -0.321217 & -0.192407 & 2.867622 \\ \mathrm{H} & 0.731511 & 1.231369 & 3.006247 \\ \mathrm{H} & -0.887948 & 1.252024 & 3.730697 \\ \mathrm{O} & -1.052185 & 2.959383 & -0.738224 \\ \mathrm{H} & -0.913914 & 2.945490 & -1.692631 \\ \mathrm{C} & -2.460401 & 2.909161 & -0.484241 \\ \mathrm{H} & -2.930091 & 2.043641 & -0.976074 \\ \mathrm{H} & -2.560136 & 2.796783 & 0.596451 \\ \mathrm{H} & -2.951571 & 3.831812 & -0.813373\end{array}$

\section{TS 3 B-4B}

\begin{tabular}{|c|c|c|c|}
\hline & 284 & 204 & -0.458319 \\
\hline $\mathrm{C}$ & 380661 & .006923 & 035032 \\
\hline 4 & -0.027898 & .146343 & 287551 \\
\hline $\mathrm{C}$ & 118 t & 2 & (1) \\
\hline & 7 & 4 & 04 \\
\hline $\mathrm{H}$ & 0.537145 & -3.452779 & 422 \\
\hline $\mathrm{H}$ & -2.207128 & 98 & 000 \\
\hline $\mathrm{H}$ & -1.601666 & -2.5 & 78947 \\
\hline $\mathrm{N}$ & 0.554639 & 59 & 124 \\
\hline $\mathrm{N}$ & -1.583939 & -1.4 & 039 \\
\hline $\mathrm{C}$ & 1.912655 & -1.961003 & 0.267827 \\
\hline $\mathrm{C}$ & 081 & -1.6 & 826 \\
\hline $\mathrm{C}$ & 2.19 & -2. & 94 \\
\hline $\mathrm{C}$ & 4.259869 & -1.688420 & 703136 \\
\hline $\mathrm{C}$ & 3. & -2.4 & 32 \\
\hline $\mathrm{C}$ & 4.57 & -2.0 & $0 \pi$ \\
\hline $\mathrm{H}$ & 5.062700 & -1.4 & \\
\hline $\mathrm{H}$ & 3 & -2.1 & -2.483964 \\
\hline $\mathrm{C}$ & -2.852 & -0.9 & 25 \\
\hline $\mathrm{C}$ & -3.598254 & -0.1 & 5744 \\
\hline $\mathrm{C}$ & -3.3683 & -1 & -0.814911 \\
\hline $\mathrm{C}$ & -4.849048 & 0.315973 & 0.832636 \\
\hline $\mathrm{C}$ & -4.614169 & -0.905223 & -1.215135 \\
\hline $\mathrm{C}$ & -5.3 & $-0 .($ & ר, \\
\hline $\mathrm{H}$ & -5.430754 & 0.968609 & 1.482378 \\
\hline 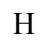 & -5.013052 & -1.221339 & -2.178060 \\
\hline $\mathrm{C}$ & 1.094338 & -2.6 & 483 \\
\hline $\mathrm{H}$ & 0.577946 & -1.7 & -2.2 \\
\hline $\mathrm{H}$ & 1.474569 & -3.118022 & -2.928247 \\
\hline$U$ & 0.329620 & -3.311161 & -1.588550 \\
\hline $\mathrm{C}$ & 2.664218 & -1.217912 & 2.573275 \\
\hline $\mathrm{H}$ & 3.138993 & -1.901082 & 3. \\
\hline $\mathrm{H}$ & 3.071175 & -0.220208 & 2.770133 \\
\hline
\end{tabular}




\begin{tabular}{|c|c|c|c|c|c|c|c|}
\hline $\mathrm{H}$ & 1.594824 & -1.180522 & 2.787329 & $\mathrm{Ru}$ & -0.580070 & 0.869889 & -0.584997 \\
\hline $\mathrm{C}$ & 5.994664 & -2.077689 & -1.079348 & $\mathrm{C}$ & -0.504489 & -0.958205 & 0.308897 \\
\hline $\mathrm{H}$ & 6.704275 & -2.078762 & -0.248048 & $\mathrm{C}$ & -0.073924 & -3.016391 & 1.368735 \\
\hline $\mathrm{H}$ & 6.203563 & -2.951097 & -1.704204 & $\mathrm{C}$ & -1.511233 & -2.609494 & 1.663549 \\
\hline $\mathrm{H}$ & 6.213248 & -1.194674 & -1.691361 & $\mathrm{H}$ & -0.005568 & -3.885595 & 0.697393 \\
\hline $\mathrm{C}$ & -3.069575 & 0.326801 & 2.597884 & $\mathrm{H}$ & 0.502515 & -3.247648 & 2.270097 \\
\hline $\mathrm{H}$ & -2.414223 & 1.192988 & 2.448376 & $\mathrm{H}$ & -2.243431 & -3.380773 & 1.402569 \\
\hline $\mathrm{H}$ & -3.882057 & 0.611548 & 3.271111 & $\mathrm{H}$ & -1.664587 & -2.345327 & 2.719872 \\
\hline $\mathrm{H}$ & -2.463398 & -0.439616 & 3.089599 & $\mathrm{~N}$ & 0.469212 & -1.824301 & 0.697626 \\
\hline $\mathrm{C}$ & -2.593779 & -2.318750 & -1.683608 & $\mathrm{~N}$ & -1.676378 & -1.426961 & 0.816026 \\
\hline $\mathrm{H}$ & -1.670283 & -1.849995 & -2.043154 & $\mathrm{C}$ & 1.838231 & -1.819199 & 0.300305 \\
\hline $\mathrm{H}$ & -2.295519 & -3.225857 & -1.143905 & $\mathrm{C}$ & 2.835580 & -1.591870 & 1.259475 \\
\hline $\mathrm{H}$ & -3.179372 & -2.624311 & -2.553661 & $\mathrm{C}$ & 2.169874 & -2.145623 & -1.027833 \\
\hline $\mathrm{C}$ & -6.683287 & 0.488941 & -0.879114 & $\mathrm{C}$ & 4.169676 & -1.619048 & 0.844899 \\
\hline $\mathrm{H}$ & -6.542638 & 1.389610 & -1.488553 & $\mathrm{C}$ & 3.513753 & -2.162759 & -1.392046 \\
\hline $\mathrm{H}$ & -7.218727 & -0.234220 & -1.500907 & $\mathrm{C}$ & 4.529977 & -1.884667 & -0.473479 \\
\hline $\mathrm{H}$ & -7.331297 & 0.765996 & -0.043336 & $\mathrm{H}$ & 4.948381 & -1.422235 & 1.580616 \\
\hline $\mathrm{C}$ & 1.374330 & 0.917092 & -0.819058 & $\mathrm{H}$ & 3.774605 & -2.403567 & -2.422035 \\
\hline $\mathrm{H}$ & 1.947959 & 0.102549 & -1.287540 & $\mathrm{C}$ & -2.961526 & -0.946047 & 0.405138 \\
\hline $\mathrm{C}$ & 2.192599 & 2.066612 & -0.483533 & $\mathrm{C}$ & -3.657142 & -0.006156 & 1.177544 \\
\hline $\mathrm{C}$ & 3.596059 & 1.924839 & -0.480015 & $\mathrm{C}$ & -3.502933 & -1.470316 & -0.782838 \\
\hline $\mathrm{C}$ & 1.638078 & 3.315221 & -0.131901 & $\mathrm{C}$ & -4.893789 & 0.438670 & 0.700633 \\
\hline $\mathrm{C}$ & 4.416157 & 2.979467 & -0.105451 & $\mathrm{C}$ & -4.735185 & -0.992661 & -1.216381 \\
\hline $\mathrm{H}$ & 4.024271 & 0.962662 & -0.760709 & $\mathrm{C}$ & -5.439378 & -0.024244 & -0.494366 \\
\hline $\mathrm{C}$ & 2.465937 & 4.367508 & 0.231354 & $\mathrm{H}$ & -5.439472 & 1.179376 & 1.283664 \\
\hline $\mathrm{H}$ & 0.558883 & 3.431459 & -0.165872 & $\mathrm{H}$ & -5.157042 & -1.383581 & -2.141290 \\
\hline $\mathrm{C}$ & 3.851427 & 4.203574 & 0.253195 & $\mathrm{C}$ & 1.105973 & -2.471544 & -2.027959 \\
\hline $\mathrm{H}$ & 5.495774 & 2.853589 & -0.096648 & $\mathrm{H}$ & 0.488948 & -1.594792 & -2.257947 \\
\hline $\mathrm{H}$ & 2.031104 & 5.328065 & 0.494568 & $\mathrm{H}$ & 1.541243 & -2.833245 & -2.962329 \\
\hline $\mathrm{H}$ & 4.492416 & 5.033681 & 0.538879 & $\mathrm{H}$ & 0.417933 & -3.240619 & -1.657755 \\
\hline $\mathrm{O}$ & -0.550436 & 1.740455 & 1.295891 & $\mathrm{C}$ & 2.518138 & -1.365635 & 2.706095 \\
\hline $\mathrm{H}$ & -0.804546 & 0.273144 & -2.176109 & $\mathrm{H}$ & 2.768133 & -2.251250 & 3.303220 \\
\hline $\mathrm{C}$ & 0.188684 & 1.333348 & 2.394737 & $\mathrm{H}$ & 3.104304 & -0.536146 & 3.112157 \\
\hline $\mathrm{H}$ & -0.055446 & 0.301623 & 2.723825 & $\mathrm{H}$ & 1.463585 & -1.135171 & 2.867789 \\
\hline $\mathrm{H}$ & 1.278387 & 1.359897 & 2.208712 & $\mathrm{C}$ & 5.962223 & -1.842292 & -0.906920 \\
\hline $\mathrm{H}$ & -0.013960 & 1.998675 & 3.249791 & $\mathrm{H}$ & 6.645560 & -2.021718 & -0.072870 \\
\hline $\mathrm{O}$ & -1.278001 & 2.450711 & -1.620161 & $\mathrm{H}$ & 6.174406 & -2.579557 & -1.686454 \\
\hline $\mathrm{H}$ & -1.066468 & 1.193577 & -2.160889 & $\mathrm{H}$ & 6.215633 & -0.859158 & -1.322399 \\
\hline $\mathrm{C}$ & -2.609501 & 2.598092 & -1.195339 & $\mathrm{C}$ & -3.085523 & 0.544351 & 2.442766 \\
\hline $\mathrm{H}$ & -3.220559 & 1.693879 & -1.384498 & $\mathrm{H}$ & -2.290608 & 1.266168 & 2.208962 \\
\hline $\mathrm{H}$ & -2.671108 & 2.811986 & -0.115618 & $\mathrm{H}$ & -3.854883 & 1.049043 & 3.032141 \\
\hline \multirow[t]{2}{*}{$\mathrm{H}$} & -3.090772 & 3.427680 & -1.732951 & $\mathrm{H}$ & -2.633878 & -0.233682 & 3.067289 \\
\hline & & & & $\mathrm{C}$ & -2.758786 & -2.507405 & -1.565894 \\
\hline & & & & $\mathrm{H}$ & -1.791150 & -2.129985 & -1.918975 \\
\hline
\end{tabular}




\begin{tabular}{crrc}
$\mathrm{H}$ & -2.537389 & -3.399872 & -0.967252 \\
$\mathrm{H}$ & -3.331210 & -2.828216 & -2.439066 \\
$\mathrm{C}$ & -6.742537 & 0.509846 & -1.003597 \\
$\mathrm{H}$ & -6.578535 & 1.293749 & -1.751362 \\
$\mathrm{H}$ & -7.340682 & -0.269006 & -1.485750 \\
$\mathrm{H}$ & -7.340573 & 0.950356 & -0.201623 \\
$\mathrm{C}$ & 1.230079 & 0.914653 & -1.087842 \\
$\mathrm{H}$ & 1.585329 & 0.283887 & -1.918898 \\
$\mathrm{C}$ & 2.294673 & 1.797048 & -0.636411 \\
$\mathrm{C}$ & 3.615457 & 1.496089 & -1.034769 \\
$\mathrm{C}$ & 2.094086 & 2.944512 & 0.159226 \\
$\mathrm{C}$ & 4.689649 & 2.270116 & -0.619071 \\
$\mathrm{H}$ & 3.781186 & 0.625232 & -1.667269 \\
$\mathrm{C}$ & 3.170263 & 3.723380 & 0.559694 \\
$\mathrm{H}$ & 1.080689 & 3.195252 & 0.458336 \\
$\mathrm{C}$ & 4.471357 & 3.389271 & 0.182578 \\
$\mathrm{H}$ & 5.698639 & 2.010128 & -0.930623 \\
$\mathrm{H}$ & 2.993738 & 4.606731 & 1.168226 \\
$\mathrm{H}$ & 5.308320 & 4.006617 & 0.498162 \\
$\mathrm{O}$ & -0.453283 & 1.785185 & 1.206563 \\
$\mathrm{H}$ & -0.680596 & 0.460989 & -2.314624 \\
$\mathrm{C}$ & 0.338901 & 1.238099 & 2.203999 \\
$\mathrm{H}$ & -0.109585 & 0.328509 & 2.659611 \\
$\mathrm{H}$ & 1.346179 & 0.955417 & 1.850396 \\
$\mathrm{H}$ & 0.473492 & 1.967986 & 3.019460 \\
$\mathrm{O}$ & -1.740122 & 2.374141 & -1.175204 \\
$\mathrm{H}$ & -1.307162 & 0.027227 & -1.960084 \\
$\mathrm{C}$ & -2.032652 & 3.448691 & -0.334631 \\
$\mathrm{H}$ & -2.597533 & 3.149907 & 0.563822 \\
$\mathrm{H}$ & -1.134037 & 3.974678 & 0.022906 \\
$\mathrm{H}$ & -2.647183 & 4.171994 & -0.891643 \\
& & & \\
\hline
\end{tabular}

$\mathbf{T S}_{3 \mathrm{C}-4 \mathrm{C}}$

$\begin{array}{rrrr}\mathrm{Ru} & -0.557535 & 0.863546 & -0.460488 \\ \mathrm{C} & -0.516034 & -0.926949 & 0.360423 \\ \mathrm{C} & -0.118187 & -3.049187 & 1.299619 \\ \mathrm{C} & -1.552842 & -2.639468 & 1.612075 \\ \mathrm{H} & -0.061895 & -3.880570 & 0.579281 \\ \mathrm{H} & 0.450165 & -3.342311 & 2.188547 \\ \mathrm{H} & -2.292576 & -3.386256 & 1.303948 \\ \mathrm{H} & -1.704509 & -2.440065 & 2.683591 \\ \mathrm{~N} & 0.448307 & -1.828933 & 0.708761 \\ \mathrm{~N} & -1.701884 & -1.409382 & 0.835557 \\ \mathrm{C} & 1.804732 & -1.856119 & 0.266657\end{array}$

\begin{tabular}{|c|c|c|c|}
\hline & & & \\
\hline & 2.084861 & 2.272550 & \\
\hline & 158489 & 6 & \\
\hline & & & \\
\hline & $46 \quad$ & -2.0 & \\
\hline & 96 & & 39 \\
\hline & & & \\
\hline & -2.97 & 0.9 & \\
\hline & .73 & 2 & \\
\hline & -3 & & \\
\hline & 7 & 0.364704 & \\
\hline & 1.7 & -0.8 & \\
\hline & -5.48 & 0.005 & 0.4 \\
\hline & -5.5 & & \\
\hline & -5 & & \\
\hline & 97 & -2.5 & \\
\hline & 0.43 & -1 & \\
\hline & 1.3 & -2 & \\
\hline & 0.23 & & \\
\hline & 2.57 & - & \\
\hline & 3.12 & & \\
\hline & 2.90 & $-0 .($ & \\
\hline & 1.51 & & \\
\hline & 5.886717 & -2. & 1.0 \\
\hline & 6.588 & -2.2 & \\
\hline & 0.046 & & \\
\hline & 6.165411 & -1.12 & \\
\hline & -3.212 & 0.3 & \\
\hline & -2.55 & & \\
\hline & & & \\
\hline & -2.6 & $=30$ & \\
\hline & -2.690411 & 0 & 1.694 \\
\hline & .78 & -1.76 & \\
\hline & -2.364 & & \\
\hline & -3.27 & 25 & -2.55 \\
\hline & -6.799061 & 21 & \\
\hline & -6.662 & 12 & 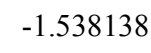 \\
\hline & -7.334 & ברת & \\
\hline & -7.4 & & -0.0798 \\
\hline & & & \\
\hline & 1.6123 & 175 & 1.00 \\
\hline & & & -0.457 \\
\hline & & 1. & 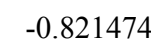 \\
\hline & 059769 & 890178 & \\
\hline
\end{tabular}




$\begin{array}{lrrr}\mathrm{C} & 4.677104 & 2.230497 & -0.357757 \\ \mathrm{H} & 3.791946 & 0.603759 & -1.458690 \\ \mathrm{C} & 3.125315 & 3.657103 & 0.820104 \\ \mathrm{H} & 1.037535 & 3.118538 & 0.665362 \\ \mathrm{C} & 4.435001 & 3.330729 & 0.463109 \\ \mathrm{H} & 5.695015 & 1.970418 & -0.638045 \\ \mathrm{H} & 2.937312 & 4.517981 & 1.456386 \\ \mathrm{H} & 5.263970 & 3.936137 & 0.820535 \\ \mathrm{O} & -0.655290 & 1.789506 & 1.328574 \\ \mathrm{H} & -0.910956 & 0.382722 & -2.183023 \\ \mathrm{C} & 0.018772 & 1.332812 & 2.448956 \\ \mathrm{H} & -0.243223 & 0.288998 & 2.721224 \\ \mathrm{H} & 1.117632 & 1.364708 & 2.335301 \\ \mathrm{H} & -0.233559 & 1.959912 & 3.320678 \\ \mathrm{O} & -1.653541 & 2.411136 & -1.532863 \\ \mathrm{H} & -1.227551 & 1.303262 & -2.156924 \\ \mathrm{C} & -0.957581 & 3.618370 & -1.691337 \\ \mathrm{H} & -0.020797 & 3.495546 & -2.261221 \\ \mathrm{H} & -1.583303 & 4.346421 & -2.227165 \\ \mathrm{H} & -0.693748 & 4.055398 & -0.715816\end{array}$

3D

$\begin{array}{rrrr}\mathrm{Ru} & -0.329695 & 0.841442 & -0.236625 \\ \mathrm{C} & -0.567443 & -0.998190 & 0.311597 \\ \mathrm{C} & -0.436634 & -3.234369 & 1.084421 \\ \mathrm{C} & -1.864208 & -2.739837 & 1.275085 \\ \mathrm{H} & -0.361042 & -4.023817 & 0.320210 \\ \mathrm{H} & 0.006494 & -3.630440 & 2.005468 \\ \mathrm{H} & -2.610709 & -3.390689 & 0.804785 \\ \mathrm{H} & -2.133379 & -2.641336 & 2.337538 \\ \mathrm{~N} & 0.273469 & -2.028960 & 0.651871 \\ \mathrm{~N} & -1.836594 & -1.429404 & 0.628764 \\ \mathrm{C} & 1.659620 & -2.146520 & 0.336723 \\ \mathrm{C} & 2.623975 & -1.844620 & 1.305318 \\ \mathrm{C} & 2.031656 & -2.596464 & -0.942467 \\ \mathrm{C} & 3.972210 & -1.984139 & 0.965061 \\ \mathrm{C} & 3.386597 & -2.727747 & -1.234675 \\ \mathrm{C} & 4.374668 & -2.417190 & -0.296036 \\ \mathrm{H} & 4.727895 & -1.740917 & 1.711046 \\ \mathrm{H} & 3.679442 & -3.062022 & -2.229577 \\ \mathrm{C} & -3.041676 & -0.839725 & 0.132371 \\ \mathrm{C} & -3.857206 & -0.057300 & 0.961722 \\ \mathrm{C} & -3.441224 & -1.158513 & -1.182031 \\ \mathrm{C} & -5.053054 & 0.441508 & 0.432812\end{array}$

\begin{tabular}{|c|c|c|c|}
\hline & & & \\
\hline & .455 & 0.175821 & \\
\hline & 690470 & & \\
\hline & 7 & & \\
\hline & 7 & & 2.0024 \\
\hline & 0.50 & & 74 \\
\hline & & & \\
\hline & 0.20 & & \\
\hline & 2.23 & & \\
\hline & 3. & & \\
\hline & 2.08 & & \\
\hline & & & \\
\hline & 5.82 & -2.5 & -0.653 \\
\hline & 6.032899 & -3 & \\
\hline & 6.15 & & \\
\hline & 6.458 & & \\
\hline & -3.482 & 0 & \\
\hline & 2.9 & & \\
\hline & & & \\
\hline & -2 & -0.44 & \\
\hline & -2.59 & -2.0 & \\
\hline & .6 & -1.5 & \\
\hline & -10 & & \\
\hline & -3.11 & -2.31 & -2.9 \\
\hline & -6.732501 & 68 & \\
\hline & . & & \\
\hline & -7.35 & & \\
\hline & -6.538 & 1.521 & \\
\hline & 43 & 716 & 07 \\
\hline & & & \\
\hline & 2 & & \\
\hline & 880 & 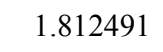 & \\
\hline & & & \\
\hline & & & \\
\hline & 3.76 & 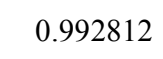 & -2.0666 \\
\hline & 3.164 & 86 & 45 \\
\hline & 1.541252 & 2.823009 & (0) \\
\hline & 4.310 & & \\
\hline & 5.417 & 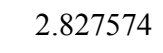 & -1.8647 \\
\hline & 3. & 99 & \\
\hline & 5.044527 & 666238 & -0.237 \\
\hline & & 633 & 5077 \\
\hline & -0 & 0. & -1.7 \\
\hline & 97 & $J 6 / 648$ & 19 \\
\hline
\end{tabular}




$\begin{array}{crcc}\mathrm{H} & -0.476676 & -0.037421 & 2.805513 \\ \mathrm{H} & 0.645287 & 1.309741 & 3.075520 \\ \mathrm{H} & -1.036838 & 1.437145 & 3.619326 \\ \mathrm{O} & -0.632622 & 3.032117 & -0.892968 \\ \mathrm{H} & -0.132074 & 3.249997 & -1.685129 \\ \mathrm{C} & -0.741978 & 4.174874 & -0.045071 \\ \mathrm{H} & -1.067883 & 3.792123 & 0.923060 \\ \mathrm{H} & 0.223434 & 4.678944 & 0.075556 \\ \mathrm{H} & -1.478565 & 4.879045 & -0.446618\end{array}$

\section{TS3D-4D}

$\mathrm{Ru} \quad-0.554540$

$\begin{array}{llll}\text { C } & -0.535655 & -1.023249 & 0.261652\end{array}$

$\begin{array}{llll}\text { C } & -0.182117 & -3.238243 & 0.984524\end{array}$

$\begin{array}{llll}\text { C } & -1.624969 & -2.850185 & 1.290251\end{array}$

$\mathrm{H} \quad-0.107038 \quad-3.994303 \quad 0.187622$

$\mathrm{H} \quad 0.354253 \quad-3.623989 \quad 1.857977$

$\mathrm{H} \quad-2.357728 \quad-3.555713 \quad 0.883768$

$\begin{array}{llll}\mathrm{H} & -1.812037 & -2.760251 & 2.371090\end{array}$

$\begin{array}{llll}\mathrm{N} & 0.408081 & -1.970402 & 0.538101\end{array}$

$\begin{array}{llll}\mathrm{N} & -1.738541 & -1.547796 & 0.638217\end{array}$

$\begin{array}{llll}\text { C } & 1.783001 & -1.933888 & 0.167254\end{array}$

$\begin{array}{llll}\text { C } & 2.766876 & -1.760084 & 1.148914\end{array}$

$\begin{array}{llll}\text { C } & 2.130254 & -2.141208 & -1.181895\end{array}$

$\begin{array}{llll}\text { C } & 4.105931 & -1.734969 & 0.746255\end{array}$

$\begin{array}{llll}\text { C } & 3.476017 & -2.110268 & -1.534295\end{array}$

$\begin{array}{llll}\text { C } & 4.480477 & -1.893455 & -0.584510\end{array}$

$\mathrm{H} \quad 4.875681 \quad-1.579854 \quad 1.501068$

$\mathrm{H} \quad 3.749028 \quad-2.254265 \quad-2.579399$

$\begin{array}{llll}\text { C } & -2.993813 & -0.941276 & 0.316859\end{array}$

$\begin{array}{llll}\text { C } & -3.686791 & -0.180510 & 1.268960\end{array}$

$\begin{array}{llll}\text { C } & -3.528674 & -1.173889 & -0.966148\end{array}$

$\begin{array}{llll}\text { C } & -4.911763 & 0.379124 & 0.893694\end{array}$

$\begin{array}{llll}\text { C } & -4.743516 & -0.582950 & -1.294806\end{array}$

$\begin{array}{llll}\text { C } & -5.447078 & 0.207000 & -0.379927\end{array}$

$\mathrm{H} \quad-5.455242 \quad 0.976957 \quad 1.624053$

$\mathrm{H} \quad-5.157093 \quad-0.747573 \quad-2.288828$

$\begin{array}{llll}\text { C } & 1.067331 & -2.350450 & -2.214499\end{array}$

$\mathrm{H} \quad 0.438330 \quad-1.456493 \quad-2.322319$

$\mathrm{H} \quad 1.502070 \quad-2.581494 \quad-3.189728$

$\begin{array}{llll}\mathrm{H} & 0.388888 & -3.168249 & -1.944828\end{array}$

$\begin{array}{llll}\text { C } & 2.428473 & -1.630398 & 2.601627\end{array}$

$\mathrm{H} \quad 2.704730 \quad-2.539184 \quad 3.149865$

$\mathrm{H} \quad 2.979212 \quad-0.804642 \quad 3.061710$

$\begin{array}{lrrr}\mathrm{H} & 1.364076 & -1.448873 & 2.761322 \\ \mathrm{C} & 5.916702 & -1.809174 & -1.000113 \\ \mathrm{H} & 6.196013 & -2.630233 & -1.667764 \\ \mathrm{H} & 6.116162 & -0.879960 & -1.547547 \\ \mathrm{H} & 6.589485 & -1.829530 & -0.139282 \\ \mathrm{C} & -3.126716 & 0.081846 & 2.628291 \\ \mathrm{H} & -2.446911 & 0.941314 & 2.579311 \\ \mathrm{H} & -3.920673 & 0.303999 & 3.345827\end{array}$

$\begin{array}{llll}\mathrm{H} & -2.541752 & -0.760714 & 3.009331\end{array}$

$\begin{array}{llll}\text { C } & -2.797155 & -2.035719 & -1.946570\end{array}$

$\mathrm{H} \quad-1.822321 \quad-1.601801 \quad-2.198692$

$\mathrm{H} \quad-2.600991 \quad-3.037892 \quad-1.545193$

$\mathrm{H} \quad-3.366543 \quad-2.151569 \quad-2.871652$

$\begin{array}{llll}\text { C } & -6.736810 & 0.860182 & -0.769505\end{array}$

$\mathrm{H} \quad-7.354338 \quad 0.203243 \quad-1.389354$

$\begin{array}{llll}\mathrm{H} & -7.323363 & 1.151767 & 0.105414\end{array}$

$\mathrm{H} \quad-6.555044 \quad 1.768484 \quad-1.354819$

$\begin{array}{llll}\text { C } & 1.226882 & 0.930414 & -0.867923\end{array}$

$\mathrm{H} \quad 1.606792 \quad 0.281522 \quad-1.674969$

$\begin{array}{llll}\text { C } & 2.275868 & 1.809370 & -0.371601\end{array}$

$\begin{array}{llll}\text { C } & 3.587560 & 1.603661 & -0.846837\end{array}$

$\begin{array}{llll}\text { C } & 2.057586 & 2.852043 & 0.552510\end{array}$

$\begin{array}{llll}\text { C } & 4.642722 & 2.391034 & -0.408161\end{array}$

$\mathrm{H} \quad 3.759329 \quad 0.800165 \quad-1.561744$

$\begin{array}{llll}\text { C } & 3.114854 & 3.640879 & 0.982284\end{array}$

$\mathrm{H} \quad 1.047794 \quad 3.007231 \quad 0.925767$

$\begin{array}{llll}\text { C } & 4.408058 & 3.415293 & 0.507740\end{array}$

$\mathrm{H} \quad 5.647629 \quad 2.212783 \quad-0.782861$

$\mathrm{H} \quad 2.932848 \quad 4.442809 \quad 1.692989$

$\mathrm{H} \quad 5.229720 \quad 4.040753 \quad 0.847053$

$\begin{array}{llll}\mathrm{O} & -0.593840 & 1.623071 & 1.477655\end{array}$

$\mathrm{H} \quad-0.926515 \quad 0.510264 \quad-2.136697$

$\begin{array}{llll}\text { C } & 0.126194 & 1.050686 & 2.513927\end{array}$

$\begin{array}{llll}\mathrm{H} & -0.213991 & 0.022540 & 2.758980\end{array}$

$\mathrm{H} \quad 1.210576 \quad 0.985261 \quad 2.305944$

$\mathrm{H} \quad 0.008087 \quad 1.650162 \quad 3.432204$

$\begin{array}{llll}\text { O } & -1.631001 & 2.492431 & -1.320820\end{array}$

$\mathrm{H} \quad-1.231541 \quad 1.428339 \quad-2.031158$

$\begin{array}{llll}\text { C } & -0.918352 & 3.695712 & -1.417721\end{array}$

$\mathrm{H} \quad-0.611986 \quad 4.060169 \quad-0.424785$

$\mathrm{H} \quad-0.004225 \quad 3.598465 \quad-2.028006$

$\mathrm{H} \quad-1.547940 \quad 4.469740 \quad-1.879553$

4D 


\begin{tabular}{|c|c|c|c|c|c|c|c|}
\hline $\mathrm{Ru}$ & -0.579943 & 0.869960 & -0.585031 & $\mathrm{H}$ & -2.537871 & -3.399897 & -0.967354 \\
\hline $\mathrm{C}$ & -0.504567 & -0.958280 & 0.308798 & $\mathrm{H}$ & -3.330927 & -2.827664 & -2.439329 \\
\hline $\mathrm{C}$ & -0.074225 & -3.016719 & 1.368292 & $\mathrm{C}$ & -6.742534 & 0.509999 & -1.003738 \\
\hline $\mathrm{C}$ & -1.511447 & -2.609649 & 1.663280 & $\mathrm{H}$ & -7.340999 & -0.269039 & -1.485198 \\
\hline $\mathrm{H}$ & -0.005991 & -3.885967 & 0.697012 & $\mathrm{H}$ & -7.340260 & 0.951224 & -0.201929 \\
\hline $\mathrm{H}$ & 0.502288 & -3.247961 & 2.269612 & $\mathrm{H}$ & -6.578514 & 1.293321 & -1.752104 \\
\hline $\mathrm{H}$ & -2.243813 & -3.380678 & 1.402039 & $\mathrm{C}$ & 1.230122 & 0.914744 & -1.087886 \\
\hline $\mathrm{H}$ & -1.664731 & -2.345830 & 2.719695 & $\mathrm{H}$ & 1.585351 & 0.284160 & -1.919100 \\
\hline $\mathrm{N}$ & 0.468984 & -1.824710 & 0.697049 & $\mathrm{C}$ & 2.294760 & 1.796990 & -0.636235 \\
\hline $\mathrm{N}$ & -1.676449 & -1.426739 & 0.816207 & $\mathrm{C}$ & 3.615549 & 1.496023 & -1.034560 \\
\hline $\mathrm{C}$ & 1.838079 & -1.819521 & 0.300030 & $\mathrm{C}$ & 2.094180 & 2.944362 & 0.159514 \\
\hline $\mathrm{C}$ & 2.835248 & -1.592462 & 1.259440 & $\mathrm{C}$ & 4.689749 & 2.269961 & -0.618745 \\
\hline $\mathrm{C}$ & 2.169955 & -2.145456 & -1.028169 & $\mathrm{H}$ & 3.781248 & 0.625178 & -1.667087 \\
\hline $\mathrm{C}$ & 4.169419 & -1.619305 & 0.845043 & $\mathrm{C}$ & 3.170368 & 3.723178 & 0.560078 \\
\hline $\mathrm{C}$ & 3.513869 & -2.162269 & -1.392198 & $\mathrm{H}$ & 1.080787 & 3.195106 & 0.458641 \\
\hline $\mathrm{C}$ & 4.529930 & -1.884332 & -0.473386 & $\mathrm{C}$ & 4.471458 & 3.389074 & 0.182973 \\
\hline $\mathrm{H}$ & 4.947993 & -1.422642 & 1.580941 & $\mathrm{H}$ & 5.698761 & 2.009945 & -0.930220 \\
\hline $\mathrm{H}$ & 3.774903 & -2.402658 & -2.422238 & $\mathrm{H}$ & 2.993828 & 4.606481 & 1.168678 \\
\hline $\mathrm{C}$ & -2.961554 & -0.945823 & 0.405147 & $\mathrm{H}$ & 5.308436 & 4.006374 & 0.498613 \\
\hline $\mathrm{C}$ & -3.657238 & -0.005980 & 1.177548 & $\mathrm{O}$ & -0.453290 & 1.784931 & 1.206730 \\
\hline $\mathrm{C}$ & -3.502896 & -1.470091 & -0.782862 & $\mathrm{H}$ & -1.306204 & 0.026789 & -1.960392 \\
\hline $\mathrm{C}$ & -4.893877 & 0.438830 & 0.700594 & $\mathrm{C}$ & 0.338837 & 1.237746 & 2.204158 \\
\hline $\mathrm{C}$ & -4.735137 & -0.992454 & -1.216441 & $\mathrm{H}$ & -0.109686 & 0.328106 & 2.659645 \\
\hline $\mathrm{C}$ & -5.439389 & -0.024062 & -0.494441 & $\mathrm{H}$ & 1.346156 & 0.955127 & 1.850613 \\
\hline $\mathrm{H}$ & -5.439594 & 1.179522 & 1.283610 & $\mathrm{H}$ & 0.473343 & 1.967549 & 3.019708 \\
\hline $\mathrm{H}$ & -5.156952 & -1.383372 & -2.141370 & $\mathrm{O}$ & -1.740018 & 2.374329 & -1.175030 \\
\hline $\mathrm{C}$ & 1.106207 & -2.471216 & -2.028500 & $\mathrm{H}$ & -0.680678 & 0.461960 & -2.314883 \\
\hline $\mathrm{H}$ & 0.489103 & -1.594474 & -2.258291 & $\mathrm{C}$ & -2.032093 & 3.448949 & -0.334307 \\
\hline $\mathrm{H}$ & 1.541594 & -2.832616 & -2.962930 & $\mathrm{H}$ & -2.597002 & 3.150284 & 0.564152 \\
\hline $\mathrm{H}$ & 0.418218 & -3.240452 & -1.658555 & $\mathrm{H}$ & -1.133284 & 3.974659 & 0.023093 \\
\hline $\mathrm{C}$ & 2.517555 & -1.366936 & 2.706111 & $\mathrm{H}$ & -2.646451 & 4.172458 & -0.891241 \\
\hline $\mathrm{H}$ & 2.766490 & -2.253223 & 3.302701 & & & & \\
\hline $\mathrm{H}$ & 3.104401 & -0.538323 & 3.112970 & $3 E$ & & & \\
\hline $\mathrm{H}$ & 1.463162 & -1.135605 & 2.867623 & $\mathrm{Ru}$ & -0.189365 & 0.641260 & 0.117340 \\
\hline $\mathrm{C}$ & 5.962196 & -1.841497 & -0.906719 & $\mathrm{C}$ & -0.291980 & -1.255176 & -0.128300 \\
\hline $\mathrm{H}$ & 6.175228 & -2.580249 & -1.684623 & $\mathrm{C}$ & 0.101357 & -3.556337 & -0.477896 \\
\hline $\mathrm{H}$ & 6.214672 & -0.859033 & -1.324353 & $\mathrm{C}$ & -1.385916 & -3.350096 & -0.231533 \\
\hline $\mathrm{H}$ & 6.645596 & -2.018445 & -0.072197 & $\mathrm{H}$ & 0.312101 & -3.839887 & -1.520125 \\
\hline $\mathrm{C}$ & -3.085695 & 0.544425 & 2.442850 & $\mathrm{H}$ & 0.546230 & -4.315636 & 0.173149 \\
\hline $\mathrm{H}$ & -2.290404 & 1.265860 & 2.209161 & $\mathrm{H}$ & -2.018015 & -3.804043 & -1.002465 \\
\hline $\mathrm{H}$ & -3.854984 & 1.049560 & 3.031937 & $\mathrm{H}$ & -1.707312 & -3.751505 & 0.742043 \\
\hline $\mathrm{H}$ & -2.634635 & -0.233774 & 3.067598 & $\mathrm{~N}$ & 0.662128 & -2.236769 & -0.178241 \\
\hline $\mathrm{C}$ & -2.758744 & -2.507214 & -1.565868 & $\mathrm{~N}$ & -1.498027 & -1.895140 & -0.258315 \\
\hline $\mathrm{H}$ & -1.790865 & -2.130006 & -1.918498 & $\mathrm{C}$ & 2.061567 & -2.078742 & 0.020770 \\
\hline
\end{tabular}




\begin{tabular}{|c|c|c|c|c|c|c|c|}
\hline $\mathrm{C}$ & 2.544906 & -1.862655 & 1.320079 & $\mathrm{C}$ & 4.009167 & 3.675860 & -0.853040 \\
\hline $\mathrm{C}$ & 2.925909 & -2.157682 & -1.082241 & $\mathrm{H}$ & 4.024530 & 1.524599 & -0.995079 \\
\hline $\mathrm{C}$ & 3.918679 & -1.673517 & 1.482352 & $\mathrm{C}$ & 1.842111 & 4.693316 & -0.546904 \\
\hline $\mathrm{C}$ & 4.291234 & -1.974518 & -0.866903 & $\mathrm{H}$ & 0.158799 & 3.358809 & -0.508560 \\
\hline $\mathrm{C}$ & 4.804724 & -1.714376 & 0.405395 & $\mathrm{C}$ & 3.224848 & 4.816625 & -0.684047 \\
\hline $\mathrm{H}$ & 4.307549 & -1.496022 & 2.484244 & $\mathrm{H}$ & 5.086503 & 3.768965 & -0.964084 \\
\hline $\mathrm{H}$ & 4.967884 & -2.013274 & -1.719933 & $\mathrm{H}$ & 1.225699 & 5.580526 & -0.428903 \\
\hline $\mathrm{C}$ & -2.766738 & -1.245783 & -0.273361 & $\mathrm{H}$ & 3.688697 & 5.799322 & -0.667286 \\
\hline $\mathrm{C}$ & -3.462108 & -1.039900 & 0.926774 & $\mathrm{O}$ & -0.426591 & 0.695953 & 2.106977 \\
\hline $\mathrm{C}$ & -3.319456 & -0.891967 & -1.516986 & $\mathrm{H}$ & -0.995318 & 0.839968 & -1.292588 \\
\hline $\mathrm{C}$ & -4.724267 & -0.442945 & 0.855571 & $\mathrm{C}$ & -0.263305 & 2.010682 & 2.552188 \\
\hline $\mathrm{C}$ & -4.572953 & -0.285790 & -1.534134 & $\mathrm{H}$ & 0.690739 & 2.144867 & 3.086425 \\
\hline $\mathrm{C}$ & -5.290614 & -0.051795 & -0.356486 & $\mathrm{H}$ & -0.276349 & 2.752978 & 1.724911 \\
\hline $\mathrm{H}$ & -5.274248 & -0.271627 & 1.780413 & $\mathrm{H}$ & -1.079632 & 2.301323 & 3.232824 \\
\hline $\mathrm{H}$ & -5.006833 & 0.000550 & -2.491546 & $\mathrm{O}$ & -2.163849 & 3.066143 & -0.151438 \\
\hline $\mathrm{C}$ & 2.392278 & -2.367956 & -2.466178 & $\mathrm{H}$ & -2.067432 & 2.317234 & -0.760793 \\
\hline $\mathrm{H}$ & 1.436356 & -1.850955 & -2.606922 & $\mathrm{C}$ & -3.073553 & 2.677318 & 0.860131 \\
\hline $\mathrm{H}$ & 3.093448 & -1.996502 & -3.217477 & $\mathrm{H}$ & -2.757160 & 1.757264 & 1.376355 \\
\hline $\mathrm{H}$ & 2.221462 & -3.428972 & -2.687274 & $\mathrm{H}$ & -3.103259 & 3.487214 & 1.593108 \\
\hline $\mathrm{C}$ & 1.615413 & -1.829567 & 2.491419 & $\mathrm{H}$ & -4.089948 & 2.522489 & 0.471025 \\
\hline $\mathrm{H}$ & 0.887284 & -2.648431 & 2.448123 & & & & \\
\hline $\mathrm{H}$ & 2.168355 & -1.914572 & 3.430370 & \multicolumn{4}{|c|}{$\mathbf{T S}_{3 \mathrm{E}-4 \mathrm{E}}$} \\
\hline $\mathrm{H}$ & 1.026949 & -0.902870 & 2.520719 & $\mathrm{Ru}$ & -0.309500 & 0.896385 & -0.420662 \\
\hline $\mathrm{C}$ & 6.267247 & -1.456005 & 0.602320 & $\mathrm{C}$ & -0.621984 & -1.030301 & -0.255798 \\
\hline $\mathrm{H}$ & 6.580911 & -1.665643 & 1.628412 & $\mathrm{C}$ & -0.242708 & -3.332911 & -0.573934 \\
\hline $\mathrm{H}$ & 6.882304 & -2.060486 & -0.070480 & $\mathrm{C}$ & -1.701919 & -3.120808 & -0.225310 \\
\hline $\mathrm{H}$ & 6.513547 & -0.406592 & 0.400850 & $\mathrm{H}$ & -0.099380 & -3.554445 & -1.642619 \\
\hline $\mathrm{C}$ & -2.862345 & -1.419666 & 2.243499 & $\mathrm{H}$ & 0.231010 & -4.131958 & 0.002397 \\
\hline $\mathrm{H}$ & -1.978808 & -0.804887 & 2.459704 & $\mathrm{H}$ & -2.392932 & -3.618821 & -0.912391 \\
\hline $\mathrm{H}$ & -3.582580 & -1.281635 & 3.053780 & $\mathrm{H}$ & -1.939497 & -3.455801 & 0.796346 \\
\hline $\mathrm{H}$ & -2.539017 & -2.466474 & 2.262429 & $\mathrm{~N}$ & 0.338138 & -2.021484 & -0.259529 \\
\hline $\mathrm{C}$ & -2.565266 & -1.165495 & -2.779966 & $\mathrm{~N}$ & -1.824899 & -1.665126 & -0.321043 \\
\hline $\mathrm{H}$ & -1.603055 & -0.640575 & -2.780929 & $\mathrm{C}$ & 1.748643 & -1.844477 & -0.339700 \\
\hline $\mathrm{H}$ & -2.340238 & -2.232762 & -2.894673 & $\mathrm{C}$ & 2.555462 & -2.468876 & 0.626555 \\
\hline $\mathrm{H}$ & -3.132525 & -0.847169 & -3.657671 & $\mathrm{C}$ & 2.331679 & -1.058836 & -1.352770 \\
\hline $\mathrm{C}$ & -6.628003 & 0.621861 & -0.400571 & $\mathrm{C}$ & 3.940640 & -2.308781 & 0.548058 \\
\hline $\mathrm{H}$ & -6.528808 & 1.688327 & -0.634571 & $\mathrm{C}$ & 3.716169 & -0.921120 & -1.383111 \\
\hline $\mathrm{H}$ & -7.273867 & 0.191604 & -1.172354 & $\mathrm{C}$ & 4.543430 & -1.536573 & -0.442003 \\
\hline $\mathrm{H}$ & -7.150181 & 0.546019 & 0.556566 & $\mathrm{H}$ & 4.563034 & -2.778057 & 1.309505 \\
\hline $\mathrm{C}$ & 1.435468 & 0.941183 & -0.712904 & $\mathrm{H}$ & 4.159366 & -0.288428 & -2.151484 \\
\hline $\mathrm{H}$ & 2.129863 & 0.153599 & -1.026041 & $\mathrm{C}$ & -3.081197 & -1.045110 & -0.034961 \\
\hline $\mathrm{C}$ & 2.021647 & 2.273973 & -0.737371 & $\mathrm{C}$ & -3.412838 & -0.750138 & 1.293756 \\
\hline $\mathrm{C}$ & 3.416277 & 2.420730 & -0.877048 & $\mathrm{C}$ & -3.959550 & -0.785537 & -1.091482 \\
\hline $\mathrm{C}$ & 1.244049 & 3.441088 & -0.580805 & $\mathrm{C}$ & -4.653772 & -0.171040 & 1.545023 \\
\hline
\end{tabular}




\begin{tabular}{|c|c|c|c|}
\hline & -5.190170 & -0.198751 & -0.793062 \\
\hline C & -5.551565 & 0.121234 & 0.515880 \\
\hline & -4.919773 & 0.074771 & 2.572608 \\
\hline & -5.881889 & 0.015642 & -1.606766 \\
\hline & 1.497809 & -0.277627 & -2.316312 \\
\hline & 1.261290 & 0.733342 & -1.905350 \\
\hline & 2.052837 & -0.038802 & -3.228246 \\
\hline & 0.567763 & -0.775201 & -2.601068 \\
\hline 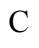 & 1.958122 & -3.240561 & 1.762960 \\
\hline $\mathrm{H}$ & 1.812385 & -4.301011 & 1.521016 \\
\hline $\mathrm{H}$ & 2.611778 & -3.207830 & 2.638734 \\
\hline 4 & 0.980513 & -2.838342 & 2.050305 \\
\hline$C$ & 6.022856 & -1.311908 & -0.462132 \\
\hline 11 & 6.546673 & -1.997021 & 0.209576 \\
\hline 10 & 6.441078 & -1.436574 & -1.465783 \\
\hline H & 6.261761 & -0.289481 & -0.143294 \\
\hline 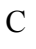 & -2.448470 & -1.028609 & 2.403679 \\
\hline$H$ & -1.476581 & -0.571189 & 2.190953 \\
\hline $\mathrm{H}$ & -2.812209 & -0.620132 & 3.349869 \\
\hline U & -2.283725 & -2.103776 & 2.552284 \\
\hline$C$ & -3.573413 & -1.117494 & -2.498786 \\
\hline $\mathrm{H}$ & -2.657236 & -0.587102 & -2.783917 \\
\hline 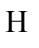 & -3.375824 & -2.188469 & -2.629387 \\
\hline $\mathrm{H}$ & -4.361977 & -0.838092 & -3.201607 \\
\hline 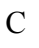 & -6.860876 & 0.787127 & 0.811220 \\
\hline$H$ & -6.739796 & 1.871980 & 0.909645 \\
\hline $\mathrm{H}$ & -7.591274 & 0.616808 & 0.015636 \\
\hline 11 & -7.294415 & 0.431448 & 1.750611 \\
\hline$r$ & 1.063246 & 0.727956 & 0.872478 \\
\hline$H$ & 1.015305 & -0.028850 & 1.678029 \\
\hline$C$ & 2.356857 & 1.380886 & 0.836965 \\
\hline $\mathrm{C}$ & 3.396764 & 0.925304 & 1.674715 \\
\hline$C$ & 2.640333 & 2.430419 & -0.066856 \\
\hline$C$ & 4.669424 & 1.473074 & 1.601563 \\
\hline $\mathrm{H}$ & 3.185887 & 0.114488 & 2.370775 \\
\hline$r$ & 3.914348 & 2.976047 & -0.133 \\
\hline $\mathrm{H}$ & 1.837044 & 2.804616 & -0.701823 \\
\hline 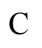 & 4.934401 & 2.496554 & 0.691195 \\
\hline 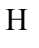 & 5.460311 & 1.099775 & 2.248219 \\
\hline $\mathrm{H}$ & 4.118514 & 3.784137 & -0.831313 \\
\hline 11 & 5.931993 & 2.924095 & 0.628298 \\
\hline$\Omega$ & -1.734043 & 1.482112 & 0.802667 \\
\hline $\mathrm{H}$ & -1.322731 & 1.092836 & -2.108952 \\
\hline $\mathrm{C}$ & -1.336839 & 2.310643 & 1.842156 \\
\hline
\end{tabular}

$\begin{array}{lrrr}\mathrm{H} & -0.656177 & 1.783382 & 2.541828 \\ \mathrm{H} & -0.811367 & 3.226875 & 1.520384 \\ \mathrm{H} & -2.235356 & 2.615021 & 2.402541 \\ \mathrm{O} & -0.301082 & 2.948105 & -1.393839 \\ \mathrm{H} & -0.973951 & 2.014545 & -1.952620 \\ \mathrm{C} & -1.128041 & 3.997986 & -0.976361 \\ \mathrm{H} & -2.068440 & 3.634765 & -0.532322 \\ \mathrm{H} & -0.618122 & 4.610578 & -0.217706 \\ \mathrm{H} & -1.380590 & 4.657251 & -1.820586\end{array}$

$4 E$

$\begin{array}{llll}\mathrm{Ru} & -0.284861 & 1.028107 & -0.364672\end{array}$

$\begin{array}{llll}\text { C } & -0.671716 & -0.965323 & -0.119758\end{array}$

$\begin{array}{llll}\text { C } & -0.302726 & -3.282844 & -0.420619\end{array}$

$\begin{array}{llll}\text { C } & -1.788875 & -3.046959 & -0.255125\end{array}$

$\mathrm{H} \quad-0.031660 \quad-3.528985 \quad-1.458964$

$\mathrm{H} \quad 0.087922 \quad-4.077451 \quad 0.222909$

$\mathrm{H} \quad-2.395896 \quad-3.508755 \quad-1.040962$

$\mathrm{H} \quad-2.166203 \quad-3.404205 \quad 0.714715$

$\begin{array}{llll}\mathrm{N} & 0.260435 & -1.974548 & -0.059876\end{array}$

$\begin{array}{llll}\mathrm{N} & -1.876522 & -1.582429 & -0.312628\end{array}$

$\begin{array}{llll}\text { C } & 1.673427 & -1.819809 & -0.146229\end{array}$

$\begin{array}{llll}\text { C } & 2.461500 & -2.362861 & 0.880219\end{array}$

$\begin{array}{llll}\text { C } & 2.268609 & -1.184394 & -1.251475\end{array}$

$\begin{array}{llll}\text { C } & 3.850395 & -2.268765 & 0.776334\end{array}$

$\begin{array}{llll}\text { C } & 3.657537 & -1.112222 & -1.308113\end{array}$

$\begin{array}{llll}\text { C } & 4.469678 & -1.649232 & -0.306739\end{array}$

$\mathrm{H} \quad 4.464451 \quad-2.669880 \quad 1.582315$

$\begin{array}{llll}\mathrm{H} & 4.119311 & -0.596259 & -2.149258\end{array}$

$\begin{array}{llll}\text { C } & -3.149837 & -0.974458 & -0.088583\end{array}$

$\begin{array}{llll}\text { C } & -3.499284 & -0.566237 & 1.209820\end{array}$

$\begin{array}{llll}\text { C } & -4.048293 & -0.868082 & -1.156584\end{array}$

$\begin{array}{llll}\text { C } & -4.752455 & 0.007498 & 1.399754\end{array}$

$\begin{array}{llll}\text { C } & -5.294127 & -0.283099 & -0.916536\end{array}$

$\begin{array}{llll}\text { C } & -5.658659 & 0.171527 & 0.348517\end{array}$

$\begin{array}{llll}\mathrm{H} & -5.029451 & 0.340415 & 2.399631\end{array}$

$\mathrm{H} \quad-5.995010 \quad-0.179867 \quad-1.744047$

$\begin{array}{llll}\text { C } & 1.453601 & -0.522246 & -2.317957\end{array}$

$\mathrm{H} \quad 1.209134 \quad 0.527091 \quad-2.054688$

$\mathrm{H} \quad 2.019383 \quad-0.437019 \quad-3.250616$

$\mathrm{H} \quad 0.514318 \quad-1.040743 \quad-2.532135$

$\begin{array}{llll}\text { C } & 1.831573 & -2.995401 & 2.082430\end{array}$

$\mathrm{H} \quad 1.578930 \quad-4.050118 \quad 1.913028$

$\begin{array}{llll}\mathrm{H} & 2.509493 & -2.968871 & 2.939517\end{array}$ 


\begin{tabular}{|c|c|c|c|c|c|c|c|}
\hline $\mathrm{H}$ & 0.901828 & -2.487516 & 2.358981 & $\mathrm{Ru}$ & 0.052484 & 0.674535 & -0.541546 \\
\hline $\mathrm{C}$ & 5.959914 & -1.536166 & -0.390883 & $\mathrm{C}$ & 0.612950 & -0.945379 & 0.332167 \\
\hline $\mathrm{H}$ & 6.440235 & -1.858341 & 0.536945 & $\mathrm{C}$ & 0.441973 & -3.182278 & 1.115661 \\
\hline $\mathrm{H}$ & 6.367626 & -2.147347 & -1.204029 & $\mathrm{C}$ & 1.901832 & -2.762622 & 1.172124 \\
\hline $\mathrm{H}$ & 6.264946 & -0.502029 & -0.583960 & $\mathrm{H}$ & 0.076378 & -3.599567 & 2.061261 \\
\hline $\mathrm{C}$ & -2.566382 & -0.783953 & 2.358686 & $\mathrm{H}$ & 0.256222 & -3.931509 & 0.330814 \\
\hline $\mathrm{H}$ & -1.585316 & -0.344494 & 2.158945 & $\mathrm{H}$ & 2.271593 & -2.665356 & 2.204562 \\
\hline $\mathrm{H}$ & -2.959654 & -0.332025 & 3.272780 & $\mathrm{H}$ & 2.570072 & -3.458564 & 0.650292 \\
\hline $\mathrm{H}$ & -2.413095 & -1.852860 & 2.557799 & $\mathrm{~N}$ & -0.246925 & -1.933159 & 0.787857 \\
\hline $\mathrm{C}$ & -3.710261 & -1.385233 & -2.522608 & $\mathrm{~N}$ & 1.884120 & -1.454685 & 0.518407 \\
\hline $\mathrm{H}$ & -2.629526 & -1.435923 & -2.686690 & $\mathrm{C}$ & -1.619712 & -2.030140 & 0.401340 \\
\hline $\mathrm{H}$ & -4.107386 & -2.396530 & -2.677467 & $\mathrm{C}$ & -1.959028 & -2.239267 & -0.946706 \\
\hline $\mathrm{H}$ & -4.142889 & -0.753945 & -3.303621 & $\mathrm{C}$ & -2.606567 & -1.973910 & 1.397644 \\
\hline $\mathrm{C}$ & -6.982592 & 0.833701 & 0.579086 & $\mathrm{C}$ & -3.311799 & -2.306410 & -1.280393 \\
\hline $\mathrm{H}$ & -6.872678 & 1.921321 & 0.655257 & $\mathrm{C}$ & -3.945560 & -2.035121 & 1.011206 \\
\hline $\mathrm{H}$ & -7.682885 & 0.635115 & -0.236527 & $\mathrm{C}$ & -4.320108 & -2.181504 & -0.324215 \\
\hline $\mathrm{H}$ & -7.446204 & 0.498377 & 1.511949 & $\mathrm{H}$ & -3.582659 & -2.456470 & -2.325527 \\
\hline $\mathrm{C}$ & 1.107548 & 0.977511 & 0.857569 & $\mathrm{H}$ & -4.717142 & -1.965233 & 1.777344 \\
\hline $\mathrm{H}$ & 0.923734 & 0.626295 & 1.890819 & $\mathrm{C}$ & 3.076676 & -0.678468 & 0.550193 \\
\hline $\mathrm{C}$ & 2.482349 & 1.395291 & 0.640732 & $\mathrm{C}$ & 4.028841 & -0.862715 & -0.464740 \\
\hline $\mathrm{C}$ & 3.472662 & 1.037813 & 1.578728 & $\mathrm{C}$ & 3.319541 & 0.192500 & 1.628938 \\
\hline $\mathrm{C}$ & 2.880507 & 2.131881 & -0.496755 & $\mathrm{C}$ & 5.199148 & -0.097727 & -0.424228 \\
\hline $\mathrm{C}$ & 4.804504 & 1.373473 & 1.379629 & $\mathrm{C}$ & 4.503415 & 0.926863 & 1.628584 \\
\hline $\mathrm{H}$ & 3.175777 & 0.468619 & 2.458733 & $\mathrm{C}$ & 5.450179 & 0.807169 & 0.606490 \\
\hline $\mathrm{C}$ & 4.211197 & 2.478051 & -0.683058 & $\mathrm{H}$ & 5.935664 & -0.221074 & -1.217389 \\
\hline $\mathrm{H}$ & 2.114126 & 2.458992 & -1.198577 & $\mathrm{H}$ & 4.694796 & 1.610592 & 2.455073 \\
\hline $\mathrm{C}$ & 5.179739 & 2.094256 & 0.245777 & $\mathrm{C}$ & -2.242427 & -1.863450 & 2.846854 \\
\hline $\mathrm{H}$ & 5.554764 & 1.072963 & 2.107513 & $\mathrm{H}$ & -1.308310 & -1.311613 & 2.990415 \\
\hline $\mathrm{H}$ & 4.498938 & 3.058237 & -1.556097 & $\mathrm{H}$ & -3.031266 & -1.365687 & 3.417796 \\
\hline $\mathrm{H}$ & 6.222003 & 2.362727 & 0.091272 & $\mathrm{H}$ & -2.100271 & -2.853009 & 3.299955 \\
\hline $\mathrm{O}$ & -1.768432 & 1.681489 & 0.798746 & $\mathrm{C}$ & -0.918532 & -2.387676 & -2.015764 \\
\hline $\mathrm{H}$ & -2.053731 & 0.664278 & -2.037157 & $\mathrm{H}$ & 0.007127 & -2.832296 & -1.635552 \\
\hline $\mathrm{C}$ & -1.451978 & 2.344691 & 1.973298 & $\mathrm{H}$ & -1.290804 & -3.018218 & -2.828539 \\
\hline $\mathrm{H}$ & -0.942718 & 1.697266 & 2.715365 & $\mathrm{H}$ & -0.644213 & -1.414230 & -2.442062 \\
\hline $\mathrm{H}$ & -0.818561 & 3.239306 & 1.836656 & $\mathrm{C}$ & -5.763178 & -2.195279 & -0.725962 \\
\hline $\mathrm{H}$ & -2.393874 & 2.681054 & 2.437273 & $\mathrm{H}$ & -5.956040 & -2.922163 & -1.520721 \\
\hline $\mathrm{O}$ & -0.161271 & 2.805060 & -1.286071 & $\mathrm{H}$ & -6.416769 & -2.434658 & 0.117198 \\
\hline $\mathrm{H}$ & -1.723145 & 1.327147 & -2.182515 & $\mathrm{H}$ & -6.076103 & -1.217508 & -1.110209 \\
\hline $\mathrm{C}$ & -0.379826 & 4.028135 & -0.667883 & $\mathrm{C}$ & 3.794184 & -1.854529 & -1.563591 \\
\hline $\mathrm{H}$ & -1.368183 & 4.065955 & -0.178921 & $\mathrm{H}$ & 4.414802 & -1.632803 & -2.435655 \\
\hline $\mathrm{H}$ & 0.376922 & 4.258329 & 0.106591 & $\mathrm{H}$ & 4.046634 & -2.872455 & -1.241203 \\
\hline \multirow[t]{2}{*}{$\mathrm{H}$} & -0.348034 & 4.840160 & -1.411114 & $\mathrm{H}$ & 2.747580 & -1.862721 & -1.878495 \\
\hline & & & & $\mathrm{C}$ & 2.329544 & 0.334189 & 2.742357 \\
\hline & & & & $\mathrm{H}$ & 1.487390 & 0.965346 & 2.436439 \\
\hline
\end{tabular}




$\begin{array}{cccc}\mathrm{H} & 1.897341 & -0.630290 & 3.031008 \\ \mathrm{H} & 2.792757 & 0.779931 & 3.626060 \\ \mathrm{C} & 6.709708 & 1.618305 & 0.635079 \\ \mathrm{H} & 7.311204 & 1.393913 & 1.522478 \\ \mathrm{H} & 7.332310 & 1.427522 & -0.242455 \\ \mathrm{H} & 6.496967 & 2.691894 & 0.665931 \\ \mathrm{C} & -1.192321 & 1.038542 & 0.790204 \\ \mathrm{H} & -1.343015 & 0.395625 & 1.671597 \\ \mathrm{C} & -2.042122 & 2.220933 & 0.825746 \\ \mathrm{C} & -3.032005 & 2.365470 & 1.816900 \\ \mathrm{C} & -1.924229 & 3.240297 & -0.142204 \\ \mathrm{C} & -3.866266 & 3.476557 & 1.841799 \\ \mathrm{H} & -3.138166 & 1.580362 & 2.564714 \\ \mathrm{C} & -2.751085 & 4.351929 & -0.112105 \\ \mathrm{H} & -1.174344 & 3.127817 & -0.924896 \\ \mathrm{C} & -3.725987 & 4.475308 & 0.879527 \\ \mathrm{H} & -4.626281 & 3.568259 & 2.613518 \\ \mathrm{H} & -2.642427 & 5.128005 & -0.865129 \\ \mathrm{H} & -4.374726 & 5.346893 & 0.901022 \\ \mathrm{O} & -0.875250 & 0.956762 & -2.310097 \\ \mathrm{H} & 1.215147 & 1.361224 & 0.338814 \\ \mathrm{C} & -2.234842 & 0.840471 & -2.575194 \\ \mathrm{H} & -2.876000 & 0.983003 & -1.686255 \\ \mathrm{H} & -2.546726 & 1.584765 & -3.327523 \\ \mathrm{H} & -2.486693 & -0.151969 & -2.991874 \\ \mathrm{O} & 1.872541 & 0.606616 & -2.119420 \\ \mathrm{H} & 2.649687 & 0.685245 & -1.549636 \\ \mathrm{C} & 1.883930 & 1.683493 & -3.054903 \\ \mathrm{H} & 0.928503 & 1.636619 & -3.575441 \\ \mathrm{H} & 1.962788 & 2.654326 & -2.549568 \\ \mathrm{H} & 2.712114 & 1.571103 & -3.764708\end{array}$

\begin{tabular}{|c|c|c|c|}
\hline $\mathrm{C}$ & 1.913766 & -2.582521 & 0.582345 \\
\hline $\mathrm{C}$ & 2.746984 & -1.517922 & -1.459864 \\
\hline $\mathrm{C}$ & 3.227930 & -2.883757 & 0.928829 \\
\hline $\mathrm{C}$ & 4.049441 & -1.825251 & -1.054886 \\
\hline $\mathrm{C}$ & 4.310914 & -2.505117 & 0.131349 \\
\hline $\mathrm{H}$ & 3.413068 & -3.412888 & 1.863350 \\
\hline $\mathrm{H}$ & 4.880747 & -1.528614 & -1.693529 \\
\hline $\mathrm{C}$ & -3.065356 & -0.657114 & -0.632101 \\
\hline $\mathrm{C}$ & -3.869041 & -1.098620 & 0.426531 \\
\hline $\mathrm{C}$ & -3.487152 & 0.350323 & -1.512188 \\
\hline $\mathrm{C}$ & -5.116835 & -0.496833 & 0.593075 \\
\hline $\mathrm{C}$ & -4.739262 & 0.925782 & -1.301735 \\
\hline $\mathrm{C}$ & -5.566611 & 0.517526 & -0.252927 \\
\hline $\mathrm{H}$ & -5.751525 & -0.828150 & 1.413861 \\
\hline $\mathrm{H}$ & -5.075885 & 1.714543 & -1.973477 \\
\hline $\mathrm{C}$ & 2.527261 & -0.798547 & -2.755374 \\
\hline $\mathrm{H}$ & 1.473670 & -0.781228 & -3.043106 \\
\hline $\mathrm{H}$ & 2.857805 & 0.244516 & -2.680043 \\
\hline $\mathrm{H}$ & 3.103262 & -1.256578 & -3.565705 \\
\hline $\mathrm{C}$ & 0.788452 & -2.937615 & 1.504659 \\
\hline $\mathrm{H}$ & -0.109838 & -3.269522 & 0.972880 \\
\hline $\mathrm{H}$ & 1.084095 & -3.729915 & 2.197049 \\
\hline $\mathrm{H}$ & 0.497434 & -2.060874 & 2.097449 \\
\hline $\mathrm{C}$ & 5.714027 & -2.799176 & 0.564929 \\
\hline $\mathrm{H}$ & 5.820516 & -3.826743 & 0.926391 \\
\hline $\mathrm{H}$ & 6.430054 & -2.650859 & -0.247398 \\
\hline $\mathrm{H}$ & 6.016632 & -2.143677 & 1.390075 \\
\hline $\mathrm{C}$ & -3.381128 & -2.153178 & 1.366623 \\
\hline $\mathrm{H}$ & -4.183187 & -2.494610 & 2.025488 \\
\hline $\mathrm{H}$ & -2.981082 & -3.026015 & 0.838255 \\
\hline $\mathrm{H}$ & -2.575042 & -1.742015 & 1.985833 \\
\hline $\mathrm{C}$ & -2.588620 & 0.825822 & -2.611244 \\
\hline $\mathrm{H}$ & -1.648035 & 1.219025 & -2.204656 \\
\hline $\mathrm{H}$ & -2.314629 & 0.023016 & -3.306117 \\
\hline $\mathrm{H}$ & -3.062012 & 1.619246 & -3.193803 \\
\hline $\mathrm{C}$ & -6.896295 & 1.170184 & -0.026902 \\
\hline $\mathrm{H}$ & -7.305345 & 1.587592 & -0.950998 \\
\hline $\mathrm{H}$ & -7.627934 & 0.467181 & 0.380880 \\
\hline $\mathrm{H}$ & -6.815662 & 1.995540 & 0.689686 \\
\hline $\mathrm{C}$ & 0.914475 & 1.421629 & -0.697473 \\
\hline $\mathrm{H}$ & 0.815622 & 1.120202 & -1.754275 \\
\hline $\mathrm{C}$ & 1.738038 & 2.609756 & -0.563167 \\
\hline $\mathrm{C}$ & 2.364069 & 3.174229 & -1.693866 \\
\hline $\mathrm{C}$ & 1.948830 & 3.235571 & 0.685457 \\
\hline
\end{tabular}

\section{TS $_{3 F-4 F}$}

$\begin{array}{rrrr}\mathrm{Ru} & -0.071124 & 0.614870 & 0.697309 \\ \mathrm{C} & -0.569648 & -0.859441 & -0.463840 \\ \mathrm{C} & -0.295996 & -2.824987 & -1.745544 \\ \mathrm{C} & -1.755072 & -2.405680 & -1.802067 \\ \mathrm{H} & 0.153010 & -2.982734 & -2.732132 \\ \mathrm{H} & -0.145844 & -3.745653 & -1.162809 \\ \mathrm{H} & -2.058219 & -2.064248 & -2.802227 \\ \mathrm{H} & -2.448720 & -3.199605 & -1.502863 \\ \mathrm{~N} & 0.340512 & -1.693223 & -1.063308 \\ \mathrm{~N} & -1.806317 & -1.293150 & -0.851633 \\ \mathrm{C} & 1.687120 & -1.893065 & -0.624190\end{array}$




\begin{tabular}{|c|c|c|c|c|c|c|c|}
\hline $\mathrm{C}$ & 3.169672 & 4.299779 & -1.585809 & $\mathrm{C}$ & 4.945568 & 0.866420 & 0.977578 \\
\hline $\mathrm{H}$ & 2.200614 & 2.708635 & -2.664954 & $\mathrm{C}$ & 5.600640 & 0.216306 & -0.070291 \\
\hline $\mathrm{C}$ & 2.746393 & 4.363679 & 0.791136 & $\mathrm{H}$ & 5.451158 & -1.363066 & -1.522882 \\
\hline $\mathrm{H}$ & 1.479024 & 2.805356 & 1.570302 & $\mathrm{H}$ & 5.439253 & 1.693058 & 1.486492 \\
\hline $\mathrm{C}$ & 3.361425 & 4.900054 & -0.342477 & $\mathrm{C}$ & -2.462237 & -0.411792 & 2.714018 \\
\hline $\mathrm{H}$ & 3.643859 & 4.716503 & -2.470640 & $\mathrm{H}$ & -1.641131 & -0.754659 & 3.353238 \\
\hline $\mathrm{H}$ & 2.894765 & 4.831429 & 1.760637 & $\mathrm{H}$ & -2.159397 & 0.570536 & 2.327491 \\
\hline $\mathrm{H}$ & 3.986051 & 5.784997 & -0.255902 & $\mathrm{H}$ & -3.344518 & -0.268014 & 3.342297 \\
\hline $\mathrm{O}$ & 1.334153 & 0.304886 & 2.033382 & $\mathrm{C}$ & -0.850069 & -3.100141 & -1.230818 \\
\hline $\mathrm{H}$ & -1.579435 & 1.341731 & 0.094716 & $\mathrm{H}$ & -0.074130 & -3.632595 & -0.666049 \\
\hline $\mathrm{C}$ & 2.721335 & 0.245610 & 1.920690 & $\mathrm{H}$ & -1.218809 & -3.778323 & -2.003950 \\
\hline $\mathrm{H}$ & 3.064533 & 0.140752 & 0.878072 & $\mathrm{H}$ & -0.350564 & -2.253185 & -1.721636 \\
\hline $\mathrm{H}$ & 3.181156 & 1.162056 & 2.328041 & $\mathrm{C}$ & -5.753506 & -2.969409 & -0.101918 \\
\hline $\mathrm{H}$ & 3.129213 & -0.604456 & 2.490658 & $\mathrm{H}$ & -5.811474 & -3.969370 & -0.541073 \\
\hline $\mathrm{O}$ & -1.702429 & 0.370086 & 2.198856 & $\mathrm{H}$ & -6.387086 & -2.952357 & 0.788964 \\
\hline $\mathrm{H}$ & -1.832008 & 1.018529 & 0.911040 & $\mathrm{H}$ & -6.200163 & -2.278551 & -0.826696 \\
\hline $\mathrm{C}$ & -1.552556 & 1.297998 & 3.236451 & $\mathrm{C}$ & 2.972757 & -2.366587 & -1.050649 \\
\hline $\mathrm{H}$ & -0.601614 & 1.164900 & 3.775472 & $\mathrm{H}$ & 3.669198 & -2.935411 & -1.671314 \\
\hline $\mathrm{H}$ & -1.579775 & 2.344785 & 2.877199 & $\mathrm{H}$ & 2.487612 & -3.062789 & -0.356351 \\
\hline \multirow[t]{2}{*}{$\mathrm{H}$} & -2.372384 & 1.193705 & 3.963873 & $\mathrm{H}$ & 2.186051 & -1.953594 & -1.700969 \\
\hline & & & & $\mathrm{C}$ & 2.948765 & 1.227814 & 2.472022 \\
\hline $4 F$ & & & & $\mathrm{H}$ & 2.045785 & 1.712517 & 2.080224 \\
\hline $\mathrm{Ru}$ & 0.088841 & 0.545971 & -0.837239 & $\mathrm{H}$ & 2.616236 & 0.566251 & 3.279756 \\
\hline $\mathrm{C}$ & 0.553313 & -0.755099 & 0.552808 & $\mathrm{H}$ & 3.581953 & 2.001235 & 2.912334 \\
\hline $\mathrm{C}$ & 0.253451 & -2.550257 & 2.048682 & $\mathrm{C}$ & 6.959964 & 0.657522 & -0.519185 \\
\hline $\mathrm{C}$ & 1.661520 & -2.006099 & 2.224285 & $\mathrm{H}$ & 7.478135 & 1.227731 & 0.256106 \\
\hline $\mathrm{H}$ & -0.308182 & -2.617627 & 2.986312 & $\mathrm{H}$ & 7.590735 & -0.192901 & -0.793664 \\
\hline $\mathrm{H}$ & 0.237376 & -3.545870 & 1.580209 & $\mathrm{H}$ & 6.894796 & 1.300245 & -1.404357 \\
\hline $\mathrm{H}$ & 1.809359 & -1.526087 & 3.202359 & $\mathrm{C}$ & -0.470950 & 1.792245 & 0.520637 \\
\hline $\mathrm{H}$ & 2.438080 & -2.769092 & 2.107131 & $\mathrm{H}$ & -0.161054 & 1.702403 & 1.578854 \\
\hline $\mathrm{N}$ & -0.362502 & -1.566087 & 1.153764 & $\mathrm{C}$ & -1.242569 & 3.003866 & 0.327399 \\
\hline $\mathrm{N}$ & 1.755756 & -1.012706 & 1.151095 & $\mathrm{C}$ & -1.639804 & 3.787784 & 1.432660 \\
\hline $\mathrm{C}$ & -1.713983 & -1.812293 & 0.758733 & $\mathrm{C}$ & -1.633054 & 3.442907 & -0.958507 \\
\hline $\mathrm{C}$ & -1.965175 & -2.637860 & -0.348941 & $\mathrm{C}$ & -2.401593 & 4.935933 & 1.268138 \\
\hline $\mathrm{C}$ & -2.746780 & -1.350281 & 1.583616 & $\mathrm{H}$ & -1.339628 & 3.467969 & 2.429746 \\
\hline $\mathrm{C}$ & -3.287453 & -3.002305 & -0.601983 & $\mathrm{C}$ & -2.388211 & 4.592142 & -1.122335 \\
\hline $\mathrm{C}$ & -4.051947 & -1.751689 & 1.296339 & $\mathrm{H}$ & -1.336474 & 2.850608 & -1.823130 \\
\hline $\mathrm{C}$ & -4.341416 & -2.580517 & 0.212029 & $\mathrm{C}$ & -2.778640 & 5.343158 & -0.010906 \\
\hline $\mathrm{H}$ & -3.498130 & -3.641439 & -1.458385 & $\mathrm{H}$ & -2.699244 & 5.519889 & 2.135009 \\
\hline $\mathrm{H}$ & -4.862625 & -1.401065 & 1.933926 & $\mathrm{H}$ & -2.677703 & 4.910956 & -2.120160 \\
\hline $\mathrm{C}$ & 3.043932 & -0.574867 & 0.720215 & $\mathrm{H}$ & -3.370012 & 6.245193 & -0.143427 \\
\hline $\mathrm{C}$ & 3.674155 & -1.260306 & -0.331960 & $\mathrm{O}$ & -1.671664 & 0.270355 & -1.648656 \\
\hline $\mathrm{C}$ & 3.668107 & 0.487551 & 1.388816 & $\mathrm{H}$ & 1.572997 & 1.448627 & -0.945350 \\
\hline $\mathrm{C}$ & 4.950809 & -0.843076 & -0.707297 & $\mathrm{C}$ & -2.944922 & 0.516350 & -1.144249 \\
\hline
\end{tabular}




$\begin{array}{cccc}\mathrm{H} & -2.940462 & 0.920778 & -0.118557 \\ \mathrm{H} & -3.458667 & 1.254246 & -1.782063 \\ \mathrm{H} & -3.554115 & -0.399227 & -1.142315 \\ \mathrm{O} & 0.858839 & -0.468458 & -2.486044 \\ \mathrm{H} & 1.819726 & 0.762389 & -0.512713 \\ \mathrm{C} & 0.234145 & -0.307462 & -3.714403 \\ \mathrm{H} & -0.700994 & -0.891214 & -3.789229 \\ \mathrm{H} & -0.051067 & 0.739844 & -3.929961 \\ \mathrm{H} & 0.899088 & -0.634093 & -4.531333\end{array}$

\section{TS 3B-4Bpy}

$\begin{array}{rrrr}\mathrm{Ru} & 0.065184 & 0.401107 & -0.577703 \\ \mathrm{C} & 0.826379 & 2.636196 & -2.648157 \\ \mathrm{C} & -0.772276 & 3.404011 & -1.178262 \\ \mathrm{C} & 0.856791 & 3.857343 & -3.310019 \\ \mathrm{H} & 1.426527 & 1.784904 & -2.961733 \\ \mathrm{C} & -0.802358 & 4.650574 & -1.786614 \\ \mathrm{H} & -1.410726 & 3.174803 & -0.329835 \\ \mathrm{H} & 1.520101 & 3.986772 & -4.159354 \\ \mathrm{H} & -1.473079 & 5.415074 & -1.408393 \\ \mathrm{~N} & 0.031969 & 2.406665 & -1.586332 \\ \mathrm{C} & 0.033678 & 4.888477 & -2.872047 \\ \mathrm{H} & 0.038473 & 5.855010 & -3.368702 \\ \mathrm{C} & 0.012775 & -1.399916 & 0.357353 \\ \mathrm{C} & -0.811162 & -3.255051 & 1.579191 \\ \mathrm{C} & 0.692544 & -3.214772 & 1.726991 \\ \mathrm{H} & -1.349382 & -3.313663 & 2.531018 \\ \mathrm{H} & -1.153319 & -4.092534 & 0.952613 \\ \mathrm{H} & 1.013808 & -2.913469 & 2.735691 \\ \mathrm{H} & 1.184777 & -4.164185 & 1.490997 \\ \mathrm{~N} & -1.100361 & -1.981819 & 0.903662 \\ \mathrm{~N} & 1.068628 & -2.175980 & 0.763021 \\ \mathrm{C} & -2.474954 & -1.823309 & 0.527350 \\ \mathrm{C} & -2.909926 & -2.376605 & -0.692397 \\ \mathrm{C} & -3.382827 & -1.285746 & 1.443154 \\ \mathrm{C} & -4.265856 & -2.331718 & -0.991971 \\ \mathrm{C} & -4.739348 & -1.258033 & 1.093566 \\ \mathrm{C} & -5.199055 & -1.764971 & -0.115624 \\ \mathrm{H} & -4.606978 & -2.746194 & -1.940100 \\ \mathrm{H} & -5.452133 & -0.832633 & 1.799371 \\ \mathrm{C} & 2.465707 & -1.945734 & 0.581617 \\ \mathrm{C} & 3.178434 & -2.806118 & -0.271726 \\ \mathrm{C} & 3.135031 & -0.970750 & 1.341552 \\ \mathrm{C} & 4.544222 & -2.583460 & -0.450712\end{array}$

$\begin{array}{lrrc}\mathrm{C} & 4.503319 & -0.794421 & 1.134390 \\ \mathrm{C} & 5.221734 & -1.568961 & 0.224017 \\ \mathrm{H} & 5.094986 & -3.233319 & -1.130053 \\ \mathrm{H} & 5.023298 & -0.030727 & 1.712108 \\ \mathrm{C} & -2.967170 & -0.739516 & 2.775848 \\ \mathrm{H} & -1.955229 & -1.041970 & 3.058978 \\ \mathrm{H} & -2.978957 & 0.357342 & 2.769275 \\ \mathrm{H} & -3.657004 & -1.059116 & 3.563509 \\ \mathrm{C} & -1.931909 & -2.951165 & -1.666500 \\ \mathrm{H} & -1.164922 & -3.568450 & -1.182706 \\ \mathrm{H} & -2.435116 & -3.566142 & -2.417137 \\ \mathrm{H} & -1.411791 & -2.133484 & -2.177097 \\ \mathrm{C} & -6.649289 & -1.699414 & -0.484667 \\ \mathrm{H} & -7.013108 & -2.654583 & -0.876688 \\ \mathrm{H} & -7.272803 & -1.427350 & 0.371054 \\ \mathrm{H} & -6.825843 & -0.952648 & -1.267644 \\ \mathrm{C} & 2.537989 & -3.988985 & -0.934663 \\ \mathrm{H} & 2.870077 & -4.090740 & -1.971871 \\ \mathrm{H} & 2.818365 & -4.916082 & -0.418960 \\ \mathrm{H} & -1.727223 & 0.039354 & -1.388751 \\ \mathrm{H} & 1.447664 & -3.928004 & -0.939932 \\ \mathrm{C} & -2.755498 & 0.956193 & -1.334195 \\ \mathrm{C} & 2.439679 & -0.134776 & 2.370374 \\ \mathrm{H} & 2.304551 & 0.896228 & 2.024643 \\ \mathrm{H} & 1.442727 & -0.509802 & 2.617613 \\ \mathrm{H} & -1.172349 & 4.411592 & 4.233336 \\ \mathrm{H} & 3.030978 & -0.091531 & 3.290732 \\ \mathrm{C} & 6.679468 & -1.322040 & -0.015914 \\ \mathrm{H} & 7.195751 & -2.227939 & -0.345438 \\ \mathrm{H} & 6.829775 & -0.566836 & -0.795844 \\ \mathrm{H} & 7.181380 & -0.953505 & 0.883387 \\ \mathrm{C} & -0.317590 & 1.094819 & 1.136602 \\ \mathrm{H} & -0.804430 & 0.446951 & 1.883585 \\ \mathrm{C} & -0.006075 & 2.387366 & 1.730654 \\ \mathrm{C} & -0.774939 & 2.841126 & 2.824005 \\ \mathrm{C} & 1.039030 & 3.223811 & 1.283994 \\ \mathrm{H} & -0.551733 & 4.082127 & 3.403735 \\ \mathrm{H} & 1.567872 & 2.197465 & 3.202855 \\ \mathrm{H} & 0.666449 & 2.452792 & 1.883241 \\ \mathrm{H} & 2.875292 & 0.465254 \\ \mathrm{H} & & \\ \mathrm{H} & & \\ \mathrm{H} & & \\ \mathrm{H} & & \end{array}$




$\begin{array}{crrr}\mathrm{H} & -2.921653 & 1.340669 & -0.304804 \\ \mathrm{H} & -2.616345 & 1.837988 & -1.987782 \\ \mathrm{H} & -3.692951 & 0.468594 & -1.654422 \\ \mathrm{O} & 1.295352 & -0.203221 & -2.402834 \\ \mathrm{H} & 1.838124 & 0.210985 & -1.172962 \\ \mathrm{C} & 1.462465 & -1.518905 & -2.816936 \\ \mathrm{H} & 1.043643 & -1.654234 & -3.828104 \\ \mathrm{H} & 2.525605 & -1.819129 & -2.862145 \\ \mathrm{H} & 0.948498 & -2.245774 & -2.167362\end{array}$

\section{TS $_{3 \mathrm{C}-4 \mathrm{Cpy}}$}

$\begin{array}{rrrr}\mathrm{Ru} & -0.093087 & 0.417004 & -0.389477 \\ \mathrm{C} & -0.320215 & 3.561821 & -0.566104 \\ \mathrm{C} & 0.469736 & 2.602817 & -2.509850 \\ \mathrm{C} & -0.405549 & 4.811333 & -1.162265 \\ \mathrm{H} & -0.596806 & 3.405680 & 0.473978 \\ \mathrm{C} & 0.416630 & 3.818924 & -3.177305 \\ \mathrm{H} & 0.789436 & 1.678223 & -2.987076 \\ \mathrm{H} & -0.761038 & 5.659351 & -0.586190 \\ \mathrm{H} & 0.717805 & 3.871919 & -4.218519 \\ \mathrm{~N} & 0.110074 & 2.467826 & -1.220351 \\ \mathrm{C} & -0.030775 & 4.945506 & -2.496092 \\ \mathrm{H} & -0.090351 & 5.909085 & -2.994126 \\ \mathrm{C} & -0.447131 & -1.445304 & 0.284524 \\ \mathrm{C} & -0.049799 & -3.614469 & 1.158892 \\ \mathrm{C} & -1.543299 & -3.341520 & 1.192756 \\ \mathrm{H} & 0.219137 & -4.427910 & 0.472712 \\ \mathrm{H} & 0.370646 & -3.864182 & 2.140600 \\ \mathrm{H} & -2.137008 & -4.082594 & 0.643218 \\ \mathrm{H} & -1.945964 & -3.293909 & 2.213923 \\ \mathrm{~N} & 0.506972 & -2.343827 & 0.672891 \\ \mathrm{~N} & -1.652368 & -2.023278 & 0.557093 \\ \mathrm{C} & 1.848515 & -2.399331 & 0.175336 \\ \mathrm{C} & 2.927633 & -2.172685 & 1.033313 \\ \mathrm{C} & 2.038622 & -2.809788 & -1.158124 \\ \mathrm{C} & 4.221324 & -2.320678 & 0.517501 \\ \mathrm{C} & 3.341460 & -2.940138 & -1.624116 \\ \mathrm{C} & 4.447522 & -2.686914 & -0.804941 \\ \mathrm{H} & 5.071261 & -2.138208 & 1.174435 \\ \mathrm{H} & 3.501462 & -3.239755 & -2.659518 \\ \mathrm{C} & -2.936519 & -1.400093 & 0.609014 \\ \mathrm{C} & -3.283914 & -0.639785 & 1.733511 \\ \mathrm{C} & -3.850149 & -1.643747 & -0.426981 \\ \mathrm{C} & -4.565114 & -0.085863 & 1.785201\end{array}$

\begin{tabular}{|c|c|c|c|}
\hline & & & \\
\hline & .490781 & .282972 & 07 \\
\hline & 815 & $=10$ & $=1$ \\
\hline & 583 & 54 & \\
\hline & 0.86 & 8 & \\
\hline & 1.19 & -3.4 & \\
\hline & & & \\
\hline & 0.27 & -2.17 & \\
\hline & 2.7 & 2 & 78 \\
\hline & & & \\
\hline & 2.95 & 37 & \\
\hline & 1.72 & -1 & \\
\hline & 5.83 & -2.7 & -1.35140 \\
\hline & 6.588193 & -2 & \\
\hline & & -3 & \\
\hline & 06 & 58 & \\
\hline & -2.297 & -0.3 & \\
\hline & -2.80 & -0 & \\
\hline & -1.6 & & \\
\hline & -1.5 & 538 & \\
\hline & -3.45 & -2.4 & \\
\hline & -2.6 & -1. & \\
\hline & -3.06 & & \\
\hline & -4.30 & -2.61 & -22 \\
\hline & -6.855 & 0.33 & \\
\hline & -6.97 & & \\
\hline & -7.6 & 0 & \\
\hline & -7.05 & 898 & \\
\hline & & & \\
\hline & & & \\
\hline & & & \\
\hline & 0 & 76 & 21 \\
\hline & & TOJ & \\
\hline & & & \\
\hline & 9 & 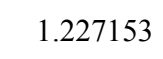 & 2818 \\
\hline & 2. & 85 & 6465 \\
\hline & 9 & 75 & -0.058 \\
\hline & $000-$ & & \\
\hline & 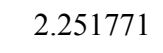 & 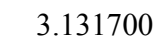 & 49003 \\
\hline & 2 & & \\
\hline & 3.426289 & 821038 & (5) \\
\hline & & -0.151997 & \\
\hline & 2.605559 & 0.398116 & \\
\hline & 543207 & 49953 & .10 \\
\hline
\end{tabular}




$\begin{array}{rrrr}\mathrm{H} & 3.271457 & -0.039137 & -2.325827 \\ \mathrm{H} & 3.058244 & 0.196427 & -0.572221 \\ \mathrm{H} & -1.668922 & 1.043446 & 0.290115 \\ \mathrm{O} & -1.816140 & 0.144792 & -1.845522 \\ \mathrm{H} & -1.928680 & 0.695662 & -0.503332 \\ \mathrm{C} & -2.276215 & 1.091616 & -2.746792 \\ \mathrm{H} & -1.600485 & 1.228265 & -3.611052 \\ \mathrm{H} & -3.258971 & 0.791561 & -3.153407 \\ \mathrm{H} & -2.418187 & 2.098006 & -2.301707\end{array}$

\section{TS 3 3D-4Dpy}

\begin{tabular}{|c|c|c|c|}
\hline $\mathrm{Ru}$ & 0.286698 & 09 & 0.08 \\
\hline $\mathrm{C}$ & 0.080074 & .518768 & $2151+>$ \\
\hline $\mathrm{C}$ & .041898 & 3.437632 & .87645 \\
\hline $\mathrm{C}$ & 1 & 2 & 264 \\
\hline $\mathrm{H}$ & 486774 & 2.981046 & 106700 \\
\hline & 1 & 4.74 & 0451 \\
\hline$\Pi$ & 1.2 & $2.0+3$ & 427 \\
\hline$x^{2}$ & 0.264609 & 5. & 447 \\
\hline $\mathrm{H}$ & 53 & 015 & 5654 \\
\hline $\mathrm{N}$ & 4 & 4 & >0 \\
\hline ( & 226006 & 3 & 0.393411 \\
\hline $\mathrm{H}$ & & & \\
\hline $\mathrm{C}$ & -0.857487 & -1.216884 & 32 \\
\hline $\mathrm{C}$ & -0.743412 & -3.440612 & 1.182669 \\
\hline $\mathrm{C}$ & -2.201968 & -3.081712 & 0.947076 \\
\hline$H$ & $47+2+3$ & -4.344038 & 0.647528 \\
\hline $\mathrm{H}$ & -0.511970 & -3.592594 & 2246854 \\
\hline$\Pi$ & -2.66 & -3.6 & 08 \\
\hline $\mathrm{H}$ & -2.82 & 5.2 & 1.0 \\
\hline $\mathrm{N}$ & -0.02 & -2.26 & 78675 \\
\hline Iv & -2.1 & -1.66 & 0446 \\
\hline $\mathrm{C}$ & 30 & 2 & 268446 \\
\hline $\mathrm{C}$ & 2.348038 & -2.216 & 080 \\
\hline$C$ & 1.03 & 2.845 & -0.02 \\
\hline$C$ & 3.682461 & -2.393040 & 0.969041 \\
\hline $\mathrm{C}$ & 3.040342 & -3.005075 & -1.252071 \\
\hline $\mathrm{C}$ & 053060 & -2.762 & 2 \\
\hline $\mathrm{H}$ & 4.456675 & -2.2241 & 1.717182 \\
\hline $\mathrm{H}$ & 3.304381 & -3.319827 & -2.261449 \\
\hline $\mathrm{C}$ & -3.346400 & -1.049432 & 0.130034 \\
\hline $\mathrm{C}$ & -4.197088 & -0.453877 & 1.071977 \\
\hline $\mathrm{C}$ & -3.710344 & -1.146237 & -1.220655 \\
\hline $\mathrm{C}$ & -5.406289 & 0.079422 & 0.027 \\
\hline
\end{tabular}

\begin{tabular}{|c|c|c|c|}
\hline$C$ & -4.926632 & -0.590887 & -1.619243 \\
\hline $\mathrm{C}$ & -5.784193 & 0.032475 & -0.714003 \\
\hline $\mathrm{H}$ & -6.068929 & 0.548877 & 1.355484 \\
\hline 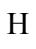 & -5.211984 & -0.653783 & -2.668800 \\
\hline $\mathrm{C}$ & 0.628327 & -3.105317 & -1.942168 \\
\hline $\mathrm{H}$ & 0.028873 & -2.208064 & -2.134792 \\
\hline $\mathrm{H}$ & 1.057868 & -3.437085 & -2.890618 \\
\hline $\mathrm{H}$ & -0.079578 & -3.876244 & -1.611627 \\
\hline $\mathrm{C}$ & 2.031633 & -1.814557 & 2.747421 \\
\hline $\mathrm{H}$ & 2.693285 & -2.324586 & 3.454334 \\
\hline $\mathrm{H}$ & 2.160366 & -0.734916 & 2.863228 \\
\hline $\mathrm{H}$ & 0.995413 & -2.027761 & 3.019270 \\
\hline $\mathrm{C}$ & 5.496619 & -2.874642 & -0.701752 \\
\hline Н & 5.626361 & -3.385447 & -1.659761 \\
\hline $\mathrm{H}$ & 5.948762 & -1.879927 & -0.795474 \\
\hline $\mathrm{H}$ & 6.076124 & -3.416163 & 0.052434 \\
\hline $\mathrm{C}$ & -3.793712 & -0.336061 & 2.505181 \\
\hline $\mathrm{H}$ & -4.651461 & -0.106749 & 3.142569 \\
\hline $\mathrm{H}$ & -3.314097 & -1.245242 & 2.882687 \\
\hline $\mathrm{H}$ & -3.057957 & 0.469221 & 2.621132 \\
\hline $\mathrm{C}$ & -2.810285 & -1.799565 & -2.218701 \\
\hline $\mathrm{H}$ & -1.962845 & -1.140086 & -2.436545 \\
\hline $\mathrm{H}$ & -2.402050 & -2.748340 & -1.853677 \\
\hline $\mathrm{H}$ & -3.337896 & -1.997012 & -3.155441 \\
\hline $\mathrm{C}$ & -7.074177 & 0.643870 & -1.169401 \\
\hline $\mathrm{H}$ & -7.399251 & 0.234527 & -2.129801 \\
\hline $\mathrm{H}$ & -7.877611 & 0.483579 & -0.444120 \\
\hline $\mathrm{H}$ & -6.977189 & 1.728115 & -1.297502 \\
\hline $\mathrm{C}$ & 1.268065 & 0.217502 & -0.868343 \\
\hline $\mathrm{H}$ & 1.134879 & -0.443176 & -1.743769 \\
\hline $\mathrm{C}$ & 2.638907 & 0.673936 & -0.762129 \\
\hline $\mathrm{C}$ & 3.570938 & 0.363845 & -1.776203 \\
\hline $\mathrm{C}$ & 3.109605 & 1.344037 & 0.390239 \\
\hline $\mathrm{C}$ & 4.905469 & 0.727271 & -1.663730 \\
\hline $\mathrm{H}$ & 3.221749 & -0.184178 & -2.650734 \\
\hline 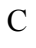 & 4.449212 & 1.688522 & 0.506061 \\
\hline$H$ & 2.403691 & 1.542559 & 1.193828 \\
\hline $\mathrm{C}$ & 5.349112 & 1.391407 & -0.518947 \\
\hline $\mathrm{H}$ & 5.605929 & 0.486263 & -2.460072 \\
\hline $\mathrm{H}$ & 4.800910 & 2.192819 & 1.402953 \\
\hline $\mathrm{H}$ & 6.395618 & 1.670799 & -0.424152 \\
\hline $\mathrm{O}$ & 0.537682 & 0.741175 & 1.958566 \\
\hline $\mathrm{C}$ & -0.312517 & 0.412820 & 2.994559 \\
\hline $\mathrm{H}$ & -0.808071 & -0.574530 & 2.87808 \\
\hline
\end{tabular}




$\begin{array}{crrr}\mathrm{H} & 0.252677 & 0.374802 & 3.942648 \\ \mathrm{H} & -1.134824 & 1.147022 & 3.142021 \\ \mathrm{C} & -1.587888 & 2.199398 & -2.403445 \\ \mathrm{H} & -0.639110 & 2.217401 & -2.960794 \\ \mathrm{H} & -2.408272 & 2.233320 & -3.134479 \\ \mathrm{H} & -1.638571 & 3.119031 & -1.798456 \\ \mathrm{O} & -1.695844 & 1.039977 & -1.622829 \\ \mathrm{H} & -2.097912 & 1.317035 & -0.530336 \\ \mathrm{H} & -2.037718 & 1.464603 & 0.535609\end{array}$

\section{TS 3E-4Epy}

$\begin{array}{rrrr}\mathrm{Ru} & -0.336589 & 0.703939 & 0.040760 \\ \mathrm{C} & -1.603244 & 3.461202 & 0.836066 \\ \mathrm{C} & -0.247580 & 3.699442 & -1.017367 \\ \mathrm{C} & -1.977172 & 4.798536 & 0.778569 \\ \mathrm{H} & -1.954972 & 2.766709 & 1.597335 \\ \mathrm{C} & -0.571698 & 5.043140 & -1.141867 \\ \mathrm{H} & 0.438975 & 3.210391 & -1.705025 \\ \mathrm{H} & -2.669812 & 5.193800 & 1.514674 \\ \mathrm{H} & -0.134464 & 5.631720 & -1.941718 \\ \mathrm{~N} & -0.756187 & 2.917986 & -0.051556 \\ \mathrm{C} & -1.458719 & 5.604611 & -0.227880 \\ \mathrm{H} & -1.737321 & 6.652339 & -0.299458 \\ \mathrm{C} & -0.292816 & -1.310511 & 0.272659 \\ \mathrm{C} & 0.355079 & -3.466162 & 1.000067 \\ \mathrm{C} & -1.152373 & -3.444371 & 0.818585 \\ \mathrm{H} & 0.853029 & -4.251895 & 0.420631 \\ \mathrm{H} & 0.651295 & -3.590761 & 2.051268 \\ \mathrm{H} & -1.494439 & -4.092369 & -0.001747 \\ \mathrm{H} & -1.702403 & -3.738786 & 1.719610 \\ \mathrm{~N} & 0.759087 & -2.140984 & 0.515895 \\ \mathrm{~N} & -1.426981 & -2.039507 & 0.497150 \\ \mathrm{C} & 2.128493 & -1.955466 & 0.162699 \\ \mathrm{C} & 3.075219 & -1.663020 & 1.152010 \\ \mathrm{C} & 2.514449 & -2.198914 & -1.165429 \\ \mathrm{C} & 4.418156 & -1.592612 & 0.780719 \\ \mathrm{C} & 3.868236 & -2.131925 & -1.486942 \\ \mathrm{C} & 4.836286 & -1.827071 & -0.528043 \\ \mathrm{H} & 5.156471 & -1.326984 & 1.536606 \\ \mathrm{H} & 4.172480 & -2.302409 & -2.519570 \\ \mathrm{C} & -2.777625 & -1.732399 & 0.120753 \\ \mathrm{C} & -3.726837 & -1.404814 & 1.093474 \\ \mathrm{C} & -3.145715 & -1.903372 & -1.226540 \\ \mathrm{C} & -5.046506 & -1.187388 & 0.679110\end{array}$

\begin{tabular}{|c|c|c|c|}
\hline $\mathrm{C}$ & -4.469396 & -1.684367 & -1.588197 \\
\hline $\mathrm{C}$ & -5.434892 & -1.306347 & -0.650581 \\
\hline $\mathrm{H}$ & -5.789980 & -0.922229 & 1.430504 \\
\hline H & -4.752989 & -1.797332 & -2.634308 \\
\hline $\mathrm{C}$ & 1.487710 & -2.480109 & -2.215050 \\
\hline $\mathrm{H}$ & 1.953608 & -2.746864 & -3.166814 \\
\hline $\mathrm{H}$ & 0.811889 & -3.294434 & -1.927416 \\
\hline $\mathrm{H}$ & 0.849568 & -1.602207 & -2.378906 \\
\hline $\mathrm{C}$ & 2.650939 & -1.364977 & 2.553721 \\
\hline $\mathrm{H}$ & 2.093168 & -2.191869 & 3.007962 \\
\hline $\mathrm{H}$ & 3.513100 & -1.153555 & 3.191358 \\
\hline $\mathrm{H}$ & 1.982514 & -0.495937 & 2.577451 \\
\hline $\mathrm{C}$ & 6.284822 & -1.743072 & -0.899279 \\
\hline $\mathrm{H}$ & 6.419833 & -1.299007 & -1.890629 \\
\hline $\mathrm{H}$ & 6.846559 & -1.136862 & -0.182944 \\
\hline $\mathrm{H}$ & 6.753885 & -2.733757 & -0.927362 \\
\hline $\mathrm{C}$ & -3.386052 & -1.269449 & 2.541997 \\
\hline $\mathrm{H}$ & -3.185790 & -0.221628 & 2.782149 \\
\hline $\mathrm{H}$ & -4.210557 & -1.620300 & 3.170224 \\
\hline $\mathrm{H}$ & -2.481001 & -1.819776 & 2.812108 \\
\hline $\mathrm{C}$ & -2.130112 & -2.281708 & -2.254590 \\
\hline $\mathrm{H}$ & -1.364389 & -1.503342 & -2.328642 \\
\hline $\mathrm{H}$ & -1.618772 & -3.222701 & -2.015677 \\
\hline $\mathrm{H}$ & -2.591684 & -2.396242 & -3.238411 \\
\hline $\mathrm{C}$ & -6.841551 & -1.018233 & -1.076935 \\
\hline $\mathrm{H}$ & -7.530846 & -1.016425 & -0.228121 \\
\hline $\mathrm{H}$ & -6.914686 & -0.034201 & -1.555881 \\
\hline $\mathrm{H}$ & -7.204676 & -1.749208 & -1.806189 \\
\hline $\mathrm{C}$ & 1.329525 & 1.251130 & 0.711515 \\
\hline $\mathrm{H}$ & 1.228432 & 1.842754 & 1.644812 \\
\hline $\mathrm{C}$ & 2.685808 & 1.379372 & 0.206305 \\
\hline $\mathrm{C}$ & 3.685819 & 1.884202 & 1.066956 \\
\hline $\mathrm{C}$ & 3.059131 & 1.080599 & -1.118902 \\
\hline $\mathrm{C}$ & 4.991250 & 2.064932 & 0.635383 \\
\hline $\mathrm{H}$ & 3.408587 & 2.129032 & 2.091552 \\
\hline $\mathrm{C}$ & 4.357632 & 1.290844 & -1.559050 \\
\hline $\mathrm{H}$ & 2.299798 & 0.689855 & -1.793157 \\
\hline $\mathrm{C}$ & 5.332027 & 1.776994 & -0.686677 \\
\hline $\mathrm{H}$ & 5.742335 & 2.447743 & 1.321746 \\
\hline $\mathrm{H}$ & 4.620379 & 1.058011 & -2.588811 \\
\hline $\mathrm{H}$ & 6.349562 & 1.932588 & -1.036734 \\
\hline $\mathrm{O}$ & -1.233608 & 0.829823 & 1.894595 \\
\hline $\mathrm{H}$ & 0.174429 & 0.724100 & -1.682214 \\
\hline $\mathrm{C}$ & -0.608684 & 0.271172 & 2.987784 \\
\hline
\end{tabular}




\begin{tabular}{|c|c|c|c|c|c|c|c|}
\hline $\mathrm{H}$ & -0.387985 & -0.811981 & 2.871896 & $\mathrm{H}$ & -0.831667 & -0.732343 & -2.416098 \\
\hline $\mathrm{H}$ & 0.361999 & 0.749903 & 3.237252 & $\mathrm{C}$ & -6.005830 & -1.752645 & -0.596364 \\
\hline $\mathrm{H}$ & -1.244333 & 0.366695 & 3.885839 & $\mathrm{H}$ & -6.570576 & -2.542902 & -0.092104 \\
\hline $\mathrm{O}$ & -2.081212 & 0.639417 & -1.426153 & $\mathrm{H}$ & -6.379271 & -0.800371 & -0.202077 \\
\hline $\mathrm{H}$ & -0.788053 & 0.805072 & -1.762989 & $\mathrm{H}$ & -6.258340 & -1.784509 & -1.659214 \\
\hline $\mathrm{C}$ & -3.092304 & 1.593140 & -1.427278 & $\mathrm{C}$ & 3.051770 & -1.161757 & -2.310118 \\
\hline $\mathrm{H}$ & -3.939709 & 1.236964 & -2.038255 & $\mathrm{H}$ & 3.798706 & -1.110179 & -3.106419 \\
\hline $\mathrm{H}$ & -3.484248 & 1.776713 & -0.410059 & $\mathrm{H}$ & 2.720797 & -2.201373 & -2.214297 \\
\hline \multirow[t]{2}{*}{$\mathrm{H}$} & -2.785579 & 2.570816 & -1.841909 & $\mathrm{H}$ & 2.178277 & -0.572120 & -2.617079 \\
\hline & & & & $\mathrm{C}$ & 2.791369 & -0.713985 & 2.712809 \\
\hline 5 & & & & $\mathrm{H}$ & 1.702032 & -0.710914 & 2.607223 \\
\hline $\mathrm{Ru}$ & 0.303481 & 0.786862 & -0.142370 & $\mathrm{H}$ & 3.087624 & -1.698254 & 3.097733 \\
\hline $\mathrm{C}$ & 0.504625 & -1.098679 & 0.144927 & $\mathrm{H}$ & 3.055905 & 0.020791 & 3.477196 \\
\hline $\mathrm{C}$ & 0.296989 & -3.437204 & 0.297943 & $\mathrm{C}$ & 6.539110 & 1.480390 & 0.180511 \\
\hline $\mathrm{C}$ & 1.740531 & -3.059797 & 0.584508 & $\mathrm{H}$ & 7.152758 & 1.292248 & -0.704432 \\
\hline $\mathrm{H}$ & -0.133356 & -4.114706 & 1.042665 & $\mathrm{H}$ & 6.290641 & 2.547869 & 0.178282 \\
\hline $\mathrm{H}$ & 0.181420 & -3.906928 & -0.691689 & $\mathrm{H}$ & 7.156351 & 1.301687 & 1.065694 \\
\hline $\mathrm{H}$ & 1.989897 & -3.163431 & 1.652494 & $\mathrm{C}$ & -1.386837 & 1.025515 & 0.572165 \\
\hline $\mathrm{H}$ & 2.464736 & -3.645873 & 0.009562 & $\mathrm{H}$ & -2.089910 & 0.210848 & 0.780616 \\
\hline $\mathrm{N}$ & -0.377898 & -2.135784 & 0.318980 & $\mathrm{C}$ & -2.005981 & 2.345376 & 0.601308 \\
\hline $\mathrm{N}$ & 1.758480 & -1.657976 & 0.182006 & $\mathrm{C}$ & -3.394941 & 2.474936 & 0.396975 \\
\hline $\mathrm{C}$ & -1.777689 & -2.078770 & 0.059751 & $\mathrm{C}$ & -1.255573 & 3.523740 & 0.791914 \\
\hline $\mathrm{C}$ & -2.248015 & -1.867065 & -1.243563 & $\mathrm{C}$ & -3.997345 & 3.724439 & 0.349843 \\
\hline $\mathrm{C}$ & -2.655679 & -2.236061 & 1.143611 & $\mathrm{H}$ & -3.985989 & 1.570048 & 0.259769 \\
\hline $\mathrm{C}$ & -3.629636 & -1.771430 & -1.429806 & $\mathrm{C}$ & -1.860639 & 4.773088 & 0.744890 \\
\hline $\mathrm{C}$ & -4.024928 & -2.140481 & 0.906838 & $\mathrm{H}$ & -0.193703 & 3.431582 & 1.016266 \\
\hline $\mathrm{C}$ & -4.530808 & -1.893988 & -0.372959 & $\mathrm{C}$ & -3.231893 & 4.878943 & 0.518118 \\
\hline $\mathrm{H}$ & -4.010873 & -1.599840 & -2.435802 & $\mathrm{H}$ & -5.068607 & 3.803695 & 0.182690 \\
\hline $\mathrm{H}$ & -4.713893 & -2.244537 & 1.744297 & $\mathrm{H}$ & -1.265431 & 5.668740 & 0.902953 \\
\hline $\mathrm{C}$ & 2.954780 & -0.879716 & 0.195772 & $\mathrm{H}$ & -3.705823 & 5.856337 & 0.486782 \\
\hline $\mathrm{C}$ & 3.604782 & -0.634379 & -1.024054 & $\mathrm{O}$ & 0.705875 & 0.977978 & -2.105132 \\
\hline $\mathrm{C}$ & 3.469575 & -0.404977 & 1.414032 & $\mathrm{H}$ & 0.994001 & 1.078553 & 1.296004 \\
\hline $\mathrm{C}$ & 4.774742 & 0.127603 & -1.001112 & $\mathrm{C}$ & 0.695255 & 2.364949 & -2.164040 \\
\hline $\mathrm{C}$ & 4.638586 & 0.353063 & 1.383162 & $\mathrm{H}$ & -0.116649 & 2.762757 & -2.793857 \\
\hline $\mathrm{C}$ & 5.301307 & 0.635728 & 0.186803 & $\mathrm{H}$ & 0.532079 & 2.831701 & -1.148939 \\
\hline $\mathrm{H}$ & 5.290701 & 0.323414 & -1.940280 & $\mathrm{H}$ & 1.655454 & 2.779308 & -2.510244 \\
\hline $\mathrm{H}$ & 5.041630 & 0.734019 & 2.320799 & & & & \\
\hline $\mathrm{C}$ & -2.117805 & -2.437103 & 2.526257 & $\mathbf{T S}_{5-6}$ & & & \\
\hline $\mathrm{H}$ & -1.618553 & -3.407208 & 2.638900 & $\mathrm{Ru}$ & 0.452059 & 0.964794 & -0.316615 \\
\hline $\mathrm{H}$ & -1.370482 & -1.674077 & 2.770825 & $\mathrm{C}$ & 0.643497 & -1.106561 & 0.088997 \\
\hline $\mathrm{H}$ & -2.915090 & -2.392180 & 3.271779 & $\mathrm{C}$ & 0.441713 & -3.354957 & 0.783594 \\
\hline $\mathrm{C}$ & -1.307414 & -1.721390 & -2.398291 & $\mathrm{C}$ & 1.887796 & -2.914150 & 0.967435 \\
\hline $\mathrm{H}$ & -0.491696 & -2.451863 & -2.355224 & $\mathrm{H}$ & -0.006491 & -3.771912 & 1.691855 \\
\hline $\mathrm{H}$ & -1.831770 & -1.859502 & -3.347302 & $\mathrm{H}$ & 0.328471 & -4.101523 & -0.015771 \\
\hline
\end{tabular}




\begin{tabular}{|c|c|c|c|c|c|c|c|}
\hline $\mathrm{H}$ & 2.162131 & -2.814061 & 2.028071 & $\mathrm{C}$ & -1.395997 & 1.000763 & -0.459685 \\
\hline $\mathrm{H}$ & 2.612144 & -3.585906 & 0.495560 & $\mathrm{H}$ & -1.820076 & 0.137921 & -0.998878 \\
\hline $\mathrm{N}$ & -0.222079 & -2.098982 & 0.406279 & $\mathrm{C}$ & -2.428304 & 1.982653 & -0.155894 \\
\hline $\mathrm{N}$ & 1.888317 & -1.600379 & 0.316434 & $\mathrm{C}$ & -3.718270 & 1.793847 & -0.692917 \\
\hline $\mathrm{C}$ & -1.634995 & -2.043939 & 0.236377 & $\mathrm{C}$ & -2.227078 & 3.086073 & 0.694839 \\
\hline $\mathrm{C}$ & -2.195296 & -2.416641 & -0.991172 & $\mathrm{C}$ & -4.753628 & 2.675455 & -0.411651 \\
\hline $\mathrm{C}$ & -2.422182 & -1.568947 & 1.295122 & $\mathrm{H}$ & -3.889781 & 0.930047 & -1.334825 \\
\hline $\mathrm{C}$ & -3.579384 & -2.307815 & -1.141656 & $\mathrm{C}$ & -3.262799 & 3.964508 & 0.979226 \\
\hline $\mathrm{C}$ & -3.797855 & -1.470502 & 1.096971 & $\mathrm{H}$ & -1.243651 & 3.222843 & 1.136289 \\
\hline $\mathrm{C}$ & -4.394339 & -1.831637 & -0.113662 & $\mathrm{C}$ & -4.528393 & 3.767154 & 0.425156 \\
\hline $\mathrm{H}$ & -4.028923 & -2.582319 & -2.095347 & $\mathrm{H}$ & -5.737940 & 2.514293 & -0.844595 \\
\hline $\mathrm{H}$ & -4.418877 & -1.081259 & 1.902949 & $\mathrm{H}$ & -3.088662 & 4.807954 & 1.642596 \\
\hline $\mathrm{C}$ & 3.052513 & -0.772587 & 0.287541 & $\mathrm{H}$ & -5.336959 & 4.457372 & 0.651412 \\
\hline $\mathrm{C}$ & 3.787054 & -0.696887 & -0.906513 & $\mathrm{O}$ & 1.718964 & 1.278459 & -1.936720 \\
\hline $\mathrm{C}$ & 3.421852 & -0.046517 & 1.429092 & $\mathrm{H}$ & 0.341409 & 1.172704 & 1.277199 \\
\hline $\mathrm{C}$ & 4.909047 & 0.125426 & -0.936216 & $\mathrm{C}$ & 1.320505 & 2.483938 & -1.549208 \\
\hline $\mathrm{C}$ & 4.546128 & 0.779304 & 1.343602 & $\mathrm{H}$ & 0.612150 & 3.019390 & -2.200674 \\
\hline $\mathrm{C}$ & 5.298612 & 0.879008 & 0.174784 & $\mathrm{H}$ & 0.337806 & 2.593019 & -0.161825 \\
\hline $\mathrm{H}$ & 5.480596 & 0.200481 & -1.860342 & $\mathrm{H}$ & 2.079978 & 3.141996 & -1.099353 \\
\hline $\mathrm{H}$ & 4.838833 & 1.358903 & 2.218073 & & & & \\
\hline $\mathrm{C}$ & -1.783920 & -1.109298 & 2.566986 & 6 & & & \\
\hline $\mathrm{H}$ & -1.081156 & -1.848150 & 2.968456 & $\mathrm{Ru}$ & 0.641790 & 1.046880 & -0.295354 \\
\hline $\mathrm{H}$ & -1.204600 & -0.193768 & 2.389114 & $\mathrm{C}$ & 0.630776 & -0.970859 & 0.207927 \\
\hline $\mathrm{H}$ & -2.532886 & -0.898098 & 3.333629 & $\mathrm{C}$ & 0.194832 & -3.158257 & 0.897354 \\
\hline $\mathrm{C}$ & -1.318792 & -2.823913 & -2.135113 & $\mathrm{C}$ & 1.664627 & -2.841274 & 1.162953 \\
\hline $\mathrm{H}$ & -0.683399 & -3.683128 & -1.892637 & $\mathrm{H}$ & -0.330329 & -3.553903 & 1.771908 \\
\hline $\mathrm{H}$ & -1.911510 & -3.088143 & -3.013640 & $\mathrm{H}$ & 0.062348 & -3.872875 & 0.072085 \\
\hline $\mathrm{H}$ & -0.640829 & -2.008594 & -2.416593 & $\mathrm{H}$ & 1.877374 & -2.720350 & 2.235432 \\
\hline $\mathrm{C}$ & -5.871007 & -1.671392 & -0.309170 & $\mathrm{H}$ & 2.351802 & -3.592523 & 0.762350 \\
\hline $\mathrm{H}$ & -6.441000 & -2.156949 & 0.489308 & $\mathrm{~N}$ & -0.345186 & -1.843320 & 0.512106 \\
\hline $\mathrm{H}$ & -6.156584 & -0.612865 & -0.298095 & $\mathrm{~N}$ & 1.817324 & -1.559010 & 0.465740 \\
\hline $\mathrm{H}$ & -6.202410 & -2.092875 & -1.261491 & $\mathrm{C}$ & -1.714586 & -1.662204 & 0.154379 \\
\hline $\mathrm{C}$ & 3.325043 & -1.426700 & -2.126274 & $\mathrm{C}$ & -2.086560 & -1.749262 & -1.195320 \\
\hline $\mathrm{H}$ & 4.095267 & -1.429016 & -2.901188 & $\mathrm{C}$ & -2.637616 & -1.357207 & 1.160933 \\
\hline $\mathrm{H}$ & 3.048919 & -2.464133 & -1.909395 & $\mathrm{C}$ & -3.429427 & -1.549854 & -1.512319 \\
\hline $\mathrm{H}$ & 2.436262 & -0.932056 & -2.534541 & $\mathrm{C}$ & -3.969475 & -1.165753 & 0.794135 \\
\hline $\mathrm{C}$ & 2.666852 & -0.167640 & 2.718214 & $\mathrm{C}$ & -4.383967 & -1.260807 & -0.533558 \\
\hline $\mathrm{H}$ & 1.599314 & -0.340245 & 2.553833 & $\mathrm{H}$ & -3.734659 & -1.601948 & -2.556881 \\
\hline $\mathrm{H}$ & 3.050803 & -0.995481 & 3.328595 & $\mathrm{H}$ & -4.693223 & -0.895991 & 1.562045 \\
\hline $\mathrm{H}$ & 2.765552 & 0.742107 & 3.315430 & $\mathrm{C}$ & 3.020147 & -0.785686 & 0.415869 \\
\hline $\mathrm{C}$ & 6.493331 & 1.780408 & 0.101097 & $\mathrm{C}$ & 3.784139 & -0.821172 & -0.763984 \\
\hline $\mathrm{H}$ & 7.387797 & 1.238816 & -0.224046 & $\mathrm{C}$ & 3.383121 & 0.015711 & 1.510989 \\
\hline $\mathrm{H}$ & 6.338373 & 2.589341 & -0.620883 & $\mathrm{C}$ & 4.923550 & -0.022294 & -0.828053 \\
\hline $\mathrm{H}$ & 6.713735 & 2.239491 & 1.068159 & $\mathrm{C}$ & 4.529436 & 0.804848 & 1.390221 \\
\hline
\end{tabular}




\begin{tabular}{|c|c|c|c|c|c|c|c|}
\hline $\mathrm{C}$ & 5.305641 & 0.804665 & 0.232109 & $\mathrm{H}$ & 1.132567 & 2.470922 & 0.336997 \\
\hline $\mathrm{H}$ & 5.522259 & -0.036157 & -1.737682 & $\mathrm{H}$ & 2.791062 & 1.935783 & -1.688512 \\
\hline $\mathrm{H}$ & 4.817505 & 1.439713 & 2.226641 & & & & \\
\hline $\mathrm{C}$ & -2.185007 & -1.181056 & 2.576729 & 7 & & & \\
\hline $\mathrm{H}$ & -1.872653 & -2.127212 & 3.036463 & $\mathrm{Ru}$ & -0.719387 & -0.980917 & -0.721378 \\
\hline $\mathrm{H}$ & -1.323815 & -0.504227 & 2.626377 & $\mathrm{C}$ & -0.709852 & 0.831157 & 0.201883 \\
\hline $\mathrm{H}$ & -2.983113 & -0.762579 & 3.194999 & $\mathrm{C}$ & -0.306127 & 2.837525 & 1.349338 \\
\hline $\mathrm{C}$ & -1.064009 & -1.978243 & -2.265122 & $\mathrm{C}$ & -1.747759 & 2.410259 & 1.603760 \\
\hline $\mathrm{H}$ & -0.352769 & -2.769441 & -2.000927 & $\mathrm{H}$ & 0.244757 & 3.068823 & 2.266599 \\
\hline $\mathrm{H}$ & -1.538905 & -2.254300 & -3.209789 & $\mathrm{H}$ & -0.243713 & 3.716436 & 0.689032 \\
\hline $\mathrm{H}$ & -0.466065 & -1.073082 & -2.438239 & $\mathrm{H}$ & -1.888333 & 2.031909 & 2.628079 \\
\hline $\mathrm{C}$ & -5.818542 & -1.044766 & -0.905352 & $\mathrm{H}$ & -2.473625 & 3.211748 & 1.434834 \\
\hline $\mathrm{H}$ & -6.345087 & -1.995491 & -1.050187 & $\mathrm{~N}$ & 0.254890 & 1.657372 & 0.677663 \\
\hline $\mathrm{H}$ & -6.352874 & -0.490065 & -0.129124 & $\mathrm{~N}$ & -1.903411 & 1.336785 & 0.622106 \\
\hline $\mathrm{H}$ & -5.910310 & -0.485026 & -1.841579 & $\mathrm{C}$ & 1.631418 & 1.565938 & 0.324703 \\
\hline $\mathrm{C}$ & 3.351570 & -1.653232 & -1.928412 & $\mathrm{C}$ & 2.037285 & 1.897248 & -0.977666 \\
\hline $\mathrm{H}$ & 4.163521 & -1.774337 & -2.649329 & $\mathrm{C}$ & 2.542012 & 1.097863 & 1.279339 \\
\hline $\mathrm{H}$ & 3.010602 & -2.647084 & -1.620024 & $\mathrm{C}$ & 3.382470 & 1.744091 & -1.305680 \\
\hline $\mathrm{H}$ & 2.511751 & -1.160029 & -2.432765 & $\mathrm{C}$ & 3.880186 & 0.966052 & 0.906948 \\
\hline $\mathrm{C}$ & 2.606957 & 0.002660 & 2.792541 & $\mathrm{C}$ & 4.317870 & 1.276213 & -0.379058 \\
\hline $\mathrm{H}$ & 1.544822 & -0.195548 & 2.628820 & $\mathrm{H}$ & 3.706319 & 1.981187 & -2.319059 \\
\hline $\mathrm{H}$ & 2.994376 & -0.759695 & 3.480552 & $\mathrm{H}$ & 4.590244 & 0.564426 & 1.628254 \\
\hline $\mathrm{H}$ & 2.683806 & 0.966262 & 3.301547 & $\mathrm{C}$ & -3.098622 & 0.599483 & 0.368152 \\
\hline $\mathrm{C}$ & 6.506709 & 1.690864 & 0.110024 & $\mathrm{C}$ & -3.729504 & 0.777702 & -0.874867 \\
\hline $\mathrm{H}$ & 7.334966 & 1.183212 & -0.392884 & $\mathrm{C}$ & -3.585798 & -0.319581 & 1.312143 \\
\hline $\mathrm{H}$ & 6.277100 & 2.583390 & -0.482940 & $\mathrm{C}$ & -4.868457 & 0.023106 & -1.152538 \\
\hline $\mathrm{H}$ & 6.859790 & 2.033038 & 1.086011 & $\mathrm{C}$ & -4.730541 & -1.047955 & 0.989339 \\
\hline $\mathrm{C}$ & -1.056722 & 1.513981 & -0.797504 & $\mathrm{C}$ & -5.381294 & -0.896286 & -0.235790 \\
\hline $\mathrm{H}$ & -1.155395 & 1.496711 & -1.900982 & $\mathrm{H}$ & -5.365108 & 0.153843 & -2.112790 \\
\hline $\mathrm{C}$ & -2.305642 & 1.838686 & -0.122752 & $\mathrm{H}$ & -5.111954 & -1.769890 & 1.709959 \\
\hline $\mathrm{C}$ & -3.464386 & 1.969785 & -0.913417 & $\mathrm{C}$ & 2.075073 & 0.702642 & 2.645296 \\
\hline $\mathrm{C}$ & -2.438699 & 2.019686 & 1.266633 & $\mathrm{H}$ & 1.868338 & 1.574386 & 3.279273 \\
\hline $\mathrm{C}$ & -4.700526 & 2.251219 & -0.345541 & $\mathrm{H}$ & 1.147438 & 0.122424 & 2.588285 \\
\hline $\mathrm{H}$ & -3.376495 & 1.833194 & -1.990193 & $\mathrm{H}$ & 2.827668 & 0.098344 & 3.157734 \\
\hline $\mathrm{C}$ & -3.673244 & 2.297052 & 1.834238 & $\mathrm{C}$ & 1.044299 & 2.347479 & -2.003284 \\
\hline $\mathrm{H}$ & -1.547751 & 1.939816 & 1.883526 & $\mathrm{H}$ & 0.337258 & 3.083813 & -1.605804 \\
\hline $\mathrm{C}$ & -4.811854 & 2.409976 & 1.033789 & $\mathrm{H}$ & 1.542330 & 2.786603 & -2.871223 \\
\hline $\mathrm{H}$ & -5.579940 & 2.341614 & -0.978624 & $\mathrm{H}$ & 0.438744 & 1.501233 & -2.354248 \\
\hline $\mathrm{H}$ & -3.752360 & 2.431572 & 2.910532 & $\mathrm{C}$ & 5.752039 & 1.093498 & -0.769609 \\
\hline $\mathrm{H}$ & -5.777390 & 2.627788 & 1.483565 & $\mathrm{H}$ & 6.270381 & 2.053902 & -0.872417 \\
\hline $\mathrm{O}$ & 1.234964 & 0.749228 & -2.392050 & $\mathrm{H}$ & 6.295646 & 0.501621 & -0.028303 \\
\hline $\mathrm{H}$ & 0.182600 & 1.190286 & 1.235038 & $\mathrm{H}$ & 5.839552 & 0.580988 & -1.733534 \\
\hline $\mathrm{C}$ & 1.717721 & 1.872020 & -1.955397 & $\mathrm{C}$ & -3.182361 & 1.749583 & -1.875023 \\
\hline $\mathrm{H}$ & 1.277642 & 2.822982 & -2.291723 & $\mathrm{H}$ & -3.876305 & 1.893873 & -2.706172 \\
\hline
\end{tabular}




\begin{tabular}{|c|c|c|c|c|c|c|c|}
\hline $\mathrm{H}$ & -2.973501 & 2.724976 & -1.422442 & $\mathrm{C}$ & 2.132369 & -2.034653 & 0.044916 \\
\hline $\mathrm{H}$ & -2.229905 & 1.396469 & -2.289556 & $\mathrm{C}$ & 2.943241 & -1.857671 & -1.091426 \\
\hline $\mathrm{C}$ & -2.890636 & -0.519607 & 2.622773 & $\mathrm{C}$ & 2.668889 & -1.966746 & 1.343814 \\
\hline $\mathrm{H}$ & -1.802874 & -0.480959 & 2.501614 & $\mathrm{C}$ & 4.290072 & -1.544935 & -0.898775 \\
\hline $\mathrm{H}$ & -3.172013 & 0.246579 & 3.356355 & $\mathrm{C}$ & 4.021748 & -1.660189 & 1.483426 \\
\hline $\mathrm{H}$ & -3.145304 & -1.489791 & 3.055168 & $\mathrm{C}$ & 4.842003 & -1.425225 & 0.377525 \\
\hline $\mathrm{C}$ & -6.579519 & -1.729318 & -0.573078 & $\mathrm{H}$ & 4.924598 & -1.397940 & -1.771858 \\
\hline $\mathrm{H}$ & -7.216468 & -1.241709 & -1.315916 & $\mathrm{H}$ & 4.443380 & -1.589327 & 2.485710 \\
\hline $\mathrm{H}$ & -6.278371 & -2.695995 & -0.992275 & $\mathrm{C}$ & -3.638198 & -2.036945 & 1.480688 \\
\hline $\mathrm{H}$ & -7.188176 & -1.941557 & 0.310329 & $\mathrm{H}$ & -3.875912 & -3.091606 & 1.287110 \\
\hline $\mathrm{C}$ & 0.989133 & -1.361352 & -1.197175 & $\mathrm{H}$ & -2.642856 & -2.004133 & 1.934407 \\
\hline $\mathrm{H}$ & 1.149808 & -1.125033 & -2.277009 & $\mathrm{H}$ & -4.360737 & -1.675224 & 2.216606 \\
\hline $\mathrm{C}$ & 2.192556 & -1.827278 & -0.523092 & $\mathrm{C}$ & -1.595525 & -0.524663 & -2.892246 \\
\hline $\mathrm{C}$ & 3.413347 & -1.863463 & -1.221893 & $\mathrm{H}$ & -1.008271 & -1.447294 & -2.828830 \\
\hline $\mathrm{C}$ & 2.197472 & -2.224822 & 0.827626 & $\mathrm{H}$ & -1.996603 & -0.440330 & -3.906466 \\
\hline $\mathrm{C}$ & 4.588984 & -2.266736 & -0.599028 & $\mathrm{H}$ & -0.878047 & 0.287164 & -2.729956 \\
\hline $\mathrm{H}$ & 3.426883 & -1.554797 & -2.266530 & $\mathrm{C}$ & -6.238920 & 0.904178 & -1.649804 \\
\hline $\mathrm{C}$ & 3.371341 & -2.621770 & 1.449835 & $\mathrm{H}$ & -6.362165 & 1.040792 & -2.727918 \\
\hline $\mathrm{H}$ & 1.253066 & -2.209472 & 1.366796 & $\mathrm{H}$ & -7.133196 & 0.403695 & -1.266594 \\
\hline $\mathrm{C}$ & 4.574941 & -2.642582 & 0.742154 & $\mathrm{H}$ & -6.218891 & 1.904816 & -1.200277 \\
\hline $\mathrm{H}$ & 5.520025 & -2.284060 & -1.160956 & $\mathrm{C}$ & 2.379933 & -2.037447 & -2.467504 \\
\hline $\mathrm{H}$ & 3.351889 & -2.922687 & 2.494720 & $\mathrm{H}$ & 3.158964 & -1.928751 & -3.225817 \\
\hline $\mathrm{H}$ & 5.492959 & -2.957383 & 1.232001 & $\mathrm{H}$ & 1.925604 & -3.027596 & -2.587192 \\
\hline $\mathrm{H}$ & -0.706433 & -1.607661 & 0.713407 & $\mathrm{H}$ & 1.598188 & -1.297491 & -2.668667 \\
\hline \multirow[t]{2}{*}{$\mathrm{H}$} & -1.518276 & -2.405876 & -1.055001 & $\mathrm{C}$ & 1.807272 & -2.229581 & 2.538499 \\
\hline & & & & $\mathrm{H}$ & 0.810274 & -1.798913 & 2.404435 \\
\hline \multicolumn{2}{|c|}{ 7-py } & & & $\mathrm{H}$ & 1.690339 & -3.306305 & 2.717112 \\
\hline $\mathrm{C}$ & -0.143860 & -1.217631 & -0.152233 & $\mathrm{H}$ & 2.242562 & -1.795336 & 3.441709 \\
\hline $\mathrm{C}$ & -1.304312 & -3.244296 & -0.484752 & $\mathrm{C}$ & 6.275009 & -1.027803 & 0.558460 \\
\hline $\mathrm{C}$ & 0.098476 & -3.574870 & -0.009952 & $\mathrm{H}$ & 6.891741 & -1.332290 & -0.291526 \\
\hline $\mathrm{H}$ & -2.087456 & -3.771654 & 0.068323 & $\mathrm{H}$ & 6.373642 & 0.061238 & 0.647936 \\
\hline $\mathrm{H}$ & -1.438472 & -3.465039 & -1.555975 & $\mathrm{H}$ & 6.706864 & -1.461002 & 1.465130 \\
\hline $\mathrm{H}$ & 0.110074 & -3.917532 & 1.038150 & $\mathrm{C}$ & -0.629681 & 1.710684 & 0.280755 \\
\hline $\mathrm{H}$ & 0.593164 & -4.337975 & -0.620366 & $\mathrm{H}$ & -0.447393 & 2.752170 & -0.058684 \\
\hline $\mathrm{N}$ & -1.386752 & -1.792563 & -0.264647 & $\mathrm{C}$ & -1.871853 & 1.597929 & 1.041470 \\
\hline $\mathrm{N}$ & 0.741446 & -2.276428 & -0.148317 & $\mathrm{C}$ & -2.977569 & 2.411822 & 0.731168 \\
\hline $\mathrm{C}$ & -2.593890 & -1.135695 & -0.639740 & $\mathrm{C}$ & -1.960953 & 0.770133 & 2.174364 \\
\hline $\mathrm{C}$ & -2.701778 & -0.497580 & -1.886910 & $\mathrm{C}$ & -4.127338 & 2.387241 & 1.510271 \\
\hline $\mathrm{C}$ & -3.700083 & -1.226493 & 0.222509 & $\mathrm{H}$ & -2.923260 & 3.062854 & -0.139997 \\
\hline $\mathrm{C}$ & -3.901696 & 0.147284 & -2.194046 & $\mathrm{C}$ & -3.100982 & 0.768193 & 2.967703 \\
\hline $\mathrm{C}$ & -4.876247 & -0.568905 & -0.130845 & $\mathrm{H}$ & -1.092003 & 0.164378 & 2.425913 \\
\hline $\mathrm{C}$ & -4.989685 & 0.145816 & -1.324068 & $\mathrm{C}$ & -4.192588 & 1.570142 & 2.638266 \\
\hline $\mathrm{H}$ & -3.989350 & 0.652732 & -3.155723 & $\mathrm{H}$ & -4.972556 & 3.019234 & 1.247144 \\
\hline $\mathrm{H}$ & -5.723051 & -0.606245 & 0.553908 & $\mathrm{H}$ & -3.139187 & 0.135944 & 3.852140 \\
\hline
\end{tabular}




\begin{tabular}{|c|c|c|c|c|c|c|c|}
\hline $\mathrm{H}$ & -5.082723 & 1.568536 & 3.262164 & $\mathrm{H}$ & -1.097068 & -1.909441 & 2.634947 \\
\hline $\mathrm{Ru}$ & 0.743053 & 0.548643 & -0.086492 & $\mathrm{H}$ & -2.691997 & -2.231358 & 3.342060 \\
\hline $\mathrm{H}$ & 1.217230 & 0.373804 & 1.518191 & $\mathrm{H}$ & -1.712649 & -3.557507 & 2.723828 \\
\hline $\mathrm{H}$ & 0.960420 & 0.513475 & -1.762459 & $\mathrm{C}$ & -1.552224 & -2.030453 & -2.449076 \\
\hline $\mathrm{C}$ & 2.019723 & 3.219174 & -1.111351 & $\mathrm{H}$ & -2.155895 & -1.973651 & -3.357972 \\
\hline $\mathrm{C}$ & 3.267121 & 2.265859 & 0.575755 & $\mathrm{H}$ & -0.889473 & -1.155035 & -2.416234 \\
\hline $\mathrm{C}$ & 2.961441 & 4.221413 & -1.301041 & $\mathrm{H}$ & -0.904019 & -2.911871 & -2.523867 \\
\hline $\mathrm{H}$ & 1.116650 & 3.161209 & -1.713018 & $\mathrm{C}$ & -6.070910 & -1.598462 & -0.281047 \\
\hline $\mathrm{C}$ & 4.259923 & 3.226955 & 0.442441 & $\mathrm{H}$ & -6.492427 & -1.986413 & -1.212752 \\
\hline $\mathrm{H}$ & 3.329146 & 1.469755 & 1.314937 & $\mathrm{H}$ & -6.632500 & -2.030952 & 0.551485 \\
\hline $\mathrm{C}$ & 4.107945 & 4.226282 & -0.513893 & $\mathrm{H}$ & -6.261559 & -0.518627 & -0.273083 \\
\hline $\mathrm{H}$ & 2.795480 & 4.979492 & -2.059592 & $\mathrm{C}$ & 2.558250 & -1.525542 & -2.495124 \\
\hline $\mathrm{H}$ & 5.131331 & 3.192442 & 1.088661 & $\mathrm{H}$ & 2.598877 & -0.838283 & -3.343945 \\
\hline $\mathrm{H}$ & 4.865627 & 4.993967 & -0.640759 & $\mathrm{H}$ & 3.030174 & -2.464126 & -2.813699 \\
\hline \multirow[t]{2}{*}{$\mathrm{N}$} & 2.161378 & 2.246606 & -0.191753 & $\mathrm{H}$ & 1.504193 & -1.723932 & -2.289484 \\
\hline & & & & $\mathrm{C}$ & 3.219287 & -1.176825 & 2.503949 \\
\hline 7-A & & & & $\mathrm{H}$ & 2.171864 & -0.917163 & 2.686066 \\
\hline $\mathrm{Ru}$ & 0.494476 & 0.500956 & -0.049404 & $\mathrm{H}$ & 3.317759 & -2.256256 & 2.666833 \\
\hline $\mathrm{C}$ & 0.475149 & -1.424629 & 0.147988 & $\mathrm{H}$ & 3.838179 & -0.668905 & 3.247586 \\
\hline $\mathrm{C}$ & 0.180282 & -3.752241 & 0.357555 & $\mathrm{C}$ & 6.273405 & 1.297351 & -0.640481 \\
\hline $\mathrm{C}$ & 1.641585 & -3.476707 & 0.035367 & $\mathrm{H}$ & 5.945962 & 2.343511 & -0.592478 \\
\hline $\mathrm{H}$ & 0.048740 & -4.075082 & 1.401530 & $\mathrm{H}$ & 7.050967 & 1.175329 & 0.118679 \\
\hline $\mathrm{H}$ & -0.273870 & -4.508496 & -0.290562 & $\mathrm{H}$ & 6.730422 & 1.151475 & -1.622820 \\
\hline $\mathrm{H}$ & 2.336222 & -4.019098 & 0.685011 & $\mathrm{C}$ & -1.311494 & 0.794159 & 0.151041 \\
\hline $\mathrm{H}$ & 1.884255 & -3.728912 & -1.010238 & $\mathrm{H}$ & -1.906789 & 0.077232 & 0.736155 \\
\hline $\mathrm{N}$ & -0.437357 & -2.442371 & 0.135462 & $\mathrm{C}$ & -2.107095 & 1.943996 & -0.275069 \\
\hline $\mathrm{N}$ & 1.710354 & -2.036514 & 0.262462 & $\mathrm{C}$ & -3.298701 & 2.244912 & 0.414641 \\
\hline $\mathrm{C}$ & -1.848258 & -2.292705 & 0.036974 & $\mathrm{C}$ & -1.755618 & 2.754745 & -1.370063 \\
\hline $\mathrm{C}$ & -2.414082 & -2.078100 & -1.228725 & $\mathrm{C}$ & -4.076952 & 3.339774 & 0.061943 \\
\hline $\mathrm{C}$ & -2.636877 & -2.332622 & 1.195537 & $\mathrm{H}$ & -3.594757 & 1.607275 & 1.247415 \\
\hline $\mathrm{C}$ & -3.790551 & -1.871141 & -1.307163 & C & -2.540531 & 3.842586 & -1.727878 \\
\hline $\mathrm{C}$ & -4.011565 & -2.130700 & 1.066601 & $\mathrm{H}$ & -0.862432 & 2.496734 & -1.931070 \\
\hline $\mathrm{C}$ & -4.604413 & -1.884135 & -0.172889 & $\mathrm{C}$ & -3.697151 & 4.146875 & -1.009630 \\
\hline $\mathrm{H}$ & -4.238546 & -1.688824 & -2.283070 & $\mathrm{H}$ & -4.984934 & 3.562216 & 0.617112 \\
\hline $\mathrm{H}$ & -4.631382 & -2.141966 & 1.962433 & $\mathrm{H}$ & -2.256143 & 4.454644 & -2.579994 \\
\hline $\mathrm{C}$ & 2.878404 & -1.253024 & 0.010596 & $\mathrm{H}$ & -4.308782 & 4.998904 & -1.294294 \\
\hline $\mathrm{C}$ & 3.269051 & -0.951106 & -1.307165 & $\mathrm{H}$ & 1.098447 & 0.760333 & 1.553478 \\
\hline $\mathrm{C}$ & 3.621685 & -0.794157 & 1.115383 & $\mathrm{O}$ & 0.995942 & 2.764511 & -0.347181 \\
\hline $\mathrm{C}$ & 4.390843 & -0.136982 & -1.490311 & $\mathrm{H}$ & 0.815343 & 2.222465 & 2.251485 \\
\hline $\mathrm{C}$ & 4.732100 & 0.012534 & 0.878948 & $\mathrm{C}$ & 2.407627 & 2.848135 & -0.477351 \\
\hline $\mathrm{C}$ & 5.122714 & 0.365466 & -0.416209 & $\mathrm{H}$ & 2.926184 & 2.286181 & 0.315238 \\
\hline $\mathrm{H}$ & 4.693105 & 0.113804 & -2.506670 & $\mathrm{H}$ & 2.671068 & 2.405806 & -1.441746 \\
\hline $\mathrm{H}$ & 5.303547 & 0.383311 & 1.729001 & $\mathrm{H}$ & 2.745019 & 3.891408 & -0.457916 \\
\hline $\mathrm{C}$ & -2.005200 & -2.516022 & 2.541274 & $\mathrm{O}$ & 0.581202 & 3.176115 & 2.293070 \\
\hline
\end{tabular}




\begin{tabular}{|c|c|c|c|c|c|c|c|}
\hline $\mathrm{H}$ & 0.756990 & 3.143029 & 0.530147 & $\mathrm{H}$ & -6.385093 & 0.196385 & 1.753603 \\
\hline $\mathrm{C}$ & -0.739610 & 3.257470 & 2.797192 & $\mathrm{H}$ & -6.541226 & 0.294610 & -0.006228 \\
\hline $\mathrm{H}$ & -1.334928 & 2.378485 & 2.517673 & $\mathrm{C}$ & 2.054484 & -1.539113 & -2.739579 \\
\hline $\mathrm{H}$ & -0.744538 & 3.354926 & 3.890917 & $\mathrm{H}$ & 2.248883 & -0.919453 & -3.618611 \\
\hline $\mathrm{H}$ & -1.223467 & 4.139255 & 2.366511 & $\mathrm{H}$ & 2.212419 & -2.583392 & -3.038717 \\
\hline \multirow[t]{2}{*}{$\mathrm{H}$} & 0.590860 & 0.410005 & -1.713343 & $\mathrm{H}$ & 0.999004 & -1.419157 & -2.477365 \\
\hline & & & & $\mathrm{C}$ & 3.113238 & -1.381432 & 2.224277 \\
\hline \multicolumn{2}{|c|}{ TSA $_{7-5}$} & & & $\mathrm{H}$ & 2.081628 & -1.722542 & 2.334576 \\
\hline $\mathrm{Ru}$ & 0.614091 & 0.683891 & 0.106695 & $\mathrm{H}$ & 3.776400 & -2.159357 & 2.620976 \\
\hline $\mathrm{C}$ & 0.240110 & -1.235364 & 0.115049 & $\mathrm{H}$ & 3.242778 & -0.489363 & 2.843655 \\
\hline $\mathrm{C}$ & -0.450660 & -3.468960 & 0.235679 & $\mathrm{C}$ & 6.296664 & 0.601580 & -1.108095 \\
\hline $\mathrm{C}$ & 1.001173 & -3.433629 & -0.228183 & $\mathrm{H}$ & 6.595627 & 1.249223 & -0.279525 \\
\hline $\mathrm{H}$ & -0.546415 & -3.846381 & 1.265023 & $\mathrm{H}$ & 7.117247 & -0.105948 & -1.275184 \\
\hline $\mathrm{H}$ & -1.096792 & -4.076859 & -0.405453 & $\mathrm{H}$ & 6.219467 & 1.216974 & -2.009353 \\
\hline $\mathrm{H}$ & 1.651703 & -4.105758 & 0.340128 & $\mathrm{C}$ & -1.106102 & 1.259644 & 0.453681 \\
\hline $\mathrm{H}$ & 1.097899 & -3.684186 & -1.296634 & $\mathrm{H}$ & -1.344959 & 1.390475 & 1.526911 \\
\hline $\mathrm{N}$ & -0.836456 & -2.054823 & 0.173203 & $\mathrm{C}$ & -2.226585 & 1.704693 & -0.369575 \\
\hline $\mathrm{N}$ & 1.345742 & -2.036248 & 0.016879 & $\mathrm{C}$ & -3.382311 & 2.173843 & 0.287547 \\
\hline $\mathrm{C}$ & -2.184381 & -1.639855 & 0.354539 & $\mathrm{C}$ & -2.242315 & 1.681194 & -1.777312 \\
\hline $\mathrm{C}$ & -2.996715 & -1.517966 & -0.780445 & $\mathrm{C}$ & -4.503465 & 2.587072 & -0.421310 \\
\hline $\mathrm{C}$ & -2.656432 & -1.347164 & 1.640040 & $\mathrm{H}$ & -3.386431 & 2.195186 & 1.376513 \\
\hline $\mathrm{C}$ & -4.316869 & -1.112381 & -0.603293 & $\mathrm{C}$ & -3.364253 & 2.084669 & -2.485809 \\
\hline $\mathrm{C}$ & -3.984977 & -0.939428 & 1.769333 & $\mathrm{H}$ & -1.348912 & 1.335729 & -2.290664 \\
\hline $\mathrm{C}$ & -4.827870 & -0.818738 & 0.663012 & $\mathrm{C}$ & -4.501484 & 2.537707 & -1.813353 \\
\hline $\mathrm{H}$ & -4.952928 & -0.985646 & -1.478569 & $\mathrm{H}$ & -5.381850 & 2.942325 & 0.112517 \\
\hline $\mathrm{H}$ & -4.364170 & -0.687221 & 2.759277 & $\mathrm{H}$ & -3.355507 & 2.052135 & -3.572749 \\
\hline $\mathrm{C}$ & 2.609626 & -1.434114 & -0.268687 & $\mathrm{H}$ & -5.377023 & 2.856093 & -2.373541 \\
\hline $\mathrm{C}$ & 2.953200 & -1.149198 & -1.606201 & $\mathrm{H}$ & 0.974685 & 0.721975 & 1.976774 \\
\hline $\mathrm{C}$ & 3.452497 & -1.074544 & 0.798034 & $\mathrm{O}$ & 2.081874 & 2.207236 & -0.029437 \\
\hline $\mathrm{C}$ & 4.158147 & -0.496335 & -1.851432 & $\mathrm{H}$ & 1.789746 & 1.226939 & 2.130935 \\
\hline $\mathrm{C}$ & 4.642132 & -0.405833 & 0.496036 & $\mathrm{C}$ & 2.737659 & 2.768005 & -1.141173 \\
\hline $\mathrm{C}$ & 5.011868 & -0.110915 & -0.814163 & $\mathrm{H}$ & 3.823398 & 2.774099 & -0.981288 \\
\hline $\mathrm{H}$ & 4.424526 & -0.255917 & -2.880224 & $\mathrm{H}$ & 2.523327 & 2.168351 & -2.031702 \\
\hline $\mathrm{H}$ & 5.281929 & -0.088385 & 1.317669 & $\mathrm{H}$ & 2.402120 & 3.798158 & -1.319660 \\
\hline $\mathrm{C}$ & -1.735080 & -1.413378 & 2.817948 & $\mathrm{O}$ & 2.794851 & 2.001696 & 2.265663 \\
\hline $\mathrm{H}$ & -0.880393 & -0.736001 & 2.685784 & $\mathrm{H}$ & 2.568422 & 2.247439 & 0.946144 \\
\hline $\mathrm{H}$ & -2.251356 & -1.137948 & 3.740679 & $\mathrm{C}$ & 2.316735 & 3.090031 & 3.008976 \\
\hline $\mathrm{H}$ & -1.316107 & -2.416732 & 2.958959 & $\mathrm{H}$ & 1.211526 & 3.122664 & 3.024279 \\
\hline $\mathrm{C}$ & -2.412298 & -1.736424 & -2.140288 & $\mathrm{H}$ & 2.659920 & 3.043374 & 4.051834 \\
\hline $\mathrm{H}$ & -3.146036 & -1.535419 & -2.924718 & $\mathrm{H}$ & 2.661708 & 4.047173 & 2.590058 \\
\hline $\mathrm{H}$ & -1.554886 & -1.068377 & -2.291624 & $\mathrm{H}$ & 0.342355 & 0.722944 & -1.493945 \\
\hline $\mathrm{H}$ & -2.040102 & -2.759340 & -2.277558 & & & & \\
\hline $\mathrm{C}$ & -6.244015 & -0.357675 & 0.820962 & 5-A & & & \\
\hline $\mathrm{H}$ & -6.945654 & -1.199995 & 0.834785 & $\mathrm{Ru}$ & 0.646219 & 0.648426 & 0.186177 \\
\hline
\end{tabular}




\begin{tabular}{|c|c|c|c|c|c|c|c|}
\hline $\mathrm{C}$ & 0.189743 & -1.298164 & 0.107304 & $\mathrm{H}$ & 3.233140 & -0.777875 & 2.851322 \\
\hline $\mathrm{C}$ & -0.596111 & -3.500635 & -0.002371 & $\mathrm{C}$ & 6.109666 & 0.755095 & -0.975154 \\
\hline $\mathrm{C}$ & 0.871710 & -3.485852 & -0.420385 & $\mathrm{H}$ & 6.042798 & 1.669412 & -0.374955 \\
\hline $\mathrm{H}$ & -0.747029 & -3.989277 & 0.971472 & $\mathrm{H}$ & 7.027461 & 0.243057 & -0.666728 \\
\hline $\mathrm{H}$ & -1.249160 & -3.995276 & -0.728374 & $\mathrm{H}$ & 6.223313 & 1.047243 & -2.022327 \\
\hline $\mathrm{H}$ & 1.479858 & -4.221638 & 0.114849 & $\mathrm{C}$ & -1.004210 & 1.369436 & 0.630813 \\
\hline $\mathrm{H}$ & 0.996050 & -3.660172 & -1.499818 & $\mathrm{H}$ & -1.134383 & 1.600142 & 1.706698 \\
\hline $\mathrm{N}$ & -0.917331 & -2.069760 & 0.093804 & $\mathrm{C}$ & -2.161508 & 1.827687 & -0.128493 \\
\hline $\mathrm{N}$ & 1.261512 & -2.122241 & -0.065739 & $\mathrm{C}$ & -3.297088 & 2.261037 & 0.586533 \\
\hline $\mathrm{C}$ & -2.254622 & -1.615025 & 0.274352 & $\mathrm{C}$ & -2.232552 & 1.861738 & -1.535271 \\
\hline $\mathrm{C}$ & -3.022673 & -1.341813 & -0.863642 & $\mathrm{C}$ & -4.451926 & 2.677231 & -0.063269 \\
\hline $\mathrm{C}$ & -2.760147 & -1.440920 & 1.567941 & $\mathrm{H}$ & -3.259804 & 2.249469 & 1.675048 \\
\hline $\mathrm{C}$ & -4.331788 & -0.900171 & -0.682208 & $\mathrm{C}$ & -3.385600 & 2.276845 & -2.185310 \\
\hline $\mathrm{C}$ & -4.075920 & -0.998648 & 1.702368 & $\mathrm{H}$ & -1.356529 & 1.558841 & -2.102736 \\
\hline $\mathrm{C}$ & -4.876749 & -0.727066 & 0.591000 & $\mathrm{C}$ & -4.504921 & 2.681472 & -1.455295 \\
\hline $\mathrm{H}$ & -4.928061 & -0.647177 & -1.557624 & $\mathrm{H}$ & -5.314054 & 2.997069 & 0.517402 \\
\hline $\mathrm{H}$ & -4.479982 & -0.843868 & 2.702284 & $\mathrm{H}$ & -3.413376 & 2.292860 & -3.272438 \\
\hline $\mathrm{C}$ & 2.538044 & -1.523971 & -0.316853 & $\mathrm{H}$ & -5.405519 & 3.008520 & -1.969099 \\
\hline $\mathrm{C}$ & 2.871709 & -1.138578 & -1.629341 & $\mathrm{H}$ & 0.891321 & 0.386114 & 2.112144 \\
\hline $\mathrm{C}$ & 3.379796 & -1.228553 & 0.771074 & $\mathrm{O}$ & 2.129060 & 1.954988 & -0.178118 \\
\hline $\mathrm{C}$ & 4.058628 & -0.436779 & -1.825620 & $\mathrm{H}$ & 1.455477 & 0.934415 & 2.007479 \\
\hline $\mathrm{C}$ & 4.553135 & -0.519894 & 0.518797 & $\mathrm{C}$ & 2.306393 & 2.562127 & -1.422713 \\
\hline $\mathrm{C}$ & 4.896892 & -0.096209 & -0.763560 & $\mathrm{H}$ & 3.263324 & 3.109622 & -1.434359 \\
\hline $\mathrm{H}$ & 4.309556 & -0.103278 & -2.832024 & $\mathrm{H}$ & 2.337058 & 1.830271 & -2.247263 \\
\hline $\mathrm{H}$ & 5.177400 & -0.232292 & 1.361379 & $\mathrm{H}$ & 1.506341 & 3.285122 & -1.647658 \\
\hline $\mathrm{C}$ & -1.885193 & -1.674947 & 2.758979 & $\mathrm{O}$ & 3.607311 & 1.917875 & 2.063377 \\
\hline $\mathrm{H}$ & -1.012878 & -1.009347 & 2.735769 & $\mathrm{H}$ & 3.216319 & 1.929601 & 1.159167 \\
\hline $\mathrm{H}$ & -2.424990 & -1.496060 & 3.691663 & $\mathrm{C}$ & 3.198833 & 3.109373 & 2.682869 \\
\hline $\mathrm{H}$ & -1.493177 & -2.698315 & 2.791422 & $\mathrm{H}$ & 2.103197 & 3.182472 & 2.791687 \\
\hline $\mathrm{C}$ & -2.414023 & -1.458622 & -2.225820 & $\mathrm{H}$ & 3.636613 & 3.141859 & 3.685533 \\
\hline $\mathrm{H}$ & -3.108880 & -1.122985 & -2.999459 & $\mathrm{H}$ & 3.533218 & 4.008875 & 2.142654 \\
\hline $\mathrm{H}$ & -1.506325 & -0.845955 & -2.293068 & $\mathrm{H}$ & 0.238943 & 0.693483 & -1.348218 \\
\hline $\mathrm{H}$ & -2.117625 & -2.488323 & -2.462626 & & & & \\
\hline $\mathrm{C}$ & -6.284356 & -0.244104 & 0.758136 & 7-B & & & \\
\hline $\mathrm{H}$ & -7.000014 & -1.074781 & 0.749716 & $\mathrm{Ru}$ & -0.676782 & 0.606493 & -0.298182 \\
\hline $\mathrm{H}$ & -6.417667 & 0.285440 & 1.706124 & $\mathrm{C}$ & -0.268625 & -1.309394 & 0.131788 \\
\hline $\mathrm{H}$ & -6.569036 & 0.436007 & -0.050347 & $\mathrm{C}$ & 0.479919 & -3.506187 & 0.445300 \\
\hline $\mathrm{C}$ & 1.973367 & -1.450344 & -2.787099 & $\mathrm{C}$ & -0.956892 & -3.548354 & -0.066367 \\
\hline $\mathrm{H}$ & 2.174010 & -0.777451 & -3.624649 & $\mathrm{H}$ & 1.159154 & -4.149660 & -0.122634 \\
\hline $\mathrm{H}$ & 2.119417 & -2.474419 & -3.154126 & $\mathrm{H}$ & 0.548947 & -3.793734 & 1.505801 \\
\hline $\mathrm{H}$ & 0.917135 & -1.339777 & -2.521032 & $\mathrm{H}$ & -1.007284 & -3.861738 & -1.121558 \\
\hline $\mathrm{C}$ & 3.038075 & -1.617085 & 2.177093 & $\mathrm{H}$ & -1.602899 & -4.211876 & 0.516840 \\
\hline $\mathrm{H}$ & 1.989932 & -1.910007 & 2.280495 & $\mathrm{~N}$ & 0.831452 & -2.088841 & 0.277552 \\
\hline $\mathrm{H}$ & 3.653091 & -2.459242 & 2.515185 & $\mathrm{~N}$ & -1.345332 & -2.148888 & 0.089863 \\
\hline
\end{tabular}




\begin{tabular}{|c|c|c|c|c|c|c|c|}
\hline $\mathrm{C}$ & 2.135073 & -1.583362 & 0.540115 & $\mathrm{C}$ & 2.369336 & 1.198490 & -2.042597 \\
\hline $\mathrm{C}$ & 2.408331 & -0.980321 & 1.778175 & $\mathrm{C}$ & 4.424110 & 2.555881 & -0.741605 \\
\hline $\mathrm{C}$ & 3.102276 & -1.657111 & -0.468926 & $\mathrm{H}$ & 3.035173 & 2.772098 & 0.888276 \\
\hline $\mathrm{C}$ & 3.679431 & -0.449339 & 1.983618 & $\mathrm{C}$ & 3.596757 & 1.369031 & -2.666594 \\
\hline $\mathrm{C}$ & 4.364503 & -1.119210 & -0.214936 & $\mathrm{H}$ & 1.557873 & 0.676749 & -2.542587 \\
\hline $\mathrm{C}$ & 4.669441 & -0.505743 & 0.998254 & $\mathrm{C}$ & 4.633288 & 2.044678 & -2.020335 \\
\hline $\mathrm{H}$ & 3.898249 & 0.039219 & 2.933050 & $\mathrm{H}$ & 5.222555 & 3.087114 & -0.228831 \\
\hline $\mathrm{H}$ & 5.113555 & -1.139002 & -1.005454 & $\mathrm{H}$ & 3.749513 & 0.973958 & -3.668668 \\
\hline $\mathrm{C}$ & -2.614420 & -1.552396 & -0.186084 & $\mathrm{H}$ & 5.593488 & 2.177080 & -2.512901 \\
\hline $\mathrm{C}$ & -3.385996 & -1.130973 & 0.915130 & $\mathrm{H}$ & -0.215841 & 0.229724 & -1.757572 \\
\hline $\mathrm{C}$ & -3.012461 & -1.288293 & -1.506952 & $\mathrm{H}$ & -1.662317 & 1.675382 & -1.181362 \\
\hline $\mathrm{C}$ & -4.552520 & -0.415442 & 0.666917 & $\mathrm{O}$ & -1.646760 & 1.438325 & 1.808813 \\
\hline $\mathrm{C}$ & -4.198696 & -0.572096 & -1.701807 & $\mathrm{H}$ & -2.430037 & 1.886037 & 1.419642 \\
\hline $\mathrm{C}$ & -4.966565 & -0.111924 & -0.635087 & $\mathrm{C}$ & -1.004119 & 2.362466 & 2.663339 \\
\hline $\mathrm{H}$ & -5.143015 & -0.060906 & 1.510651 & $\mathrm{H}$ & -0.656375 & 3.258183 & 2.129046 \\
\hline $\mathrm{H}$ & -4.508294 & -0.343069 & -2.720477 & $\mathrm{H}$ & -1.665548 & 2.674764 & 3.481388 \\
\hline $\mathrm{C}$ & 2.768666 & -2.251979 & -1.801275 & $\mathrm{H}$ & -0.130512 & 1.868082 & 3.097605 \\
\hline $\mathrm{H}$ & 2.744069 & -3.348973 & -1.771170 & $\mathrm{O}$ & -3.477706 & 2.785945 & 0.258793 \\
\hline $\mathrm{H}$ & 1.781642 & -1.920080 & -2.142539 & $\mathrm{H}$ & -2.994421 & 2.277906 & -0.432806 \\
\hline $\mathrm{H}$ & 3.507689 & -1.965927 & -2.554023 & $\mathrm{C}$ & -2.955597 & 4.102384 & 0.198628 \\
\hline $\mathrm{C}$ & 1.340677 & -0.865520 & 2.821081 & $\mathrm{H}$ & -3.216416 & 4.610965 & 1.131041 \\
\hline $\mathrm{H}$ & 0.869224 & -1.831296 & 3.038621 & $\mathrm{H}$ & -1.861564 & 4.101374 & 0.088695 \\
\hline $\mathrm{H}$ & 1.740885 & -0.467362 & 3.757084 & $\mathrm{H}$ & -3.383729 & 4.672903 & -0.635509 \\
\hline $\mathrm{H}$ & 0.534839 & -0.204343 & 2.474501 & & & & \\
\hline $\mathrm{C}$ & 6.021900 & 0.090583 & 1.240423 & \multicolumn{4}{|c|}{$\operatorname{TSB}_{7-5}$} \\
\hline $\mathrm{H}$ & 5.947858 & 1.044999 & 1.771788 & $\mathrm{Ru}$ & -0.739149 & -0.601060 & 0.330113 \\
\hline $\mathrm{H}$ & 6.651588 & -0.566225 & 1.852085 & $\mathrm{C}$ & -0.239746 & 1.283665 & -0.016130 \\
\hline $\mathrm{H}$ & 6.552005 & 0.272353 & 0.301290 & $\mathrm{C}$ & 0.534566 & 3.465276 & -0.326819 \\
\hline $\mathrm{C}$ & -2.941035 & -1.443350 & 2.309320 & $\mathrm{C}$ & -0.919216 & 3.525108 & 0.131326 \\
\hline $\mathrm{H}$ & -3.648338 & -1.049053 & 3.042568 & $\mathrm{H}$ & 1.197592 & 4.115029 & 0.252339 \\
\hline $\mathrm{H}$ & -2.849604 & -2.523806 & 2.469823 & $\mathrm{H}$ & 0.643481 & 3.731222 & -1.389330 \\
\hline $\mathrm{H}$ & -1.961267 & -1.001900 & 2.514255 & $\mathrm{H}$ & -1.009550 & 3.858450 & 1.176990 \\
\hline $\mathrm{C}$ & -2.219372 & -1.779444 & -2.678617 & $\mathrm{H}$ & -1.541496 & 4.177141 & -0.489277 \\
\hline $\mathrm{H}$ & -1.152658 & -1.837343 & -2.448713 & $\mathrm{~N}$ & 0.877378 & 2.048075 & -0.122288 \\
\hline $\mathrm{H}$ & -2.556442 & -2.774777 & -2.995940 & $\mathrm{~N}$ & -1.308880 & 2.125637 & -0.007988 \\
\hline $\mathrm{H}$ & -2.333605 & -1.107991 & -3.532888 & $\mathrm{C}$ & 2.154783 & 1.536071 & -0.489313 \\
\hline $\mathrm{C}$ & -6.175578 & 0.740044 & -0.862129 & $\mathrm{C}$ & 2.330225 & 0.973573 & -1.764102 \\
\hline $\mathrm{H}$ & -7.018373 & 0.426430 & -0.238374 & $\mathrm{C}$ & 3.196498 & 1.580670 & 0.444777 \\
\hline $\mathrm{H}$ & -5.952923 & 1.780768 & -0.600975 & $\mathrm{C}$ & 3.575720 & 0.430847 & -2.075037 \\
\hline $\mathrm{H}$ & -6.498416 & 0.719144 & -1.906015 & $\mathrm{C}$ & 4.427371 & 1.031445 & 0.085171 \\
\hline $\mathrm{C}$ & 0.879606 & 1.531477 & -0.023511 & $\mathrm{C}$ & 4.633110 & 0.443224 & -1.162117 \\
\hline $\mathrm{H}$ & 0.887991 & 2.098297 & 0.932447 & $\mathrm{H}$ & 3.719029 & -0.026950 & -3.053382 \\
\hline $\mathrm{C}$ & 2.138000 & 1.695897 & -0.746282 & $\mathrm{H}$ & 5.233905 & 1.025134 & 0.817319 \\
\hline $\mathrm{C}$ & 3.194707 & 2.382802 & -0.117027 & $\mathrm{C}$ & -2.593915 & 1.533498 & 0.209155 \\
\hline
\end{tabular}




\begin{tabular}{|c|c|c|c|c|c|c|c|}
\hline $\mathrm{C}$ & -3.344113 & 1.161917 & -0.923672 & $\mathrm{H}$ & -0.284291 & -0.251616 & 1.813868 \\
\hline $\mathrm{C}$ & -3.022095 & 1.226721 & 1.514406 & $\mathrm{H}$ & -1.690311 & -1.851294 & 1.254570 \\
\hline $\mathrm{C}$ & -4.507090 & 0.426585 & -0.724994 & $\mathrm{O}$ & -1.625215 & -1.362392 & -1.689309 \\
\hline $\mathrm{C}$ & -4.192857 & 0.475818 & 1.654672 & $\mathrm{H}$ & -2.489205 & -1.954597 & -1.165710 \\
\hline$C$ & -4.924473 & 0.039841 & 0.551376 & $\mathrm{C}$ & -0.970165 & -2.204708 & -2.590522 \\
\hline $\mathrm{H}$ & -5.074009 & 0.096284 & -1.593596 & $\mathrm{H}$ & -0.570021 & -3.122046 & -2.121330 \\
\hline$U$ & -4.518248 & 0.196565 & 2.655745 & $\mathrm{H}$ & -1.644379 & -2.522431 & -3.400926 \\
\hline & 2.976262 & 2.161691 & 1.806733 & $\mathrm{H}$ & -0.118672 & -1.691345 & -3.062330 \\
\hline & 2.936530 & 3.258009 & 1.784153 & $\mathrm{O}$ & -3.264341 & -2.534365 & -0.364551 \\
\hline & 2.027274 & 1.818654 & 2.232662 & $\mathrm{H}$ & -2.281998 & -2.028996 & 0.620442 \\
\hline $\mathrm{H}$ & 3.782112 & 1.879766 & 2.488942 & $\mathrm{C}$ & -3.023824 & -3.909676 & -0.405688 \\
\hline & 1.198660 & 0.925302 & -2.743282 & $\mathrm{H}$ & -3.118329 & -4.316597 & -1.425718 \\
\hline & 0.756081 & 1.914664 & -2.910028 & $\mathrm{H}$ & -2.008726 & -4.177002 & -0.049359 \\
\hline & 1.530942 & 0.541632 & -3.711061 & $\mathrm{H}$ & -3.739229 & -4.453991 & 0.227963 \\
\hline & 0.385224 & 0.284860 & -2.378148 & & & & \\
\hline & 5.954217 & -0.168280 & -1.513116 & 5-B & & & \\
\hline & 5.828669 & -1.081948 & -2.102875 & $\mathrm{Ru}$ & -0.273408 & 1.107163 & 0.036787 \\
\hline & 6.570344 & 0.513210 & -2.111171 & $\mathrm{C}$ & -0.549534 & -0.903498 & -0.358590 \\
\hline & 6.526930 & -0.423149 & -0.616979 & $\mathrm{C}$ & -0.136341 & -3.167190 & -0.899297 \\
\hline & -2.856563 & 1.482395 & -2.300042 & $\mathrm{C}$ & -1.611012 & -2.873270 & -1.117390 \\
\hline $\mathrm{H}$ & -3.671807 & 1.428161 & -3.025737 & $\mathrm{H}$ & 0.362755 & -3.572845 & -1.786255 \\
\hline & -2.400776 & 2.475766 & -2.359331 & $\mathrm{H}$ & 0.041920 & -3.868448 & -0.071467 \\
\hline & -2.098966 & 0.745474 & -2.592475 & $\mathrm{H}$ & -1.908666 & -2.934282 & -2.173069 \\
\hline & -2.280781 & 1.719243 & 2.719677 & $\mathrm{H}$ & -2.276771 & -3.531444 & -0.548436 \\
\hline $\mathrm{H}$ & -1.209935 & 1.821144 & 2.526834 & $\mathrm{~N}$ & 0.408528 & -1.845378 & -0.565386 \\
\hline 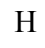 & -2.663605 & 2.695910 & 3.043016 & $\mathrm{~N}$ & -1.735168 & -1.491796 & -0.638560 \\
\hline $\mathrm{H}$ & -2.398683 & 1.029868 & 3.559221 & $\mathrm{C}$ & 1.786847 & -1.769937 & -0.214054 \\
\hline & -6.079591 & -0.893666 & 0.710836 & $\mathrm{C}$ & 2.158085 & -2.005834 & 1.119656 \\
\hline & -6.904746 & -0.646989 & 0.035875 & $\mathrm{C}$ & 2.731970 & -1.498785 & -1.209758 \\
\hline $\mathrm{H}$ & -5.743975 & -1.907277 & 0.460430 & $\mathrm{C}$ & 3.514545 & -1.968962 & 1.435077 \\
\hline 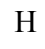 & -6.462559 & -0.905085 & 1.734623 & $\mathrm{C}$ & 4.078732 & -1.471753 & -0.846315 \\
\hline $\mathrm{C}$ & 0.856356 & -1.465737 & 0.029537 & $\mathrm{C}$ & 4.488693 & -1.708129 & 0.465684 \\
\hline $\mathrm{H}$ & 0.890750 & -1.992152 & -0.946219 & $\mathrm{H}$ & 3.818210 & -2.124699 & 2.470027 \\
\hline & 2.099166 & -1.650260 & 0.768712 & $\mathrm{H}$ & 4.824111 & -1.226962 & -1.602260 \\
\hline & 3.160330 & -2.327929 & 0.136251 & $\mathrm{C}$ & -3.040551 & -0.940274 & -0.465962 \\
\hline & 2.305986 & -1.191336 & 2.083493 & $\mathrm{C}$ & -3.666609 & -1.097819 & 0.775451 \\
\hline$G$ & 4.375681 & -2.528653 & 0.778630 & $\mathrm{C}$ & -3.663659 & -0.288571 & -1.536796 \\
\hline $\mathrm{H}$ & 3.014596 & -2.684986 & -0.882608 & $\mathrm{C}$ & -4.954533 & -0.580516 & 0.924914 \\
\hline & 3.519140 & -1.390975 & 2.725229 & $\mathrm{C}$ & -4.948828 & 0.209886 & -1.340781 \\
\hline $\mathrm{H}$ & 1.488073 & -0.677900 & 2.581144 & $\mathrm{C}$ & -5.608544 & 0.076968 & -0.116203 \\
\hline & 4.560374 & -2.057397 & 2.076195 & $\mathrm{H}$ & -5.455596 & -0.689001 & 1.886173 \\
\hline $\mathrm{H}$ & 5.180424 & -3.050406 & 0.266854 & $\mathrm{H}$ & -5.443447 & 0.727700 & -2.162160 \\
\hline $\mathrm{H}$ & 3.657800 & -1.027757 & 3.740823 & $\mathrm{C}$ & 2.283031 & -1.160989 & -2.595327 \\
\hline & 5.509178 & -2.213356 & 2.583560 & $\mathrm{H}$ & 1.787934 & -2.006039 & -3.088814 \\
\hline
\end{tabular}




\begin{tabular}{|c|c|c|c|c|c|c|c|}
\hline $\mathrm{H}$ & 1.551875 & -0.342514 & -2.567961 & $\mathrm{H}$ & -0.146813 & 2.878111 & 0.407630 \\
\hline $\mathrm{H}$ & 3.125021 & -0.853989 & -3.220522 & $\mathrm{C}$ & -2.993755 & 3.070247 & 0.290647 \\
\hline $\mathrm{C}$ & 1.106089 & -2.171059 & 2.172022 & $\mathrm{H}$ & -3.965515 & 3.197315 & 0.787286 \\
\hline $\mathrm{H}$ & 0.444911 & -3.022314 & 1.968570 & $\mathrm{H}$ & -2.288448 & 3.803835 & 0.711554 \\
\hline $\mathrm{H}$ & 1.552952 & -2.326167 & 3.156847 & $\mathrm{H}$ & -3.123109 & 3.291942 & -0.773446 \\
\hline $\mathrm{H}$ & 0.455764 & -1.283495 & 2.223177 & & & & \\
\hline $\mathrm{C}$ & 5.938093 & -1.654089 & 0.840062 & 7-C & & & \\
\hline $\mathrm{H}$ & 6.094983 & -1.060528 & 1.747092 & $\mathrm{C}$ & 0.547391 & -1.212095 & -0.138454 \\
\hline $\mathrm{H}$ & 6.340978 & -2.652721 & 1.043984 & $\mathrm{C}$ & 0.319877 & -3.560372 & -0.140788 \\
\hline $\mathrm{H}$ & 6.542526 & -1.212132 & 0.043468 & $\mathrm{C}$ & 1.772023 & -3.214440 & 0.146276 \\
\hline $\mathrm{C}$ & -2.943333 & -1.730951 & 1.921564 & $\mathrm{H}$ & -0.109086 & -4.271769 & 0.572216 \\
\hline $\mathrm{H}$ & -3.620104 & -1.919110 & 2.758854 & $\mathrm{H}$ & 0.194713 & -3.974744 & -1.152901 \\
\hline $\mathrm{H}$ & -2.476093 & -2.683929 & 1.644671 & $\mathrm{H}$ & 2.028074 & -3.368066 & 1.207844 \\
\hline $\mathrm{H}$ & -2.138863 & -1.064103 & 2.265665 & $\mathrm{H}$ & 2.481062 & -3.789057 & -0.458833 \\
\hline $\mathrm{C}$ & -2.931874 & -0.069489 & -2.823008 & $\mathrm{~N}$ & -0.335405 & -2.255418 & -0.025446 \\
\hline $\mathrm{H}$ & -2.061959 & 0.581057 & -2.663862 & $\mathrm{~N}$ & 1.798537 & -1.798885 & -0.203912 \\
\hline $\mathrm{H}$ & -2.545081 & -1.002475 & -3.248997 & $\mathrm{C}$ & -1.751524 & -2.162596 & 0.064900 \\
\hline $\mathrm{H}$ & -3.576871 & 0.396682 & -3.571892 & $\mathrm{C}$ & -2.539841 & -2.395403 & -1.071170 \\
\hline $\mathrm{C}$ & -6.977532 & 0.655546 & 0.077016 & $\mathrm{C}$ & -2.330184 & -1.837678 & 1.302414 \\
\hline $\mathrm{H}$ & -7.445881 & 0.289200 & 0.994205 & $\mathrm{C}$ & -3.925930 & -2.283327 & -0.949971 \\
\hline $\mathrm{H}$ & -6.940687 & 1.749157 & 0.145479 & $\mathrm{C}$ & -3.717783 & -1.725189 & 1.371713 \\
\hline $\mathrm{H}$ & -7.641978 & 0.416813 & -0.759391 & $\mathrm{C}$ & -4.533591 & -1.945220 & 0.259406 \\
\hline $\mathrm{C}$ & 1.519760 & 0.978174 & 0.485895 & $\mathrm{H}$ & -4.545384 & -2.443580 & -1.831947 \\
\hline $\mathrm{H}$ & 1.713060 & 0.451575 & 1.438052 & $\mathrm{H}$ & -4.174781 & -1.458862 & 2.324299 \\
\hline $\mathrm{C}$ & 2.734669 & 1.603486 & -0.016356 & $\mathrm{C}$ & 2.961049 & -0.991233 & -0.014829 \\
\hline $\mathrm{C}$ & 3.891227 & 1.598241 & 0.788165 & $\mathrm{C}$ & 3.708843 & -0.622803 & -1.148837 \\
\hline $\mathrm{C}$ & 2.832470 & 2.147526 & -1.311682 & $\mathrm{C}$ & 3.349175 & -0.594713 & 1.276341 \\
\hline $\mathrm{C}$ & 5.094776 & 2.110632 & 0.321787 & $\mathrm{C}$ & 4.834133 & 0.176172 & -0.969463 \\
\hline $\mathrm{H}$ & 3.825702 & 1.165163 & 1.785513 & $\mathrm{C}$ & 4.484844 & 0.210757 & 1.402779 \\
\hline $\mathrm{C}$ & 4.036440 & 2.653469 & -1.779985 & $\mathrm{C}$ & 5.233387 & 0.611162 & 0.298225 \\
\hline $\mathrm{H}$ & 1.945774 & 2.131096 & -1.941362 & $\mathrm{H}$ & 5.415944 & 0.468583 & -1.842700 \\
\hline $\mathrm{C}$ & 5.171665 & 2.635124 & -0.967061 & $\mathrm{H}$ & 4.791100 & 0.530034 & 2.398400 \\
\hline $\mathrm{H}$ & 5.976377 & 2.095526 & 0.957650 & $\mathrm{C}$ & -1.472795 & -1.583070 & 2.499421 \\
\hline $\mathrm{H}$ & 4.098892 & 3.058454 & -2.786883 & $\mathrm{H}$ & -0.754378 & -2.395630 & 2.661989 \\
\hline $\mathrm{H}$ & 6.114430 & 3.026164 & -1.340593 & $\mathrm{H}$ & -0.878828 & -0.668206 & 2.363028 \\
\hline $\mathrm{H}$ & -0.039134 & 1.228327 & -1.579330 & $\mathrm{H}$ & -2.076687 & -1.475075 & 3.403866 \\
\hline $\mathrm{H}$ & -0.073562 & 2.780782 & -0.437641 & $\mathrm{C}$ & -1.904717 & -2.689521 & -2.395437 \\
\hline $\mathrm{O}$ & -0.940920 & 0.826079 & 2.128493 & $\mathrm{H}$ & -1.603952 & -3.740858 & -2.484837 \\
\hline $\mathrm{H}$ & -2.240991 & 1.524094 & 1.333863 & $\mathrm{H}$ & -2.595210 & -2.485096 & -3.217368 \\
\hline $\mathrm{C}$ & -0.304362 & 1.546888 & 3.120652 & $\mathrm{H}$ & -1.003485 & -2.084351 & -2.544782 \\
\hline $\mathrm{H}$ & -0.317835 & 2.646545 & 2.949911 & $\mathrm{C}$ & -6.024007 & -1.839044 & 0.371013 \\
\hline $\mathrm{H}$ & -0.767358 & 1.373041 & 4.108742 & $\mathrm{H}$ & -6.496993 & -1.750305 & -0.610544 \\
\hline $\mathrm{H}$ & 0.767276 & 1.278911 & 3.232557 & $\mathrm{H}$ & -6.452890 & -2.722315 & 0.858704 \\
\hline $\mathrm{O}$ & -2.533333 & 1.746462 & 0.400534 & $\mathrm{H}$ & -6.323446 & -0.972957 & 0.968538 \\
\hline
\end{tabular}




\begin{tabular}{|c|c|c|c|c|c|c|c|}
\hline $\mathrm{C}$ & 3.292351 & -1.092560 & -2.506381 & $\mathrm{~N}$ & 1.768333 & -1.792241 & 0.220853 \\
\hline $\mathrm{H}$ & 3.958009 & -0.701636 & -3.279792 & $\mathrm{C}$ & -1.803331 & -1.802264 & 0.190550 \\
\hline $\mathrm{H}$ & 3.300824 & -2.186321 & -2.574669 & $\mathrm{C}$ & -2.510943 & -2.147569 & -0.969588 \\
\hline $\mathrm{H}$ & 2.270646 & -0.765457 & -2.727533 & $\mathrm{C}$ & -2.435967 & -1.190548 & 1.282798 \\
\hline $\mathrm{C}$ & 2.620774 & -1.058228 & 2.501797 & $\mathrm{C}$ & -3.878090 & -1.876824 & -1.011525 \\
\hline $\mathrm{H}$ & 1.563964 & -1.252141 & 2.305760 & $\mathrm{C}$ & -3.804506 & -0.938762 & 1.195882 \\
\hline $\mathrm{H}$ & 3.070005 & -1.977213 & 2.900939 & $\mathrm{C}$ & -4.541832 & -1.274228 & 0.059953 \\
\hline $\mathrm{H}$ & 2.671565 & -0.305080 & 3.292029 & $\mathrm{H}$ & -4.434875 & -2.120124 & -1.916052 \\
\hline $\mathrm{C}$ & 6.429307 & 1.499077 & 0.457894 & $\mathrm{H}$ & -4.300613 & -0.434440 & 2.024280 \\
\hline $\mathrm{H}$ & 6.205459 & 2.526240 & 0.148571 & $\mathrm{C}$ & 2.961146 & -1.002193 & 0.250251 \\
\hline $\mathrm{H}$ & 6.766825 & 1.539730 & 1.496712 & $\mathrm{C}$ & 3.759850 & -0.963529 & -0.906450 \\
\hline $\mathrm{H}$ & 7.269262 & 1.161837 & -0.157385 & $\mathrm{C}$ & 3.290874 & -0.257578 & 1.396972 \\
\hline $\mathrm{C}$ & -1.340960 & 0.873509 & -0.452947 & $\mathrm{C}$ & 4.888699 & -0.149090 & -0.900494 \\
\hline $\mathrm{H}$ & -1.909839 & 0.099242 & -0.985913 & $\mathrm{C}$ & 4.431510 & 0.547337 & 1.349899 \\
\hline $\mathrm{C}$ & -2.117053 & 2.081058 & -0.161412 & $\mathrm{C}$ & 5.234064 & 0.622228 & 0.213007 \\
\hline $\mathrm{C}$ & -3.055377 & 2.575282 & -1.087675 & $\mathrm{H}$ & 5.509564 & -0.105235 & -1.793978 \\
\hline $\mathrm{C}$ & -1.942847 & 2.798624 & 1.039419 & $\mathrm{H}$ & 4.690206 & 1.139316 & 2.226395 \\
\hline $\mathrm{C}$ & -3.732946 & 3.766569 & -0.855822 & $\mathrm{C}$ & -1.637030 & -0.752317 & 2.467597 \\
\hline $\mathrm{H}$ & -3.222855 & 2.020418 & -2.008912 & $\mathrm{H}$ & -1.034324 & -1.568961 & 2.883178 \\
\hline $\mathrm{C}$ & -2.622122 & 3.990209 & 1.269780 & $\mathrm{H}$ & -0.929873 & 0.039130 & 2.178292 \\
\hline $\mathrm{H}$ & -1.279167 & 2.370528 & 1.791126 & $\mathrm{H}$ & -2.280296 & -0.360133 & 3.259151 \\
\hline $\mathrm{C}$ & -3.510991 & 4.486878 & 0.317488 & $\mathrm{C}$ & -1.794608 & -2.732743 & -2.146748 \\
\hline $\mathrm{H}$ & -4.437534 & 4.138940 & -1.595326 & $\mathrm{H}$ & -1.494618 & -3.773150 & -1.971171 \\
\hline $\mathrm{H}$ & -2.474227 & 4.524686 & 2.205303 & $\mathrm{H}$ & -2.426673 & -2.725351 & -3.037818 \\
\hline $\mathrm{H}$ & -4.045169 & 5.415652 & 0.498232 & $\mathrm{H}$ & -0.878715 & -2.172807 & -2.369876 \\
\hline $\mathrm{Ru}$ & 0.461311 & 0.717201 & -0.131520 & $\mathrm{C}$ & -6.008559 & -0.981188 & -0.016046 \\
\hline $\mathrm{H}$ & 0.573533 & 0.834410 & 1.548871 & $\mathrm{H}$ & -6.312066 & -0.717662 & -1.033931 \\
\hline $\mathrm{H}$ & 1.075185 & 0.850116 & -1.699041 & $\mathrm{H}$ & -6.609174 & -1.848833 & 0.281127 \\
\hline $\mathrm{O}$ & 0.842004 & 3.005525 & -0.082658 & $\mathrm{H}$ & -6.285772 & -0.153254 & 0.642577 \\
\hline $\mathrm{H}$ & 0.271806 & 3.354832 & 0.614976 & $\mathrm{C}$ & 3.384471 & -1.770122 & -2.108535 \\
\hline $\mathrm{C}$ & 0.575953 & 3.737799 & -1.278100 & $\mathrm{H}$ & 4.131522 & -1.671038 & -2.899300 \\
\hline $\mathrm{H}$ & 1.168458 & 3.264680 & -2.061003 & $\mathrm{H}$ & 3.280663 & -2.833554 & -1.866866 \\
\hline $\mathrm{H}$ & -0.484023 & 3.685043 & -1.556040 & $\mathrm{H}$ & 2.417435 & -1.437945 & -2.502256 \\
\hline \multirow[t]{2}{*}{$\mathrm{H}$} & 0.877237 & 4.785212 & -1.163717 & $\mathrm{C}$ & 2.475163 & -0.346212 & 2.651496 \\
\hline & & & & $\mathrm{H}$ & 1.405504 & -0.433597 & 2.438500 \\
\hline \multicolumn{2}{|c|}{$\mathbf{T S C}_{7-5}$} & & & $\mathrm{H}$ & 2.769975 & -1.212208 & 3.258449 \\
\hline $\mathrm{C}$ & 0.576307 & -1.149668 & 0.006396 & $\mathrm{H}$ & 2.612217 & 0.545456 & 3.267443 \\
\hline $\mathrm{C}$ & 0.136411 & -3.381486 & 0.574875 & $\mathrm{C}$ & 6.420097 & 1.535123 & 0.169883 \\
\hline $\mathrm{C}$ & 1.590431 & -3.067201 & 0.909646 & $\mathrm{H}$ & 6.151701 & 2.499480 & -0.275964 \\
\hline $\mathrm{H}$ & -0.411006 & -3.839292 & 1.404479 & $\mathrm{H}$ & 6.811359 & 1.740767 & 1.169822 \\
\hline $\mathrm{H}$ & 0.052508 & -4.047562 & -0.296983 & $\mathrm{H}$ & 7.231237 & 1.119267 & -0.434755 \\
\hline $\mathrm{H}$ & 1.747479 & -2.958230 & 1.995065 & $\mathrm{C}$ & -1.061383 & 0.907632 & -0.928981 \\
\hline $\mathrm{H}$ & 2.290931 & -3.821813 & 0.538789 & $\mathrm{H}$ & -1.394931 & 0.356288 & -1.824700 \\
\hline $\mathrm{N}$ & -0.405383 & -2.053455 & 0.265657 & $\mathrm{C}$ & -2.177424 & 1.673611 & -0.377276 \\
\hline
\end{tabular}




\begin{tabular}{|c|c|c|c|c|c|c|c|}
\hline $\mathrm{C}$ & -3.388287 & 1.672720 & -1.099709 & $\mathrm{H}$ & 5.471850 & 0.010401 & -1.631508 \\
\hline $\mathrm{C}$ & -2.142101 & 2.379277 & 0.840907 & $\mathrm{H}$ & 4.706833 & 1.006602 & 2.467260 \\
\hline $\mathrm{C}$ & -4.510085 & 2.345834 & -0.634843 & $\mathrm{C}$ & -1.944958 & -1.108732 & 2.484795 \\
\hline $\mathrm{H}$ & -3.432558 & 1.117077 & -2.035420 & $\mathrm{H}$ & -1.453819 & -2.018072 & 2.852091 \\
\hline $\mathrm{C}$ & -3.266680 & 3.042624 & 1.310691 & $\mathrm{H}$ & -1.159021 & -0.345976 & 2.398416 \\
\hline $\mathrm{H}$ & -1.217550 & 2.379322 & 1.412669 & $\mathrm{H}$ & -2.656818 & -0.775957 & 3.244118 \\
\hline $\mathrm{C}$ & -4.454819 & 3.030159 & 0.577234 & $\mathrm{C}$ & -1.559757 & -2.119726 & -2.408754 \\
\hline $\mathrm{H}$ & -5.431491 & 2.329731 & -1.211899 & $\mathrm{H}$ & -1.071907 & -3.097326 & -2.316358 \\
\hline $\mathrm{H}$ & -3.221360 & 3.575342 & 2.257382 & $\mathrm{H}$ & -2.138631 & -2.124626 & -3.335174 \\
\hline $\mathrm{H}$ & -5.331991 & 3.552953 & 0.950337 & $\mathrm{H}$ & -0.756786 & -1.377041 & -2.506880 \\
\hline $\mathrm{Ru}$ & 0.717215 & 0.747274 & -0.454488 & $\mathrm{C}$ & -6.030074 & -0.861513 & -0.449329 \\
\hline $\mathrm{H}$ & 0.693096 & 1.737373 & 1.204810 & $\mathrm{H}$ & -6.240126 & -0.472790 & -1.450410 \\
\hline $\mathrm{H}$ & 0.951894 & 0.228877 & -1.973933 & $\mathrm{H}$ & -6.645346 & -1.759500 & -0.317934 \\
\hline $\mathrm{O}$ & 1.748684 & 2.674829 & -0.508570 & $\mathrm{H}$ & -6.376966 & -0.121151 & 0.277279 \\
\hline $\mathrm{H}$ & 1.166785 & 2.375867 & 0.552060 & $\mathrm{C}$ & 3.310616 & -1.579056 & -2.041417 \\
\hline $\mathrm{C}$ & 1.166931 & 3.713650 & -1.254434 & $\mathrm{H}$ & 4.042992 & -1.415889 & -2.835570 \\
\hline $\mathrm{H}$ & 1.157543 & 3.463370 & -2.324600 & $\mathrm{H}$ & 3.209304 & -2.659681 & -1.890261 \\
\hline $\mathrm{H}$ & 0.126307 & 3.914649 & -0.952878 & $\mathrm{H}$ & 2.338084 & -1.215199 & -2.392213 \\
\hline \multirow[t]{2}{*}{$\mathrm{H}$} & 1.740497 & 4.642036 & -1.122412 & $\mathrm{C}$ & 2.448693 & -0.446205 & 2.802180 \\
\hline & & & & $\mathrm{H}$ & 1.383379 & -0.560849 & 2.574633 \\
\hline 5-C & & & & $\mathrm{H}$ & 2.748061 & -1.321219 & 3.392832 \\
\hline $\mathrm{C}$ & 0.528646 & -1.048644 & 0.070939 & $\mathrm{H}$ & 2.561664 & 0.428743 & 3.447461 \\
\hline $\mathrm{C}$ & 0.046543 & -3.308160 & 0.464463 & $\mathrm{C}$ & 6.420335 & 1.511484 & 0.420188 \\
\hline $\mathrm{C}$ & 1.495066 & -3.041776 & 0.854276 & $\mathrm{H}$ & 6.169172 & 2.484403 & -0.017989 \\
\hline $\mathrm{H}$ & -0.528846 & -3.813176 & 1.246277 & $\mathrm{H}$ & 6.790343 & 1.698879 & 1.431681 \\
\hline $\mathrm{H}$ & -0.030654 & -3.909167 & -0.453904 & $\mathrm{H}$ & 7.242272 & 1.099091 & -0.172346 \\
\hline $\mathrm{H}$ & 1.633283 & -3.033778 & 1.947480 & $\mathrm{C}$ & -1.143577 & 1.126783 & -0.862205 \\
\hline $\mathrm{H}$ & 2.198092 & -3.764702 & 0.429554 & $\mathrm{H}$ & -1.441720 & 0.846940 & -1.886453 \\
\hline $\mathrm{N}$ & -0.467822 & -1.952467 & 0.231049 & $\mathrm{C}$ & -2.277647 & 1.754175 & -0.184221 \\
\hline $\mathrm{N}$ & 1.702747 & -1.706618 & 0.296641 & $\mathrm{C}$ & -3.453496 & 1.967936 & -0.935215 \\
\hline $\mathrm{C}$ & -1.856826 & -1.697339 & 0.048840 & $\mathrm{C}$ & -2.323148 & 2.101171 & 1.180105 \\
\hline $\mathrm{C}$ & -2.423305 & -1.804453 & -1.228114 & $\mathrm{C}$ & -4.604728 & 2.491339 & -0.361807 \\
\hline $\mathrm{C}$ & -2.613065 & -1.313169 & 1.162028 & $\mathrm{H}$ & -3.446160 & 1.694896 & -1.989412 \\
\hline $\mathrm{C}$ & -3.784520 & -1.534707 & -1.363042 & $\mathrm{C}$ & -3.473596 & 2.616879 & 1.758494 \\
\hline $\mathrm{C}$ & -3.969427 & -1.051331 & 0.981278 & $\mathrm{H}$ & -1.445009 & 1.945742 & 1.799315 \\
\hline $\mathrm{C}$ & -4.573247 & -1.159873 & -0.272452 & $\mathrm{C}$ & -4.623671 & 2.814961 & 0.993033 \\
\hline $\mathrm{H}$ & -4.236265 & -1.592646 & -2.352998 & $\mathrm{H}$ & -5.492962 & 2.640783 & -0.971438 \\
\hline $\mathrm{H}$ & -4.560559 & -0.718307 & 1.833275 & $\mathrm{H}$ & -3.477704 & 2.865523 & 2.817021 \\
\hline $\mathrm{C}$ & 2.924635 & -0.969181 & 0.372092 & $\mathrm{H}$ & -5.523855 & 3.218800 & 1.449096 \\
\hline $\mathrm{C}$ & 3.714003 & -0.873471 & -0.785384 & $\mathrm{Ru}$ & 0.658358 & 0.880325 & -0.432967 \\
\hline $\mathrm{C}$ & 3.276945 & -0.310277 & 1.560456 & $\mathrm{H}$ & 0.343628 & 1.333821 & 1.425084 \\
\hline $\mathrm{C}$ & 4.861333 & -0.086485 & -0.734583 & $\mathrm{H}$ & 0.775492 & 0.367961 & -1.939861 \\
\hline $\mathrm{C}$ & 4.431717 & 0.473167 & 1.558393 & $\mathrm{O}$ & 2.032801 & 2.276608 & -0.772340 \\
\hline $\mathrm{C}$ & 5.225901 & 0.608671 & 0.420756 & $\mathrm{H}$ & 0.895367 & 1.869665 & 1.213352 \\
\hline
\end{tabular}




\begin{tabular}{|c|c|c|c|c|c|c|c|}
\hline $\mathrm{C}$ & 2.619239 & 2.460473 & -2.023471 & $\mathrm{C}$ & -3.102377 & 0.381074 & -2.405506 \\
\hline $\mathrm{H}$ & 2.892010 & 1.512784 & -2.520261 & $\mathrm{H}$ & -3.800376 & -0.069763 & -3.114817 \\
\hline $\mathrm{H}$ & 1.965897 & 3.017801 & -2.714716 & $\mathrm{H}$ & -2.906934 & 1.412377 & -2.720552 \\
\hline \multirow[t]{2}{*}{$\mathrm{H}$} & 3.549020 & 3.043001 & -1.909035 & $\mathrm{H}$ & -2.153152 & -0.161053 & -2.479151 \\
\hline & & & & $\mathrm{C}$ & -2.742408 & 1.664820 & 2.457194 \\
\hline 7-D & & & & $\mathrm{H}$ & -1.659002 & 1.540342 & 2.357185 \\
\hline $\mathrm{Ru}$ & -0.728669 & -0.987852 & 0.335601 & $\mathrm{H}$ & -2.958457 & 2.740750 & 2.459906 \\
\hline $\mathrm{C}$ & -0.592825 & 0.940891 & -0.234639 & $\mathrm{H}$ & -3.037003 & 1.272119 & 3.432902 \\
\hline $\mathrm{C}$ & -0.099349 & 3.158254 & -0.818590 & $\mathrm{C}$ & -6.565532 & -1.208056 & 0.890894 \\
\hline $\mathrm{C}$ & -1.535743 & 3.096337 & -0.311124 & $\mathrm{H}$ & -7.236349 & -1.256056 & 0.028646 \\
\hline $\mathrm{H}$ & 0.493396 & 3.943485 & -0.338797 & $\mathrm{H}$ & -6.319128 & -2.240657 & 1.163049 \\
\hline $\mathrm{H}$ & -0.050777 & 3.316650 & -1.907386 & $\mathrm{H}$ & -7.120891 & -0.781625 & 1.730653 \\
\hline $\mathrm{H}$ & -1.634837 & 3.543747 & 0.690374 & $\mathrm{C}$ & 0.984411 & -1.615885 & 0.238314 \\
\hline $\mathrm{H}$ & -2.251693 & 3.589584 & -0.976269 & $\mathrm{H}$ & 1.189013 & -2.204894 & -0.682346 \\
\hline $\mathrm{N}$ & 0.412090 & 1.823862 & -0.477044 & $\mathrm{C}$ & 2.185151 & -1.477784 & 1.056776 \\
\hline $\mathrm{N}$ & -1.755325 & 1.652940 & -0.268720 & $\mathrm{C}$ & 3.396044 & -2.017038 & 0.581800 \\
\hline $\mathrm{C}$ & 1.758061 & 1.451507 & -0.750222 & $\mathrm{C}$ & 2.213713 & -0.806775 & 2.293896 \\
\hline $\mathrm{C}$ & 2.055833 & 0.725218 & -1.914566 & $\mathrm{C}$ & 4.580029 & -1.885242 & 1.297721 \\
\hline $\mathrm{C}$ & 2.753478 & 1.796051 & 0.173632 & $\mathrm{H}$ & 3.394337 & -2.533764 & -0.377796 \\
\hline $\mathrm{C}$ & 3.377918 & 0.338393 & -2.126152 & $\mathrm{C}$ & 3.395482 & -0.670738 & 3.006947 \\
\hline $\mathrm{C}$ & 4.063649 & 1.394551 & -0.086012 & $\mathrm{H}$ & 1.281950 & -0.398018 & 2.675019 \\
\hline $\mathrm{C}$ & 4.393927 & 0.658064 & -1.222436 & $\mathrm{C}$ & 4.586903 & -1.205929 & 2.513431 \\
\hline $\mathrm{H}$ & 3.618478 & -0.242828 & -3.016210 & $\mathrm{H}$ & 5.500930 & -2.309253 & 0.903779 \\
\hline $\mathrm{H}$ & 4.838006 & 1.623262 & 0.645058 & $\mathrm{H}$ & 3.391113 & -0.145434 & 3.959535 \\
\hline $\mathrm{C}$ & -2.979618 & 0.988323 & 0.037457 & $\mathrm{H}$ & 5.510478 & -1.100569 & 3.077187 \\
\hline $\mathrm{C}$ & -3.643086 & 0.325019 & -1.010051 & $\mathrm{H}$ & -0.535330 & -0.441928 & 1.800702 \\
\hline $\mathrm{C}$ & -3.466819 & 0.958039 & 1.354389 & $\mathrm{H}$ & -1.598185 & -2.228771 & 1.103253 \\
\hline $\mathrm{C}$ & -4.812754 & -0.373328 & -0.710379 & $\mathrm{O}$ & -1.425584 & -2.373619 & -1.659265 \\
\hline $\mathrm{C}$ & -4.646277 & 0.254579 & 1.600739 & $\mathrm{H}$ & -1.930238 & -2.716109 & -0.902439 \\
\hline $\mathrm{C}$ & -5.327139 & -0.421509 & 0.587413 & $\mathrm{C}$ & -0.609356 & -3.427794 & -2.147196 \\
\hline $\mathrm{H}$ & -5.338797 & -0.886849 & -1.514019 & $\mathrm{H}$ & -0.044433 & -3.916368 & -1.343571 \\
\hline $\mathrm{H}$ & -5.031997 & 0.218335 & 2.618582 & $\mathrm{H}$ & -1.206785 & -4.178632 & -2.677922 \\
\hline $\mathrm{C}$ & 2.407076 & 2.539597 & 1.425802 & $\mathrm{H}$ & 0.101616 & -2.989569 & -2.851674 \\
\hline $\mathrm{H}$ & 2.223619 & 3.604918 & 1.235244 & & & & \\
\hline $\mathrm{H}$ & 1.498044 & 2.136327 & 1.885309 & 5-D & & & \\
\hline $\mathrm{H}$ & 3.217303 & 2.476291 & 2.156295 & $\mathrm{Ru}$ & 0.561783 & 0.672215 & 1.409116 \\
\hline $\mathrm{C}$ & 0.977778 & 0.333348 & -2.876750 & $\mathrm{C}$ & 0.459928 & 0.206381 & -0.544800 \\
\hline $\mathrm{H}$ & 0.300302 & 1.165137 & -3.101853 & $\mathrm{C}$ & -0.020426 & 0.540811 & -2.819484 \\
\hline $\mathrm{H}$ & 1.401190 & -0.024184 & -3.819118 & $\mathrm{C}$ & 1.307959 & -0.176978 & -2.709399 \\
\hline $\mathrm{H}$ & 0.346846 & -0.462011 & -2.456622 & $\mathrm{H}$ & -0.704770 & 0.086356 & -3.539228 \\
\hline $\mathrm{C}$ & 5.800974 & 0.202763 & -1.459198 & $\mathrm{H}$ & 0.101338 & 1.601409 & -3.085418 \\
\hline $\mathrm{H}$ & 6.321128 & 0.844725 & -2.179957 & $\mathrm{H}$ & 1.249510 & -1.232517 & -3.020422 \\
\hline $\mathrm{H}$ & 6.383076 & 0.211522 & -0.533464 & $\mathrm{H}$ & 2.107900 & 0.298141 & -3.283479 \\
\hline $\mathrm{H}$ & 5.829437 & -0.814914 & -1.862203 & $\mathrm{~N}$ & -0.538063 & 0.444567 & -1.447440 \\
\hline
\end{tabular}




\begin{tabular}{|c|c|c|c|c|c|c|c|}
\hline $\mathrm{N}$ & 1.571882 & -0.087928 & -1.270347 & $\mathrm{C}$ & -3.341828 & -1.048867 & 1.936610 \\
\hline $\mathrm{C}$ & -1.927937 & 0.640808 & -1.192977 & $\mathrm{C}$ & -1.570884 & -2.164358 & 0.745075 \\
\hline $\mathrm{C}$ & -2.402980 & 1.786924 & -0.536558 & $\mathrm{C}$ & -4.228797 & -2.048144 & 1.558727 \\
\hline $\mathrm{C}$ & -2.818383 & -0.352303 & -1.648912 & $\mathrm{H}$ & -3.684755 & -0.217243 & 2.550841 \\
\hline $\mathrm{C}$ & -3.773046 & 1.874595 & -0.275737 & $\mathrm{C}$ & -2.448274 & -3.180065 & 0.392918 \\
\hline $\mathrm{C}$ & -4.174867 & -0.211017 & -1.369111 & $\mathrm{H}$ & -0.528174 & -2.194026 & 0.441574 \\
\hline $\mathrm{C}$ & -4.671729 & 0.884023 & -0.662549 & $\mathrm{C}$ & -3.784205 & -3.123837 & 0.790717 \\
\hline $\mathrm{H}$ & -4.144510 & 2.757773 & 0.243828 & $\mathrm{H}$ & -5.268470 & -1.999052 & 1.873783 \\
\hline $\mathrm{H}$ & -4.858947 & -0.995648 & -1.690703 & $\mathrm{H}$ & -2.092743 & -4.019119 & -0.201442 \\
\hline $\mathrm{C}$ & 2.858832 & -0.435920 & -0.758090 & $\mathrm{H}$ & -4.471968 & -3.918528 & 0.512877 \\
\hline $\mathrm{C}$ & 3.901698 & 0.502720 & -0.860541 & $\mathrm{H}$ & 1.209486 & -0.748912 & 1.755236 \\
\hline $\mathrm{C}$ & 3.089013 & -1.726437 & -0.257637 & $\mathrm{H}$ & 1.103354 & 0.894923 & 3.119388 \\
\hline $\mathrm{C}$ & 5.162833 & 0.138370 & -0.391473 & $\mathrm{O}$ & 1.416128 & 2.350645 & 0.624285 \\
\hline $\mathrm{C}$ & 4.370739 & -2.041970 & 0.196125 & $\mathrm{H}$ & 0.581746 & 1.534433 & 3.019038 \\
\hline $\mathrm{C}$ & 5.416572 & -1.122208 & 0.152201 & $\mathrm{C}$ & 1.609920 & 3.517030 & 1.348472 \\
\hline $\mathrm{H}$ & 5.972742 & 0.863869 & -0.457675 & $\mathrm{H}$ & 1.790040 & 3.353997 & 2.427965 \\
\hline $\mathrm{H}$ & 4.554693 & -3.041685 & 0.588211 & $\mathrm{H}$ & 2.484176 & 4.065851 & 0.957449 \\
\hline $\mathrm{C}$ & -2.346881 & -1.546361 & -2.422406 & $\mathrm{H}$ & 0.747311 & 4.205898 & 1.268935 \\
\hline $\mathrm{H}$ & -2.292458 & -1.338818 & -3.499207 & & & & \\
\hline $\mathrm{H}$ & -1.353257 & -1.877258 & -2.101987 & 8 & & & \\
\hline $\mathrm{H}$ & -3.035501 & -2.385155 & -2.295225 & $\mathrm{Ru}$ & -0.115439 & 0.434515 & -0.402941 \\
\hline $\mathrm{C}$ & -1.508506 & 2.909564 & -0.118111 & $\mathrm{C}$ & 0.665323 & 3.478386 & -0.482933 \\
\hline $\mathrm{H}$ & -0.550630 & 2.908873 & -0.642063 & $\mathrm{C}$ & 1.604876 & 2.345322 & -2.257555 \\
\hline $\mathrm{H}$ & -2.007309 & 3.871824 & -0.271729 & $\mathrm{C}$ & 1.290406 & 4.662117 & -0.847964 \\
\hline $\mathrm{H}$ & -1.258776 & 2.843922 & 0.949499 & $\mathrm{H}$ & 0.025089 & 3.413675 & 0.393142 \\
\hline $\mathrm{C}$ & -6.121291 & 0.972633 & -0.299573 & $\mathrm{C}$ & 2.256135 & 3.491074 & -2.694457 \\
\hline $\mathrm{H}$ & -6.464115 & 2.009423 & -0.238739 & $\mathrm{H}$ & 1.711425 & 1.376559 & -2.743918 \\
\hline $\mathrm{H}$ & -6.755571 & 0.446224 & -1.018345 & $\mathrm{H}$ & 1.139282 & 5.556171 & -0.251596 \\
\hline $\mathrm{H}$ & -6.305951 & 0.517868 & 0.681790 & $\mathrm{H}$ & 2.882314 & 3.447907 & -3.580055 \\
\hline $\mathrm{C}$ & 3.671266 & 1.851660 & -1.464342 & $\mathrm{~N}$ & 0.819323 & 2.329058 & -1.164856 \\
\hline $\mathrm{H}$ & 4.513763 & 2.517323 & -1.260337 & $\mathrm{C}$ & 2.103352 & 4.672620 & -1.977254 \\
\hline $\mathrm{H}$ & 3.566120 & 1.794647 & -2.555892 & $\mathrm{H}$ & 2.607907 & 5.582857 & -2.289522 \\
\hline $\mathrm{H}$ & 2.757931 & 2.298317 & -1.057497 & $\mathrm{C}$ & -0.975322 & -1.206209 & 0.284592 \\
\hline $\mathrm{C}$ & 2.008029 & -2.758778 & -0.191897 & $\mathrm{C}$ & -1.195849 & -3.443967 & 1.019469 \\
\hline $\mathrm{H}$ & 1.239102 & -2.608978 & -0.956612 & $\mathrm{C}$ & -2.543808 & -2.754592 & 1.149826 \\
\hline $\mathrm{H}$ & 2.419483 & -3.764679 & -0.313124 & $\mathrm{H}$ & -1.183428 & -4.176054 & 0.198208 \\
\hline $\mathrm{H}$ & 1.497696 & -2.727250 & 0.778124 & $\mathrm{H}$ & -0.877392 & -3.960077 & 1.931680 \\
\hline $\mathrm{C}$ & 6.773743 & -1.472957 & 0.680226 & $\mathrm{H}$ & -3.362657 & -3.299466 & 0.666562 \\
\hline $\mathrm{H}$ & 6.953817 & -2.551134 & 0.650152 & $\mathrm{H}$ & -2.832763 & -2.590938 & 2.199283 \\
\hline $\mathrm{H}$ & 7.569646 & -0.980103 & 0.114663 & $\mathrm{~N}$ & -0.299636 & -2.326776 & 0.707021 \\
\hline $\mathrm{H}$ & 6.885710 & -1.159185 & 1.724510 & $\mathrm{~N}$ & -2.302095 & -1.471997 & 0.486252 \\
\hline $\mathrm{C}$ & -1.058240 & -0.068278 & 2.010199 & $\mathrm{C}$ & 1.036090 & -2.633662 & 0.289263 \\
\hline $\mathrm{H}$ & -1.456540 & 0.406291 & 2.927440 & $\mathrm{C}$ & 2.083086 & -2.573304 & 1.219819 \\
\hline $\mathrm{C}$ & -1.991483 & -1.079901 & 1.534485 & $\mathrm{C}$ & 1.263924 & -3.045034 & -1.037130 \\
\hline
\end{tabular}




\begin{tabular}{|c|c|c|c|c|c|c|c|}
\hline $\mathrm{C}$ & 3.377734 & -2.861691 & 0.781436 & $\mathrm{C}$ & 4.213404 & 2.113839 & 0.900847 \\
\hline $\mathrm{C}$ & 2.573853 & -3.313334 & -1.426752 & $\mathrm{H}$ & 3.009577 & 0.793489 & -0.315432 \\
\hline $\mathrm{C}$ & 3.646782 & -3.212394 & -0.539544 & $\mathrm{C}$ & 4.302565 & 2.818120 & 2.101423 \\
\hline $\mathrm{H}$ & 4.197717 & -2.805501 & 1.496883 & $\mathrm{H}$ & 3.327118 & 3.275754 & 3.967587 \\
\hline $\mathrm{H}$ & 2.760401 & -3.608944 & -2.458758 & $\mathrm{H}$ & 5.035332 & 2.146431 & 0.189449 \\
\hline $\mathrm{C}$ & -3.369460 & -0.528714 & 0.424221 & $\mathrm{H}$ & 5.187782 & 3.406844 & 2.326344 \\
\hline $\mathrm{C}$ & -3.601671 & 0.341093 & 1.498685 & $\mathrm{O}$ & 0.614897 & -0.393251 & -2.214178 \\
\hline $\mathrm{C}$ & -4.208741 & -0.548219 & -0.697668 & $\mathrm{C}$ & -0.501504 & 0.029953 & -2.877806 \\
\hline $\mathrm{C}$ & -4.693348 & 1.206892 & 1.417981 & $\mathrm{H}$ & -1.087249 & -0.792715 & -3.338299 \\
\hline $\mathrm{C}$ & -5.286100 & 0.335546 & -0.735986 & $\mathrm{H}$ & -0.305071 & 0.790431 & -3.662775 \\
\hline $\mathrm{C}$ & -5.546025 & 1.219510 & 0.313612 & $\mathrm{H}$ & -1.262087 & 0.555264 & -2.204246 \\
\hline $\mathrm{H}$ & -4.881791 & 1.891970 & 2.244077 & $\mathrm{H}$ & -1.329913 & 1.306266 & 0.201001 \\
\hline $\mathrm{H}$ & -5.939396 & 0.332552 & -1.607738 & & & & \\
\hline $\mathrm{C}$ & 0.144067 & -3.251845 & -2.011002 & TS 8 -9 & & & \\
\hline $\mathrm{H}$ & 0.475630 & -3.033326 & -3.028020 & $\mathrm{Ru}$ & -0.105058 & 0.364878 & -0.492503 \\
\hline $\mathrm{H}$ & -0.206162 & -4.293009 & -1.991644 & $\mathrm{C}$ & 0.813014 & 3.359600 & -0.597495 \\
\hline $\mathrm{H}$ & -0.711902 & -2.603574 & -1.808420 & $\mathrm{C}$ & 1.676999 & 2.197036 & -2.387750 \\
\hline $\mathrm{C}$ & 1.857282 & -2.222709 & 2.659663 & $\mathrm{C}$ & 1.484137 & 4.517097 & -0.963239 \\
\hline $\mathrm{H}$ & 2.339290 & -2.953896 & 3.317131 & $\mathrm{H}$ & 0.191623 & 3.319276 & 0.293697 \\
\hline $\mathrm{H}$ & 2.287517 & -1.243707 & 2.902319 & $\mathrm{C}$ & 2.374983 & 3.315188 & -2.826089 \\
\hline $\mathrm{H}$ & 0.794421 & -2.179484 & 2.909170 & $\mathrm{H}$ & 1.738994 & 1.234128 & -2.890723 \\
\hline $\mathrm{C}$ & 5.048329 & -3.470594 & -1.002398 & $\mathrm{H}$ & 1.385242 & 5.409770 & -0.354106 \\
\hline $\mathrm{H}$ & 5.727152 & -3.641776 & -0.162611 & $\mathrm{H}$ & 2.988848 & 3.248052 & -3.718791 \\
\hline $\mathrm{H}$ & 5.103623 & -4.340508 & -1.664256 & $\mathrm{~N}$ & 0.907279 & 2.207197 & -1.284962 \\
\hline $\mathrm{H}$ & 5.442537 & -2.619291 & -1.569310 & $\mathrm{C}$ & 2.285119 & 4.498047 & -2.100436 \\
\hline $\mathrm{C}$ & -2.677087 & 0.378699 & 2.674216 & $\mathrm{H}$ & 2.830748 & 5.384601 & -2.410991 \\
\hline $\mathrm{H}$ & -3.139988 & 0.879279 & 3.528401 & $\mathrm{C}$ & -0.975613 & -1.211440 & 0.317780 \\
\hline $\mathrm{H}$ & -2.363288 & -0.621750 & 2.992477 & $\mathrm{C}$ & -1.228131 & -3.378559 & 1.230315 \\
\hline $\mathrm{H}$ & -1.757706 & 0.917157 & 2.412299 & $\mathrm{C}$ & -2.545392 & -2.637714 & 1.371332 \\
\hline $\mathrm{C}$ & -3.933839 & -1.489940 & -1.828271 & $\mathrm{H}$ & -1.277190 & -4.162239 & 0.459118 \\
\hline $\mathrm{H}$ & -2.940870 & -1.315113 & -2.257734 & $\mathrm{H}$ & -0.885187 & -3.844975 & 2.159910 \\
\hline $\mathrm{H}$ & -3.944008 & -2.537369 & -1.503786 & $\mathrm{H}$ & -3.406849 & -3.200493 & 0.995381 \\
\hline $\mathrm{H}$ & -4.673339 & -1.380418 & -2.625013 & $\mathrm{H}$ & -2.761639 & -2.359773 & 2.415114 \\
\hline $\mathrm{C}$ & -6.731404 & 2.135232 & 0.268575 & $\mathrm{~N}$ & -0.315970 & -2.311918 & 0.810374 \\
\hline $\mathrm{H}$ & -6.602764 & 2.995397 & 0.930959 & $\mathrm{~N}$ & -2.306944 & -1.436806 & 0.568769 \\
\hline $\mathrm{H}$ & -6.914196 & 2.511961 & -0.741947 & $\mathrm{C}$ & 1.002610 & -2.679314 & 0.388557 \\
\hline $\mathrm{H}$ & -7.646475 & 1.620431 & 0.584689 & $\mathrm{C}$ & 2.071374 & -2.589253 & 1.290500 \\
\hline $\mathrm{C}$ & 0.792334 & 0.535149 & 1.215609 & $\mathrm{C}$ & 1.189002 & -3.180875 & -0.912470 \\
\hline $\mathrm{H}$ & 0.423180 & -0.006471 & 2.100928 & $\mathrm{C}$ & 3.346788 & -2.939968 & 0.842204 \\
\hline $\mathrm{C}$ & 2.002056 & 1.303861 & 1.511017 & $\mathrm{C}$ & 2.481281 & -3.510201 & -1.314346 \\
\hline $\mathrm{C}$ & 2.131310 & 1.981392 & 2.737862 & $\mathrm{C}$ & 3.576781 & -3.381289 & -0.458941 \\
\hline $\mathrm{C}$ & 3.080342 & 1.364572 & 0.609278 & $\mathrm{H}$ & 4.184302 & -2.860770 & 1.534865 \\
\hline $\mathrm{C}$ & 3.258274 & 2.743022 & 3.022104 & $\mathrm{H}$ & 2.634956 & -3.879698 & -2.327836 \\
\hline $\mathrm{H}$ & 1.317964 & 1.917225 & 3.459740 & $\mathrm{C}$ & -3.350643 & -0.473676 & 0.456849 \\
\hline
\end{tabular}




\begin{tabular}{|c|c|c|c|c|c|c|c|}
\hline $\mathrm{C}$ & -3.485756 & 0.538588 & 1.417350 & $\mathrm{O}$ & 0.602185 & -0.560395 & -2.311059 \\
\hline $\mathrm{C}$ & -4.254567 & -0.594002 & -0.609373 & $\mathrm{C}$ & -0.548959 & -0.072811 & -2.690305 \\
\hline $\mathrm{C}$ & -4.539644 & 1.443991 & 1.275465 & $\mathrm{H}$ & -1.370987 & -0.784536 & -2.889831 \\
\hline $\mathrm{C}$ & -5.289123 & 0.333956 & -0.712815 & $\mathrm{H}$ & -0.549148 & 0.806690 & -3.360270 \\
\hline $\mathrm{C}$ & -5.448913 & 1.360795 & 0.221220 & $\mathrm{H}$ & -1.491495 & 0.832745 & -1.461081 \\
\hline $\mathrm{H}$ & -4.652244 & 2.236785 & 2.014303 & $\mathrm{H}$ & -1.553332 & 1.237406 & -0.535179 \\
\hline $\mathrm{H}$ & -5.987868 & 0.253543 & -1.544883 & & & & \\
\hline $\mathrm{C}$ & 0.037158 & -3.411767 & -1.842676 & 9 & & & \\
\hline $\mathrm{H}$ & 0.359663 & -3.338749 & -2.883307 & $\mathrm{Ru}$ & -0.151229 & 0.394398 & -0.501964 \\
\hline $\mathrm{H}$ & -0.392875 & -4.412368 & -1.700071 & $\mathrm{C}$ & 0.723706 & 3.410739 & -0.525770 \\
\hline $\mathrm{H}$ & -0.762658 & -2.680712 & -1.698977 & $\mathrm{C}$ & 1.616245 & 2.299069 & -2.332957 \\
\hline $\mathrm{C}$ & 1.891581 & -2.141715 & 2.709324 & $\mathrm{C}$ & 1.366020 & 4.590377 & -0.873233 \\
\hline $\mathrm{H}$ & 2.373411 & -2.841584 & 3.400312 & $\mathrm{H}$ & 0.103855 & 3.341559 & 0.365098 \\
\hline $\mathrm{H}$ & 2.350277 & -1.160301 & 2.875159 & $\mathrm{C}$ & 2.290138 & 3.439112 & -2.751013 \\
\hline $\mathrm{H}$ & 0.837458 & -2.052931 & 2.982672 & $\mathrm{H}$ & 1.710494 & 1.345456 & -2.847278 \\
\hline $\mathrm{C}$ & 4.961622 & -3.702973 & -0.932047 & $\mathrm{H}$ & 1.241119 & 5.472598 & -0.253680 \\
\hline $\mathrm{H}$ & 5.635225 & -3.916172 & -0.097459 & $\mathrm{H}$ & 2.909194 & 3.399043 & -3.641670 \\
\hline $\mathrm{H}$ & 4.971309 & -4.566655 & -1.603780 & $\mathrm{~N}$ & 0.840016 & 2.273061 & -1.234473 \\
\hline $\mathrm{H}$ & 5.395647 & -2.864842 & -1.489536 & $\mathrm{C}$ & 2.168003 & 4.609148 & -2.010002 \\
\hline $\mathrm{C}$ & -2.502163 & 0.685209 & 2.534048 & $\mathrm{H}$ & 2.690423 & 5.514155 & -2.307107 \\
\hline $\mathrm{H}$ & -2.929002 & 1.249242 & 3.367471 & $\mathrm{C}$ & -0.973726 & -1.223040 & 0.280990 \\
\hline $\mathrm{H}$ & -2.152145 & -0.280720 & 2.913481 & $\mathrm{C}$ & -1.179206 & -3.413814 & 1.150865 \\
\hline $\mathrm{H}$ & -1.603859 & 1.209951 & 2.183185 & $\mathrm{C}$ & -2.508535 & -2.701176 & 1.320524 \\
\hline $\mathrm{C}$ & -4.090134 & -1.689214 & -1.616680 & $\mathrm{H}$ & -1.220365 & -4.179802 & 0.361304 \\
\hline $\mathrm{H}$ & -3.080504 & -1.688804 & -2.042805 & $\mathrm{H}$ & -0.819375 & -3.896019 & 2.065971 \\
\hline $\mathrm{H}$ & -4.231867 & -2.681459 & -1.171455 & $\mathrm{H}$ & -3.363022 & -3.270894 & 0.938629 \\
\hline $\mathrm{H}$ & -4.808761 & -1.589008 & -2.433451 & $\mathrm{H}$ & -2.720131 & -2.453951 & 2.372982 \\
\hline $\mathrm{C}$ & -6.588183 & 2.327320 & 0.107717 & $\mathrm{~N}$ & -0.290261 & -2.320791 & 0.749633 \\
\hline $\mathrm{H}$ & -6.401865 & 3.240574 & 0.678814 & $\mathrm{~N}$ & -2.298394 & -1.479745 & 0.543287 \\
\hline $\mathrm{H}$ & -6.775837 & 2.611644 & -0.931871 & $\mathrm{C}$ & 1.040462 & -2.655924 & 0.341305 \\
\hline $\mathrm{H}$ & -7.520039 & 1.893120 & 0.488447 & $\mathrm{C}$ & 2.091482 & -2.570860 & 1.265112 \\
\hline $\mathrm{C}$ & 0.821857 & 0.553309 & 1.177949 & $\mathrm{C}$ & 1.258049 & -3.131301 & -0.964239 \\
\hline $\mathrm{H}$ & 0.458102 & 0.056956 & 2.092936 & $\mathrm{C}$ & 3.379949 & -2.894297 & 0.834202 \\
\hline $\mathrm{C}$ & 1.958877 & 1.418871 & 1.500875 & $\mathrm{C}$ & 2.561979 & -3.435144 & -1.348106 \\
\hline $\mathrm{C}$ & 1.968818 & 2.227068 & 2.654515 & $\mathrm{C}$ & 3.639916 & -3.306470 & -0.470853 \\
\hline $\mathrm{C}$ & 3.090722 & 1.470051 & 0.664273 & $\mathrm{H}$ & 4.203299 & -2.817066 & 1.543857 \\
\hline $\mathrm{C}$ & 3.021991 & 3.094435 & 2.919997 & $\mathrm{H}$ & 2.739525 & -3.782508 & -2.365589 \\
\hline $\mathrm{H}$ & 1.115316 & 2.183480 & 3.330896 & $\mathrm{C}$ & -3.359641 & -0.534471 & 0.455097 \\
\hline $\mathrm{C}$ & 4.154605 & 2.319163 & 0.940785 & $\mathrm{C}$ & -3.522204 & 0.442051 & 1.447797 \\
\hline $\mathrm{H}$ & 3.108092 & 0.814069 & -0.205155 & $\mathrm{C}$ & -4.243499 & -0.628193 & -0.629662 \\
\hline $\mathrm{C}$ & 4.119975 & 3.148497 & 2.061682 & $\mathrm{C}$ & -4.588345 & 1.335488 & 1.323208 \\
\hline $\mathrm{H}$ & 2.993602 & 3.725345 & 3.805450 & $\mathrm{C}$ & -5.294110 & 0.283825 & -0.712997 \\
\hline $\mathrm{H}$ & 5.016440 & 2.338324 & 0.277348 & $\mathrm{C}$ & -5.483729 & 1.273265 & 0.255038 \\
\hline $\mathrm{H}$ & 4.948193 & 3.819581 & 2.273859 & $\mathrm{H}$ & -4.722444 & 2.101037 & 2.086683 \\
\hline
\end{tabular}




\begin{tabular}{|c|c|c|c|c|c|c|c|}
\hline $\mathrm{H}$ & -5.978995 & 0.223518 & -1.558127 & & & & \\
\hline $\mathrm{C}$ & 0.127206 & -3.361176 & -1.920143 & 10 & & & \\
\hline $\mathrm{H}$ & 0.460207 & -3.231554 & -2.952139 & $\mathrm{Ru}$ & -0.412285 & 0.712356 & -0.808063 \\
\hline $\mathrm{H}$ & -0.267215 & -4.382121 & -1.827840 & $\mathrm{C}$ & -0.494770 & -1.113224 & -0.080451 \\
\hline $\mathrm{H}$ & -0.702422 & -2.668240 & -1.756279 & $\mathrm{C}$ & -0.146191 & -3.419973 & 0.364075 \\
\hline $\mathrm{C}$ & 1.879232 & -2.160645 & 2.691114 & $\mathrm{C}$ & -1.577180 & -3.066271 & 0.742240 \\
\hline $\mathrm{H}$ & 2.347483 & -2.877634 & 3.373911 & $\mathrm{H}$ & -0.113056 & -4.055868 & -0.536807 \\
\hline $\mathrm{H}$ & 2.331801 & -1.183125 & 2.893673 & $\mathrm{H}$ & 0.391609 & -3.945664 & 1.161701 \\
\hline $\mathrm{H}$ & 0.818979 & -2.082110 & 2.943142 & $\mathrm{H}$ & -2.317510 & -3.734202 & 0.284008 \\
\hline $\mathrm{C}$ & 5.037586 & -3.598073 & -0.925439 & $\mathrm{H}$ & -1.734327 & -3.098816 & 1.833763 \\
\hline $\mathrm{H}$ & 5.701843 & -3.808742 & -0.082779 & $\mathrm{~N}$ & 0.446861 & -2.109924 & 0.113429 \\
\hline $\mathrm{H}$ & 5.073257 & -4.453792 & -1.606559 & $\mathrm{~N}$ & -1.703106 & -1.712271 & 0.221776 \\
\hline $\mathrm{H}$ & 5.464901 & -2.746129 & -1.466965 & $\mathrm{C}$ & 1.841110 & -1.902740 & 0.242142 \\
\hline $\mathrm{C}$ & -2.551915 & 0.563496 & 2.579826 & $\mathrm{C}$ & 2.331029 & -1.140494 & 1.317559 \\
\hline $\mathrm{H}$ & -2.965591 & 1.164118 & 3.393888 & $\mathrm{C}$ & 2.712851 & -2.460902 & -0.705886 \\
\hline $\mathrm{H}$ & -2.259185 & -0.410163 & 2.987656 & $\mathrm{C}$ & 3.706165 & -0.920907 & 1.394513 \\
\hline $\mathrm{H}$ & -1.622283 & 1.036595 & 2.236979 & $\mathrm{C}$ & 4.082241 & -2.232331 & -0.573964 \\
\hline $\mathrm{C}$ & -4.028551 & -1.667604 & -1.685252 & $\mathrm{C}$ & 4.596491 & -1.450292 & 0.460817 \\
\hline $\mathrm{H}$ & -3.040193 & -1.557394 & -2.146822 & $\mathrm{H}$ & 4.088148 & -0.285409 & 2.193840 \\
\hline $\mathrm{H}$ & -4.061917 & -2.684960 & -1.277745 & $\mathrm{H}$ & 4.758910 & -2.637802 & -1.327245 \\
\hline $\mathrm{H}$ & -4.783580 & -1.597729 & -2.471759 & $\mathrm{C}$ & -2.961394 & -1.042974 & 0.216783 \\
\hline $\mathrm{C}$ & -6.639316 & 2.222520 & 0.162998 & $\mathrm{C}$ & -3.471757 & -0.524098 & 1.418453 \\
\hline $\mathrm{H}$ & -6.464215 & 3.130588 & 0.745911 & $\mathrm{C}$ & -3.694574 & -0.963544 & -0.978920 \\
\hline $\mathrm{H}$ & -6.839543 & 2.517960 & -0.870994 & $\mathrm{C}$ & -4.726069 & 0.084669 & 1.396564 \\
\hline $\mathrm{H}$ & -7.560753 & 1.767534 & 0.544992 & $\mathrm{C}$ & -4.940455 & -0.336561 & -0.952249 \\
\hline $\mathrm{C}$ & 0.811783 & 0.542012 & 1.163183 & $\mathrm{C}$ & -5.470166 & 0.197997 & 0.222190 \\
\hline $\mathrm{H}$ & 0.469108 & 0.021401 & 2.073534 & $\mathrm{H}$ & -5.115990 & 0.508363 & 2.322533 \\
\hline $\mathrm{C}$ & 1.966230 & 1.380997 & 1.488768 & $\mathrm{H}$ & -5.509513 & -0.261375 & -1.879042 \\
\hline $\mathrm{C}$ & 2.009727 & 2.151464 & 2.667220 & $\mathrm{C}$ & 2.166017 & -3.213528 & -1.878489 \\
\hline $\mathrm{C}$ & 3.080840 & 1.442281 & 0.630234 & $\mathrm{H}$ & 1.289501 & -2.699691 & -2.289913 \\
\hline $\mathrm{C}$ & 3.081560 & 2.992895 & 2.940076 & $\mathrm{H}$ & 2.915308 & -3.310196 & -2.669411 \\
\hline $\mathrm{H}$ & 1.168719 & 2.098001 & 3.358462 & $\mathrm{H}$ & 1.842538 & -4.228969 & -1.612559 \\
\hline $\mathrm{C}$ & 4.167237 & 2.258629 & 0.918641 & $\mathrm{C}$ & 1.401084 & -0.548660 & 2.325455 \\
\hline $\mathrm{H}$ & 3.066200 & 0.818670 & -0.262852 & $\mathrm{H}$ & 0.686040 & -1.296140 & 2.693901 \\
\hline $\mathrm{C}$ & 4.166335 & 3.052244 & 2.065646 & $\mathrm{H}$ & 1.957146 & -0.151037 & 3.179778 \\
\hline $\mathrm{H}$ & 3.079336 & 3.596981 & 3.844435 & $\mathrm{H}$ & 0.788935 & 0.263030 & 1.903999 \\
\hline $\mathrm{H}$ & 5.017841 & 2.284128 & 0.241091 & $\mathrm{C}$ & 6.065754 & -1.170603 & 0.559019 \\
\hline $\mathrm{H}$ & 5.010986 & 3.700048 & 2.285065 & $\mathrm{H}$ & 6.606649 & -1.974842 & 1.074657 \\
\hline $\mathrm{O}$ & 0.711115 & -0.505395 & -2.251673 & $\mathrm{H}$ & 6.520471 & -1.066304 & -0.432050 \\
\hline $\mathrm{C}$ & -0.428916 & -0.066905 & -2.700331 & $\mathrm{H}$ & 6.256176 & -0.244220 & 1.109204 \\
\hline $\mathrm{H}$ & -1.223875 & -0.799326 & -2.924068 & $\mathrm{C}$ & -2.648983 & -0.544932 & 2.664954 \\
\hline $\mathrm{H}$ & -0.440766 & 0.836522 & -3.336023 & $\mathrm{H}$ & -1.728515 & 0.026203 & 2.465374 \\
\hline $\mathrm{H}$ & -1.672596 & 0.936613 & -1.298362 & $\mathrm{H}$ & -3.187309 & -0.082518 & 3.497298 \\
\hline $\mathrm{H}$ & -1.646450 & 1.315602 & -0.503053 & $\mathrm{H}$ & -2.364631 & -1.558910 & 2.970730 \\
\hline
\end{tabular}




\begin{tabular}{|c|c|c|c|c|c|c|c|}
\hline $\mathrm{C}$ & -3.136286 & -1.513139 & -2.251789 & $\mathrm{C}$ & -1.655401 & 1.700037 & 0.383410 \\
\hline $\mathrm{H}$ & -2.293760 & -0.893432 & -2.587833 & $\mathrm{C}$ & -2.300462 & 1.134247 & 1.490147 \\
\hline $\mathrm{H}$ & -2.735081 & -2.524485 & -2.116503 & $\mathrm{C}$ & -2.369614 & 2.179456 & -0.722855 \\
\hline $\mathrm{H}$ & -3.895487 & -1.540101 & -3.038844 & $\mathrm{C}$ & -3.696472 & 1.093062 & 1.481367 \\
\hline $\mathrm{C}$ & -6.786904 & 0.913943 & 0.213921 & $\mathrm{C}$ & -3.760563 & 2.114518 & -0.687044 \\
\hline $\mathrm{H}$ & -7.311989 & 0.812028 & 1.169260 & $\mathrm{C}$ & -4.441636 & 1.582441 & 0.410544 \\
\hline $\mathrm{H}$ & -6.652448 & 1.988076 & 0.037866 & $\mathrm{H}$ & -4.212173 & 0.623179 & 2.318591 \\
\hline $\mathrm{H}$ & -7.446974 & 0.542212 & -0.576585 & $\mathrm{H}$ & -4.326953 & 2.455203 & -1.554598 \\
\hline $\mathrm{C}$ & 1.399019 & 0.669020 & -1.188298 & $\mathrm{C}$ & 3.169712 & 0.857274 & 0.256545 \\
\hline $\mathrm{H}$ & 1.876867 & -0.023468 & -1.905541 & $\mathrm{C}$ & 3.490905 & 0.063423 & 1.365418 \\
\hline $\mathrm{C}$ & 2.390080 & 1.563295 & -0.590333 & $\mathrm{C}$ & 4.071330 & 1.067798 & -0.787974 \\
\hline $\mathrm{C}$ & 3.723963 & 1.539155 & -1.048505 & $\mathrm{C}$ & 4.767764 & -0.495425 & 1.415571 \\
\hline $\mathrm{C}$ & 2.087794 & 2.415644 & 0.495264 & $\mathrm{C}$ & 5.338533 & 0.493524 & -0.694390 \\
\hline $\mathrm{C}$ & 4.709495 & 2.321667 & -0.460128 & $\mathrm{C}$ & 5.701876 & -0.292164 & 0.399360 \\
\hline $\mathrm{H}$ & 3.975406 & 0.870574 & -1.872160 & $\mathrm{H}$ & 5.031829 & -1.122026 & 2.267803 \\
\hline $\mathrm{C}$ & 3.077970 & 3.190901 & 1.083993 & $\mathrm{H}$ & 6.050382 & 0.645489 & -1.506372 \\
\hline $\mathrm{H}$ & 1.065365 & 2.411197 & 0.880021 & $\mathrm{C}$ & -1.636906 & 2.663035 & -1.934247 \\
\hline $\mathrm{C}$ & 4.392705 & 3.153295 & 0.614124 & $\mathrm{H}$ & -0.941943 & 1.887634 & -2.284152 \\
\hline $\mathrm{H}$ & 5.732193 & 2.277397 & -0.833135 & $\mathrm{H}$ & -2.331066 & 2.905412 & -2.744152 \\
\hline $\mathrm{H}$ & 2.824552 & 3.830931 & 1.927459 & $\mathrm{H}$ & -1.035904 & 3.559135 & -1.732593 \\
\hline $\mathrm{H}$ & 5.162655 & 3.764554 & 1.081319 & $\mathrm{C}$ & -1.502246 & 0.521763 & 2.595008 \\
\hline $\mathrm{O}$ & -0.904306 & 1.503895 & 1.179611 & $\mathrm{H}$ & -0.776328 & 1.229043 & 3.016419 \\
\hline $\mathrm{H}$ & -0.470507 & 0.017419 & -2.261535 & $\mathrm{H}$ & -2.154329 & 0.178377 & 3.404180 \\
\hline $\mathrm{C}$ & -1.882337 & 2.444208 & 1.403669 & $\mathrm{H}$ & -0.921774 & -0.339427 & 2.219180 \\
\hline $\mathrm{H}$ & -1.479788 & 3.424195 & 1.746593 & $\mathrm{C}$ & -5.938810 & 1.517637 & 0.423928 \\
\hline $\mathrm{H}$ & -2.504538 & 2.662577 & 0.513056 & $\mathrm{H}$ & -6.386635 & 2.441965 & 0.812603 \\
\hline $\mathrm{H}$ & -2.592051 & 2.122695 & 2.201410 & $\mathrm{H}$ & -6.339403 & 1.364917 & -0.583607 \\
\hline $\mathrm{O}$ & -1.311644 & 2.322969 & -1.675164 & $\mathrm{H}$ & -6.293658 & 0.691555 & 1.047367 \\
\hline $\mathrm{C}$ & -0.837324 & 3.571453 & -1.331561 & $\mathrm{C}$ & 2.466517 & -0.215235 & 2.413817 \\
\hline $\mathrm{H}$ & -0.419456 & 3.639404 & -0.307071 & $\mathrm{H}$ & 1.606056 & -0.756947 & 1.973247 \\
\hline $\mathrm{H}$ & -0.024270 & 3.909769 & -2.009423 & $\mathrm{H}$ & 2.886787 & -0.819057 & 3.224059 \\
\hline \multirow[t]{2}{*}{$\mathrm{H}$} & -1.635672 & 4.339157 & -1.390305 & $\mathrm{H}$ & 2.066814 & 0.707488 & 2.857288 \\
\hline & & & & $\mathrm{C}$ & 3.641861 & 1.833784 & -1.998861 \\
\hline \multicolumn{2}{|c|}{$\mathbf{T S}_{10-11}$} & & & $\mathrm{H}$ & 2.790045 & 1.330016 & -2.472261 \\
\hline $\mathrm{Ru}$ & 0.296060 & -0.855642 & -0.991290 & $\mathrm{H}$ & 3.305502 & 2.849555 & -1.755681 \\
\hline $\mathrm{C}$ & 0.693615 & 0.924428 & 0.016203 & $\mathrm{H}$ & 4.452697 & 1.912422 & -2.729046 \\
\hline $\mathrm{C}$ & 0.326081 & 3.116440 & 0.841111 & $\mathrm{C}$ & 7.052404 & -0.941130 & 0.460725 \\
\hline $\mathrm{C}$ & 1.813832 & 2.814988 & 0.893237 & $\mathrm{H}$ & 7.437249 & -0.986844 & 1.485148 \\
\hline $\mathrm{H}$ & 0.080693 & 3.909707 & 0.117683 & $\mathrm{H}$ & 7.015972 & -1.973103 & 0.090949 \\
\hline $\mathrm{H}$ & -0.091724 & 3.417091 & 1.810053 & $\mathrm{H}$ & 7.787087 & -0.407790 & -0.151404 \\
\hline $\mathrm{H}$ & 2.429623 & 3.566656 & 0.382755 & $\mathrm{C}$ & -1.555354 & -0.895320 & -1.402037 \\
\hline $\mathrm{H}$ & 2.189918 & 2.727678 & 1.924408 & $\mathrm{H}$ & -1.865229 & -0.538991 & -2.407885 \\
\hline $\mathrm{N}$ & -0.238188 & 1.834714 & 0.413030 & $\mathrm{C}$ & -2.762727 & -1.392998 & -0.736003 \\
\hline $\mathrm{N}$ & 1.905007 & 1.517786 & 0.215942 & $\mathrm{C}$ & -4.010772 & -1.235695 & -1.378279 \\
\hline
\end{tabular}




\begin{tabular}{|c|c|c|c|c|c|c|c|}
\hline $\mathrm{C}$ & -2.763176 & -2.021220 & 0.529369 & $\mathrm{C}$ & 5.411538 & 0.464579 & -0.684256 \\
\hline $\mathrm{C}$ & -5.198182 & -1.651253 & -0.788867 & $\mathrm{C}$ & 5.759433 & -0.351803 & 0.391547 \\
\hline $\mathrm{H}$ & -4.028319 & -0.753765 & -2.356771 & $\mathrm{H}$ & 5.064940 & -1.222994 & 2.231668 \\
\hline $\mathrm{C}$ & -3.951312 & -2.440598 & 1.113152 & $\mathrm{H}$ & 6.134094 & 0.638155 & -1.482282 \\
\hline $\mathrm{H}$ & -1.791351 & -2.128084 & 1.018711 & $\mathrm{C}$ & -1.563504 & 2.683194 & -1.926303 \\
\hline $\mathrm{C}$ & -5.176608 & -2.255038 & 0.467722 & $\mathrm{H}$ & -0.928375 & 1.879991 & -2.321459 \\
\hline $\mathrm{H}$ & -6.145403 & -1.501860 & -1.306230 & $\mathrm{H}$ & -2.253960 & 2.999423 & -2.713371 \\
\hline $\mathrm{H}$ & -3.926203 & -2.915276 & 2.093718 & $\mathrm{H}$ & -0.899276 & 3.527660 & -1.702750 \\
\hline $\mathrm{H}$ & -6.103958 & -2.581853 & 0.935991 & $\mathrm{C}$ & -1.474726 & 0.541378 & 2.608076 \\
\hline $\mathrm{O}$ & 0.106481 & -1.804150 & 1.064733 & $\mathrm{H}$ & -0.739456 & 1.241392 & 3.026380 \\
\hline $\mathrm{H}$ & 0.528098 & 0.063091 & -2.317003 & $\mathrm{H}$ & -2.136671 & 0.217635 & 3.417107 \\
\hline $\mathrm{C}$ & 0.623319 & -3.047323 & 1.302512 & $\mathrm{H}$ & -0.909937 & -0.330248 & 2.237990 \\
\hline $\mathrm{H}$ & 0.066037 & -3.875796 & 0.797728 & $\mathrm{C}$ & -5.885330 & 1.605033 & 0.422313 \\
\hline $\mathrm{H}$ & 1.685694 & -3.161458 & 0.990039 & $\mathrm{H}$ & -6.313872 & 2.513358 & 0.866824 \\
\hline $\mathrm{H}$ & 0.606105 & -3.302421 & 2.387091 & $\mathrm{H}$ & -6.290055 & 1.518119 & -0.591091 \\
\hline $\mathrm{O}$ & 2.439750 & -1.617583 & -0.954000 & $\mathrm{H}$ & -6.252757 & 0.749407 & 0.996374 \\
\hline $\mathrm{C}$ & 1.707995 & -2.468818 & -1.595483 & $\mathrm{C}$ & 2.502857 & -0.307859 & 2.372752 \\
\hline $\mathrm{H}$ & 0.438789 & -1.648505 & -2.406037 & $\mathrm{H}$ & 1.627489 & -0.795303 & 1.907769 \\
\hline $\mathrm{H}$ & 1.962286 & -2.684070 & -2.655563 & $\mathrm{H}$ & 2.900323 & -0.964505 & 3.152499 \\
\hline \multirow[t]{2}{*}{$\mathrm{H}$} & 1.317462 & -3.367528 & -1.082197 & $\mathrm{H}$ & 2.133005 & 0.604562 & 2.861705 \\
\hline & & & & $\mathrm{C}$ & 3.744800 & 1.854558 & -1.969366 \\
\hline 11 & & & & $\mathrm{H}$ & 2.914423 & 1.363797 & -2.490463 \\
\hline $\mathrm{Ru}$ & 0.251378 & -0.893476 & -0.974243 & $\mathrm{H}$ & 3.391361 & 2.857333 & -1.698950 \\
\hline $\mathrm{C}$ & 0.748682 & 0.894598 & -0.011259 & $\mathrm{H}$ & 4.574598 & 1.967611 & -2.673181 \\
\hline $\mathrm{C}$ & 0.400001 & 3.065165 & 0.882531 & $\mathrm{C}$ & 7.106111 & -1.008579 & 0.450945 \\
\hline $\mathrm{C}$ & 1.883677 & 2.747901 & 0.932257 & $\mathrm{H}$ & 7.480680 & -1.080294 & 1.477650 \\
\hline $\mathrm{H}$ & 0.164157 & 3.882553 & 0.182788 & $\mathrm{H}$ & 7.068283 & -2.031361 & 0.056647 \\
\hline $\mathrm{H}$ & -0.021057 & 3.339197 & 1.857843 & $\mathrm{H}$ & 7.849390 & -0.464734 & -0.141180 \\
\hline $\mathrm{H}$ & 2.510494 & 3.509799 & 0.451525 & $\mathrm{C}$ & -1.623626 & -0.965337 & -1.394842 \\
\hline $\mathrm{H}$ & 2.253463 & 2.620411 & 1.961295 & $\mathrm{H}$ & -1.932379 & -0.520298 & -2.365169 \\
\hline $\mathrm{N}$ & -0.174999 & 1.802752 & 0.412045 & $\mathrm{C}$ & -2.847010 & -1.404106 & -0.729683 \\
\hline $\mathrm{N}$ & 1.965904 & 1.472306 & 0.209322 & $\mathrm{C}$ & -4.093194 & -1.217399 & -1.375975 \\
\hline $\mathrm{C}$ & -1.597026 & 1.695110 & 0.380959 & $\mathrm{C}$ & -2.889308 & -2.012363 & 0.548067 \\
\hline $\mathrm{C}$ & -2.256262 & 1.153188 & 1.491183 & $\mathrm{C}$ & -5.296536 & -1.591177 & -0.792562 \\
\hline $\mathrm{C}$ & -2.302557 & 2.198081 & -0.719625 & $\mathrm{H}$ & -4.093247 & -0.744284 & -2.359232 \\
\hline $\mathrm{C}$ & -3.652488 & 1.142164 & 1.482121 & $\mathrm{C}$ & -4.093418 & -2.379734 & 1.131971 \\
\hline $\mathrm{C}$ & -3.694096 & 2.160695 & -0.686407 & $\mathrm{H}$ & -1.937614 & -2.142580 & 1.058372 \\
\hline $\mathrm{C}$ & -4.387324 & 1.640834 & 0.408945 & $\mathrm{C}$ & -5.310053 & -2.171862 & 0.475854 \\
\hline $\mathrm{H}$ & -4.177569 & 0.685710 & 2.320801 & $\mathrm{H}$ & -6.233249 & -1.417944 & -1.322735 \\
\hline $\mathrm{H}$ & -4.252369 & 2.517597 & -1.552651 & $\mathrm{H}$ & -4.087488 & -2.833695 & 2.123067 \\
\hline $\mathrm{C}$ & 3.229055 & 0.805932 & 0.245803 & $\mathrm{H}$ & -6.250507 & -2.461622 & 0.941958 \\
\hline $\mathrm{C}$ & 3.537986 & -0.013310 & 1.338991 & $\mathrm{O}$ & 0.028517 & -1.732460 & 0.979075 \\
\hline $\mathrm{C}$ & 4.147312 & 1.045814 & -0.776742 & $\mathrm{H}$ & 0.607712 & 0.073691 & -2.373382 \\
\hline $\mathrm{C}$ & 4.812601 & -0.575391 & 1.391631 & $\mathrm{C}$ & 0.503274 & -3.000955 & 1.220802 \\
\hline
\end{tabular}




$\begin{array}{crrc}\mathrm{H} & -0.043827 & -3.797229 & 0.667468 \\ \mathrm{H} & 1.578224 & -3.128551 & 0.977834 \\ \mathrm{H} & 0.397474 & -3.248384 & 2.298310 \\ \mathrm{O} & 2.400374 & -1.564412 & -0.924885 \\ \mathrm{C} & 1.620967 & -2.400136 & -1.568110 \\ \mathrm{H} & 0.295847 & -0.681538 & -2.676152 \\ \mathrm{H} & 1.746561 & -2.491092 & -2.669244 \\ \mathrm{H} & 1.382019 & -3.377510 & -1.106715\end{array}$

12

$\mathrm{Ru}$$$
\mathrm{H}
$$

$$
\begin{aligned}
& \mathrm{H} \\
& \mathrm{H} \\
& \mathrm{H}
\end{aligned}
$$

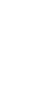

C$$
\text { C }
$$

$\mathrm{c}$

C

C

c

c$$
\begin{array}{llll}
\mathrm{C} & 5.682577 & -0.609072 & 0.177657 \\
\mathrm{H} & 5.165026 & -0.725836 & 2.261583
\end{array}
$$$$
\begin{array}{llll}
\mathrm{H} & 5.898579 & -0.325456 & -1.944806
\end{array}
$$$$
\begin{array}{llll}
\text { C } & -1.257621 & 1.226361 & -2.415713
\end{array}
$$$$
\begin{array}{llll}
\mathrm{H} & -0.797263 & 0.234614 & -2.305826
\end{array}
$$$$
\begin{array}{llll}
\mathrm{H} & -1.873765 & 1.227809 & -3.319445
\end{array}
$$$$
\begin{array}{llll}
\mathrm{H} & -0.427660 & 1.926769 & -2.567255
\end{array}
$$$$
\begin{array}{llll}
\text { C } & -1.565637 & 2.129943 & 2.519416
\end{array}
$$$$
\begin{array}{llll}
\mathrm{H} & -0.782243 & 2.896738 & 2.520588
\end{array}
$$$$
\begin{array}{llll}
\mathrm{H} & -2.280686 & 2.363340 & 3.314269
\end{array}
$$

\begin{tabular}{|c|c|c|c|}
\hline & -5.759722 & 1.728009 & -0.245003 \\
\hline & -6.133904 & 2.209683 & -1.154945 \\
\hline & -6.090771 & 0.682242 & -0.273805 \\
\hline & -6.242532 & 2.204144 & 0.614483 \\
\hline & 2.770033 & 0.522874 & 2.358101 \\
\hline & 1.894251 & -0.143640 & 2.378417 \\
\hline & 3.342086 & 0.372802 & 3.279135 \\
\hline & 2.357637 & 1.539252 & 2.373211 \\
\hline & 3.575714 & 0.926900 & -2.598781 \\
\hline & 2.625526 & 0.414608 & -2.791994 \\
\hline H & 3.391547 & 2.006006 & -2.674649 \\
\hline & 4.299585 & 0.657146 & -3.373533 \\
\hline & 6.968210 & -1.365258 & 0.330107 \\
\hline & 7.406145 & -1.226545 & 1.323891 \\
\hline & 6.817396 & -2.443852 & 0.198227 \\
\hline $\mathrm{H}$ & 7.711649 & -1.056382 & -0.411873 \\
\hline C & -1.120007 & -1.905314 & -0.827070 \\
\hline $\mathrm{H}$ & -0.891342 & -2.180525 & -1.874272 \\
\hline C & -2.538908 & -1.894585 & -0.556208 \\
\hline c & -3.440982 & -2.499386 & -1.470460 \\
\hline $\mathrm{C}$ & -3.117161 & -1.334749 & 0.611730 \\
\hline C & -4.804229 & -2.574730 & -1.221942 \\
\hline $\mathrm{H}$ & -3.032979 & -2.918145 & -2.390137 \\
\hline C & -4.478906 & -1.409954 & 0.853850 \\
\hline $\mathrm{H}$ & -2.459954 & -0.819633 & 1.309870 \\
\hline C & -5.343392 & -2.032931 & -0.053122 \\
\hline & -5.458558 & -3.051349 & -1.951843 \\
\hline & -4.882659 & -0.953859 & 1.758620 \\
\hline $\mathrm{H}$ & -6.412816 & -2.084221 & 0.143016 \\
\hline C & -0.258586 & -0.957670 & 2.072413 \\
\hline C & -0.701077 & -2.111081 & 2.709749 \\
\hline H & -1.346423 & -2.739185 & 2.070455 \\
\hline $\mathrm{H}$ & 0.135722 & -2.753977 & 3.046995 \\
\hline & -1.289518 & -1.858665 & 3.614 \\
\hline C & 1.333526 & -1.217318 & -1.769936 \\
\hline C & 1.782606 & -2.076038 & -0.875355 \\
\hline $\mathrm{H}$ & 1.552180 & -3.147153 & -1.028340 \\
\hline & 2.792582 & -1.918910 & -0.439361 \\
\hline \multicolumn{4}{|c|}{13} \\
\hline & -0.500477 & 0.377880 & 0.218360 \\
\hline C & -3.034686 & 1.747332 & 1.203996 \\
\hline ( & -2.266680 & 2.805696 & -0.709944 \\
\hline C & -4.198738 & 2.506940 & 1.198515 \\
\hline
\end{tabular}$$
\begin{array}{llll}
\mathrm{H} & -1.070500 & 1.174112 & 2.747993
\end{array}
$$ 


\begin{tabular}{|c|c|c|c|c|c|c|c|}
\hline $\mathrm{H}$ & -2.789708 & 1.002609 & 1.963779 & $\mathrm{H}$ & 7.026568 & -0.501082 & 1.560533 \\
\hline $\mathrm{C}$ & -3.396126 & 3.606907 & -0.771082 & $\mathrm{C}$ & -2.494528 & -2.418137 & 1.950238 \\
\hline $\mathrm{H}$ & -1.469822 & 2.865802 & -1.445960 & $\mathrm{H}$ & -3.317745 & -2.645810 & 2.632894 \\
\hline $\mathrm{H}$ & -4.943052 & 2.350859 & 1.973372 & $\mathrm{H}$ & -1.901393 & -3.325924 & 1.801751 \\
\hline $\mathrm{H}$ & -3.496894 & 4.335501 & -1.568688 & $\mathrm{H}$ & -1.839980 & -1.668958 & 2.425451 \\
\hline $\mathrm{N}$ & -2.084054 & 1.889705 & 0.259737 & $\mathrm{C}$ & -1.830755 & -1.408843 & -2.958336 \\
\hline $\mathrm{C}$ & -4.386122 & 3.451754 & 0.197978 & $\mathrm{H}$ & -0.783773 & -1.193838 & -2.724161 \\
\hline $\mathrm{H}$ & -5.287354 & 4.058151 & 0.168244 & $\mathrm{H}$ & -1.878488 & -2.389600 & -3.448878 \\
\hline $\mathrm{C}$ & 0.127504 & -1.437032 & -0.160914 & $\mathrm{H}$ & -2.167314 & -0.666996 & -3.686751 \\
\hline $\mathrm{C}$ & 1.111083 & -3.525826 & -0.543544 & $\mathrm{C}$ & -6.158364 & -0.206619 & -0.705899 \\
\hline $\mathrm{C}$ & -0.352772 & -3.593463 & -0.961495 & $\mathrm{H}$ & -6.812006 & -0.777455 & -1.374689 \\
\hline $\mathrm{H}$ & 1.796578 & -3.908784 & -1.306320 & $\mathrm{H}$ & -6.637535 & -0.175004 & 0.276409 \\
\hline $\mathrm{H}$ & 1.299552 & -4.078735 & 0.389732 & $\mathrm{H}$ & -6.127590 & 0.816954 & -1.095490 \\
\hline $\mathrm{H}$ & -0.463207 & -3.603150 & -2.057608 & $\mathrm{C}$ & 1.130411 & 1.172643 & 0.514380 \\
\hline $\mathrm{H}$ & -0.877315 & -4.465580 & -0.558279 & $\mathrm{H}$ & 1.387952 & 1.260561 & 1.588425 \\
\hline $\mathrm{N}$ & 1.310233 & -2.087780 & -0.331428 & $\mathrm{C}$ & 2.158628 & 1.782315 & -0.321410 \\
\hline $\mathrm{N}$ & -0.866446 & -2.353211 & -0.388770 & $\mathrm{C}$ & 3.242801 & 2.418128 & 0.316073 \\
\hline $\mathrm{C}$ & 2.570824 & -1.562336 & 0.066819 & $\mathrm{C}$ & 2.148733 & 1.758737 & -1.729315 \\
\hline $\mathrm{C}$ & 2.830709 & -1.345879 & 1.430971 & $\mathrm{C}$ & 4.267243 & 3.008226 & -0.413777 \\
\hline $\mathrm{C}$ & 3.508656 & -1.244564 & -0.924336 & $\mathrm{H}$ & 3.267883 & 2.431095 & 1.405018 \\
\hline $\mathrm{C}$ & 4.051687 & -0.765372 & 1.774720 & $\mathrm{C}$ & 3.177473 & 2.337100 & -2.458073 \\
\hline $\mathrm{C}$ & 4.718707 & -0.675710 & -0.528712 & $\mathrm{H}$ & 1.315343 & 1.263966 & -2.221972 \\
\hline $\mathrm{C}$ & 5.002047 & -0.416359 & 0.811927 & $\mathrm{C}$ & 4.240223 & 2.965827 & -1.805823 \\
\hline $\mathrm{H}$ & 4.258546 & -0.567041 & 2.826247 & $\mathrm{H}$ & 5.091077 & 3.494819 & 0.102641 \\
\hline $\mathrm{H}$ & 5.442028 & -0.390369 & -1.292010 & $\mathrm{H}$ & 3.155447 & 2.300238 & -3.544844 \\
\hline $\mathrm{C}$ & -2.210225 & -1.878590 & -0.499803 & $\mathrm{H}$ & 5.041437 & 3.422361 & -2.381555 \\
\hline $\mathrm{C}$ & -3.011315 & -1.881654 & 0.655241 & $\mathrm{O}$ & -0.920464 & 0.149856 & 2.365905 \\
\hline $\mathrm{C}$ & -2.682157 & -1.370336 & -1.726893 & $\mathrm{H}$ & -0.392199 & 0.669752 & -1.377642 \\
\hline $\mathrm{C}$ & -4.295537 & -1.333914 & 0.558823 & $\mathrm{C}$ & -0.457914 & 1.072847 & 3.271766 \\
\hline $\mathrm{C}$ & -3.969049 & -0.839507 & -1.767748 & $\mathrm{H}$ & 0.628752 & 0.979765 & 3.502803 \\
\hline $\mathrm{C}$ & -4.786109 & -0.803840 & -0.632936 & $\mathrm{H}$ & -0.600141 & 2.132080 & 2.953451 \\
\hline $\mathrm{H}$ & -4.924407 & -1.317850 & 1.448444 & $\mathrm{H}$ & -0.968246 & 0.976843 & 4.249379 \\
\hline $\mathrm{H}$ & -4.339767 & -0.433169 & -2.708237 & & & & \\
\hline $\mathrm{C}$ & 3.196873 & -1.465061 & -2.371891 & \multicolumn{4}{|c|}{$\mathbf{T S}_{2-14}$} \\
\hline $\mathrm{H}$ & 2.156099 & -1.206495 & -2.593762 & $\mathrm{Ru}$ & -0.440426 & 0.084109 & 0.128762 \\
\hline $\mathrm{H}$ & 3.846029 & -0.859364 & -3.009431 & $\mathrm{C}$ & -3.005911 & 1.377932 & 1.221754 \\
\hline $\mathrm{H}$ & 3.336555 & -2.512918 & -2.667961 & $\mathrm{C}$ & -2.386841 & 2.332855 & -0.788528 \\
\hline $\mathrm{C}$ & 1.812643 & -1.692394 & 2.471117 & $\mathrm{C}$ & -4.261061 & 1.965996 & 1.159759 \\
\hline $\mathrm{H}$ & 1.460941 & -2.725985 & 2.362304 & $\mathrm{H}$ & -2.708519 & 0.729736 & 2.044805 \\
\hline $\mathrm{H}$ & 2.230985 & -1.587569 & 3.475527 & $\mathrm{C}$ & -3.623867 & 2.942378 & -0.925898 \\
\hline $\mathrm{H}$ & 0.912919 & -1.060238 & 2.402212 & $\mathrm{H}$ & -1.598871 & 2.452455 & -1.526685 \\
\hline $\mathrm{C}$ & 6.291836 & 0.233351 & 1.210255 & $\mathrm{H}$ & -4.971507 & 1.794318 & 1.963035 \\
\hline $\mathrm{H}$ & 6.743170 & 0.771730 & 0.371904 & $\mathrm{H}$ & -3.821333 & 3.558974 & -1.797265 \\
\hline $\mathrm{H}$ & 6.144635 & 0.948048 & 2.026524 & $\mathrm{~N}$ & -2.068083 & 1.541643 & 0.261704 \\
\hline
\end{tabular}




\begin{tabular}{|c|c|c|c|c|c|c|c|}
\hline $\mathrm{C}$ & -4.588334 & 2.758629 & 0.063327 & $\mathrm{H}$ & -0.628217 & -1.352438 & -2.785458 \\
\hline $\mathrm{H}$ & -5.566439 & 3.224277 & -0.017219 & $\mathrm{H}$ & -1.939815 & -2.269571 & -3.560261 \\
\hline $\mathrm{C}$ & 0.495099 & -1.569983 & -0.317423 & $\mathrm{H}$ & -2.049763 & -0.515342 & -3.396430 \\
\hline $\mathrm{C}$ & 1.659727 & -3.499659 & -0.979631 & $\mathrm{C}$ & -5.845486 & -0.877245 & -0.100623 \\
\hline $\mathrm{C}$ & 0.178943 & -3.704542 & -1.283296 & $\mathrm{H}$ & -6.539202 & -1.555388 & -0.611070 \\
\hline $\mathrm{H}$ & 2.314594 & -3.742983 & -1.822827 & $\mathrm{H}$ & -6.157053 & -0.821304 & 0.946128 \\
\hline $\mathrm{H}$ & 1.985535 & -4.100883 & -0.115921 & $\mathrm{H}$ & -5.981779 & 0.113939 & -0.545220 \\
\hline $\mathrm{H}$ & -0.031714 & -3.622742 & -2.362271 & $\mathrm{C}$ & 1.109549 & 1.182713 & 0.609308 \\
\hline $\mathrm{H}$ & -0.202282 & -4.670458 & -0.935184 & $\mathrm{H}$ & 2.068221 & 0.657995 & 0.719539 \\
\hline $\mathrm{N}$ & 1.720528 & -2.067243 & -0.658931 & $\mathrm{C}$ & 1.302702 & 2.561067 & 0.123088 \\
\hline $\mathrm{N}$ & -0.399602 & -2.594685 & -0.536811 & $\mathrm{C}$ & 2.436115 & 2.859885 & -0.653162 \\
\hline $\mathrm{C}$ & 2.950648 & -1.480461 & -0.246101 & $\mathrm{C}$ & 0.389409 & 3.597830 & 0.373687 \\
\hline $\mathrm{C}$ & 3.310346 & -1.502455 & 1.109422 & $\mathrm{C}$ & 2.622493 & 4.127513 & -1.192988 \\
\hline $\mathrm{C}$ & 3.755627 & -0.862109 & -1.211895 & $\mathrm{H}$ & 3.167221 & 2.072441 & -0.834551 \\
\hline $\mathrm{C}$ & 4.519254 & -0.907254 & 1.475404 & $\mathrm{C}$ & 0.587551 & 4.871043 & -0.146420 \\
\hline $\mathrm{C}$ & 4.955188 & -0.282489 & -0.800220 & $\mathrm{H}$ & -0.479286 & 3.392946 & 0.993955 \\
\hline $\mathrm{C}$ & 5.356268 & -0.299347 & 0.538498 & $\mathrm{C}$ & 1.698926 & 5.140744 & -0.943452 \\
\hline $\mathrm{H}$ & 4.807913 & -0.907699 & 2.525720 & $\mathrm{H}$ & 3.500376 & 4.330236 & -1.801906 \\
\hline $\mathrm{H}$ & 5.583625 & 0.211079 & -1.541232 & $\mathrm{H}$ & -0.129783 & 5.659036 & 0.071052 \\
\hline $\mathrm{C}$ & -1.779402 & -2.219444 & -0.478732 & $\mathrm{H}$ & 1.849304 & 6.134213 & -1.357293 \\
\hline $\mathrm{C}$ & -2.467565 & -2.431410 & 0.733924 & $\mathrm{O}$ & -0.542440 & -0.137324 & 2.322522 \\
\hline $\mathrm{C}$ & -2.403844 & -1.587486 & -1.574346 & $\mathrm{H}$ & -0.228784 & 0.521435 & -1.419587 \\
\hline $\mathrm{C}$ & -3.785438 & -1.989532 & 0.831035 & $\mathrm{C}$ & -0.089171 & 0.973777 & 2.832463 \\
\hline $\mathrm{C}$ & -3.725201 & -1.156719 & -1.418905 & $\mathrm{H}$ & 0.363069 & 0.925646 & 3.841589 \\
\hline $\mathrm{C}$ & -4.428026 & -1.344258 & -0.229678 & $\mathrm{H}$ & 0.842668 & 1.317979 & 1.981328 \\
\hline $\mathrm{H}$ & -4.320115 & -2.135895 & 1.768996 & $\mathrm{H}$ & -0.684872 & 1.903287 & 2.698314 \\
\hline $\mathrm{H}$ & -4.210043 & -0.646885 & -2.251041 & & & & \\
\hline $\mathrm{C}$ & 3.288202 & -0.772860 & -2.630914 & 14 & & & \\
\hline $\mathrm{H}$ & 2.307867 & -0.285158 & -2.681201 & $\mathrm{Ru}$ & -0.689438 & 0.214644 & 0.172909 \\
\hline $\mathrm{H}$ & 3.990401 & -0.203050 & -3.244622 & $\mathrm{C}$ & -3.102713 & 2.074827 & 0.592853 \\
\hline $\mathrm{H}$ & 3.168004 & -1.760845 & -3.090273 & $\mathrm{C}$ & -1.331152 & 3.229693 & -0.331673 \\
\hline $\mathrm{C}$ & 2.377605 & -2.062995 & 2.137679 & $\mathrm{C}$ & -3.931643 & 3.183624 & 0.503302 \\
\hline $\mathrm{H}$ & 2.021953 & -3.066149 & 1.874004 & $\mathrm{H}$ & -3.457033 & 1.126862 & 0.995575 \\
\hline $\mathrm{H}$ & 2.860601 & -2.124515 & 3.116097 & $\mathrm{C}$ & -2.097420 & 4.379363 & -0.458295 \\
\hline $\mathrm{H}$ & 1.476870 & -1.439244 & 2.235974 & $\mathrm{H}$ & -0.288875 & 3.196419 & -0.641363 \\
\hline $\mathrm{C}$ & 6.657439 & 0.316385 & 0.953771 & $\mathrm{H}$ & -4.956731 & 3.118347 & 0.855693 \\
\hline $\mathrm{H}$ & 6.821119 & 1.282227 & 0.466120 & $\mathrm{H}$ & -1.651866 & 5.275646 & -0.878446 \\
\hline $\mathrm{H}$ & 6.702131 & 0.474277 & 2.034359 & $\mathrm{~N}$ & -1.820778 & 2.075002 & 0.168778 \\
\hline $\mathrm{H}$ & 7.506969 & -0.321252 & 0.683058 & $\mathrm{C}$ & -3.425089 & 4.362699 & -0.038127 \\
\hline $\mathrm{C}$ & -1.776730 & -3.076102 & 1.892842 & $\mathrm{H}$ & -4.047980 & 5.248112 & -0.124871 \\
\hline $\mathrm{H}$ & -2.490913 & -3.332490 & 2.679296 & $\mathrm{C}$ & 0.044801 & -1.568487 & -0.132984 \\
\hline $\mathrm{H}$ & -1.246664 & -3.986403 & 1.594871 & $\mathrm{C}$ & 1.049323 & -3.619084 & -0.695886 \\
\hline $\mathrm{H}$ & -1.034236 & -2.391061 & 2.317951 & $\mathrm{C}$ & -0.443732 & -3.714134 & -0.998612 \\
\hline $\mathrm{C}$ & -1.712363 & -1.426552 & -2.894769 & $\mathrm{H}$ & 1.682090 & -3.933170 & -1.532927 \\
\hline
\end{tabular}




\begin{tabular}{|c|c|c|c|c|c|c|c|}
\hline $\mathrm{H}$ & 1.329586 & -4.225492 & 0.179912 & $\mathrm{H}$ & -6.373037 & 0.358895 & 0.377289 \\
\hline $\mathrm{H}$ & -0.643455 & -3.660041 & -2.081250 & $\mathrm{C}$ & 2.112006 & 1.795210 & 0.237340 \\
\hline $\mathrm{H}$ & -0.901446 & -4.633016 & -0.616682 & $\mathrm{C}$ & 3.275515 & 2.115574 & 0.956688 \\
\hline $\mathrm{N}$ & 1.220974 & -2.190426 & -0.412862 & $\mathrm{C}$ & 2.114967 & 2.089422 & -1.133039 \\
\hline $\mathrm{N}$ & -0.939755 & -2.527831 & -0.303481 & $\mathrm{C}$ & 4.379456 & 2.701221 & 0.347618 \\
\hline $\mathrm{C}$ & 2.505357 & -1.644365 & -0.130802 & $\mathrm{H}$ & 3.307305 & 1.888958 & 2.022315 \\
\hline $\mathrm{C}$ & 3.000115 & -1.680172 & 1.180171 & $\mathrm{C}$ & 3.222813 & 2.663546 & -1.753300 \\
\hline $\mathrm{C}$ & 3.241776 & -1.082886 & -1.180338 & $\mathrm{H}$ & 1.236036 & 1.825090 & -1.716459 \\
\hline $\mathrm{C}$ & 4.275180 & -1.168316 & 1.414707 & $\mathrm{C}$ & 4.363071 & 2.975151 & -1.018342 \\
\hline $\mathrm{C}$ & 4.510178 & -0.577912 & -0.899364 & $\mathrm{H}$ & 5.261525 & 2.935745 & 0.940322 \\
\hline $\mathrm{C}$ & 5.047287 & -0.622347 & 0.386639 & $\mathrm{H}$ & 3.193519 & 2.863133 & -2.822904 \\
\hline $\mathrm{H}$ & 4.667602 & -1.175919 & 2.431638 & $\mathrm{H}$ & 5.227702 & 3.423867 & -1.501828 \\
\hline $\mathrm{H}$ & 5.076904 & -0.101884 & -1.698238 & $\mathrm{O}$ & -1.387391 & -0.017949 & 2.230866 \\
\hline $\mathrm{C}$ & -2.253575 & -1.973477 & -0.437932 & $\mathrm{H}$ & -0.172137 & 0.537101 & -1.314784 \\
\hline $\mathrm{C}$ & -3.162369 & -2.135541 & 0.628803 & $\mathrm{C}$ & -1.529791 & 0.864164 & 3.084337 \\
\hline $\mathrm{C}$ & -2.603239 & -1.234385 & -1.587093 & $\mathrm{H}$ & -1.284288 & 1.919459 & 2.872238 \\
\hline $\mathrm{C}$ & -4.404060 & -1.514435 & 0.547983 & $\mathrm{H}$ & -1.899998 & 0.611351 & 4.089563 \\
\hline $\mathrm{C}$ & -3.860419 & -0.610265 & -1.607088 & $\mathrm{C}$ & 0.931056 & 1.239175 & 0.986364 \\
\hline $\mathrm{C}$ & -4.763821 & -0.730254 & -0.557210 & $\mathrm{H}$ & 1.321834 & 0.623940 & 1.815149 \\
\hline $\mathrm{H}$ & -5.102884 & -1.624322 & 1.376778 & $\mathrm{H}$ & 0.442658 & 2.102728 & 1.483266 \\
\hline $\mathrm{H}$ & -4.131185 & -0.015493 & -2.478848 & & & & \\
\hline $\mathrm{C}$ & 2.640515 & -0.982067 & -2.547116 & 15 & & & \\
\hline $\mathrm{H}$ & 1.678683 & -0.455939 & -2.501294 & $\mathrm{Ru}$ & -0.350771 & 0.620675 & 0.003375 \\
\hline $\mathrm{H}$ & 3.298787 & -0.437860 & -3.229286 & $\mathrm{C}$ & -0.277569 & 3.238988 & -1.547530 \\
\hline $\mathrm{H}$ & 2.440440 & -1.968095 & -2.985149 & $\mathrm{C}$ & -0.533776 & 3.699775 & 0.694837 \\
\hline $\mathrm{C}$ & 2.148954 & -2.204658 & 2.295836 & $\mathrm{C}$ & -0.358946 & 4.581616 & -1.874943 \\
\hline $\mathrm{H}$ & 1.832776 & -3.241031 & 2.126275 & $\mathrm{H}$ & -0.135835 & 2.470090 & -2.302993 \\
\hline $\mathrm{H}$ & 2.681633 & -2.171771 & 3.249596 & $\mathrm{C}$ & -0.612512 & 5.062609 & 0.448653 \\
\hline $\mathrm{H}$ & 1.228085 & -1.615329 & 2.395380 & $\mathrm{H}$ & -0.595712 & 3.308445 & 1.708293 \\
\hline $\mathrm{C}$ & 6.409558 & -0.065737 & 0.665065 & $\mathrm{H}$ & -0.277712 & 4.881973 & -2.915078 \\
\hline $\mathrm{H}$ & 6.684757 & 0.696361 & -0.070014 & $\mathrm{H}$ & -0.738165 & 5.748497 & 1.280854 \\
\hline $\mathrm{H}$ & 6.460136 & 0.393064 & 1.657739 & $\mathrm{~N}$ & -0.372463 & 2.774859 & -0.277311 \\
\hline $\mathrm{H}$ & 7.181121 & -0.844487 & 0.633288 & $\mathrm{C}$ & -0.529410 & 5.524253 & -0.861462 \\
\hline $\mathrm{C}$ & -2.768508 & -2.937248 & 1.827982 & $\mathrm{H}$ & -0.590047 & 6.584547 & -1.086537 \\
\hline $\mathrm{H}$ & -3.565820 & -2.944798 & 2.575537 & $\mathrm{C}$ & -0.433331 & -1.310996 & 0.144351 \\
\hline $\mathrm{H}$ & -2.542374 & -3.976209 & 1.564434 & $\mathrm{C}$ & 0.035986 & -3.638082 & 0.170972 \\
\hline $\mathrm{H}$ & -1.865997 & -2.523096 & 2.286860 & $\mathrm{C}$ & -1.391328 & -3.434675 & 0.638583 \\
\hline $\mathrm{C}$ & -1.753314 & -1.206785 & -2.823827 & $\mathrm{H}$ & 0.079034 & -4.066775 & -0.844703 \\
\hline $\mathrm{H}$ & -0.710239 & -1.457649 & -2.627438 & $\mathrm{H}$ & 0.619419 & -4.288779 & 0.830685 \\
\hline $\mathrm{H}$ & -2.145739 & -1.914818 & -3.566446 & $\mathrm{H}$ & -2.103890 & -4.115831 & 0.162145 \\
\hline $\mathrm{H}$ & -1.762966 & -0.214135 & -3.280673 & $\mathrm{H}$ & -1.488187 & -3.554512 & 1.729503 \\
\hline $\mathrm{C}$ & -6.095139 & -0.044024 & -0.602538 & $\mathrm{~N}$ & 0.556152 & -2.271405 & 0.172872 \\
\hline $\mathrm{H}$ & -6.097447 & 0.782002 & -1.319271 & $\mathrm{~N}$ & -1.625671 & -2.041053 & 0.259809 \\
\hline $\mathrm{H}$ & -6.896014 & -0.733048 & -0.895383 & $\mathrm{C}$ & 1.879545 & -2.048995 & -0.312935 \\
\hline
\end{tabular}




\begin{tabular}{|c|c|c|c|c|c|c|c|}
\hline $\mathrm{C}$ & 2.954652 & -2.167635 & 0.579103 & $\mathrm{C}$ & 4.368585 & 1.840880 & 0.037660 \\
\hline $\mathrm{C}$ & 2.092097 & -1.789162 & -1.675958 & $\mathrm{H}$ & 2.309035 & 1.908114 & -0.591504 \\
\hline $\mathrm{C}$ & 4.244117 & -1.941706 & 0.101557 & $\mathrm{C}$ & 5.268170 & 1.459657 & 1.030840 \\
\hline $\mathrm{C}$ & 3.399504 & -1.560304 & -2.105757 & $\mathrm{H}$ & 5.463445 & 0.570005 & 2.985306 \\
\hline $\mathrm{C}$ & 4.484813 & -1.609099 & -1.231234 & $\mathrm{H}$ & 4.735892 & 2.296090 & -0.880475 \\
\hline $\mathrm{H}$ & 5.079334 & -1.996273 & 0.798479 & $\mathrm{H}$ & 6.336289 & 1.616848 & 0.898639 \\
\hline $\mathrm{H}$ & 3.574605 & -1.343286 & -3.159526 & $\mathrm{H}$ & 0.759304 & 0.549804 & -1.095986 \\
\hline $\mathrm{C}$ & -2.918566 & -1.476084 & 0.407818 & $\mathrm{C}$ & 1.015729 & 0.812648 & 1.584476 \\
\hline $\mathrm{C}$ & -3.202694 & -0.523583 & 1.409904 & $\mathrm{H}$ & 0.909950 & -0.001030 & 2.323237 \\
\hline $\mathrm{C}$ & -3.935526 & -1.897111 & -0.470716 & $\mathrm{H}$ & 0.641398 & 1.709306 & 2.122734 \\
\hline $\mathrm{C}$ & -4.505536 & -0.039310 & 1.524987 & $\mathrm{O}$ & -1.910133 & 0.565736 & -1.869807 \\
\hline $\mathrm{C}$ & -5.224607 & -1.376618 & -0.318279 & $\mathrm{H}$ & -2.109547 & -0.374982 & -1.780286 \\
\hline $\mathrm{C}$ & -5.535569 & -0.454641 & 0.677030 & $\mathrm{C}$ & -3.127528 & 1.288907 & -1.710062 \\
\hline $\mathrm{H}$ & -4.720731 & 0.686734 & 2.308550 & $\mathrm{H}$ & -3.540972 & 1.171057 & -0.700832 \\
\hline $\mathrm{H}$ & -6.004179 & -1.702470 & -1.006324 & $\mathrm{H}$ & -2.887783 & 2.341735 & -1.874370 \\
\hline $\mathrm{C}$ & 0.963111 & -1.763266 & -2.661587 & $\mathrm{H}$ & -3.876236 & 0.977715 & -2.448829 \\
\hline $\mathrm{H}$ & 0.494526 & -0.772978 & -2.699481 & & & & \\
\hline $\mathrm{H}$ & 1.317289 & -2.013761 & -3.665831 & \multicolumn{2}{|c|}{$\mathbf{T S}_{15-16}$} & & \\
\hline $\mathrm{H}$ & 0.170067 & -2.471255 & -2.395762 & $\mathrm{Ru}$ & 0.440573 & 0.488388 & 0.190880 \\
\hline $\mathrm{C}$ & 2.722763 & -2.525985 & 2.013486 & $\mathrm{C}$ & 0.171747 & 3.364964 & 1.441405 \\
\hline $\mathrm{H}$ & 2.499855 & -3.594384 & 2.135808 & $\mathrm{C}$ & 1.342658 & 3.345226 & -0.538131 \\
\hline $\mathrm{H}$ & 3.604354 & -2.304961 & 2.620309 & $\mathrm{C}$ & 0.350790 & 4.732187 & 1.582134 \\
\hline $\mathrm{H}$ & 1.870783 & -1.975045 & 2.426095 & $\mathrm{H}$ & -0.382688 & 2.784524 & 2.174962 \\
\hline $\mathrm{C}$ & 5.869902 & -1.287916 & -1.699668 & $\mathrm{C}$ & 1.561700 & 4.713968 & -0.477506 \\
\hline $\mathrm{H}$ & 5.961448 & -1.367391 & -2.786580 & $\mathrm{H}$ & 1.728217 & 2.751131 & -1.366108 \\
\hline $\mathrm{H}$ & 6.140951 & -0.262232 & -1.421288 & $\mathrm{H}$ & -0.068141 & 5.241239 & 2.444234 \\
\hline $\mathrm{H}$ & 6.617893 & -1.946008 & -1.246754 & $\mathrm{H}$ & 2.119171 & 5.204743 & -1.268610 \\
\hline $\mathrm{C}$ & -2.146518 & -0.012478 & 2.337213 & $\mathrm{~N}$ & 0.659194 & 2.659584 & 0.400109 \\
\hline $\mathrm{H}$ & -2.588803 & 0.337573 & 3.274928 & $\mathrm{C}$ & 1.059134 & 5.428047 & 0.605187 \\
\hline $\mathrm{H}$ & -1.381317 & -0.758480 & 2.564315 & $\mathrm{H}$ & 1.212828 & 6.499828 & 0.685581 \\
\hline $\mathrm{H}$ & -1.618595 & 0.866072 & 1.921096 & $\mathrm{C}$ & 0.371108 & -1.451562 & -0.041096 \\
\hline $\mathrm{C}$ & -3.660121 & -2.871554 & -1.576860 & $\mathrm{C}$ & -0.206456 & -3.733665 & -0.079892 \\
\hline $\mathrm{H}$ & -2.616039 & -2.832745 & -1.905884 & $\mathrm{C}$ & 1.216290 & -3.585552 & -0.592545 \\
\hline $\mathrm{H}$ & -3.852611 & -3.907733 & -1.271507 & $\mathrm{H}$ & -0.234928 & -4.153894 & 0.938568 \\
\hline $\mathrm{H}$ & -4.303740 & -2.677275 & -2.439591 & $\mathrm{H}$ & -0.838270 & -4.357674 & -0.719840 \\
\hline $\mathrm{C}$ & -6.927331 & 0.074082 & 0.841608 & $\mathrm{H}$ & 1.921024 & -4.285198 & -0.132072 \\
\hline $\mathrm{H}$ & -7.571788 & -0.221679 & 0.009835 & $\mathrm{H}$ & 1.276592 & -3.716504 & -1.685311 \\
\hline $\mathrm{H}$ & -7.390825 & -0.297913 & 1.762325 & $\mathrm{~N}$ & -0.667045 & -2.343571 & -0.062976 \\
\hline $\mathrm{H}$ & -6.941691 & 1.166830 & 0.904801 & $\mathrm{~N}$ & 1.510198 & -2.199542 & -0.230884 \\
\hline $\mathrm{C}$ & 2.485377 & 1.032844 & 1.358421 & $\mathrm{C}$ & -1.991575 & -2.027439 & 0.359491 \\
\hline $\mathrm{C}$ & 3.410657 & 0.670234 & 2.351221 & $\mathrm{C}$ & -3.008344 & -2.017966 & -0.609916 \\
\hline $\mathrm{C}$ & 3.001492 & 1.631187 & 0.199689 & $\mathrm{C}$ & -2.264782 & -1.733543 & 1.702510 \\
\hline $\mathrm{C}$ & 4.778078 & 0.873497 & 2.195874 & $\mathrm{C}$ & -4.293995 & -1.652614 & -0.224317 \\
\hline $\mathrm{H}$ & 3.036132 & 0.212116 & 3.266794 & $\mathrm{C}$ & -3.571092 & -1.368648 & 2.040801 \\
\hline
\end{tabular}




\begin{tabular}{|c|c|c|c|c|c|c|c|}
\hline $\mathrm{C}$ & -4.591154 & -1.302576 & 1.094852 & $\mathrm{H}$ & -4.619426 & 2.712952 & 0.637911 \\
\hline $\mathrm{H}$ & -5.075168 & -1.598020 & -0.981330 & $\mathrm{H}$ & -6.133638 & 2.011692 & -1.206372 \\
\hline $\mathrm{H}$ & -3.792334 & -1.120757 & 3.078874 & $\mathrm{H}$ & -0.981466 & 0.457835 & 0.807385 \\
\hline $\mathrm{C}$ & 2.790394 & -1.617846 & -0.461920 & $\mathrm{C}$ & -0.837752 & 0.892128 & -1.476311 \\
\hline $\mathrm{C}$ & 3.029283 & -0.822854 & -1.591184 & $\mathrm{H}$ & -0.726666 & 0.098509 & -2.231147 \\
\hline $\mathrm{C}$ & 3.800012 & -1.842215 & 0.493796 & $\mathrm{H}$ & -0.320723 & 1.773389 & -1.906481 \\
\hline $\mathrm{C}$ & 4.291555 & -0.229430 & -1.729304 & $\mathrm{O}$ & 1.872027 & 0.083560 & 1.870538 \\
\hline $\mathrm{C}$ & 5.043934 & -1.253861 & 0.298719 & $\mathrm{H}$ & 0.456931 & 0.307679 & 1.801911 \\
\hline $\mathrm{C}$ & 5.309146 & -0.432883 & -0.803116 & $\mathrm{C}$ & 2.636629 & 1.165233 & 2.301597 \\
\hline $\mathrm{H}$ & 4.485242 & 0.388892 & -2.605937 & $\mathrm{H}$ & 3.143416 & 1.698679 & 1.474487 \\
\hline $\mathrm{H}$ & 5.822611 & -1.415975 & 1.043384 & $\mathrm{H}$ & 2.060192 & 1.921912 & 2.863277 \\
\hline $\mathrm{C}$ & -1.216482 & -1.791415 & 2.773081 & $\mathrm{H}$ & 3.422433 & 0.797244 & 2.981274 \\
\hline $\mathrm{H}$ & -1.044102 & -0.801023 & 3.208175 & & & & \\
\hline $\mathrm{H}$ & -1.533177 & -2.448043 & 3.591112 & 16 & & & \\
\hline $\mathrm{H}$ & -0.248011 & -2.140085 & 2.408183 & $\mathrm{Ru}$ & -0.258382 & 0.582665 & -0.317226 \\
\hline $\mathrm{C}$ & -2.704506 & -2.375487 & -2.031307 & $\mathrm{C}$ & 0.699543 & 3.278710 & -1.614069 \\
\hline $\mathrm{H}$ & -2.496994 & -3.446968 & -2.148843 & $\mathrm{C}$ & -0.658357 & 3.614265 & 0.211767 \\
\hline $\mathrm{H}$ & -3.544691 & -2.129864 & -2.685587 & $\mathrm{C}$ & 0.748316 & 4.638493 & -1.881482 \\
\hline $\mathrm{H}$ & -1.818796 & -1.841138 & -2.392950 & $\mathrm{H}$ & 1.221230 & 2.554660 & -2.234809 \\
\hline $\mathrm{C}$ & -5.966531 & -0.841952 & 1.466256 & $\mathrm{C}$ & -0.658376 & 4.988536 & 0.018267 \\
\hline $\mathrm{H}$ & -6.177067 & 0.137012 & 1.019127 & $\mathrm{H}$ & -1.227712 & 3.159359 & 1.020142 \\
\hline $\mathrm{H}$ & -6.738989 & -1.527504 & 1.102714 & $\mathrm{H}$ & 1.321921 & 4.997954 & -2.729622 \\
\hline $\mathrm{H}$ & -6.084196 & -0.745368 & 2.548889 & $\mathrm{H}$ & -1.219819 & 5.626813 & 0.692676 \\
\hline $\mathrm{C}$ & 1.998394 & -0.608575 & -2.660845 & $\mathrm{~N}$ & 0.005055 & 2.757338 & -0.585319 \\
\hline $\mathrm{H}$ & 2.449486 & -0.704433 & -3.653655 & $\mathrm{C}$ & 0.055933 & 5.516096 & -1.051779 \\
\hline $\mathrm{H}$ & 1.166989 & -1.314065 & -2.590510 & $\mathrm{H}$ & 0.072063 & 6.586086 & -1.236447 \\
\hline $\mathrm{H}$ & 1.554376 & 0.393674 & -2.615418 & $\mathrm{C}$ & -0.588313 & -1.371078 & -0.226149 \\
\hline $\mathrm{C}$ & 3.503482 & -2.626872 & 1.730810 & $\mathrm{C}$ & -0.332943 & -3.690509 & -0.595219 \\
\hline $\mathrm{H}$ & 2.686184 & -2.139811 & 2.273250 & $\mathrm{C}$ & -1.692909 & -3.441022 & 0.025109 \\
\hline $\mathrm{H}$ & 3.191616 & -3.655549 & 1.515958 & $\mathrm{H}$ & -0.406127 & -3.939684 & -1.666207 \\
\hline $\mathrm{H}$ & 4.378657 & -2.677478 & 2.383518 & $\mathrm{H}$ & 0.235832 & -4.484641 & -0.101516 \\
\hline $\mathrm{C}$ & 6.656668 & 0.198130 & -0.979331 & $\mathrm{H}$ & -2.514876 & -3.937645 & -0.500158 \\
\hline $\mathrm{H}$ & 7.442167 & -0.555976 & -1.099987 & $\mathrm{H}$ & -1.730654 & -3.751474 & 1.082437 \\
\hline $\mathrm{H}$ & 6.685873 & 0.847824 & -1.857774 & $\mathrm{~N}$ & 0.309140 & -2.389441 & -0.418612 \\
\hline $\mathrm{H}$ & 6.935307 & 0.801949 & -0.109350 & $\mathrm{~N}$ & -1.806072 & -1.983685 & -0.072386 \\
\hline $\mathrm{C}$ & -2.296840 & 1.217007 & -1.375146 & $\mathrm{C}$ & 1.699175 & -2.213449 & -0.671382 \\
\hline $\mathrm{C}$ & -3.172320 & 0.851211 & -2.410258 & $\mathrm{C}$ & 2.572514 & -2.256967 & 0.426050 \\
\hline $\mathrm{C}$ & -2.857146 & 1.902975 & -0.286779 & $\mathrm{C}$ & 2.162602 & -1.973140 & -1.969799 \\
\hline $\mathrm{C}$ & -4.534717 & 1.126611 & -2.356298 & $\mathrm{C}$ & 3.923060 & -2.001133 & 0.209320 \\
\hline $\mathrm{H}$ & -2.763967 & 0.325366 & -3.272854 & $\mathrm{C}$ & 3.526873 & -1.724258 & -2.141122 \\
\hline $\mathrm{C}$ & -4.217503 & 2.189410 & -0.227515 & $\mathrm{C}$ & 4.416534 & -1.718008 & -1.067212 \\
\hline $\mathrm{H}$ & -2.207954 & 2.194677 & 0.536169 & $\mathrm{H}$ & 4.602743 & -1.999870 & 1.060571 \\
\hline $\mathrm{C}$ & -5.069055 & 1.796116 & -1.258234 & $\mathrm{H}$ & 3.901307 & -1.514108 & -3.142784 \\
\hline $\mathrm{H}$ & -5.181803 & 0.814180 & -3.173671 & $\mathrm{C}$ & -3.018359 & -1.367962 & 0.359783 \\
\hline
\end{tabular}




\begin{tabular}{|c|c|c|c|c|c|c|c|}
\hline $\mathrm{C}$ & -3.170740 & -0.931468 & 1.678645 & $\mathrm{H}$ & 0.340948 & 0.083263 & 2.279337 \\
\hline$C$ & -4.068010 & -1.260744 & -0.570356 & $\mathrm{H}$ & 0.215141 & 1.792645 & 1.879285 \\
\hline $\mathrm{C}$ & -4.388423 & -0.340424 & 2.042590 & $\mathrm{O}$ & -2.054691 & 0.764780 & -1.255465 \\
\hline C & -5.260722 & -0.680023 & -0.161039 & $\mathrm{H}$ & 0.537521 & 0.381443 & -1.650664 \\
\hline $\mathrm{C}$ & -5.438638 & -0.201752 & 1.143130 & $\mathrm{C}$ & -2.474795 & 1.749045 & -2.128051 \\
\hline $\mathrm{H}$ & -4.514876 & 0.009398 & 3.067241 & $\mathrm{H}$ & -2.290481 & 2.786527 & -1.786869 \\
\hline $\mathrm{H}$ & -6.070077 & -0.577197 & -0.883479 & $\mathrm{H}$ & -1.999014 & 1.659937 & -3.123757 \\
\hline $\mathrm{C}$ & 1.222215 & -1.969122 & -3.135993 & $\mathrm{H}$ & -3.564007 & 1.660503 & -2.291386 \\
\hline $\mathrm{H}$ & 1.625770 & -1.383975 & -3.966528 & & & & \\
\hline $\mathrm{H}$ & 1.046694 & -2.983813 & -3.515704 & \multicolumn{2}{|c|}{$\mathbf{T S}_{2-17}$} & & \\
\hline $\mathrm{H}$ & 0.248093 & -1.547492 & -2.865648 & $\mathrm{Ru}$ & 0.977770 & -0.834701 & 0.433939 \\
\hline $\mathrm{C}$ & 2.042183 & -2.559137 & 1.792136 & $\mathrm{C}$ & 0.474020 & 1.010690 & -0.015267 \\
\hline $\mathrm{H}$ & 1.598263 & -3.561412 & 1.842542 & $\mathrm{C}$ & -0.193681 & 3.242184 & -0.346190 \\
\hline $\mathrm{H}$ & 2.830885 & -2.498509 & 2.545573 & $\mathrm{C}$ & 1.266186 & 3.113424 & -0.777663 \\
\hline $\mathrm{H}$ & 1.251570 & -1.853375 & 2.070605 & $\mathrm{H}$ & -0.822393 & 3.726926 & -1.100251 \\
\hline $\mathrm{C}$ & 5.869246 & -1.410485 & -1.264848 & $\mathrm{H}$ & -0.300303 & 3.808386 & 0.592469 \\
\hline $\mathrm{H}$ & 6.179308 & -0.561388 & -0.646254 & $\mathrm{H}$ & 1.368177 & 3.174616 & -1.872381 \\
\hline $\mathrm{H}$ & 6.504657 & -2.255809 & -0.978418 & $\mathrm{H}$ & 1.912447 & 3.878690 & -0.335262 \\
\hline $\mathrm{H}$ & 6.093704 & -1.166126 & -2.306321 & $\mathrm{~N}$ & -0.597331 & 1.844949 & -0.141706 \\
\hline $\mathrm{C}$ & -2.097918 & -1.087483 & 2.714239 & $\mathrm{~N}$ & 1.582282 & 1.784322 & -0.275563 \\
\hline $\mathrm{H}$ & -2.476836 & -1.626958 & 3.589282 & $\mathrm{C}$ & -1.932076 & 1.524950 & 0.227158 \\
\hline $\mathrm{H}$ & -1.223942 & -1.625628 & 2.338455 & $\mathrm{C}$ & -2.250638 & 1.288812 & 1.574581 \\
\hline $\mathrm{H}$ & -1.745443 & -0.114894 & 3.075114 & $\mathrm{C}$ & -2.910419 & 1.466148 & -0.775618 \\
\hline $\mathrm{C}$ & -3.865793 & -1.710060 & -1.981190 & $\mathrm{C}$ & -3.567175 & 0.960392 & 1.891808 \\
\hline $\mathrm{H}$ & -3.009082 & -1.177244 & -2.406296 & $\mathrm{C}$ & -4.217297 & 1.147185 & -0.407097 \\
\hline $\mathrm{H}$ & -3.652626 & -2.783769 & -2.052836 & $\mathrm{C}$ & -4.563467 & 0.880488 & 0.916028 \\
\hline $\mathrm{H}$ & -4.749420 & -1.507041 & -2.591708 & $\mathrm{H}$ & -3.821244 & 0.760800 & 2.932740 \\
\hline $\mathrm{C}$ & -6.733241 & 0.432658 & 1.551157 & $\mathrm{H}$ & -4.976479 & 1.063456 & -1.183679 \\
\hline $\mathrm{H}$ & -7.577805 & -0.252974 & 1.420532 & $\mathrm{C}$ & 2.832319 & 1.102915 & -0.260700 \\
\hline $\mathrm{H}$ & -6.718458 & 0.743510 & 2.598955 & $\mathrm{C}$ & 3.370388 & 0.711487 & 0.983294 \\
\hline $\mathrm{H}$ & -6.955026 & 1.318284 & 0.945861 & $\mathrm{C}$ & 3.462131 & 0.742696 & -1.471254 \\
\hline $\mathrm{C}$ & 2.187042 & 0.999309 & 1.802955 & $\mathrm{C}$ & 4.543875 & -0.058066 & 0.978114 \\
\hline $\mathrm{C}$ & 2.804536 & 0.437829 & 2.929487 & $\mathrm{C}$ & 4.642184 & 0.012826 & -1.415335 \\
\hline $\mathrm{C}$ & 3.008562 & 1.695778 & 0.903164 & $\mathrm{C}$ & 5.191485 & -0.412922 & -0.198777 \\
\hline $\mathrm{C}$ & 4.176650 & 0.545256 & 3.142555 & $\mathrm{H}$ & 4.964684 & -0.370986 & 1.932658 \\
\hline $\mathrm{H}$ & 2.189213 & -0.106295 & 3.645385 & $\mathrm{H}$ & 5.126382 & -0.273705 & -2.348254 \\
\hline $\mathrm{C}$ & 4.377223 & 1.815277 & 1.114275 & $\mathrm{C}$ & -2.556987 & 1.718662 & -2.208470 \\
\hline $\mathrm{H}$ & 2.556621 & 2.121066 & 0.009476 & $\mathrm{H}$ & -1.570271 & 1.309961 & -2.450568 \\
\hline $\mathrm{C}$ & 4.973208 & 1.234881 & 2.233223 & $\mathrm{H}$ & -3.289493 & 1.260343 & -2.877778 \\
\hline $\mathrm{H}$ & 4.623604 & 0.086338 & 4.021919 & $\mathrm{H}$ & -2.529437 & 2.791600 & -2.440312 \\
\hline $\mathrm{H}$ & 4.987520 & 2.354223 & 0.392497 & $\mathrm{C}$ & -1.208351 & 1.382655 & 2.645088 \\
\hline $\mathrm{H}$ & 6.045421 & 1.318747 & 2.391471 & $\mathrm{H}$ & -0.595593 & 2.285409 & 2.542471 \\
\hline $\mathrm{H}$ & 1.269182 & 0.201101 & -0.317863 & $\mathrm{H}$ & -1.663873 & 1.394430 & 3.638554 \\
\hline $\mathrm{C}$ & 0.714881 & 0.849505 & 1.588806 & $\mathrm{H}$ & -0.512067 & 0.535402 & 2.601687 \\
\hline
\end{tabular}




\begin{tabular}{|c|c|c|c|c|c|c|c|}
\hline $\mathrm{C}$ & -5.963380 & 0.497269 & 1.285866 & $\mathrm{H}$ & 1.650231 & -1.504676 & -3.274043 \\
\hline $\mathrm{H}$ & -5.980560 & -0.417775 & 1.888139 & $\mathrm{~N}$ & -0.807333 & -0.374895 & -1.508253 \\
\hline $\mathrm{H}$ & -6.455028 & 1.274330 & 1.882099 & $\mathrm{~N}$ & 1.360986 & -0.656671 & -1.354213 \\
\hline $\mathrm{H}$ & -6.579295 & 0.322979 & 0.399530 & $\mathrm{C}$ & -2.147655 & 0.034326 & -1.291526 \\
\hline $\mathrm{C}$ & 2.791309 & 1.158898 & 2.295954 & $\mathrm{C}$ & -2.499252 & 1.393018 & -1.233924 \\
\hline $\mathrm{H}$ & 3.550545 & 1.677996 & 2.892849 & $\mathrm{C}$ & -3.131865 & -0.963868 & -1.151323 \\
\hline $\mathrm{H}$ & 1.944369 & 1.834443 & 2.158283 & $\mathrm{C}$ & -3.839339 & 1.730744 & -1.034414 \\
\hline $\mathrm{H}$ & 2.448217 & 0.308374 & 2.896400 & $\mathrm{C}$ & -4.458134 & -0.576504 & -0.972090 \\
\hline $\mathrm{C}$ & 2.829011 & 1.087142 & -2.782041 & $\mathrm{C}$ & -4.836367 & 0.765260 & -0.906133 \\
\hline $\mathrm{H}$ & 1.756324 & 0.859425 & -2.760957 & $\mathrm{H}$ & -4.110510 & 2.785117 & -0.990901 \\
\hline $\mathrm{H}$ & 2.931335 & 2.150168 & -3.031950 & $\mathrm{H}$ & -5.213569 & -1.351964 & -0.846271 \\
\hline $\mathrm{H}$ & 3.281473 & 0.517649 & -3.596981 & $\mathrm{C}$ & 2.705823 & -0.552634 & -0.918520 \\
\hline $\mathrm{C}$ & 6.431762 & -1.252276 & -0.177521 & $\mathrm{C}$ & 3.352247 & 0.696228 & -0.971957 \\
\hline $\mathrm{H}$ & 6.866492 & -1.305789 & 0.823736 & $\mathrm{C}$ & 3.376270 & -1.682288 & -0.427446 \\
\hline $\mathrm{H}$ & 6.218213 & -2.279189 & -0.494207 & $\mathrm{C}$ & 4.686216 & 0.777835 & -0.582471 \\
\hline $\mathrm{H}$ & 7.195611 & -0.864150 & -0.858377 & $\mathrm{C}$ & 4.717518 & -1.553539 & -0.055629 \\
\hline $\mathrm{C}$ & -0.786020 & -1.670923 & 0.637157 & $\mathrm{C}$ & 5.394913 & -0.339660 & -0.134129 \\
\hline $\mathrm{H}$ & -1.158025 & -1.576366 & 1.678533 & $\mathrm{H}$ & 5.187338 & 1.744169 & -0.631432 \\
\hline $\mathrm{C}$ & -1.907451 & -1.780135 & -0.321364 & $\mathrm{H}$ & 5.238740 & -2.430066 & 0.328502 \\
\hline $\mathrm{C}$ & -3.193606 & -2.108847 & 0.142898 & $\mathrm{C}$ & -2.766789 & -2.416121 & -1.134040 \\
\hline $\mathrm{C}$ & -1.741108 & -1.602095 & -1.704502 & $\mathrm{H}$ & -1.810794 & -2.575255 & -0.621351 \\
\hline $\mathrm{C}$ & -4.265200 & -2.249796 & -0.729864 & $\mathrm{H}$ & -3.525778 & -2.998380 & -0.606548 \\
\hline $\mathrm{H}$ & -3.344162 & -2.244524 & 1.213563 & $\mathrm{H}$ & -2.680545 & -2.841394 & -2.142247 \\
\hline $\mathrm{C}$ & -2.812835 & -1.740832 & -2.579275 & $\mathrm{C}$ & -1.479926 & 2.481368 & -1.363090 \\
\hline $\mathrm{H}$ & -0.752349 & -1.333575 & -2.070131 & $\mathrm{H}$ & -0.663029 & 2.214207 & -2.038900 \\
\hline $\mathrm{C}$ & -4.080803 & -2.064300 & -2.098668 & $\mathrm{H}$ & -1.937265 & 3.406944 & -1.725887 \\
\hline $\mathrm{H}$ & -5.250080 & -2.499724 & -0.341008 & $\mathrm{H}$ & -1.036978 & 2.759062 & -0.384607 \\
\hline $\mathrm{H}$ & -2.658808 & -1.590937 & -3.645768 & $\mathrm{C}$ & -6.267144 & 1.150874 & -0.685665 \\
\hline $\mathrm{H}$ & -4.916864 & -2.172566 & -2.785054 & $\mathrm{H}$ & -6.629444 & 0.808089 & 0.290027 \\
\hline $\mathrm{O}$ & 1.577886 & -2.646122 & 1.226924 & $\mathrm{H}$ & -6.402607 & 2.234835 & -0.723044 \\
\hline $\mathrm{H}$ & 1.055509 & -1.068462 & -1.118560 & $\mathrm{H}$ & -6.927745 & 0.706302 & -1.437501 \\
\hline $\mathrm{C}$ & 0.807158 & -3.628066 & 0.871220 & $\mathrm{C}$ & 2.621715 & 1.927679 & -1.409063 \\
\hline $\mathrm{H}$ & 0.706256 & -4.478203 & 1.567762 & $\mathrm{H}$ & 3.319617 & 2.711162 & -1.719119 \\
\hline $\mathrm{H}$ & -0.401762 & -2.930740 & 0.796325 & $\mathrm{H}$ & 1.929628 & 1.730169 & -2.233281 \\
\hline \multirow[t]{2}{*}{$\mathrm{H}$} & 0.764419 & -3.921144 & -0.191508 & $\mathrm{H}$ & 2.033103 & 2.391387 & -0.588431 \\
\hline & & & & $\mathrm{C}$ & 2.669117 & -2.989935 & -0.241829 \\
\hline 17 & & & & $\mathrm{H}$ & 1.624604 & -2.837680 & 0.051970 \\
\hline $\mathrm{Ru}$ & 0.459244 & 1.390059 & 0.572084 & $\mathrm{H}$ & 2.664728 & -3.600231 & -1.153763 \\
\hline $\mathrm{C}$ & 0.300729 & 0.018377 & -0.779591 & $\mathrm{H}$ & 3.157059 & -3.585183 & 0.534461 \\
\hline $\mathrm{C}$ & -0.427971 & -1.031109 & -2.760591 & $\mathrm{C}$ & 6.839962 & -0.230815 & 0.245654 \\
\hline $\mathrm{C}$ & 0.953400 & -1.558590 & -2.431929 & $\mathrm{H}$ & 7.034216 & 0.659199 & 0.852036 \\
\hline $\mathrm{H}$ & -1.133168 & -1.817235 & -3.039625 & $\mathrm{H}$ & 7.173887 & -1.103003 & 0.813766 \\
\hline $\mathrm{H}$ & -0.404880 & -0.292728 & -3.579031 & $\mathrm{H}$ & 7.482986 & -0.154737 & -0.639069 \\
\hline $\mathrm{H}$ & 0.911178 & -2.602840 & -2.089518 & $\mathrm{C}$ & -1.058033 & 0.741048 & 1.814838 \\
\hline
\end{tabular}




\begin{tabular}{|c|c|c|c|c|c|c|c|}
\hline $\mathrm{H}$ & -1.983252 & 1.288237 & 1.558745 & $\mathrm{H}$ & -4.742114 & 1.809933 & 1.254129 \\
\hline $\mathrm{C}$ & -1.377435 & -0.713181 & 1.942303 & $\mathrm{H}$ & -5.711926 & -0.999392 & -1.831155 \\
\hline $\mathrm{C}$ & -2.711248 & -1.136305 & 2.038427 & $\mathrm{C}$ & 1.007437 & -2.315441 & -2.173632 \\
\hline $\mathrm{C}$ & -0.382572 & -1.702590 & 2.007480 & $\mathrm{H}$ & 0.416732 & -1.401462 & -2.303771 \\
\hline $\mathrm{C}$ & -3.042553 & -2.477943 & 2.205548 & $\mathrm{H}$ & 1.449170 & -2.585724 & -3.136106 \\
\hline $\mathrm{H}$ & -3.500901 & -0.389003 & 1.968197 & $\mathrm{H}$ & 0.301467 & -3.105139 & -1.886974 \\
\hline $\mathrm{C}$ & -0.708736 & -3.045242 & 2.175872 & $\mathrm{C}$ & 2.344715 & -1.948898 & 2.675596 \\
\hline $\mathrm{H}$ & 0.656866 & -1.392271 & 1.912239 & $\mathrm{H}$ & 2.197710 & -2.961510 & 3.072685 \\
\hline $\mathrm{C}$ & -2.041467 & -3.443077 & 2.277467 & $\mathrm{H}$ & 3.130036 & -1.480526 & 3.274367 \\
\hline $\mathrm{H}$ & -4.089335 & -2.769959 & 2.267945 & $\mathrm{H}$ & 1.412198 & -1.399493 & 2.842716 \\
\hline $\mathrm{H}$ & 0.085269 & -3.788205 & 2.227121 & $\mathrm{C}$ & 5.861697 & -1.893357 & -0.914816 \\
\hline $\mathrm{H}$ & -2.295022 & -4.492746 & 2.403138 & $\mathrm{H}$ & 6.030351 & -2.282845 & -1.922549 \\
\hline $\mathrm{O}$ & 0.720820 & 3.062317 & 1.854615 & $\mathrm{H}$ & 6.199691 & -0.849958 & -0.911325 \\
\hline $\mathrm{H}$ & 1.513700 & 0.543247 & 1.388185 & $\mathrm{H}$ & 6.515565 & -2.438326 & -0.227336 \\
\hline $\mathrm{C}$ & 0.850603 & 3.135431 & 3.080160 & $\mathrm{C}$ & -2.402233 & 0.751641 & 2.026474 \\
\hline $\mathrm{H}$ & 0.963293 & 4.115958 & 3.564625 & $\mathrm{H}$ & -2.956317 & 1.332422 & 2.770510 \\
\hline $\mathrm{H}$ & -0.744571 & 1.122420 & 2.804888 & $\mathrm{H}$ & -1.856961 & -0.049816 & 2.532768 \\
\hline \multirow[t]{2}{*}{$\mathrm{H}$} & 0.861927 & 2.234143 & 3.713045 & $\mathrm{H}$ & -1.635660 & 1.444437 & 1.631224 \\
\hline & & & & $\mathrm{C}$ & -3.508494 & -2.521651 & -1.680300 \\
\hline 18 & & & & $\mathrm{H}$ & -2.437075 & -2.522703 & -1.907456 \\
\hline $\mathrm{Ru}$ & -0.436691 & 0.790226 & -0.233370 & $\mathrm{H}$ & -3.744049 & -3.493782 & -1.229665 \\
\hline $\mathrm{C}$ & -0.567354 & -1.037176 & 0.284093 & $\mathrm{H}$ & -4.054855 & -2.469853 & -2.625370 \\
\hline $\mathrm{C}$ & -0.260740 & -3.215985 & 1.185926 & $\mathrm{C}$ & -6.681331 & 1.201592 & -0.556661 \\
\hline $\mathrm{C}$ & -1.744409 & -3.039912 & 0.871598 & $\mathrm{H}$ & -7.072741 & 0.994434 & -1.556231 \\
\hline $\mathrm{H}$ & 0.160892 & -4.125929 & 0.743784 & $\mathrm{H}$ & -7.455421 & 0.902127 & 0.159927 \\
\hline $\mathrm{H}$ & -0.073675 & -3.257045 & 2.269824 & $\mathrm{H}$ & -6.564034 & 2.284787 & -0.458470 \\
\hline $\mathrm{H}$ & -2.060389 & -3.694158 & 0.046499 & $\mathrm{C}$ & 2.110164 & 2.080596 & 0.551347 \\
\hline $\mathrm{H}$ & -2.388917 & -3.258347 & 1.730432 & $\mathrm{C}$ & 2.129392 & 3.467904 & 0.784028 \\
\hline $\mathrm{N}$ & 0.347870 & -2.023685 & 0.597510 & $\mathrm{C}$ & 3.088168 & 1.560804 & -0.318906 \\
\hline $\mathrm{N}$ & -1.810995 & -1.628783 & 0.508605 & $\mathrm{C}$ & 3.068283 & 4.299568 & 0.170431 \\
\hline $\mathrm{C}$ & 1.719994 & -2.018536 & 0.229357 & $\mathrm{H}$ & 1.410760 & 3.891359 & 1.487035 \\
\hline $\mathrm{C}$ & 2.711567 & -1.975604 & 1.223225 & $\mathrm{C}$ & 4.029030 & 2.383920 & -0.918987 \\
\hline $\mathrm{C}$ & 2.063050 & -2.129697 & -1.129452 & $\mathrm{H}$ & 3.078165 & 0.491726 & -0.525308 \\
\hline $\mathrm{C}$ & 4.049598 & -1.957343 & 0.830053 & $\mathrm{C}$ & 4.023482 & 3.762425 & -0.685643 \\
\hline $\mathrm{C}$ & 3.414609 & -2.098480 & -1.475520 & $\mathrm{H}$ & 3.058762 & 5.367718 & 0.376155 \\
\hline $\mathrm{C}$ & 4.421739 & -1.992764 & -0.514686 & $\mathrm{H}$ & 4.771592 & 1.951606 & -1.586419 \\
\hline $\mathrm{H}$ & 4.821066 & -1.895328 & 1.596781 & $\mathrm{H}$ & 4.758811 & 4.404874 & -1.162104 \\
\hline $\mathrm{H}$ & 3.687930 & -2.168100 & -2.527925 & $\mathrm{H}$ & 0.617534 & 0.472476 & -1.360838 \\
\hline $\mathrm{C}$ & -3.015662 & -0.933898 & 0.231359 & $\mathrm{C}$ & 1.080844 & 1.199743 & 1.148529 \\
\hline $\mathrm{C}$ & -3.321554 & 0.233320 & 0.962219 & $\mathrm{H}$ & 1.528595 & 0.264318 & 1.491543 \\
\hline $\mathrm{C}$ & -3.871461 & -1.368625 & -0.796090 & $\mathrm{H}$ & 0.617832 & 1.692163 & 2.024423 \\
\hline $\mathrm{C}$ & -4.504969 & 0.914975 & 0.678804 & $\mathrm{O}$ & -0.444558 & 2.997878 & -0.880943 \\
\hline $\mathrm{C}$ & -5.052877 & -0.660494 & -1.032351 & $\mathrm{H}$ & 0.487113 & 3.263495 & -0.804906 \\
\hline $\mathrm{C}$ & -5.394552 & 0.477269 & -0.304735 & $\mathrm{C}$ & -1.222984 & 3.854174 & -0.056717 \\
\hline
\end{tabular}




\begin{tabular}{|c|c|c|c|c|c|c|c|}
\hline $\mathrm{H}$ & -0.907980 & 3.807458 & 0.995478 & $\mathrm{H}$ & 1.703166 & -2.207620 & -3.555638 \\
\hline $\mathrm{H}$ & -1.172114 & 4.893384 & -0.401014 & $\mathrm{H}$ & 0.336087 & -2.723120 & -2.549941 \\
\hline \multirow[t]{2}{*}{$\mathrm{H}$} & -2.255889 & 3.504615 & -0.129622 & $\mathrm{C}$ & 2.048615 & -2.972391 & 2.289834 \\
\hline & & & & $\mathrm{H}$ & 1.697240 & -4.005977 & 2.403831 \\
\hline 19a & & & & $\mathrm{H}$ & 2.834444 & -2.818118 & 3.033918 \\
\hline $\mathrm{Ru}$ & -0.292980 & 0.545058 & -0.026419 & $\mathrm{H}$ & 1.201041 & -2.323267 & 2.541990 \\
\hline $\mathrm{C}$ & 0.433569 & 3.351386 & 1.031025 & $\mathrm{C}$ & 5.893933 & -2.201651 & -0.848367 \\
\hline $\mathrm{C}$ & 0.005939 & 3.435656 & -1.235582 & $\mathrm{H}$ & 6.479379 & -2.861616 & -0.201424 \\
\hline $\mathrm{C}$ & 0.779008 & 4.697234 & 1.054961 & $\mathrm{H}$ & 6.167613 & -2.412558 & -1.885911 \\
\hline $\mathrm{H}$ & 0.424243 & 2.731242 & 1.923056 & $\mathrm{H}$ & 6.215597 & -1.175067 & -0.633647 \\
\hline $\mathrm{C}$ & 0.324110 & 4.784404 & -1.291822 & $\mathrm{C}$ & -3.490033 & -1.166101 & 2.459089 \\
\hline $\mathrm{H}$ & -0.300905 & 2.875883 & -2.115441 & $\mathrm{H}$ & -3.560097 & -0.242893 & 3.041523 \\
\hline $\mathrm{H}$ & 1.082555 & 5.156364 & 1.990616 & $\mathrm{H}$ & -4.141010 & -1.907096 & 2.939104 \\
\hline $\mathrm{H}$ & 0.261284 & 5.313582 & -2.237416 & $\mathrm{H}$ & -2.457838 & -1.509906 & 2.531915 \\
\hline $\mathrm{N}$ & 0.066009 & 2.719138 & -0.099120 & $\mathrm{C}$ & -2.647741 & -1.525782 & -2.507378 \\
\hline $\mathrm{C}$ & 0.729681 & 5.429849 & -0.125295 & $\mathrm{H}$ & -1.745192 & -0.900654 & -2.524144 \\
\hline $\mathrm{H}$ & 1.000622 & 6.481645 & -0.138109 & $\mathrm{H}$ & -2.302221 & -2.562721 & -2.418629 \\
\hline $\mathrm{C}$ & -0.630618 & -1.418892 & 0.056543 & $\mathrm{H}$ & -3.160149 & -1.416754 & -3.466199 \\
\hline $\mathrm{C}$ & -0.392522 & -3.783123 & 0.140965 & $\mathrm{C}$ & -6.852429 & 0.642259 & -0.804770 \\
\hline $\mathrm{C}$ & -1.847181 & -3.434878 & 0.405506 & $\mathrm{H}$ & -6.746028 & 1.418204 & -1.569886 \\
\hline $\mathrm{H}$ & -0.249538 & -4.289695 & -0.826551 & $\mathrm{H}$ & -7.615643 & -0.054354 & -1.169867 \\
\hline $\mathrm{H}$ & 0.043150 & -4.425767 & 0.914195 & $\mathrm{H}$ & -7.248123 & 1.113613 & 0.098944 \\
\hline $\mathrm{H}$ & -2.543750 & -3.952074 & -0.264818 & $\mathrm{C}$ & 1.428894 & 0.252847 & 0.596610 \\
\hline $\mathrm{H}$ & -2.156055 & -3.655707 & 1.438453 & $\mathrm{H}$ & 1.549831 & -0.439426 & 1.446701 \\
\hline $\mathrm{N}$ & 0.259826 & -2.471530 & 0.120557 & $\mathrm{C}$ & 2.677399 & 0.939799 & 0.303272 \\
\hline $\mathrm{N}$ & -1.869727 & -1.993177 & 0.166849 & $\mathrm{C}$ & 3.722205 & 0.928000 & 1.248676 \\
\hline $\mathrm{C}$ & 1.660173 & -2.443961 & -0.149171 & $\mathrm{C}$ & 2.905743 & 1.590910 & -0.925073 \\
\hline $\mathrm{C}$ & 2.553891 & -2.700681 & 0.906780 & $\mathrm{C}$ & 4.924781 & 1.576601 & 0.999272 \\
\hline $\mathrm{C}$ & 2.130890 & -2.231694 & -1.455594 & $\mathrm{H}$ & 3.565121 & 0.397483 & 2.187812 \\
\hline $\mathrm{C}$ & 3.921826 & -2.643785 & 0.652233 & $\mathrm{C}$ & 4.110427 & 2.230724 & -1.177942 \\
\hline $\mathrm{C}$ & 3.511387 & -2.176197 & -1.658240 & $\mathrm{H}$ & 2.115230 & 1.550396 & -1.672157 \\
\hline $\mathrm{C}$ & 4.422315 & -2.353830 & -0.618993 & $\mathrm{C}$ & 5.121831 & 2.232500 & -0.215571 \\
\hline $\mathrm{H}$ & 4.617158 & -2.803069 & 1.476096 & $\mathrm{H}$ & 5.714642 & 1.565247 & 1.746417 \\
\hline $\mathrm{H}$ & 3.882477 & -1.981046 & -2.664027 & $\mathrm{H}$ & 4.272256 & 2.722847 & -2.133834 \\
\hline $\mathrm{C}$ & -3.111561 & -1.332673 & -0.041172 & $\mathrm{H}$ & 6.066475 & 2.730896 & -0.418139 \\
\hline $\mathrm{C}$ & -3.901408 & -0.932061 & 1.040727 & $\mathrm{O}$ & -1.156441 & 0.955152 & 1.896140 \\
\hline $\mathrm{C}$ & -3.532284 & -1.128640 & -1.369433 & $\mathrm{O}$ & -2.436291 & 1.408649 & -0.693808 \\
\hline $\mathrm{C}$ & -5.107039 & -0.275300 & 0.763502 & $\mathrm{C}$ & -0.582705 & 0.309292 & 2.960497 \\
\hline $\mathrm{C}$ & -4.752944 & -0.499982 & -1.595906 & $\mathrm{H}$ & 0.436295 & 0.688773 & 3.214282 \\
\hline $\mathrm{C}$ & -5.552360 & -0.054948 & -0.540652 & $\mathrm{H}$ & -1.189620 & 0.408236 & 3.881736 \\
\hline $\mathrm{H}$ & -5.719044 & 0.066736 & 1.598344 & $\mathrm{H}$ & -0.439533 & -0.787171 & 2.800476 \\
\hline $\mathrm{H}$ & -5.073500 & -0.326684 & -2.622502 & $\mathrm{C}$ & -3.164383 & 1.928541 & 0.137049 \\
\hline $\mathrm{C}$ & 1.193754 & -2.043355 & -2.602475 & $\mathrm{H}$ & -2.927013 & 1.862398 & 1.214408 \\
\hline $\mathrm{H}$ & 0.778611 & -1.026927 & -2.594923 & $\mathrm{H}$ & -4.070182 & 2.478449 & -0.189020 \\
\hline
\end{tabular}




\begin{tabular}{|c|c|c|c|c|c|c|c|}
\hline \multirow[t]{2}{*}{$\mathrm{H}$} & 0.030188 & 0.512842 & -1.644442 & $\mathrm{C}$ & -1.856833 & -2.318404 & -2.793859 \\
\hline & & & & $\mathrm{H}$ & -1.267054 & -3.237290 & -2.897120 \\
\hline $19 b$ & & & & $\mathrm{H}$ & -2.580984 & -2.295226 & -3.612394 \\
\hline $\mathrm{Ru}$ & 0.380720 & 0.567458 & -0.314582 & $\mathrm{H}$ & -1.153113 & -1.481282 & -2.902846 \\
\hline $\mathrm{C}$ & 1.693739 & 3.401449 & -0.257099 & $\mathrm{C}$ & -6.054925 & -1.953399 & -0.023183 \\
\hline $\mathrm{C}$ & -0.537969 & 3.602271 & -0.785937 & $\mathrm{H}$ & -6.476123 & -1.415943 & -0.878798 \\
\hline $\mathrm{C}$ & 1.862318 & 4.779150 & -0.270403 & $\mathrm{H}$ & -6.522363 & -2.945275 & -0.009798 \\
\hline $\mathrm{H}$ & 2.509460 & 2.710719 & -0.057787 & $\mathrm{H}$ & -6.369704 & -1.434627 & 0.887003 \\
\hline $\mathrm{C}$ & -0.449418 & 4.986853 & -0.827275 & $\mathrm{C}$ & 3.246119 & -1.389219 & -2.242336 \\
\hline $\mathrm{H}$ & -1.477724 & 3.094924 & -0.985172 & $\mathrm{H}$ & 3.944139 & -1.034498 & -3.005008 \\
\hline $\mathrm{H}$ & 2.841215 & 5.198848 & -0.061912 & $\mathrm{H}$ & 3.008130 & -2.434649 & -2.477698 \\
\hline $\mathrm{H}$ & -1.331324 & 5.571569 & -1.067185 & $\mathrm{H}$ & 2.307036 & -0.822744 & -2.326491 \\
\hline $\mathrm{N}$ & 0.508952 & 2.804131 & -0.501497 & $\mathrm{C}$ & 2.704040 & -1.695888 & 2.747129 \\
\hline $\mathrm{C}$ & 0.773130 & 5.593536 & -0.560985 & $\mathrm{H}$ & 1.781323 & -1.105676 & 2.739575 \\
\hline $\mathrm{H}$ & 0.875004 & 6.674734 & -0.584663 & $\mathrm{H}$ & 2.400931 & -2.750169 & 2.766844 \\
\hline $\mathrm{C}$ & 0.590720 & -1.422370 & -0.145652 & $\mathrm{H}$ & 3.239271 & -1.491376 & 3.678318 \\
\hline $\mathrm{C}$ & 0.131859 & -3.739713 & -0.182598 & $\mathrm{C}$ & 7.058008 & -0.090009 & 0.782854 \\
\hline $\mathrm{C}$ & 1.633780 & -3.538189 & -0.084236 & $\mathrm{H}$ & 7.273745 & 0.144211 & 1.828714 \\
\hline $\mathrm{H}$ & -0.296823 & -4.176518 & 0.731719 & $\mathrm{H}$ & 7.794793 & -0.832719 & 0.455713 \\
\hline $\mathrm{H}$ & -0.168615 & -4.375565 & -1.022528 & $\mathrm{H}$ & 7.243869 & 0.813027 & 0.192283 \\
\hline $\mathrm{H}$ & 2.092424 & -4.071432 & 0.756176 & $\mathrm{C}$ & -1.579100 & 0.635692 & -0.488818 \\
\hline $\mathrm{H}$ & 2.161714 & -3.843533 & -0.998318 & $\mathrm{H}$ & -1.882975 & 0.472568 & -1.540634 \\
\hline $\mathrm{N}$ & -0.366503 & -2.371251 & -0.366614 & $\mathrm{C}$ & -2.709724 & 1.108599 & 0.288346 \\
\hline $\mathrm{N}$ & 1.749438 & -2.088299 & 0.097823 & $\mathrm{C}$ & -3.986174 & 1.237493 & -0.302535 \\
\hline $\mathrm{C}$ & -1.780867 & -2.199097 & -0.288640 & $\mathrm{C}$ & -2.600159 & 1.381818 & 1.669219 \\
\hline $\mathrm{C}$ & -2.540112 & -2.246511 & -1.466595 & $\mathrm{C}$ & -5.097749 & 1.599078 & 0.447498 \\
\hline $\mathrm{C}$ & -2.383563 & -2.076422 & 0.972944 & $\mathrm{H}$ & -4.085927 & 1.020063 & -1.364990 \\
\hline $\mathrm{C}$ & -3.928285 & -2.163454 & -1.352764 & $\mathrm{C}$ & -3.708315 & 1.745740 & 2.419032 \\
\hline $\mathrm{C}$ & -3.773482 & -2.002031 & 1.036819 & $\mathrm{H}$ & -1.624839 & 1.276064 & 2.135575 \\
\hline $\mathrm{C}$ & -4.562576 & -2.045878 & -0.113665 & $\mathrm{C}$ & -4.962545 & 1.851590 & 1.811694 \\
\hline $\mathrm{H}$ & -4.529819 & -2.176495 & -2.261260 & $\mathrm{H}$ & -6.073233 & 1.679854 & -0.026330 \\
\hline $\mathrm{H}$ & -4.250306 & -1.871194 & 2.007811 & $\mathrm{H}$ & -3.604685 & 1.943983 & 3.483039 \\
\hline $\mathrm{C}$ & 3.058110 & -1.538560 & 0.260129 & $\mathrm{H}$ & -5.830532 & 2.132665 & 2.402323 \\
\hline $\mathrm{C}$ & 3.829282 & -1.245275 & -0.874322 & $\mathrm{O}$ & 0.295621 & 0.160262 & -2.344059 \\
\hline $\mathrm{C}$ & 3.555541 & -1.378507 & 1.556489 & $\mathrm{O}$ & 0.730875 & 0.862639 & 1.721383 \\
\hline $\mathrm{C}$ & 5.124059 & -0.772176 & -0.675060 & $\mathrm{C}$ & 0.579649 & 1.232401 & -3.163340 \\
\hline $\mathrm{C}$ & 4.858040 & -0.895529 & 1.706068 & $\mathrm{H}$ & 1.577014 & 1.680413 & -2.965731 \\
\hline $\mathrm{C}$ & 5.655792 & -0.588890 & 0.604618 & $\mathrm{H}$ & 0.572544 & 0.906391 & -4.218132 \\
\hline $\mathrm{H}$ & 5.731487 & -0.528094 & -1.545995 & $\mathrm{H}$ & -0.153764 & 2.065510 & -3.090229 \\
\hline $\mathrm{H}$ & 5.258520 & -0.760170 & 2.710382 & $\mathrm{C}$ & 0.847626 & 1.820785 & 2.504463 \\
\hline $\mathrm{C}$ & -1.540518 & -1.928344 & 2.198735 & $\mathrm{H}$ & 1.246480 & 1.646985 & 3.512525 \\
\hline $\mathrm{H}$ & -0.917678 & -1.026329 & 2.121994 & $\mathrm{H}$ & 0.579320 & 2.848517 & 2.214155 \\
\hline $\mathrm{H}$ & -2.157390 & -1.846711 & 3.097363 & $\mathrm{H}$ & 2.061038 & 0.789668 & -0.585098 \\
\hline $\mathrm{H}$ & -0.848310 & -2.768180 & 2.339254 & & & & \\
\hline
\end{tabular}


20

$\mathrm{Ru}$

C

$\mathrm{C}$

$$
\text { H }
$$

$$
\mathrm{H}
$$

$-0.655991$

1.819579

1.732537

N -0.707105

$\mathrm{N} \quad 1.412481$

C $\quad-2.106114$

C -2.911002

C $\quad-2.646256$

C -4.282810

C -4.015022

C -4.851034

H $\quad-4.917958$

H $\quad-4.444561$

C 2.746313

C $\quad 3.438431$

C $\quad 3.359845$

C 4.772532

C 4.687402

C $\quad 5.411408$

$\mathrm{H} \quad 5.322418$

H 5.168419

C -1.757487

H -1.063792

H $\quad-2.337692$

$\mathrm{H} \quad-1.142111$

C $\quad-2.326525$

H $\quad-3.039269$

H $\quad-2.068590$

H $\quad-1.403291$

C -6.321338

H -6.811138

H $\quad-6.817582$

H $\quad-6.520630$

0.951797

2.629633

0.927712

2.545593

1.702304

0.260784

3.164537

0.982247

0.031414

1.674627

$-0.229400$

1.386781

0.432484

$-0.972808$

1.909380

3.525607

2.923259

4.167369

4.167404

0.001462

$-0.226895$

$-0.942618$

0.370507

1.647231

0.847041

2.586997

1.481815

$-0.727375$

C 2.787964

0.727375
-0.935995

H 3.499127

$-0.193989$

H 1.930556

H 2.405912

$-1.690761$

2.637132
$-0.979180$

0.311215

1.930948

1.866130

1.778341

2.887148

1.452503

2.839355

0.837001

0.966047

588331

1.353522

$-0.411578$

1.089628

$-0.662912$

0.080993

1.671294

$-1.450428$

0.595412

1.348611

$-0.463127$

1.012824

$-0.755269$

$-0.030705$

1.589260

$-1.581425$

$-1.213943$

$-1.814866$

$-1.881487$

$-0.573450$

2.350423

3.147337

1.853388

2.806803

$-0.202589$

0.357743

0.064185

$-1.266802$

2.464503

3.269009

2.885291

2.105705

$-1.291820$

$\begin{array}{lrrr}\mathrm{H} & 1.772949 & 2.100597 & -1.820637 \\ \mathrm{H} & 2.086175 & 3.417561 & -0.691049 \\ \mathrm{H} & 3.202997 & 3.132289 & -2.032195 \\ \mathrm{C} & 6.835656 & 0.129823 & -0.384381 \\ \mathrm{H} & 6.910768 & -0.335221 & -1.373779 \\ \mathrm{H} & 7.446306 & 1.038155 & -0.419621 \\ \mathrm{H} & 7.294390 & -0.553572 & 0.334752 \\ \mathrm{C} & 0.050017 & -1.709789 & 0.425143 \\ \mathrm{H} & 0.243587 & -1.446336 & 1.478110 \\ \mathrm{C} & -0.562883 & -3.016043 & 0.327159 \\ \mathrm{C} & -0.804006 & -3.813937 & 1.468040 \\ \mathrm{C} & -0.926195 & -3.562708 & -0.926867 \\ \mathrm{C} & -1.368918 & -5.077420 & 1.364881 \\ \mathrm{H} & -0.532226 & -3.412550 & 2.443818 \\ \mathrm{C} & -1.509221 & -4.815944 & -1.030321 \\ \mathrm{H} & -0.731616 & -2.980365 & -1.829385 \\ \mathrm{C} & -1.731249 & -5.582112 & 0.115908 \\ \mathrm{H} & -1.538294 & -5.671797 & 2.259480 \\ \mathrm{H} & -1.781181 & -5.209785 & -2.006440 \\ \mathrm{H} & -2.177781 & -6.569172 & 0.033211 \\ \mathrm{O} & 1.796297 & -0.320509 & -1.701527 \\ \mathrm{O} & -2.196372 & -0.656234 & -0.937102 \\ \mathrm{C} & 2.436036 & -1.557724 & -1.760827 \\ \mathrm{H} & 1.808382 & -2.373441 & -2.165720 \\ \mathrm{H} & 3.319554 & -1.471667 & -2.410694 \\ \mathrm{H} & -2.7988239 & -0.227986 & -1.763631 \\ \mathrm{H} & -2.700386 & 0.536709 & -2.499041 \\ \mathrm{H} & -0.620462 & -1.776867 \\ \mathrm{H} & -2.110095\end{array}$

\section{TS 20-12}

$\begin{array}{llll}\mathrm{Ru} & -0.362508 & -0.698346 & 0.888267\end{array}$

$\begin{array}{llll}\text { C } & -0.688004 & 0.879657 & -0.352363\end{array}$

$\begin{array}{llll}\text { C } & -0.460196 & 2.975634 & -1.413811\end{array}$

$\begin{array}{llll}\text { C } & -1.835247 & 2.415720 & -1.731067\end{array}$

$\mathrm{H} \quad-0.507816 \quad 3.823486 \quad-0.712800$

$\mathrm{H} \quad 0.094997 \quad 3.300346 \quad-2.298932$

$\mathrm{H} \quad-2.649597 \quad 3.116616 \quad-1.522152$

$\begin{array}{llll}\mathrm{H} & -1.925656 & 2.099202 & -2.782138\end{array}$

$\begin{array}{llll}\mathrm{N} & 0.191853 & 1.828954 & -0.777189\end{array}$

$\begin{array}{llll}\mathrm{N} & -1.903455 & 1.253562 & -0.842478\end{array}$

$\begin{array}{llll}\text { C } & 1.594147 & 1.825451 & -0.529980\end{array}$

$\begin{array}{llll}\text { C } & 2.428473 & 1.177457 & -1.452362\end{array}$ 


\begin{tabular}{|c|c|c|c|c|c|c|c|}
\hline $\mathrm{C}$ & 2.107365 & 2.471254 & 0.598847 & $\mathrm{H}$ & 2.070008 & -2.316129 & -2.626955 \\
\hline $\mathrm{C}$ & 3.791790 & 1.117008 & -1.177491 & $\mathrm{C}$ & 4.079104 & -2.791993 & 0.639009 \\
\hline $\mathrm{C}$ & 3.483146 & 2.400608 & 0.826324 & $\mathrm{H}$ & 2.239652 & -2.324244 & 1.653920 \\
\hline $\mathrm{C}$ & 4.334899 & 1.706175 & -0.032173 & $\mathrm{C}$ & 4.710009 & -2.967117 & -0.593440 \\
\hline $\mathrm{H}$ & 4.446475 & 0.588677 & -1.869421 & $\mathrm{H}$ & 4.456479 & -2.965713 & -2.734985 \\
\hline $\mathrm{H}$ & 3.894205 & 2.872897 & 1.718887 & $\mathrm{H}$ & 4.632632 & -2.965659 & 1.558750 \\
\hline $\mathrm{C}$ & -3.143329 & 0.584946 & -0.630083 & $\mathrm{H}$ & 5.756367 & -3.258572 & -0.634695 \\
\hline $\mathrm{C}$ & -3.508683 & -0.476172 & -1.470011 & $\mathrm{O}$ & -0.945736 & -1.989234 & 2.439995 \\
\hline $\mathrm{C}$ & -3.985207 & 1.033820 & 0.396772 & $\mathrm{O}$ & 1.514413 & -0.054780 & 1.797146 \\
\hline $\mathrm{C}$ & -4.731999 & -1.106871 & -1.242807 & $\mathrm{C}$ & -1.888071 & -2.131925 & 1.534582 \\
\hline $\mathrm{C}$ & -5.201462 & 0.376357 & 0.583848 & $\mathrm{H}$ & -1.931702 & -3.096942 & 0.995995 \\
\hline $\mathrm{C}$ & -5.591881 & -0.695103 & -0.221978 & $\mathrm{H}$ & -2.889419 & -1.727906 & 1.768300 \\
\hline $\mathrm{H}$ & -5.020266 & -1.941463 & -1.880895 & $\mathrm{H}$ & -1.758694 & -1.370236 & 0.313372 \\
\hline $\mathrm{H}$ & -5.859066 & 0.708700 & 1.386233 & $\mathrm{C}$ & 1.552850 & 0.312020 & 2.964721 \\
\hline $\mathrm{C}$ & 1.187098 & 3.145772 & 1.568020 & $\mathrm{H}$ & 0.625969 & 0.466541 & 3.542537 \\
\hline $\mathrm{H}$ & 0.305826 & 2.520846 & 1.771040 & $\mathrm{H}$ & 2.529871 & 0.497087 & 3.442767 \\
\hline $\mathrm{H}$ & 1.693535 & 3.356081 & 2.514921 & $\mathrm{H}$ & -1.020768 & 0.597832 & 1.874976 \\
\hline $\mathrm{H}$ & 0.815890 & 4.105163 & 1.187040 & & & & \\
\hline $\mathrm{C}$ & 1.851041 & 0.590433 & -2.702342 & $21 \mathrm{a}$ & & & \\
\hline $\mathrm{H}$ & 1.411832 & 1.366289 & -3.342082 & $\mathrm{Ru}$ & -0.212383 & -1.033082 & 0.504178 \\
\hline $\mathrm{H}$ & 2.615584 & 0.070039 & -3.283742 & $\mathrm{C}$ & -0.869461 & 0.882593 & -0.184828 \\
\hline $\mathrm{H}$ & 1.048516 & -0.121660 & -2.476771 & $\mathrm{C}$ & -0.757671 & 3.150091 & -0.863802 \\
\hline $\mathrm{C}$ & 5.795595 & 1.566804 & 0.269111 & $\mathrm{C}$ & -2.188458 & 2.657920 & -1.024277 \\
\hline $\mathrm{H}$ & 6.417319 & 1.861357 & -0.582496 & $\mathrm{H}$ & -0.656982 & 3.917290 & -0.082467 \\
\hline $\mathrm{H}$ & 6.093324 & 2.174282 & 1.127665 & $\mathrm{H}$ & -0.332538 & 3.558167 & -1.787042 \\
\hline $\mathrm{H}$ & 6.048405 & 0.524522 & 0.496352 & $\mathrm{H}$ & -2.921831 & 3.269758 & -0.487568 \\
\hline $\mathrm{C}$ & -2.579565 & -0.938997 & -2.547891 & $\mathrm{H}$ & -2.505410 & 2.605773 & -2.075651 \\
\hline $\mathrm{H}$ & -3.027939 & -1.734862 & -3.146854 & $\mathrm{~N}$ & -0.047072 & 1.925036 & -0.467629 \\
\hline $\mathrm{H}$ & -2.297481 & -0.124820 & -3.225795 & $\mathrm{~N}$ & -2.126902 & 1.305927 & -0.448613 \\
\hline $\mathrm{H}$ & -1.644712 & -1.318012 & -2.117585 & $\mathrm{C}$ & 1.377663 & 1.892028 & -0.490872 \\
\hline $\mathrm{C}$ & -3.564285 & 2.167578 & 1.275618 & $\mathrm{C}$ & 2.023929 & 1.330817 & -1.606610 \\
\hline $\mathrm{H}$ & -2.616105 & 1.921511 & 1.771059 & $\mathrm{C}$ & 2.099841 & 2.488010 & 0.549519 \\
\hline $\mathrm{H}$ & -3.398740 & 3.090216 & 0.705450 & $\mathrm{C}$ & 3.414874 & 1.357465 & -1.641341 \\
\hline $\mathrm{H}$ & -4.317383 & 2.379337 & 2.038197 & $\mathrm{C}$ & 3.495766 & 2.494212 & 0.467379 \\
\hline $\mathrm{C}$ & -6.915905 & -1.366257 & -0.017554 & $\mathrm{C}$ & 4.169749 & 1.933247 & -0.615097 \\
\hline $\mathrm{H}$ & -6.897507 & -2.407524 & -0.350391 & $\mathrm{H}$ & 3.926983 & 0.906534 & -2.490499 \\
\hline $\mathrm{H}$ & -7.218497 & -1.352517 & 1.033015 & $\mathrm{H}$ & 4.068409 & 2.944880 & 1.277664 \\
\hline $\mathrm{H}$ & -7.709024 & -0.863868 & -0.583806 & $\mathrm{C}$ & -3.338151 & 0.591023 & -0.212193 \\
\hline $\mathrm{C}$ & 0.601688 & -1.773969 & -0.436989 & $\mathrm{C}$ & -3.950290 & -0.107810 & -1.258980 \\
\hline $\mathrm{H}$ & 0.155612 & -1.955901 & -1.435093 & $\mathrm{C}$ & -3.934248 & 0.691406 & 1.053890 \\
\hline $\mathrm{C}$ & 1.994694 & -2.192743 & -0.477897 & $\mathrm{C}$ & -5.159276 & -0.756086 & -0.995750 \\
\hline $\mathrm{C}$ & 2.643925 & -2.434700 & -1.709132 & $\mathrm{C}$ & -5.143054 & 0.034420 & 1.267661 \\
\hline $\mathrm{C}$ & 2.743791 & -2.419198 & 0.697559 & $\mathrm{C}$ & -5.765929 & -0.704476 & 0.258459 \\
\hline $\mathrm{C}$ & 3.980891 & -2.800523 & -1.771055 & $\mathrm{H}$ & -5.641544 & -1.313098 & -1.798492 \\
\hline
\end{tabular}




\begin{tabular}{|c|c|c|c|c|c|c|c|}
\hline $\mathrm{H}$ & -5.611218 & 0.099666 & 2.249131 & $\mathrm{H}$ & -1.984345 & -3.403024 & -2.097854 \\
\hline $\mathrm{C}$ & 1.402349 & 3.118570 & 1.715463 & $\mathrm{H}$ & -0.893094 & -3.847939 & -0.608670 \\
\hline $\mathrm{H}$ & 0.454037 & 2.618894 & 1.937844 & $\mathrm{H}$ & -1.504593 & -0.873238 & 1.610868 \\
\hline $\mathrm{H}$ & 2.026532 & 3.086708 & 2.612989 & $\mathrm{H}$ & -0.315427 & -2.431837 & 1.340582 \\
\hline $\mathrm{H}$ & 1.173740 & 4.175527 & 1.528066 & & & & \\
\hline $\mathrm{C}$ & 1.232974 & 0.717053 & -2.718444 & $21 b$ & & & \\
\hline $\mathrm{H}$ & 0.439375 & 1.385145 & -3.075199 & $\mathrm{Ru}$ & 0.347188 & 1.012719 & 0.291208 \\
\hline $\mathrm{H}$ & 1.874636 & 0.468586 & -3.567096 & $\mathrm{C}$ & 0.785769 & -0.880338 & -0.124445 \\
\hline $\mathrm{H}$ & 0.742980 & -0.202340 & -2.373851 & $\mathrm{C}$ & 0.622797 & -3.150003 & -0.815401 \\
\hline $\mathrm{C}$ & 5.665610 & 1.913147 & -0.675541 & $\mathrm{C}$ & 2.074383 & -2.832983 & -0.503743 \\
\hline $\mathrm{H}$ & 6.040968 & 2.379588 & -1.592564 & $\mathrm{H}$ & 0.242015 & -4.016061 & -0.264220 \\
\hline $\mathrm{H}$ & 6.113128 & 2.436859 & 0.173534 & $\mathrm{H}$ & 0.453991 & -3.335368 & -1.886881 \\
\hline $\mathrm{H}$ & 6.040899 & 0.883303 & -0.667549 & $\mathrm{H}$ & 2.441660 & -3.352023 & 0.394671 \\
\hline $\mathrm{C}$ & -3.349179 & -0.138425 & -2.630217 & $\mathrm{H}$ & 2.755813 & -3.076636 & -1.325775 \\
\hline $\mathrm{H}$ & -3.679704 & -1.021466 & -3.184091 & $\mathrm{~N}$ & -0.067416 & -1.927904 & -0.390220 \\
\hline $\mathrm{H}$ & -3.653535 & 0.735405 & -3.220639 & $\mathrm{~N}$ & 2.044180 & -1.388045 & -0.275643 \\
\hline $\mathrm{H}$ & -2.257336 & -0.150506 & -2.591313 & $\mathrm{C}$ & -1.445203 & -1.991065 & -0.046175 \\
\hline $\mathrm{C}$ & -3.266938 & 1.468956 & 2.142943 & $\mathrm{C}$ & -2.414733 & -2.074231 & -1.056748 \\
\hline $\mathrm{H}$ & -2.324239 & 0.978464 & 2.417287 & $\mathrm{C}$ & -1.799012 & -2.051632 & 1.311819 \\
\hline $\mathrm{H}$ & -3.014737 & 2.490154 & 1.830884 & $\mathrm{C}$ & -3.758151 & -2.083008 & -0.683257 \\
\hline $\mathrm{H}$ & -3.899544 & 1.532541 & 3.031512 & $\mathrm{C}$ & -3.155786 & -2.045108 & 1.636314 \\
\hline $\mathrm{C}$ & -7.046118 & -1.435782 & 0.525734 & $\mathrm{C}$ & -4.149347 & -2.025182 & 0.654892 \\
\hline $\mathrm{H}$ & -6.859147 & -2.395320 & 1.021458 & $\mathrm{H}$ & -4.519973 & -2.103409 & -1.462764 \\
\hline $\mathrm{H}$ & -7.709118 & -0.865697 & 1.183303 & $\mathrm{H}$ & -3.444448 & -2.059157 & 2.686692 \\
\hline $\mathrm{H}$ & -7.590222 & -1.651703 & -0.397807 & $\mathrm{C}$ & 3.291341 & -0.695985 & -0.263694 \\
\hline $\mathrm{C}$ & 1.330773 & -1.906964 & -0.303334 & $\mathrm{C}$ & 3.829682 & -0.277346 & -1.484474 \\
\hline $\mathrm{H}$ & 1.038665 & -2.668500 & -1.066596 & $\mathrm{C}$ & 3.970403 & -0.515386 & 0.948029 \\
\hline $\mathrm{C}$ & 2.741652 & -2.083453 & 0.011866 & $\mathrm{C}$ & 5.079658 & 0.342349 & -1.474776 \\
\hline $\mathrm{C}$ & 3.448334 & -3.221741 & -0.431989 & $\mathrm{C}$ & 5.217333 & 0.104264 & 0.909826 \\
\hline $\mathrm{C}$ & 3.477901 & -1.098038 & 0.702591 & $\mathrm{C}$ & 5.788795 & 0.538952 & -0.289544 \\
\hline $\mathrm{C}$ & 4.804715 & -3.382976 & -0.179873 & $\mathrm{H}$ & 5.506113 & 0.686310 & -2.416451 \\
\hline $\mathrm{H}$ & 2.902318 & -3.988056 & -0.980150 & $\mathrm{H}$ & 5.751965 & 0.264686 & 1.845567 \\
\hline $\mathrm{C}$ & 4.834255 & -1.254445 & 0.949171 & $\mathrm{C}$ & -0.741310 & -2.162993 & 2.368069 \\
\hline $\mathrm{H}$ & 2.966974 & -0.192301 & 1.012288 & $\mathrm{H}$ & -0.061667 & -1.302246 & 2.388800 \\
\hline $\mathrm{C}$ & 5.505719 & -2.399358 & 0.515958 & $\mathrm{H}$ & -1.190865 & -2.257512 & 3.359729 \\
\hline $\mathrm{H}$ & 5.320399 & -4.274454 & -0.528901 & $\mathrm{H}$ & -0.115069 & -3.049125 & 2.200654 \\
\hline $\mathrm{H}$ & 5.377103 & -0.471039 & 1.475366 & $\mathrm{C}$ & -2.020461 & -2.158510 & -2.499394 \\
\hline $\mathrm{H}$ & 6.568400 & -2.519299 & 0.711630 & $\mathrm{H}$ & -1.797495 & -3.192162 & -2.793425 \\
\hline $\mathrm{O}$ & 0.923738 & -0.160405 & 2.012132 & $\mathrm{H}$ & -2.824539 & -1.811974 & -3.155191 \\
\hline $\mathrm{O}$ & -1.304729 & -1.880742 & -0.969291 & $\mathrm{H}$ & -1.122706 & -1.567642 & -2.711040 \\
\hline $\mathrm{C}$ & 0.612168 & -0.002609 & 3.192196 & $\mathrm{C}$ & -5.597407 & -1.919412 & 1.020279 \\
\hline $\mathrm{H}$ & -0.372623 & -0.324028 & 3.565749 & $\mathrm{H}$ & -6.226675 & -2.553718 & 0.388656 \\
\hline $\mathrm{H}$ & 1.332721 & 0.467426 & 3.877193 & $\mathrm{H}$ & -5.776196 & -2.194946 & 2.062952 \\
\hline $\mathrm{C}$ & -1.381250 & -3.091924 & -1.235721 & $\mathrm{H}$ & -5.953821 & -0.889939 & 0.890331 \\
\hline
\end{tabular}




\begin{tabular}{|c|c|c|c|c|c|c|c|}
\hline $\mathrm{C}$ & 3.047673 & -0.450335 & -2.748550 & $\mathrm{H}$ & -2.476328 & 1.819650 & -2.804335 \\
\hline $\mathrm{H}$ & 2.066403 & 0.033240 & -2.662728 & $\mathrm{~N}$ & 0.006104 & 1.601369 & -0.881927 \\
\hline $\mathrm{H}$ & 3.572287 & -0.017920 & -3.604055 & $\mathrm{~N}$ & -2.069446 & 0.944818 & -0.916427 \\
\hline $\mathrm{H}$ & 2.855633 & -1.506287 & -2.978259 & $\mathrm{C}$ & 1.428706 & 1.581154 & -0.767191 \\
\hline $\mathrm{C}$ & 3.321764 & -0.909013 & 2.235287 & $\mathrm{C}$ & 2.183160 & 0.681095 & -1.532176 \\
\hline $\mathrm{H}$ & 2.421719 & -0.304231 & 2.406789 & $\mathrm{C}$ & 2.047032 & 2.528304 & 0.066128 \\
\hline $\mathrm{H}$ & 2.999993 & -1.957358 & 2.231343 & $\mathrm{C}$ & 3.573597 & 0.709876 & -1.398286 \\
\hline $\mathrm{H}$ & 3.998436 & -0.768344 & 3.081616 & $\mathrm{C}$ & 3.435722 & 2.516215 & 0.167698 \\
\hline $\mathrm{C}$ & 7.138850 & 1.189564 & -0.297684 & $\mathrm{C}$ & 4.217273 & 1.608266 & -0.552450 \\
\hline $\mathrm{H}$ & 7.216322 & 1.967933 & 0.467932 & $\mathrm{H}$ & 4.167549 & -0.013304 & -1.955615 \\
\hline $\mathrm{H}$ & 7.935526 & 0.465870 & -0.089814 & $\mathrm{H}$ & 3.920999 & 3.236583 & 0.826205 \\
\hline $\mathrm{H}$ & 7.360057 & 1.647983 & -1.265216 & $\mathrm{C}$ & -3.326151 & 0.449270 & -0.455542 \\
\hline $\mathrm{C}$ & -1.314599 & 0.889682 & -0.792341 & $\mathrm{C}$ & -4.014406 & -0.508001 & -1.208925 \\
\hline $\mathrm{H}$ & -1.230162 & 0.302782 & -1.720110 & $\mathrm{C}$ & -3.896164 & 1.018817 & 0.696147 \\
\hline $\mathrm{C}$ & -2.608169 & 1.545433 & -0.798663 & $\mathrm{C}$ & -5.247562 & -0.962244 & -0.733702 \\
\hline $\mathrm{C}$ & -3.581338 & 1.146981 & -1.744803 & $\mathrm{C}$ & -5.130596 & 0.541711 & 1.126648 \\
\hline $\mathrm{C}$ & -2.985761 & 2.537563 & 0.132641 & $\mathrm{C}$ & -5.812598 & -0.465758 & 0.438584 \\
\hline $\mathrm{C}$ & -4.868288 & 1.663695 & -1.729555 & $\mathrm{H}$ & -5.782391 & -1.721871 & -1.302799 \\
\hline $\mathrm{H}$ & -3.301158 & 0.401569 & -2.487389 & $\mathrm{H}$ & -5.574520 & 0.969613 & 2.024779 \\
\hline $\mathrm{C}$ & -4.258388 & 3.086790 & 0.122558 & $\mathrm{C}$ & 1.236217 & 3.545390 & 0.811164 \\
\hline $\mathrm{H}$ & -2.251477 & 2.866436 & 0.861171 & $\mathrm{H}$ & 0.272133 & 3.142454 & 1.141145 \\
\hline $\mathrm{C}$ & -5.211423 & 2.641072 & -0.796191 & $\mathrm{H}$ & 1.775442 & 3.919157 & 1.686141 \\
\hline $\mathrm{H}$ & -5.602450 & 1.319365 & -2.453593 & $\mathrm{H}$ & 1.010819 & 4.419154 & 0.185716 \\
\hline $\mathrm{H}$ & -4.520960 & 3.856391 & 0.843874 & $\mathrm{C}$ & 1.559134 & -0.292808 & -2.483630 \\
\hline $\mathrm{H}$ & -6.214983 & 3.058367 & -0.786035 & $\mathrm{H}$ & 0.481532 & -0.143005 & -2.595878 \\
\hline $\mathrm{O}$ & 0.209122 & 3.188559 & 0.684251 & $\mathrm{H}$ & 2.021308 & -0.217552 & -3.474086 \\
\hline $\mathrm{O}$ & 0.425855 & 0.952737 & 2.483423 & $\mathrm{H}$ & 1.711009 & -1.318991 & -2.128721 \\
\hline $\mathrm{C}$ & 0.435093 & 4.089633 & -0.107521 & $\mathrm{C}$ & 5.709470 & 1.613747 & -0.422485 \\
\hline $\mathrm{H}$ & 0.700294 & 3.883177 & -1.158396 & $\mathrm{H}$ & 6.142729 & 2.551227 & -0.789777 \\
\hline $\mathrm{H}$ & 0.383223 & 5.140701 & 0.220681 & $\mathrm{H}$ & 6.019697 & 1.509474 & 0.622721 \\
\hline $\mathrm{C}$ & -0.818580 & 1.108182 & 2.197460 & $\mathrm{H}$ & 6.161883 & 0.790451 & -0.980733 \\
\hline $\mathrm{H}$ & -1.281123 & 2.089746 & 2.388265 & $\mathrm{C}$ & -3.490087 & -0.998139 & -2.524007 \\
\hline $\mathrm{H}$ & -1.516040 & 0.257238 & 2.179652 & $\mathrm{H}$ & -3.730655 & -2.053248 & -2.683040 \\
\hline $\mathrm{H}$ & 1.983339 & 1.286968 & 0.347216 & $\mathrm{H}$ & -3.943087 & -0.446168 & -3.357391 \\
\hline \multirow[t]{2}{*}{$\mathrm{H}$} & 0.860061 & 1.339159 & -1.200062 & $\mathrm{H}$ & -2.406667 & -0.873296 & -2.607281 \\
\hline & & & & $\mathrm{C}$ & -3.195022 & 2.111880 & 1.439158 \\
\hline $22 a$ & & & & $\mathrm{H}$ & -2.297343 & 1.717252 & 1.930922 \\
\hline $\mathrm{Ru}$ & -0.283022 & -0.908918 & 0.905741 & $\mathrm{H}$ & -2.860482 & 2.919505 & 0.776545 \\
\hline $\mathrm{C}$ & -0.851426 & 0.680325 & -0.366985 & $\mathrm{H}$ & -3.843563 & 2.549545 & 2.201678 \\
\hline $\mathrm{C}$ & -0.605317 & 2.500523 & -1.870388 & $\mathrm{C}$ & -7.115769 & -0.996721 & 0.952183 \\
\hline $\mathrm{C}$ & -2.073379 & 2.101945 & -1.824103 & $\mathrm{H}$ & -6.955985 & -1.741383 & 1.740143 \\
\hline $\mathrm{H}$ & -0.431460 & 3.549236 & -1.599641 & $\mathrm{H}$ & -7.733514 & -0.205128 & 1.386641 \\
\hline $\mathrm{H}$ & -0.148002 & 2.334444 & -2.854736 & $\mathrm{H}$ & -7.694635 & -1.482991 & 0.162436 \\
\hline $\mathrm{H}$ & -2.720530 & 2.892596 & -1.421456 & $\mathrm{C}$ & 1.225167 & -2.023285 & 0.395418 \\
\hline
\end{tabular}




\begin{tabular}{|c|c|c|c|c|c|c|c|}
\hline $\mathrm{H}$ & 0.910520 & -2.907414 & -0.207086 & $\mathrm{C}$ & 4.129428 & -0.554041 & 0.829882 \\
\hline $\mathrm{C}$ & 2.680508 & -2.005311 & 0.445712 & $\mathrm{C}$ & 4.701699 & 0.093039 & -1.828507 \\
\hline $\mathrm{C}$ & 3.414478 & -2.716395 & -0.533001 & $\mathrm{C}$ & 5.333982 & 0.061935 & 0.485997 \\
\hline $\mathrm{C}$ & 3.435848 & -1.276720 & 1.390901 & $\mathrm{C}$ & 5.635348 & 0.398686 & -0.834241 \\
\hline $\mathrm{C}$ & 4.801282 & -2.664719 & -0.600110 & $\mathrm{H}$ & 4.925748 & 0.341507 & -2.865280 \\
\hline $\mathrm{H}$ & 2.860562 & -3.313760 & -1.257638 & $\mathrm{H}$ & 6.051903 & 0.291320 & 1.272653 \\
\hline $\mathrm{C}$ & 4.821167 & -1.241373 & 1.336836 & $\mathrm{C}$ & -1.294508 & -0.826272 & 2.699688 \\
\hline $\mathrm{H}$ & 2.911918 & -0.739587 & 2.171083 & $\mathrm{H}$ & -1.052901 & 0.231140 & 2.533574 \\
\hline $\mathrm{C}$ & 5.517603 & -1.920828 & 0.336003 & $\mathrm{H}$ & -1.810626 & -0.907694 & 3.660346 \\
\hline $\mathrm{H}$ & 5.326512 & -3.217871 & -1.375712 & $\mathrm{H}$ & -0.325963 & -1.329750 & 2.778605 \\
\hline $\mathrm{H}$ & 5.370736 & -0.675615 & 2.086971 & $\mathrm{C}$ & -1.773181 & -2.905903 & -1.887300 \\
\hline $\mathrm{H}$ & 6.603550 & -1.883221 & 0.297502 & $\mathrm{H}$ & -1.516057 & -3.968922 & -1.788806 \\
\hline $\mathrm{O}$ & -1.428008 & -2.308676 & -0.067605 & $\mathrm{H}$ & -2.470694 & -2.826244 & -2.725646 \\
\hline $\mathrm{O}$ & 0.937256 & 0.294625 & 2.061794 & $\mathrm{H}$ & -0.850876 & -2.379349 & -2.156975 \\
\hline $\mathrm{C}$ & -1.223426 & -2.743732 & -1.208318 & $\mathrm{C}$ & -5.843536 & -1.568044 & 0.728219 \\
\hline $\mathrm{H}$ & -1.862000 & -3.547908 & -1.596346 & $\mathrm{H}$ & -6.375463 & -2.339028 & 0.163333 \\
\hline $\mathrm{H}$ & -0.436416 & -2.325116 & -1.853824 & $\mathrm{H}$ & -6.199087 & -1.597909 & 1.762173 \\
\hline $\mathrm{C}$ & 0.644318 & 1.184538 & 2.859985 & $\mathrm{H}$ & -6.144708 & -0.598536 & 0.311975 \\
\hline $\mathrm{H}$ & -0.406311 & 1.466606 & 3.044844 & $\mathrm{C}$ & 2.514495 & -0.874565 & -2.618038 \\
\hline $\mathrm{H}$ & 1.445540 & 1.707036 & 3.400850 & $\mathrm{H}$ & 2.848907 & -0.489081 & -3.584040 \\
\hline $\mathrm{H}$ & -1.622216 & -0.296702 & 1.812143 & $\mathrm{H}$ & 2.395776 & -1.960093 & -2.722707 \\
\hline \multirow[t]{2}{*}{$\mathrm{H}$} & -1.058646 & -1.430517 & 2.242404 & $\mathrm{H}$ & 1.517987 & -0.466148 & -2.413276 \\
\hline & & & & $\mathrm{C}$ & 3.801371 & -0.896717 & 2.251075 \\
\hline $22 b$ & & & & $\mathrm{H}$ & 2.818514 & -0.505869 & 2.538896 \\
\hline $\mathrm{Ru}$ & 0.331906 & 1.052258 & 0.118254 & $\mathrm{H}$ & 3.763981 & -1.981700 & 2.407404 \\
\hline $\mathrm{C}$ & 0.744972 & -0.872903 & 0.148074 & $\mathrm{H}$ & 4.550231 & -0.493450 & 2.937203 \\
\hline $\mathrm{C}$ & 0.496422 & -3.202982 & 0.488264 & $\mathrm{C}$ & 6.940938 & 1.044519 & -1.185390 \\
\hline $\mathrm{C}$ & 1.929490 & -2.926656 & 0.086830 & $\mathrm{H}$ & 7.399832 & 1.526243 & -0.318023 \\
\hline $\mathrm{H}$ & 0.403808 & -3.469109 & 1.552346 & $\mathrm{H}$ & 7.660712 & 0.309732 & -1.564455 \\
\hline $\mathrm{H}$ & 0.025798 & -3.999594 & -0.096607 & $\mathrm{H}$ & 6.821225 & 1.800305 & -1.967075 \\
\hline $\mathrm{H}$ & 2.667429 & -3.380550 & 0.756433 & $\mathrm{C}$ & -1.205601 & 0.581849 & -0.930548 \\
\hline $\mathrm{H}$ & 2.150509 & -3.269433 & -0.936482 & $\mathrm{H}$ & -1.139019 & -0.225020 & -1.679141 \\
\hline $\mathrm{N}$ & -0.151998 & -1.913529 & 0.240495 & $\mathrm{C}$ & -2.504028 & 1.229481 & -1.051480 \\
\hline $\mathrm{N}$ & 1.985249 & -1.466132 & 0.155685 & $\mathrm{C}$ & -3.277399 & 1.065787 & -2.220420 \\
\hline $\mathrm{C}$ & -1.569199 & -1.849606 & 0.403761 & $\mathrm{C}$ & -3.099958 & 1.926488 & 0.020086 \\
\hline $\mathrm{C}$ & -2.376466 & -2.351977 & -0.632942 & $\mathrm{C}$ & -4.551906 & 1.603732 & -2.331839 \\
\hline $\mathrm{C}$ & -2.137813 & -1.358933 & 1.589250 & $\mathrm{H}$ & -2.850826 & 0.497270 & -3.046193 \\
\hline $\mathrm{C}$ & -3.760650 & -2.277840 & -0.493886 & $\mathrm{C}$ & -4.377469 & 2.459737 & -0.085346 \\
\hline $\mathrm{C}$ & -3.529217 & -1.309500 & 1.681049 & $\mathrm{H}$ & -2.552533 & 1.989291 & 0.958603 \\
\hline $\mathrm{C}$ & -4.358091 & -1.736421 & 0.644666 & $\mathrm{C}$ & -5.107590 & 2.309597 & -1.264681 \\
\hline $\mathrm{H}$ & -4.389363 & -2.623421 & -1.314016 & $\mathrm{H}$ & -5.121192 & 1.466842 & -3.248245 \\
\hline $\mathrm{H}$ & -3.976291 & -0.901636 & 2.587554 & $\mathrm{H}$ & -4.818428 & 2.980208 & 0.761740 \\
\hline $\mathrm{C}$ & 3.207745 & -0.823600 & -0.190126 & $\mathrm{H}$ & -6.110266 & 2.721714 & -1.343676 \\
\hline $\mathrm{C}$ & 3.489269 & -0.529126 & -1.533580 & $\mathrm{O}$ & -0.363409 & 3.173789 & 0.232857 \\
\hline
\end{tabular}




\begin{tabular}{|c|c|c|c|c|c|c|c|}
\hline $\mathrm{O}$ & 0.943995 & 1.386964 & 2.210987 & $\mathrm{C}$ & -5.289123 & 0.333956 & -0.712815 \\
\hline $\mathrm{C}$ & -0.358428 & 3.884993 & 1.230180 & $\mathrm{C}$ & -5.448913 & 1.360795 & 0.221220 \\
\hline $\mathrm{H}$ & -0.667400 & 4.940723 & 1.153448 & $\mathrm{H}$ & -4.652244 & 2.236785 & 2.014303 \\
\hline $\mathrm{H}$ & -0.075113 & 3.479969 & 2.217918 & $\mathrm{H}$ & -5.987868 & 0.253543 & -1.544883 \\
\hline $\mathrm{C}$ & 1.903791 & 1.940268 & 1.519704 & $\mathrm{C}$ & 0.037158 & -3.411767 & -1.842676 \\
\hline $\mathrm{H}$ & 2.908936 & 1.498038 & 1.564884 & $\mathrm{H}$ & 0.359663 & -3.338749 & -2.883307 \\
\hline $\mathrm{H}$ & 1.916612 & 3.046917 & 1.431081 & $\mathrm{H}$ & -0.392875 & -4.412368 & -1.700071 \\
\hline $\mathrm{H}$ & 1.905746 & 1.653106 & -0.298010 & $\mathrm{H}$ & -0.762658 & -2.680712 & -1.698977 \\
\hline \multirow[t]{2}{*}{$\mathrm{H}$} & 1.408387 & 1.400326 & -1.119115 & $\mathrm{C}$ & 1.891581 & -2.141715 & 2.709324 \\
\hline & & & & $\mathrm{H}$ & 2.373411 & -2.841584 & 3.400312 \\
\hline $22 \mathrm{c}$ & & & & $\mathrm{H}$ & 2.350277 & -1.160301 & 2.875159 \\
\hline $\mathrm{Ru}$ & -0.105058 & 0.364878 & -0.492503 & $\mathrm{H}$ & 0.837458 & -2.052931 & 2.982672 \\
\hline $\mathrm{C}$ & 0.813014 & 3.359600 & -0.597495 & $\mathrm{C}$ & 4.961622 & -3.702973 & -0.932047 \\
\hline $\mathrm{C}$ & 1.676999 & 2.197036 & -2.387750 & $\mathrm{H}$ & 5.635225 & -3.916172 & -0.097459 \\
\hline $\mathrm{C}$ & 1.484137 & 4.517097 & -0.963239 & $\mathrm{H}$ & 4.971309 & -4.566655 & -1.603780 \\
\hline $\mathrm{H}$ & 0.191623 & 3.319276 & 0.293697 & $\mathrm{H}$ & 5.395647 & -2.864842 & -1.489536 \\
\hline $\mathrm{C}$ & 2.374983 & 3.315188 & -2.826089 & $\mathrm{C}$ & -2.502163 & 0.685209 & 2.534048 \\
\hline $\mathrm{H}$ & 1.738994 & 1.234128 & -2.890723 & $\mathrm{H}$ & -2.929002 & 1.249242 & 3.367471 \\
\hline $\mathrm{H}$ & 1.385242 & 5.409770 & -0.354106 & $\mathrm{H}$ & -2.152145 & -0.280720 & 2.913481 \\
\hline $\mathrm{H}$ & 2.988848 & 3.248052 & -3.718791 & $\mathrm{H}$ & -1.603859 & 1.209951 & 2.183185 \\
\hline $\mathrm{N}$ & 0.907279 & 2.207197 & -1.284962 & $\mathrm{C}$ & -4.090134 & -1.689214 & -1.616680 \\
\hline $\mathrm{C}$ & 2.285119 & 4.498047 & -2.100436 & $\mathrm{H}$ & -3.080504 & -1.688804 & -2.042805 \\
\hline $\mathrm{H}$ & 2.830748 & 5.384601 & -2.410991 & $\mathrm{H}$ & -4.231867 & -2.681459 & -1.171455 \\
\hline $\mathrm{C}$ & -0.975613 & -1.211440 & 0.317780 & $\mathrm{H}$ & -4.808761 & -1.589008 & -2.433451 \\
\hline $\mathrm{C}$ & -1.228131 & -3.378559 & 1.230315 & $\mathrm{C}$ & -6.588183 & 2.327320 & 0.107717 \\
\hline C & -2.545392 & -2.637714 & 1.371332 & $\mathrm{H}$ & -6.401865 & 3.240574 & 0.678814 \\
\hline $\mathrm{H}$ & -1.277190 & -4.162239 & 0.459118 & $\mathrm{H}$ & -6.775837 & 2.611644 & -0.931871 \\
\hline $\mathrm{H}$ & -0.885187 & -3.844975 & 2.159910 & $\mathrm{H}$ & -7.520039 & 1.893120 & 0.488447 \\
\hline $\mathrm{H}$ & -3.406849 & -3.200493 & 0.995381 & $\mathrm{C}$ & 0.821857 & 0.553309 & 1.177949 \\
\hline $\mathrm{H}$ & -2.761639 & -2.359773 & 2.415114 & $\mathrm{H}$ & 0.458102 & 0.056956 & 2.092936 \\
\hline $\mathrm{N}$ & -0.315970 & -2.311918 & 0.810374 & $\mathrm{C}$ & 1.958877 & 1.418871 & 1.500875 \\
\hline $\mathrm{N}$ & -2.306944 & -1.436806 & 0.568769 & $\mathrm{C}$ & 1.968818 & 2.227068 & 2.654515 \\
\hline $\mathrm{C}$ & 1.002610 & -2.679314 & 0.388557 & $\mathrm{C}$ & 3.090722 & 1.470051 & 0.664273 \\
\hline $\mathrm{C}$ & 2.071374 & -2.589253 & 1.290500 & $\mathrm{C}$ & 3.021991 & 3.094435 & 2.919997 \\
\hline $\mathrm{C}$ & 1.189002 & -3.180875 & -0.912470 & $\mathrm{H}$ & 1.115316 & 2.183480 & 3.330896 \\
\hline $\mathrm{C}$ & 3.346788 & -2.939968 & 0.842204 & $\mathrm{C}$ & 4.154605 & 2.319163 & 0.940785 \\
\hline $\mathrm{C}$ & 2.481281 & -3.510201 & -1.314346 & $\mathrm{H}$ & 3.108092 & 0.814069 & -0.205155 \\
\hline $\mathrm{C}$ & 3.576781 & -3.381289 & -0.458941 & $\mathrm{C}$ & 4.119975 & 3.148497 & 2.061682 \\
\hline $\mathrm{H}$ & 4.184302 & -2.860770 & 1.534865 & $\mathrm{H}$ & 2.993602 & 3.725345 & 3.805450 \\
\hline $\mathrm{H}$ & 2.634956 & -3.879698 & -2.327836 & $\mathrm{H}$ & 5.016440 & 2.338324 & 0.277348 \\
\hline $\mathrm{C}$ & -3.350643 & -0.473676 & 0.456849 & $\mathrm{H}$ & 4.948193 & 3.819581 & 2.273859 \\
\hline $\mathrm{C}$ & -3.485756 & 0.538588 & 1.417350 & $\mathrm{O}$ & 0.602185 & -0.560395 & -2.311059 \\
\hline $\mathrm{C}$ & -4.254567 & -0.594002 & -0.609373 & $\mathrm{C}$ & -0.548959 & -0.072811 & -2.690305 \\
\hline $\mathrm{C}$ & -4.539644 & 1.443991 & 1.275465 & $\mathrm{H}$ & -1.370987 & -0.784536 & -2.889831 \\
\hline
\end{tabular}




\begin{tabular}{|c|c|c|c|c|c|c|c|}
\hline $\mathrm{H}$ & -0.549148 & 0.806690 & -3.360270 & $\mathrm{H}$ & 2.894417 & 0.339677 & 3.356366 \\
\hline $\mathrm{H}$ & -1.491495 & 0.832745 & -1.461081 & $\mathrm{H}$ & 1.493582 & -0.169419 & 2.393648 \\
\hline $\mathrm{H}$ & -1.553332 & 1.237406 & -0.53517 & $\mathrm{C}$ & 3.553690 & 1.753527 & -1.879574 \\
\hline $23 \mathbf{a}$ & & & & $\mathrm{H}$ & 2.509377 & 1.683935 & -2.201607 \\
\hline $\mathrm{Ru}$ & 0.742655 & -0.914574 & -0.788385 & $\mathrm{H}$ & 3.697465 & 2.774988 & -1.508425 \\
\hline $\mathrm{C}$ & 0.724769 & 0.952577 & 0.079716 & $\mathrm{H}$ & 4.192379 & 1.624080 & -2.756181 \\
\hline $\mathrm{C}$ & 0.318488 & 2.980542 & 1.179623 & $\mathrm{C}$ & 6.421288 & -2.007699 & -0.157370 \\
\hline $\mathrm{C}$ & 1.783314 & 2.598644 & 1.380638 & $\mathrm{H}$ & 6.110776 & -2.890961 & -0.726615 \\
\hline $\mathrm{H}$ & 0.200985 & 3.854209 & 0.522533 & $\mathrm{H}$ & 7.243067 & -1.544897 & -0.711119 \\
\hline $\mathrm{H}$ & -0.207033 & 3.194781 & 2.115928 & $\mathrm{H}$ & 6.813270 & -2.363787 & 0.798826 \\
\hline $\mathrm{H}$ & 2.477575 & 3.408704 & 1.136383 & $\mathrm{C}$ & -0.967611 & -1.345175 & -1.316836 \\
\hline $\mathrm{H}$ & 1.988416 & 2.273769 & 2.411565 & $\mathrm{H}$ & -1.182046 & -1.161009 & -2.387507 \\
\hline $\mathrm{N}$ & -0.234148 & 1.780336 & 0.534509 & $\mathrm{C}$ & -2.161067 & -1.830743 & -0.626636 \\
\hline $\mathrm{N}$ & 1.925161 & 1.479503 & 0.444339 & $\mathrm{C}$ & -3.390168 & -1.761910 & -1.311377 \\
\hline $\mathrm{C}$ & -1.634043 & 1.590737 & 0.341057 & $\mathrm{C}$ & -2.171428 & -2.364757 & 0.675232 \\
\hline $\mathrm{C}$ & -2.364847 & 0.945838 & 1.345995 & $\mathrm{C}$ & -4.576225 & -2.176953 & -0.719032 \\
\hline $\mathrm{C}$ & -2.232751 & 2.017307 & -0.851101 & $\mathrm{H}$ & -3.399507 & -1.357254 & -2.322753 \\
\hline $\mathrm{C}$ & -3.731569 & 0.757226 & 1.146492 & $\mathrm{C}$ & -3.355496 & -2.778965 & 1.267970 \\
\hline $\mathrm{C}$ & -3.602637 & 1.810903 & -1.005777 & $\mathrm{H}$ & -1.224237 & -2.450142 & 1.200362 \\
\hline $\mathrm{C}$ & -4.368704 & 1.190223 & -0.016139 & $\mathrm{C}$ & -4.565822 & -2.681984 & 0.578513 \\
\hline $\mathrm{H}$ & -4.304995 & 0.223834 & 1.903158 & $\mathrm{H}$ & -5.511435 & -2.105957 & -1.269804 \\
\hline $\mathrm{H}$ & -4.081134 & 2.126580 & -1.932478 & $\mathrm{H}$ & -3.337219 & -3.189253 & 2.275252 \\
\hline $\mathrm{C}$ & 3.082892 & 0.644592 & 0.334818 & $\mathrm{H}$ & -5.490910 & -3.010911 & 1.045917 \\
\hline $\mathrm{C}$ & 3.375829 & -0.284302 & 1.348770 & $\mathrm{H}$ & 1.032406 & -0.174572 & -2.472606 \\
\hline $\mathrm{C}$ & 3.870961 & 0.738216 & -0.825759 & $\mathrm{H}$ & 1.355321 & -0.948495 & -2.515090 \\
\hline $\mathrm{C}$ & 4.480727 & -1.118605 & 1.173715 & $\mathrm{H}$ & 0.401619 & -1.438624 & 0.690743 \\
\hline $\mathrm{C}$ & 4.957584 & -0.124653 & -0.956345 & $\mathrm{H}$ & 1.419886 & -2.424729 & -0.845419 \\
\hline $\mathrm{C}$ & 5.275417 & -1.062107 & 0.029441 & $23 b$ & & & \\
\hline $\mathrm{H}$ & 4.712073 & -1.847963 & 1.948537 & $\mathrm{Ru}$ & 0.702320 & -0.970762 & -0.705561 \\
\hline $\mathrm{H}$ & 5.570628 & -0.064942 & -1.854435 & $\mathrm{C}$ & 0.747956 & 0.908489 & 0.137726 \\
\hline $\mathrm{C}$ & -1.403459 & 2.620276 & -1.941210 & $\mathrm{C}$ & 0.406061 & 3.076978 & 0.953886 \\
\hline $\mathrm{H}$ & -0.651984 & 1.902939 & -2.296270 & $\mathrm{C}$ & 1.872799 & 2.701912 & 1.152413 \\
\hline $\mathrm{H}$ & -2.020695 & 2.916472 & -2.792510 & $\mathrm{H}$ & 0.274190 & 3.839244 & 0.171790 \\
\hline $\mathrm{H}$ & -0.852236 & 3.505666 & -1.603954 & $\mathrm{H}$ & -0.076027 & 3.444084 & 1.865223 \\
\hline $\mathrm{C}$ & -1.669364 & 0.413257 & 2.558707 & $\mathrm{H}$ & 2.568240 & 3.459796 & 0.779053 \\
\hline $\begin{array}{l}\mathrm{H} \\
\mathrm{H}\end{array}$ & -2.369944 & -0.095397 & 3.225525 & $\mathrm{H}$ & 2.110751 & 2.513064 & 2.210772 \\
\hline $\begin{array}{l}\mathrm{H} \\
\mathrm{H}\end{array}$ & -0.895358 & -0.306183 & 2.261426 & $\mathrm{~N}$ & -0.185892 & 1.800016 & 0.527437 \\
\hline $\begin{array}{l}\mathrm{H} \\
\mathrm{C}\end{array}$ & $\begin{array}{r}-1.166519 \\
5820887\end{array}$ & 1.201594 & 3.132846 & $\mathrm{~N}$ & 1.963796 & 1.464354 & 0.373698 \\
\hline $\mathrm{C}$ & -5.839887 & 0.981814 & -0.204172 & C & -1.589784 & 1.631633 & 0.341718 \\
\hline $\begin{array}{l}\mathrm{H} \\
\mathrm{H}\end{array}$ & -6.413231 & 1.867908 & 0.092955 & $\mathrm{C}$ & -2.339602 & 1.064516 & 1.378355 \\
\hline $\begin{array}{l}\mathrm{H} \\
\mathrm{H}\end{array}$ & -6.086151 & 0.778533 & -1.250918 & $\mathrm{C}$ & -2.170927 & 1.983385 & -0.883603 \\
\hline $\mathrm{H}$ & -6.202904 & 0.141934 & 0.395221 & $\mathrm{C}$ & -3.707462 & 0.883157 & 1.180210 \\
\hline $\mathrm{H}$ & 2.549470 & -0.371576 & 2.594800 & $\mathrm{C}$ & -3.543105 & 1.788486 & -1.035826 \\
\hline $\mathrm{H}$ & 2.614278 & -1.370209 & 3.032542 & $\mathrm{C}$ & -4.327645 & 1.243895 & -0.016276 \\
\hline \multicolumn{8}{|c|}{158} \\
\hline
\end{tabular}




\begin{tabular}{|c|c|c|c|c|c|c|c|}
\hline $\mathrm{H}$ & -4.296041 & 0.414284 & 1.967596 & $\mathrm{H}$ & -5.542896 & -2.186557 & -1.391171 \\
\hline $\mathrm{H}$ & -4.007364 & 2.041422 & -1.988709 & $\mathrm{H}$ & -3.517729 & -3.143392 & 2.275899 \\
\hline $\mathrm{C}$ & 3.122084 & 0.630666 & 0.280190 & $\mathrm{H}$ & -5.607731 & -3.079566 & 0.930547 \\
\hline $\mathrm{C}$ & 3.443696 & -0.240728 & 1.334147 & $\mathrm{H}$ & 0.901669 & -0.255038 & -2.143508 \\
\hline $\mathrm{C}$ & 3.873289 & 0.660323 & -0.907626 & $\mathrm{H}$ & 0.437785 & -1.661414 & 0.978681 \\
\hline $\mathrm{C}$ & 4.538389 & -1.091866 & 1.170472 & $\mathrm{H}$ & 1.436800 & -2.209678 & -1.538799 \\
\hline $\mathrm{C}$ & 4.959472 & -0.204594 & -1.018899 & $\mathrm{H}$ & 0.780706 & -2.287733 & 0.524955 \\
\hline $\mathrm{C}$ & 5.302537 & -1.092464 & 0.003900 & & & & \\
\hline $\mathrm{H}$ & 4.794810 & -1.777333 & 1.977184 & $23 c$ & & & \\
\hline $\mathrm{H}$ & 5.545379 & -0.195115 & -1.936624 & $\mathrm{Ru}$ & -0.514349 & -0.976125 & -0.220100 \\
\hline $\mathrm{C}$ & -1.316160 & 2.463837 & -2.013806 & $\mathrm{C}$ & -0.702421 & 1.015221 & 0.012194 \\
\hline $\mathrm{H}$ & -0.597819 & 1.684803 & -2.302644 & $\mathrm{C}$ & -0.623369 & 3.339026 & 0.383982 \\
\hline $\mathrm{H}$ & -1.919510 & 2.717264 & -2.888433 & $\mathrm{C}$ & -2.061072 & 2.867824 & 0.555373 \\
\hline $\mathrm{H}$ & -0.726272 & 3.348604 & -1.747443 & $\mathrm{H}$ & -0.253299 & 3.928783 & 1.228611 \\
\hline $\mathrm{C}$ & -1.663046 & 0.599055 & 2.629182 & $\mathrm{H}$ & -0.494044 & 3.937929 & -0.528860 \\
\hline $\mathrm{H}$ & -2.380494 & 0.170149 & 3.332981 & $\mathrm{H}$ & -2.375611 & 2.869182 & 1.610942 \\
\hline $\mathrm{H}$ & -0.915942 & -0.170908 & 2.393734 & $\mathrm{H}$ & -2.782816 & 3.464416 & -0.011837 \\
\hline $\mathrm{H}$ & -1.125264 & 1.406505 & 3.140310 & $\mathrm{~N}$ & 0.110248 & 2.072139 & 0.275952 \\
\hline $\mathrm{C}$ & -5.798718 & 1.037659 & -0.207573 & $\mathrm{~N}$ & -1.980239 & 1.501456 & 0.042262 \\
\hline $\mathrm{H}$ & -6.374953 & 1.908621 & 0.126355 & $\mathrm{C}$ & 1.534336 & 2.040099 & 0.248607 \\
\hline $\mathrm{H}$ & -6.046388 & 0.872744 & -1.260465 & $\mathrm{C}$ & 2.213711 & 2.384053 & -0.928492 \\
\hline $\mathrm{H}$ & -6.156349 & 0.173519 & 0.360003 & $\mathrm{C}$ & 2.218063 & 1.603827 & 1.392793 \\
\hline $\mathrm{C}$ & 2.668048 & -0.242643 & 2.616858 & $\mathrm{C}$ & 3.603128 & 2.254766 & -0.947918 \\
\hline $\mathrm{H}$ & 2.731906 & -1.215895 & 3.110063 & $\mathrm{C}$ & 3.605106 & 1.482629 & 1.322160 \\
\hline $\mathrm{H}$ & 3.060979 & 0.500048 & 3.322553 & $\mathrm{C}$ & 4.314064 & 1.788796 & 0.158938 \\
\hline $\mathrm{H}$ & 1.608745 & -0.016115 & 2.460396 & $\mathrm{H}$ & 4.140533 & 2.496613 & -1.864245 \\
\hline $\mathrm{C}$ & 3.488932 & 1.582484 & -2.021232 & $\mathrm{H}$ & 4.144880 & 1.120913 & 2.196353 \\
\hline $\mathrm{H}$ & 2.501040 & 1.312166 & -2.413494 & $\mathrm{C}$ & -3.089905 & 0.600757 & 0.032564 \\
\hline $\mathrm{H}$ & 3.425619 & 2.623302 & -1.685318 & $\mathrm{C}$ & -3.753154 & 0.378208 & -1.188766 \\
\hline $\mathrm{H}$ & 4.209337 & 1.532028 & -2.840628 & $\mathrm{C}$ & -3.495942 & -0.039199 & 1.215349 \\
\hline $\mathrm{C}$ & 6.445305 & -2.045177 & -0.165921 & $\mathrm{C}$ & -4.806220 & -0.529924 & -1.212203 \\
\hline $\mathrm{H}$ & 6.143342 & -2.917809 & -0.755616 & $\mathrm{C}$ & -4.554045 & -0.950542 & 1.135903 \\
\hline $\mathrm{H}$ & 7.283745 & -1.581655 & -0.693802 & $\mathrm{C}$ & -5.212735 & -1.215179 & -0.062336 \\
\hline $\mathrm{H}$ & 6.811544 & -2.415281 & 0.795065 & $\mathrm{H}$ & -5.319249 & -0.715621 & -2.154889 \\
\hline $\mathrm{C}$ & -1.047783 & -1.222591 & -1.230229 & $\mathrm{H}$ & -4.871568 & -1.463172 & 2.043079 \\
\hline $\mathrm{H}$ & -1.304449 & -0.845376 & -2.233733 & $\mathrm{C}$ & 1.467023 & 1.215391 & 2.625962 \\
\hline $\mathrm{C}$ & -2.239492 & -1.780267 & -0.582849 & $\mathrm{H}$ & 0.738287 & 1.979994 & 2.918606 \\
\hline $\mathrm{C}$ & -3.439136 & -1.756650 & -1.322657 & $\mathrm{H}$ & 0.897672 & 0.291988 & 2.452701 \\
\hline $\mathrm{C}$ & -2.303967 & -2.296223 & 0.724762 & $\mathrm{H}$ & 2.145193 & 1.048991 & 3.466265 \\
\hline $\mathrm{C}$ & -4.636100 & -2.220126 & -0.791968 & $\mathrm{C}$ & 1.464092 & 2.793382 & -2.159010 \\
\hline $\mathrm{H}$ & -3.413192 & -1.351263 & -2.333109 & $\mathrm{H}$ & 1.056672 & 3.808626 & -2.080650 \\
\hline $\mathrm{C}$ & -3.498834 & -2.754790 & 1.260252 & $\mathrm{H}$ & 2.112857 & 2.775261 & -3.037582 \\
\hline $\mathrm{H}$ & -1.403667 & -2.325214 & 1.330059 & $\mathrm{H}$ & 0.618500 & 2.122347 & -2.348079 \\
\hline $\mathrm{C}$ & -4.673790 & -2.718918 & 0.507234 & $\mathrm{C}$ & 5.794792 & 1.575800 & 0.088459 \\
\hline
\end{tabular}




\begin{tabular}{|c|c|c|c|c|c|c|c|}
\hline $\mathrm{H}$ & 6.028142 & 0.516860 & -0.075678 & $\mathrm{C}$ & 1.589094 & 1.884679 & -0.205979 \\
\hline $\mathrm{H}$ & 6.248135 & 2.138992 & -0.731497 & $\mathrm{C}$ & 2.441090 & 1.723642 & -1.309776 \\
\hline $\mathrm{H}$ & 6.293276 & 1.868172 & 1.017342 & $\mathrm{C}$ & 2.093202 & 2.259544 & 1.050232 \\
\hline $\mathrm{C}$ & -3.317351 & 1.101417 & -2.422963 & $\mathrm{C}$ & 3.816822 & 1.835539 & -1.109063 \\
\hline $\mathrm{H}$ & -3.933346 & 0.823977 & -3.281304 & $\mathrm{C}$ & 3.476199 & 2.357146 & 1.202316 \\
\hline $\mathrm{H}$ & -3.379547 & 2.188579 & -2.299009 & $\mathrm{C}$ & 4.356071 & 2.126661 & 0.143909 \\
\hline $\mathrm{H}$ & -2.273625 & 0.863298 & -2.655383 & $\mathrm{H}$ & 4.485148 & 1.682942 & -1.956145 \\
\hline $\mathrm{C}$ & -2.875237 & 0.278048 & 2.542233 & $\mathrm{H}$ & 3.876365 & 2.633194 & 2.177321 \\
\hline $\mathrm{H}$ & -1.845697 & 0.629632 & 2.444828 & $\mathrm{C}$ & -3.146604 & 0.502618 & -0.208769 \\
\hline $\mathrm{H}$ & -3.453421 & 1.049698 & 3.066933 & $\mathrm{C}$ & -3.455612 & -0.285744 & -1.329921 \\
\hline $\mathrm{H}$ & -2.856982 & -0.604570 & 3.186091 & $\mathrm{C}$ & -4.018443 & 0.600571 & 0.881778 \\
\hline $\mathrm{C}$ & -6.324122 & -2.217398 & -0.127700 & $\mathrm{C}$ & -4.646471 & -1.005515 & -1.319282 \\
\hline $\mathrm{H}$ & -7.181119 & -1.834616 & -0.690699 & $\mathrm{C}$ & -5.202864 & -0.139869 & 0.848074 \\
\hline $\mathrm{H}$ & -6.001336 & -3.134536 & -0.632931 & $\mathrm{C}$ & -5.531285 & -0.950448 & -0.237410 \\
\hline $\mathrm{H}$ & -6.673545 & -2.500235 & 0.868519 & $\mathrm{H}$ & -4.892805 & -1.626494 & -2.179709 \\
\hline $\mathrm{C}$ & 1.310658 & -1.010026 & -0.481816 & $\mathrm{H}$ & -5.883719 & -0.083928 & 1.696682 \\
\hline $\mathrm{H}$ & 1.778422 & -0.096049 & -0.868540 & $\mathrm{C}$ & 1.175811 & 2.566033 & 2.189439 \\
\hline $\mathrm{C}$ & 2.279476 & -2.093621 & -0.333484 & $\mathrm{H}$ & 0.345177 & 3.211712 & 1.879929 \\
\hline $\mathrm{C}$ & 3.462096 & -2.060097 & -1.099267 & $\mathrm{H}$ & 0.723834 & 1.647712 & 2.583769 \\
\hline $\mathrm{C}$ & 2.127506 & -3.143450 & 0.590491 & $\mathrm{H}$ & 1.710676 & 3.064539 & 3.001388 \\
\hline $\mathrm{C}$ & 4.421725 & -3.057682 & -0.984411 & $\mathrm{C}$ & 1.901101 & 1.445773 & -2.680742 \\
\hline $\mathrm{H}$ & 3.610228 & -1.233128 & -1.792840 & $\mathrm{H}$ & 1.743415 & 2.374699 & -3.243610 \\
\hline $\mathrm{C}$ & 3.092993 & -4.133765 & 0.713333 & $\mathrm{H}$ & 2.598072 & 0.838481 & -3.265509 \\
\hline $\mathrm{H}$ & 1.255349 & -3.141678 & 1.239368 & $\mathrm{H}$ & 0.940499 & 0.921734 & -2.646839 \\
\hline $\mathrm{C}$ & 4.239691 & -4.102232 & -0.079198 & $\mathrm{C}$ & 5.838510 & 2.186113 & 0.349759 \\
\hline $\mathrm{H}$ & 5.318758 & -3.018630 & -1.597434 & $\mathrm{H}$ & 6.232589 & 1.212871 & 0.664378 \\
\hline $\mathrm{H}$ & 2.959649 & -4.928659 & 1.442699 & $\mathrm{H}$ & 6.365925 & 2.462887 & -0.567418 \\
\hline $\mathrm{H}$ & 4.995916 & -4.876009 & 0.021553 & $\mathrm{H}$ & 6.111235 & 2.905142 & 1.127624 \\
\hline $\mathrm{H}$ & -0.639312 & -1.154443 & 1.441300 & $\mathrm{C}$ & -2.510845 & -0.354405 & -2.487181 \\
\hline $\mathrm{H}$ & -0.482196 & -2.739231 & 0.064504 & $\mathrm{H}$ & -2.941820 & -0.916502 & -3.319010 \\
\hline $\mathrm{H}$ & -0.695517 & -2.673236 & -0.750431 & $\mathrm{H}$ & -2.242655 & 0.643912 & -2.854071 \\
\hline \multirow[t]{2}{*}{$\mathrm{H}$} & -1.106275 & -0.872859 & -1.795582 & $\mathrm{H}$ & -1.575006 & -0.843637 & -2.188042 \\
\hline & & & & $\mathrm{C}$ & -3.680220 & 1.468493 & 2.054757 \\
\hline $23 d$ & & & & $\mathrm{H}$ & -2.605861 & 1.454516 & 2.269014 \\
\hline $\mathrm{Ru}$ & -0.231865 & -0.933394 & 0.769097 & $\mathrm{H}$ & -3.954084 & 2.515991 & 1.875807 \\
\hline $\mathrm{C}$ & -0.671447 & 0.777186 & 0.009621 & $\mathrm{H}$ & -4.214633 & 1.148822 & 2.952860 \\
\hline $\mathrm{C}$ & -0.522528 & 2.910158 & -1.013337 & $\mathrm{C}$ & -6.801019 & -1.745820 & -0.252738 \\
\hline $\mathrm{C}$ & -1.966159 & 2.664560 & -0.611239 & $\mathrm{H}$ & -7.414768 & -1.507804 & -1.127898 \\
\hline $\mathrm{H}$ & -0.124658 & 3.857902 & -0.636366 & $\mathrm{H}$ & -6.599847 & -2.821529 & -0.295910 \\
\hline $\mathrm{H}$ & -0.383105 & 2.898983 & -2.105419 & $\mathrm{H}$ & -7.405700 & -1.558335 & 0.638029 \\
\hline $\mathrm{H}$ & -2.279863 & 3.299279 & 0.232183 & $\mathrm{C}$ & 1.234059 & -1.196640 & -0.349442 \\
\hline $\mathrm{H}$ & -2.676013 & 2.820572 & -1.429931 & $\mathrm{H}$ & 1.429983 & -0.607494 & -1.252540 \\
\hline $\mathrm{N}$ & 0.178225 & 1.784199 & -0.391359 & $\mathrm{C}$ & 2.204301 & -2.273182 & -0.181673 \\
\hline $\mathrm{N}$ & -1.940942 & 1.257664 & -0.216605 & $\mathrm{C}$ & 3.226675 & -2.462499 & -1.129250 \\
\hline
\end{tabular}




\begin{tabular}{|c|c|c|c|c|c|c|c|}
\hline $\mathrm{C}$ & 2.167735 & -3.150950 & 0.921717 & $\mathrm{C}$ & -1.751447 & 0.988408 & 2.605359 \\
\hline $\mathrm{C}$ & 4.161407 & -3.481878 & -0.989173 & $\mathrm{H}$ & -2.412101 & 1.010167 & 3.475692 \\
\hline $\mathrm{H}$ & 3.273701 & -1.789717 & -1.984277 & $\mathrm{H}$ & -1.256330 & 0.009194 & 2.585095 \\
\hline $\mathrm{C}$ & 3.092767 & -4.172706 & 1.059395 & $\mathrm{H}$ & -0.959280 & 1.731077 & 2.745838 \\
\hline $\mathrm{H}$ & 1.405608 & -3.007223 & 1.689535 & $\mathrm{C}$ & -6.107849 & 0.938053 & 0.108210 \\
\hline $\mathrm{C}$ & 4.096879 & -4.343382 & 0.102793 & $\mathrm{H}$ & -6.617479 & 1.485408 & -0.688989 \\
\hline $\mathrm{H}$ & 4.943061 & -3.606234 & -1.734289 & $\mathrm{H}$ & -6.289241 & -0.129493 & -0.068213 \\
\hline $\mathrm{H}$ & 3.042943 & -4.836667 & 1.918347 & $\mathrm{H}$ & -6.592547 & 1.188437 & 1.056376 \\
\hline $\mathrm{H}$ & 4.826699 & -5.140317 & 0.215286 & $\mathrm{C}$ & 2.933446 & 0.784429 & 2.606979 \\
\hline $\mathrm{H}$ & 0.311495 & -0.233721 & 2.199741 & $\mathrm{H}$ & 3.319011 & 0.123752 & 3.387766 \\
\hline $\mathrm{H}$ & -1.572735 & -0.811004 & 2.197804 & $\mathrm{H}$ & 3.121286 & 1.813372 & 2.939452 \\
\hline $\mathrm{H}$ & -2.013350 & -1.072697 & 1.578212 & $\mathrm{H}$ & 1.846931 & 0.655117 & 2.556766 \\
\hline $\mathrm{H}$ & -1.354081 & -1.830383 & -0.088331 & $\mathrm{C}$ & 2.799370 & 0.860520 & -2.419589 \\
\hline & & & & $\mathrm{H}$ & 1.860429 & 0.294489 & -2.466607 \\
\hline 24 & & & & $\mathrm{H}$ & 2.522889 & 1.920340 & -2.469648 \\
\hline $\mathrm{C}$ & 0.468145 & 0.848855 & 0.088520 & $\mathrm{H}$ & 3.396694 & 0.621070 & -3.302511 \\
\hline $\mathrm{C}$ & 0.126107 & 3.197237 & 0.119442 & $\mathrm{C}$ & 6.791087 & -1.082158 & -0.037319 \\
\hline $\mathrm{C}$ & 1.590842 & 2.906595 & 0.407903 & $\mathrm{H}$ & 6.775517 & -1.981764 & -0.661049 \\
\hline $\mathrm{H}$ & -0.011562 & 3.636574 & -0.880813 & $\mathrm{H}$ & 7.550911 & -0.418398 & -0.464963 \\
\hline $\mathrm{H}$ & -0.333254 & 3.872026 & 0.849621 & $\mathrm{H}$ & 7.133031 & -1.373749 & 0.959029 \\
\hline $\mathrm{H}$ & 2.277755 & 3.490088 & -0.213924 & $\mathrm{C}$ & -0.873766 & -1.126116 & -1.984845 \\
\hline $\mathrm{H}$ & 1.849290 & 3.096531 & 1.462450 & $\mathrm{H}$ & -1.520736 & -0.314476 & -2.319232 \\
\hline $\mathrm{N}$ & -0.461897 & 1.862445 & 0.195945 & $\mathrm{C}$ & -1.507174 & -2.144935 & -1.170616 \\
\hline $\mathrm{N}$ & 1.685876 & 1.480545 & 0.099886 & $\mathrm{C}$ & -2.709614 & -1.879524 & -0.447586 \\
\hline $\mathrm{C}$ & -1.866848 & 1.649142 & 0.167011 & $\mathrm{C}$ & -0.765064 & -3.303665 & -0.782783 \\
\hline $\mathrm{C}$ & -2.516378 & 1.226106 & 1.340808 & $\mathrm{C}$ & -3.171473 & -2.749431 & 0.525140 \\
\hline $\mathrm{C}$ & -2.590700 & 1.888709 & -1.012610 & $\mathrm{H}$ & -3.273860 & -0.982785 & -0.697642 \\
\hline $\mathrm{C}$ & -3.895499 & 1.023661 & 1.300854 & $\mathrm{C}$ & -1.272061 & -4.181585 & 0.190296 \\
\hline $\mathrm{C}$ & -3.970023 & 1.674502 & -1.003101 & $\mathrm{H}$ & 0.122430 & -3.577517 & -1.346316 \\
\hline $\mathrm{C}$ & -4.639697 & 1.232545 & 0.138759 & $\mathrm{C}$ & -2.465936 & -3.920648 & 0.842294 \\
\hline $\mathrm{H}$ & -4.402356 & 0.688921 & 2.205635 & $\mathrm{H}$ & -4.100559 & -2.517114 & 1.043420 \\
\hline $\mathrm{H}$ & -4.533408 & 1.843703 & -1.920221 & $\mathrm{H}$ & -0.711633 & -5.083569 & 0.425291 \\
\hline $\mathrm{C}$ & 2.948140 & 0.825368 & 0.085119 & $\mathrm{H}$ & -2.844730 & -4.603574 & 1.596414 \\
\hline $\mathrm{C}$ & 3.582338 & 0.495239 & 1.287880 & $\mathrm{Ru}$ & 0.012096 & -1.033790 & -0.029056 \\
\hline $\mathrm{C}$ & 3.534624 & 0.537409 & -1.157411 & $\mathrm{H}$ & 0.294223 & -1.392282 & 1.683860 \\
\hline $\mathrm{C}$ & 4.831796 & -0.127276 & 1.225510 & $\mathrm{H}$ & -0.183484 & -1.465006 & -2.757243 \\
\hline $\mathrm{C}$ & 4.779905 & -0.084105 & -1.171961 & $\mathrm{H}$ & 1.066711 & -1.464988 & 1.281675 \\
\hline $\mathrm{C}$ & 5.447316 & -0.420703 & 0.009930 & $\mathrm{H}$ & 1.443193 & -1.234408 & -0.637967 \\
\hline $\mathrm{H}$ & 5.331987 & -0.398737 & 2.154768 & & & & \\
\hline $\mathrm{H}$ & 5.240738 & -0.317963 & -2.131258 & \multicolumn{2}{|l|}{ O-Br' } & & \\
\hline $\mathrm{C}$ & -1.903275 & 2.349521 & -2.260810 & $\mathrm{Ru}$ & -0.139833 & 0.055727 & -0.034898 \\
\hline $\mathrm{H}$ & -0.896578 & 1.926320 & -2.341327 & $\mathrm{C}$ & 2.527470 & 1.360955 & -0.222891 \\
\hline $\mathrm{H}$ & -2.467434 & 2.056445 & -3.150112 & $\mathrm{C}$ & 1.002325 & 3.078647 & -0.481400 \\
\hline $\mathrm{H}$ & -1.804397 & 3.441699 & -2.291787 & $\mathrm{C}$ & 3.574826 & 2.221232 & -0.512167 \\
\hline
\end{tabular}




\begin{tabular}{|c|c|c|c|c|c|c|c|}
\hline $\mathrm{H}$ & 2.698913 & 0.303391 & -0.014853 & $\mathrm{H}$ & 2.209288 & -2.447248 & -2.900663 \\
\hline $\mathrm{C}$ & 2.010244 & 3.989963 & -0.761194 & $\mathrm{H}$ & 1.666276 & -4.025040 & -2.324119 \\
\hline $\mathrm{H}$ & -0.043846 & 3.368628 & -0.477035 & $\mathrm{H}$ & 0.588576 & -2.602474 & -2.248915 \\
\hline $\mathrm{N}$ & 1.251851 & 1.786841 & -0.209906 & $\mathrm{C}$ & 1.001546 & -2.670203 & 2.877653 \\
\hline $\mathrm{C}$ & 3.334685 & 3.561409 & -0.783400 & $\mathrm{H}$ & 0.261804 & -1.862073 & 2.898541 \\
\hline $\mathrm{H}$ & 4.149416 & 4.241577 & -1.006798 & $\mathrm{H}$ & 0.454533 & -3.620424 & 2.871274 \\
\hline $\mathrm{C}$ & -0.923831 & -1.774467 & 0.008364 & $\mathrm{H}$ & 1.595652 & -2.630910 & 3.794453 \\
\hline $\mathrm{C}$ & -2.178961 & -3.759723 & -0.004130 & $\mathrm{C}$ & 5.555470 & -2.008110 & 0.850497 \\
\hline $\mathrm{C}$ & -0.700928 & -4.121371 & -0.000091 & $\mathrm{H}$ & 5.759629 & -1.123820 & 1.462881 \\
\hline $\mathrm{H}$ & -2.664531 & -3.976580 & 0.959227 & $\mathrm{H}$ & 6.031247 & -2.857105 & 1.354045 \\
\hline $\mathrm{H}$ & -2.750594 & -4.266650 & -0.787953 & $\mathrm{H}$ & 6.055790 & -1.870681 & -0.111393 \\
\hline $\mathrm{H}$ & -0.432191 & -4.841112 & 0.779747 & $\mathrm{C}$ & -1.716487 & 0.968396 & -0.372825 \\
\hline $\mathrm{H}$ & -0.367723 & -4.526847 & -0.967183 & $\mathrm{H}$ & -2.392415 & 0.548092 & -1.132781 \\
\hline $\mathrm{N}$ & -2.148734 & -2.309175 & -0.231200 & $\mathrm{C}$ & -2.227449 & 2.192822 & 0.204629 \\
\hline $\mathrm{N}$ & -0.079717 & -2.819283 & 0.245207 & $\mathrm{C}$ & -3.363654 & 2.806634 & -0.369071 \\
\hline $\mathrm{C}$ & -3.370321 & -1.576685 & -0.264369 & $\mathrm{C}$ & -1.695685 & 2.758306 & 1.384211 \\
\hline $\mathrm{C}$ & -4.014953 & -1.420197 & -1.496867 & $\mathrm{C}$ & -3.921266 & 3.946863 & 0.189859 \\
\hline $\mathrm{C}$ & -3.867691 & -0.999699 & 0.914295 & $\mathrm{H}$ & -3.804475 & 2.354918 & -1.257161 \\
\hline $\mathrm{C}$ & -5.187480 & -0.664235 & -1.534770 & $\mathrm{C}$ & -2.264146 & 3.894443 & 1.944212 \\
\hline $\mathrm{C}$ & -5.038927 & -0.249055 & 0.825300 & $\mathrm{H}$ & -0.856025 & 2.257118 & 1.856617 \\
\hline $\mathrm{C}$ & -5.708743 & -0.064931 & -0.386923 & $\mathrm{C}$ & -3.371780 & 4.498792 & 1.348822 \\
\hline $\mathrm{H}$ & -5.691904 & -0.520336 & -2.489624 & $\mathrm{H}$ & -4.790801 & 4.407946 & -0.272989 \\
\hline $\mathrm{H}$ & -5.425638 & 0.225186 & 1.726818 & $\mathrm{H}$ & -1.847230 & 4.309867 & 2.858367 \\
\hline $\mathrm{C}$ & 1.335221 & -2.651322 & 0.374795 & $\mathrm{H}$ & -3.812447 & 5.388395 & 1.791101 \\
\hline $\mathrm{C}$ & 2.155707 & -2.642222 & -0.766137 & $\mathrm{O}$ & -0.042357 & 0.077306 & 2.025246 \\
\hline $\mathrm{C}$ & 1.874733 & -2.536545 & 1.671230 & $\mathrm{O}$ & 0.359585 & -0.333658 & -1.975633 \\
\hline $\mathrm{C}$ & 3.523057 & -2.409197 & -0.585090 & $\mathrm{C}$ & 1.022586 & 0.719427 & 2.632651 \\
\hline $\mathrm{C}$ & 3.244941 & -2.313213 & 1.795096 & $\mathrm{H}$ & 1.004452 & 0.534482 & 3.719995 \\
\hline $\mathrm{C}$ & 4.084053 & -2.232708 & 0.679497 & $\mathrm{H}$ & 2.013635 & 0.365278 & 2.276649 \\
\hline $\mathrm{H}$ & 4.167387 & -2.377551 & -1.462854 & $\mathrm{H}$ & 1.024416 & 1.820847 & 2.494009 \\
\hline $\mathrm{H}$ & 3.669147 & -2.209227 & 2.793148 & $\mathrm{C}$ & 0.324880 & 0.673701 & -2.920331 \\
\hline $\mathrm{C}$ & -3.121795 & -1.120714 & 2.204913 & $\mathrm{H}$ & -0.676673 & 1.147718 & -2.995902 \\
\hline $\mathrm{H}$ & -2.201051 & -0.521266 & 2.190508 & $\mathrm{H}$ & 1.040326 & 1.500505 & -2.740652 \\
\hline $\mathrm{H}$ & -3.734742 & -0.784200 & 3.044664 & $\mathrm{H}$ & 0.568784 & 0.263600 & -3.915638 \\
\hline $\mathrm{H}$ & -2.808355 & -2.151766 & 2.408411 & $\mathrm{H}$ & 1.761020 & 5.024844 & -0.971400 \\
\hline $\mathrm{C}$ & -3.391946 & -1.964203 & -2.744773 & $\mathrm{Br}$ & 5.381245 & 1.524448 & -0.536797 \\
\hline $\mathrm{H}$ & -3.277082 & -3.054055 & -2.715358 & & & & \\
\hline $\mathrm{H}$ & -3.989358 & -1.718352 & -3.625841 & 0-Br & & & \\
\hline $\mathrm{H}$ & -2.383599 & -1.553426 & -2.883360 & $\mathrm{Ru}$ & 0.125921 & 0.396936 & 0.038836 \\
\hline $\mathrm{C}$ & -6.936310 & 0.791339 & -0.452039 & $\mathrm{C}$ & -2.720453 & 1.245585 & 0.004966 \\
\hline $\mathrm{H}$ & -7.412678 & 0.743477 & -1.434676 & $\mathrm{C}$ & -2.791733 & -1.057064 & -0.179258 \\
\hline $\mathrm{H}$ & -7.678730 & 0.494944 & 0.295937 & $\mathrm{C}$ & -4.088646 & 1.354897 & -0.187413 \\
\hline $\mathrm{H}$ & -6.693316 & 1.841670 & -0.252225 & $\mathrm{H}$ & -2.095792 & 2.128731 & 0.148732 \\
\hline $\mathrm{C}$ & 1.617701 & -2.945275 & -2.129091 & $\mathrm{C}$ & -4.166594 & -1.013247 & -0.358398 \\
\hline
\end{tabular}




\begin{tabular}{|c|c|c|c|c|c|c|c|}
\hline $\mathrm{H}$ & -2.242392 & -1.993479 & -0.192586 & $\mathrm{H}$ & 1.685064 & 4.370854 & -2.590311 \\
\hline $\mathrm{H}$ & -4.557799 & 2.334012 & -0.195614 & $\mathrm{H}$ & 1.448159 & 2.610894 & -2.409891 \\
\hline $\mathrm{N}$ & -2.077852 & 0.062722 & 0.009255 & $\mathrm{C}$ & 0.948289 & 3.440123 & 2.655297 \\
\hline $\mathrm{C}$ & -4.843570 & 0.199276 & -0.369729 & $\mathrm{H}$ & 1.093739 & 2.357868 & 2.746859 \\
\hline $\mathrm{H}$ & -5.917266 & 0.239362 & -0.518686 & $\mathrm{H}$ & 1.934339 & 3.917547 & 2.699672 \\
\hline $\mathrm{C}$ & 1.930367 & 1.230384 & -0.007581 & $\mathrm{H}$ & 0.368216 & 3.792004 & 3.512549 \\
\hline $\mathrm{C}$ & 4.192849 & 1.862269 & 0.003391 & $\mathrm{C}$ & -2.882635 & 5.574363 & 0.182768 \\
\hline $\mathrm{C}$ & 3.338452 & 3.118299 & -0.107258 & $\mathrm{H}$ & -3.596856 & 5.111886 & 0.872353 \\
\hline $\mathrm{H}$ & 4.674518 & 1.769478 & 0.988168 & $\mathrm{H}$ & -2.756464 & 6.610799 & 0.515970 \\
\hline $\mathrm{H}$ & 4.975480 & 1.798535 & -0.759299 & $\mathrm{H}$ & -3.341864 & 5.605572 & -0.808960 \\
\hline $\mathrm{H}$ & 3.602013 & 3.887232 & 0.625859 & $\mathrm{C}$ & 0.657345 & -1.349254 & -0.268618 \\
\hline $\mathrm{H}$ & 3.393477 & 3.568292 & -1.110161 & $\mathrm{H}$ & 1.401174 & -1.528125 & -1.059674 \\
\hline $\mathrm{N}$ & 3.202334 & 0.793465 & -0.171451 & $\mathrm{C}$ & 0.182057 & -2.572222 & 0.342280 \\
\hline $\mathrm{N}$ & 1.998073 & 2.587139 & 0.139929 & $\mathrm{C}$ & 0.429810 & -3.809306 & -0.292573 \\
\hline $\mathrm{C}$ & 3.599965 & -0.573548 & -0.215293 & $\mathrm{C}$ & -0.474151 & -2.588290 & 1.592136 \\
\hline $\mathrm{C}$ & 3.896974 & -1.132420 & -1.463728 & $\mathrm{C}$ & 0.000004 & -5.001634 & 0.270947 \\
\hline $\mathrm{C}$ & 3.617937 & -1.332884 & 0.963838 & $\mathrm{H}$ & 0.964892 & -3.808687 & -1.241505 \\
\hline $\mathrm{C}$ & 4.218999 & -2.488920 & -1.517536 & $\mathrm{C}$ & -0.887972 & -3.786738 & 2.158576 \\
\hline $\mathrm{C}$ & 3.940923 & -2.685393 & 0.859524 & $\mathrm{H}$ & -0.611593 & -1.641077 & 2.106402 \\
\hline $\mathrm{C}$ & 4.233953 & -3.282598 & -0.368919 & $\mathrm{C}$ & -0.665444 & -4.995764 & 1.498698 \\
\hline $\mathrm{H}$ & 4.434269 & -2.942768 & -2.484288 & $\mathrm{H}$ & 0.185952 & -5.942214 & -0.242159 \\
\hline $\mathrm{H}$ & 3.932273 & -3.296449 & 1.761149 & $\mathrm{H}$ & -1.386118 & -3.781519 & 3.125040 \\
\hline $\mathrm{C}$ & 0.820111 & 3.400102 & 0.135226 & $\mathrm{H}$ & -0.998828 & -5.929966 & 1.942522 \\
\hline $\mathrm{C}$ & 0.262043 & 3.824637 & -1.083203 & $\mathrm{O}$ & 0.145352 & 0.550778 & 2.098594 \\
\hline $\mathrm{C}$ & 0.254865 & 3.767727 & 1.371712 & $\mathrm{O}$ & -0.084122 & 0.918034 & -1.924702 \\
\hline $\mathrm{C}$ & -0.943424 & 4.529598 & -1.038817 & $\mathrm{C}$ & -1.044896 & 0.783730 & 2.765209 \\
\hline $\mathrm{C}$ & -0.953437 & 4.465991 & 1.359192 & $\mathrm{H}$ & -0.850430 & 0.946082 & 3.838812 \\
\hline $\mathrm{C}$ & -1.577236 & 4.837945 & 0.164807 & $\mathrm{H}$ & -1.581241 & 1.688568 & 2.406384 \\
\hline $\mathrm{H}$ & -1.401278 & 4.838046 & -1.977878 & $\mathrm{H}$ & -1.773400 & -0.050270 & 2.692227 \\
\hline $\mathrm{H}$ & -1.412747 & 4.731697 & 2.310969 & $\mathrm{C}$ & -0.803765 & 0.110304 & -2.783325 \\
\hline $\mathrm{C}$ & 3.220171 & -0.726545 & 2.271822 & $\mathrm{H}$ & -0.423063 & -0.933505 & -2.803501 \\
\hline $\mathrm{H}$ & 2.143722 & -0.507614 & 2.298496 & $\mathrm{H}$ & -1.884563 & 0.035952 & -2.548790 \\
\hline $\mathrm{H}$ & 3.452600 & -1.397840 & 3.102146 & $\mathrm{H}$ & -0.735604 & 0.502795 & -3.812930 \\
\hline $\mathrm{H}$ & 3.728377 & 0.227064 & 2.457989 & $\mathrm{Br}$ & -5.125483 & -2.676562 & -0.613077 \\
\hline $\mathrm{C}$ & 3.747950 & -0.314609 & -2.708841 & & & & \\
\hline $\mathrm{H}$ & 4.357344 & 0.596292 & -2.691227 & \multicolumn{4}{|c|}{ 3-Br-py } \\
\hline $\mathrm{H}$ & 4.030172 & -0.887026 & -3.595585 & $\mathrm{C}$ & -2.828203 & -0.090727 & 0.000074 \\
\hline $\mathrm{H}$ & 2.707290 & 0.014988 & -2.829352 & $\mathrm{C}$ & -0.825421 & -1.192416 & 0.000017 \\
\hline $\mathrm{C}$ & 4.505769 & -4.753399 & -0.451284 & $\mathrm{C}$ & -2.213144 & 1.157611 & -0.000038 \\
\hline $\mathrm{H}$ & 4.914894 & -5.037505 & -1.424374 & $\mathrm{H}$ & -3.913916 & -0.169772 & -0.000226 \\
\hline $\mathrm{H}$ & 5.209376 & -5.081698 & 0.320004 & $\mathrm{C}$ & -0.132515 & 0.014489 & 0.000096 \\
\hline $\mathrm{H}$ & 3.583779 & -5.328241 & -0.301221 & $\mathrm{H}$ & -0.288444 & -2.138829 & -0.000042 \\
\hline $\mathrm{C}$ & 0.948418 & 3.581309 & -2.389950 & $\mathrm{H}$ & -2.805066 & 2.067977 & -0.000318 \\
\hline $\mathrm{H}$ & 0.230071 & 3.587913 & -3.212641 & $\mathrm{~N}$ & -2.157889 & -1.246198 & -0.000037 \\
\hline
\end{tabular}




\begin{tabular}{|c|c|c|c|c|c|c|c|}
\hline $\mathrm{C}$ & -0.822907 & 1.217940 & 0.000087 & $\mathrm{H}$ & -1.757860 & 1.969145 & 2.250354 \\
\hline $\mathrm{H}$ & -0.294291 & 2.165508 & -0.000005 & $\mathrm{C}$ & 0.154918 & 3.660425 & -2.446578 \\
\hline \multirow[t]{2}{*}{$\mathrm{Br}$} & 1.809717 & 0.004489 & -0.000016 & $\mathrm{H}$ & -0.359567 & 4.539668 & -2.854733 \\
\hline & & & & $\mathrm{H}$ & 1.125785 & 3.598013 & -2.945823 \\
\hline \multicolumn{2}{|c|}{$\mathrm{TS}_{0-1 \mathrm{Br}}$} & & & $\mathrm{H}$ & -0.431807 & 2.781615 & -2.726174 \\
\hline $\mathrm{Ru}$ & -0.324725 & -0.189855 & 0.298285 & $\mathrm{C}$ & 2.706839 & 5.459640 & 1.478840 \\
\hline $\mathrm{C}$ & 0.944922 & -2.382802 & 2.102428 & $\mathrm{H}$ & 3.581621 & 4.798544 & 1.494585 \\
\hline $\mathrm{C}$ & 2.215975 & -2.117061 & 0.187027 & $\mathrm{H}$ & 2.977403 & 6.324023 & 0.864814 \\
\hline $\mathrm{C}$ & 1.740081 & -3.437205 & 2.531633 & $\mathrm{H}$ & 2.542891 & 5.808601 & 2.501882 \\
\hline $\mathrm{H}$ & 0.088289 & -2.008597 & 2.663111 & $\mathrm{C}$ & -3.389410 & -0.896559 & -2.909730 \\
\hline $\mathrm{C}$ & 3.053107 & -3.150528 & 0.575098 & $\mathrm{H}$ & -3.442101 & -1.709976 & -3.638182 \\
\hline $\mathrm{H}$ & 2.355652 & -1.587587 & -0.750596 & $\mathrm{H}$ & -3.939019 & -0.047731 & -3.336168 \\
\hline $\mathrm{H}$ & 1.511613 & -3.940347 & 3.465764 & $\mathrm{H}$ & -2.342479 & -0.592031 & -2.804835 \\
\hline $\mathrm{N}$ & 1.178603 & -1.730727 & 0.947711 & $\mathrm{C}$ & -4.041102 & -0.035114 & 2.010227 \\
\hline $\mathrm{C}$ & 2.825269 & -3.837847 & 1.759882 & $\mathrm{H}$ & -2.993352 & -0.016114 & 2.335996 \\
\hline $\mathrm{H}$ & 3.468205 & -4.657160 & 2.063731 & $\mathrm{H}$ & -4.323983 & 1.004192 & 1.802077 \\
\hline $\mathrm{C}$ & -1.803312 & 1.055278 & -0.274340 & $\mathrm{H}$ & -4.653274 & -0.385123 & 2.845462 \\
\hline $\mathrm{C}$ & -2.898813 & 3.020518 & -1.038486 & $\mathrm{C}$ & -5.815618 & -4.264932 & -0.068305 \\
\hline $\mathrm{C}$ & -3.894950 & 1.869764 & -1.020865 & $\mathrm{H}$ & -5.212350 & -5.097531 & -0.445525 \\
\hline $\mathrm{H}$ & -3.192158 & 3.850287 & -0.383385 & $\mathrm{H}$ & -6.069649 & -4.484278 & 0.971891 \\
\hline $\mathrm{H}$ & -2.739920 & 3.436348 & -2.042069 & $\mathrm{H}$ & -6.748343 & -4.269524 & -0.643531 \\
\hline $\mathrm{H}$ & -4.721604 & 2.024974 & -0.314338 & $\mathrm{C}$ & 0.736901 & 0.573067 & -1.065737 \\
\hline $\mathrm{H}$ & -4.338704 & 1.671084 & -2.003431 & $\mathrm{H}$ & 0.240749 & 0.983657 & -1.961068 \\
\hline $\mathrm{N}$ & -1.668087 & 2.392604 & -0.547352 & $\mathrm{C}$ & 2.140937 & 0.938356 & -1.052726 \\
\hline $\mathrm{N}$ & -3.081238 & 0.728157 & -0.588825 & $\mathrm{C}$ & 2.742128 & 1.399307 & -2.247730 \\
\hline $\mathrm{C}$ & -0.608411 & 3.220155 & -0.069411 & $\mathrm{C}$ & 2.952626 & 0.921576 & 0.105285 \\
\hline $\mathrm{C}$ & 0.314014 & 3.785352 & -0.962844 & $\mathrm{C}$ & 4.065466 & 1.810782 & -2.287523 \\
\hline $\mathrm{C}$ & -0.565248 & 3.515335 & 1.303879 & $\mathrm{H}$ & 2.136438 & 1.423248 & -3.152177 \\
\hline $\mathrm{C}$ & 1.375682 & 4.519942 & -0.434379 & $\mathrm{C}$ & 4.271842 & 1.352529 & 0.067114 \\
\hline $\mathrm{C}$ & 0.509573 & 4.262867 & 1.784833 & $\mathrm{H}$ & 2.527000 & 0.578087 & 1.044655 \\
\hline $\mathrm{C}$ & 1.510467 & 4.740829 & 0.936864 & $\mathrm{C}$ & 4.839185 & 1.800014 & -1.125925 \\
\hline $\mathrm{H}$ & 2.123300 & 4.922670 & -1.117041 & $\mathrm{H}$ & 4.496428 & 2.152108 & -3.225612 \\
\hline $\mathrm{H}$ & 0.566121 & 4.473954 & 2.852494 & $\mathrm{H}$ & 4.865565 & 1.340584 & 0.977869 \\
\hline $\mathrm{C}$ & -3.740514 & -0.532001 & -0.444316 & $\mathrm{H}$ & 5.872314 & 2.134723 & -1.150940 \\
\hline $\mathrm{C}$ & -3.938297 & -1.323523 & -1.585134 & $\mathrm{O}$ & -0.844843 & -0.092299 & 2.386743 \\
\hline $\mathrm{C}$ & -4.229317 & -0.908196 & 0.809848 & $\mathrm{O}$ & -1.202423 & -1.570094 & -0.793448 \\
\hline $\mathrm{C}$ & -4.611647 & -2.531439 & -1.438845 & $\mathrm{C}$ & 0.292011 & 0.636920 & 2.666109 \\
\hline $\mathrm{C}$ & -4.897464 & -2.133399 & 0.907463 & $\mathrm{H}$ & 0.128811 & 1.427980 & 3.421606 \\
\hline $\mathrm{C}$ & -5.093352 & -2.958205 & -0.197054 & $\mathrm{H}$ & 1.155576 & 0.026919 & 3.006783 \\
\hline $\mathrm{H}$ & -4.752675 & -3.164417 & -2.314769 & $\mathrm{H}$ & 0.668040 & 1.224227 & 1.776619 \\
\hline $\mathrm{H}$ & -5.279649 & -2.444174 & 1.879173 & $\mathrm{C}$ & -0.451782 & -2.443370 & -1.563907 \\
\hline $\mathrm{C}$ & -1.667373 & 3.061742 & 2.211681 & $\mathrm{H}$ & 0.237176 & -1.902689 & -2.242743 \\
\hline $\mathrm{H}$ & -1.506376 & 3.423941 & 3.230422 & $\mathrm{H}$ & 0.154714 & -3.160231 & -0.983647 \\
\hline $\mathrm{H}$ & -2.638516 & 3.447072 & 1.875136 & $\mathrm{H}$ & -1.146331 & -3.035350 & -2.181696 \\
\hline
\end{tabular}




\begin{tabular}{|c|c|c|c|c|c|c|c|}
\hline \multirow[t]{2}{*}{$\mathrm{Br}$} & 4.541574 & -3.624388 & -0.568110 & $\mathrm{H}$ & -0.374619 & 4.474587 & -2.809595 \\
\hline & & & & $\mathrm{H}$ & 1.215230 & 3.717824 & -2.879141 \\
\hline 1-Br & & & & $\mathrm{H}$ & -0.240892 & 2.720272 & -2.703195 \\
\hline $\mathrm{Ru}$ & -0.290803 & -0.174111 & 0.339159 & $\mathrm{C}$ & 2.661537 & 5.423586 & 1.620805 \\
\hline $\mathrm{C}$ & 0.958141 & -2.376525 & 2.123702 & $\mathrm{H}$ & 3.542261 & 4.770364 & 1.632286 \\
\hline $\mathrm{C}$ & 2.259571 & -2.079600 & 0.232756 & $\mathrm{H}$ & 2.936173 & 6.309399 & 1.039956 \\
\hline $\mathrm{C}$ & 1.744523 & -3.440254 & 2.545660 & $\mathrm{H}$ & 2.475167 & 5.739002 & 2.650741 \\
\hline $\mathrm{H}$ & 0.086487 & -2.018266 & 2.672025 & $\mathrm{C}$ & -3.256977 & -0.921493 & -2.920906 \\
\hline $\mathrm{C}$ & 3.087729 & -3.122537 & 0.615261 & $\mathrm{H}$ & -3.408687 & -1.680719 & -3.692445 \\
\hline $\mathrm{H}$ & 2.414018 & -1.538529 & -0.695249 & $\mathrm{H}$ & -3.665534 & 0.021748 & -3.304487 \\
\hline $\mathrm{H}$ & 1.498763 & -3.961037 & 3.465587 & $\mathrm{H}$ & -2.179977 & -0.773657 & -2.785728 \\
\hline $\mathrm{N}$ & 1.212222 & -1.703587 & 0.984770 & $\mathrm{C}$ & -4.125916 & -0.030446 & 1.955896 \\
\hline $\mathrm{C}$ & 2.840586 & -3.830111 & 1.783777 & $\mathrm{H}$ & -3.084544 & -0.002706 & 2.301092 \\
\hline $\mathrm{H}$ & 3.476479 & -4.656722 & 2.082446 & $\mathrm{H}$ & -4.418731 & 1.005664 & 1.744311 \\
\hline $\mathrm{C}$ & -1.785875 & 1.051705 & -0.276135 & $\mathrm{H}$ & -4.751113 & -0.389222 & 2.777650 \\
\hline $\mathrm{C}$ & -2.860803 & 2.994314 & -1.125223 & $\mathrm{C}$ & -5.840068 & -4.256924 & -0.180349 \\
\hline $\mathrm{C}$ & -3.872783 & 1.863202 & -1.030768 & $\mathrm{H}$ & -5.234892 & -5.090747 & -0.551619 \\
\hline $\mathrm{H}$ & -3.148010 & 3.877359 & -0.542703 & $\mathrm{H}$ & -6.116905 & -4.482387 & 0.852758 \\
\hline $\mathrm{H}$ & -2.681667 & 3.323868 & -2.157653 & $\mathrm{H}$ & -6.761224 & -4.250842 & -0.774115 \\
\hline $\mathrm{H}$ & -4.660388 & 2.049698 & -0.286790 & $\mathrm{C}$ & 0.728343 & 0.516119 & -1.104658 \\
\hline $\mathrm{H}$ & -4.367902 & 1.645641 & -1.983501 & $\mathrm{H}$ & 0.214647 & 0.831666 & -2.030932 \\
\hline $\mathrm{N}$ & -1.648437 & 2.385007 & -0.568553 & $\mathrm{C}$ & 2.120586 & 0.929194 & -1.145061 \\
\hline $\mathrm{N}$ & -3.057000 & 0.718352 & -0.607241 & $\mathrm{C}$ & 2.693102 & 1.345847 & -2.368500 \\
\hline $\mathrm{C}$ & -0.611343 & 3.217710 & -0.055850 & $\mathrm{C}$ & 2.945390 & 0.991594 & 0.002344 \\
\hline $\mathrm{C}$ & 0.346480 & 3.777782 & -0.913352 & $\mathrm{C}$ & 4.003760 & 1.793788 & -2.445107 \\
\hline $\mathrm{C}$ & -0.619912 & 3.510400 & 1.317943 & $\mathrm{H}$ & 2.073809 & 1.315773 & -3.263641 \\
\hline $\mathrm{C}$ & 1.394523 & 4.500590 & -0.342934 & $\mathrm{C}$ & 4.250872 & 1.454643 & -0.072145 \\
\hline $\mathrm{C}$ & 0.443361 & 4.245377 & 1.842416 & $\mathrm{H}$ & 2.533835 & 0.686163 & 0.962274 \\
\hline $\mathrm{C}$ & 1.480734 & 4.714211 & 1.033329 & $\mathrm{C}$ & 4.790036 & 1.859054 & -1.294377 \\
\hline $\mathrm{H}$ & 2.174726 & 4.891440 & -0.995497 & $\mathrm{H}$ & 4.414107 & 2.104620 & -3.402750 \\
\hline $\mathrm{H}$ & 0.460774 & 4.456774 & 2.911354 & $\mathrm{H}$ & 4.856340 & 1.503096 & 0.829523 \\
\hline $\mathrm{C}$ & -3.727773 & -0.539562 & -0.480877 & $\mathrm{H}$ & 5.812533 & 2.222103 & -1.348860 \\
\hline $\mathrm{C}$ & -3.880761 & -1.333687 & -1.625953 & $\mathrm{O}$ & -0.946145 & -0.173672 & 2.434155 \\
\hline $\mathrm{C}$ & -4.275101 & -0.904667 & 0.751280 & $\mathrm{O}$ & -1.224822 & -1.628355 & -0.628609 \\
\hline $\mathrm{C}$ & -4.568779 & -2.535791 & -1.505617 & $\mathrm{C}$ & 0.162619 & 0.593245 & 2.602885 \\
\hline $\mathrm{C}$ & -4.954835 & -2.125330 & 0.824360 & $\mathrm{H}$ & 0.020348 & 1.491688 & 3.232897 \\
\hline $\mathrm{C}$ & -5.106010 & -2.954495 & -0.283671 & $\mathrm{H}$ & 1.060631 & 0.055808 & 2.978250 \\
\hline $\mathrm{H}$ & -4.674654 & -3.171818 & -2.384272 & $\mathrm{H}$ & 0.522854 & 1.111254 & 1.623203 \\
\hline $\mathrm{H}$ & -5.378603 & -2.430530 & 1.780509 & $\mathrm{C}$ & -0.487554 & -2.512275 & -1.395191 \\
\hline $\mathrm{C}$ & -1.766229 & 3.068455 & 2.177287 & $\mathrm{H}$ & 0.205050 & -1.983732 & -2.082839 \\
\hline $\mathrm{H}$ & -1.644226 & 3.424817 & 3.203555 & $\mathrm{H}$ & 0.123255 & -3.224227 & -0.810452 \\
\hline $\mathrm{H}$ & -2.716605 & 3.469014 & 1.800918 & $\mathrm{H}$ & -1.182445 & -3.112071 & -2.006578 \\
\hline $\mathrm{H}$ & -1.879805 & 1.977659 & 2.210277 & $\mathrm{Br}$ & 4.589636 & -3.583435 & -0.516070 \\
\hline $\mathrm{C}$ & 0.234203 & 3.657693 & -2.400907 & & & & \\
\hline
\end{tabular}


TS1-2Br

\begin{tabular}{|c|c|c|c|}
\hline $\mathrm{ku}$ & -0.372004 & 0.306694 & -0.2 \\
\hline $\mathrm{C}$ & 0083201 & 3.327848 & 0224206 \\
\hline $\mathrm{C}$ & 2.043695 & 406922 & $810>-2$ \\
\hline & 0.4 & & 184154 \\
\hline $\mathrm{H}$ & 147043 & .112408 & 224011 مـ \\
\hline & 2.613865 & 466 & 853 \\
\hline $\mathrm{H}$ & 1 & 3 & 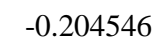 \\
\hline $\mathrm{H}$ & 7311 & 87 & 135 \\
\hline $\mathrm{N}$ & & & 5742 \\
\hline $\mathrm{C}$ & 5 & 6 & 61 \\
\hline $\mathrm{H}$ & 2.248999 & 278 & 546 \\
\hline C & -1.637182 & 1.277626 & -0.279759 \\
\hline C & 35 & 2 & 12 \\
\hline $\mathrm{C}$ & -3.65 & 1 & \\
\hline$\Pi$ & -2.3 & 3 & -1.677798 \\
\hline $\mathrm{H}$ & -2.58 & 2 & 15 \\
\hline $\mathrm{H}$ & -4.305241 & -2.494519 & -1.465386 \\
\hline $\mathrm{H}$ & -4.200141 & 8930 & 127 \\
\hline$N$ & -1347638 & 2613553 & 957 \\
\hline $\mathbf{N}$ & -2.980238 & -1.172774 & 53 \\
\hline$C$ & -0.0 & -3.2 & 2 \\
\hline $\mathrm{C}$ & 0.352773 & -3.80 & 093 \\
\hline $\mathrm{C}$ & 0 & -3.323244 & 38 \\
\hline $\mathrm{C}$ & 1 & 4426 & 76 \\
\hline 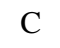 & 31 & 8 & 96 \\
\hline$C$ & 2.42 & T. & 0.120. \\
\hline $\mathrm{H}$ & 828 & -4.8 & 20 \\
\hline $\mathrm{H}$ & 591037 & 30 & 50 \\
\hline$C$ & 3000520 & 0062367 & 0002700 \\
\hline $\mathrm{C}$ & -4.021839 & 157479 & 77622 \\
\hline $\mathrm{C}$ & -4.467478 & 0.676483 & J \\
\hline $\mathrm{C}$ & -4.877615 & 40 & 45111 \\
\hline $\mathrm{C}$ & -5.314480 & .705264 & -0.654496 \\
\hline $\mathrm{C}$ & -5.521347 & 1.986824 & \\
\hline $\mathrm{H}$ & -5.047446 & .379451 & 04767 \\
\hline $\mathrm{H}$ & -5.830950 & 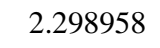 & 08419 \\
\hline $\mathrm{C}$ & 9 & 0 & 25 \\
\hline $\mathrm{H}$ & 0.972362 & -2.008144 & -3 \\
\hline $\mathrm{H}$ & 219 & 6 & 31 \\
\hline $\mathrm{H}$ & -0.710319 & 2.296967 & -2.679194 \\
\hline $\mathrm{C}$ & -0.510951 & -3.721435 & 2.170188 \\
\hline $\mathrm{H}$ & -1.453082 & -4.269930 & 2.0466 \\
\hline $\mathrm{H}$ & -0.000253 & 57 & 3. \\
\hline
\end{tabular}

\begin{tabular}{|c|c|c|c|}
\hline & -0.790489 & -2.687324 & 2.398820 \\
\hline & 98 & -5.135908 & 3136 \\
\hline & 3.899362 & -5.933215 & 0.789105 \\
\hline & 4.567248 & 9803 & -0.242469 \\
\hline & 3.966896 & -5.565108 & 0.939446 \\
\hline & -3.377599 & -0.712452 & 2.311731 \\
\hline & -3.648584 & -0.384385 & 3.318580 \\
\hline & -3.677559 & -1.765012 & 2.214362 \\
\hline & -2.289175 & -0.6 & 2.223026 \\
\hline $\mathrm{C}$ & -4.325026 & 0.365531 & -2.531009 \\
\hline $\mathrm{H}$ & -3.492860 & -0.316362 & -2.713680 \\
\hline $\mathrm{H}$ & -5.243395 & -0.086065 & -2.927756 \\
\hline & -4.132994 & 1.273978 & -3.107569 \\
\hline C & -6.408129 & 3.117799 & 1.118540 \\
\hline F & -7.085360 & 3.424102 & 0.316414 \\
\hline $\mathrm{H}$ & -7.015023 & 2. & 1.989939 \\
\hline H & -5.821592 & 3.999799 & 1.400662 \\
\hline $\mathrm{C}$ & 0.849959 & -0.670677 & 0.854421 \\
\hline $\mathrm{H}$ & 0.476983 & -1.049191 & 1.825342 \\
\hline C & 2.258676 & -0.998085 & 0.709254 \\
\hline $\mathrm{C}$ & 7373 & -1.4 & .837 \\
\hline C & 2.958735 & -0.887600 & -0.510174 \\
\hline C & 4.346704 & -1.702889 & 1.762417 \\
\hline $\mathrm{H}$ & 2.455768 & -1.544662 & 2.781800 \\
\hline$C$ & 4.314670 & -1.170197 & -0.591071 \\
\hline $\mathrm{H}$ & 2.3 & 5230 & -1.394168 \\
\hline $\mathrm{C}$ & 5.017918 & -1.569852 & 0.547033 \\
\hline H & 4.886390 & -2.023953 & 2.649570 \\
\hline $\mathrm{H}$ & 4.830121 & -1.080313 & -1.544150 \\
\hline $\mathrm{H}$ & 6.081330 & -1.785627 & 0.483848 \\
\hline $\mathrm{O}$ & -1.621978 & 1.278119 & -1.739752 \\
\hline $\mathrm{O}$ & -1.292824 & 762 & 388 \\
\hline C & -0.619847 & 0.763427 & -2.389577 \\
\hline $\mathrm{H}$ & -0.837035 & -0.042719 & -3.119274 \\
\hline $\mathrm{H}$ & 0.178336 & 1.439704 & -2.756420 \\
\hline $\mathrm{H}$ & 0.371338 & -0.176839 & -1.639875 \\
\hline & -0.5 & 1. & 55 \\
\hline $\mathrm{H}$ & -0.096305 & 0.654522 & 3.029577 \\
\hline H & 0.300899 & 2.235787 & 2.342714 \\
\hline & -1.187375 & 2.031563 & 3.288650 \\
\hline & 4.544739 & 3.827836 & -0.129793 \\
\hline \multicolumn{4}{|c|}{ E-BS } \\
\hline & .385251 & 0.373718 & -0.138852 \\
\hline
\end{tabular}




\begin{tabular}{|c|c|c|c|c|c|c|c|}
\hline $\mathrm{C}$ & -0.164755 & 3.423943 & 0.097444 & $\mathrm{H}$ & 4.566308 & -5.075472 & -1.356925 \\
\hline $\mathrm{C}$ & 1.937115 & 2.515467 & -0.285501 & $\mathrm{H}$ & 4.772356 & -4.434838 & 0.277475 \\
\hline $\mathrm{C}$ & 0.353538 & 4.713808 & 0.083664 & $\mathrm{H}$ & 4.046746 & -6.019527 & 0.045504 \\
\hline $\mathrm{H}$ & -1.208380 & 3.180293 & 0.281907 & $\mathrm{C}$ & -3.267479 & -1.053879 & 2.182025 \\
\hline $\mathrm{C}$ & 2.504961 & 3.780248 & -0.307101 & $\mathrm{H}$ & -3.555076 & -0.810911 & 3.208153 \\
\hline $\mathrm{H}$ & 2.527793 & 1.613453 & -0.424465 & $\mathrm{H}$ & -3.467123 & -2.122732 & 2.021992 \\
\hline $\mathrm{H}$ & -0.305605 & 5.562343 & 0.236217 & $\mathrm{H}$ & -2.188597 & -0.894373 & 2.089789 \\
\hline $\mathrm{N}$ & 0.622984 & 2.351168 & -0.085459 & $\mathrm{C}$ & -4.310618 & 0.221805 & -2.581857 \\
\hline $\mathrm{C}$ & 1.714305 & 4.907067 & -0.124277 & $\mathrm{H}$ & -3.274323 & -0.038156 & -2.809829 \\
\hline $\mathrm{H}$ & 2.149591 & 5.900590 & -0.144453 & $\mathrm{H}$ & -4.953191 & -0.574782 & -2.980707 \\
\hline $\mathrm{C}$ & -1.496734 & -1.302548 & -0.423767 & $\mathrm{H}$ & -4.554409 & 1.134414 & -3.131447 \\
\hline $\mathrm{C}$ & -2.168405 & -3.528235 & -0.830636 & $\mathrm{C}$ & -6.671374 & 2.518995 & 1.214915 \\
\hline $\mathrm{C}$ & -3.343436 & -2.602838 & -1.091744 & $\mathrm{H}$ & -7.482329 & 2.675870 & 0.497698 \\
\hline $\mathrm{H}$ & -1.940000 & -4.188784 & -1.673272 & $\mathrm{H}$ & -7.116182 & 2.227318 & 2.170864 \\
\hline $\mathrm{H}$ & -2.307783 & -4.159389 & 0.059665 & $\mathrm{H}$ & -6.191856 & 3.492331 & 1.371613 \\
\hline $\mathrm{H}$ & -3.605712 & -2.547324 & -2.157827 & $\mathrm{C}$ & 0.780616 & -0.347332 & 1.183881 \\
\hline $\mathrm{H}$ & -4.245987 & -2.876003 & -0.537672 & $\mathrm{H}$ & 0.366629 & -0.269058 & 2.203626 \\
\hline $\mathrm{N}$ & -1.068479 & -2.580577 & -0.617359 & $\mathrm{C}$ & 2.139217 & -0.844284 & 1.241750 \\
\hline $\mathrm{N}$ & -2.839273 & -1.302295 & -0.626814 & $\mathrm{C}$ & 2.683015 & -1.182593 & 2.502181 \\
\hline $\mathrm{C}$ & 0.255162 & -3.102701 & -0.540464 & $\mathrm{C}$ & 2.973115 & -0.994331 & 0.113291 \\
\hline $\mathrm{C}$ & 0.707818 & -3.637836 & 0.676271 & $\mathrm{C}$ & 3.984063 & -1.646887 & 2.630624 \\
\hline $\mathrm{C}$ & 1.024355 & -3.194199 & -1.708682 & $\mathrm{H}$ & 2.051513 & -1.071424 & 3.382214 \\
\hline $\mathrm{C}$ & 1.956873 & -4.254206 & 0.704890 & $\mathrm{C}$ & 4.277976 & -1.443134 & 0.242656 \\
\hline $\mathrm{C}$ & 2.266477 & -3.827205 & -1.632897 & $\mathrm{H}$ & 2.563114 & -0.763516 & -0.867408 \\
\hline $\mathrm{C}$ & 2.749619 & -4.363197 & -0.439261 & $\mathrm{C}$ & 4.789617 & -1.773213 & 1.499097 \\
\hline $\mathrm{H}$ & 2.327163 & -4.647267 & 1.651287 & $\mathrm{H}$ & 4.376826 & -1.902466 & 3.611113 \\
\hline $\mathrm{H}$ & 2.875118 & -3.900146 & -2.533803 & $\mathrm{H}$ & 4.899960 & -1.555274 & -0.642408 \\
\hline $\mathrm{C}$ & -3.777970 & -0.321232 & -0.175200 & $\mathrm{H}$ & 5.813091 & -2.127637 & 1.594304 \\
\hline $\mathrm{C}$ & -4.002859 & -0.196138 & 1.202373 & $\mathrm{O}$ & -1.716378 & 1.450509 & -1.435357 \\
\hline $\mathrm{C}$ & -4.513716 & 0.411656 & -1.111578 & $\mathrm{O}$ & -1.587265 & 1.297407 & 1.400453 \\
\hline $\mathrm{C}$ & -4.957781 & 0.724363 & 1.629084 & $\mathrm{C}$ & -0.774894 & 0.949838 & -2.173866 \\
\hline $\mathrm{C}$ & -5.459283 & 1.320198 & -0.637024 & $\mathrm{H}$ & -1.008302 & 0.092697 & -2.834340 \\
\hline $\mathrm{C}$ & -5.685510 & 1.500891 & 0.727765 & $\mathrm{H}$ & 0.018937 & 1.608479 & -2.570519 \\
\hline $\mathrm{H}$ & -5.130345 & 0.841006 & 2.698592 & $\mathrm{H}$ & 0.729664 & -0.293949 & -1.087755 \\
\hline $\mathrm{H}$ & -6.028398 & 1.908841 & -1.355941 & $\mathrm{C}$ & -1.142938 & 1.555180 & 2.665316 \\
\hline $\mathrm{C}$ & 0.542641 & -2.606358 & -2.997287 & $\mathrm{H}$ & -1.005727 & 0.650780 & 3.309286 \\
\hline $\mathrm{H}$ & 0.668305 & -1.516515 & -2.993356 & $\mathrm{H}$ & -0.169891 & 2.101700 & 2.719807 \\
\hline $\mathrm{H}$ & 1.095454 & -3.008646 & -3.849835 & $\mathrm{H}$ & -1.875347 & 2.178763 & 3.213092 \\
\hline $\mathrm{H}$ & -0.525335 & -2.785875 & -3.161911 & $\mathrm{Br}$ & 4.411443 & 3.956286 & -0.602689 \\
\hline $\mathrm{C}$ & -0.119504 & -3.524585 & 1.919031 & & & & \\
\hline $\mathrm{H}$ & -0.916256 & -4.278445 & 1.955218 & \multicolumn{4}{|c|}{$\mathrm{TS}^{\prime}{ }_{1-2 \mathrm{Br}}$} \\
\hline $\mathrm{H}$ & 0.495608 & -3.664138 & 2.812114 & $\mathrm{Ru}$ & -0.009661 & 0.142060 & -0.206730 \\
\hline $\mathrm{H}$ & -0.607943 & -2.546742 & 1.987930 & $\mathrm{C}$ & -2.439164 & 1.940722 & -0.234881 \\
\hline $\mathrm{C}$ & 4.098942 & -5.008276 & -0.371008 & $\mathrm{C}$ & -0.564160 & 3.306169 & -0.251445 \\
\hline
\end{tabular}




\begin{tabular}{|c|c|c|c|c|c|c|c|}
\hline $\mathrm{C}$ & -3.261624 & 3.058427 & -0.201269 & $\mathrm{C}$ & -0.771596 & -2.859149 & 2.399559 \\
\hline $\mathrm{H}$ & -2.809751 & 0.920898 & -0.221006 & $\mathrm{H}$ & -1.220784 & -2.731225 & 3.387720 \\
\hline $\mathrm{C}$ & -1.339325 & 4.456289 & -0.214922 & $\mathrm{H}$ & -0.153926 & -3.767538 & 2.435624 \\
\hline $\mathrm{H}$ & 0.521750 & 3.347537 & -0.262778 & $\mathrm{H}$ & -0.113608 & -2.004251 & 2.224969 \\
\hline $\mathrm{N}$ & -1.101975 & 2.077979 & -0.259727 & $\mathrm{C}$ & -2.106980 & -3.216218 & -2.464692 \\
\hline $\mathrm{C}$ & -2.725870 & 4.338065 & -0.188608 & $\mathrm{H}$ & -1.073598 & -2.914255 & -2.645399 \\
\hline $\mathrm{H}$ & -3.368044 & 5.211814 & -0.158979 & $\mathrm{H}$ & -2.240845 & -4.241439 & -2.833127 \\
\hline $\mathrm{C}$ & 0.678970 & -1.764269 & -0.238464 & $\mathrm{H}$ & -2.744226 & -2.565528 & -3.068583 \\
\hline $\mathrm{C}$ & 2.072698 & -3.649015 & -0.550753 & $\mathrm{C}$ & -5.618636 & -3.236394 & 1.123901 \\
\hline $\mathrm{C}$ & 0.635264 & -4.118505 & -0.418175 & $\mathrm{H}$ & -6.232603 & -3.711240 & 0.353353 \\
\hline $\mathrm{H}$ & 2.468180 & -3.778020 & -1.569384 & $\mathrm{H}$ & -5.757819 & -3.790649 & 2.057172 \\
\hline $\mathrm{H}$ & 2.764397 & -4.149510 & 0.136168 & $\mathrm{H}$ & -6.028178 & -2.232940 & 1.288302 \\
\hline $\mathrm{H}$ & 0.299989 & -4.743503 & -1.253050 & $\mathrm{C}$ & 1.449256 & 0.683479 & 0.868001 \\
\hline $\mathrm{H}$ & 0.456792 & -4.679143 & 0.509810 & $\mathrm{H}$ & 1.555285 & 0.184066 & 1.850455 \\
\hline $\mathrm{N}$ & 1.968637 & -2.220902 & -0.239096 & $\mathrm{C}$ & 2.479932 & 1.697912 & 0.721048 \\
\hline $\mathrm{N}$ & -0.116333 & -2.858063 & -0.387059 & $\mathrm{C}$ & 3.232504 & 2.089250 & 1.849251 \\
\hline $\mathrm{C}$ & 3.164285 & -1.447235 & -0.268018 & $\mathrm{C}$ & 2.780846 & 2.325451 & -0.505860 \\
\hline $\mathrm{C}$ & 3.911118 & -1.346982 & 0.918083 & $\mathrm{C}$ & 4.204956 & 3.076560 & 1.766767 \\
\hline $\mathrm{C}$ & 3.618619 & -0.873108 & -1.464013 & $\mathrm{H}$ & 3.031662 & 1.595039 & 2.799295 \\
\hline $\mathrm{C}$ & 5.095864 & -0.614725 & 0.896160 & $\mathrm{C}$ & 3.759832 & 3.304607 & -0.593671 \\
\hline $\mathrm{C}$ & 4.812070 & -0.150586 & -1.435481 & $\mathrm{H}$ & 2.235172 & 2.001887 & -1.391422 \\
\hline $\mathrm{C}$ & 5.558089 & 0.003216 & -0.267012 & $\mathrm{C}$ & 4.470477 & 3.692626 & 0.543814 \\
\hline $\mathrm{H}$ & 5.666435 & -0.508436 & 1.818314 & $\mathrm{H}$ & 4.764141 & 3.363559 & 2.653453 \\
\hline $\mathrm{H}$ & 5.162883 & 0.317209 & -2.355791 & $\mathrm{H}$ & 3.978976 & 3.765714 & -1.553844 \\
\hline $\mathrm{C}$ & -1.499434 & -2.938270 & -0.021393 & $\mathrm{H}$ & 5.235342 & 4.461807 & 0.473433 \\
\hline $\mathrm{C}$ & -1.830836 & -2.939917 & 1.343565 & $\mathrm{O}$ & -1.489268 & -0.426437 & -1.727192 \\
\hline $\mathrm{C}$ & -2.467075 & -3.114919 & -1.014974 & $\mathrm{O}$ & -1.295060 & -0.154762 & 1.406496 \\
\hline $\mathrm{C}$ & -3.174180 & -3.050703 & 1.689809 & $\mathrm{C}$ & -0.531772 & 0.157986 & -2.385113 \\
\hline $\mathrm{C}$ & -3.801437 & -3.220517 & -0.617009 & $\mathrm{H}$ & 0.041980 & -0.450970 & -3.111505 \\
\hline $\mathrm{C}$ & -4.175822 & -3.173004 & 0.724387 & $\mathrm{H}$ & -0.696311 & 1.189413 & -2.755976 \\
\hline $\mathrm{H}$ & -3.446434 & -3.032386 & 2.744530 & $\mathrm{H}$ & 0.794850 & 0.527146 & -1.637327 \\
\hline $\mathrm{H}$ & -4.568648 & -3.340910 & -1.381171 & $\mathrm{C}$ & -1.180191 & 0.646555 & 2.517810 \\
\hline $\mathrm{C}$ & 2.874361 & -1.024139 & -2.755243 & $\mathrm{H}$ & -0.208771 & 0.529563 & 3.050146 \\
\hline $\mathrm{H}$ & 2.594169 & -0.047179 & -3.165935 & $\mathrm{H}$ & -1.275489 & 1.736438 & 2.316490 \\
\hline $\mathrm{H}$ & 3.495491 & -1.519051 & -3.510327 & $\mathrm{H}$ & -1.967919 & 0.392666 & 3.249843 \\
\hline $\mathrm{H}$ & 1.951867 & -1.598594 & -2.641377 & $\mathrm{H}$ & -0.865202 & 5.432300 & -0.200357 \\
\hline $\mathrm{C}$ & 3.430288 & -1.993935 & 2.179880 & $\mathrm{Br}$ & -5.181576 & 2.807789 & -0.163207 \\
\hline $\mathrm{H}$ & 3.495205 & -3.088137 & 2.131038 & & & & \\
\hline $\mathrm{H}$ & 4.024674 & -1.671029 & 3.038346 & 2-Br' & & & \\
\hline $\mathrm{H}$ & 2.379748 & -1.758519 & 2.380418 & $\mathrm{Ru}$ & 0.064934 & -0.154129 & -0.256278 \\
\hline $\mathrm{C}$ & 6.802954 & 0.834914 & -0.257496 & $\mathrm{C}$ & 2.488883 & -2.000417 & -0.279171 \\
\hline $\mathrm{H}$ & 7.424185 & 0.648370 & -1.138835 & $\mathrm{C}$ & 0.596002 & -3.245501 & -0.789424 \\
\hline $\mathrm{H}$ & 6.556114 & 1.903642 & -0.260566 & $\mathrm{C}$ & 3.278489 & -3.133142 & -0.424016 \\
\hline $\mathrm{H}$ & 7.409701 & 0.644773 & 0.631727 & $\mathrm{H}$ & 2.853759 & -1.022728 & 0.029515 \\
\hline
\end{tabular}




\begin{tabular}{|c|c|c|c|c|c|c|c|}
\hline $\mathrm{C}$ & 1.338618 & -4.406748 & -0.946132 & $\mathrm{H}$ & -0.194798 & 3.535009 & 2.354358 \\
\hline $\mathrm{H}$ & -0.484130 & -3.227509 & -0.916217 & $\mathrm{H}$ & 0.153515 & 1.798313 & 2.263293 \\
\hline $\mathrm{N}$ & 1.161014 & -2.074163 & -0.463872 & $\mathrm{C}$ & 2.007947 & 3.422539 & -2.379608 \\
\hline $\mathrm{C}$ & 2.718191 & -4.355572 & -0.764105 & $\mathrm{H}$ & 1.120950 & 2.831009 & -2.618127 \\
\hline $\mathrm{H}$ & 3.336388 & -5.239783 & -0.880634 & $\mathrm{H}$ & 1.816826 & 4.458935 & -2.688210 \\
\hline $\mathrm{C}$ & -0.719275 & 1.723122 & -0.280929 & $\mathrm{H}$ & 2.829439 & 3.049085 & -2.996316 \\
\hline $\mathrm{C}$ & -2.211839 & 3.550276 & -0.393829 & $\mathrm{C}$ & 5.425898 & 3.677047 & 1.293796 \\
\hline $\mathrm{C}$ & -0.799160 & 4.052569 & -0.629580 & $\mathrm{H}$ & 5.991457 & 4.294269 & 0.589710 \\
\hline $\mathrm{H}$ & -2.911763 & 3.831723 & -1.187468 & $\mathrm{H}$ & 5.491918 & 4.142986 & 2.281504 \\
\hline $\mathrm{H}$ & -2.636170 & 3.893456 & 0.561452 & $\mathrm{H}$ & 5.944107 & 2.713436 & 1.362335 \\
\hline $\mathrm{H}$ & -0.636613 & 4.395908 & -1.661192 & $\mathrm{C}$ & -1.142008 & -0.894352 & 1.016600 \\
\hline $\mathrm{H}$ & -0.507740 & 4.864332 & 0.043529 & $\mathrm{H}$ & -0.816036 & -0.726020 & 2.056644 \\
\hline $\mathrm{N}$ & -2.025880 & 2.094262 & -0.373871 & $\mathrm{C}$ & -2.306199 & -1.755552 & 1.000416 \\
\hline $\mathrm{N}$ & 0.017397 & 2.859944 & -0.362359 & $\mathrm{C}$ & -2.843530 & -2.188269 & 2.234141 \\
\hline $\mathrm{C}$ & -3.193121 & 1.276416 & -0.383665 & $\mathrm{C}$ & -2.934946 & -2.211516 & -0.177922 \\
\hline $\mathrm{C}$ & -3.862852 & 1.034022 & 0.825330 & $\mathrm{C}$ & -3.948263 & -3.025836 & 2.291032 \\
\hline $\mathrm{C}$ & -3.721648 & 0.851523 & -1.610116 & $\mathrm{H}$ & -2.368813 & -1.844799 & 3.151938 \\
\hline $\mathrm{C}$ & -5.070111 & 0.339865 & 0.785667 & $\mathrm{C}$ & -4.031335 & -3.057458 & -0.121770 \\
\hline $\mathrm{C}$ & -4.936618 & 0.163700 & -1.601081 & $\mathrm{H}$ & -2.546311 & -1.870764 & -1.134906 \\
\hline $\mathrm{C}$ & -5.625715 & -0.100108 & -0.417186 & $\mathrm{C}$ & -4.544795 & -3.467267 & 1.110230 \\
\hline $\mathrm{H}$ & -5.583843 & 0.125270 & 1.722546 & $\mathrm{H}$ & -4.342706 & -3.342509 & 3.252768 \\
\hline $\mathrm{H}$ & -5.355973 & -0.174611 & -2.548210 & $\mathrm{H}$ & -4.504620 & -3.388772 & -1.043433 \\
\hline $\mathrm{C}$ & 1.378807 & 3.045251 & 0.037178 & $\mathrm{H}$ & -5.407533 & -4.127776 & 1.148192 \\
\hline $\mathrm{C}$ & 1.678819 & 3.021043 & 1.406240 & $\mathrm{O}$ & 1.666523 & 0.516346 & -1.519757 \\
\hline $\mathrm{C}$ & 2.349964 & 3.335356 & -0.925495 & $\mathrm{O}$ & 1.534898 & 0.141811 & 1.296387 \\
\hline $\mathrm{C}$ & 2.997800 & 3.245147 & 1.792162 & $\mathrm{C}$ & 0.711880 & 0.098794 & -2.291529 \\
\hline $\mathrm{C}$ & 3.657536 & 3.551906 & -0.491658 & $\mathrm{H}$ & 0.112209 & 0.839619 & -2.854119 \\
\hline $\mathrm{C}$ & 4.003940 & 3.495481 & 0.858029 & $\mathrm{H}$ & 0.811482 & -0.872366 & -2.809285 \\
\hline $\mathrm{H}$ & 3.246764 & 3.213333 & 2.852281 & $\mathrm{H}$ & -1.125728 & -0.599424 & -1.243388 \\
\hline $\mathrm{H}$ & 4.428886 & 3.758013 & -1.232997 & $\mathrm{C}$ & 1.534834 & -0.518944 & 2.492122 \\
\hline $\mathrm{C}$ & -2.994015 & 1.110190 & -2.891230 & $\mathrm{H}$ & 0.741015 & -0.190773 & 3.208577 \\
\hline $\mathrm{H}$ & -2.115943 & 0.457912 & -2.973009 & $\mathrm{H}$ & 1.420347 & -1.627745 & 2.417423 \\
\hline $\mathrm{H}$ & -3.635699 & 0.929155 & -3.756973 & $\mathrm{H}$ & 2.486888 & -0.347100 & 3.029219 \\
\hline $\mathrm{H}$ & -2.614963 & 2.136731 & -2.953448 & $\mathrm{H}$ & 0.846710 & -5.338443 & -1.205956 \\
\hline $\mathrm{C}$ & -3.279004 & 1.490512 & 2.126090 & $\mathrm{Br}$ & 5.187335 & -2.989449 & -0.129475 \\
\hline $\mathrm{H}$ & -3.410777 & 2.568808 & 2.281718 & & & & \\
\hline $\mathrm{H}$ & -3.753524 & 0.980765 & 2.968494 & \multicolumn{2}{|c|}{$\mathbf{T S}_{\mathbf{0 - 1 \mathrm { Br }}}^{\prime}$} & & \\
\hline $\mathrm{H}$ & -2.201531 & 1.297128 & 2.169241 & $\mathrm{Ru}$ & -0.004143 & 0.122496 & 0.027434 \\
\hline $\mathrm{C}$ & -6.911100 & -0.867708 & -0.425828 & $\mathrm{C}$ & 2.404894 & 1.984430 & 0.225733 \\
\hline $\mathrm{H}$ & -7.291224 & -1.012013 & -1.440647 & $\mathrm{C}$ & 0.654981 & 3.229239 & -0.639822 \\
\hline $\mathrm{H}$ & -6.775862 & -1.860494 & 0.020875 & $\mathrm{C}$ & 3.272448 & 3.049129 & 0.029382 \\
\hline $\mathrm{H}$ & -7.689616 & -0.365072 & 0.156777 & $\mathrm{H}$ & 2.705191 & 1.027844 & 0.651760 \\
\hline $\mathrm{C}$ & 0.604231 & 2.783483 & 2.419132 & $\mathrm{C}$ & 1.477371 & 4.324802 & -0.858237 \\
\hline $\mathrm{H}$ & 1.008535 & 2.811262 & 3.434312 & $\mathrm{H}$ & -0.396569 & 3.245097 & -0.910675 \\
\hline
\end{tabular}




\begin{tabular}{|c|c|c|c|}
\hline & 6 & 2.083692 & -0.10073 \\
\hline & 824684 & 4.242730 & 5181 \\
\hline & 502 & 074743 & 831 \\
\hline C & 60 & 755478 & 26332 \\
\hline & 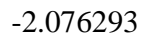 & .572030 & $835>2>$ \\
\hline $\mathrm{C}$ & 7 & & 365 \\
\hline $\mathrm{H}$ & -2.288582 & -3.605994 & 014820 \\
\hline $\mathrm{H}$ & 5436 & -4.05 & 397 \\
\hline $\mathrm{H}$ & -0.2 & -4.7 & 206 \\
\hline $\mathrm{H}$ & 8 & -4.659149 & 55 \\
\hline $\mathrm{N}$ & -1.9 & -2.177493 & 92910 \\
\hline $\mathrm{N}$ & 9 & 98 & 71 \\
\hline $\mathrm{C}$ & -3.16 & -1.37 & 218 \\
\hline $\mathrm{C}$ & -4.097963 & -1.4 & \\
\hline $\mathrm{C}$ & 3 & -0. & 84 \\
\hline$\Gamma$ & -5.264063 & -0.727861 & -0.517241 \\
\hline $\mathrm{C}$ & -4.5 & 84 & \\
\hline $\mathrm{C}$ & -5 & 3 & 48 \\
\hline $\mathrm{H}$ & -5.974241 & -0.784948 & 33 \\
\hline $\mathrm{H}$ & -4.78 & 17 & \\
\hline $\mathrm{C}$ & 1.462620 & -2.977 & 134 \\
\hline 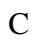 & 1.804665 & 349 & \\
\hline $\mathrm{C}$ & 2 & -3.046534 & \\
\hline $\mathrm{C}$ & 3.149107 & -3.252472 & \\
\hline $\mathrm{C}$ & 3 & 3 & 45 \\
\hline $\mathrm{C}$ & 4.146062 & -3.271422 & 51 \\
\hline $\mathrm{H}$ & 811 & -3.339256 & \\
\hline$H$ & 4.525945 & -3.223489 & 1.415765 \\
\hline$C$ & 474940 & 0460 & 66 \\
\hline $\mathrm{H}$ & -2.280911 & - & 078 \\
\hline $\mathrm{H}$ & -2.9003 & 6 & 0 \\
\hline${ }^{2}$ & -1.509949 & -0.943539 & 528 \\
\hline$G^{\prime}$ & -3.824635 & -2.364298 & -1.762057 \\
\hline $\mathrm{H}$ & 13 & 50 & 40 \\
\hline $\mathrm{H}$ & -4.451665 & -2.086960 & 67 \\
\hline $\mathrm{H}$ & -2.776171 & -2.30 & -2.072104 \\
\hline $\mathrm{C}$ & -6.761325 & 46 & 8983 \\
\hline 11 & -6.900577 & & \\
\hline$H_{-}$ & -6.698819 & 1.763262 & -0.194115 \\
\hline $\mathrm{H}$ & -7.662317 & 0.394328 & 0.354851 \\
\hline C & 0.753780 & -3.141102 & -2.415910 \\
\hline 11 & 1.193297 & 19. & 240 \\
\hline $\mathrm{H}$ & 0.255336 & 8323 & -2.474010 \\
\hline $\mathrm{H}$ & 0.010256 & 22 & 40 \\
\hline
\end{tabular}

\begin{tabular}{|c|c|c|c|}
\hline $\mathrm{C}$ & 2.059185 & -3.028451 & 2.4712 \\
\hline $\mathrm{H}$ & 1.028016 & -2.707256 & 26260 \\
\hline $\mathrm{H}$ & 2.192640 & -4.023167 & 2.916573 \\
\hline $\mathrm{H}$ & 2.696544 & -2.333610 & 023236 \\
\hline $\mathrm{C}$ & 5.590536 & -3.375329 & 1.081261 \\
\hline $\mathrm{H}$ & 6.213310 & -3.680686 & -0.236115 \\
\hline 11 & 5.744107 & -4.095093 & -1.891549 \\
\hline $\mathrm{H}$ & 5.977303 & -2.413584 & 37366 \\
\hline $\mathrm{C}$ & -1.532159 & 0.554562 & -1.016087 \\
\hline $\mathrm{H}$ & -1.896618 & -0.139390 & -1.799914 \\
\hline 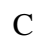 & -2.481536 & 1.643863 & -0.844819 \\
\hline $\mathrm{C}$ & -3.506908 & 1.856069 & -1.789984 \\
\hline $\mathrm{C}$ & -2.455169 & 2.499720 & 0.279115 \\
\hline $\mathrm{C}$ & -4.431768 & 2.880742 & -1.640663 \\
\hline $\mathrm{H}$ & -3.563242 & 1.186627 & -2.647637 \\
\hline $\mathrm{C}$ & -3.393752 & 3.507838 & 340 \\
\hline 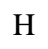 & -1.681836 & 2.339260 & 1.033915 \\
\hline$C$ & -4.384229 & 3.709082 & -0.519136 \\
\hline $\mathrm{H}$ & -5.205758 & 3.026106 & -2.390728 \\
\hline$H$ & -3.358081 & 4.141625 & 1.326950 \\
\hline 11 & -5.117867 & 4.500632 & -0.391529 \\
\hline 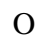 & 1.299582 & -0.281599 & 1.652715 \\
\hline $\mathrm{O}$ & 1.207401 & -0.407865 & -1.455659 \\
\hline $\mathrm{C}$ & 0.810484 & 0.588549 & 2.593086 \\
\hline $\mathrm{H}$ & 0.460304 & 0.086876 & 3.518019 \\
\hline $\mathrm{H}$ & 1.535089 & 1.371535 & 2.900470 \\
\hline $\mathrm{H}$ & -0.09 & 1.1 & 2. \\
\hline$C$ & 1.132985 & 0.300846 & -2.641965 \\
\hline $\mathrm{H}$ & 0.139502 & 0.214653 & -3.124548 \\
\hline 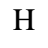 & 1.349034 & 1.381236 & -2.546347 \\
\hline $\mathrm{H}$ & 1.880579 & -0.120122 & -3.334705 \\
\hline $\mathrm{H}$ & 1.070639 & 5.229698 & -1.297883 \\
\hline & 5.132797 & 2.846504 & 0.527862 \\
\hline
\end{tabular}

1-Br'

$\begin{array}{rrrr}\mathrm{Ru} & 0.101037 & 0.059952 & -0.021744 \\ \mathrm{C} & 2.410967 & 2.108506 & 0.205244 \\ \mathrm{C} & 0.696380 & 3.031132 & -1.047582 \\ \mathrm{C} & 3.187936 & 3.238070 & -0.009152 \\ \mathrm{H} & 2.739108 & 1.239016 & 0.776986 \\ \mathrm{C} & 1.429649 & 4.181515 & -1.294035 \\ \mathrm{H} & -0.300189 & 2.893267 & -1.455736 \\ \mathrm{~N} & 1.171403 & 2.012892 & -0.309251 \\ \mathrm{C} & 2.711903 & 4.299002 & -0.765554\end{array}$




\begin{tabular}{|c|c|c|c|c|c|c|c|}
\hline $\mathrm{H}$ & 3.320173 & 5.179841 & -0.941266 & $\mathrm{H}$ & 2.417870 & -3.972414 & 3.023116 \\
\hline $\mathrm{C}$ & -0.668424 & -1.775371 & 0.321965 & $\mathrm{H}$ & 2.789237 & -2.244857 & 2.919221 \\
\hline $\mathrm{C}$ & -2.087772 & -3.510019 & 1.066893 & $\mathrm{C}$ & 5.441869 & -3.867235 & -1.229194 \\
\hline $\mathrm{C}$ & -0.738050 & -4.097795 & 0.716633 & $\mathrm{H}$ & 6.066917 & -4.277507 & -0.430925 \\
\hline $\mathrm{H}$ & -2.264190 & -3.475230 & 2.153300 & $\mathrm{H}$ & 5.484171 & -4.554547 & -2.079651 \\
\hline $\mathrm{H}$ & -2.932300 & -4.036940 & 0.610761 & $\mathrm{H}$ & 5.911208 & -2.931588 & -1.554912 \\
\hline $\mathrm{H}$ & -0.305700 & -4.716988 & 1.508889 & $\mathrm{C}$ & -1.500499 & 0.543633 & -0.908936 \\
\hline $\mathrm{H}$ & -0.768979 & -4.698291 & -0.204933 & $\mathrm{H}$ & -1.902182 & -0.153589 & -1.669409 \\
\hline $\mathrm{N}$ & -1.972167 & -2.145643 & 0.541250 & $\mathrm{C}$ & -2.398202 & 1.676815 & -0.759204 \\
\hline $\mathrm{N}$ & 0.076778 & -2.897573 & 0.499756 & $\mathrm{C}$ & -3.413402 & 1.901989 & -1.712400 \\
\hline $\mathrm{C}$ & -3.136945 & -1.323769 & 0.567608 & $\mathrm{C}$ & -2.320624 & 2.571961 & 0.329374 \\
\hline $\mathrm{C}$ & -4.031551 & -1.428373 & -0.515843 & $\mathrm{C}$ & -4.289518 & 2.972685 & -1.599559 \\
\hline $\mathrm{C}$ & -3.422265 & -0.497120 & 1.660599 & $\mathrm{H}$ & -3.500180 & 1.206308 & -2.546509 \\
\hline $\mathrm{C}$ & -5.184991 & -0.653022 & -0.503705 & $\mathrm{C}$ & -3.211096 & 3.627999 & 0.457348 \\
\hline $\mathrm{C}$ & -4.582597 & 0.281694 & 1.615517 & $\mathrm{H}$ & -1.549532 & 2.401501 & 1.081374 \\
\hline $\mathrm{C}$ & -5.470591 & 0.226856 & 0.546599 & $\mathrm{C}$ & -4.197979 & 3.836992 & -0.508394 \\
\hline $\mathrm{H}$ & -5.868700 & -0.714261 & -1.350162 & $\mathrm{H}$ & -5.058107 & 3.127354 & -2.353174 \\
\hline $\mathrm{H}$ & -4.793634 & 0.953423 & 2.447548 & $\mathrm{H}$ & -3.141172 & 4.294757 & 1.313284 \\
\hline $\mathrm{C}$ & 1.427357 & -3.099957 & 0.068718 & $\mathrm{H}$ & -4.894627 & 4.665044 & -0.406685 \\
\hline $\mathrm{C}$ & 1.671455 & -3.344857 & -1.293055 & $\mathrm{O}$ & 1.613760 & -0.347502 & 1.496256 \\
\hline $\mathrm{C}$ & 2.445270 & -3.184236 & 1.025265 & $\mathrm{O}$ & 1.020231 & -0.587340 & -1.686581 \\
\hline $\mathrm{C}$ & 2.982704 & -3.597765 & -1.687472 & $\mathrm{C}$ & 0.790397 & 0.371971 & 2.274091 \\
\hline $\mathrm{C}$ & 3.742775 & -3.444747 & 0.578689 & $\mathrm{H}$ & 0.417573 & -0.148533 & 3.181587 \\
\hline $\mathrm{C}$ & 4.034409 & -3.636356 & -0.769931 & $\mathrm{H}$ & 1.158713 & 1.380315 & 2.573029 \\
\hline $\mathrm{H}$ & 3.187585 & -3.771635 & -2.743532 & $\mathrm{H}$ & -0.249481 & 0.649526 & 1.769378 \\
\hline $\mathrm{H}$ & 4.547402 & -3.500097 & 1.311439 & $\mathrm{C}$ & 0.941416 & 0.186276 & -2.824711 \\
\hline $\mathrm{C}$ & -2.538426 & -0.429238 & 2.866567 & $\mathrm{H}$ & -0.107274 & 0.423567 & -3.109641 \\
\hline $\mathrm{H}$ & -2.151967 & 0.585662 & 3.017443 & $\mathrm{H}$ & 1.471186 & 1.156682 & -2.758988 \\
\hline $\mathrm{H}$ & -3.097047 & -0.689762 & 3.772288 & $\mathrm{H}$ & 1.395939 & -0.365132 & -3.665135 \\
\hline $\mathrm{H}$ & -1.677963 & -1.098380 & 2.794120 & $\mathrm{H}$ & 1.004893 & 4.974835 & -1.900525 \\
\hline $\mathrm{C}$ & -3.717288 & -2.323770 & -1.673962 & $\mathrm{Br}$ & 4.966589 & 3.313011 & 0.755047 \\
\hline $\mathrm{H}$ & -3.636581 & -3.376995 & -1.378651 & & & & \\
\hline $\mathrm{H}$ & -4.488784 & -2.255837 & -2.444770 & \multicolumn{2}{|c|}{ 7-py-Br } & & \\
\hline $\mathrm{H}$ & -2.754858 & -2.063562 & -2.130329 & $\mathrm{C}$ & 1.025196 & 1.426413 & -0.445528 \\
\hline $\mathrm{C}$ & -6.687893 & 1.096869 & 0.502744 & $\mathrm{C}$ & 2.740111 & 2.691568 & -1.451766 \\
\hline $\mathrm{H}$ & -6.857737 & 1.602363 & 1.456976 & $\mathrm{C}$ & 1.624003 & 3.639946 & -1.055238 \\
\hline $\mathrm{H}$ & -6.583046 & 1.873957 & -0.263581 & $\mathrm{H}$ & 3.730342 & 3.020722 & -1.122339 \\
\hline $\mathrm{H}$ & -7.588622 & 0.525126 & 0.257133 & $\mathrm{H}$ & 2.781334 & 2.534679 & -2.541140 \\
\hline $\mathrm{C}$ & 0.560185 & -3.393741 & -2.297039 & $\mathrm{H}$ & 1.887708 & 4.244234 & -0.170815 \\
\hline $\mathrm{H}$ & 0.941768 & -3.200491 & -3.302808 & $\mathrm{H}$ & 1.333324 & 4.325449 & -1.858301 \\
\hline $\mathrm{H}$ & 0.083146 & -4.383593 & -2.320614 & $\mathrm{~N}$ & 2.350793 & 1.438925 & -0.786344 \\
\hline $\mathrm{H}$ & -0.206340 & -2.642993 & -2.091154 & $\mathrm{~N}$ & 0.562630 & 2.691843 & -0.749124 \\
\hline $\mathrm{C}$ & 2.177763 & -3.042959 & 2.491767 & $\mathrm{C}$ & 3.229844 & 0.325248 & -0.914506 \\
\hline $\mathrm{H}$ & 1.136117 & -2.783407 & 2.690233 & $\mathrm{C}$ & 3.002800 & -0.659986 & -1.888104 \\
\hline
\end{tabular}




\begin{tabular}{|c|c|c|c|c|c|c|c|}
\hline $\mathrm{C}$ & 4.383780 & 0.299315 & -0.112025 & $\mathrm{H}$ & 2.022383 & -3.459001 & 0.592416 \\
\hline $\mathrm{C}$ & 3.920334 & -1.709070 & -1.988686 & $\mathrm{C}$ & 3.510762 & -0.821022 & 2.997553 \\
\hline $\mathrm{C}$ & 5.267601 & -0.767136 & -0.250407 & $\mathrm{H}$ & 1.802501 & 0.394041 & 2.448727 \\
\hline $\mathrm{C}$ & 5.047886 & -1.789362 & -1.175091 & $\mathrm{C}$ & 4.167794 & -2.036155 & 2.812437 \\
\hline $\mathrm{H}$ & 3.748720 & -2.481258 & -2.738055 & $\mathrm{H}$ & 4.137695 & -3.930860 & 1.784790 \\
\hline $\mathrm{H}$ & 6.146211 & -0.806211 & 0.392971 & $\mathrm{H}$ & 3.913017 & -0.085908 & 3.691120 \\
\hline $\mathrm{C}$ & -0.767816 & 3.063825 & -0.395961 & $\mathrm{H}$ & 5.082932 & -2.253745 & 3.357518 \\
\hline $\mathrm{C}$ & -1.774669 & 2.962353 & -1.374454 & $\mathrm{Ru}$ & -0.408216 & 0.222475 & 0.193513 \\
\hline $\mathrm{C}$ & -1.054208 & 3.526913 & 0.901749 & $\mathrm{H}$ & -0.522704 & 0.939130 & 1.713669 \\
\hline $\mathrm{C}$ & -3.085278 & 3.272114 & -1.008364 & $\mathrm{H}$ & -0.838644 & -0.039295 & -1.420156 \\
\hline $\mathrm{C}$ & -2.379085 & 3.826515 & 1.217587 & $\mathrm{C}$ & -2.712507 & -1.873100 & -0.004808 \\
\hline $\mathrm{C}$ & -3.408622 & 3.688700 & 0.284135 & $\mathrm{C}$ & -3.249703 & -0.144477 & 1.430926 \\
\hline $\mathrm{H}$ & -3.872201 & 3.190953 & -1.756879 & $\mathrm{C}$ & -3.988357 & -2.402496 & 0.128151 \\
\hline $\mathrm{H}$ & -2.612862 & 4.171674 & 2.224433 & $\mathrm{H}$ & -1.961643 & -2.333394 & -0.639427 \\
\hline $\mathrm{C}$ & 4.677239 & 1.395539 & 0.865279 & $\mathrm{C}$ & -4.540611 & -0.623775 & 1.598371 \\
\hline $\mathrm{H}$ & 5.220313 & 2.224402 & 0.392212 & $\mathrm{H}$ & -2.892433 & 0.749059 & 1.937298 \\
\hline $\mathrm{H}$ & 3.761325 & 1.810848 & 1.296675 & $\mathrm{C}$ & -4.933890 & -1.781082 & 0.931349 \\
\hline $\mathrm{H}$ & 5.303776 & 1.029976 & 1.683164 & $\mathrm{H}$ & -5.231240 & -0.102136 & 2.253553 \\
\hline $\mathrm{C}$ & 1.829142 & -0.601566 & -2.812242 & $\mathrm{H}$ & -5.934296 & -2.186391 & 1.035923 \\
\hline $\mathrm{H}$ & 1.583940 & 0.426782 & -3.099707 & $\mathrm{~N}$ & -2.347883 & -0.746711 & 0.633031 \\
\hline $\mathrm{H}$ & 2.022052 & -1.177677 & -3.721452 & $\mathrm{Br}$ & -4.437185 & -4.024152 & -0.833500 \\
\hline $\mathrm{H}$ & 0.923167 & -0.992230 & -2.335762 & & & & \\
\hline $\mathrm{C}$ & 5.994222 & -2.945613 & -1.272365 & \multicolumn{4}{|c|}{ 7-py-Br' } \\
\hline $\mathrm{H}$ & 5.917410 & -3.592931 & -0.390552 & $\mathrm{C}$ & 1.046735 & 1.360212 & -0.088931 \\
\hline $\mathrm{H}$ & 5.789868 & -3.564141 & -2.150183 & $\mathrm{C}$ & 2.578557 & 3.150507 & -0.124859 \\
\hline $\mathrm{H}$ & 7.036934 & -2.616507 & -1.327537 & $\mathrm{C}$ & 1.226333 & 3.692820 & 0.298762 \\
\hline $\mathrm{C}$ & -1.435768 & 2.569692 & -2.778874 & $\mathrm{H}$ & 3.398546 & 3.460036 & 0.530052 \\
\hline $\mathrm{H}$ & -2.324746 & 2.580629 & -3.413860 & $\mathrm{H}$ & 2.836940 & 3.445413 & -1.154316 \\
\hline $\mathrm{H}$ & -0.699092 & 3.252514 & -3.217101 & $\mathrm{H}$ & 1.191386 & 3.918905 & 1.377514 \\
\hline $\mathrm{H}$ & -1.002301 & 1.564709 & -2.808869 & $\mathrm{H}$ & 0.930795 & 4.595201 & -0.246761 \\
\hline $\mathrm{C}$ & 0.038721 & 3.717890 & 1.906439 & $\mathrm{~N}$ & 2.375322 & 1.694593 & -0.063141 \\
\hline $\mathrm{H}$ & 0.804174 & 2.942641 & 1.810878 & $\mathrm{~N}$ & 0.370730 & 2.562042 & -0.031525 \\
\hline $\mathrm{H}$ & 0.524712 & 4.694214 & 1.782018 & $\mathrm{C}$ & 3.483232 & 0.861328 & -0.391423 \\
\hline $\mathrm{H}$ & -0.352758 & 3.677287 & 2.925694 & $\mathrm{C}$ & 3.620490 & 0.328049 & -1.682882 \\
\hline $\mathrm{C}$ & -4.830673 & 3.959898 & 0.669489 & $\mathrm{C}$ & 4.482365 & 0.676122 & 0.579361 \\
\hline $\mathrm{H}$ & -5.427835 & 4.288253 & -0.185496 & $\mathrm{C}$ & 4.739348 & -0.464182 & -1.951293 \\
\hline $\mathrm{H}$ & -5.311826 & 3.056201 & 1.063992 & $\mathrm{C}$ & 5.578930 & -0.121204 & 0.262749 \\
\hline $\mathrm{H}$ & -4.902548 & 4.723909 & 1.448533 & $\mathrm{C}$ & 5.718921 & -0.714344 & -0.992787 \\
\hline $\mathrm{C}$ & 0.477511 & -1.265940 & 0.799776 & $\mathrm{H}$ & 4.850994 & -0.887243 & -2.949328 \\
\hline $\mathrm{H}$ & -0.130703 & -2.195189 & 0.774942 & $\mathrm{H}$ & 6.338362 & -0.294339 & 1.024636 \\
\hline $\mathrm{C}$ & 1.778376 & -1.486826 & 1.427297 & $\mathrm{C}$ & -1.050968 & 2.565318 & 0.081608 \\
\hline $\mathrm{C}$ & 2.447108 & -2.714058 & 1.263172 & $\mathrm{C}$ & -1.817530 & 2.695754 & -1.092276 \\
\hline $\mathrm{C}$ & 2.339200 & -0.542292 & 2.304785 & $\mathrm{C}$ & -1.662375 & 2.447058 & 1.344090 \\
\hline $\mathrm{C}$ & 3.634261 & -2.978209 & 1.933604 & $\mathrm{C}$ & -3.207838 & 2.639952 & -0.982158 \\
\hline
\end{tabular}




\begin{tabular}{|c|c|c|c|c|c|c|c|}
\hline $\mathrm{C}$ & -3.055010 & 2.398135 & 1.400976 & $\mathrm{C}$ & -1.886713 & -2.409802 & -1.655278 \\
\hline $\mathrm{C}$ & -3.843475 & 2.477805 & 0.250363 & $\mathrm{C}$ & -3.059284 & -1.312046 & 0.005561 \\
\hline $\mathrm{H}$ & -3.810092 & 2.737738 & -1.884493 & $\mathrm{C}$ & -3.003871 & -3.145126 & -2.024861 \\
\hline $\mathrm{H}$ & -3.537388 & 2.290943 & 2.372053 & $\mathrm{H}$ & -0.933879 & -2.518032 & -2.165104 \\
\hline $\mathrm{C}$ & 4.387029 & 1.330011 & 1.923302 & $\mathrm{C}$ & -4.211119 & -2.008686 & -0.327818 \\
\hline $\mathrm{H}$ & 4.794574 & 2.349598 & 1.906349 & $\mathrm{H}$ & -3.031981 & -0.581183 & 0.810558 \\
\hline $\mathrm{H}$ & 3.349268 & 1.400473 & 2.264589 & $\mathrm{C}$ & -4.205782 & -2.945369 & -1.353270 \\
\hline $\mathrm{H}$ & 4.957024 & 0.772001 & 2.670738 & $\mathrm{H}$ & -5.102114 & -3.497141 & -1.614095 \\
\hline $\mathrm{C}$ & 2.625075 & 0.604814 & -2.763595 & $\mathrm{~N}$ & -1.907022 & -1.503990 & -0.660155 \\
\hline $\mathrm{H}$ & 2.210608 & 1.616332 & -2.690147 & $\mathrm{H}$ & -2.936831 & -3.863494 & -2.835658 \\
\hline $\mathrm{H}$ & 3.083328 & 0.490444 & -3.749890 & $\mathrm{Br}$ & -5.837993 & -1.672005 & 0.672477 \\
\hline $\mathrm{H}$ & 1.761779 & -0.066311 & -2.697836 & & & & \\
\hline $\mathrm{C}$ & 6.878953 & -1.613876 & -1.287592 & $\mathbf{3}_{\mathrm{w}}$ & & & \\
\hline $\mathrm{H}$ & 7.020753 & -1.754898 & -2.362537 & $\mathrm{Ru}$ & -0.389457 & 0.645578 & 0.017155 \\
\hline $\mathrm{H}$ & 7.813669 & -1.226206 & -0.870877 & $\mathrm{C}$ & -0.386867 & -1.267304 & 0.287365 \\
\hline $\mathrm{H}$ & 6.727249 & -2.607173 & -0.847705 & $\mathrm{C}$ & -0.078316 & -3.562018 & 0.747440 \\
\hline $\mathrm{C}$ & -1.155633 & 2.919807 & -2.416668 & $\mathrm{C}$ & -1.562825 & -3.309446 & 0.537131 \\
\hline $\mathrm{H}$ & -1.890158 & 3.181949 & -3.182071 & $\mathrm{H}$ & 0.314674 & -4.385364 & 0.141001 \\
\hline $\mathrm{H}$ & -0.413073 & 3.723350 & -2.362833 & $\mathrm{H}$ & 0.159427 & -3.780800 & 1.800195 \\
\hline $\mathrm{H}$ & -0.623241 & 2.019202 & -2.742003 & $\mathrm{H}$ & -1.918511 & -3.716823 & -0.423011 \\
\hline $\mathrm{C}$ & -0.839569 & 2.401257 & 2.593635 & $\mathrm{H}$ & -2.189177 & -3.728428 & 1.331893 \\
\hline $\mathrm{H}$ & 0.088771 & 1.844473 & 2.436482 & $\mathrm{~N}$ & 0.519949 & -2.287381 & 0.344764 \\
\hline $\mathrm{H}$ & -0.578180 & 3.412134 & 2.932622 & $\mathrm{~N}$ & -1.623752 & -1.848206 & 0.532699 \\
\hline $\mathrm{H}$ & -1.386235 & 1.916623 & 3.405880 & $\mathrm{C}$ & 1.921413 & -2.207913 & 0.099702 \\
\hline $\mathrm{C}$ & -5.335206 & 2.374514 & 0.333986 & $\mathrm{C}$ & 2.814988 & -1.947257 & 1.146087 \\
\hline $\mathrm{H}$ & -5.825045 & 2.923982 & -0.474610 & $\mathrm{C}$ & 2.374532 & -2.405901 & -1.213824 \\
\hline $\mathrm{H}$ & -5.662850 & 1.330337 & 0.256492 & $\mathrm{C}$ & 4.177361 & -1.876584 & 0.848641 \\
\hline $\mathrm{H}$ & -5.715931 & 2.756508 & 1.285253 & $\mathrm{C}$ & 3.743487 & -2.328322 & -1.464340 \\
\hline $\mathrm{C}$ & 0.890538 & -1.654441 & -0.001245 & $\mathrm{C}$ & 4.661155 & -2.058243 & -0.446620 \\
\hline $\mathrm{H}$ & 0.500606 & -2.585378 & -0.465946 & $\mathrm{H}$ & 4.878875 & -1.665623 & 1.654928 \\
\hline $\mathrm{C}$ & 2.094193 & -1.918682 & 0.783430 & $\mathrm{H}$ & 4.101863 & -2.473105 & -2.482861 \\
\hline $\mathrm{C}$ & 3.001366 & -2.910457 & 0.366633 & $\mathrm{C}$ & -2.832958 & -1.185637 & 0.135076 \\
\hline $\mathrm{C}$ & 2.320865 & -1.296327 & 2.023234 & $\mathrm{C}$ & -3.677134 & -0.629321 & 1.111027 \\
\hline $\mathrm{C}$ & 4.098094 & -3.253424 & 1.146519 & $\mathrm{C}$ & -3.187156 & -1.141846 & -1.230907 \\
\hline $\mathrm{H}$ & 2.833718 & -3.407689 & -0.587409 & $\mathrm{C}$ & -4.837233 & 0.027643 & 0.692007 \\
\hline $\mathrm{C}$ & 3.402138 & -1.662051 & 2.815823 & $\mathrm{C}$ & -4.350577 & -0.467079 & -1.596480 \\
\hline $\mathrm{H}$ & 1.599004 & -0.551218 & 2.354172 & $\mathrm{C}$ & -5.181608 & 0.139311 & -0.652498 \\
\hline $\mathrm{C}$ & 4.300204 & -2.634914 & 2.379831 & $\mathrm{H}$ & -5.485492 & 0.470770 & 1.447406 \\
\hline $\mathrm{H}$ & 4.790732 & -4.016903 & 0.799803 & $\mathrm{H}$ & -4.614692 & -0.415889 & -2.652310 \\
\hline $\mathrm{H}$ & 3.544019 & -1.186605 & 3.783866 & $\mathrm{C}$ & 1.397443 & -2.611782 & -2.327700 \\
\hline $\mathrm{H}$ & 5.144613 & -2.919562 & 3.002343 & $\mathrm{H}$ & 0.793539 & -1.706883 & -2.474384 \\
\hline $\mathrm{Ru}$ & -0.175862 & -0.184348 & -0.266005 & $\mathrm{H}$ & 1.906968 & -2.837762 & -3.267471 \\
\hline $\mathrm{H}$ & -0.701774 & -0.120457 & 1.332155 & $\mathrm{H}$ & 0.692000 & -3.424811 & -2.119751 \\
\hline $\mathrm{H}$ & -0.257485 & 0.109966 & -1.928243 & $\mathrm{C}$ & 2.323437 & -1.667542 & 2.529012 \\
\hline
\end{tabular}




\begin{tabular}{|c|c|c|c|c|c|c|c|}
\hline $\mathrm{H}$ & 3.110852 & -1.819511 & 3.271190 & $\mathrm{O}$ & 0.259307 & 2.691551 & -2.786770 \\
\hline $\mathrm{H}$ & 1.985588 & -0.627367 & 2.609006 & $\mathrm{H}$ & -0.737221 & 3.017148 & -1.309158 \\
\hline $\mathrm{H}$ & 1.464306 & -2.287361 & 2.801623 & $\mathrm{H}$ & 1.138403 & 2.919360 & -2.461555 \\
\hline $\mathrm{C}$ & 6.124108 & -1.932000 & -0.743918 & & & & \\
\hline $\mathrm{H}$ & 6.738668 & -2.248033 & 0.103444 & \multicolumn{2}{|c|}{$\mathbf{T S}_{\mathrm{w3-4}}$} & & \\
\hline $\mathrm{H}$ & 6.414742 & -2.526084 & -1.614514 & $\mathrm{Ru}$ & -0.397798 & 0.631188 & -0.429293 \\
\hline $\mathrm{H}$ & 6.394426 & -0.891869 & -0.961417 & $\mathrm{C}$ & -0.268769 & -1.061611 & 0.554275 \\
\hline $\mathrm{C}$ & -3.364174 & -0.698945 & 2.570232 & $\mathrm{C}$ & 0.218498 & -3.052896 & 1.703378 \\
\hline $\mathrm{H}$ & -2.999855 & 0.273006 & 2.916810 & $\mathrm{C}$ & -1.292603 & -2.881951 & 1.643241 \\
\hline $\mathrm{H}$ & -4.257075 & -0.953554 & 3.149320 & $\mathrm{H}$ & 0.562208 & -4.033271 & 1.356656 \\
\hline $\mathrm{H}$ & -2.579428 & -1.426606 & 2.786862 & $\mathrm{H}$ & 0.614800 & -2.898376 & 2.718291 \\
\hline $\mathrm{C}$ & -2.379355 & -1.831427 & -2.287351 & $\mathrm{H}$ & -1.762358 & -3.590475 & 0.943008 \\
\hline $\mathrm{H}$ & -1.338077 & -1.966192 & -1.993441 & $\mathrm{H}$ & -1.780015 & -2.997869 & 2.616587 \\
\hline $\mathrm{H}$ & -2.798752 & -2.819537 & -2.517551 & $\mathrm{~N}$ & 0.691262 & -1.988903 & 0.811473 \\
\hline $\mathrm{H}$ & -2.382955 & -1.256761 & -3.217448 & $\mathrm{~N}$ & -1.429411 & -1.511416 & 1.143970 \\
\hline $\mathrm{C}$ & -6.388853 & 0.916749 & -1.078144 & $\mathrm{C}$ & 2.047267 & -1.958818 & 0.370946 \\
\hline $\mathrm{H}$ & -7.153985 & 0.939534 & -0.297525 & $\mathrm{C}$ & 3.035092 & -1.322278 & 1.134537 \\
\hline $\mathrm{H}$ & -6.126015 & 1.958688 & -1.298968 & $\mathrm{C}$ & 2.360807 & -2.600406 & -0.837999 \\
\hline $\mathrm{H}$ & -6.839944 & 0.505808 & -1.985775 & $\mathrm{C}$ & 4.345815 & -1.328747 & 0.654022 \\
\hline $\mathrm{C}$ & 1.420064 & 0.886029 & -0.272239 & $\mathrm{C}$ & 3.682813 & -2.576691 & -1.279929 \\
\hline $\mathrm{H}$ & 2.085136 & 0.086079 & -0.624698 & $\mathrm{C}$ & 4.689143 & -1.938930 & -0.552457 \\
\hline $\mathrm{C}$ & 2.141223 & 2.136873 & -0.048618 & $\mathrm{H}$ & 5.116625 & -0.823587 & 1.235583 \\
\hline $\mathrm{C}$ & 3.411820 & 2.318552 & -0.635007 & $\mathrm{H}$ & 3.932124 & -3.066600 & -2.220352 \\
\hline $\mathrm{C}$ & 1.628456 & 3.172153 & 0.761000 & $\mathrm{C}$ & -2.709456 & -1.043373 & 0.684557 \\
\hline $\mathrm{C}$ & 4.120876 & 3.499835 & -0.457096 & $\mathrm{C}$ & -3.493184 & -0.231616 & 1.522134 \\
\hline $\mathrm{H}$ & 3.831137 & 1.510025 & -1.234069 & $\mathrm{C}$ & -3.172720 & -1.429850 & -0.588821 \\
\hline $\mathrm{C}$ & 2.351038 & 4.341479 & 0.950600 & $\mathrm{C}$ & -4.726025 & 0.216025 & 1.046228 \\
\hline $\mathrm{H}$ & 0.664317 & 3.024168 & 1.241608 & $\mathrm{C}$ & -4.404006 & -0.938432 & -1.023859 \\
\hline $\mathrm{C}$ & 3.591934 & 4.517122 & 0.336326 & $\mathrm{C}$ & -5.191976 & -0.110035 & -0.226632 \\
\hline $\mathrm{H}$ & 5.093424 & 3.624923 & -0.926424 & $\mathrm{H}$ & -5.333150 & 0.851369 & 1.690137 \\
\hline $\mathrm{H}$ & 1.946806 & 5.125073 & 1.586451 & $\mathrm{H}$ & -4.749503 & -1.211429 & -2.020157 \\
\hline $\mathrm{H}$ & 4.149805 & 5.437951 & 0.484973 & $\mathrm{C}$ & 1.291620 & -3.250615 & -1.658016 \\
\hline $\mathrm{O}$ & -0.952064 & 1.188892 & 1.875792 & $\mathrm{H}$ & 0.608394 & -2.499499 & -2.072899 \\
\hline $\mathrm{H}$ & -0.669484 & 0.326173 & -1.601180 & $\mathrm{H}$ & 1.718845 & -3.813373 & -2.491337 \\
\hline $\mathrm{C}$ & -0.426289 & 0.592802 & 3.010858 & $\mathrm{H}$ & 0.673455 & -3.937294 & -1.068252 \\
\hline $\mathrm{H}$ & -0.418457 & -0.514684 & 2.963312 & $\mathrm{C}$ & 2.703132 & -0.588850 & 2.393147 \\
\hline $\mathrm{H}$ & 0.615530 & 0.911235 & 3.206322 & $\mathrm{H}$ & 3.546357 & -0.591086 & 3.088709 \\
\hline $\mathrm{H}$ & -1.014268 & 0.869085 & 3.903969 & $\mathrm{H}$ & 2.464158 & 0.458367 & 2.169640 \\
\hline $\mathrm{O}$ & -1.065488 & 2.840274 & -0.399781 & $\mathrm{H}$ & 1.828592 & -1.001903 & 2.902333 \\
\hline $\mathrm{H}$ & 0.175277 & 1.751234 & -2.545210 & $\mathrm{C}$ & 6.096026 & -1.883075 & -1.063855 \\
\hline $\mathrm{C}$ & -2.487810 & 2.782642 & -0.410897 & $\mathrm{H}$ & 6.825165 & -2.051093 & -0.265829 \\
\hline $\mathrm{H}$ & -2.860823 & 2.037261 & -1.130012 & $\mathrm{H}$ & 6.273192 & -2.625062 & -1.846385 \\
\hline $\mathrm{H}$ & -2.795908 & 2.485989 & 0.594733 & $\mathrm{H}$ & 6.321288 & -0.899640 & -1.493591 \\
\hline $\mathrm{H}$ & -2.920023 & 3.761758 & -0.648495 & $\mathrm{C}$ & -3.025931 & 0.199505 & 2.873647 \\
\hline
\end{tabular}




\begin{tabular}{|c|c|c|c|c|c|c|c|}
\hline $\mathrm{H}$ & -2.605241 & 1.207380 & 2.803340 & $\mathrm{C}$ & -1.218641 & -2.749778 & 1.866776 \\
\hline $\mathrm{H}$ & -3.853719 & 0.223557 & 3.588361 & $\mathrm{H}$ & 0.608687 & -3.938223 & 1.546615 \\
\hline $\mathrm{H}$ & -2.235322 & -0.443711 & 3.267039 & $\mathrm{H}$ & 0.743300 & -2.739804 & 2.845030 \\
\hline $\mathrm{C}$ & -2.419114 & -2.364049 & -1.486981 & $\mathrm{H}$ & -1.737154 & -3.508730 & 1.261415 \\
\hline $\mathrm{H}$ & -1.413044 & -2.584199 & -1.127830 & $\mathrm{H}$ & -1.653956 & -2.765909 & 2.870952 \\
\hline $\mathrm{H}$ & -2.955607 & -3.314928 & -1.590811 & $\mathrm{~N}$ & 0.734699 & -1.924913 & 0.893551 \\
\hline $\mathrm{H}$ & -2.323171 & -1.932301 & -2.487872 & $\mathrm{~N}$ & -1.373533 & -1.428626 & 1.249049 \\
\hline $\mathrm{C}$ & -6.488852 & 0.440285 & -0.735438 & $\mathrm{C}$ & 2.072387 & -1.925232 & 0.399401 \\
\hline $\mathrm{H}$ & -7.215597 & 0.578850 & 0.070006 & $\mathrm{C}$ & 3.092662 & -1.276942 & 1.108230 \\
\hline $\mathrm{H}$ & -6.342245 & 1.420459 & -1.204270 & $\mathrm{C}$ & 2.336663 & -2.613349 & -0.795288 \\
\hline $\mathrm{H}$ & -6.937087 & -0.210234 & -1.491167 & $\mathrm{C}$ & 4.387203 & -1.323651 & 0.588822 \\
\hline $\mathrm{C}$ & 1.413368 & 0.886824 & -0.715675 & $\mathrm{C}$ & 3.643235 & -2.620014 & -1.282767 \\
\hline $\mathrm{H}$ & 2.030821 & 0.127360 & -1.223589 & $\mathrm{C}$ & 4.682276 & -1.976361 & -0.608742 \\
\hline $\mathrm{C}$ & 2.179511 & 2.078745 & -0.380571 & $\mathrm{H}$ & 5.183615 & -0.813715 & 1.130191 \\
\hline $\mathrm{C}$ & 3.585076 & 2.025266 & -0.484023 & $\mathrm{H}$ & 3.854684 & -3.144517 & -2.213602 \\
\hline $\mathrm{C}$ & 1.590215 & 3.284256 & 0.056068 & $\mathrm{C}$ & -2.673647 & -0.982718 & 0.823539 \\
\hline $\mathrm{C}$ & 4.373046 & 3.113622 & -0.134478 & $\mathrm{C}$ & -3.414878 & -0.115729 & 1.642792 \\
\hline $\mathrm{H}$ & 4.044277 & 1.100944 & -0.832297 & $\mathrm{C}$ & -3.185440 & -1.437822 & -0.406371 \\
\hline $\mathrm{C}$ & 2.382632 & 4.371531 & 0.394658 & $\mathrm{C}$ & -4.653413 & 0.328536 & 1.181081 \\
\hline $\mathrm{H}$ & 0.507023 & 3.332639 & 0.140182 & $\mathrm{C}$ & -4.418398 & -0.943036 & -0.832755 \\
\hline $\mathrm{C}$ & 3.773428 & 4.292036 & 0.306571 & $\mathrm{C}$ & -5.160819 & -0.052072 & -0.061042 \\
\hline $\mathrm{H}$ & 5.455395 & 3.047520 & -0.211231 & $\mathrm{H}$ & -5.228991 & 1.009723 & 1.807051 \\
\hline $\mathrm{H}$ & 1.914660 & 5.293683 & 0.729665 & $\mathrm{H}$ & -4.792013 & -1.253629 & -1.806563 \\
\hline $\mathrm{H}$ & 4.386332 & 5.148884 & 0.573371 & $\mathrm{C}$ & 1.236993 & -3.298126 & -1.545095 \\
\hline $\mathrm{O}$ & -0.815750 & 1.740716 & 1.202731 & $\mathrm{H}$ & 0.516922 & -2.576900 & -1.948814 \\
\hline $\mathrm{H}$ & -0.273804 & -0.389424 & -1.886228 & $\mathrm{H}$ & 1.631003 & -3.878053 & -2.382699 \\
\hline $\mathrm{C}$ & -0.082576 & 1.591971 & 2.368230 & $\mathrm{H}$ & 0.661000 & -3.978157 & -0.906834 \\
\hline $\mathrm{H}$ & -0.039595 & 0.543064 & 2.724149 & $\mathrm{C}$ & 2.809236 & -0.486494 & 2.344074 \\
\hline $\mathrm{H}$ & 0.962308 & 1.940334 & 2.265235 & $\mathrm{H}$ & 3.677862 & -0.459274 & 3.006787 \\
\hline $\mathrm{H}$ & -0.535926 & 2.187833 & 3.179112 & $\mathrm{H}$ & 2.564544 & 0.550047 & 2.078905 \\
\hline $\mathrm{O}$ & -1.584550 & 2.022390 & -1.531186 & $\mathrm{H}$ & 1.953474 & -0.871730 & 2.904578 \\
\hline $\mathrm{H}$ & -0.892264 & -0.019341 & -2.475138 & $\mathrm{C}$ & 6.073181 & -1.962069 & -1.164000 \\
\hline $\mathrm{C}$ & -2.568250 & 2.852538 & -0.963694 & $\mathrm{H}$ & 6.819638 & -2.182338 & -0.394933 \\
\hline $\mathrm{H}$ & -3.577937 & 2.508438 & -1.233561 & $\mathrm{H}$ & 6.195251 & -2.688681 & -1.970976 \\
\hline $\mathrm{H}$ & -2.477838 & 2.849449 & 0.128612 & $\mathrm{H}$ & 6.325045 & -0.976540 & -1.573013 \\
\hline $\mathrm{H}$ & -2.454019 & 3.886802 & -1.319393 & $\mathrm{C}$ & -2.905821 & 0.366831 & 2.962613 \\
\hline $\mathrm{O}$ & -1.816666 & 0.489260 & -3.328573 & $\mathrm{H}$ & -2.554275 & 1.398650 & 2.868969 \\
\hline $\mathrm{H}$ & -1.847499 & 1.382893 & -2.523437 & $\mathrm{H}$ & -3.697284 & 0.351483 & 3.718129 \\
\hline \multirow[t]{2}{*}{$\mathrm{H}$} & -1.319372 & 0.813629 & -4.086657 & $\mathrm{H}$ & -2.062065 & -0.223714 & 3.326869 \\
\hline & & & & $\mathrm{C}$ & -2.480150 & -2.436879 & -1.273775 \\
\hline $4 w$ & & & & $\mathrm{H}$ & -1.486476 & -2.699213 & -0.904158 \\
\hline $\mathrm{Ru}$ & -0.384831 & 0.613317 & -0.547222 & $\mathrm{H}$ & -3.062101 & -3.363558 & -1.342777 \\
\hline $\mathrm{C}$ & -0.237970 & -1.028591 & 0.599327 & $\mathrm{H}$ & -2.379401 & -2.038111 & -2.289925 \\
\hline $\mathrm{C}$ & 0.292853 & -2.938020 & 1.861602 & $\mathrm{C}$ & -6.454554 & 0.509311 & -0.564952 \\
\hline
\end{tabular}




\begin{tabular}{|c|c|c|c|c|c|c|c|}
\hline $\mathrm{H}$ & -7.168482 & 0.678585 & 0.246380 & $\mathrm{C}$ & 2.296111 & 3.777304 & -0.125710 \\
\hline $\mathrm{H}$ & -6.294316 & 1.476473 & -1.055718 & $\mathrm{C}$ & 0.828630 & 4.201872 & -0.097791 \\
\hline $\mathrm{H}$ & -6.922985 & -0.148557 & -1.301475 & $\mathrm{H}$ & 2.827177 & 4.046834 & 0.798046 \\
\hline $\mathrm{C}$ & 1.455895 & 0.895905 & -0.771140 & $\mathrm{H}$ & 2.854274 & 4.203018 & -0.966473 \\
\hline $\mathrm{H}$ & 2.090769 & 0.160307 & -1.290161 & $\mathrm{H}$ & 0.606693 & 4.924894 & 0.693800 \\
\hline $\mathrm{C}$ & 2.194647 & 2.099109 & -0.427010 & $\mathrm{H}$ & 0.498924 & 4.634673 & -1.053481 \\
\hline $\mathrm{C}$ & 3.603727 & 2.033745 & -0.378532 & $\mathrm{~N}$ & 2.208941 & 2.316141 & -0.244524 \\
\hline $\mathrm{C}$ & 1.578471 & 3.336187 & -0.143855 & $\mathrm{~N}$ & 0.148209 & 2.929964 & 0.144856 \\
\hline $\mathrm{C}$ & 4.361115 & 3.135760 & -0.006547 & $\mathrm{C}$ & 3.377391 & 1.510395 & -0.377972 \\
\hline $\mathrm{H}$ & 4.089861 & 1.089702 & -0.621839 & $\mathrm{C}$ & 3.825000 & 1.210410 & -1.672695 \\
\hline $\mathrm{C}$ & 2.342616 & 4.438890 & 0.209716 & $\mathrm{C}$ & 4.010324 & 0.998215 & 0.762753 \\
\hline $\mathrm{H}$ & 0.495261 & 3.401017 & -0.196682 & $\mathrm{C}$ & 4.923675 & 0.365230 & -1.811750 \\
\hline $\mathrm{C}$ & 3.732654 & 4.344108 & 0.290331 & $\mathrm{C}$ & 5.105084 & 0.152278 & 0.572542 \\
\hline $\mathrm{H}$ & 5.444388 & 3.057954 & 0.040353 & $\mathrm{C}$ & 5.570952 & -0.180868 & -0.699380 \\
\hline $\mathrm{H}$ & 1.852027 & 5.385450 & 0.421157 & $\mathrm{H}$ & 5.263451 & 0.101507 & -2.812868 \\
\hline $\mathrm{H}$ & 4.323723 & 5.212329 & 0.568654 & $\mathrm{H}$ & 5.588268 & -0.282198 & 1.447206 \\
\hline $\mathrm{O}$ & -0.756454 & 1.787923 & 1.031093 & $\mathrm{C}$ & -1.271692 & 2.777224 & 0.255230 \\
\hline $\mathrm{H}$ & -0.674589 & -0.567977 & -1.839118 & $\mathrm{C}$ & -2.065221 & 2.719548 & -0.903659 \\
\hline $\mathrm{C}$ & -0.000148 & 1.682123 & 2.189028 & $\mathrm{C}$ & -1.833373 & 2.672758 & 1.542116 \\
\hline $\mathrm{H}$ & 0.053702 & 0.648671 & 2.583802 & $\mathrm{C}$ & -3.424028 & 2.435391 & -0.748580 \\
\hline $\mathrm{H}$ & 1.039748 & 2.032638 & 2.059620 & $\mathrm{C}$ & -3.194777 & 2.385325 & 1.639642 \\
\hline $\mathrm{H}$ & -0.446392 & 2.306741 & 2.980346 & $\mathrm{C}$ & -4.000798 & 2.241334 & 0.507734 \\
\hline $\mathrm{O}$ & -1.639639 & 1.749346 & -1.664573 & $\mathrm{H}$ & -4.046366 & 2.354654 & -1.639258 \\
\hline $\mathrm{H}$ & -0.016143 & -0.132683 & -2.115115 & $\mathrm{H}$ & -3.629572 & 2.266058 & 2.630477 \\
\hline $\mathrm{C}$ & -2.431918 & 2.771337 & -1.128345 & $\mathrm{C}$ & 3.492618 & 1.304605 & 2.131823 \\
\hline $\mathrm{H}$ & -3.500936 & 2.523729 & -1.236426 & $\mathrm{H}$ & 2.466997 & 0.935664 & 2.268886 \\
\hline $\mathrm{H}$ & -2.230756 & 2.943616 & -0.062481 & $\mathrm{H}$ & 4.125184 & 0.848173 & 2.897483 \\
\hline $\mathrm{H}$ & -2.254404 & 3.715826 & -1.667799 & $\mathrm{H}$ & 3.467797 & 2.384000 & 2.324478 \\
\hline $\mathrm{O}$ & -2.596127 & -0.048029 & -3.489326 & $\mathrm{C}$ & 3.058769 & 1.703086 & -2.860696 \\
\hline $\mathrm{H}$ & -2.365427 & 0.653470 & -2.838651 & $\mathrm{H}$ & 2.985174 & 2.796613 & -2.886351 \\
\hline \multirow[t]{2}{*}{$\mathrm{H}$} & -2.214331 & 0.278764 & -4.306707 & $\mathrm{H}$ & 3.520014 & 1.376986 & -3.795754 \\
\hline & & & & $\mathrm{H}$ & 2.026301 & 1.328010 & -2.839902 \\
\hline $0 \mathrm{I}-\mathrm{Br}$ & & & & $\mathrm{C}$ & 6.732403 & -1.110876 & -0.871089 \\
\hline $\mathrm{Ru}$ & 0.111890 & 0.054497 & 0.144792 & $\mathrm{H}$ & 6.874433 & -1.740380 & 0.011657 \\
\hline $\mathrm{C}$ & -2.566406 & -1.290639 & -0.240175 & $\mathrm{H}$ & 6.594133 & -1.769228 & -1.734631 \\
\hline $\mathrm{C}$ & -1.174317 & -2.840802 & 0.777515 & $\mathrm{H}$ & 7.667819 & -0.563304 & -1.035345 \\
\hline $\mathrm{C}$ & -3.597830 & -2.215440 & -0.269308 & $\mathrm{C}$ & -1.502321 & 2.998848 & -2.261596 \\
\hline $\mathrm{H}$ & -2.657682 & -0.297940 & -0.676975 & $\mathrm{H}$ & -2.050157 & 2.450363 & -3.030732 \\
\hline $\mathrm{C}$ & -2.164865 & -3.811280 & 0.752478 & $\mathrm{H}$ & -1.582050 & 4.068620 & -2.497532 \\
\hline $\mathrm{H}$ & -0.183985 & -3.044413 & 1.177458 & $\mathrm{H}$ & -0.455352 & 2.700708 & -2.340006 \\
\hline $\mathrm{N}$ & -1.367907 & -1.600119 & 0.296403 & $\mathrm{C}$ & -1.004214 & 2.907053 & 2.763362 \\
\hline $\mathrm{C}$ & -3.415196 & -3.498655 & 0.225792 & $\mathrm{H}$ & -0.129605 & 2.250457 & 2.771500 \\
\hline $\mathrm{H}$ & -4.215939 & -4.229509 & 0.194187 & $\mathrm{H}$ & -0.654983 & 3.946745 & 2.799607 \\
\hline $\mathrm{C}$ & 0.958287 & 1.852538 & -0.016961 & $\mathrm{H}$ & -1.582676 & 2.708765 & 3.667463 \\
\hline
\end{tabular}




$\begin{array}{rrrr}\mathrm{C} & -5.454613 & 1.907572 & 0.649269 \\ \mathrm{H} & -5.596669 & 0.965325 & 1.189506 \\ \mathrm{H} & -5.989004 & 2.677613 & 1.215979 \\ \mathrm{H} & -5.943140 & 1.806556 & -0.322989 \\ \mathrm{C} & 1.587135 & -0.875383 & -0.455761 \\ \mathrm{H} & 1.998628 & -0.560882 & -1.430507 \\ \mathrm{C} & 2.307008 & -2.006787 & 0.094822 \\ \mathrm{C} & 3.144336 & -2.763894 & -0.751292 \\ \mathrm{C} & 2.272235 & -2.329649 & 1.466640 \\ \mathrm{C} & 3.893305 & -3.820062 & -0.252869 \\ \mathrm{H} & 3.198422 & -2.497766 & -1.806150 \\ \mathrm{C} & 3.043955 & -3.371401 & 1.963896 \\ \mathrm{H} & 1.672085 & -1.702079 & 2.120438 \\ \mathrm{C} & 3.847811 & -4.125509 & 1.107953 \\ \mathrm{H} & 4.526350 & -4.399416 & -0.920126 \\ \mathrm{H} & 3.023924 & -3.598167 & 3.026798 \\ \mathrm{H} & 4.443593 & -4.945027 & 1.501199 \\ \mathrm{O} & 0.421697 & 0.206915 & 2.203787 \\ \mathrm{O} & -0.684200 & 0.269763 & -1.765334 \\ \mathrm{C} & -0.338499 & -0.508265 & 3.085823 \\ \mathrm{H} & -0.011676 & -0.295885 & 4.117401 \\ \mathrm{H} & -0.236251 & -1.608158 & 2.927127 \\ \mathrm{C} & -0.364772 & -0.569850 & -2.783821 \\ \mathrm{H} & -0.960059 & -0.315945 & -3.676358 \\ \mathrm{H} & 0.713187 & -0.482390 & -3.063929 \\ \mathrm{H} & -1.960426 & -4.803493 & 1.140714 \\ \mathrm{Br} & -5.290082 & -1.703948 & -1.054825 \\ \mathrm{O} & -0.643118 & -1.954238 & -2.560895 \\ \mathrm{H} & -0.092308 & -2.206382 & -1.807710 \\ \mathrm{O} & -1.722672 & -0.174012 & 3.097205 \\ \mathrm{H} & -2.046192 & -0.312442 & 2.198711 \\ & & & \end{array}$

$\begin{array}{cccc}\mathrm{CO}_{2} & & & \\ \mathrm{C} & 0.000000 & 0.000000 & 0.000000 \\ \mathrm{O} & 0.000000 & 0.000000 & 1.168443 \\ \mathrm{O} & 0.000000 & 0.000000 & -1.168443\end{array}$

\section{$\mathrm{HCOOH}$}

$\begin{array}{cccc}\mathrm{C} & 0.000000 & 0.419125 & 0.000000 \\ \mathrm{H} & -0.391679 & 1.448657 & 0.000000 \\ \mathrm{O} & -1.033681 & -0.439886 & 0.000000 \\ \mathrm{H} & -0.647658 & -1.330420 & 0.000000 \\ \mathrm{O} & 1.163599 & 0.110762 & 0.000000\end{array}$

\section{$\mathrm{HOCH}_{2} \mathrm{OH}$}

C $\quad 0.007566$

$\begin{array}{ll}0.518417 & 0.026617\end{array}$

$\begin{array}{llll}\mathrm{H} & -0.056435 & 1.083450 & 0.978293\end{array}$

$\begin{array}{llll}\mathrm{H} & 0.041948 & 1.243569 & -0.799760\end{array}$

$\begin{array}{llll}\text { O } & 1.178472 & -0.213851 & -0.072534\end{array}$

$\begin{array}{llll}\mathrm{H} & 1.117103 & -0.915707 & 0.585123\end{array}$

$\begin{array}{llll}\text { O } & -1.091045 & -0.358385 & -0.085287\end{array}$

$\begin{array}{llll}\mathrm{H} & -1.847433 & 0.056072 & 0.339210\end{array}$

TSI $\mathbf{0}_{0-1 \mathrm{Br}}$

$\begin{array}{llll}\mathrm{Ru} & -0.111174 & 0.118234 & 0.020564\end{array}$

$\begin{array}{llll}\text { C } & 2.400570 & 1.748393 & -0.080635\end{array}$

$\begin{array}{llll}\text { C } & 0.697284 & 3.307961 & 0.037161\end{array}$

$\begin{array}{llll}\text { C } & 3.346624 & 2.759667 & -0.008874\end{array}$

$\begin{array}{llll}\mathrm{H} & 2.669344 & 0.700101 & -0.160418\end{array}$

$\begin{array}{llll}\text { C } & 1.597616 & 4.360305 & 0.110834\end{array}$

$\begin{array}{llll}\mathrm{H} & -0.375813 & 3.482196 & 0.041584\end{array}$

$\begin{array}{llll}\mathrm{N} & 1.086658 & 2.027731 & -0.048500\end{array}$

$\begin{array}{llll}\text { C } & 2.961947 & 4.086095 & 0.094862\end{array}$

$\begin{array}{llll}\mathrm{H} & 3.699443 & 4.878865 & 0.158529\end{array}$

$\begin{array}{llll}\text { C } & -0.780770 & -1.767084 & 0.229669\end{array}$

$\begin{array}{llll}\text { C } & -2.135181 & -3.628743 & 0.744142\end{array}$

$\begin{array}{llll}\text { C } & -0.719344 & -4.105933 & 0.494431\end{array}$

$\begin{array}{llll}\mathrm{H} & -2.411839 & -3.667791 & 1.808967\end{array}$

$\begin{array}{llll}\mathrm{H} & -2.894307 & -4.183783 & 0.183711\end{array}$

$\begin{array}{llll}\mathrm{H} & -0.319785 & -4.739855 & 1.292295\end{array}$

$\begin{array}{llll}\mathrm{H} & -0.624419 & -4.654157 & -0.454493\end{array}$

$\begin{array}{llll}\mathrm{N} & -2.069317 & -2.230855 & 0.303696\end{array}$

$\begin{array}{llll}\mathrm{N} & 0.025268 & -2.845579 & 0.415770\end{array}$

$\begin{array}{llll}\text { C } & -3.261770 & -1.455288 & 0.389987\end{array}$

$\begin{array}{llll}\text { C } & -4.189362 & -1.551384 & -0.662917\end{array}$

$\begin{array}{llll}\text { C } & -3.518427 & -0.658518 & 1.513017\end{array}$

$\begin{array}{llll}\text { C } & -5.364599 & -0.811777 & -0.579947\end{array}$

$\begin{array}{llll}\text { C } & -4.709368 & 0.070861 & 1.547472\end{array}$

$\begin{array}{llll}\text { C } & -5.641038 & 0.014171 & 0.514213\end{array}$

$\mathrm{H} \quad-6.072652 \quad-0.856796 \quad-1.407166$

$\begin{array}{llll}\mathrm{H} & -4.902440 & 0.717849 & 2.402786\end{array}$

$\begin{array}{llll}\text { C } & 1.424842 & -2.912359 & 0.114714\end{array}$

$\begin{array}{llll}\text { C } & 1.818449 & -3.060868 & -1.225412\end{array}$

$\begin{array}{llll}\text { C } & 2.358186 & -2.908658 & 1.157620\end{array}$

$\begin{array}{llll}\text { C } & 3.179761 & -3.107199 & -1.510329\end{array}$

$\begin{array}{llll}\text { C } & 3.713354 & -2.957754 & 0.819895\end{array}$

$\begin{array}{llll}\text { C } & 4.143894 & -3.032364 & -0.502651\end{array}$

$\begin{array}{llll}\mathrm{H} & 3.496334 & -3.192848 & -2.549509\end{array}$ 


\begin{tabular}{|c|c|c|c|c|c|c|c|}
\hline $\mathrm{H}$ & 4.452110 & -2.930321 & 1.620188 & $\mathrm{H}$ & 1.522975 & -0.317433 & -3.467123 \\
\hline $\mathrm{C}$ & -2.516814 & -0.517677 & 2.613910 & $\mathrm{H}$ & -0.229941 & -0.446913 & -3.111200 \\
\hline $\mathrm{H}$ & -1.822794 & 0.308442 & 2.400638 & $\mathrm{H}$ & 1.236674 & 5.381409 & 0.177991 \\
\hline $\mathrm{H}$ & -3.001087 & -0.285754 & 3.566609 & $\mathrm{Br}$ & 5.224049 & 2.294139 & -0.034544 \\
\hline $\mathrm{H}$ & -1.895411 & -1.408993 & 2.744686 & $\mathrm{O}$ & 0.649261 & 1.424545 & -2.992893 \\
\hline $\mathrm{C}$ & -3.884833 & -2.380309 & -1.871572 & $\mathrm{H}$ & -0.032429 & 1.730932 & -2.378011 \\
\hline $\mathrm{H}$ & -3.881145 & -3.454772 & -1.651713 & $\mathrm{O}$ & 1.965481 & 1.450447 & 2.877292 \\
\hline $\mathrm{H}$ & -4.622860 & -2.213117 & -2.659841 & $\mathrm{H}$ & 1.809756 & 1.839058 & 3.744093 \\
\hline $\mathrm{H}$ & -2.892624 & -2.145156 & -2.274118 & & & & \\
\hline $\mathrm{C}$ & -6.900249 & 0.823232 & 0.560396 & 1I-Br & & & \\
\hline $\mathrm{H}$ & -6.880297 & 1.551741 & 1.375371 & $\mathrm{Ru}$ & 0.035248 & -0.078435 & 0.156121 \\
\hline $\mathrm{H}$ & -7.049491 & 1.375583 & -0.373348 & $\mathrm{C}$ & -2.390841 & -1.871311 & 0.139827 \\
\hline $\mathrm{H}$ & -7.784395 & 0.191110 & 0.701564 & $\mathrm{C}$ & -0.533228 & -3.245070 & 0.109410 \\
\hline $\mathrm{C}$ & 0.814837 & -3.204789 & -2.328412 & $\mathrm{C}$ & -3.223414 & -2.977143 & 0.057116 \\
\hline $\mathrm{H}$ & 1.240650 & -2.891298 & -3.285135 & $\mathrm{H}$ & -2.766513 & -0.855100 & 0.182408 \\
\hline $\mathrm{H}$ & 0.498489 & -4.249612 & -2.449115 & $\mathrm{C}$ & -1.315992 & -4.387209 & 0.025294 \\
\hline $\mathrm{H}$ & -0.079019 & -2.599692 & -2.154220 & $\mathrm{H}$ & 0.550573 & -3.303611 & 0.114705 \\
\hline $\mathrm{C}$ & 1.952033 & -2.892078 & 2.598623 & $\mathrm{~N}$ & -1.057660 & -2.016534 & 0.173773 \\
\hline $\mathrm{H}$ & 0.882770 & -2.706198 & 2.716461 & $\mathrm{C}$ & -2.700265 & -4.259782 & -0.000112 \\
\hline $\mathrm{H}$ & 2.201262 & -3.846278 & 3.080786 & $\mathrm{H}$ & -3.349640 & -5.126400 & -0.065058 \\
\hline $\mathrm{H}$ & 2.475756 & -2.098227 & 3.136214 & $\mathrm{C}$ & 0.696610 & 1.825831 & 0.207581 \\
\hline $\mathrm{C}$ & 5.603865 & -3.022821 & -0.839505 & $\mathrm{C}$ & 2.063084 & 3.719183 & 0.564196 \\
\hline $\mathrm{H}$ & 5.948458 & -2.009166 & -1.075244 & $\mathrm{C}$ & 0.632617 & 4.176935 & 0.369735 \\
\hline $\mathrm{H}$ & 6.214090 & -3.382392 & -0.006279 & $\mathrm{H}$ & 2.395654 & 3.806430 & 1.610713 \\
\hline $\mathrm{H}$ & 5.823528 & -3.643036 & -1.713687 & $\mathrm{H}$ & 2.788091 & 4.253684 & -0.059043 \\
\hline $\mathrm{C}$ & -1.656937 & 0.549796 & -0.982423 & $\mathrm{H}$ & 0.272740 & 4.851272 & 1.153289 \\
\hline $\mathrm{H}$ & -2.011764 & -0.124835 & -1.787649 & $\mathrm{H}$ & 0.484339 & 4.674445 & -0.600247 \\
\hline $\mathrm{C}$ & -2.614025 & 1.632348 & -0.794023 & $\mathrm{~N}$ & 1.981761 & 2.306976 & 0.184773 \\
\hline $\mathrm{C}$ & -3.630956 & 1.850420 & -1.745292 & $\mathrm{~N}$ & -0.108994 & 2.910907 & 0.395865 \\
\hline $\mathrm{C}$ & -2.611384 & 2.456598 & 0.351270 & $\mathrm{C}$ & 3.183781 & 1.543611 & 0.188840 \\
\hline $\mathrm{C}$ & -4.573273 & 2.857105 & -1.580494 & $\mathrm{C}$ & 3.924510 & 1.485816 & -1.007111 \\
\hline $\mathrm{H}$ & -3.666787 & 1.202120 & -2.619782 & $\mathrm{C}$ & 3.640448 & 0.920005 & 1.355547 \\
\hline $\mathrm{C}$ & -3.564495 & 3.449098 & 0.529461 & $\mathrm{C}$ & 5.105123 & 0.752455 & -1.021856 \\
\hline $\mathrm{H}$ & -1.854546 & 2.277590 & 1.117946 & $\mathrm{C}$ & 4.831887 & 0.191957 & 1.290373 \\
\hline $\mathrm{C}$ & -4.547544 & 3.658235 & -0.439681 & $\mathrm{C}$ & 5.570259 & 0.083545 & 0.116127 \\
\hline $\mathrm{H}$ & -5.341700 & 3.009172 & -2.334375 & $\mathrm{H}$ & 5.669953 & 0.681570 & -1.951188 \\
\hline $\mathrm{H}$ & -3.549701 & 4.059779 & 1.428662 & $\mathrm{H}$ & 5.179597 & -0.325112 & 2.184696 \\
\hline $\mathrm{H}$ & -5.294363 & 4.435129 & -0.300162 & $\mathrm{C}$ & -1.506395 & 2.973396 & 0.080833 \\
\hline $\mathrm{O}$ & 1.192307 & -0.299689 & 1.654645 & $\mathrm{C}$ & -1.891075 & 3.021503 & -1.269315 \\
\hline $\mathrm{O}$ & 1.061907 & -0.379597 & -1.526974 & $\mathrm{C}$ & -2.442446 & 3.093639 & 1.114214 \\
\hline $\mathrm{C}$ & 0.856699 & 0.614543 & 2.571417 & $\mathrm{C}$ & -3.249276 & 3.106085 & -1.562619 \\
\hline $\mathrm{H}$ & 0.469655 & 0.159529 & 3.516334 & $\mathrm{C}$ & -3.792587 & 3.184535 & 0.768306 \\
\hline $\mathrm{H}$ & 0.001588 & 1.281694 & 2.203449 & $\mathrm{C}$ & -4.216922 & 3.172275 & -0.558451 \\
\hline $\mathrm{C}$ & 0.725207 & 0.027472 & -2.790155 & $\mathrm{H}$ & -3.559533 & 3.118965 & -2.606925 \\
\hline
\end{tabular}




\begin{tabular}{|c|c|c|c|c|c|c|c|}
\hline $\mathrm{H}$ & -4.532810 & 3.261513 & 1.564023 & $\mathrm{H}$ & -1.542881 & 0.047510 & -3.463590 \\
\hline $\mathrm{C}$ & 2.887736 & 0.991530 & 2.648245 & $\mathrm{H}$ & 0.196917 & 0.271921 & -3.082509 \\
\hline $\mathrm{H}$ & 2.610831 & -0.013137 & 2.991956 & $\mathrm{H}$ & -0.847599 & -5.364818 & -0.026214 \\
\hline $\mathrm{H}$ & 3.501965 & 1.436908 & 3.438840 & $\mathrm{Br}$ & -5.142684 & -2.706745 & 0.012232 \\
\hline $\mathrm{H}$ & 1.964311 & 1.570911 & 2.567013 & $\mathrm{O}$ & -0.546515 & -1.639012 & -2.751852 \\
\hline $\mathrm{C}$ & 3.415304 & 2.162247 & -2.242144 & $\mathrm{H}$ & -0.167455 & -1.855936 & -3.610178 \\
\hline $\mathrm{H}$ & 3.206013 & 3.225432 & -2.076046 & $\mathrm{O}$ & -0.892239 & -1.292627 & 3.148604 \\
\hline $\mathrm{H}$ & 4.134530 & 2.084286 & -3.061044 & $\mathrm{H}$ & -0.271687 & -1.445986 & 3.869842 \\
\hline $\mathrm{H}$ & 2.468842 & 1.719180 & -2.577608 & & & & \\
\hline $\mathrm{C}$ & 6.819593 & -0.739378 & 0.057902 & \multicolumn{2}{|c|}{ TSI $_{1-2 \mathrm{Br}}$} & & \\
\hline $\mathrm{H}$ & 7.045085 & -1.197911 & 1.024300 & $\mathrm{Ru}$ & 0.033513 & -0.074889 & 0.186828 \\
\hline $\mathrm{H}$ & 6.719582 & -1.548573 & -0.674176 & $\mathrm{C}$ & -2.396051 & -1.866655 & 0.176307 \\
\hline $\mathrm{H}$ & 7.687273 & -0.142038 & -0.242034 & $\mathrm{C}$ & -0.534855 & -3.234194 & 0.246098 \\
\hline $\mathrm{C}$ & -0.884860 & 3.038694 & -2.379173 & $\mathrm{C}$ & -3.223227 & -2.978004 & 0.113033 \\
\hline $\mathrm{H}$ & -1.327453 & 2.666040 & -3.306550 & $\mathrm{H}$ & -2.775589 & -0.851033 & 0.165777 \\
\hline $\mathrm{H}$ & -0.529361 & 4.058483 & -2.581866 & $\mathrm{C}$ & -1.311738 & -4.381536 & 0.179973 \\
\hline $\mathrm{H}$ & -0.014648 & 2.414886 & -2.161689 & $\mathrm{H}$ & 0.548646 & -3.287519 & 0.283013 \\
\hline $\mathrm{C}$ & -2.038611 & 3.140316 & 2.555311 & $\mathrm{~N}$ & -1.063994 & -2.006550 & 0.249747 \\
\hline $\mathrm{H}$ & -0.960288 & 3.015124 & 2.677105 & $\mathrm{C}$ & -2.695291 & -4.259903 & 0.112934 \\
\hline $\mathrm{H}$ & -2.334338 & 4.090997 & 3.015299 & $\mathrm{H}$ & -3.340326 & -5.130559 & 0.060357 \\
\hline $\mathrm{H}$ & -2.519246 & 2.332626 & 3.112560 & $\mathrm{C}$ & 0.700280 & 1.829121 & 0.213613 \\
\hline $\mathrm{C}$ & -5.674639 & 3.216712 & -0.902133 & $\mathrm{C}$ & 2.077270 & 3.721597 & 0.551752 \\
\hline $\mathrm{H}$ & -6.269999 & 3.638692 & -0.087582 & $\mathrm{C}$ & 0.640425 & 4.182525 & 0.407031 \\
\hline $\mathrm{H}$ & -5.862276 & 3.810746 & -1.801839 & $\mathrm{H}$ & 2.448882 & 3.814918 & 1.584167 \\
\hline $\mathrm{H}$ & -6.065668 & 2.211864 & -1.099240 & $\mathrm{H}$ & 2.779531 & 4.253298 & -0.099938 \\
\hline $\mathrm{C}$ & 1.547321 & -0.638921 & -0.801698 & $\mathrm{H}$ & 0.301455 & 4.829191 & 1.223195 \\
\hline $\mathrm{H}$ & 1.787201 & -0.142758 & -1.763446 & $\mathrm{H}$ & 0.466030 & 4.716411 & -0.538365 \\
\hline $\mathrm{C}$ & 2.530030 & -1.685143 & -0.545410 & $\mathrm{~N}$ & 1.985198 & 2.307526 & 0.182753 \\
\hline $\mathrm{C}$ & 3.387577 & -2.100772 & -1.582463 & $\mathrm{~N}$ & -0.103087 & 2.916950 & 0.407949 \\
\hline $\mathrm{C}$ & 2.687147 & -2.300491 & 0.712553 & $\mathrm{C}$ & 3.183641 & 1.539461 & 0.176795 \\
\hline $\mathrm{C}$ & 4.334433 & -3.097693 & -1.385784 & $\mathrm{C}$ & 3.899302 & 1.452624 & -1.032187 \\
\hline $\mathrm{H}$ & 3.290027 & -1.617888 & -2.554390 & $\mathrm{C}$ & 3.659669 & 0.937456 & 1.347057 \\
\hline $\mathrm{C}$ & 3.647347 & -3.280832 & 0.920002 & $\mathrm{C}$ & 5.076912 & 0.713765 & -1.053974 \\
\hline $\mathrm{H}$ & 2.039654 & -1.974045 & 1.527314 & $\mathrm{C}$ & 4.845351 & 0.201638 & 1.273922 \\
\hline $\mathrm{C}$ & 4.472139 & -3.687958 & -0.129969 & $\mathrm{C}$ & 5.560496 & 0.065708 & 0.087812 \\
\hline $\mathrm{H}$ & 4.976544 & -3.407882 & -2.206635 & $\mathrm{H}$ & 5.622762 & 0.620187 & -1.992665 \\
\hline $\mathrm{H}$ & 3.758918 & -3.731672 & 1.902927 & $\mathrm{H}$ & 5.208795 & -0.297724 & 2.172191 \\
\hline $\mathrm{H}$ & 5.222956 & -4.456669 & 0.032521 & $\mathrm{C}$ & -1.492056 & 2.986328 & 0.060394 \\
\hline $\mathrm{O}$ & -1.442409 & 0.405737 & 1.738164 & $\mathrm{C}$ & -1.844875 & 2.996930 & -1.299371 \\
\hline $\mathrm{O}$ & -1.141599 & 0.209052 & -1.519126 & $\mathrm{C}$ & -2.449437 & 3.146835 & 1.067542 \\
\hline $\mathrm{C}$ & -0.525854 & -0.089650 & 2.509561 & $\mathrm{C}$ & -3.194937 & 3.084445 & -1.625864 \\
\hline $\mathrm{H}$ & -0.068477 & 0.612386 & 3.251741 & $\mathrm{C}$ & -3.790827 & 3.236500 & 0.689506 \\
\hline $\mathrm{H}$ & 0.523440 & -0.384527 & 1.885468 & $\mathrm{C}$ & -4.184643 & 3.187236 & -0.645991 \\
\hline $\mathrm{C}$ & -0.751507 & -0.213791 & -2.728111 & $\mathrm{H}$ & -3.481519 & 3.068780 & -2.676800 \\
\hline
\end{tabular}




\begin{tabular}{|c|c|c|c|c|c|c|c|}
\hline $\mathrm{H}$ & -4.548215 & 3.343391 & 1.465255 & $\mathrm{H}$ & -1.656326 & -0.116877 & -3.430598 \\
\hline $\mathrm{C}$ & 2.938411 & 1.049965 & 2.655014 & $\mathrm{H}$ & 0.086206 & 0.162100 & -3.105477 \\
\hline $\mathrm{H}$ & 2.672601 & 0.057209 & 3.039061 & $\mathrm{H}$ & -0.839546 & -5.358750 & 0.175187 \\
\hline $\mathrm{H}$ & 3.570197 & 1.524290 & 3.414540 & $\mathrm{Br}$ & -5.141269 & -2.715579 & 0.014534 \\
\hline $\mathrm{H}$ & 2.011446 & 1.623811 & 2.575613 & $\mathrm{O}$ & -0.606090 & -1.745401 & -2.662258 \\
\hline $\mathrm{C}$ & 3.369760 & 2.104191 & -2.272152 & $\mathrm{H}$ & -0.310519 & -2.002145 & -3.542208 \\
\hline $\mathrm{H}$ & 3.138070 & 3.164159 & -2.117016 & $\mathrm{O}$ & -0.827674 & -1.204564 & 3.164042 \\
\hline $\mathrm{H}$ & 4.086333 & 2.031019 & -3.093888 & $\mathrm{H}$ & -0.107829 & -1.383581 & 3.780378 \\
\hline $\mathrm{H}$ & 2.431692 & 1.636961 & -2.598098 & & & & \\
\hline $\mathrm{C}$ & 6.806672 & -0.761661 & 0.023437 & \multicolumn{2}{|c|}{ 2I-Br } & & \\
\hline $\mathrm{H}$ & 7.042464 & -1.210945 & 0.991785 & $\mathrm{Ru}$ & -0.022092 & -0.088207 & 0.228186 \\
\hline $\mathrm{H}$ & 6.696713 & -1.577478 & -0.699688 & $\mathrm{C}$ & -2.424902 & -1.967187 & 0.251589 \\
\hline $\mathrm{H}$ & 7.672516 & -0.169399 & -0.291785 & $\mathrm{C}$ & -0.513010 & -3.148732 & 0.809637 \\
\hline $\mathrm{C}$ & -0.804389 & 2.961792 & -2.376093 & $\mathrm{C}$ & -3.188802 & -3.112750 & 0.421173 \\
\hline $\mathrm{H}$ & -1.257449 & 2.733453 & -3.344345 & $\mathrm{H}$ & -2.825140 & -1.013288 & -0.078542 \\
\hline $\mathrm{H}$ & -0.293135 & 3.929030 & -2.479488 & $\mathrm{C}$ & -1.225724 & -4.325012 & 0.983627 \\
\hline $\mathrm{H}$ & -0.045392 & 2.200220 & -2.181207 & $\mathrm{H}$ & 0.564315 & -3.104155 & 0.948493 \\
\hline $\mathrm{C}$ & -2.070147 & 3.249531 & 2.512115 & $\mathrm{~N}$ & -1.101051 & -1.999405 & 0.456833 \\
\hline $\mathrm{H}$ & -1.021808 & 2.987864 & 2.670757 & $\mathrm{C}$ & -2.604659 & -4.313260 & 0.792595 \\
\hline $\mathrm{H}$ & -2.233751 & 4.266542 & 2.890338 & $\mathrm{H}$ & -3.202745 & -5.208880 & 0.925540 \\
\hline $\mathrm{H}$ & -2.672727 & 2.571836 & 3.121884 & $\mathrm{C}$ & 0.771986 & 1.787246 & 0.272859 \\
\hline $\mathrm{C}$ & -5.633603 & 3.231271 & -1.024747 & $\mathrm{C}$ & 2.304517 & 3.570313 & 0.566671 \\
\hline $\mathrm{H}$ & -6.245403 & 3.678417 & -0.236246 & $\mathrm{C}$ & 0.895960 & 4.136437 & 0.525764 \\
\hline $\mathrm{H}$ & -5.795908 & 3.802065 & -1.944247 & $\mathrm{H}$ & 2.789459 & 3.699364 & 1.544543 \\
\hline $\mathrm{H}$ & -6.026383 & 2.223924 & -1.204749 & $\mathrm{H}$ & 2.973236 & 4.003616 & -0.187723 \\
\hline $\mathrm{C}$ & 1.513797 & -0.653588 & -0.799539 & $\mathrm{H}$ & 0.632567 & 4.718162 & 1.416986 \\
\hline $\mathrm{H}$ & 1.687632 & -0.192072 & -1.791939 & $\mathrm{H}$ & 0.720678 & 4.776979 & -0.348080 \\
\hline $\mathrm{C}$ & 2.518161 & -1.683497 & -0.563217 & $\mathrm{~N}$ & 2.086120 & 2.143138 & 0.308001 \\
\hline $\mathrm{C}$ & 3.331481 & -2.109645 & -1.631349 & $\mathrm{~N}$ & 0.052757 & 2.935286 & 0.429172 \\
\hline $\mathrm{C}$ & 2.730538 & -2.282445 & 0.693866 & $\mathrm{C}$ & 3.231471 & 1.297187 & 0.355886 \\
\hline $\mathrm{C}$ & 4.289789 & -3.100781 & -1.462857 & $\mathrm{C}$ & 3.942583 & 1.085209 & -0.837241 \\
\hline $\mathrm{H}$ & 3.189720 & -1.640532 & -2.604556 & $\mathrm{C}$ & 3.704322 & 0.813338 & 1.581333 \\
\hline $\mathrm{C}$ & 3.698279 & -3.261396 & 0.871002 & $\mathrm{C}$ & 5.129422 & 0.361534 & -0.783704 \\
\hline $\mathrm{H}$ & 2.120011 & -1.938868 & 1.529157 & $\mathrm{C}$ & 4.906847 & 0.099774 & 1.587900 \\
\hline $\mathrm{C}$ & 4.478640 & -3.679367 & -0.208188 & $\mathrm{C}$ & 5.630817 & -0.137476 & 0.421847 \\
\hline $\mathrm{H}$ & 4.898741 & -3.417815 & -2.305877 & $\mathrm{H}$ & 5.673456 & 0.172371 & -1.708617 \\
\hline $\mathrm{H}$ & 3.851892 & -3.701199 & 1.853328 & $\mathrm{H}$ & 5.283214 & -0.287528 & 2.534707 \\
\hline $\mathrm{H}$ & 5.236386 & -4.445922 & -0.068864 & $\mathrm{C}$ & -1.295168 & 3.154340 & -0.008952 \\
\hline $\mathrm{O}$ & -1.472060 & 0.455713 & 1.771308 & $\mathrm{C}$ & -1.551060 & 3.173404 & -1.388795 \\
\hline $\mathrm{O}$ & -1.204018 & 0.147665 & -1.499576 & $\mathrm{C}$ & -2.289293 & 3.450861 & 0.926168 \\
\hline $\mathrm{C}$ & -0.544143 & -0.012723 & 2.505847 & $\mathrm{C}$ & -2.847958 & 3.444303 & -1.812981 \\
\hline $\mathrm{H}$ & 0.022568 & 0.687675 & 3.160781 & $\mathrm{C}$ & -3.575692 & 3.717346 & 0.453198 \\
\hline $\mathrm{H}$ & 0.659023 & -0.376136 & 1.756870 & $\mathrm{C}$ & -3.878293 & 3.704004 & -0.906281 \\
\hline $\mathrm{C}$ & -0.844774 & -0.320292 & -2.697190 & $\mathrm{H}$ & -3.061699 & 3.442236 & -2.881464 \\
\hline
\end{tabular}




\begin{tabular}{|c|c|c|c|}
\hline $\mathrm{H}$ & .365263 & .930704 & 1. \\
\hline$C$ & 2.951317 & 1.020724 & 8504 \\
\hline & .513850 & .075499 & 204080 \\
\hline & 607048 & .384317 & 6573 \\
\hline & 1 & 3 & 37 \\
\hline & 3.406796 & 1.603659 & 2.135408 \\
\hline $\mathrm{H}$ & 3.364879 & 5 & -2.155893 \\
\hline $\mathrm{H}$ & 4.02 & 71 & 449 \\
\hline $\mathrm{H}$ & 2. & 5 & \\
\hline $\mathrm{C}$ & 6.894138 & 1 & 764 \\
\hline $\mathrm{H}$ & 7.218223 & 59956 & 4583 \\
\hline $\mathrm{H}$ & 6.7 & 3 & 68 \\
\hline $\mathrm{H}$ & 7.713834 & 74 & 83 \\
\hline$c$ & -0.453321 & 2.921250 & -2.373969 \\
\hline $\mathrm{H}$ & 481 & 26 & 51 \\
\hline $\mathrm{H}$ & .348920 & 3 & -2.300987 \\
\hline 11 & -0.003277 & 1.937213 & -2.2 \\
\hline e & -2.006661 & 3.4 & 93 \\
\hline $\mathrm{H}$ & -1.016338 & 3.090358 & 98 \\
\hline $\mathrm{H}$ & -2.0. & $r$ & \\
\hline $\mathrm{H}$ & -2.73 & 1 & 2 \\
\hline $\mathrm{C}$ & -5.276826 & 3.943532 & 7306 \\
\hline $\mathrm{H}$ & 0 & 4.433997 & 99 \\
\hline $\mathrm{H}$ & -5.296612 & 4.564 & -2.288527 \\
\hline $\mathrm{H}$ & -5.7 & 96 & 12 \\
\hline $\mathrm{C}$ & 1 & 0 & 77 \\
\hline $\mathrm{H}$ & 0.900355 & -0.566688 & 0161 \\
\hline C & 2 & -1.709791 & 1.020138 \\
\hline$C$ & 482 & 43 & 6961 \\
\hline $\mathrm{C}$ & 2.931826 & 36 & 39 \\
\hline$C$ & 3.884960 & 3053657 & 2321387 \\
\hline $\mathrm{H}$ & 2.351779 & -1.802367 & -3.170148 \\
\hline$c$ & 1 & -5.0011 & 0 \\
\hline $\mathrm{H}$ & .568143 & -1.820256 & 1.109773 \\
\hline $\mathrm{C}$ & 4.472139 & -3.518052 & 4709 \\
\hline$H$ & 4.255787 & 2301057 & -3.285450 \\
\hline $\mathrm{H}$ & 4.458 & -3.429915 & 1.008492 \\
\hline $\mathrm{H}$ & 5.304118 & -4.216719 & -1.188940 \\
\hline $\mathrm{O}$ & .680218 & 0622002 & 1520005 \\
\hline $\mathrm{O}$ & -1.521229 & .218397 & -1.336769 \\
\hline C & -0.804588 & 0.241401 & 2.338253 \\
\hline $\mathrm{H}$ & 185 & .956750 & 2.82 \\
\hline $\mathrm{H}$ & 1.111162 & -0.524275 & 1.295401 \\
\hline $\mathrm{C}$ & -1.437623 & -0.265840 & 2.56755 \\
\hline
\end{tabular}

$\begin{array}{rrrc}\mathrm{H} & -2.328132 & 0.029030 & -3.170753 \\ \mathrm{H} & -0.540818 & 0.095088 & -3.150869 \\ \mathrm{H} & -0.711299 & -5.237919 & 1.265894 \\ \mathrm{Br} & -5.099491 & -3.017810 & 0.108458 \\ \mathrm{O} & -1.360210 & -1.718702 & -2.556027 \\ \mathrm{H} & -1.409603 & -2.012061 & -3.472326 \\ \mathrm{O} & -1.060924 & -0.864241 & 3.113391 \\ \mathrm{H} & -0.268696 & -1.042067 & 3.633360\end{array}$

\section{I}

$\begin{array}{llll}\mathrm{Ru} & -0.414999 & 0.745979 & 0.233371\end{array}$

$\begin{array}{llll}\text { C } & -0.516623 & -1.170819 & -0.104544\end{array}$

$\begin{array}{llll}\text { C } & -0.239713 & -3.464705 & -0.575155\end{array}$

C $\quad-1.723411 \quad-3.177033 \quad-0.421476$

$\mathrm{H} \quad 0.039479 \quad-3.663398 \quad-1.622136$

$\mathrm{H} \quad 0.105835 \quad-4.311574 \quad 0.026912$

$\mathrm{H} \quad-2.331897 \quad-3.576938 \quad-1.239202$

$\begin{array}{llll}\mathrm{H} & -2.122435 & -3.574232 & 0.525169\end{array}$

$\begin{array}{llll}\mathrm{N} & 0.370659 & -2.213868 & -0.114633\end{array}$

$\begin{array}{llll}\mathrm{N} & -1.736597 & -1.717693 & -0.421637\end{array}$

$\begin{array}{llll}\text { C } & 1.789733 & -2.104822 & -0.098751\end{array}$

$\begin{array}{llll}\text { C } & 2.465313 & -2.408238 & 1.089587\end{array}$

$\begin{array}{llll}\text { C } & 2.475495 & -1.686422 & -1.249797\end{array}$

$\begin{array}{llll}\text { C } & 3.853575 & -2.267551 & 1.117154\end{array}$

$\begin{array}{llll}\text { C } & 3.861743 & -1.558266 & -1.174542\end{array}$

$\begin{array}{llll}\text { C } & 4.567428 & -1.834091 & -0.001097\end{array}$

$\mathrm{H} \quad 4.388466 \quad-2.487076 \quad 2.040473$

$\mathrm{H} \quad 4.404921 \quad-1.219700 \quad-2.056259$

$\begin{array}{llll}\text { C } & -2.943237 & -0.956420 & -0.379877\end{array}$

$\begin{array}{llll}\text { C } & -3.651592 & -0.828704 & 0.825244\end{array}$

$\begin{array}{llll}\text { C } & -3.384524 & -0.342673 & -1.562563\end{array}$

$\begin{array}{llll}\text { C } & -4.806421 & -0.049315 & 0.827066\end{array}$

$\begin{array}{llll}\text { C } & -4.542719 & 0.433666 & -1.508544\end{array}$

$\begin{array}{llll}\text { C } & -5.260092 & 0.599331 & -0.323141\end{array}$

$\mathrm{H} \quad-5.353838 \quad 0.073586 \quad 1.760976$

H $\quad-4.893519 \quad 0.919582 \quad-2.418156$

$\begin{array}{llll}\text { C } & 1.738506 & -1.312479 & -2.497133\end{array}$

$\mathrm{H} \quad 1.284705 \quad-0.319434 \quad-2.386591$

$\mathrm{H} \quad 2.408022 \quad-1.289517 \quad-3.360294$

$\mathrm{H} \quad 0.915868 \quad-2.000207 \quad-2.720069$

$\begin{array}{llll}\text { C } & 1.697276 & -2.789782 & 2.317076\end{array}$

$\mathrm{H} \quad 1.033630 \quad-3.644269 \quad 2.144291$

$\mathrm{H} \quad 2.367504 \quad-3.046242 \quad 3.140906$

$\begin{array}{llll}\mathrm{H} & 1.054065 & -1.964636 & 2.645863\end{array}$ 


\begin{tabular}{|c|c|c|c|c|c|c|c|}
\hline $\mathrm{C}$ & 6.051475 & -1.638131 & 0.056451 & $\mathrm{H}$ & 0.525931 & 3.398848 & -0.155165 \\
\hline $\mathrm{H}$ & 6.560777 & -2.145044 & -0.769262 & $\mathrm{~N}$ & -1.066127 & 2.095517 & -0.298079 \\
\hline $\mathrm{H}$ & 6.311884 & -0.575878 & -0.020347 & $\mathrm{C}$ & -2.741352 & 4.311944 & -0.042834 \\
\hline $\mathrm{H}$ & 6.473925 & -2.013562 & 0.991918 & $\mathrm{H}$ & -3.401800 & 5.166433 & 0.059772 \\
\hline $\mathrm{C}$ & -3.162652 & -1.487019 & 2.076220 & $\mathrm{C}$ & 0.735853 & -1.736845 & -0.256346 \\
\hline $\mathrm{H}$ & -3.620187 & -1.033806 & 2.957847 & $\mathrm{C}$ & 2.109168 & -3.628722 & -0.562096 \\
\hline $\mathrm{H}$ & -3.400717 & -2.558201 & 2.093353 & $\mathrm{C}$ & 0.683117 & -4.087729 & -0.335101 \\
\hline $\mathrm{H}$ & -2.078834 & -1.376073 & 2.177883 & $\mathrm{H}$ & 2.431778 & -3.751723 & -1.607219 \\
\hline $\mathrm{C}$ & -2.630055 & -0.520443 & -2.843747 & $\mathrm{H}$ & 2.842793 & -4.133693 & 0.075319 \\
\hline $\mathrm{H}$ & -1.645028 & -0.042638 & -2.789722 & $\mathrm{H}$ & 0.317921 & -4.787976 & -1.092557 \\
\hline $\mathrm{H}$ & -2.450498 & -1.578578 & -3.065274 & $\mathrm{H}$ & 0.546328 & -4.551987 & 0.653579 \\
\hline $\mathrm{H}$ & -3.174413 & -0.084050 & -3.684685 & $\mathrm{~N}$ & 2.023568 & -2.202604 & -0.236779 \\
\hline $\mathrm{C}$ & -6.473445 & 1.477073 & -0.275418 & $\mathrm{~N}$ & -0.067223 & -2.827849 & -0.390907 \\
\hline $\mathrm{H}$ & -6.898970 & 1.636127 & -1.269749 & $\mathrm{C}$ & 3.233117 & -1.454418 & -0.141653 \\
\hline $\mathrm{H}$ & -7.254560 & 1.056522 & 0.364842 & $\mathrm{C}$ & 3.897191 & -1.446894 & 1.102455 \\
\hline $\mathrm{H}$ & -6.228855 & 2.464668 & 0.132859 & $\mathrm{C}$ & 3.775150 & -0.796096 & -1.251202 \\
\hline $\mathrm{C}$ & 1.423567 & 0.903022 & 0.341369 & $\mathrm{C}$ & 5.084597 & -0.734408 & 1.218545 \\
\hline $\mathrm{H}$ & 1.993538 & 0.123257 & 0.868174 & $\mathrm{C}$ & 4.967995 & -0.085920 & -1.081231 \\
\hline $\mathrm{C}$ & 2.260432 & 2.055509 & 0.019284 & $\mathrm{C}$ & 5.632184 & -0.031919 & 0.138694 \\
\hline $\mathrm{C}$ & 3.574314 & 2.118552 & 0.526951 & $\mathrm{H}$ & 5.586624 & -0.703865 & 2.185403 \\
\hline $\mathrm{C}$ & 1.830432 & 3.103570 & -0.821213 & $\mathrm{H}$ & 5.376729 & 0.458025 & -1.932826 \\
\hline $\mathrm{C}$ & 4.412595 & 3.184944 & 0.226208 & $\mathrm{C}$ & -1.456978 & -2.888472 & -0.038314 \\
\hline $\mathrm{H}$ & 3.924620 & 1.306069 & 1.162628 & $\mathrm{C}$ & -1.817181 & -2.860229 & 1.318243 \\
\hline $\mathrm{C}$ & 2.667921 & 4.168975 & -1.117149 & $\mathrm{C}$ & -2.409386 & -3.089179 & -1.047785 \\
\hline $\mathrm{H}$ & 0.827269 & 3.049029 & -1.239418 & $\mathrm{C}$ & -3.173113 & -2.952458 & 1.635597 \\
\hline $\mathrm{C}$ & 3.961530 & 4.217318 & -0.593823 & $\mathrm{C}$ & -3.751628 & -3.171116 & -0.677660 \\
\hline $\mathrm{H}$ & 5.421633 & 3.212002 & 0.630401 & $\mathrm{C}$ & -4.154778 & -3.088341 & 0.655507 \\
\hline $\mathrm{H}$ & 2.317180 & 4.965707 & -1.768157 & $\mathrm{H}$ & -3.465694 & -2.914069 & 2.684977 \\
\hline $\mathrm{H}$ & 4.615846 & 5.051420 & -0.832430 & $\mathrm{H}$ & -4.501672 & -3.308711 & -1.455890 \\
\hline $\mathrm{O}$ & -1.601408 & 1.107945 & 1.883277 & $\mathrm{C}$ & 3.106066 & -0.801537 & -2.588200 \\
\hline $\mathrm{H}$ & -0.365878 & 0.969680 & -1.360958 & $\mathrm{H}$ & 2.877682 & 0.226652 & -2.899793 \\
\hline $\mathrm{C}$ & -1.648996 & 2.455627 & 1.911921 & $\mathrm{H}$ & 3.762549 & -1.226927 & -3.355975 \\
\hline $\mathrm{H}$ & -2.640380 & 2.865146 & 1.648666 & $\mathrm{H}$ & 2.165107 & -1.353971 & -2.588176 \\
\hline $\mathrm{H}$ & -1.322416 & 2.899683 & 2.872115 & $\mathrm{C}$ & 3.299589 & -2.137876 & 2.288757 \\
\hline $\mathrm{O}$ & -0.750739 & 2.973948 & 0.856267 & $\mathrm{H}$ & 3.064866 & -3.189947 & 2.090345 \\
\hline \multirow[t]{2}{*}{$\mathrm{H}$} & 0.084857 & 3.231251 & 1.267319 & $\mathrm{H}$ & 3.974490 & -2.101777 & 3.147216 \\
\hline & & & & $\mathrm{H}$ & 2.352752 & -1.669614 & 2.586320 \\
\hline \multicolumn{2}{|c|}{ TSI-1-2Br } & & & $\mathrm{C}$ & 6.888665 & 0.764014 & 0.306701 \\
\hline $\mathrm{Ru}$ & 0.049030 & 0.159342 & -0.255872 & $\mathrm{H}$ & 7.154698 & 1.294643 & -0.611259 \\
\hline $\mathrm{C}$ & -2.402340 & 1.936047 & -0.303979 & $\mathrm{H}$ & 6.776167 & 1.512813 & 1.098723 \\
\hline $\mathrm{C}$ & -0.558023 & 3.330697 & -0.169088 & $\mathrm{H}$ & 7.737435 & 0.130203 & 0.585920 \\
\hline $\mathrm{C}$ & -3.249316 & 3.027928 & -0.178726 & $\mathrm{C}$ & -0.813511 & -2.782850 & 2.428367 \\
\hline $\mathrm{H}$ & -2.761332 & 0.915925 & -0.392874 & $\mathrm{H}$ & -1.159763 & -2.090225 & 3.199887 \\
\hline $\mathrm{C}$ & -1.357584 & 4.457317 & -0.041568 & $\mathrm{H}$ & -0.665285 & -3.767714 & 2.891703 \\
\hline
\end{tabular}




$\begin{array}{cccc}\mathrm{H} & 0.157770 & -2.417347 & 2.088003 \\ \mathrm{C} & -2.016607 & -3.257097 & -2.482528 \\ \mathrm{H} & -0.990310 & -2.937419 & -2.663383 \\ \mathrm{H} & -2.120773 & -4.305252 & -2.792296 \\ \mathrm{H} & -2.658233 & -2.660290 & -3.135537 \\ \mathrm{C} & -5.607359 & -3.135751 & 1.021706 \\ \mathrm{H} & -6.165342 & -3.827230 & 0.382886 \\ \mathrm{H} & -5.753292 & -3.443019 & 2.061168 \\ \mathrm{H} & -6.079380 & -2.152642 & 0.908277 \\ \mathrm{C} & 1.540156 & 0.705818 & 0.745063 \\ \mathrm{H} & 1.759554 & 0.153717 & 1.678715 \\ \mathrm{C} & 2.521935 & 1.764786 & 0.557073 \\ \mathrm{C} & 3.358617 & 2.140734 & 1.627394 \\ \mathrm{C} & 2.716529 & 2.414911 & -0.679064 \\ \mathrm{C} & 4.319491 & 3.133131 & 1.479993 \\ \mathrm{H} & 3.235659 & 1.632119 & 2.582775 \\ \mathrm{C} & 3.692231 & 3.388610 & -0.835626 \\ \mathrm{H} & 2.092761 & 2.106512 & -1.518190 \\ \mathrm{C} & 4.495751 & 3.756377 & 0.245253 \\ \mathrm{H} & 4.944989 & 3.412087 & 2.324380 \\ \mathrm{H} & 3.835340 & 3.862319 & -1.803710 \\ \mathrm{H} & 5.260363 & 4.518736 & 0.122469 \\ \mathrm{O} & -1.417915 & -0.396115 & -1.858149 \\ \mathrm{O} & -1.198528 & -0.159022 & 1.369747 \\ \mathrm{C} & -0.513129 & 0.124024 & -2.588880 \\ \mathrm{H} & 0.682519 & 0.469666 & -1.838019 \\ \mathrm{C} & -1.035997 & 0.596459 & 2.489910 \\ \mathrm{H} & -0.036354 & 0.462613 & 2.962570 \\ \mathrm{H} & -1.147893 & 1.691503 & 2.291534 \\ \mathrm{H} & -0.902692 & 5.435943 & 0.069612 \\ \mathrm{Br} & -5.162778 & 2.732127 & -0.177232 \\ \mathrm{O} & -1.977435 & 0.194130 & 3.473759 \\ \mathrm{H} & -2.760168 & -0.036793 & 2.958735 \\ \mathrm{H} & -0.659678 & 1.152653 & -2.986337 \\ \mathrm{O} & 0.026513 & -0.722263 & -3.554430 \\ \mathrm{H} & 0.619436 & -0.203661 & -4.110867 \\ & & & \end{array}$

\section{TSI-21-2Br}

$\begin{array}{rrrr}\mathrm{Ru} & -0.074133 & -0.104586 & -0.155173 \\ \mathrm{C} & 2.390107 & -1.851068 & -0.064419 \\ \mathrm{C} & 0.567096 & -3.272447 & -0.200635 \\ \mathrm{C} & 3.252135 & -2.935480 & -0.133606 \\ \mathrm{H} & 2.732345 & -0.825229 & 0.039653 \\ \mathrm{C} & 1.381857 & -4.392296 & -0.277667\end{array}$

\begin{tabular}{|c|c|c|c|}
\hline $\mathrm{H}$ & -0.515932 & -3.355884 & -0.213953 \\
\hline $\mathrm{N}$ & 1.057856 & -2.030046 & -0.098859 \\
\hline C & 2.763192 & -4.228794 & -0.247352 \\
\hline H & 3.436092 & -5.077618 & -0.307232 \\
\hline $\mathrm{C}$ & -0.770503 & 1.779930 & -0.287409 \\
\hline $\mathrm{C}$ & -2.168207 & 3.658268 & -0.608447 \\
\hline $\mathrm{C}$ & -0.739497 & 4.130563 & -0.426414 \\
\hline $\mathrm{H}$ & -2.523310 & 3.773510 & -1.644387 \\
\hline $\mathrm{H}$ & -2.885901 & 4.164284 & 0.046280 \\
\hline $\mathrm{H}$ & -0.397091 & 4.814985 & -1.209107 \\
\hline $\mathrm{H}$ & -0.582611 & 4.621417 & 0.545414 \\
\hline $\mathrm{N}$ & -2.065131 & 2.235060 & -0.275321 \\
\hline $\mathrm{N}$ & 0.018383 & 2.875084 & -0.467487 \\
\hline $\mathrm{C}$ & -3.257631 & 1.455165 & -0.288638 \\
\hline $\mathrm{C}$ & -4.037683 & 1.419083 & 0.882877 \\
\hline $\mathrm{C}$ & -3.673680 & 0.791215 & -1.450920 \\
\hline $\mathrm{C}$ & -5.200320 & 0.654675 & 0.884494 \\
\hline $\mathrm{C}$ & -4.850729 & 0.040998 & -1.400727 \\
\hline $\mathrm{C}$ & -5.622323 & -0.051290 & -0.245249 \\
\hline $\mathrm{H}$ & -5.785581 & 0.591599 & 1.801613 \\
\hline $\mathrm{H}$ & -5.161791 & -0.504392 & -2.291898 \\
\hline $\mathrm{C}$ & 1.417179 & 2.945799 & -0.155273 \\
\hline $\mathrm{C}$ & 1.810634 & 2.984746 & 1.191985 \\
\hline $\mathrm{C}$ & 2.346314 & 3.054660 & -1.197304 \\
\hline $\mathrm{C}$ & 3.173498 & 3.051531 & 1.471972 \\
\hline $\mathrm{C}$ & 3.699858 & 3.123447 & -0.864036 \\
\hline $\mathrm{C}$ & 4.132604 & 3.102809 & 0.460901 \\
\hline $\mathrm{H}$ & 3.490623 & 3.036596 & 2.513487 \\
\hline $\mathrm{H}$ & 4.435171 & 3.187748 & -1.665573 \\
\hline $\mathrm{C}$ & -2.905269 & 0.866793 & -2.734075 \\
\hline $\mathrm{H}$ & -2.612004 & -0.133405 & -3.075597 \\
\hline $\mathrm{H}$ & -3.516489 & 1.306077 & -3.530619 \\
\hline $\mathrm{H}$ & -1.992293 & 1.459497 & -2.640245 \\
\hline $\mathrm{C}$ & -3.614220 & 2.164079 & 2.110906 \\
\hline $\mathrm{H}$ & -3.879142 & 3.227543 & 2.052675 \\
\hline $\mathrm{H}$ & -4.102543 & 1.762661 & 3.002940 \\
\hline $\mathrm{H}$ & -2.530778 & 2.120204 & 2.260289 \\
\hline $\mathrm{C}$ & -6.873216 & -0.873954 & -0.212270 \\
\hline $\mathrm{H}$ & -6.879669 & -1.627669 & -1.004485 \\
\hline $\mathrm{H}$ & -6.985039 & -1.392944 & 0.744568 \\
\hline $\mathrm{H}$ & -7.767279 & -0.253319 & -0.345186 \\
\hline $\mathrm{C}$ & 0.826293 & 2.989348 & 2.321688 \\
\hline $\mathrm{H}$ & 1.229881 & 2.433506 & 3.170856 \\
\hline $\mathrm{H}$ & 0.609580 & 4.014477 & 2.653729 \\
\hline
\end{tabular}




\begin{tabular}{cccc}
$\mathrm{H}$ & -0.118653 & 2.510680 & 2.050651 \\
$\mathrm{C}$ & 1.926902 & 3.124509 & -2.633565 \\
$\mathrm{H}$ & 0.879484 & 2.843980 & -2.764012 \\
$\mathrm{H}$ & 2.061982 & 4.137366 & -3.034177 \\
$\mathrm{H}$ & 2.526559 & 2.447910 & -3.247597 \\
$\mathrm{C}$ & 5.593997 & 3.112816 & 0.792255 \\
$\mathrm{H}$ & 6.193002 & 3.520633 & -0.027138 \\
$\mathrm{H}$ & 5.803224 & 3.701023 & 1.690913 \\
$\mathrm{H}$ & 5.960823 & 2.098236 & 0.986339 \\
$\mathrm{C}$ & -1.570097 & -0.562879 & 0.879486 \\
$\mathrm{H}$ & -1.815468 & 0.083671 & 1.743697 \\
$\mathrm{C}$ & -2.526254 & -1.658109 & 0.791419 \\
$\mathrm{C}$ & -3.309457 & -1.996411 & 1.913941 \\
$\mathrm{C}$ & -2.747783 & -2.384927 & -0.395568 \\
$\mathrm{C}$ & -4.237919 & -3.027743 & 1.864837 \\
$\mathrm{H}$ & -3.168964 & -1.427426 & 2.832583 \\
$\mathrm{C}$ & -3.691042 & -3.401241 & -0.453871 \\
$\mathrm{H}$ & -2.175793 & -2.102022 & -1.279463 \\
$\mathrm{C}$ & -4.435580 & -3.733564 & 0.678465 \\
$\mathrm{H}$ & -4.821412 & -3.274899 & 2.748110 \\
$\mathrm{H}$ & -3.856218 & -3.934471 & -1.387240 \\
$\mathrm{H}$ & -5.174990 & -4.528816 & 0.632007 \\
$\mathrm{O}$ & 1.384497 & 0.312067 & -1.820342 \\
$\mathrm{O}$ & 1.188575 & 0.326355 & 1.423288 \\
$\mathrm{C}$ & 0.470753 & -0.245787 & -2.502592 \\
$\mathrm{H}$ & -0.128799 & 0.370505 & -3.207893 \\
$\mathrm{H}$ & -0.712728 & -0.567106 & -1.691021 \\
$\mathrm{C}$ & 1.027684 & -0.370571 & 2.568410 \\
$\mathrm{H}$ & -0.001197 & -0.226425 & 2.996454 \\
$\mathrm{H}$ & 1.987507 & -0.493737 & 4.245949 \\
$\mathrm{H}$ & 1.156021 & -1.475467 & 2.441427 \\
$\mathrm{H}$ & 0.941689 & -5.380960 & -0.357711 \\
& 0.064683 & -1.738200 & -3.643810 \\
\hline & 5.161741 & -2.620191 & -0.072241 \\
$\mathrm{H}$ & &
\end{tabular}

\section{TSI-31-2Br}

$\begin{array}{rrrr}\mathrm{Ru} & -0.020878 & 0.125908 & 0.249489 \\ \mathrm{C} & 2.385988 & 1.933181 & 0.286002 \\ \mathrm{C} & 0.512994 & 3.281552 & 0.451218 \\ \mathrm{C} & 3.198705 & 3.054153 & 0.201952 \\ \mathrm{H} & 2.774235 & 0.921520 & 0.243214 \\ \mathrm{C} & 1.275911 & 4.436948 & 0.364933\end{array}$

\begin{tabular}{|c|c|c|c|}
\hline $\mathrm{H}$ & -0.569297 & 3.321313 & 0.531044 \\
\hline $\mathrm{N}$ & 1.055263 & 2.057862 & 0.419459 \\
\hline$C$ & 2.656851 & 4.330258 & 0.237104 \\
\hline $\mathrm{H}$ & 3.289552 & 5.208612 & 0.164698 \\
\hline C & -0.655862 & -1.795633 & 0.186546 \\
\hline $\mathrm{C}$ & -2.000664 & -3.706791 & 0.520930 \\
\hline $\mathrm{C}$ & -0.560667 & -4.146653 & 0.329464 \\
\hline $\mathrm{H}$ & -2.342894 & -3.8 & 1.559410 \\
\hline $\mathrm{H}$ & -2.709987 & -4.229694 & -0.130001 \\
\hline $\mathrm{H}$ & -0.192322 & -4.799159 & 1.127756 \\
\hline $\mathrm{H}$ & -0.404327 & -4.664995 & -0.628352 \\
\hline $\mathrm{N}$ & -1.933344 & 1084 & 34501 \\
\hline $\mathrm{N}$ & 0.167823 & -2.872858 & 0.328269 \\
\hline $\mathrm{C}$ & -3.141828 & -1.551030 & 0.019168 \\
\hline 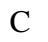 & -3.665173 & -1.426836 & -1.280972 \\
\hline C & -3.812246 & -1.015086 & 1.125698 \\
\hline $\mathrm{C}$ & -4.847820 & -0.714567 & -1.453827 \\
\hline $\mathrm{C}$ & -4.996403 & -0.308715 & 0.901635 \\
\hline $\mathrm{C}$ & -5.524267 & -0.136937 & -0.375326 \\
\hline $\mathrm{H}$ & -5.245145 & -0.590391 & -2.461007 \\
\hline $\mathrm{H}$ & -5.503583 & 7746 & 1.751315 \\
\hline$c$ & 1.556957 & -2.920343 & -0.025751 \\
\hline $\mathrm{C}$ & 1.906975 & -2.861723 & 5905 \\
\hline$r$ & 2.518827 & -3.144850 & 0.965889 \\
\hline $\mathrm{C}$ & 3.255549 & -2.934446 & -1.723000 \\
\hline $\mathrm{C}$ & 3.858354 & -3.216141 & 0.576277 \\
\hline $\mathrm{C}$ & 4.249200 & -3.091559 & -0.754582 \\
\hline$n$ & 3.536599 & -2.869386 & -2.773654 \\
\hline $\mathrm{H}$ & 4.617005 & -3.372474 & 1.342539 \\
\hline $\mathrm{C}$ & -3.265289 & -1.164816 & 2.510205 \\
\hline 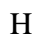 & -3.555543 & -0.313560 & 3.134869 \\
\hline $\mathrm{H}$ & -3.654762 & -2.065366 & 3.002001 \\
\hline $\mathrm{H}$ & -2.174105 & -1.242194 & 2.508309 \\
\hline C & -2.937327 & -2.006801 & -2.453188 \\
\hline $\mathrm{H}$ & -2.681010 & -3.061809 & -2.303498 \\
\hline H & -3.534149 & -1.929800 & -3.365303 \\
\hline$H$ & -1.985797 & -1.487625 & -2.630273 \\
\hline $\mathrm{C}$ & -6.782773 & 0.644212 & -0.594228 \\
\hline- & -7.024849 & 1.265913 & 0.271812 \\
\hline $\mathrm{H}$ & -6.694516 & 1.304154 & -1.463245 \\
\hline $\mathrm{H}$ & -7.640296 & -0.013504 & -0.778413 \\
\hline$c$ & 0.864270 & -2.781601 & -2.45703 \\
\hline $\mathrm{H}$ & 1.314728 & -2.522818 & -3.418959 \\
\hline $\mathrm{H}$ & 0.345605 & -3.741592 & 885 \\
\hline
\end{tabular}




\begin{tabular}{|c|c|c|c|c|c|c|c|}
\hline $\mathrm{H}$ & 0.113165 & -2.022059 & -2.230899 & $\mathrm{H}$ & -0.537451 & 3.373841 & 0.642152 \\
\hline $\mathrm{C}$ & 2.151870 & -3.340032 & 2.403328 & $\mathrm{~N}$ & 1.063982 & 2.114558 & 0.327908 \\
\hline $\mathrm{H}$ & 1.095692 & -3.144538 & 2.586233 & $\mathrm{C}$ & 2.689876 & 4.374854 & 0.282096 \\
\hline $\mathrm{H}$ & 2.388594 & -4.361344 & 2.727834 & $\mathrm{H}$ & 3.331772 & 5.249081 & 0.262650 \\
\hline $\mathrm{H}$ & 2.713243 & -2.654703 & 3.043613 & $\mathrm{C}$ & -0.622939 & -1.752636 & 0.279007 \\
\hline $\mathrm{C}$ & 5.696757 & -3.119486 & -1.140365 & $\mathrm{C}$ & -1.960849 & -3.684237 & 0.564133 \\
\hline $\mathrm{H}$ & 6.309180 & -3.613343 & -0.380658 & $\mathrm{C}$ & -0.492559 & -4.075277 & 0.676076 \\
\hline $\mathrm{H}$ & 5.853904 & -3.637470 & -2.091549 & $\mathrm{H}$ & -2.529280 & -3.891104 & 1.479429 \\
\hline $\mathrm{H}$ & 6.093745 & -2.105226 & -1.264383 & $\mathrm{H}$ & -2.480278 & -4.184555 & -0.264752 \\
\hline $\mathrm{C}$ & -1.510816 & 0.777015 & -0.681502 & $\mathrm{H}$ & -0.232292 & -4.489170 & 1.658007 \\
\hline $\mathrm{H}$ & -1.669848 & 0.443046 & -1.724668 & $\mathrm{H}$ & -0.183896 & -4.803560 & -0.083809 \\
\hline $\mathrm{C}$ & -2.526930 & 1.762374 & -0.331268 & $\mathrm{~N}$ & -1.893303 & -2.240234 & 0.328412 \\
\hline $\mathrm{C}$ & -3.334402 & 2.312807 & -1.345633 & $\mathrm{~N}$ & 0.213648 & -2.806183 & 0.463806 \\
\hline $\mathrm{C}$ & -2.753573 & 2.200571 & 0.988713 & $\mathrm{C}$ & -3.099340 & -1.529208 & 0.070533 \\
\hline $\mathrm{C}$ & -4.305328 & 3.265480 & -1.062818 & $\mathrm{C}$ & -3.555449 & -1.443885 & -1.256133 \\
\hline $\mathrm{H}$ & -3.177529 & 1.971659 & -2.368424 & $\mathrm{C}$ & -3.829699 & -0.979288 & 1.132727 \\
\hline $\mathrm{C}$ & -3.735862 & 3.136169 & 1.278214 & $\mathrm{C}$ & -4.753922 & -0.773466 & -1.495874 \\
\hline $\mathrm{H}$ & -2.144759 & 1.763890 & 1.779888 & $\mathrm{C}$ & -5.019410 & -0.311813 & 0.840556 \\
\hline $\mathrm{C}$ & -4.514990 & 3.675522 & 0.252690 & $\mathrm{C}$ & -5.496719 & -0.195164 & -0.464657 \\
\hline $\mathrm{H}$ & -4.910224 & 3.680914 & -1.865201 & $\mathrm{H}$ & -5.107849 & -0.683313 & -2.522596 \\
\hline $\mathrm{H}$ & -3.902482 & 3.448081 & 2.306526 & $\mathrm{H}$ & -5.573299 & 0.154406 & 1.655040 \\
\hline $\mathrm{H}$ & -5.284275 & 4.409194 & 0.479345 & $\mathrm{C}$ & 1.604676 & -2.868947 & 0.121205 \\
\hline $\mathrm{O}$ & 1.500409 & -0.501644 & 1.775121 & $\mathrm{C}$ & 1.943495 & -2.935933 & -1.240913 \\
\hline $\mathrm{O}$ & 1.183473 & 0.028875 & -1.475790 & $\mathrm{C}$ & 2.571732 & -3.008684 & 1.124294 \\
\hline $\mathrm{C}$ & 0.571602 & -0.072690 & 2.538500 & $\mathrm{C}$ & 3.292852 & -3.019437 & -1.577536 \\
\hline $\mathrm{H}$ & -0.631523 & 0.314452 & 1.846789 & $\mathrm{C}$ & 3.910122 & -3.090054 & 0.734909 \\
\hline $\mathrm{C}$ & 0.793523 & 0.554786 & -2.637911 & $\mathrm{C}$ & 4.292937 & -3.070648 & -0.605036 \\
\hline $\mathrm{H}$ & 1.590957 & 0.405971 & -3.398896 & $\mathrm{H}$ & 3.567228 & -3.058399 & -2.631054 \\
\hline $\mathrm{H}$ & -0.139461 & 0.081846 & -3.054270 & $\mathrm{H}$ & 4.673686 & -3.177882 & 1.507293 \\
\hline $\mathrm{H}$ & 0.794340 & 5.409133 & 0.389958 & $\mathrm{C}$ & -3.332658 & -1.073704 & 2.541144 \\
\hline $\mathrm{Br}$ & 5.111272 & 2.812388 & 0.019558 & $\mathrm{H}$ & -3.729116 & -0.252478 & 3.146918 \\
\hline $\mathrm{O}$ & 0.534076 & 1.973158 & -2.516139 & $\mathrm{H}$ & -3.651606 & -2.007163 & 3.022068 \\
\hline $\mathrm{H}$ & 0.217265 & 2.275246 & -3.374145 & $\mathrm{H}$ & -2.239204 & -1.050961 & 2.584892 \\
\hline $\mathrm{H}$ & 0.699883 & 0.912351 & 3.039617 & $\mathrm{C}$ & -2.752760 & -2.015651 & -2.381804 \\
\hline $\mathrm{O}$ & 0.041787 & -1.022556 & 3.420645 & $\mathrm{H}$ & -2.480006 & -3.062245 & -2.204375 \\
\hline \multirow[t]{2}{*}{$\mathrm{H}$} & -0.549725 & -0.562798 & 4.028255 & $\mathrm{H}$ & -3.312004 & -1.972318 & -3.319826 \\
\hline & & & & $\mathrm{H}$ & -1.811890 & -1.469580 & -2.531020 \\
\hline \multicolumn{2}{|c|}{ TSI-41-2Br } & & & $\mathrm{C}$ & -6.774340 & 0.532046 & -0.751753 \\
\hline $\mathrm{Ru}$ & -0.015191 & 0.191280 & 0.244477 & $\mathrm{H}$ & -6.879659 & 1.418499 & -0.118767 \\
\hline $\mathrm{C}$ & 2.387633 & 1.984887 & 0.126994 & $\mathrm{H}$ & -6.824647 & 0.857383 & -1.794820 \\
\hline $\mathrm{C}$ & 0.539719 & 3.337023 & 0.497900 & $\mathrm{H}$ & -7.649772 & -0.101405 & -0.566571 \\
\hline $\mathrm{C}$ & 3.212076 & 3.101228 & 0.105369 & $\mathrm{C}$ & 0.890170 & -2.957871 & -2.300974 \\
\hline $\mathrm{H}$ & 2.749946 & 0.974086 & -0.031098 & $\mathrm{H}$ & 1.308033 & -3.289338 & -3.255344 \\
\hline $\mathrm{C}$ & 1.316830 & 4.486736 & 0.481538 & $\mathrm{H}$ & 0.065903 & -3.635428 & -2.043600 \\
\hline
\end{tabular}




\begin{tabular}{|c|c|c|c|c|c|c|c|}
\hline $\mathrm{H}$ & 0.470185 & -1.958494 & -2.447984 & $\mathrm{H}$ & -0.499106 & 3.347723 & 0.480564 \\
\hline $\mathrm{C}$ & 2.208816 & -3.122234 & 2.571298 & $\mathrm{~N}$ & 1.065996 & 2.052399 & 0.138334 \\
\hline $\mathrm{H}$ & 1.192703 & -2.782362 & 2.768214 & $\mathrm{C}$ & 2.748244 & 4.270015 & 0.110573 \\
\hline $\mathrm{H}$ & 2.310791 & -4.160638 & 2.913947 & $\mathrm{H}$ & 3.412482 & 5.127606 & 0.101170 \\
\hline $\mathrm{H}$ & 2.872883 & -2.511515 & 3.188485 & $\mathrm{C}$ & -0.663297 & -1.787042 & 0.192592 \\
\hline $\mathrm{C}$ & 5.739422 & -3.112415 & -0.993911 & $\mathrm{C}$ & -1.998114 & -3.728181 & 0.317286 \\
\hline $\mathrm{H}$ & 6.355369 & -3.574722 & -0.217127 & $\mathrm{C}$ & -0.548938 & -4.138167 & 0.150136 \\
\hline $\mathrm{H}$ & 5.893652 & -3.667841 & -1.924094 & $\mathrm{H}$ & -2.378593 & -3.924283 & 1.332228 \\
\hline $\mathrm{H}$ & 6.135978 & -2.103671 & -1.158944 & $\mathrm{H}$ & -2.678835 & -4.210378 & -0.392118 \\
\hline $\mathrm{C}$ & -1.518428 & 0.832033 & -0.681527 & $\mathrm{H}$ & -0.209032 & -4.869564 & 0.890141 \\
\hline $\mathrm{H}$ & -1.638156 & 0.547153 & -1.742083 & $\mathrm{H}$ & -0.342489 & -4.543003 & -0.851275 \\
\hline $\mathrm{C}$ & -2.553268 & 1.786353 & -0.300585 & $\mathrm{~N}$ & -1.931995 & -2.283670 & 0.084991 \\
\hline $\mathrm{C}$ & -3.387926 & 2.323442 & -1.301700 & $\mathrm{~N}$ & 0.159585 & -2.864353 & 0.318105 \\
\hline C & -2.773283 & 2.217329 & 1.024088 & $\mathrm{C}$ & -3.145996 & -1.542377 & 0.116635 \\
\hline $\mathrm{C}$ & -4.376837 & 3.252751 & -1.002662 & $\mathrm{C}$ & -3.868145 & -1.423596 & -1.086132 \\
\hline $\mathrm{H}$ & -3.241195 & 1.985437 & -2.326715 & $\mathrm{C}$ & -3.626015 & -0.997481 & 1.313692 \\
\hline $\mathrm{C}$ & -3.768901 & 3.133224 & 1.329125 & $\mathrm{C}$ & -5.070010 & -0.723488 & -1.066674 \\
\hline $\mathrm{H}$ & -2.149624 & 1.791400 & 1.809256 & $\mathrm{C}$ & -4.833842 & -0.295368 & 1.278486 \\
\hline $\mathrm{C}$ & -4.574216 & 3.658108 & 0.315865 & $\mathrm{C}$ & -5.565639 & -0.140881 & 0.104666 \\
\hline $\mathrm{H}$ & -5.005460 & 3.652194 & -1.794784 & $\mathrm{H}$ & -5.624043 & -0.604408 & -1.997759 \\
\hline $\mathrm{H}$ & -3.927051 & 3.440038 & 2.360378 & $\mathrm{H}$ & -5.202293 & 0.160148 & 2.197742 \\
\hline $\mathrm{H}$ & -5.354340 & 4.376196 & 0.555529 & $\mathrm{C}$ & 1.586686 & -2.881979 & 0.178646 \\
\hline $\mathrm{O}$ & 1.522309 & -0.346692 & 1.790484 & $\mathrm{C}$ & 2.151901 & -2.919035 & -1.105596 \\
\hline $\mathrm{O}$ & 1.083217 & 0.016133 & -1.549495 & $\mathrm{C}$ & 2.379180 & -2.970287 & 1.331632 \\
\hline $\mathrm{C}$ & 0.572658 & 0.098435 & 2.521509 & $\mathrm{C}$ & 3.543458 & -2.942411 & -1.204255 \\
\hline $\mathrm{H}$ & -0.632221 & 0.434611 & 1.819697 & $\mathrm{C}$ & 3.763764 & -2.999560 & 1.179299 \\
\hline $\mathrm{C}$ & 0.776743 & 0.755980 & -2.619190 & $\mathrm{C}$ & 4.365781 & -2.960497 & -0.078426 \\
\hline $\mathrm{H}$ & 0.318081 & 1.747175 & -2.367394 & $\mathrm{H}$ & 3.994933 & -2.946982 & -2.195976 \\
\hline $\mathrm{H}$ & 1.684622 & 0.959809 & -3.228678 & $\mathrm{H}$ & 4.389069 & -3.041724 & 2.070424 \\
\hline $\mathrm{H}$ & 0.853405 & 5.458081 & 0.618413 & $\mathrm{C}$ & -2.883334 & -1.118315 & 2.609026 \\
\hline $\mathrm{Br}$ & 5.108607 & 2.859258 & -0.189702 & $\mathrm{H}$ & -2.620143 & -0.125468 & 2.995386 \\
\hline $\mathrm{O}$ & -0.169857 & 0.044531 & -3.462880 & $\mathrm{H}$ & -3.499647 & -1.603183 & 3.374367 \\
\hline $\mathrm{H}$ & -0.235537 & 0.528977 & -4.292814 & $\mathrm{H}$ & -1.951480 & -1.681075 & 2.512204 \\
\hline $\mathrm{H}$ & 0.685693 & 1.101171 & 2.992519 & $\mathrm{C}$ & -3.316524 & -1.991466 & -2.356757 \\
\hline $\mathrm{O}$ & 0.043860 & -0.826378 & 3.435591 & $\mathrm{H}$ & -3.157098 & -3.074558 & -2.289182 \\
\hline \multirow[t]{2}{*}{$\mathrm{H}$} & -0.560992 & -0.352781 & 4.018821 & $\mathrm{H}$ & -3.998166 & -1.810759 & -3.192204 \\
\hline & & & & $\mathrm{H}$ & -2.340683 & -1.553909 & -2.605402 \\
\hline \multicolumn{2}{|c|}{ TSI-51-2Br } & & & $\mathrm{C}$ & -6.850826 & 0.627948 & 0.090007 \\
\hline $\mathrm{Ru}$ & -0.045007 & 0.138369 & 0.184958 & $\mathrm{H}$ & -6.989261 & 1.197455 & 1.013206 \\
\hline $\mathrm{C}$ & 2.383029 & 1.891528 & -0.068720 & $\mathrm{H}$ & -6.880034 & 1.336166 & -0.744445 \\
\hline $\mathrm{C}$ & 0.574346 & 3.284077 & 0.321403 & $\mathrm{H}$ & -7.717220 & -0.033971 & -0.023331 \\
\hline $\mathrm{C}$ & 3.236157 & 2.985547 & -0.079956 & $\mathrm{C}$ & 1.316267 & -3.003530 & -2.344608 \\
\hline $\mathrm{H}$ & 2.720926 & 0.873692 & -0.235989 & $\mathrm{H}$ & 1.776140 & -2.446050 & -3.163058 \\
\hline $\mathrm{C}$ & 1.378674 & 4.414450 & 0.311543 & $\mathrm{H}$ & 1.221685 & -4.048476 & -2.672707 \\
\hline
\end{tabular}




\begin{tabular}{|c|c|c|c|c|c|c|c|}
\hline $\mathrm{H}$ & 0.319560 & -2.580112 & -2.214883 & $\mathrm{H}$ & 2.591098 & -1.098338 & -0.870375 \\
\hline $\mathrm{C}$ & 1.781885 & -3.054576 & 2.702191 & $\mathrm{H}$ & 2.489056 & -2.715181 & 3.753347 \\
\hline $\mathrm{H}$ & 0.719264 & -2.801105 & 2.698327 & $\mathrm{~N}$ & 1.553355 & -1.152358 & 0.923986 \\
\hline $\mathrm{H}$ & 1.885783 & -4.065461 & 3.116597 & $\mathrm{C}$ & 3.646449 & -2.705956 & 1.933931 \\
\hline $\mathrm{H}$ & 2.285078 & -2.366758 & 3.386518 & $\mathrm{H}$ & 4.462148 & -3.311178 & 2.314716 \\
\hline $\mathrm{C}$ & 5.857438 & -2.923260 & -0.214638 & $\mathrm{C}$ & -2.054769 & 0.594178 & -0.410966 \\
\hline $\mathrm{H}$ & 6.353708 & -3.468717 & 0.593485 & $\mathrm{C}$ & -3.758413 & 2.008198 & -1.250536 \\
\hline $\mathrm{H}$ & 6.189158 & -3.349941 & -1.165581 & $\mathrm{C}$ & -4.360002 & 0.653248 & -0.928058 \\
\hline $\mathrm{H}$ & 6.230690 & -1.892963 & -0.178899 & $\mathrm{H}$ & -4.279930 & 2.840633 & -0.767149 \\
\hline $\mathrm{C}$ & -1.563933 & 0.731865 & -0.741787 & $\mathrm{H}$ & -3.731950 & 2.212047 & -2.330702 \\
\hline $\mathrm{H}$ & -1.720573 & 0.346273 & -1.764586 & $\mathrm{H}$ & -5.021530 & 0.677847 & -0.048133 \\
\hline $\mathrm{C}$ & -2.581535 & 1.725006 & -0.419733 & $\mathrm{H}$ & -4.929615 & 0.219867 & -1.756276 \\
\hline $\mathrm{C}$ & -3.448966 & 2.160148 & -1.442027 & $\mathrm{~N}$ & -2.390946 & 1.885092 & -0.725201 \\
\hline $\mathrm{C}$ & -2.754563 & 2.291411 & 0.859314 & $\mathrm{~N}$ & -3.172817 & -0.156453 & -0.634871 \\
\hline $\mathrm{C}$ & -4.425154 & 3.120535 & -1.209445 & $\mathrm{C}$ & -1.701654 & 3.072920 & -0.327448 \\
\hline $\mathrm{H}$ & -3.338410 & 1.715515 & -2.430481 & $\mathrm{C}$ & -0.999869 & 3.839471 & -1.268927 \\
\hline $\mathrm{C}$ & -3.737529 & 3.240870 & 1.099963 & $\mathrm{C}$ & -1.849982 & 3.515995 & 1.000359 \\
\hline $\mathrm{H}$ & -2.100456 & 1.949388 & 1.661131 & $\mathrm{C}$ & -0.331919 & 4.983470 & -0.826367 \\
\hline $\mathrm{C}$ & -4.575011 & 3.662910 & 0.065673 & $\mathrm{C}$ & -1.175497 & 4.670952 & 1.390969 \\
\hline $\mathrm{H}$ & -5.077412 & 3.440956 & -2.018144 & $\mathrm{C}$ & -0.386070 & 5.402982 & 0.501304 \\
\hline $\mathrm{H}$ & -3.858901 & 3.655817 & 2.097616 & $\mathrm{H}$ & 0.240277 & 5.566220 & -1.547593 \\
\hline $\mathrm{H}$ & -5.344992 & 4.406031 & 0.255990 & $\mathrm{H}$ & -1.271697 & 5.008635 & 2.422763 \\
\hline $\mathrm{O}$ & 1.525007 & -0.324974 & 1.722665 & C & -3.379643 & -1.530793 & -0.301889 \\
\hline $\mathrm{O}$ & 1.030125 & -0.170004 & -1.607214 & $\mathrm{C}$ & -3.538232 & -2.461387 & -1.337825 \\
\hline $\mathrm{C}$ & 0.602963 & 0.161593 & 2.456588 & $\mathrm{C}$ & -3.466868 & -1.910979 & 1.043460 \\
\hline $\mathrm{H}$ & 0.075104 & -0.518267 & 3.163195 & $\mathrm{C}$ & -3.777344 & -3.792633 & -0.997471 \\
\hline $\mathrm{H}$ & -0.651674 & 0.464390 & 1.740951 & $\mathrm{C}$ & -3.684052 & -3.259503 & 1.335868 \\
\hline $\mathrm{C}$ & 0.638174 & 0.388166 & -2.754131 & $\mathrm{C}$ & -3.845203 & -4.215127 & 0.333545 \\
\hline $\mathrm{H}$ & 0.171769 & 1.400325 & -2.630347 & $\mathrm{H}$ & -3.893377 & -4.524260 & -1.797203 \\
\hline $\mathrm{H}$ & 1.502704 & 0.503675 & -3.444522 & $\mathrm{H}$ & -3.743677 & -3.565100 & 2.379799 \\
\hline $\mathrm{H}$ & 0.940253 & 5.395740 & 0.459828 & $\mathrm{C}$ & -2.719290 & 2.773958 & 1.966303 \\
\hline $\mathrm{Br}$ & 5.128338 & 2.692089 & -0.369994 & $\mathrm{H}$ & -2.828078 & 3.333158 & 2.899446 \\
\hline $\mathrm{O}$ & -0.342934 & -0.455919 & -3.425002 & $\mathrm{H}$ & -3.725182 & 2.604442 & 1.561302 \\
\hline $\mathrm{H}$ & -0.600505 & 0.004573 & -4.231581 & $\mathrm{H}$ & -2.281482 & 1.795974 & 2.196866 \\
\hline $\mathrm{O}$ & 0.880345 & 1.392153 & 3.051249 & $\mathrm{C}$ & -0.980934 & 3.496869 & -2.728051 \\
\hline \multirow[t]{2}{*}{$\mathrm{H}$} & 0.182310 & 1.576207 & 3.690629 & $\mathrm{H}$ & -1.571646 & 4.220455 & -3.302449 \\
\hline & & & & $\mathrm{H}$ & 0.036868 & 3.531280 & -3.130132 \\
\hline \multicolumn{2}{|c|}{ OI-Br' } & & & $\mathrm{H}$ & -1.388519 & 2.503422 & -2.928443 \\
\hline $\mathrm{Ru}$ & -0.279627 & -0.132261 & 0.187512 & $\mathrm{C}$ & 0.379855 & 6.604725 & 0.962419 \\
\hline $\mathrm{C}$ & 1.533845 & -1.595921 & 2.192489 & $\mathrm{H}$ & -0.129066 & 7.120497 & 1.781675 \\
\hline $\mathrm{C}$ & 2.604555 & -1.467642 & 0.150446 & $\mathrm{H}$ & 1.372886 & 6.322977 & 1.331863 \\
\hline $\mathrm{C}$ & 2.550274 & -2.376759 & 2.724329 & $\mathrm{H}$ & 0.534389 & 7.323651 & 0.153094 \\
\hline $\mathrm{H}$ & 0.682783 & -1.272241 & 2.783509 & $\mathrm{C}$ & -3.318604 & -2.073557 & -2.763880 \\
\hline $\mathrm{C}$ & 3.658445 & -2.226425 & 0.631071 & $\mathrm{H}$ & -3.759863 & -2.802047 & -3.448768 \\
\hline
\end{tabular}




\begin{tabular}{|c|c|c|c|}
\hline $\mathrm{H}$ & -3.718482 & -1.084349 & -3.004728 \\
\hline $\mathrm{H}$ & -2.238196 & -2.043223 & -2.948043 \\
\hline & -3.324041 & -0.909365 & 2.146934 \\
\hline H & -3.931628 & -0.016209 & 1.963140 \\
\hline $\mathrm{H}$ & -3.636309 & -1.336117 & 3.103363 \\
\hline $\mathrm{H}$ & -2.294332 & -0.548629 & 2.266807 \\
\hline $\mathrm{C}$ & -4.122518 & -5.648501 & 0.670925 \\
\hline $\mathrm{H}$ & -3.655219 & -6.331495 & -0.044403 \\
\hline $\mathrm{H}$ & -3.760180 & -5.905674 & 1.669849 \\
\hline $\mathrm{H}$ & -5.197890 & -5.861471 & 0.653996 \\
\hline $\mathrm{C}$ & 0.591865 & 1.051540 & -0.936949 \\
\hline $\mathrm{H}$ & 0.000214 & 1.426373 & -1.781600 \\
\hline $\mathrm{C}$ & 1.985489 & 1.466466 & -1.030670 \\
\hline $\mathrm{C}$ & 2.481893 & 1.866222 & -2.289593 \\
\hline $\mathrm{C}$ & 2.867159 & 1.523325 & 0.068560 \\
\hline $\mathrm{C}$ & 3.802471 & 2.262721 & -2.454947 \\
\hline $\mathrm{H}$ & 1.810741 & 1.835572 & -3.146784 \\
\hline $\mathrm{C}$ & 4.184200 & 1.930086 & -0.099831 \\
\hline $\mathrm{H}$ & 2.507153 & 1.242338 & 1.056201 \\
\hline $\mathrm{C}$ & 4.662338 & 2.294706 & -1.358547 \\
\hline $\mathrm{H}$ & 4.162762 & 2.548972 & -3.439762 \\
\hline $\mathrm{H}$ & 4.848348 & 1.964203 & 0.760642 \\
\hline $\mathrm{H}$ & 5.695374 & 2.608544 & -1.482257 \\
\hline $\mathrm{O}$ & -0.394096 & 0.764982 & 2.031682 \\
\hline $\mathrm{O}$ & -0.688538 & -1.781268 & -0.868275 \\
\hline $\mathrm{C}$ & 0.431438 & 1.722149 & 2.501231 \\
\hline $\mathrm{H}$ & -0.090022 & 2.307118 & 3.291143 \\
\hline $\mathrm{H}$ & 0.750849 & 2.433506 & 1.709874 \\
\hline $\mathrm{C}$ & 0.174025 & -2.781329 & -1.253029 \\
\hline $\mathrm{H}$ & 0.962856 & -2.423411 & -1.941224 \\
\hline $\mathrm{H}$ & 0.676899 & -3.250249 & -0.378606 \\
\hline $\mathrm{Br}$ & 5.146521 & -2.604956 & -0.548096 \\
\hline $\mathrm{O}$ & 1.609862 & 1.129551 & 3.087933 \\
\hline $\mathrm{H}$ & 2.179148 & 1.856722 & 3.364077 \\
\hline $\mathrm{O}$ & -0.533469 & -3.758088 & -1.980937 \\
\hline $\mathrm{H}$ & -1.304215 & -3.967562 & -1.435546 \\
\hline
\end{tabular}

TSI' ${ }_{0-1 B r}$

$\begin{array}{rrrr}\mathrm{Ru} & -0.263818 & -0.057304 & 0.312055 \\ \mathrm{C} & 1.386278 & -1.781550 & 2.327302 \\ \mathrm{C} & 2.483318 & -1.717819 & 0.295746 \\ \mathrm{C} & 2.306130 & -2.687171 & 2.840221 \\ \mathrm{H} & 0.561380 & -1.389494 & 2.914363 \\ \mathrm{C} & 3.445694 & -2.597928 & 0.761962\end{array}$

\begin{tabular}{|c|c|c|c|}
\hline $\mathrm{H}$ & 2.504998 & -1.344164 & -0.722990 \\
\hline $\mathrm{H}$ & 2.192818 & -3.053476 & 3.855302 \\
\hline $\mathrm{N}$ & 1.472044 & -1.301224 & 1.074649 \\
\hline${ }^{2}$ & 3.370688 & -3.106686 & 2.051242 \\
\hline $\mathrm{H}$ & 4.113174 & -3.805725 & 2.421224 \\
\hline $\mathrm{C}$ & -1.933920 & 0.848525 & -0.336500 \\
\hline C & -3.352972 & 2.515296 & -1.259563 \\
\hline $\mathrm{C}$ & -4.155382 & 1.242903 & -1.049160 \\
\hline $\mathrm{H}$ & -3.772886 & 3.381538 & -0.735548 \\
\hline $\mathrm{H}$ & -3.253380 & 2.788431 & -2.319171 \\
\hline $\mathrm{H}$ & -4.928343 & 1.344482 & -0.273194 \\
\hline $\mathrm{H}$ & -4.650976 & 0.888767 & -1.959268 \\
\hline $\mathrm{N}$ & -2.041519 & 2.165691 & -0.701923 \\
\hline $\mathrm{N}$ & -3.138343 & 0.280215 & -0.611541 \\
\hline $\mathrm{C}$ & -1.180340 & 3.223899 & -0.281146 \\
\hline $\mathrm{C}$ & -0.299500 & 3.830865 & -1.187014 \\
\hline $\mathrm{C}$ & -1.325891 & 3.709082 & 1.031496 \\
\hline $\mathrm{C}$ & 0.531200 & 4.849722 & -0.713372 \\
\hline $\mathrm{C}$ & -0.486446 & 4.738937 & 1.452290 \\
\hline $\mathrm{C}$ & 0.469240 & 5.302578 & 0.603289 \\
\hline $\mathrm{H}$ & 1.237318 & 5.310487 & -1.403346 \\
\hline $\mathrm{H}$ & -0.583687 & 5.111741 & 2.471835 \\
\hline $\mathrm{C}$ & -3.580209 & -1.058352 & -0.379549 \\
\hline $\mathrm{C}$ & -3.699631 & -1.919284 & -1.477105 \\
\hline $\mathrm{C}$ & -3.926353 & -1.455675 & 0.915731 \\
\hline $\mathrm{C}$ & -4.176069 & -3.209215 & -1.250858 \\
\hline $\mathrm{C}$ & -4.392023 & -2.760529 & 1.095649 \\
\hline $\mathrm{C}$ & -4.527181 & -3.649453 & 0.029329 \\
\hline $\mathrm{H}$ & -4.255557 & -3.894653 & -2.094694 \\
\hline $\mathrm{H}$ & -4.659225 & -3.087277 & 2.100010 \\
\hline $\mathrm{C}$ & -2.356999 & 3.126890 & 1.949394 \\
\hline $\mathrm{H}$ & -2.396035 & 3.680629 & 2.891058 \\
\hline $\mathrm{H}$ & -3.359509 & 3.159024 & 1.504413 \\
\hline $\mathrm{H}$ & -2.144133 & 2.075388 & 2.182263 \\
\hline $\mathrm{C}$ & -0.261170 & 3.469666 & -2.640036 \\
\hline $\mathrm{H}$ & -0.518338 & 4.339122 & -3.255324 \\
\hline $\mathrm{H}$ & 0.741558 & 3.150676 & -2.942803 \\
\hline $\mathrm{H}$ & -0.955169 & 2.663460 & -2.885082 \\
\hline $\mathrm{C}$ & 1.397615 & 6.371203 & 1.094084 \\
\hline $\mathrm{H}$ & 0.963535 & 6.934012 & 1.925039 \\
\hline $\mathrm{H}$ & 2.340483 & 5.943431 & 1.454184 \\
\hline $\mathrm{H}$ & 1.655523 & 7.080071 & 0.302311 \\
\hline $\mathrm{C}$ & -3.223872 & -1.489163 & -2.827314 \\
\hline $\mathrm{H}$ & -3.267920 & -2.312764 & -3.542915 \\
\hline
\end{tabular}




$\begin{array}{cccc}\mathrm{H} & -3.812491 & -0.658119 & -3.235711 \\ \mathrm{H} & -2.182485 & -1.155638 & -2.761680 \\ \mathrm{C} & -3.774691 & -0.520391 & 2.073519 \\ \mathrm{H} & -4.270609 & 0.440112 & 1.887340 \\ \mathrm{H} & -4.207147 & -0.947193 & 2.981812 \\ \mathrm{H} & -2.722538 & -0.286345 & 2.276702 \\ \mathrm{C} & -5.063637 & -5.032447 & 0.243018 \\ \mathrm{H} & -4.629994 & -5.748944 & -0.460527 \\ \mathrm{H} & -4.865318 & -5.391574 & 1.256565 \\ \mathrm{H} & -6.150028 & -5.066048 & 0.098496 \\ \mathrm{C} & 0.621400 & 0.620056 & -1.201493 \\ \mathrm{H} & 0.038482 & 0.883847 & -2.097752 \\ \mathrm{C} & 2.008603 & 1.028059 & -1.323088 \\ \mathrm{C} & 2.556692 & 1.223926 & -2.612247 \\ \mathrm{C} & 2.860497 & 1.257913 & -0.217704 \\ \mathrm{C} & 3.884496 & 1.584971 & -2.789529 \\ \mathrm{H} & 1.915227 & 1.061485 & -3.477436 \\ \mathrm{C} & 4.182023 & 1.643157 & -0.401299 \\ \mathrm{H} & 2.478725 & 1.113814 & 0.790505 \\ \mathrm{C} & 4.707101 & 1.800022 & -1.683242 \\ \mathrm{H} & 4.281107 & 1.705860 & -3.794403 \\ \mathrm{H} & 4.814072 & 1.815303 & 0.466690 \\ \mathrm{H} & 5.744975 & 2.090617 & -1.819841 \\ \mathrm{O} & -0.594651 & 0.429531 & 2.407638 \\ \mathrm{O} & -0.955724 & -1.736260 & -0.449371 \\ \mathrm{C} & 0.341096 & 1.394419 & 2.425633 \\ \mathrm{H} & -0.013113 & 2.338006 & 2.897531 \\ \mathrm{H} & 0.637137 & 1.716816 & 1.368970 \\ \mathrm{C} & -0.148750 & -2.664201 & -1.078567 \\ \mathrm{H} & 0.554296 & -2.178377 & -1.782972 \\ \mathrm{H} & 0.448999 & -3.247059 & -0.345450 \\ \mathrm{Br} & 4.891823 & -3.109172 & -0.420345 \\ \mathrm{O} & 1.527074 & 0.967095 & 3.097211 \\ \mathrm{H} & 2.063163 & 1.752542 & 3.252609 \\ \mathrm{O} & -0.948540 & -3.522682 & -1.848878 \\ \mathrm{H} & -1.686637 & -3.768002 & -1.275259\end{array}$

\begin{tabular}{|c|c|c|c|}
\hline $\mathrm{H}$ & -2.572486 & -1.642135 & 0.050880 \\
\hline $\mathrm{H}$ & 0.697785 & -5.279147 & -0.243956 \\
\hline$N$ & -0.572114 & -2.169608 & -0.070845 \\
\hline C & -1.408202 & -4.831723 & -0.145757 \\
\hline $\mathrm{H}$ & -1.743634 & -5.862756 & -0.178747 \\
\hline $\mathrm{C}$ & 1.515510 & 1.496733 & -0.272396 \\
\hline $\mathrm{C}$ & 2.265226 & 3.701627 & -0.657452 \\
\hline $\mathrm{C}$ & 3.465182 & 2.805842 & -0.427132 \\
\hline $\mathrm{H}$ & 2.163680 & 4.008705 & -1.710107 \\
\hline $\mathrm{H}$ & 2.267335 & 4.609389 & -0.045112 \\
\hline $\mathrm{H}$ & 4.237037 & 2.894091 & -1.197893 \\
\hline $\mathrm{H}$ & 3.937635 & 2.982337 & 0.551093 \\
\hline $\mathrm{N}$ & 1.153596 & 2.818725 & -0.289640 \\
\hline $\mathrm{N}$ & 2.864033 & 1.468408 & -0.445735 \\
\hline $\mathrm{C}$ & -0.165528 & 3.356247 & -0.273886 \\
\hline $\mathrm{C}$ & -0.599729 & 3.979647 & 0.911085 \\
\hline $\mathrm{C}$ & -0.987630 & 3.305228 & -1.407068 \\
\hline $\mathrm{C}$ & -1.890521 & 4.495633 & 0.956121 \\
\hline $\mathrm{C}$ & -2.276648 & 3.834921 & -1.311388 \\
\hline $\mathrm{C}$ & -2.753145 & 4.420108 & -0.141367 \\
\hline $\mathrm{H}$ & -2.245228 & 4.945148 & 1.883110 \\
\hline $\mathrm{H}$ & -2.934641 & 3.766557 & -2.177661 \\
\hline $\mathrm{C}$ & 3.722178 & 0.354602 & -0.167007 \\
\hline $\mathrm{C}$ & 4.040568 & 0.056487 & 1.166250 \\
\hline $\mathrm{C}$ & 4.291682 & -0.351217 & -1.237254 \\
\hline $\mathrm{C}$ & 4.890995 & -1.024348 & 1.407456 \\
\hline $\mathrm{C}$ & 5.131068 & -1.424268 & -0.942302 \\
\hline $\mathrm{C}$ & 5.434291 & -1.782793 & 0.372409 \\
\hline $\mathrm{H}$ & 5.130303 & -1.279366 & 2.439903 \\
\hline $\mathrm{H}$ & 5.561220 & -1.994279 & -1.765440 \\
\hline $\mathrm{C}$ & -0.534806 & 2.699885 & -2.699766 \\
\hline $\mathrm{H}$ & -1.199650 & 1.881967 & -3.003142 \\
\hline $\mathrm{H}$ & -0.558285 & 3.439362 & -3.507944 \\
\hline $\mathrm{H}$ & 0.477619 & 2.292972 & -2.643207 \\
\hline $\mathrm{C}$ & 0.292622 & 4.032800 & 2.112170 \\
\hline $\mathrm{H}$ & 1.207857 & 4.607312 & 1.923796 \\
\hline $\mathrm{H}$ & -0.217026 & 4.494417 & 2.961230 \\
\hline $\mathrm{H}$ & 0.620499 & 3.031267 & 2.414519 \\
\hline $\mathrm{C}$ & -4.147025 & 4.962426 & -0.064338 \\
\hline $\mathrm{H}$ & -4.192759 & 6.005334 & -0.399648 \\
\hline $\mathrm{H}$ & -4.834442 & 4.389623 & -0.693843 \\
\hline $\mathrm{H}$ & -4.531050 & 4.935889 & 0.959417 \\
\hline $\mathrm{C}$ & 3.533299 & 0.863712 & 2.322264 \\
\hline I & 3.186192 & 0.202904 & 3.120762 \\
\hline
\end{tabular}




$\begin{array}{rrrc}\mathrm{H} & 4.326017 & 1.506538 & 2.727939 \\ \mathrm{H} & 2.685063 & 1.496377 & 2.049224 \\ \mathrm{C} & 4.049533 & 0.031811 & -2.664961 \\ \mathrm{H} & 3.227564 & 0.743480 & -2.760842 \\ \mathrm{H} & 4.949207 & 0.479556 & -3.106423 \\ \mathrm{H} & 3.788377 & -0.845337 & -3.261396 \\ \mathrm{C} & 6.348440 & -2.936111 & 0.657880 \\ \mathrm{H} & 7.386891 & -2.700127 & 0.397970 \\ \mathrm{H} & 6.333398 & -3.211209 & 1.715779 \\ \mathrm{H} & 6.075275 & -3.822520 & 0.076660 \\ \mathrm{C} & -0.908081 & 0.817949 & 0.861377 \\ \mathrm{H} & -0.562715 & 1.404854 & 1.733485 \\ \mathrm{C} & -2.349175 & 0.961762 & 0.712159 \\ \mathrm{C} & -3.121479 & 1.443562 & 1.788829 \\ \mathrm{C} & -3.026200 & 0.660042 & -0.487308 \\ \mathrm{C} & -4.500408 & 1.575565 & 1.688623 \\ \mathrm{H} & -2.609498 & 1.709433 & 2.713023 \\ \mathrm{C} & -4.400784 & 0.813198 & -0.598270 \\ \mathrm{H} & -2.436031 & 0.315341 & -1.337301 \\ \mathrm{C} & -5.146243 & 1.262039 & 0.493273 \\ \mathrm{H} & -5.074570 & 1.934206 & 2.539215 \\ \mathrm{H} & -4.898375 & 0.581765 & -1.536865 \\ \mathrm{H} & -6.223496 & 1.376065 & 0.407979 \\ \mathrm{O} & 1.505999 & -1.074522 & -1.797772 \\ \mathrm{O} & 1.421657 & -0.852045 & 1.452803 \\ \mathrm{C} & 0.505820 & -0.655664 & -2.508722 \\ \mathrm{H} & 0.737521 & 0.108084 & -3.291823 \\ \mathrm{H} & -0.304164 & 0.025622 & -1.840212 \\ \mathrm{C} & 0.751424 & -1.119794 & 2.613087 \\ \mathrm{H} & 0.321507 & -0.207577 & 3.080785 \\ \mathrm{H} & -0.091866 & -1.834070 & 2.463110 \\ \mathrm{H} & -4.222339 & -4.131025 & 0.001700 \\ \mathrm{H} & -0.285128 & -1.686078 & -3.061535 \\ & 1.665678 & -1.642241 & 3.561805 \\ & & -1.298500 & -3.745312 \\ & & \\ \mathrm{H}\end{array}$

\begin{tabular}{|c|c|c|c|}
\hline & 4981 & & \\
\hline & 0.612446 & 5.323 & \\
\hline & 603 & 10 & 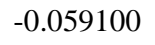 \\
\hline & -1.486999 & -4.835 & \\
\hline & .84 & 9 & \\
\hline & 1.50 & 09 & 34 \\
\hline & & & \\
\hline & 3.44 & & \\
\hline & 2.11 & 86 & \\
\hline & 2.25 & & \\
\hline & 4.206239 & 2.854052 & \\
\hline & & & \\
\hline & 1.14 & 783045 & -0.3574 \\
\hline & 2.852367 & 08 & \\
\hline & -0.17 & & \\
\hline & -0.58 & & \\
\hline & -1.009 & 1 & \\
\hline & -1.86 & & \\
\hline & -2.28 & & \\
\hline & -2.74 & 34 & \\
\hline & -2.20 & & \\
\hline & -2.9 & & \\
\hline & 3. & & \\
\hline & 4.009 & 6 & \\
\hline & 4.345453 & -0.37 & \\
\hline & 4.89 & & \\
\hline & 5.21 & $-1.40 s$ & \\
\hline & 5.494118 & -17 & 67 \\
\hline & 5.10 & & \\
\hline & & & \\
\hline & -0.57 & 2630 & \\
\hline & -1.218 & 1 & -2.9916 \\
\hline & -0.654112 & $\mathrm{J4}$ & \\
\hline & & & \\
\hline & 328 & 4 & \\
\hline & 4 & & 19 \\
\hline & -0.164 & 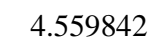 & 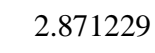 \\
\hline & 0.607 & & \\
\hline & -4.1231 & 5.013163 & 1140 \\
\hline & -4.160 & 3 & -0.534 \\
\hline & -4.841877 & 4.406693 & -0.673 \\
\hline & -4.4741 & 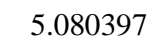 & \\
\hline & & & \\
\hline & 157005 & 28910 & \\
\hline
\end{tabular}

TSI' ${ }_{1-2 B r}$

$\begin{array}{rrrr}\mathrm{Ru} & 0.347635 & -0.179319 & -0.155981 \\ \mathrm{C} & 0.276645 & -3.205899 & -0.090618 \\ \mathrm{C} & -1.913606 & -2.465077 & -0.055326 \\ \mathrm{C} & -0.129575 & -4.532037 & -0.124689 \\ \mathrm{H} & 1.320962 & -2.911331 & -0.084741 \\ \mathrm{C} & -2.376304 & -3.771224 & -0.092686\end{array}$




\begin{tabular}{|c|c|c|c|c|c|c|c|}
\hline $\mathrm{H}$ & 4.184763 & 1.655376 & 2.631176 & $\mathrm{H}$ & -2.526805 & -1.638019 & -0.268981 \\
\hline $\mathrm{H}$ & 2.547184 & 1.476065 & 1.968695 & $\mathrm{H}$ & 0.492531 & -5.456881 & 0.310967 \\
\hline $\mathrm{C}$ & 4.124296 & -0.060494 & -2.669795 & $\mathrm{~N}$ & -0.589713 & -2.288402 & 0.061070 \\
\hline $\mathrm{H}$ & 3.290346 & 0.629839 & -2.809778 & $\mathrm{C}$ & -1.555951 & -4.893959 & -0.047514 \\
\hline $\mathrm{H}$ & 5.022031 & 0.387458 & -3.114152 & $\mathrm{H}$ & -1.941875 & -5.906496 & -0.099058 \\
\hline $\mathrm{H}$ & 3.894599 & -0.966575 & -3.236195 & $\mathrm{C}$ & 1.351663 & 1.441051 & -0.479891 \\
\hline $\mathrm{C}$ & 6.439573 & -2.819204 & 0.818124 & $\mathrm{C}$ & 1.860421 & 3.660739 & -1.104067 \\
\hline $\mathrm{H}$ & 7.472815 & -2.566921 & 0.553086 & $\mathrm{C}$ & 3.152394 & 2.889460 & -0.932752 \\
\hline $\mathrm{H}$ & 6.422927 & -3.039797 & 1.888707 & $\mathrm{H}$ & 1.646712 & 3.907664 & -2.155038 \\
\hline $\mathrm{H}$ & 6.196541 & -3.742293 & 0.281902 & $\mathrm{H}$ & 1.824493 & 4.594948 & -0.533043 \\
\hline $\mathrm{C}$ & -0.870762 & 0.806140 & 0.873195 & $\mathrm{H}$ & 3.825925 & 2.956027 & -1.793324 \\
\hline $\mathrm{H}$ & -0.466832 & 1.372190 & 1.734205 & $\mathrm{H}$ & 3.713264 & 3.203517 & -0.041065 \\
\hline $\mathrm{C}$ & -2.314411 & 0.980658 & 0.802923 & $\mathrm{~N}$ & 0.863720 & 2.706272 & -0.608752 \\
\hline $\mathrm{C}$ & -3.009111 & 1.473862 & 1.926692 & $\mathrm{~N}$ & 2.685907 & 1.507140 & -0.746541 \\
\hline $\mathrm{C}$ & -3.067334 & 0.707605 & -0.356735 & $\mathrm{C}$ & -0.492448 & 3.141898 & -0.574558 \\
\hline $\mathrm{C}$ & -4.386654 & 1.645817 & 1.907481 & $\mathrm{C}$ & -0.946832 & 3.759821 & 0.603450 \\
\hline $\mathrm{H}$ & -2.437204 & 1.718919 & 2.820921 & $\mathrm{C}$ & -1.295403 & 3.078028 & -1.719664 \\
\hline $\mathrm{C}$ & -4.442218 & 0.893269 & -0.383955 & $\mathrm{C}$ & -2.233327 & 4.287116 & 0.623457 \\
\hline $\mathrm{H}$ & -2.531784 & 0.363898 & -1.241896 & $\mathrm{C}$ & -2.5776661 & 3.632221 & -1.655823 \\
\hline C & -5.110032 & 1.354828 & 0.751544 & $\mathrm{C}$ & -3.066007 & 4.234620 & -0.498612 \\
\hline $\mathrm{H}$ & -4.899418 & 2.017525 & 2.791082 & $\mathrm{H}$ & -2.601522 & 4.741990 & 1.542564 \\
\hline $\mathrm{H}$ & -4.999988 & 0.681543 & -1.292921 & $\mathrm{H}$ & -3.214474 & 3.584564 & -2.539093 \\
\hline $\mathrm{H}$ & -6.187019 & 1.498776 & 0.730249 & $\mathrm{C}$ & 3.690308 & 0.569726 & -0.331822 \\
\hline $\mathrm{O}$ & 1.539181 & -1.161244 & -1.799698 & $\mathrm{C}$ & 4.007440 & 0.467860 & 1.029604 \\
\hline $\mathrm{O}$ & 1.446755 & -0.936553 & 1.430319 & $\mathrm{C}$ & 4.399243 & -0.141658 & -1.308194 \\
\hline $\mathrm{C}$ & 0.563493 & -0.742752 & -2.493505 & $\mathrm{C}$ & 5.038661 & -0.399204 & 1.396148 \\
\hline $\mathrm{H}$ & 0.721158 & 0.105217 & -3.194741 & $\mathrm{C}$ & 5.417717 & -0.997216 & -0.892094 \\
\hline $\mathrm{H}$ & -0.412669 & 0.049900 & -1.683130 & $\mathrm{C}$ & 5.749479 & -1.142840 & 0.455706 \\
\hline $\mathrm{C}$ & 0.808794 & -1.169934 & 2.609793 & $\mathrm{H}$ & 5.278204 & -0.501132 & 2.454275 \\
\hline $\mathrm{H}$ & 0.381135 & -0.246367 & 3.062053 & $\mathrm{H}$ & 5.962666 & -1.568742 & -1.643010 \\
\hline $\mathrm{H}$ & -0.036534 & -1.892752 & 2.505155 & $\mathrm{C}$ & -0.830260 & 2.418871 & -2.981559 \\
\hline $\mathrm{Br}$ & -4.290954 & -4.083790 & -0.088750 & $\mathrm{H}$ & -1.306604 & 1.437361 & -3.099394 \\
\hline $\mathrm{O}$ & -0.266135 & -1.713028 & -3.035598 & $\mathrm{H}$ & -1.089662 & 3.014197 & -3.862420 \\
\hline $\mathrm{H}$ & -0.895830 & -1.278524 & -3.623035 & $\mathrm{H}$ & 0.249082 & 2.242598 & -2.989138 \\
\hline $\mathrm{O}$ & 1.742393 & -1.662091 & 3.561464 & $\mathrm{C}$ & -0.072979 & 3.802885 & 1.818149 \\
\hline \multirow[t]{2}{*}{$\mathrm{H}$} & 2.350982 & -2.185992 & 3.026599 & $\mathrm{H}$ & 0.873757 & 4.324578 & 1.632696 \\
\hline & & & & $\mathrm{H}$ & -0.575953 & 4.304625 & 2.648302 \\
\hline \multicolumn{2}{|c|}{ 2I-Br' } & & & $\mathrm{H}$ & 0.198964 & 2.792434 & 2.146573 \\
\hline $\mathrm{Ru}$ & 0.319188 & -0.276022 & -0.103901 & $\mathrm{C}$ & -4.457146 & 4.783949 & -0.438749 \\
\hline $\mathrm{C}$ & 0.244322 & -3.326705 & 0.225911 & $\mathrm{H}$ & -4.469844 & 5.824996 & -0.100315 \\
\hline $\mathrm{C}$ & -1.893165 & -2.512081 & -0.144867 & $\mathrm{H}$ & -4.952130 & 4.741238 & -1.412580 \\
\hline $\mathrm{C}$ & -0.208038 & -4.639260 & 0.176622 & $\mathrm{H}$ & -5.070517 & 4.214228 & 0.270029 \\
\hline $\mathrm{H}$ & 1.276887 & -3.041825 & 0.413013 & $\mathrm{C}$ & 3.305496 & 1.268266 & 2.081998 \\
\hline $\mathrm{C}$ & -2.398307 & -3.800970 & -0.201594 & $\mathrm{H}$ & 3.310065 & 0.718084 & 3.026411 \\
\hline
\end{tabular}




\begin{tabular}{|c|c|c|c|}
\hline $\mathrm{H}$ & 3.796476 & 2.237143 & 2.248818 \\
\hline $\mathrm{H}$ & 2.260239 & 1.456289 & 1.822713 \\
\hline c & 4.093356 & 0.000988 & -2.767578 \\
\hline $\mathrm{H}$ & 3.169933 & 0.560520 & -2.934798 \\
\hline $\mathrm{H}$ & 4.903762 & 0.517242 & -3.296527 \\
\hline $\mathrm{H}$ & 3.974517 & -0.979330 & -3.237115 \\
\hline$C$ & 6.851478 & -2.067726 & 0.876266 \\
\hline 11 & 7.827911 & -1.719191 & 0.520846 \\
\hline $\mathrm{H}$ & 6.910915 & -2.155569 & 1.964084 \\
\hline $\mathrm{H}$ & 6.711679 & -3.073623 & 0.467124 \\
\hline $\mathrm{C}$ & -0.795437 & 0.465449 & 1.223095 \\
\hline $\mathrm{H}$ & -0.304451 & 0.513035 & 2.212001 \\
\hline $\mathrm{C}$ & -2.182795 & 0.870466 & 1.338093 \\
\hline $\mathrm{C}$ & -2.675229 & 1.231767 & 2.612368 \\
\hline $\mathrm{C}$ & -3.086074 & 0.910440 & 0.255834 \\
\hline $\mathrm{C}$ & -3.997903 & 1.607631 & 2.798330 \\
\hline $\mathrm{H}$ & -1.988752 & 1.208718 & 3.457177 \\
\hline $\mathrm{C}$ & -4.409836 & 1.277057 & 0.442220 \\
\hline $\mathrm{H}$ & -2.712300 & 0.663231 & -0.735191 \\
\hline $\mathrm{C}$ & -4.871425 & 1.629018 & 1.711732 \\
\hline $\mathrm{H}$ & -4.352023 & 1.880698 & 3.788645 \\
\hline $\mathrm{H}$ & -5.086253 & 1.307547 & -0.408865 \\
\hline $\mathrm{H}$ & -5.910117 & 1.918191 & 1.852355 \\
\hline $\mathrm{O}$ & 1.689114 & -1.346550 & -1.481571 \\
\hline $\mathrm{O}$ & 1.691865 & -1.095028 & 1.371586 \\
\hline C & 0.739615 & -0.984425 & -2.223283 \\
\hline $\mathrm{H}$ & 0.809772 & -0.081050 & -2.855863 \\
\hline $\mathrm{H}$ & -0.849492 & 0.246990 & -1.086876 \\
\hline $\mathrm{C}$ & 1.445424 & -1.240066 & 2.688093 \\
\hline $\mathrm{H}$ & 1.129463 & -0.297603 & 3.205762 \\
\hline $\mathrm{H}$ & 0.646508 & -1.994898 & 2.910781 \\
\hline $\mathrm{Br}$ & -4.295230 & -4.058089 & -0.509781 \\
\hline $\mathrm{O}$ & -0.089149 & -1.947949 & -2.748208 \\
\hline $\mathrm{H}$ & -0.775094 & -1.498316 & -3.255388 \\
\hline $\mathrm{O}$ & 2.644368 & -1.648124 & 3.356484 \\
\hline $\mathrm{H}$ & 3.209196 & -1.895061 & 2.612396 \\
\hline
\end{tabular}

\section{TSI-1' 1-2Br}

$\begin{array}{rrrc}\mathrm{Ru} & 0.348895 & -0.169778 & -0.101487 \\ \mathrm{C} & 0.360735 & -3.189345 & 0.145396 \\ \mathrm{C} & -1.848820 & -2.519255 & -0.007724 \\ \mathrm{C} & -0.002673 & -4.528361 & 0.112504 \\ \mathrm{H} & 1.390890 & -2.859192 & 0.242247 \\ \mathrm{C} & -2.267036 & -3.839584 & -0.051481\end{array}$

\begin{tabular}{|c|c|c|c|}
\hline & -2.556041 & -1.696535 & -0.039918 \\
\hline $\mathrm{H}$ & 0.762412 & -5.295680 & 0.168556 \\
\hline N & -0.551412 & -2.201839 & 0.081861 \\
\hline $\mathrm{C}$ & -1.345249 & -4.875269 & 0.005078 \\
\hline & -1.664156 & -5.911497 & -0.030369 \\
\hline $\mathrm{C}$ & 1.475137 & 1.479037 & -0.369447 \\
\hline C & 2.174511 & 3.670681 & -0.902914 \\
\hline $\mathrm{C}$ & 3.396636 & 2.802750 & -0.687631 \\
\hline $\mathrm{H}$ & 2.015400 & 3.921189 & -1.963531 \\
\hline $\mathrm{H}$ & 2.193759 & 4.610059 & -0.340331 \\
\hline $\mathrm{H}$ & 4.128383 & 2.859621 & -1.499570 \\
\hline $\mathrm{H}$ & 3.914893 & 3.036960 & 0.254664 \\
\hline $\mathrm{N}$ & 1.095533 & 2.796044 & -0.434262 \\
\hline $\mathrm{N}$ & 2.816412 & 1.458284 & -0.605927 \\
\hline $\mathrm{C}$ & -0.222957 & 3.330675 & -0.374981 \\
\hline C & -0.592849 & 4.020132 & 0.797109 \\
\hline $\mathrm{C}$ & -1.099357 & 3.234536 & -1.462292 \\
\hline $\mathrm{C}$ & -1.870945 & 4.559605 & 0.876186 \\
\hline $\mathrm{C}$ & -2.376989 & 3.788065 & -1.332024 \\
\hline C & -2.787603 & 4.440488 & -0.174464 \\
\hline $\mathrm{H}$ & -2.170157 & 5.070829 & 1.790988 \\
\hline $\mathrm{H}$ & -3.076460 & 3.690570 & -2.162191 \\
\hline $\mathrm{C}$ & 3.704679 & 0.386215 & -0.261513 \\
\hline $\mathrm{C}$ & 4.012065 & 0.159230 & 1.089819 \\
\hline $\mathrm{C}$ & 4.318635 & -0.341365 & -1.289009 \\
\hline $\mathrm{C}$ & 4.890429 & -0.879834 & 1.391622 \\
\hline $\mathrm{C}$ & 5.197661 & -1.364492 & -0.934860 \\
\hline $\mathrm{C}$ & 5.480435 & -1.660892 & 0.398336 \\
\hline $\mathrm{H}$ & 5.104837 & -1.090261 & 2.438489 \\
\hline $\mathrm{H}$ & 5.669276 & -1.948928 & -1.724427 \\
\hline $\mathrm{C}$ & -0.720042 & 2.552306 & -2.739298 \\
\hline $\mathrm{H}$ & -1.343381 & 1.664457 & -2.904467 \\
\hline $\mathrm{H}$ & -0.873427 & 3.213779 & -3.598848 \\
\hline $\mathrm{H}$ & 0.321217 & 2.220246 & -2.742901 \\
\hline $\mathrm{C}$ & 0.356429 & 4.120913 & 1.950536 \\
\hline $\mathrm{H}$ & 1.285693 & 4.638232 & 1.683382 \\
\hline $\mathrm{H}$ & -0.095124 & 4.662686 & 2.785130 \\
\hline $\mathrm{H}$ & 0.656667 & 3.129697 & 2.311224 \\
\hline $\mathrm{C}$ & -4.174960 & 4.986113 & -0.039245 \\
\hline $\mathrm{H}$ & -4.739156 & 4.889728 & -0.970526 \\
\hline $\mathrm{H}$ & -4.730889 & 4.449970 & 0.738657 \\
\hline $\mathrm{H}$ & -4.170260 & 6.043702 & 0.244421 \\
\hline $\mathrm{C}$ & 3.481491 & 1.018795 & 2.196718 \\
\hline $\mathrm{H}$ & 3.250028 & 0.405868 & 3.070572 \\
\hline
\end{tabular}




\begin{tabular}{|c|c|c|c|c|c|c|c|}
\hline $\mathrm{H}$ & 4.223139 & 1.774321 & 2.491961 & $\mathrm{H}$ & 2.523153 & -1.751017 & 0.120511 \\
\hline $\mathrm{H}$ & 2.560074 & 1.536385 & 1.918103 & $\mathrm{H}$ & -0.776215 & -5.275777 & -0.729026 \\
\hline $\mathrm{C}$ & 4.079064 & -0.028292 & -2.734215 & $\mathrm{~N}$ & 0.533978 & -2.226422 & -0.200265 \\
\hline $\mathrm{H}$ & 3.197305 & 0.601264 & -2.871419 & $\mathrm{C}$ & 1.317769 & -4.891536 & -0.395399 \\
\hline $\mathrm{H}$ & 4.940268 & 0.491896 & -3.173332 & $\mathrm{H}$ & 1.632851 & -5.927640 & -0.460973 \\
\hline $\mathrm{H}$ & 3.923947 & -0.943395 & -3.311790 & $\mathrm{C}$ & -1.512836 & 1.411353 & 0.350743 \\
\hline $\mathrm{C}$ & 6.384522 & -2.800820 & 0.757852 & $\mathrm{C}$ & -2.261523 & 3.602128 & 0.817722 \\
\hline $\mathrm{H}$ & 5.809007 & -3.690244 & 1.039349 & $\mathrm{C}$ & -3.465155 & 2.695888 & 0.654815 \\
\hline $\mathrm{H}$ & 7.029888 & -3.085626 & -0.077924 & $\mathrm{H}$ & -2.104878 & 3.917254 & 1.861061 \\
\hline $\mathrm{H}$ & 7.023956 & -2.558049 & 1.611957 & $\mathrm{H}$ & -2.302593 & 4.506689 & 0.201223 \\
\hline $\mathrm{C}$ & -0.893124 & 0.845603 & 0.869601 & $\mathrm{H}$ & -4.175838 & 2.752797 & 1.485799 \\
\hline $\mathrm{H}$ & -0.509492 & 1.476425 & 1.693219 & $\mathrm{H}$ & -4.015544 & 2.894668 & -0.276377 \\
\hline $\mathrm{C}$ & -2.340737 & 0.984389 & 0.782923 & $\mathrm{~N}$ & -1.162731 & 2.729866 & 0.394586 \\
\hline $\mathrm{C}$ & -3.048307 & 1.545926 & 1.865660 & $\mathrm{~N}$ & -2.855082 & 1.362591 & 0.587665 \\
\hline $\mathrm{C}$ & -3.085233 & 0.623038 & -0.357850 & $\mathrm{C}$ & 0.146674 & 3.282744 & 0.341770 \\
\hline $\mathrm{C}$ & -4.428275 & 1.696625 & 1.829857 & $\mathrm{C}$ & 0.542496 & 3.901341 & -0.857148 \\
\hline $\mathrm{H}$ & -2.483312 & 1.862544 & 2.741725 & $\mathrm{C}$ & 0.979786 & 3.269711 & 1.469142 \\
\hline $\mathrm{C}$ & -4.462355 & 0.788886 & -0.404342 & $\mathrm{C}$ & 1.807549 & 4.481296 & -0.911957 \\
\hline $\mathrm{H}$ & -2.542935 & 0.227842 & -1.217055 & $\mathrm{C}$ & 2.240104 & 3.859942 & 1.362099 \\
\hline $\mathrm{C}$ & -5.142595 & 1.318686 & 0.693157 & $\mathrm{C}$ & 2.675940 & 4.461318 & 0.182008 \\
\hline $\mathrm{H}$ & -4.950118 & 2.121438 & 2.684045 & $\mathrm{H}$ & 2.135684 & 4.942173 & -1.843305 \\
\hline $\mathrm{H}$ & -5.012085 & 0.510326 & -1.299951 & $\mathrm{H}$ & 2.907920 & 3.830080 & 2.223125 \\
\hline $\mathrm{H}$ & -6.221195 & 1.446287 & 0.657330 & $\mathrm{C}$ & -3.730920 & 0.269121 & 0.278220 \\
\hline $\mathrm{O}$ & 1.530554 & -1.203953 & -1.726744 & $\mathrm{C}$ & -4.104258 & 0.049722 & -1.056517 \\
\hline $\mathrm{O}$ & 1.462134 & -0.839625 & 1.506799 & $\mathrm{C}$ & -4.284774 & -0.477530 & 1.327580 \\
\hline $\mathrm{C}$ & 0.537924 & -0.825697 & -2.418871 & $\mathrm{C}$ & -4.971470 & -1.010737 & -1.324417 \\
\hline $\mathrm{H}$ & 0.671694 & -0.002983 & -3.155643 & $\mathrm{C}$ & -5.154960 & -1.517997 & 1.008540 \\
\hline $\mathrm{H}$ & -0.429129 & -0.027708 & -1.626180 & $\mathrm{C}$ & -5.491906 & -1.815474 & -0.312485 \\
\hline $\mathrm{C}$ & 0.821373 & -1.025786 & 2.679535 & $\mathrm{H}$ & -5.250766 & -1.205966 & -2.359303 \\
\hline $\mathrm{H}$ & 0.357631 & -0.073040 & 3.053518 & $\mathrm{H}$ & -5.575767 & -2.116527 & 1.816153 \\
\hline $\mathrm{H}$ & -0.011194 & -1.772277 & 2.619530 & $\mathrm{C}$ & 0.576627 & 2.607590 & 2.750442 \\
\hline $\mathrm{Br}$ & -4.166733 & -4.211372 & -0.188221 & $\mathrm{H}$ & 1.188879 & 1.714118 & 2.928700 \\
\hline $\mathrm{O}$ & -0.288058 & -1.826917 & -2.911528 & $\mathrm{H}$ & 0.728112 & 3.273275 & 3.606638 \\
\hline $\mathrm{H}$ & -0.918462 & -1.424590 & -3.520692 & $\mathrm{H}$ & -0.466841 & 2.282156 & 2.746781 \\
\hline $\mathrm{O}$ & 1.788712 & -1.482448 & 3.622137 & $\mathrm{C}$ & -0.357078 & 3.880870 & -2.053154 \\
\hline \multirow[t]{2}{*}{$\mathrm{H}$} & 1.310720 & -1.694134 & 4.429760 & $\mathrm{H}$ & -1.347300 & 4.297377 & -1.832683 \\
\hline & & & & $\mathrm{H}$ & 0.071063 & 4.462882 & -2.873600 \\
\hline \multicolumn{2}{|c|}{ TSI-2' ${ }_{1-2 B r}$} & & & $\mathrm{H}$ & -0.529775 & 2.856911 & -2.412042 \\
\hline $\mathrm{Ru}$ & -0.341920 & -0.213560 & 0.076017 & $\mathrm{C}$ & 4.045632 & 5.060612 & 0.092227 \\
\hline $\mathrm{C}$ & -0.374139 & -3.187410 & -0.443112 & $\mathrm{H}$ & 4.125735 & 5.981140 & 0.681480 \\
\hline $\mathrm{C}$ & 1.822423 & -2.559598 & -0.066321 & $\mathrm{H}$ & 4.803825 & 4.368683 & 0.473851 \\
\hline $\mathrm{C}$ & -0.015696 & -4.525030 & -0.542174 & $\mathrm{H}$ & 4.309192 & 5.306424 & -0.940030 \\
\hline $\mathrm{H}$ & -1.395891 & -2.839280 & -0.557955 & $\mathrm{C}$ & -3.669987 & 0.954527 & -2.167146 \\
\hline $\mathrm{C}$ & 2.236850 & -3.878596 & -0.160355 & $\mathrm{H}$ & -3.517299 & 0.395846 & -3.092285 \\
\hline
\end{tabular}




$\begin{array}{rrrc}\mathrm{H} & -4.438962 & 1.715115 & -2.364157 \\ \mathrm{H} & -2.723351 & 1.455820 & -1.961144 \\ \mathrm{C} & -3.987778 & -0.169648 & 2.762668 \\ \mathrm{H} & -3.159254 & 0.535409 & 2.861859 \\ \mathrm{H} & -4.863468 & 0.263953 & 3.262066 \\ \mathrm{H} & -3.717117 & -1.077626 & 3.307559 \\ \mathrm{C} & -6.386099 & -2.974469 & -0.633740 \\ \mathrm{H} & -7.167632 & -3.106950 & 0.120583 \\ \mathrm{H} & -6.871691 & -2.853722 & -1.606134 \\ \mathrm{H} & -5.822386 & -3.914087 & -0.671798 \\ \mathrm{C} & 1.006731 & 0.761678 & -0.790053 \\ \mathrm{H} & 0.758945 & 1.231876 & -1.758045 \\ \mathrm{C} & 2.418951 & 0.952158 & -0.480485 \\ \mathrm{C} & 3.258856 & 1.535940 & -1.450470 \\ \mathrm{C} & 3.008666 & 0.583217 & 0.746293 \\ \mathrm{C} & 4.614928 & 1.727861 & -1.217935 \\ \mathrm{H} & 2.813038 & 1.845885 & -2.394762 \\ \mathrm{C} & 4.361049 & 0.776338 & 0.984947 \\ \mathrm{H} & 2.366736 & 0.143928 & 1.509147 \\ \mathrm{C} & 5.171613 & 1.348896 & 0.002031 \\ \mathrm{H} & 5.239283 & 2.182342 & -1.983231 \\ \mathrm{H} & 4.791198 & 0.484353 & 1.939790 \\ \mathrm{H} & 6.231396 & 1.501234 & 0.189682 \\ \mathrm{O} & -1.611375 & -1.357561 & 1.542952 \\ \mathrm{O} & -1.281415 & -0.713341 & -1.751522 \\ \mathrm{C} & -0.655016 & -0.983715 & 2.297172 \\ \mathrm{H} & -0.863398 & -0.222537 & 3.082877 \\ \mathrm{H} & 0.328065 & -0.103517 & 1.637526 \\ \mathrm{C} & -0.632609 & -0.617793 & -2.912916 \\ \mathrm{H} & 0.460163 & -0.857420 & -2.845689 \\ \mathrm{H} & -1.076813 & -1.310041 & -3.662812 \\ \mathrm{O} \mathrm{O} & 4.122434 & -4.282705 & 0.049029 \\ \mathrm{H} & 0.172655 & -1.996250 & 2.779858 \\ \mathrm{O} & -0.742453 & -1.610672 & 3.437936 \\ \mathrm{H} & -0.227065 & 0.732518 & -3.452829 \\ & & & -4.267121 \\ \end{array}$

\section{TSI-3' 1-2Br}

$\begin{array}{rrrr}\mathrm{Ru} & -0.397180 & -0.195416 & 0.183026 \\ \mathrm{C} & -0.277978 & -3.217081 & 0.051896 \\ \mathrm{C} & 1.891091 & -2.430948 & 0.148520 \\ \mathrm{C} & 0.158121 & -4.525134 & -0.103075 \\ \mathrm{H} & -1.329313 & -2.953561 & 0.069155 \\ \mathrm{C} & 2.384516 & -3.717004 & -0.005247\end{array}$

\begin{tabular}{|c|c|c|c|}
\hline & & & \\
\hline & -0.565811 & -5.326856 & .2046 \\
\hline & 0.577462 & 2.190471 & 1821 \\
\hline & 1. & & \\
\hline & 00 & -5.80 & \\
\hline & -1.601950 & $1.41 \mathrm{~s}$ & 27 \\
\hline & -2 & & \\
\hline & -3.58 & & 0.23 \\
\hline & -2.33 & 4.01 & \\
\hline & -2.4 & & \\
\hline & -4.370 & 2.809191 & \\
\hline & -4.05 & 6 & -0 . \\
\hline & -1.27 & 274 & 01156 \\
\hline & -2.958716 & 56 & \\
\hline & & & \\
\hline & 0.47 & 3.843267 & -1.1311 \\
\hline & 0.787 & 3.42 & .26 \\
\hline & 1.73 & 4.438 & -1 \\
\hline & 2.04 & & \\
\hline & 2.542230 & 4.52 & -0 . \\
\hline & 2.109627 & 4.81 & -2.1 \\
\hline & 669 & 3 & 2.062 \\
\hline & -3.79 & 0.2327 & 07 \\
\hline & -4.043957 & -0.136 & 1.254 \\
\hline & -4.427167 & -0.41 & 14 \\
\hline & -4.879 & & 1. \\
\hline & $-5.25 \mathrm{c}$ & -1.4 & קor \\
\hline & -5.482639 & -1.929 & -0.44148 \\
\hline & -5.064365 & -1.53 & -2.5 \\
\hline & -5.74 & & \\
\hline & 31 & 2.89 & 2576 \\
\hline & 0.981 & 03 & 92 \\
\hline & 5217 & 00 & .547 \\
\hline & -0.6 & & \\
\hline & -0.35 & 3.697036 & 23681 \\
\hline & -1.347011 & 6283 & -2.26003 \\
\hline & 133641 & 163424 & -5.2204 \\
\hline & -0.5237 & 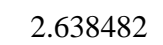 & \\
\hline & 3 & 9 & -0 \\
\hline & 1 & 81 & 0.33 \\
\hline & 4.639677 & 4.541489 & 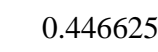 \\
\hline & 254 & 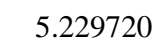 & -1.13 \\
\hline & -3.504 & & -2.4 \\
\hline & -3.364169 & .004485 & 3.282 \\
\hline
\end{tabular}




\begin{tabular}{|c|c|c|c|}
\hline $\mathrm{H}$ & -4.202661 & 1.444451 & -2.705005 \\
\hline $\mathrm{H}$ & -2.537811 & 1.105399 & -2.188740 \\
\hline $\mathrm{C}$ & -4.255473 & 0.047983 & 2.560976 \\
\hline $\mathrm{H}$ & -3.404030 & 0.723889 & 2.665398 \\
\hline $\mathrm{H}$ & -5.151269 & 0.573033 & 2.916904 \\
\hline $\mathrm{H}$ & -4.089479 & -0.801385 & 3.228456 \\
\hline $\mathrm{C}$ & -6.336691 & -3.129859 & -0.716528 \\
\hline $\mathrm{H}$ & -7.109739 & -3.262152 & 0.045972 \\
\hline $\mathrm{H}$ & -6.829697 & -3.062028 & -1.690636 \\
\hline $\mathrm{H}$ & -5.738859 & -4.048718 & -0.726339 \\
\hline $\mathrm{C}$ & 0.976620 & 0.720620 & -0.701469 \\
\hline $\mathrm{H}$ & 0.778452 & 1.149238 & -1.703166 \\
\hline $\mathrm{C}$ & 2.373322 & 0.952926 & -0.347245 \\
\hline $\mathrm{C}$ & 3.263685 & 1.440784 & -1.323364 \\
\hline $\mathrm{C}$ & 2.888571 & 0.728899 & 0.945008 \\
\hline $\mathrm{C}$ & 4.602114 & 1.675859 & -1.032299 \\
\hline $\mathrm{H}$ & 2.874564 & 1.633475 & -2.322595 \\
\hline $\mathrm{C}$ & 4.220755 & 0.972054 & 1.243441 \\
\hline $\mathrm{H}$ & 2.205107 & 0.359593 & 1.708789 \\
\hline $\mathrm{C}$ & 5.085337 & 1.444951 & 0.254136 \\
\hline $\mathrm{H}$ & 5.269102 & 2.050516 & -1.804793 \\
\hline $\mathrm{H}$ & 4.593471 & 0.794062 & 2.249039 \\
\hline $\mathrm{H}$ & 6.129674 & 1.635494 & 0.487438 \\
\hline $\mathrm{O}$ & -1.739464 & -1.189371 & 1.694439 \\
\hline $\mathrm{O}$ & -1.216935 & -1.011005 & -1.582105 \\
\hline $\mathrm{C}$ & -0.851298 & -0.699215 & 2.463233 \\
\hline $\mathrm{H}$ & -1.132547 & 0.149393 & 3.128054 \\
\hline $\mathrm{H}$ & 0.153923 & 0.125693 & 1.763317 \\
\hline $\mathrm{C}$ & -0.610571 & -0.838205 & -2.757194 \\
\hline $\mathrm{H}$ & -1.195925 & -1.344375 & -3.557166 \\
\hline $\mathrm{H}$ & -0.519167 & 0.242557 & -3.062848 \\
\hline $\mathrm{Br}$ & 4.307587 & -3.984871 & -0.039058 \\
\hline $\mathrm{O}$ & -0.038602 & -1.615278 & 3.121204 \\
\hline $\mathrm{H}$ & 0.500553 & -1.133974 & 3.759771 \\
\hline $\mathrm{O}$ & 0.724387 & -1.394209 & -2.750617 \\
\hline $\mathrm{H}$ & 1.137659 & -1.149210 & -3.585562 \\
\hline
\end{tabular}

\section{TSI-4' ${ }_{1-2 B r}$}

$\begin{array}{rrrr}\mathrm{Ru} & -0.372562 & -0.253958 & 0.263444 \\ \mathrm{C} & -0.047225 & -3.269065 & 0.367382 \\ \mathrm{C} & 2.062318 & -2.322887 & 0.349509 \\ \mathrm{C} & 0.480361 & -4.547710 & 0.251014 \\ \mathrm{H} & -1.114224 & -3.077889 & 0.407181 \\ \mathrm{C} & 2.644089 & -3.575140 & 0.229808\end{array}$

\begin{tabular}{|c|c|c|c|}
\hline & 2.660708 & -1.417814 & 0.365 \\
\hline & 183842 & .404545 & 0.208656 \\
\hline & 0.735241 & -2.177629 & 0.423669 \\
\hline C & 1.858415 & -4.716709 & 0.176715 \\
\hline & 2.305928 & -5.699629 & 076723 \\
\hline & -1.662333 & 1.301523 & 0.208149 \\
\hline C & -2.568151 & 3.464317 & 0.503396 \\
\hline & -3.707278 & 2.467161 & 0.382971 \\
\hline & -2.470396 & 3.874234 & 1.519567 \\
\hline & -2.651050 & 4.311462 & -0.187471 \\
\hline $\mathrm{H}$ & -4.414679 & 2.509853 & 616 \\
\hline $\mathrm{H}$ & -4.27 & 2.592898 & -0.547319 \\
\hline $\mathrm{N}$ & -1.398952 & 2.64 & 93 \\
\hline$\Lambda$ & -3.010126 & 1.174793 & 0.365951 \\
\hline C & -0.127526 & 3.275006 & 0.072072 \\
\hline C & 0.272842 & 3.722476 & -1.201864 \\
\hline C & 0.676441 & 3.484701 & 1.198072 \\
\hline C & 1.514103 & 4.337274 & -1.330280 \\
\hline C & 1.913208 & 4.111123 & 1.018618 \\
\hline C & 2.356787 & 4.531731 & -0.230981 \\
\hline $\mathrm{H}$ & 1.842788 & 4.658538 & -2.3 \\
\hline $\mathrm{H}$ & 2.560947 & 4.246447 & 1.884760 \\
\hline C & -3.783772 & 0.029015 & -0.015627 \\
\hline $\mathrm{C}$ & -3.915799 & .258520 & -1.385255 \\
\hline $\mathrm{C}$ & -4.487530 & -0.692338 & 0.954093 \\
\hline $\mathrm{C}$ & -4.702873 & -1.342961 & -1.759208 \\
\hline $\mathrm{C}$ & -5.266467 & -1.771935 & 0.528235 \\
\hline C & -5.374365 & -2.123332 & -0.815016 \\
\hline $\mathrm{H}$ & -4.7938 & 0 & 4 \\
\hline $\mathrm{H}$ & -5.806323 & -2.352400 & 1.275957 \\
\hline $\mathrm{C}$ & 0.259524 & 3.041782 & 2.565213 \\
\hline $\mathrm{H}$ & 1.072704 & 2.488924 & 3.052119 \\
\hline $\mathrm{H}$ & 0.037290 & 3.901368 & 3.209192 \\
\hline $\mathrm{H}$ & -0.618183 & 2.392903 & 2.548239 \\
\hline $\mathrm{C}$ & -0.604085 & 3.497140 & -2.393915 \\
\hline 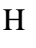 & -1.603597 & 3.927461 & -2.260136 \\
\hline $\mathrm{H}$ & -0.167151 & 3.937703 & -3.293446 \\
\hline $\mathrm{H}$ & -0.760297 & 2.427406 & -2.581883 \\
\hline $\mathrm{C}$ & 3.698477 & 5.174984 & -0.400588 \\
\hline $\mathrm{H}$ & 3.610166 & 6.248693 & -0.602648 \\
\hline $\mathrm{H}$ & 4.314966 & 5.056792 & 0.494357 \\
\hline $\mathrm{H}$ & 4.245062 & 4.736163 & -1.241465 \\
\hline $\mathrm{C}$ & -3.265632 & 0.603588 & -2.421674 \\
\hline $\mathrm{H}$ & -3.351935 & 0.151850 & -3.41340 \\
\hline
\end{tabular}




\begin{tabular}{|c|c|c|c|c|c|c|c|}
\hline $\mathrm{H}$ & -3.727688 & 1.599372 & -2.473467 & $\mathrm{H}$ & 2.592627 & -1.705504 & 0.272911 \\
\hline $\mathrm{H}$ & -2.202042 & 0.740878 & -2.211887 & $\mathrm{H}$ & -0.613596 & -5.348347 & -0.435635 \\
\hline $\mathrm{C}$ & -4.459660 & -0.310322 & 2.400074 & $\mathrm{~N}$ & 0.605406 & -2.254321 & 0.060225 \\
\hline $\mathrm{H}$ & -3.614215 & 0.336933 & 2.629825 & $\mathrm{C}$ & 1.480066 & -4.887517 & -0.209984 \\
\hline $\mathrm{H}$ & -5.389663 & 0.199297 & 2.685505 & $\mathrm{H}$ & 1.830706 & -5.909122 & -0.311954 \\
\hline $\mathrm{H}$ & -4.369203 & -1.195645 & 3.034631 & $\mathrm{C}$ & -1.542275 & 1.368352 & 0.241761 \\
\hline $\mathrm{C}$ & -6.186781 & -3.308157 & -1.241753 & $\mathrm{C}$ & -2.346979 & 3.560308 & 0.564874 \\
\hline $\mathrm{H}$ & -6.860499 & -3.643226 & -0.448368 & $\mathrm{C}$ & -3.523344 & 2.629737 & 0.351108 \\
\hline $\mathrm{H}$ & -6.791981 & -3.087860 & -2.126971 & $\mathrm{H}$ & -2.266523 & 3.905667 & 1.606697 \\
\hline $\mathrm{H}$ & -5.545848 & -4.157796 & -1.503875 & $\mathrm{H}$ & -2.359751 & 4.444211 & -0.081720 \\
\hline $\mathrm{C}$ & 0.911591 & 0.672567 & -0.736800 & $\mathrm{H}$ & -4.296818 & 2.712228 & 1.121030 \\
\hline $\mathrm{H}$ & 0.633506 & 1.011348 & -1.754588 & $\mathrm{H}$ & -3.999597 & 2.775963 & -0.629812 \\
\hline $\mathrm{C}$ & 2.310246 & 0.996464 & -0.488991 & $\mathrm{~N}$ & -1.212617 & 2.691023 & 0.240472 \\
\hline $\mathrm{C}$ & 3.135302 & 1.347785 & -1.574759 & $\mathrm{~N}$ & -2.892613 & 1.305151 & 0.394970 \\
\hline $\mathrm{C}$ & 2.896736 & 0.966006 & 0.792185 & $\mathrm{C}$ & 0.087535 & 3.263023 & 0.152551 \\
\hline $\mathrm{C}$ & 4.484448 & 1.625649 & -1.399697 & $\mathrm{C}$ & 0.504310 & 3.747124 & -1.100678 \\
\hline $\mathrm{H}$ & 2.687592 & 1.390807 & -2.567269 & $\mathrm{C}$ & 0.892109 & 3.390151 & 1.292204 \\
\hline $\mathrm{C}$ & 4.239603 & 1.264258 & 0.974387 & $\mathrm{C}$ & 1.763607 & 4.335078 & -1.192778 \\
\hline $\mathrm{H}$ & 2.256738 & 0.718746 & 1.639391 & $\mathrm{C}$ & 2.151724 & 3.974775 & 1.144643 \\
\hline $\mathrm{C}$ & 5.040721 & 1.589575 & -0.121594 & $\mathrm{C}$ & 2.607062 & 4.447872 & -0.084286 \\
\hline $\mathrm{H}$ & 5.103222 & 1.882579 & -2.255626 & $\mathrm{H}$ & 2.106402 & 4.694782 & -2.162676 \\
\hline $\mathrm{H}$ & 4.669247 & 1.245557 & 1.973012 & $\mathrm{H}$ & 2.803539 & 4.041877 & 2.015445 \\
\hline $\mathrm{H}$ & 6.093158 & 1.819654 & 0.022482 & $\mathrm{C}$ & -3.738004 & 0.177172 & 0.126968 \\
\hline $\mathrm{O}$ & -1.653800 & -1.190572 & 1.838962 & $\mathrm{C}$ & -4.027928 & -0.157528 & -1.206625 \\
\hline $\mathrm{O}$ & -1.151493 & -1.259649 & -1.403790 & $\mathrm{C}$ & -4.364229 & -0.474049 & 1.197788 \\
\hline $\mathrm{C}$ & -0.777575 & -0.623077 & 2.579509 & $\mathrm{C}$ & -4.869848 & -1.244462 & -1.438701 \\
\hline $\mathrm{H}$ & 0.135922 & 0.179221 & 1.867916 & $\mathrm{C}$ & -5.206907 & -1.549027 & 0.911363 \\
\hline $\mathrm{C}$ & -0.531912 & -1.255674 & -2.587174 & $\mathrm{C}$ & -5.452880 & -1.964988 & -0.395442 \\
\hline $\mathrm{H}$ & -1.122815 & -1.861131 & -3.309039 & $\mathrm{H}$ & -5.084848 & -1.525259 & -2.469333 \\
\hline $\mathrm{H}$ & -0.429164 & -0.229265 & -3.035570 & $\mathrm{H}$ & -5.684187 & -2.074310 & 1.738227 \\
\hline $\mathrm{Br}$ & 4.577458 & -3.702096 & 0.131215 & $\mathrm{C}$ & 0.435713 & 2.908152 & 2.633925 \\
\hline $\mathrm{O}$ & 0.799024 & -1.813424 & -2.495526 & $\mathrm{H}$ & 1.288626 & 2.562530 & 3.228358 \\
\hline $\mathrm{H}$ & 1.223306 & -1.672377 & -3.348562 & $\mathrm{H}$ & -0.039115 & 3.714466 & 3.207188 \\
\hline $\mathrm{H}$ & 0.000976 & -1.252897 & 3.064871 & $\mathrm{H}$ & -0.286045 & 2.089851 & 2.562620 \\
\hline $\mathrm{O}$ & -1.296758 & 0.314374 & 3.483087 & $\mathrm{C}$ & -0.368279 & 3.582280 & -2.305293 \\
\hline \multirow[t]{2}{*}{$\mathrm{H}$} & -0.580830 & 0.593377 & 4.065854 & $\mathrm{H}$ & -1.371992 & 3.994780 & -2.148015 \\
\hline & & & & $\mathrm{H}$ & 0.065463 & 4.086969 & -3.172680 \\
\hline \multicolumn{2}{|c|}{ TSI-5' ${ }_{1-2 \mathrm{Br}}$} & & & $\mathrm{H}$ & -0.507747 & 2.523530 & -2.563234 \\
\hline $\mathrm{Ru}$ & -0.324237 & -0.251917 & 0.151206 & $\mathrm{C}$ & 3.963063 & 5.070583 & -0.215657 \\
\hline $\mathrm{C}$ & -0.277348 & -3.250170 & -0.143828 & $\mathrm{H}$ & 3.916556 & 6.161414 & -0.114763 \\
\hline $\mathrm{C}$ & 1.913090 & -2.540319 & 0.122260 & $\mathrm{H}$ & 4.650014 & 4.702523 & 0.551580 \\
\hline $\mathrm{C}$ & 0.126821 & -4.571571 & -0.276579 & $\mathrm{H}$ & 4.407796 & 4.858736 & -1.192746 \\
\hline $\mathrm{H}$ & -1.316516 & -2.943889 & -0.203904 & $\mathrm{C}$ & -3.537150 & 0.664247 & -2.357013 \\
\hline $\mathrm{C}$ & 2.371866 & -3.842446 & -0.009123 & $\mathrm{H}$ & -3.398146 & 0.048648 & -3.247727 \\
\hline
\end{tabular}




$\begin{array}{cccc}\mathrm{H} & -4.268128 & 1.446218 & -2.608461 \\ \mathrm{H} & -2.572655 & 1.132564 & -2.160063 \\ \mathrm{C} & -4.179969 & -0.034539 & 2.616164 \\ \mathrm{H} & -3.447877 & 0.768655 & 2.703950 \\ \mathrm{H} & -5.132327 & 0.305852 & 3.041578 \\ \mathrm{H} & -3.816621 & -0.857527 & 3.236272 \\ \mathrm{C} & -6.323131 & -3.152443 & -0.676607 \\ \mathrm{H} & -6.971163 & -3.389497 & 0.171844 \\ \mathrm{H} & -6.958768 & -2.991977 & -1.552985 \\ \mathrm{H} & -5.723862 & -4.047099 & -0.882959 \\ \mathrm{C} & 1.055353 & 0.670408 & -0.729029 \\ \mathrm{H} & 0.859733 & 1.051320 & -1.746817 \\ \mathrm{C} & 2.446539 & 0.902920 & -0.360876 \\ \mathrm{C} & 3.349197 & 1.363460 & -1.341134 \\ \mathrm{C} & 2.955575 & 0.686035 & 0.935853 \\ \mathrm{C} & 4.690852 & 1.577930 & -1.049074 \\ \mathrm{H} & 2.965481 & 1.552895 & -2.342887 \\ \mathrm{C} & 4.291022 & 0.910184 & 1.234611 \\ \mathrm{H} & 2.263687 & 0.349028 & 1.706649 \\ \mathrm{C} & 5.166212 & 1.355419 & 0.241635 \\ \mathrm{H} & 5.366636 & 1.929960 & -1.824519 \\ \mathrm{H} & 4.657669 & 0.742067 & 2.244221 \\ \mathrm{H} & 6.212804 & 1.531993 & 0.475698 \\ \mathrm{O} & -1.642399 & -1.271374 & 1.658844 \\ \mathrm{O} & -1.158315 & -0.904910 & -1.675450 \\ \mathrm{C} & -0.678119 & -0.872011 & 2.398438 \\ \mathrm{H} & 0.252395 & 0.004056 & 1.741341 \\ \mathrm{C} & -0.435970 & -0.927630 & -2.797038 \\ \mathrm{H} & 0.653235 & -1.137867 & -2.630696 \\ \mathrm{H} & -0.821244 & -1.708692 & -3.490162 \\ \mathrm{Br} & 4.278055 & -4.176686 & 0.088432 \\ \mathrm{O} & -0.526193 & 0.349980 & -3.488299 \\ \mathrm{H} & 0.025274 & 0.281812 & -4.275660 \\ \mathrm{H} & 0.087527 & -1.610844 & 2.727095 \\ \mathrm{O} & -1.053260 & -0.037493 & 3.457710 \\ \mathrm{H} & -0.270169 & 0.122061 & 3.998045\end{array}$

TSI-6' ${ }_{1-2 B r}$

$\begin{array}{rrrr}\mathrm{Ru} & 0.329797 & -0.201846 & -0.244354 \\ \mathrm{C} & 0.255685 & -3.223035 & -0.265990 \\ \mathrm{C} & -1.945065 & -2.500840 & -0.241452 \\ \mathrm{C} & -0.140230 & -4.552313 & -0.209808 \\ \mathrm{H} & 1.297220 & -2.920181 & -0.277729 \\ \mathrm{C} & -2.395352 & -3.811232 & -0.189728\end{array}$

\begin{tabular}{|c|c|c|c|}
\hline & 27 & & \\
\hline & 0.609372 & 5.336678 & \\
\hline & .636277 & תו & 21 \\
\hline & th & & \\
\hline & -1.838 & 43 & \\
\hline & 1.511401 & 1.4 & 64 \\
\hline & & & \\
\hline & 3.478692 & 2.717 & \\
\hline & 2.211505 & 97 & \\
\hline & 2.25 & & \\
\hline & 4.264637 & 02 & \\
\hline & 3.9 & & \\
\hline & 1.16 & 75 & -0.2476 \\
\hline & 2.862208 & 23 & \\
\hline & -0.13 & & \\
\hline & -0.492 & 236 & \\
\hline & -0.997 & 13 & $1.2 \mathrm{r}$ \\
\hline & -1.75 & & \\
\hline & -2.25 & 05 & \\
\hline & -2.66 & 85 & \\
\hline & -2.051575 & 4 & \\
\hline & -2.95 & & \\
\hline & 3. & & \\
\hline & 3.877288 & -0.06 & 7710 \\
\hline & 4.390131 & -0.3 & -1 \\
\hline & 4.70 & & \\
\hline & 5.21 & - & \\
\hline & 5.376955 & -1.85 & 5755 \\
\hline & 4.823029 & -1 & \\
\hline & & & \\
\hline & -0.62 & 28750 & 2 \\
\hline & -1.350 & 96 & 8934 \\
\hline & -0.656587 & $0.00 / 4 \angle 4$ & \\
\hline & & & \\
\hline & 434 & 1 & \\
\hline & 1.4513 & & 948 \\
\hline & 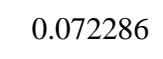 & & (1) \\
\hline & 531462 & & \\
\hline & -4.024 & 5.089683 & 3696 \\
\hline & -4.006 & 2 & 0.2. \\
\hline & -4.732422 & 4.698486 & -0.3663 \\
\hline & -4.425597 & & 2652 \\
\hline & & 716066 & \\
\hline & 02479 & 05812 & .22 \\
\hline
\end{tabular}




\begin{tabular}{|c|c|c|c|c|c|c|c|}
\hline $\mathrm{H}$ & 3.932638 & 1.504977 & 2.741964 & $\mathrm{H}$ & 1.783352 & -3.943681 & 0.715740 \\
\hline $\mathrm{H}$ & 2.314175 & 1.183744 & 2.084848 & $\mathrm{H}$ & 1.946772 & -4.106232 & -1.043049 \\
\hline $\mathrm{C}$ & 4.282809 & 0.028938 & -2.535614 & $\mathrm{~N}$ & -0.656926 & -2.483509 & -0.079925 \\
\hline $\mathrm{H}$ & 3.529273 & 0.804405 & -2.677109 & $\mathrm{~N}$ & 1.490939 & -2.148486 & -0.369193 \\
\hline $\mathrm{H}$ & 5.247256 & 0.396763 & -2.907591 & $\mathrm{C}$ & -2.064374 & -2.305723 & 0.056985 \\
\hline $\mathrm{H}$ & 3.986252 & -0.813665 & -3.165449 & $\mathrm{C}$ & -2.864970 & -2.114157 & -1.076563 \\
\hline $\mathrm{C}$ & 6.234700 & -3.037501 & 0.927991 & $\mathrm{C}$ & -2.609426 & -2.329220 & 1.349437 \\
\hline $\mathrm{H}$ & 6.875107 & -3.335922 & 0.093307 & $\mathrm{C}$ & -4.234729 & -1.926027 & -0.883929 \\
\hline $\mathrm{H}$ & 6.878146 & -2.828028 & 1.788477 & $\mathrm{C}$ & -3.982814 & -2.136526 & 1.492474 \\
\hline $\mathrm{H}$ & 5.624883 & -3.907099 & 1.198475 & $\mathrm{C}$ & -4.810690 & -1.925787 & 0.387130 \\
\hline $\mathrm{C}$ & -0.955583 & 0.739301 & 0.748809 & $\mathrm{H}$ & -4.866556 & -1.763612 & -1.756438 \\
\hline $\mathrm{H}$ & -0.617216 & 1.272584 & 1.656496 & $\mathrm{H}$ & -4.415659 & -2.140789 & 2.492213 \\
\hline $\mathrm{C}$ & -2.391173 & 0.915844 & 0.582646 & $\mathrm{C}$ & 2.725672 & -1.494785 & -0.048352 \\
\hline $\mathrm{C}$ & -3.168430 & 1.366340 & 1.668802 & $\mathrm{C}$ & 3.571388 & -1.062757 & -1.085164 \\
\hline $\mathrm{C}$ & -3.053013 & 0.694481 & -0.642620 & $\mathrm{C}$ & 3.095895 & -1.329879 & 1.304260 \\
\hline $\mathrm{C}$ & -4.540136 & 1.548916 & 1.550352 & $\mathrm{C}$ & 4.755361 & -0.404906 & -0.745793 \\
\hline $\mathrm{H}$ & -2.666037 & 1.570856 & 2.613672 & $\mathrm{C}$ & 4.284070 & -0.655892 & 1.587880 \\
\hline $\mathrm{C}$ & -4.419349 & 0.899235 & -0.770270 & $\mathrm{C}$ & 5.119828 & -0.173139 & 0.579401 \\
\hline $\mathrm{H}$ & -2.451902 & 0.380336 & -1.496031 & $\mathrm{H}$ & 5.402936 & -0.050317 & -1.546596 \\
\hline $\mathrm{C}$ & -5.171342 & 1.319991 & 0.328300 & $\mathrm{H}$ & 4.564413 & -0.507013 & 2.630169 \\
\hline $\mathrm{H}$ & -5.119041 & 1.885294 & 2.406842 & $\mathrm{C}$ & -1.714161 & -2.477356 & 2.539707 \\
\hline $\mathrm{H}$ & -4.905144 & 0.734012 & -1.728739 & $\mathrm{H}$ & -1.023195 & -1.626783 & 2.608357 \\
\hline $\mathrm{H}$ & -6.242144 & 1.476287 & 0.228828 & $\mathrm{H}$ & -2.289257 & -2.528609 & 3.467246 \\
\hline $\mathrm{O}$ & 1.595267 & -1.100278 & -1.869151 & $\mathrm{H}$ & -1.090383 & -3.377206 & 2.479211 \\
\hline $\mathrm{O}$ & 1.300003 & -1.057892 & 1.368586 & $\mathrm{C}$ & -2.277061 & -2.033576 & -2.448128 \\
\hline $\mathrm{C}$ & 0.654862 & -0.629070 & -2.589164 & $\mathrm{H}$ & -2.978720 & -2.402626 & -3.200995 \\
\hline $\mathrm{H}$ & -0.288927 & 0.137922 & -1.831660 & $\mathrm{H}$ & -2.035572 & -0.993077 & -2.699247 \\
\hline $\mathrm{C}$ & 0.589689 & -1.331367 & 2.481886 & $\mathrm{H}$ & -1.346583 & -2.601363 & -2.538449 \\
\hline $\mathrm{H}$ & 0.129048 & -0.403950 & 2.918109 & $\mathrm{C}$ & -6.279024 & -1.681884 & 0.559193 \\
\hline $\mathrm{H}$ & -0.258051 & -2.044303 & 2.308965 & $\mathrm{H}$ & -6.875189 & -2.304041 & -0.115511 \\
\hline $\mathrm{Br}$ & -4.305066 & -4.141771 & -0.129786 & $\mathrm{H}$ & -6.606517 & -1.886234 & 1.581803 \\
\hline $\mathrm{O}$ & 1.490407 & -1.902173 & 3.427414 & $\mathrm{H}$ & -6.537347 & -0.640716 & 0.334077 \\
\hline $\mathrm{H}$ & 0.966583 & -2.144754 & 4.197078 & $\mathrm{C}$ & 3.241841 & -1.281550 & -2.527403 \\
\hline $\mathrm{H}$ & -0.111597 & -1.323025 & -3.002043 & $\mathrm{H}$ & 2.991811 & -0.330828 & -3.008965 \\
\hline $\mathrm{O}$ & 1.052908 & 0.308147 & -3.542585 & $\mathrm{H}$ & 4.098310 & -1.705137 & -3.061268 \\
\hline \multirow[t]{2}{*}{$\mathrm{H}$} & 0.287516 & 0.521809 & -4.089206 & $\mathrm{H}$ & 2.384403 & -1.944879 & -2.656155 \\
\hline & & & & $\mathrm{C}$ & 2.294007 & -1.904191 & 2.434175 \\
\hline $3 I$ & & & & $\mathrm{H}$ & 1.258326 & -2.111203 & 2.160190 \\
\hline $\mathrm{Ru}$ & 0.398297 & 0.425833 & 0.068160 & $\mathrm{H}$ & 2.745857 & -2.839205 & 2.789363 \\
\hline $\mathrm{C}$ & 0.285645 & -1.504590 & -0.143680 & $\mathrm{H}$ & 2.271329 & -1.216876 & 3.284402 \\
\hline $\mathrm{C}$ & -0.120650 & -3.819418 & -0.354882 & $\mathrm{C}$ & 6.353222 & 0.608180 & 0.911519 \\
\hline $\mathrm{C}$ & 1.381554 & -3.603389 & -0.251082 & $\mathrm{H}$ & 7.140870 & 0.460535 & 0.167833 \\
\hline $\mathrm{H}$ & -0.507828 & -4.546311 & 0.367713 & $\mathrm{H}$ & 6.138288 & 1.683405 & 0.935366 \\
\hline $\mathrm{H}$ & -0.427610 & -4.153807 & -1.356982 & $\mathrm{H}$ & 6.753829 & 0.339508 & 1.892731 \\
\hline
\end{tabular}




\begin{tabular}{|c|c|c|c|c|c|c|c|}
\hline $\mathrm{C}$ & -1.400431 & 0.790657 & 0.241005 & $\mathrm{~N}$ & 1.262441 & -2.072805 & -0.724825 \\
\hline $\mathrm{H}$ & -2.115007 & 0.044862 & 0.613255 & $\mathrm{C}$ & -2.257356 & -2.038749 & -0.082536 \\
\hline $\mathrm{C}$ & -2.066005 & 2.050336 & -0.070728 & $\mathrm{C}$ & -3.205526 & -1.709739 & -1.060718 \\
\hline $\mathrm{C}$ & -3.404421 & 2.216272 & 0.339072 & $\mathrm{C}$ & -2.623799 & -2.219918 & 1.261943 \\
\hline $\mathrm{C}$ & -1.447898 & 3.111844 & -0.764566 & $\mathrm{C}$ & -4.528533 & -1.518334 & -0.652499 \\
\hline $\mathrm{C}$ & -4.087645 & 3.400807 & 0.099171 & $\mathrm{C}$ & -3.954852 & -2.017736 & 1.619863 \\
\hline $\mathrm{H}$ & -3.892850 & 1.394370 & 0.861507 & $\mathrm{C}$ & -4.920949 & -1.653922 & 0.677656 \\
\hline $\mathrm{C}$ & -2.140110 & 4.288340 & -1.016313 & $\mathrm{H}$ & -5.271749 & -1.252436 & -1.403225 \\
\hline $\mathrm{H}$ & -0.414632 & 2.999101 & -1.082196 & $\mathrm{H}$ & -4.244821 & -2.146078 & 2.662065 \\
\hline $\mathrm{C}$ & -3.456498 & 4.442418 & -0.579227 & $\mathrm{C}$ & 2.581278 & -1.570876 & -0.490767 \\
\hline $\mathrm{H}$ & -5.115950 & 3.512412 & 0.433596 & $\mathrm{C}$ & 3.304130 & -0.949374 & -1.519381 \\
\hline $\mathrm{H}$ & -1.649397 & 5.096230 & -1.552440 & $\mathrm{C}$ & 3.135918 & -1.730427 & 0.797890 \\
\hline $\mathrm{H}$ & -3.990655 & 5.368546 & -0.774377 & $\mathrm{C}$ & 4.568658 & -0.435074 & -1.217347 \\
\hline $\mathrm{O}$ & 1.018600 & 0.913894 & -1.862606 & $\mathrm{C}$ & 4.383988 & -1.171205 & 1.054827 \\
\hline $\mathrm{H}$ & 0.478869 & 0.083302 & 1.686267 & $\mathrm{C}$ & 5.110841 & -0.505729 & 0.062807 \\
\hline $\mathrm{C}$ & 0.428306 & 0.389073 & -2.979286 & $\mathrm{H}$ & 5.128592 & 0.064365 & -2.007041 \\
\hline $\mathrm{H}$ & 1.024567 & 0.677956 & -3.869510 & $\mathrm{H}$ & 4.803177 & -1.261649 & 2.055670 \\
\hline $\mathrm{H}$ & 0.366114 & -0.716889 & -2.958900 & $\mathrm{C}$ & -1.600173 & -2.560401 & 2.299388 \\
\hline $\mathrm{O}$ & 1.228725 & 2.549119 & 0.505501 & $\mathrm{H}$ & -0.965759 & -1.693081 & 2.523826 \\
\hline $\mathrm{H}$ & 0.187470 & 1.476495 & 2.691018 & $\mathrm{H}$ & -2.073220 & -2.877113 & 3.231953 \\
\hline $\mathrm{C}$ & 2.617500 & 2.474641 & 0.104961 & $\mathrm{H}$ & -0.924005 & -3.359375 & 1.975258 \\
\hline $\mathrm{H}$ & 3.205439 & 3.169268 & 0.717910 & $\mathrm{C}$ & -2.831473 & -1.530312 & -2.496204 \\
\hline $\mathrm{H}$ & 2.963211 & 1.438559 & 0.303417 & $\mathrm{H}$ & -3.664347 & -1.795490 & -3.152994 \\
\hline $\mathrm{O}$ & 0.396542 & 2.345249 & 3.086339 & $\mathrm{H}$ & -2.566888 & -0.484706 & -2.692033 \\
\hline $\mathrm{H}$ & 1.157133 & 2.657611 & 1.480202 & $\mathrm{H}$ & -1.963718 & -2.133441 & -2.779596 \\
\hline $\mathrm{C}$ & -0.801009 & 3.106404 & 3.128820 & $\mathrm{C}$ & -6.335563 & -1.390354 & 1.093710 \\
\hline $\mathrm{H}$ & -1.124136 & 3.431974 & 2.129952 & $\mathrm{H}$ & -7.035513 & -1.534469 & 0.266517 \\
\hline $\mathrm{H}$ & -1.624150 & 2.548475 & 3.592525 & $\mathrm{H}$ & -6.645019 & -2.038822 & 1.918281 \\
\hline $\mathrm{H}$ & -0.601275 & 3.991199 & 3.736420 & $\mathrm{H}$ & -6.458169 & -0.357178 & 1.440884 \\
\hline $\mathrm{O}$ & 2.735518 & 2.830910 & -1.206346 & $\mathrm{C}$ & 2.765711 & -0.799803 & -2.906203 \\
\hline $\mathrm{H}$ & 2.195888 & 2.168571 & -1.701953 & $\mathrm{H}$ & 2.598664 & 0.258314 & -3.131049 \\
\hline $\mathrm{O}$ & -0.917676 & 0.806236 & -3.152720 & $\mathrm{H}$ & 3.472173 & -1.191509 & -3.645009 \\
\hline \multirow[t]{2}{*}{$\mathrm{H}$} & -0.941826 & 1.733791 & -2.886604 & $\mathrm{H}$ & 1.808010 & -1.306523 & -3.038250 \\
\hline & & & & $\mathrm{C}$ & 2.419954 & -2.516570 & 1.853141 \\
\hline \multicolumn{2}{|c|}{ TSI3-4 } & & & $\mathrm{H}$ & 1.368622 & -2.228339 & 1.937128 \\
\hline $\mathrm{Ru}$ & 0.471347 & 0.455425 & 0.298583 & $\mathrm{H}$ & 2.440794 & -3.591095 & 1.629423 \\
\hline $\mathrm{C}$ & 0.144347 & -1.406022 & -0.307032 & $\mathrm{H}$ & 2.886501 & -2.375022 & 2.830665 \\
\hline $\mathrm{C}$ & -0.475709 & -3.605743 & -0.869762 & $\mathrm{C}$ & 6.418988 & 0.148611 & 0.380234 \\
\hline $\mathrm{C}$ & 0.979395 & -3.397252 & -1.275084 & $\mathrm{H}$ & 7.064399 & 0.216393 & -0.499396 \\
\hline $\mathrm{H}$ & -0.585627 & -4.303903 & -0.025990 & $\mathrm{H}$ & 6.260662 & 1.172001 & 0.741267 \\
\hline $\mathrm{H}$ & -1.103534 & -3.975111 & -1.687208 & $\mathrm{H}$ & 6.963103 & -0.384626 & 1.164580 \\
\hline $\mathrm{H}$ & 1.656253 & -4.148326 & -0.854445 & $\mathrm{C}$ & -1.273316 & 0.873893 & 0.726600 \\
\hline $\mathrm{H}$ & 1.113857 & -3.398728 & -2.367259 & $\mathrm{H}$ & -1.907266 & 0.205826 & 1.331448 \\
\hline $\mathrm{N}$ & -0.900739 & -2.258112 & -0.466226 & $\mathrm{C}$ & -1.956003 & 2.090765 & 0.324873 \\
\hline
\end{tabular}




\begin{tabular}{|c|c|c|c|c|c|c|c|}
\hline $\mathrm{C}$ & -3.363707 & 2.095257 & 0.278582 & $\mathrm{C}$ & -2.461420 & -2.659836 & 0.845601 \\
\hline $\mathrm{C}$ & -1.261483 & 3.256917 & -0.058512 & $\mathrm{C}$ & -4.512249 & -1.373408 & -0.542583 \\
\hline $\mathrm{C}$ & -4.054386 & 3.206826 & -0.184577 & $\mathrm{C}$ & -3.752680 & -2.599807 & 1.370364 \\
\hline $\mathrm{H}$ & -3.899347 & 1.195272 & 0.579070 & $\mathrm{C}$ & -4.791213 & -1.959003 & 0.693631 \\
\hline $\mathrm{C}$ & -1.962177 & 4.373557 & -0.497333 & $\mathrm{H}$ & -5.309625 & -0.867034 & -1.085642 \\
\hline $\mathrm{H}$ & -0.175318 & 3.273026 & 0.006685 & $\mathrm{H}$ & -3.950718 & -3.067414 & 2.333749 \\
\hline $\mathrm{C}$ & -3.355591 & 4.348986 & -0.575252 & $\mathrm{C}$ & 2.538995 & -1.256570 & -0.954183 \\
\hline $\mathrm{H}$ & -5.139813 & 3.187729 & -0.238770 & $\mathrm{C}$ & 3.306805 & -0.450046 & -1.810941 \\
\hline $\mathrm{H}$ & -1.416064 & 5.269600 & -0.780449 & $\mathrm{C}$ & 3.053317 & -1.698732 & 0.281388 \\
\hline $\mathrm{H}$ & -3.897114 & 5.222427 & -0.928966 & $\mathrm{C}$ & 4.566526 & -0.037351 & -1.375946 \\
\hline $\mathrm{O}$ & 0.722728 & 1.195064 & -1.609701 & $\mathrm{C}$ & 4.305817 & -1.232354 & 0.680755 \\
\hline $\mathrm{H}$ & 0.542929 & -0.250935 & 1.901401 & $\mathrm{C}$ & 5.070065 & -0.389318 & -0.124386 \\
\hline $\mathrm{C}$ & -0.035479 & 0.795518 & -2.673466 & $\mathrm{H}$ & 5.159380 & 0.601804 & -2.029005 \\
\hline $\mathrm{H}$ & 0.471404 & 1.125561 & -3.604071 & $\mathrm{H}$ & 4.685506 & -1.534317 & 1.655261 \\
\hline $\mathrm{H}$ & -0.155718 & -0.306593 & -2.732690 & $\mathrm{C}$ & -1.364600 & -3.340546 & 1.604249 \\
\hline $\mathrm{O}$ & 1.715360 & 2.073608 & 0.952203 & $\mathrm{H}$ & -0.648047 & -2.620052 & 2.016736 \\
\hline $\mathrm{H}$ & 1.101401 & 0.329775 & 2.398063 & $\mathrm{H}$ & -1.764429 & -3.918194 & 2.440723 \\
\hline $\mathrm{C}$ & 2.646455 & 2.644060 & 0.067613 & $\mathrm{H}$ & -0.783571 & -4.021614 & 0.972287 \\
\hline $\mathrm{H}$ & 3.344534 & 3.272048 & 0.640263 & $\mathrm{C}$ & -2.956830 & -0.685131 & -2.386913 \\
\hline $\mathrm{H}$ & 3.224126 & 1.842223 & -0.433688 & $\mathrm{H}$ & -3.872286 & -0.550083 & -2.968284 \\
\hline $\mathrm{O}$ & 1.849034 & 1.128316 & 3.129656 & $\mathrm{H}$ & -2.534005 & 0.311136 & -2.200230 \\
\hline $\mathrm{H}$ & 1.982668 & 1.705835 & 2.162326 & $\mathrm{H}$ & -2.233590 & -1.216506 & -3.013167 \\
\hline $\mathrm{C}$ & 0.962666 & 1.875529 & 3.944241 & $\mathrm{C}$ & -6.174776 & -1.914821 & 1.265751 \\
\hline $\mathrm{H}$ & 0.039811 & 2.137557 & 3.404201 & $\mathrm{H}$ & -6.852404 & -2.584023 & 0.723842 \\
\hline $\mathrm{H}$ & 0.698544 & 1.291302 & 4.831011 & $\mathrm{H}$ & -6.187935 & -2.217093 & 2.315612 \\
\hline $\mathrm{H}$ & 1.442155 & 2.804196 & 4.270741 & $\mathrm{H}$ & -6.605862 & -0.911002 & 1.199195 \\
\hline $\mathrm{O}$ & 2.032889 & 3.462346 & -0.876306 & $\mathrm{C}$ & 2.812665 & -0.007670 & -3.150693 \\
\hline $\mathrm{H}$ & 1.510438 & 2.833231 & -1.413195 & $\mathrm{H}$ & 2.578314 & 1.061696 & -3.135128 \\
\hline $\mathrm{O}$ & -1.363200 & 1.289479 & -2.653836 & $\mathrm{H}$ & 3.576010 & -0.162459 & -3.919248 \\
\hline \multirow[t]{2}{*}{$\mathrm{H}$} & -1.313008 & 2.180796 & -2.283976 & $\mathrm{H}$ & 1.903068 & -0.530755 & -3.452763 \\
\hline & & & & $\mathrm{C}$ & 2.331788 & -2.662349 & 1.176220 \\
\hline $4 I$ & & & & $\mathrm{H}$ & 1.321211 & -2.893630 & 0.832915 \\
\hline $\mathrm{Ru}$ & 0.360706 & 0.448565 & 0.409476 & $\mathrm{H}$ & 2.882459 & -3.608012 & 1.242084 \\
\hline $\mathrm{C}$ & 0.109708 & -1.215477 & -0.690058 & $\mathrm{H}$ & 2.264483 & -2.252757 & 2.190915 \\
\hline $\mathrm{C}$ & -0.502129 & -3.159445 & -1.856202 & $\mathrm{C}$ & 6.378650 & 0.155869 & 0.356294 \\
\hline $\mathrm{C}$ & 1.012052 & -3.007998 & -1.919553 & $\mathrm{H}$ & 7.087901 & 0.297070 & -0.464054 \\
\hline $\mathrm{H}$ & -0.830077 & -4.136003 & -1.485099 & $\mathrm{H}$ & 6.237054 & 1.135559 & 0.827847 \\
\hline $\mathrm{H}$ & -0.981793 & -2.993633 & -2.830923 & $\mathrm{H}$ & 6.841401 & -0.494604 & 1.102798 \\
\hline $\mathrm{H}$ & 1.534768 & -3.758505 & -1.307873 & $\mathrm{C}$ & -1.451321 & 0.788843 & 0.738448 \\
\hline $\mathrm{H}$ & 1.411093 & -3.066862 & -2.937048 & $\mathrm{H}$ & -2.108587 & 0.048181 & 1.217868 \\
\hline $\mathrm{N}$ & -0.890732 & -2.094705 & -0.920339 & $\mathrm{C}$ & -2.103653 & 2.061260 & 0.504778 \\
\hline $\mathrm{N}$ & 1.219470 & -1.670428 & -1.351736 & $\mathrm{C}$ & -3.497605 & 2.082268 & 0.297554 \\
\hline $\mathrm{C}$ & -2.214885 & -2.040465 & -0.389018 & $\mathrm{C}$ & -1.398507 & 3.282247 & 0.471665 \\
\hline $\mathrm{C}$ & -3.234182 & -1.395775 & -1.103894 & $\mathrm{C}$ & -4.150159 & 3.265614 & -0.016791 \\
\hline
\end{tabular}




$\begin{array}{rrrr}\mathrm{H} & -4.049202 & 1.144540 & 0.352389 \\ \mathrm{C} & -2.065749 & 4.466802 & 0.184521 \\ \mathrm{H} & -0.332382 & 3.289229 & 0.691236 \\ \mathrm{C} & -3.436120 & 4.461983 & -0.080229 \\ \mathrm{H} & -5.220531 & 3.260084 & -0.205522 \\ \mathrm{H} & -1.511365 & 5.401587 & 0.172388 \\ \mathrm{H} & -3.949968 & 5.390320 & -0.315031 \\ \mathrm{O} & 0.694162 & 1.627497 & -1.222860 \\ \mathrm{H} & 0.003667 & -0.331570 & 1.937043 \\ \mathrm{C} & 0.033576 & 1.489437 & -2.416947 \\ \mathrm{H} & 0.645816 & 1.977865 & -3.200748 \\ \mathrm{H} & -0.112349 & 0.431576 & -2.711233 \\ \mathrm{O} & 1.651361 & 1.606497 & 1.495733 \\ \mathrm{H} & 0.740970 & -0.668598 & 1.708852 \\ \mathrm{C} & 2.527875 & 2.475469 & 0.838803 \\ \mathrm{H} & 3.223099 & 2.888087 & 1.586127 \\ \mathrm{H} & 3.119693 & 1.917101 & 0.085992 \\ \mathrm{O} & 2.402526 & -0.206164 & 3.380113 \\ \mathrm{H} & 2.308399 & 0.497781 & 2.697870 \\ \mathrm{C} & 1.533296 & 0.149096 & 4.422500 \\ \mathrm{H} & 0.473386 & 0.169470 & 4.108884 \\ \mathrm{H} & 1.630848 & -0.594122 & 5.219530 \\ \mathrm{H} & 1.760815 & 1.136527 & 4.849570 \\ \mathrm{O} & 1.897390 & 3.568870 & 0.233904 \\ \mathrm{H} & 1.425632 & 3.165472 & -0.518006 \\ \mathrm{O} & -1.270309 & 2.038366 & -2.434339 \\ \mathrm{H} & -1.227862 & 2.855598 & -1.920660 \\ & & & \end{array}$

\section{TSI-13-4}

$\begin{array}{rrrr}\mathrm{Ru} & 0.412926 & 0.400249 & 0.479268 \\ \mathrm{C} & 0.131262 & -1.305585 & -0.494381 \\ \mathrm{C} & -0.515138 & -3.274379 & -1.618409 \\ \mathrm{C} & 0.976044 & -3.043777 & -1.858058 \\ \mathrm{H} & -0.718108 & -4.180637 & -1.029439 \\ \mathrm{H} & -1.090731 & -3.352136 & -2.547479 \\ \mathrm{H} & 1.598394 & -3.889288 & -1.542482 \\ \mathrm{H} & 1.203080 & -2.840681 & -2.913753 \\ \mathrm{~N} & -0.921548 & -2.080002 & -0.866698 \\ \mathrm{~N} & 1.251976 & -1.864185 & -1.037724 \\ \mathrm{C} & -2.259997 & -1.983328 & -0.381903 \\ \mathrm{C} & -3.249631 & -1.372672 & -1.163282 \\ \mathrm{C} & -2.566585 & -2.550097 & 0.868181 \\ \mathrm{C} & -4.553100 & -1.326768 & -0.660214 \\ \mathrm{C} & -3.878928 & -2.474782 & 1.329085\end{array}$

$$
\begin{array}{lrrr}
\mathrm{C} & -4.887427 & -1.860344 & 0.582867 \\
\mathrm{H} & -5.325352 & -0.845182 & -1.259768 \\
\mathrm{H} & -4.120307 & -2.906219 & 2.299713 \\
\mathrm{C} & 2.574409 & -1.472450 & -0.647898 \\
\mathrm{C} & 3.377483 & -0.700308 & -1.502571 \\
\mathrm{C} & 3.056837 & -1.925164 & 0.597706 \\
\mathrm{C} & 4.644847 & -0.325906 & -1.046025 \\
\mathrm{C} & 4.316720 & -1.503968 & 1.014230 \\
\mathrm{C} & 5.119430 & -0.687650 & 0.212626 \\
\mathrm{H} & 5.269876 & 0.284873 & -1.696038
\end{array}
$$$$
\begin{array}{llll}
\mathrm{H} & 4.687234 & -1.833934 & 1.983560
\end{array}
$$$$
\begin{array}{llll}
\text { C } & -1.500591 & -3.168281 & 1.716066
\end{array}
$$$$
\begin{array}{llll}
\mathrm{H} & -0.854741 & -2.394063 & 2.148010
\end{array}
$$$$
\begin{array}{llll}
\mathrm{H} & -1.932760 & -3.741044 & 2.539852
\end{array}
$$$$
\begin{array}{llll}
\mathrm{H} & -0.842697 & -3.833950 & 1.147413
\end{array}
$$$$
\begin{array}{llll}
\text { C } & -2.943546 & -0.729354 & -2.477977
\end{array}
$$

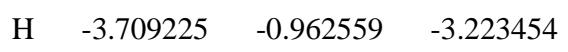$$
\begin{array}{llll}
\mathrm{H} & -2.923685 & 0.362476 & -2.369234
\end{array}
$$$$
\begin{array}{llll}
\mathrm{H} & -1.968321 & -1.022388 & -2.872130
\end{array}
$$$$
\begin{array}{llll}
\text { C } & -6.282696 & -1.749788 & 1.116270
\end{array}
$$$$
\begin{array}{llll}
\mathrm{H} & -7.028916 & -1.829727 & 0.320927
\end{array}
$$$$
\begin{array}{llll}
\mathrm{H} & -6.495220 & -2.521616 & 1.860458
\end{array}
$$$$
\begin{array}{llll}
\mathrm{H} & -6.441719 & -0.781098 & 1.604542
\end{array}
$$$$
\begin{array}{llll}
\text { C } & 2.915801 & -0.251456 & -2.849784
\end{array}
$$$$
\begin{array}{llll}
\mathrm{H} & 2.515422 & 0.766914 & -2.802142
\end{array}
$$$$
\begin{array}{llll}
\mathrm{H} & 3.741899 & -0.258141 & -3.566378
\end{array}
$$$$
\text { H } \quad 2.111242 \quad-0.875020 \quad-3.247437
$$$$
\begin{array}{llll}
\text { C } & 2.249521 & -2.861946 & 1.441489
\end{array}
$$$$
\begin{array}{llll}
\mathrm{H} & 1.321603 & -2.396347 & 1.787672
\end{array}
$$$$
\begin{array}{llll}
\mathrm{H} & 1.960893 & -3.761121 & 0.883336
\end{array}
$$$$
\begin{array}{llll}
\mathrm{H} & 2.814000 & -3.181434 & 2.320224
\end{array}
$$$$
\begin{array}{llll}
\text { C } & 6.445509 & -0.195719 & 0.703511
\end{array}
$$$$
\text { H } \quad 7.160610 \quad-0.068288 \quad-0.113743
$$$$
\begin{array}{llll}
\mathrm{H} & 6.340719 & 0.781405 & 1.189820
\end{array}
$$$$
\begin{array}{llll}
\mathrm{H} & 6.884658 & -0.873859 & 1.440045
\end{array}
$$$$
\begin{array}{llll}
\text { C } & -1.370882 & 0.804069 & 0.738077
\end{array}
$$$$
\begin{array}{llll}
\mathrm{H} & -2.072433 & 0.096109 & 1.209537
\end{array}
$$$$
\begin{array}{llll}
\text { C } & -1.996489 & 2.052866 & 0.333969
\end{array}
$$$$
\begin{array}{llll}
\text { C } & -3.391215 & 2.081809 & 0.127941
\end{array}
$$$$
\begin{array}{llll}
\text { C } & -1.255882 & 3.230592 & 0.100890
\end{array}
$$$$
\begin{array}{llll}
\text { C } & -4.014383 & 3.222246 & -0.360294
\end{array}
$$$$
\begin{array}{llll}
\mathrm{H} & -3.967920 & 1.179459 & 0.329525
\end{array}
$$$$
\begin{array}{llll}
\text { C } & -1.890917 & 4.372918 & -0.367252
\end{array}
$$$$
\begin{array}{llll}
\mathrm{H} & -0.187871 & 3.233241 & 0.303691
\end{array}
$$ 


$\begin{array}{crrr}\mathrm{C} & -3.263895 & 4.369864 & -0.615809 \\ \mathrm{H} & -5.086810 & 3.221575 & -0.538253 \\ \mathrm{H} & -1.309960 & 5.276026 & -0.536385 \\ \mathrm{H} & -3.751634 & 5.265161 & -0.992950 \\ \mathrm{O} & 0.807774 & 1.419331 & -1.261677 \\ \mathrm{H} & 0.320033 & -0.544423 & 1.956358 \\ \mathrm{C} & 0.016286 & 1.204604 & -2.364460 \\ \mathrm{H} & 0.041289 & 0.147356 & -2.699325 \\ \mathrm{H} & -1.042114 & 1.459225 & -2.143098 \\ \mathrm{O} & 1.538273 & 1.905877 & 1.486867 \\ \mathrm{H} & 0.705648 & 0.022889 & 2.626943 \\ \mathrm{C} & 2.596228 & 2.561839 & 0.835470 \\ \mathrm{H} & 3.182931 & 3.118986 & 1.579675 \\ \mathrm{H} & 3.259724 & 1.812663 & 0.357686 \\ \mathrm{O} & 1.224611 & 0.779775 & 3.550994 \\ \mathrm{H} & 1.555521 & 1.439069 & 2.689621 \\ \mathrm{C} & 0.146908 & 1.443887 & 4.188744 \\ \mathrm{H} & -0.676225 & 1.643549 & 3.484957 \\ \mathrm{H} & -0.229996 & 0.826362 & 5.009207 \\ \mathrm{H} & 0.483686 & 2.401619 & 4.597928 \\ \mathrm{O} & 2.140405 & 3.483228 & -0.102419 \\ \mathrm{H} & 1.680692 & 2.930021 & -0.765938 \\ \mathrm{O} & 0.479924 & 1.945215 & -3.469246 \\ \mathrm{H} & 0.452049 & 2.869314 & -3.197121\end{array}$

\section{TSI-23-4}

$\begin{array}{rrrr}\mathrm{Ru} & 0.224256 & 0.653251 & 0.326805 \\ \mathrm{C} & 0.548259 & -1.230837 & -0.239249 \\ \mathrm{C} & 0.370221 & -3.458437 & -1.012529 \\ \mathrm{C} & 1.838407 & -3.117226 & -0.847563 \\ \mathrm{H} & 0.074225 & -4.381867 & -0.503320 \\ \mathrm{H} & 0.075794 & -3.550772 & -2.069202 \\ \mathrm{H} & 2.295955 & -3.630721 & 0.012850 \\ \mathrm{H} & 2.443505 & -3.344548 & -1.731416 \\ \mathrm{~N} & -0.285565 & -2.297404 & -0.407042 \\ \mathrm{~N} & 1.798842 & -1.673305 & -0.598874 \\ \mathrm{C} & -1.683395 & -2.342288 & -0.141811 \\ \mathrm{C} & -2.607014 & -1.972894 & -1.129698 \\ \mathrm{C} & -2.096313 & -2.760583 & 1.130803 \\ \mathrm{C} & -3.962656 & -2.010119 & -0.804579 \\ \mathrm{C} & -3.462865 & -2.778724 & 1.411324 \\ \mathrm{C} & -4.410826 & -2.401416 & 0.458785 \\ \mathrm{H} & -4.688123 & -1.698520 & -1.555489 \\ \mathrm{H} & -3.794204 & -3.086847 & 2.402071\end{array}$

\begin{tabular}{|c|c|c|c|}
\hline $\mathrm{C}$ & 3.041283 & -1.032137 & -0.2 \\
\hline $\mathrm{C}$ & 3.856889 & -0.597220 & -1.354623 \\
\hline$C$ & 3.490521 & -0.960283 & 1.035066 \\
\hline $\mathrm{C}$ & 5.094106 & -0.026419 & -1.051074 \\
\hline $\mathrm{C}$ & 4.740823 & -0.398146 & 1.283452 \\
\hline $\mathrm{C}$ & 5.550999 & 0.091536 & 0.260149 \\
\hline $\mathrm{H}$ & 5.722061 & 0.325606 & -1.869001 \\
\hline 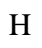 & 5.084587 & -0.330180 & 2.314856 \\
\hline $\mathrm{C}$ & -1.087198 & -3.1 & 2.178395 \\
\hline $\mathrm{H}$ & -0.497330 & -2.235677 & 2.466561 \\
\hline $\mathrm{H}$ & -1.569115 & -3.505938 & 3.077313 \\
\hline $\mathrm{H}$ & -0.369268 & -3.8 & 7318 \\
\hline $\mathrm{C}$ & -2.158997 & -1.459607 & -2.459774 \\
\hline $\mathrm{H}$ & -2.970569 & -1.507964 & -3.190847 \\
\hline $\mathrm{H}$ & -1.839129 & -0.410436 & -2.385546 \\
\hline $\mathrm{H}$ & -1.307970 & -2.021265 & -2.859667 \\
\hline $\mathrm{C}$ & -5.875238 & -2.417209 & 0.774850 \\
\hline $\mathrm{H}$ & -6.056892 & -2.542164 & 1.845202 \\
\hline $\mathrm{H}$ & -6.363216 & -1.490114 & 0.456417 \\
\hline $\mathrm{H}$ & -6.389250 & -3.234856 & 0.257339 \\
\hline $\mathrm{C}$ & 3.454883 & -0.758611 & -2.788128 \\
\hline $\mathrm{H}$ & 3.544469 & 0.187465 & -3.331028 \\
\hline $\mathrm{H}$ & 4.105798 & -1.478452 & -3.298304 \\
\hline $\mathrm{H}$ & 2.423704 & -1.101842 & -2.887739 \\
\hline $\mathrm{C}$ & 2.666233 & -1.434445 & 2.190904 \\
\hline $\mathrm{H}$ & 2.141697 & -0.591774 & 2.654950 \\
\hline $\mathrm{H}$ & 1.903907 & -2.164317 & 1.903579 \\
\hline $\mathrm{H}$ & 3.300227 & -1.885364 & 2.959921 \\
\hline $\mathrm{C}$ & 6.864729 & 0.741293 & 0.566854 \\
\hline $\mathrm{H}$ & 7.523680 & 0.757455 & -0.305283 \\
\hline $\mathrm{H}$ & 6.723706 & 1.780292 & 0805817 \\
\hline $\mathrm{H}$ & 7.389712 & 0.231000 & 1.379697 \\
\hline $\mathrm{C}$ & -1.636504 & 0.634360 & 0.310631 \\
\hline $\mathrm{H}$ & -2.213258 & -0.143316 & 0.834169 \\
\hline $\mathrm{C}$ & -2.506263 & 1.681747 & -0.192952 \\
\hline $\mathrm{C}$ & -3.898060 & 1.544135 & 0.000651 \\
\hline $\mathrm{C}$ & -2.032882 & 2.826796 & -0.866045 \\
\hline $\mathrm{C}$ & -4.784866 & 2.504947 & -0.463155 \\
\hline $\mathrm{H}$ & -4.263298 & 0.659957 & 0.523250 \\
\hline $\mathrm{C}$ & -2.924053 & 3.785826 & -1.323252 \\
\hline $\mathrm{H}$ & -0.966124 & 2.931536 & -1.027231 \\
\hline $\mathrm{C}$ & -4.296845 & 3.630724 & -1.126403 \\
\hline $\mathrm{H}$ & -5.853745 & 2.382466 & -0.307565 \\
\hline $\mathrm{H}$ & -2.548387 & 4.662977 & -1.843375 \\
\hline
\end{tabular}




$\begin{array}{rrrr}\mathrm{H} & -4.987474 & 4.387445 & -1.490451 \\ \mathrm{O} & 0.930509 & 1.388179 & -1.462152 \\ \mathrm{H} & 0.052076 & -0.050563 & 1.924612 \\ \mathrm{C} & 0.581758 & 0.882359 & -2.666040 \\ \mathrm{H} & 1.349810 & 1.150249 & -3.421611 \\ \mathrm{H} & 0.493703 & -0.230681 & -2.652241 \\ \mathrm{O} & 0.309778 & 2.754378 & 1.073522 \\ \mathrm{H} & -0.031661 & 0.727721 & 2.416282 \\ \mathrm{C} & 1.644368 & 3.063553 & 0.926998 \\ \mathrm{H} & 1.801222 & 3.734979 & 0.070238 \\ \mathrm{H} & 2.049478 & 3.529648 & 1.837337 \\ \mathrm{O} & -0.138568 & 1.833178 & 3.241686 \\ \mathrm{H} & 0.071531 & 2.433136 & 2.262073 \\ \mathrm{C} & -1.491490 & 1.989222 & 3.596268 \\ \mathrm{H} & -2.169274 & 1.824971 & 2.741636 \\ \mathrm{H} & -1.758737 & 1.276258 & 4.385569 \\ \mathrm{H} & -1.678538 & 3.001118 & 3.977030 \\ \mathrm{O} & 2.411971 & 1.861916 & 0.721713 \\ \mathrm{H} & 2.486616 & 1.743908 & -0.241429 \\ \mathrm{O} & -0.676039 & 1.425894 & -3.075241 \\ \mathrm{H} & -0.871389 & 1.066230 & -3.947519\end{array}$

\section{TSI-33-4}

$\begin{array}{rrrr}\mathrm{Ru} & 0.399223 & 0.858114 & 0.113507 \\ \mathrm{C} & 0.468511 & -1.128816 & -0.084125 \\ \mathrm{C} & 0.059068 & -3.403283 & -0.543055 \\ \mathrm{C} & 1.486510 & -3.073002 & -0.936778 \\ \mathrm{H} & -0.000699 & -4.047982 & 0.348268 \\ \mathrm{H} & -0.507731 & -3.884341 & -1.345817 \\ \mathrm{H} & 2.226397 & -3.768552 & -0.528991 \\ \mathrm{H} & 1.605672 & -3.026800 & -2.027783 \\ \mathrm{~N} & -0.501754 & -2.079874 & -0.241489 \\ \mathrm{~N} & 1.659268 & -1.738277 & -0.358176 \\ \mathrm{C} & -1.866257 & -2.026038 & 0.175489 \\ \mathrm{C} & -2.893764 & -2.147468 & -0.773514 \\ \mathrm{C} & -2.164172 & -1.997786 & 1.553948 \\ \mathrm{C} & -4.217443 & -2.131472 & -0.323753 \\ \mathrm{C} & -3.498647 & -1.991825 & 1.951476 \\ \mathrm{C} & -4.544594 & -2.038046 & 1.025436 \\ \mathrm{H} & -5.015342 & -2.203746 & -1.062042 \\ \mathrm{H} & -3.727480 & -1.956437 & 3.016119 \\ \mathrm{C} & 2.961355 & -1.222938 & -0.070382 \\ \mathrm{C} & 3.799851 & -0.793152 & -1.107641 \\ \mathrm{C} & 3.412847 & -1.244605 & 1.261688\end{array}$

\begin{tabular}{|c|c|c|c|}
\hline & & & \\
\hline & 4.693126 & .769585 & \\
\hline & 541347 & & \\
\hline & & & \\
\hline & .043 & & \\
\hline & .073 & 9 & 2.57608 \\
\hline & 4 & & \\
\hline & -1.47 & 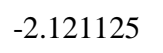 & \\
\hline & -0.31 & -2.72 & \\
\hline & -2.63 & & \\
\hline & -2.884244 & 3 & -2.52 \\
\hline & -3.2 & & -2.8 \\
\hline & -1.59 & -2.1 & -2.5 \\
\hline & -5.971112 & -1.9 & \\
\hline & -6.6 & & \\
\hline & -6.12 & -2 & 20002 \\
\hline & -6.28 & -0. & \\
\hline & 3. & & \\
\hline & & & \\
\hline & 3.93 & -0.1 & -3 \\
\hline & 3.35 & -1.77 & -2.9 \\
\hline & 2.55 & -1 & \\
\hline & & & \\
\hline & 2.153189 & -2.7 & \\
\hline & 3.12 & -1.88 & 3.28 \\
\hline & 6.901 & & \\
\hline & 6.8 & & \\
\hline & 7.370708 & -0.32 & \\
\hline & 7.570 & & -0.0 \\
\hline & & & \\
\hline & 17 & & \\
\hline & -2.43 & 9 & .00 \\
\hline & -3.7653 & 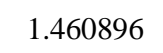 & \\
\hline & -2.204 & & \\
\hline & -4.82 & 2.110544 & -0.2048 \\
\hline & -3.940732 & 43 & 18 \\
\hline & -3.272197 & 5 & -1.0 \\
\hline & -1.178989 & & 1.28 \\
\hline & -4.583 & 3.049151 & -1.20688 \\
\hline & -5.848 & 3 & 0.1 \\
\hline & -3.084764 & 42 & -2.360 \\
\hline & -5.413118 & & - \\
\hline & & 115438 & -1.94 \\
\hline & 34025 & 64339 & \\
\hline
\end{tabular}




\begin{tabular}{|c|c|c|c|c|c|c|c|}
\hline $\mathrm{C}$ & -0.540792 & 0.351111 & -2.716906 & $\mathrm{H}$ & 5.622913 & 0.326711 & -1.921469 \\
\hline $\mathrm{H}$ & -1.452922 & 0.036859 & -2.182503 & $\mathrm{H}$ & 5.121664 & -0.543889 & 2.243748 \\
\hline $\mathrm{H}$ & -0.847146 & 0.927793 & -3.610402 & $\mathrm{C}$ & -1.441309 & -3.234469 & 2.016489 \\
\hline $\mathrm{O}$ & 0.755290 & 3.061975 & 0.160079 & $\mathrm{H}$ & -0.867544 & -2.400432 & 2.438104 \\
\hline $\mathrm{H}$ & 0.802930 & 1.536505 & 2.079353 & $\mathrm{H}$ & -2.023926 & -3.683939 & 2.823976 \\
\hline $\mathrm{C}$ & 2.009853 & 3.198557 & -0.408406 & $\mathrm{H}$ & -0.705262 & -3.972426 & 1.678289 \\
\hline $\mathrm{H}$ & 1.970682 & 3.866720 & -1.283324 & $\mathrm{C}$ & -2.002031 & -1.253140 & -2.591063 \\
\hline $\mathrm{H}$ & 2.740935 & 3.593685 & 0.312821 & $\mathrm{H}$ & -2.664282 & -1.400175 & -3.448360 \\
\hline $\mathrm{O}$ & 0.843475 & 2.816802 & 2.540787 & $\mathrm{H}$ & -1.857855 & -0.171552 & -2.469310 \\
\hline $\mathrm{H}$ & 0.824692 & 3.105295 & 1.409204 & $\mathrm{H}$ & -1.020471 & -1.669766 & -2.833340 \\
\hline $\mathrm{C}$ & -0.408375 & 3.133334 & 3.102047 & $\mathrm{C}$ & -6.038868 & -2.241696 & 0.216167 \\
\hline $\mathrm{H}$ & -1.236239 & 2.962535 & 2.393636 & $\mathrm{H}$ & -6.520457 & -3.067847 & -0.319101 \\
\hline $\mathrm{H}$ & -0.590060 & 2.519255 & 3.993201 & $\mathrm{H}$ & -6.322294 & -2.327555 & 1.268237 \\
\hline $\mathrm{H}$ & -0.437638 & 4.186598 & 3.405928 & $\mathrm{H}$ & -6.474680 & -1.317222 & -0.175975 \\
\hline $\mathrm{O}$ & 2.520134 & 1.925502 & -0.810186 & $\mathrm{C}$ & 3.283853 & -0.620102 & -2.813154 \\
\hline $\mathrm{H}$ & 2.035271 & 1.692767 & -1.626426 & $\mathrm{H}$ & 2.916033 & 0.366801 & -3.120283 \\
\hline $\mathrm{O}$ & 0.044067 & -0.886349 & -3.157130 & $\mathrm{H}$ & 4.099437 & -0.893740 & -3.489601 \\
\hline \multirow[t]{2}{*}{$\mathrm{H}$} & 0.705568 & -0.654965 & -3.816778 & $\mathrm{H}$ & 2.460579 & -1.324631 & -2.961036 \\
\hline & & & & $\mathrm{C}$ & 2.665666 & -1.593121 & 2.139923 \\
\hline \multicolumn{2}{|c|}{ TSI-43-4 } & & & $\mathrm{H}$ & 2.104274 & -0.771145 & 2.599695 \\
\hline $\mathrm{Ru}$ & 0.172207 & 0.574531 & 0.488928 & $\mathrm{H}$ & 1.933893 & -2.352688 & 1.848407 \\
\hline $\mathrm{C}$ & 0.486452 & -1.254538 & -0.248335 & $\mathrm{H}$ & 3.311877 & -2.019164 & 2.912465 \\
\hline $\mathrm{C}$ & 0.282463 & -3.455023 & -1.087081 & $\mathrm{C}$ & 6.861799 & 0.581484 & 0.485836 \\
\hline $\mathrm{C}$ & 1.751645 & -3.139063 & -0.895230 & $\mathrm{H}$ & 7.504061 & 0.586876 & -0.398863 \\
\hline $\mathrm{H}$ & -0.031102 & -4.394173 & -0.619305 & $\mathrm{H}$ & 6.761331 & 1.621460 & 0.817026 \\
\hline $\mathrm{H}$ & -0.002914 & -3.499427 & -2.149603 & $\mathrm{H}$ & 7.385428 & 0.045886 & 1.282966 \\
\hline $\mathrm{H}$ & 2.187721 & -3.673118 & -0.036311 & $\mathrm{C}$ & -1.674948 & 0.660943 & 0.301420 \\
\hline $\mathrm{H}$ & 2.368835 & -3.357524 & -1.772495 & $\mathrm{H}$ & -2.346494 & -0.114741 & 0.699423 \\
\hline $\mathrm{N}$ & -0.360911 & -2.309932 & -0.438304 & $\mathrm{C}$ & -2.427239 & 1.794225 & -0.214104 \\
\hline $\mathrm{N}$ & 1.727451 & -1.698993 & -0.622284 & $\mathrm{C}$ & -3.835845 & 1.711076 & -0.223224 \\
\hline $\mathrm{C}$ & -1.777165 & -2.301105 & -0.299503 & $\mathrm{C}$ & -1.822467 & 2.970625 & -0.707985 \\
\hline $\mathrm{C}$ & -2.587900 & -1.833369 & -1.343680 & $\mathrm{C}$ & -4.613266 & 2.752205 & -0.710836 \\
\hline $\mathrm{C}$ & -2.327032 & -2.768583 & 0.903136 & $\mathrm{H}$ & -4.302922 & 0.805774 & 0.164000 \\
\hline $\mathrm{C}$ & -3.969677 & -1.828179 & -1.151387 & $\mathrm{C}$ & -2.605566 & 4.006875 & -1.193476 \\
\hline $\mathrm{C}$ & -3.713456 & -2.735749 & 1.053217 & $\mathrm{H}$ & -0.738307 & 3.043056 & -0.692212 \\
\hline $\mathrm{C}$ & -4.551362 & -2.266739 & 0.039216 & $\mathrm{C}$ & -3.998044 & 3.904676 & -1.197313 \\
\hline $\mathrm{H}$ & -4.607750 & -1.445146 & -1.947536 & $\mathrm{H}$ & -5.697131 & 2.670945 & -0.708457 \\
\hline $\mathrm{H}$ & -4.150033 & -3.080945 & 1.989490 & $\mathrm{H}$ & -2.129778 & 4.909027 & -1.568959 \\
\hline $\mathrm{C}$ & 2.982369 & -1.076130 & -0.329670 & $\mathrm{H}$ & -4.602787 & 4.725526 & -1.574532 \\
\hline $\mathrm{C}$ & 3.760015 & -0.591209 & -1.394380 & $\mathrm{O}$ & 0.959513 & 1.555846 & -1.122088 \\
\hline $\mathrm{C}$ & 3.474753 & -1.084283 & 0.988108 & $\mathrm{H}$ & -0.152423 & -0.340516 & 1.948362 \\
\hline $\mathrm{C}$ & 5.017658 & -0.060288 & -1.102532 & $\mathrm{C}$ & 0.542406 & 1.234455 & -2.374804 \\
\hline $\mathrm{C}$ & 4.741479 & -0.553229 & 1.223033 & $\mathrm{H}$ & 0.639972 & 0.141006 & -2.577723 \\
\hline $\mathrm{C}$ & 5.522659 & -0.023692 & 0.196598 & $\mathrm{H}$ & -0.529078 & 1.500299 & -2.533577 \\
\hline
\end{tabular}




$\begin{array}{rrrr}\mathrm{O} & 0.246596 & 2.549041 & 1.521765 \\ \mathrm{H} & -0.259901 & 0.380916 & 2.527618 \\ \mathrm{C} & 1.593377 & 2.837429 & 1.483162 \\ \mathrm{H} & 1.810373 & 3.590786 & 0.712718 \\ \mathrm{H} & 1.955656 & 3.190945 & 2.459411 \\ \mathrm{O} & -0.447891 & 1.353241 & 3.475909 \\ \mathrm{H} & -0.099383 & 2.083522 & 2.628198 \\ \mathrm{C} & -1.841729 & 1.468313 & 3.644379 \\ \mathrm{H} & -2.329428 & 1.936298 & 2.773906 \\ \mathrm{H} & -2.293093 & 0.474769 & 3.784910 \\ \mathrm{H} & -2.074796 & 2.071723 & 4.529700 \\ \mathrm{O} & 2.342421 & 1.641852 & 1.182763 \\ \mathrm{H} & 2.521121 & 1.658222 & 0.226380 \\ \mathrm{O} & 1.346288 & 1.969316 & -3.289078 \\ \mathrm{H} & 0.948484 & 1.868370 & -4.159395\end{array}$

\section{TSI-53-4}

$\begin{array}{rrrc}\mathrm{Ru} & 0.354319 & 0.758074 & 0.129812 \\ \mathrm{C} & 0.504604 & -1.199932 & -0.221859 \\ \mathrm{C} & 0.239632 & -3.524239 & -0.561702 \\ \mathrm{C} & 1.637885 & -3.130505 & -0.998347 \\ \mathrm{H} & 0.233901 & -4.126699 & 0.360329 \\ \mathrm{H} & -0.314322 & -4.081301 & -1.323967 \\ \mathrm{H} & 2.426227 & -3.730986 & -0.533066 \\ \mathrm{H} & 1.768878 & -3.190088 & -2.089260 \\ \mathrm{~N} & -0.394942 & -2.224556 & -0.315598 \\ \mathrm{~N} & 1.721160 & -1.736234 & -0.553389 \\ \mathrm{C} & -1.778849 & -2.236171 & 0.035558 \\ \mathrm{C} & -2.746831 & -2.240445 & -0.978841 \\ \mathrm{C} & -2.138892 & -2.342037 & 1.389441 \\ \mathrm{C} & -4.091718 & -2.278132 & -0.601792 \\ \mathrm{C} & -3.493362 & -2.374629 & 1.716316 \\ \mathrm{C} & -4.486238 & -2.326816 & 0.734278 \\ \mathrm{H} & -4.851372 & -2.264753 & -1.382436 \\ \mathrm{H} & -3.780167 & -2.436438 & 2.765361 \\ \mathrm{C} & 2.999443 & -1.157546 & -0.282482 \\ \mathrm{C} & 3.755144 & -0.582272 & -1.310780 \\ \mathrm{C} & 3.510383 & -1.268618 & 1.024441 \\ \mathrm{C} & 5.025682 & -0.092694 & -0.999209 \\ \mathrm{C} & 4.772154 & -0.742082 & 1.292063 \\ \mathrm{C} & 5.547836 & -0.150613 & 0.289980 \\ \mathrm{H} & 5.615186 & 0.366209 & -1.791907 \\ \mathrm{H} & 5.170598 & -0.818522 & 2.303411 \\ \mathrm{C} & -1.097636 & -2.339647 & 2.463940 \\ & & & \end{array}$

\begin{tabular}{|c|c|c|c|}
\hline & & & \\
\hline & 1.525980 & 2.616223 & 430 \\
\hline & .272957 & & 10 \\
\hline & -2.374266 & -2.17 & \\
\hline & .131 & 2.66 & $30-2$ \\
\hline & -2.28 & -1.13 & 2.7 \\
\hline & & & \\
\hline & -5.937226 & -2.38 & \\
\hline & -6.54 & -2.8 & \\
\hline & -6.1 & & \\
\hline & -6.324 & 3 & \\
\hline & 3.2 & & \\
\hline & 2.65 & .527178 & -2 \\
\hline & 4.014288 & -0.3 & \\
\hline & 2.50 & -1.2 & \\
\hline & 2.723 & -1 & 20968 \\
\hline & 1.786466 & -1.4 & 2.3 \\
\hline & 2.44 & -2.9 & \\
\hline & & & \\
\hline & 6.920130 & 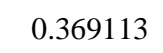 & 0.59 \\
\hline & 7.249946 & 1.09 & -0.1 \\
\hline & 6.964 & 0.848 & \\
\hline & 7. & -0.4 & \\
\hline & -1.469132 & 0.77 & 0.4639 \\
\hline & -1.93 & 8 & \\
\hline & -2.44 & & \\
\hline & -3.8 & 1.508965 & \\
\hline & -2.131231 & 2.890050 & -0.7 \\
\hline & -4.802834 & 15 & -0. \\
\hline & & & \\
\hline & -3.1 & 8717 & 11 \\
\hline & -1.086 & 28 & 0.96982 \\
\hline & -4.471607 & 5.400094 & -0.00350 \\
\hline & -5.8414 & & \\
\hline & -2.8783 & 62 & 7. \\
\hline & -5.250923 & 4.164 & -1.2 \\
\hline & 0.555888 & 3 & -1.06 \\
\hline & & & \\
\hline & 0. & 0.5860 & -2 \\
\hline & & 31 & -3. \\
\hline & 0.177162 & -0.512687 & -2.7728 \\
\hline & & & \\
\hline & & 11 & 2.2. \\
\hline & 939399 & .099798. & 1.12 \\
\hline
\end{tabular}




$\begin{array}{cccc}\mathrm{H} & 1.966165 & 3.328012 & -0.945022 \\ \mathrm{H} & 2.494052 & 3.864361 & 0.693028 \\ \mathrm{O} & 0.571600 & 2.367778 & 2.873880 \\ \mathrm{H} & 0.603433 & 2.823793 & 1.763474 \\ \mathrm{C} & -0.719892 & 2.535009 & 3.406327 \\ \mathrm{H} & -1.377684 & 1.683611 & 3.155097 \\ \mathrm{H} & -0.677187 & 2.616139 & 4.498660 \\ \mathrm{H} & -1.203152 & 3.439769 & 3.011078 \\ \mathrm{O} & 2.625888 & 1.830471 & 0.230705 \\ \mathrm{H} & 2.940585 & 1.719372 & 1.137043 \\ \mathrm{O} & -1.363651 & 0.749828 & -3.017088 \\ \mathrm{H} & -1.553172 & 1.658798 & -2.752385\end{array}$

\section{TSI-63-4}

\begin{tabular}{rrrr}
$\mathrm{Ru}$ & 0.279321 & 0.678646 & 0.366878 \\
$\mathrm{C}$ & 0.460115 & -1.146416 & -0.402508 \\
$\mathrm{C}$ & 0.141630 & -3.254797 & -1.432203 \\
$\mathrm{C}$ & 1.603314 & -2.850022 & -1.585383 \\
$\mathrm{H}$ & 0.008234 & -4.170374 & -0.838563 \\
$\mathrm{H}$ & -0.361092 & -3.414513 & -2.394068 \\
$\mathrm{H}$ & 2.298270 & -3.569482 & -1.134974 \\
$\mathrm{H}$ & 1.899548 & -2.718514 & -2.634648 \\
$\mathrm{~N}$ & -0.450469 & -2.106455 & -0.739581 \\
$\mathrm{~N}$ & 1.668894 & -1.570770 & -0.874077 \\
$\mathrm{C}$ & -1.811715 & -2.197048 & -0.324300 \\
$\mathrm{C}$ & -2.843642 & -1.781426 & -1.173662 \\
$\mathrm{C}$ & -2.089900 & -2.742969 & 0.943447 \\
$\mathrm{C}$ & -4.161416 & -1.894859 & -0.718365 \\
$\mathrm{C}$ & -3.416110 & -2.833737 & 1.353455 \\
$\mathrm{C}$ & -4.469956 & -2.408742 & 0.536987 \\
$\mathrm{H}$ & -4.967131 & -1.546624 & -1.364087 \\
$\mathrm{H}$ & -3.633612 & -3.238600 & 2.341193 \\
$\mathrm{C}$ & 2.946568 & -1.082674 & -0.449428 \\
$\mathrm{C}$ & 3.738539 & -0.323916 & -1.321955 \\
$\mathrm{C}$ & 3.418843 & -1.471008 & 0.816171 \\
$\mathrm{C}$ & 5.007287 & 0.062975 & -0.885325 \\
$\mathrm{C}$ & 4.687007 & -1.048469 & 1.210886 \\
$\mathrm{C}$ & 5.494872 & -0.274302 & 0.375088 \\
$\mathrm{H}$ & 5.627839 & 0.656990 & -1.555052 \\
$\mathrm{H}$ & 5.060450 & -1.348415 & 2.189705 \\
$\mathrm{C}$ & -0.978156 & -3.153290 & 1.855467 \\
$\mathrm{H}$ & -0.362004 & -2.289402 & 2.131374 \\
$\mathrm{H}$ & -1.364603 & -3.601451 & 2.773640 \\
$\mathrm{H}$ & -0.301249 & -3.877592 & 1.387568 \\
& & & \\
\hline
\end{tabular}

\begin{tabular}{|c|c|c|c|}
\hline $\mathrm{C}$ & -2.580722 & -1.170998 & -2.512512 \\
\hline $\mathrm{H}$ & -3.218618 & -1.614714 & -3.283232 \\
\hline $\mathrm{H}$ & -2.804058 & -0.096756 & -2.489576 \\
\hline H & -1.536721 & -1.272508 & -2.816367 \\
\hline $\mathrm{C}$ & -5.888728 & -2.507230 & 1.006993 \\
\hline $\mathrm{H}$ & -6.003499 & -2.117150 & 2.023319 \\
\hline $\mathrm{H}$ & -6.568103 & -1.951455 & 0.355660 \\
\hline $\mathrm{H}$ & -6.233743 & -3.547052 & 1.029880 \\
\hline $\mathrm{C}$ & 3.236545 & 0.119873 & -2.654813 \\
\hline $\mathrm{H}$ & 2.619579 & 1.019421 & -2.545682 \\
\hline $\mathrm{H}$ & 4.063411 & 0.350672 & -3.331549 \\
\hline $\mathrm{H}$ & 2.596874 & -0.627711 & -3.134985 \\
\hline $\mathrm{C}$ & 2.585585 & -2.312591 & 1.731428 \\
\hline $\mathrm{H}$ & 1.785703 & -1.721609 & 2.194293 \\
\hline $\mathrm{H}$ & 2.089961 & -3.134621 & 1.202515 \\
\hline $\mathrm{H}$ & 3.191135 & -2.741396 & 2.533652 \\
\hline $\mathrm{C}$ & 6.844462 & 0.192754 & 0.827726 \\
\hline $\mathrm{H}$ & 7.541782 & 0.288135 & -0.009269 \\
\hline $\mathrm{H}$ & 6.783299 & 1.178965 & 1.302492 \\
\hline $\mathrm{H}$ & 7.285591 & -0.487770 & 1.561316 \\
\hline $\mathrm{C}$ & -1.571644 & 0.668647 & 0.533567 \\
\hline $\mathrm{H}$ & -2.095381 & -0.127872 & 1.085369 \\
\hline $\mathrm{C}$ & -2.496775 & 1.695077 & 0.082714 \\
\hline $\mathrm{C}$ & -3.875637 & 1.500494 & 0.311942 \\
\hline $\mathrm{C}$ & -2.090469 & 2.855525 & -0.611138 \\
\hline $\mathrm{C}$ & -4.815306 & 2.413033 & -0.146784 \\
\hline $\mathrm{H}$ & -4.191700 & 0.605643 & 0.847095 \\
\hline $\mathrm{C}$ & -3.035220 & 3.763418 & -1.066394 \\
\hline $\mathrm{H}$ & -1.028950 & 3.010931 & -0.783621 \\
\hline $\mathrm{C}$ & -4.395926 & 3.548369 & -0.839112 \\
\hline $\mathrm{H}$ & -5.873521 & 2.243775 & 0.035809 \\
\hline $\mathrm{H}$ & -2.711157 & 4.650845 & -1.603444 \\
\hline $\mathrm{H}$ & -5.127882 & 4.267094 & -1.198579 \\
\hline $\mathrm{O}$ & 0.639025 & 1.550654 & -1.423639 \\
\hline $\mathrm{H}$ & 0.313434 & -0.103306 & 1.956928 \\
\hline $\mathrm{C}$ & 0.068382 & 1.089232 & -2.566056 \\
\hline $\mathrm{H}$ & 0.288259 & 0.001936 & -2.738958 \\
\hline $\mathrm{H}$ & -1.040468 & 1.195074 & -2.550549 \\
\hline $\mathrm{O}$ & 0.493445 & 2.779033 & 1.179945 \\
\hline $\mathrm{H}$ & 0.320758 & 0.662059 & 2.472729 \\
\hline $\mathrm{C}$ & 1.817005 & 2.966823 & 0.850367 \\
\hline $\mathrm{H}$ & 1.910841 & 3.314092 & -0.182634 \\
\hline H & 2.322506 & 3.659471 & 1.541742 \\
\hline O & 0.383195 & 1.749575 & 3.340357 \\
\hline
\end{tabular}




$\begin{array}{cccc}\mathrm{H} & 0.420432 & 2.422474 & 2.327164 \\ \mathrm{C} & -0.876976 & 1.890930 & 3.944349 \\ \mathrm{H} & -1.702758 & 1.738294 & 3.226650 \\ \mathrm{H} & -0.998704 & 1.164149 & 4.757710 \\ \mathrm{H} & -0.995608 & 2.895026 & 4.372366 \\ \mathrm{O} & 2.505167 & 1.691495 & 0.860652 \\ \mathrm{H} & 2.710160 & 1.467612 & 1.777732 \\ \mathrm{O} & 0.608018 & 1.855490 & -3.630311 \\ \mathrm{H} & 0.221476 & 1.520482 & -4.444990\end{array}$

TSI-73-4

$\begin{array}{rrrr}\mathrm{Ru} & 0.357932 & 0.834113 & 0.004289 \\ \mathrm{C} & 0.473691 & -1.152900 & -0.111104 \\ \mathrm{C} & 0.117082 & -3.462784 & -0.429456 \\ \mathrm{C} & 1.549836 & -3.133392 & -0.808951 \\ \mathrm{H} & 0.045453 & -4.070569 & 0.486298 \\ \mathrm{H} & -0.426191 & -3.985775 & -1.222786 \\ \mathrm{H} & 2.290141 & -3.772890 & -0.317266 \\ \mathrm{H} & 1.708175 & -3.189354 & -1.894405 \\ \mathrm{~N} & -0.469054 & -2.137766 & -0.197753 \\ \mathrm{~N} & 1.679451 & -1.747535 & -0.352837 \\ \mathrm{C} & -1.840333 & -2.084076 & 0.195242 \\ \mathrm{C} & -2.854141 & -2.238328 & -0.762528 \\ \mathrm{C} & -2.153731 & -2.011937 & 1.567374 \\ \mathrm{C} & -4.183238 & -2.191791 & -0.330635 \\ \mathrm{C} & -3.492282 & -1.984169 & 1.948750 \\ \mathrm{C} & -4.526156 & -2.046496 & 1.010029 \\ \mathrm{H} & -4.972746 & -2.282848 & -1.075627 \\ \mathrm{H} & -3.734512 & -1.911244 & 3.008626 \\ \mathrm{C} & 2.965986 & -1.192957 & -0.070899 \\ \mathrm{C} & 3.769462 & -0.693352 & -1.104754 \\ \mathrm{C} & 3.433943 & -1.235333 & 1.254795 \\ \mathrm{C} & 5.043224 & -0.221649 & -0.785971 \\ \mathrm{C} & 4.704636 & -0.731164 & 1.529252 \\ \mathrm{C} & 5.528780 & -0.225628 & 0.520978 \\ \mathrm{H} & 5.664211 & 0.181634 & -1.584857 \\ \mathrm{H} & 5.066940 & -0.752494 & 2.556374 \\ \mathrm{C} & -1.070849 & -1.938941 & 2.596763 \\ \mathrm{H} & -0.531089 & -0.984310 & 2.545774 \\ \mathrm{H} & -1.478276 & -2.043624 & 3.605047 \\ \mathrm{H} & -0.316038 & -2.719977 & 2.452097 \\ \mathrm{C} & -2.568022 & -2.512953 & -2.206455 \\ \mathrm{H} & -2.809692 & -3.556339 & -2.445585 \\ \mathrm{H} & -3.195164 & -1.894696 & -2.856117\end{array}$

\begin{tabular}{|c|c|c|c|}
\hline $\mathrm{H}$ & -1.528859 & -2.331073 & -2.480708 \\
\hline C & -5.957436 & -1.940562 & 1.436051 \\
\hline $\mathrm{H}$ & -6.631375 & -2.418466 & 0.720163 \\
\hline $\mathrm{H}$ & -6.125589 & -2.393568 & 2.417327 \\
\hline $\mathrm{H}$ & -6.267232 & -0.890971 & 1.514074 \\
\hline $\mathrm{C}$ & 3.245581 & -0.581128 & -2.497899 \\
\hline H & 2.448890 & 0.174398 & -2.523063 \\
\hline $\mathrm{H}$ & 4.035141 & -0.281816 & -3.191741 \\
\hline $\mathrm{H}$ & 2.815225 & -1.523908 & -2.852972 \\
\hline $\mathrm{C}$ & 2.588122 & -1.808146 & 2.347410 \\
\hline $\mathrm{H}$ & 1.700104 & -1.191833 & 2.526311 \\
\hline $\mathrm{H}$ & 2.221975 & -2.810623 & 2.094595 \\
\hline $\mathrm{H}$ & 3.146103 & -1.878399 & 3.283961 \\
\hline $\mathrm{C}$ & 6.909645 & 0.265214 & 0.832159 \\
\hline $\mathrm{H}$ & 6.963939 & 0.723176 & 1.823837 \\
\hline $\mathrm{H}$ & 7.635402 & -0.556576 & 0.822743 \\
\hline $\mathrm{H}$ & 7.252102 & 1.001199 & 0.099832 \\
\hline $\mathrm{C}$ & -1.407812 & 0.904948 & 0.566080 \\
\hline $\mathrm{H}$ & -1.750462 & 0.357609 & 1.456104 \\
\hline $\mathrm{C}$ & -2.478612 & 1.717872 & 0.014912 \\
\hline $\mathrm{C}$ & -3.763819 & 1.598195 & 0.586008 \\
\hline $\mathrm{C}$ & -2.311454 & 2.596276 & -1.076963 \\
\hline $\mathrm{C}$ & -4.842782 & 2.310502 & 0.083360 \\
\hline $\mathrm{H}$ & -3.895467 & 0.917167 & 1.426420 \\
\hline $\mathrm{C}$ & -3.394554 & 3.307952 & -1.572892 \\
\hline $\mathrm{H}$ & -1.321559 & 2.698412 & -1.510328 \\
\hline $\mathrm{C}$ & -4.659544 & 3.169344 & -0.999793 \\
\hline $\mathrm{H}$ & -5.826091 & 2.201964 & 0.533882 \\
\hline $\mathrm{H}$ & -3.254072 & 3.984693 & -2.411858 \\
\hline $\mathrm{H}$ & -5.500618 & 3.734295 & -1.393961 \\
\hline $\mathrm{O}$ & 0.205382 & 1.052401 & -2.012446 \\
\hline $\mathrm{H}$ & 0.838848 & 0.664726 & 1.709115 \\
\hline $\mathrm{C}$ & -0.572750 & 0.218462 & -2.757875 \\
\hline $\mathrm{H}$ & -1.489827 & -0.102623 & -2.233373 \\
\hline $\mathrm{H}$ & -0.872218 & 0.744087 & -3.685742 \\
\hline $\mathrm{O}$ & 0.613887 & 3.087047 & -0.002268 \\
\hline $\mathrm{H}$ & 0.885756 & 1.562475 & 1.944900 \\
\hline $\mathrm{C}$ & 1.809648 & 3.153665 & -0.673867 \\
\hline $\mathrm{H}$ & 1.642880 & 3.193036 & -1.754940 \\
\hline $\mathrm{H}$ & 2.426001 & 4.007300 & -0.349150 \\
\hline $\mathrm{O}$ & 1.021590 & 2.866610 & 2.348564 \\
\hline $\mathrm{H}$ & 0.808112 & 3.143514 & 1.183599 \\
\hline $\mathrm{C}$ & -0.104588 & 3.194368 & 3.122217 \\
\hline $\mathrm{H}$ & -1.023451 & 2.707547 & 2.748677 \\
\hline
\end{tabular}




$\begin{array}{crrc}\mathrm{H} & 0.041996 & 2.884392 & 4.164504 \\ \mathrm{H} & -0.282388 & 4.277600 & 3.115213 \\ \mathrm{O} & 2.555574 & 1.925655 & -0.477565 \\ \mathrm{H} & 3.016387 & 1.974051 & 0.370447 \\ \mathrm{O} & 0.071582 & -1.011376 & -3.118250 \\ \mathrm{H} & 0.868043 & -0.750521 & -3.592323\end{array}$

3I $\mathbf{w}_{\mathrm{w}}$

$\mathrm{Ru}$

$$
\text { C }
$$$$
\begin{array}{llll}
\text { C } & -1.060991 & -3.376972 & 1.169981
\end{array}
$$$$
\begin{array}{llll}
\mathrm{H} & 0.578500 & -4.278771 & 0.025712
\end{array}
$$$$
\begin{array}{llll}
\mathrm{H} & 0.983984 & -3.973160 & 1.722592
\end{array}
$$$$
\begin{array}{llll}
\mathrm{H} & -1.703740 & -4.154883 & 0.743830
\end{array}
$$$$
\begin{array}{llll}
\mathrm{H} & -1.261483 & -3.330503 & 2.251938
\end{array}
$$$$
\begin{array}{llll}
\mathrm{N} & 0.875300 & -2.239083 & 0.514049
\end{array}
$$$$
\begin{array}{llll}
\mathrm{N} & -1.306733 & -2.084816 & 0.539683
\end{array}
$$$$
\begin{array}{llll}
\text { C } & 2.242782 & -2.047117 & 0.154826
\end{array}
$$$$
\begin{array}{llll}
\text { C } & 3.173879 & -1.702244 & 1.142698
\end{array}
$$$$
\begin{array}{llll}
\text { C } & 2.629116 & -2.244419 & -1.181240
\end{array}
$$$$
\begin{array}{llll}
\text { C } & 4.504338 & -1.521511 & 0.755136
\end{array}
$$$$
\begin{array}{llll}
\text { C } & 3.967429 & -2.054590 & -1.519239
\end{array}
$$$$
\begin{array}{llll}
\text { C } & 4.918730 & -1.681494 & -0.565763
\end{array}
$$$$
\mathrm{H} \quad 5.235445 \quad-1.243796 \quad 1.513441
$$$$
\begin{array}{llll}
\mathrm{H} & 4.273684 & -2.193673 & -2.555552
\end{array}
$$$$
\begin{array}{llll}
\text { C } & -2.600628 & -1.537051 & 0.284978
\end{array}
$$$$
\begin{array}{llll}
\text { C } & -3.337072 & -0.912270 & 1.303197
\end{array}
$$$$
\begin{array}{llll}
\text { C } & -3.121591 & -1.653583 & -1.022524
\end{array}
$$$$
\begin{array}{llll}
\text { C } & -4.565801 & -0.333555 & 0.967786
\end{array}
$$$$
\begin{array}{llll}
\text { C } & -4.338308 & -1.042113 & -1.309882
\end{array}
$$$$
\begin{array}{llll}
\text { C } & -5.067540 & -0.359504 & -0.330807
\end{array}
$$$$
\begin{array}{llll}
\mathrm{H} & -5.133119 & 0.170874 & 1.749090
\end{array}
$$$$
\mathrm{H} \quad-4.731274 \quad-1.103986 \quad-2.323683
$$$$
\begin{array}{llll}
\text { C } & 1.612174 & -2.560815 & -2.232940
\end{array}
$$$$
\mathrm{H} \quad 0.971087 \quad-1.688643 \quad-2.418689
$$$$
\mathrm{H} \quad 2.090735 \quad-2.840937 \quad-3.174588
$$$$
\begin{array}{llll}
\mathrm{H} & 0.941648 & -3.376529 & -1.938991
\end{array}
$$$$
\begin{array}{llll}
\text { C } & 2.764053 & -1.484605 & 2.563152
\end{array}
$$$$
\begin{array}{llll}
\mathrm{H} & 3.592146 & -1.688767 & 3.247123
\end{array}
$$$$
\mathrm{H} \quad 2.452308 \quad-0.444121 \quad 2.711797
$$$$
\mathrm{H} \quad 1.914724-2.112156 \quad 2.849925
$$$$
\begin{array}{llll}
\text { C } & 6.342201 & -1.431208 & -0.960122
\end{array}
$$$$
\mathrm{H} \quad 7.032063-1.624243 \quad-0.134140
$$

$\begin{array}{lrrc}\mathrm{H} & 6.644847 & -2.051705 & -1.808210 \\ \mathrm{H} & 6.491177 & -0.386982 & -1.260819 \\ \mathrm{C} & -2.868380 & -0.865903 & 2.722835 \\ \mathrm{H} & -2.893217 & 0.158173 & 3.104535 \\ \mathrm{H} & -3.518240 & -1.474333 & 3.362611 \\ \mathrm{H} & -1.845351 & -1.227000 & 2.836357 \\ \mathrm{C} & -2.399270 & -2.454133 & -2.061358 \\ \mathrm{H} & -1.372953 & -2.100863 & -2.197745 \\ \mathrm{H} & -2.335850 & -3.510927 & -1.773716 \\ \mathrm{H} & -2.913764 & -2.402016 & -3.024155 \\ \mathrm{C} & -6.338198 & 0.350801 & -0.680079 \\ \mathrm{H} & -6.991045 & 0.467947 & 0.188754 \\ \mathrm{H} & -6.127876 & 1.356831 & -1.063086 \\ \mathrm{H} & -6.896997 & -0.173330 & -1.460376 \\ \mathrm{C} & 1.294545 & 0.879082 & -0.720256 \\ \mathrm{H} & 1.991917 & 0.133419 & -1.127544 \\ \mathrm{C} & 1.951003 & 2.165651 & -0.525621 \\ \mathrm{C} & 3.323816 & 2.259588 & -0.832331 \\ \mathrm{C} & 1.310214 & 3.307420 & 0.002912 \\ \mathrm{C} & 4.029421 & 3.437340 & -0.625842 \\ \mathrm{H} & 1.222801 & 1.352892 & 2.658725 \\ \mathrm{H} & 3.827233 & 1.377516 & -1.227423 \\ \mathrm{C} & 2.020623 & 4.481842 & 0.207879 \\ \mathrm{H} & 0.250797 & 3.265821 & 0.241217 \\ \mathrm{C} & 3.379537 & 4.553976 & -0.104283 \\ \mathrm{H} & 5.088472 & 3.485225 & -0.866028 \\ \mathrm{H} & 1.510522 & 5.351515 & 0.614238 \\ \mathrm{H} & 3.928820 & 5.477037 & 0.061931 \\ \mathrm{O} & -0.863344 & 1.175122 & 1.628112 \\ \mathrm{H} & -0.619922 & -0.121555 & -1.847124 \\ \mathrm{C} & -0.099456 & 0.841354 & 2.709868 \\ \mathrm{H} & -0.602562 & 1.202139 & 3.631061 \\ \mathrm{H} & 0.045118 & -0.253882 & 2.814400 \\ \mathrm{O} & -1.508756 & 2.379799 & -1.010923 \\ \mathrm{H} & -1.724203 & 0.510281 & -2.925624 \\ \mathrm{C} & -2.606968 & 2.794559 & -0.162165 \\ \mathrm{H} & -3.214057 & 3.522302 & -0.715113 \\ \mathrm{H} & -3.21742 & 1.898624 & 0.061599 \\ \mathrm{H} & 1.207416 & -3.386186 \\ \mathrm{H} & -154625 & -1.901646 \\ \mathrm{H} & & -3.073310\end{array}$




\section{TSI $_{\mathrm{w} 3-4}$}$$
\mathrm{Ru} \quad-0.495388
$$$$
\text { C } \quad-0.218248
$$$$
\text { C } \quad 0.333320
$$$$
0.523866
$$$$
-0.451932
$$$$
-1.257594
$$$$
0.378334
$$$$
\text { C }-1.089231
$$$$
-3.396130
$$$$
1.191742
$$$$
\mathrm{H} \quad 0.375198
$$$$
-3.066945
$$$$
1.628210
$$$$
\mathrm{H} \quad 0.981412
$$$$
\text { H } \quad-1.819100
$$$$
-4.197768
$$$$
0.438729
$$$$
2.023212
$$$$
1.329976
$$$$
\text { H } \quad-1.172242 \quad-2.932269
$$$$
2.716552
$$$$
\mathrm{N} \quad 0.796218 \quad-2.131173
$$$$
0.603453
$$$$
\mathrm{N} \quad-1.343290
$$$$
-1.801639
$$$$
0.936460
$$$$
\begin{array}{lll}
\text { C } & 2.158689 & -2.009948
\end{array}
$$$$
0.194979
$$$$
\text { C } 3.130242
$$$$
\text { C } 2.508249
$$$$
-1.651331
$$$$
1.139945
$$$$
\text { C } 4.458335
$$$$
\text { C } 3.844872
$$$$
-2.325432
$$$$
-1.128850
$$$$
-1.564266
$$$$
0.715267
$$$$
-2.216539
$$$$
-1.507157
$$$$
\text { C } 4.834573
$$$$
-1.825564
$$$$
-0.601521
$$$$
\mathrm{H} \quad 5.218461
$$$$
-1.275751
$$$$
1.440256
$$$$
\text { H } 4.120241
$$$$
-2.445563
$$$$
-2.535780
$$$$
\text { C } \quad-2.654689
$$$$
-1.359945
$$$$
0.565945
$$$$
\text { C } \quad-3.415757
$$$$
-0.550560
$$$$
1.421981
$$$$
\text { C }-3.166822
$$$$
-1.798749
$$$$
-0.673310
$$$$
\text { C }-4.675063
$$$$
-0.131806
$$$$
0.977025
$$$$
\text { C } \quad-4.417201
$$$$
-1.342642
$$$$
-1.074064
$$$$
\text { C } \quad-5.181001
$$$$
-0.492083
$$$$
-0.268301
$$$$
\text { H } \quad-5.269409
$$$$
0.507416
$$$$
1.628800
$$$$
\text { H } \quad-4.807252
$$$$
-1.658527
$$$$
-2.040215
$$$$
\text { C } \quad 1.461878
$$$$
-2.720853
$$$$
-2.122256
$$$$
\text { H } \quad 0.795717 \quad-1.878329
$$$$
\text { H } 1.911829 \quad-3.055078
$$$$
-2.347214
$$$$
-3.059922
$$$$
-1.750337
$$$$
\text { H } 0.819665
$$$$
\begin{array}{lll}
\text { C } & 2.776745 & -1.332314
\end{array}
$$$$
\text { H } \quad 3.596121 \quad-1.590449
$$$$
2.556199
$$$$
3.232370
$$$$
2.658815
$$$$
\text { H } \quad 2.575346 \quad-0.260670
$$$$
\text { H } 1.875494 \quad-1.853695
$$$$
\begin{array}{lll}
\text { C } & 6.257137 & -1.663206
\end{array}
$$$$
2.891476
$$$$
-1.041007
$$$$
\mathrm{H} \quad 6.960463 \quad-1.904808 \quad-0.239328
$$$$
\mathrm{H} \quad 6.491824 \quad-2.295061 \quad-1.901810
$$$$
\mathrm{H} \quad 6.461118 \quad-0.627654 \quad-1.340025
$$$$
\begin{array}{llll}
\text { C } & -2.922990 & -0.110197 & 2.762758
\end{array}
$$$$
\begin{array}{llll}
\mathrm{H} & -2.529196 & 0.910236 & 2.701345
\end{array}
$$$$
\mathrm{H} \quad-3.732547 \quad-0.119547 \quad 3.498046
$$$$
\begin{array}{llll}
\mathrm{H} & -2.108769 & -0.737583 & 3.133735
\end{array}
$$

\begin{tabular}{|c|c|c|c|}
\hline$C$ & -2.387078 & -2.7 & 114 \\
\hline F & -1.419804 & -2.326391 & -1.822354 \\
\hline 1 & -2.174053 & -3.687063 & -1.003237 \\
\hline $\mathrm{H}$ & -2.936169 & -2.994282 & -2.440506 \\
\hline$C$ & -6.501707 & 0.029666 & -0.743075 \\
\hline 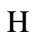 & -7.144936 & 0.323989 & 90367 \\
\hline $\mathrm{H}$ & -6.366343 & 0.915285 & -1.374582 \\
\hline $\mathrm{H}$ & -7.038779 & -0.709144 & -1.344725 \\
\hline C & 1.253686 & 0.803772 & -0.961063 \\
\hline $\mathrm{H}$ & 1.820004 & 0.060916 & -1.544339 \\
\hline C & 2.033044 & 1.990975 & -0.660238 \\
\hline C & 3.437511 & 1.888826 & -0.655493 \\
\hline C & 1.442740 & 3.231023 & -0.341024 \\
\hline C & 4.226840 & 2.971077 & -0.291046 \\
\hline $\mathrm{H}$ & 3.891077 & 0.931523 & -0.910 \\
\hline$C$ & 2.241194 & 4.316861 & -0.003629 \\
\hline $\mathrm{H}$ & 0.359420 & 3.328315 & -0.377191 \\
\hline C & 3.630910 & 4.188519 & 0.036890 \\
\hline П & 5.308971 & 2.869875 & -0.267236 \\
\hline $\mathrm{H}$ & 1.776125 & 5.2 & 14 \\
\hline $\mathrm{H}$ & 4.248249 & 5.039498 & 0.311825 \\
\hline ? & -0.630268 & 1.503124 & 1.357571 \\
\hline$U$ & -0.628404 & -0.346852 & -1.966979 \\
\hline $\mathrm{C}$ & 0.081356 & 1.156364 & 2.471862 \\
\hline $\mathrm{H}$ & -0.405699 & 1.626198 & 3.351643 \\
\hline $\mathrm{H}$ & 0.105422 & 0.061454 & 2.650507 \\
\hline $\mathrm{O}$ & -1.693136 & 2.100610 & -1.256113 \\
\hline $\mathrm{H}$ & -1.243020 & 0.151202 & -2.488003 \\
\hline $\mathrm{C}$ & -2.553066 & 2.845759 & -0.428862 \\
\hline $\mathrm{H}$ & -3.211592 & 3.458302 & -1.061736 \\
\hline $\mathrm{H}$ & -3.185116 & 2.155580 & 0.166055 \\
\hline $\mathrm{O}$ & -2.123456 & 0.818499 & -3.216499 \\
\hline $\mathrm{H}$ & -2.083403 & 1.576779 & -2.359684 \\
\hline $\mathrm{O}$ & -1.853883 & 3.714078 & 0.400984 \\
\hline $\mathrm{H}$ & -1.353337 & 3.114171 & 0.990846 \\
\hline $\mathrm{O}$ & 1.445347 & 1.533804 & 2.42524 \\
\hline $\mathrm{H}$ & 1.469165 & 2.396965 & 1.991932 \\
\hline $\mathrm{H}$ & -1.633187 & 1.207268 & -3.949014 \\
\hline
\end{tabular}

$4 I_{w}$

$\begin{array}{rrrr}\mathrm{Ru} & -0.524616 & 0.490297 & -0.498727 \\ \mathrm{C} & -0.188226 & -1.348051 & 0.296156 \\ \mathrm{C} & 0.439290 & -3.432609 & 1.171134 \\ \mathrm{C} & -1.081622 & -3.346920 & 1.153411\end{array}$




\begin{tabular}{|c|c|c|c|c|c|c|c|}
\hline $\mathrm{H}$ & 0.833032 & -4.323634 & 0.671992 & $\mathrm{H}$ & -6.182863 & 1.462395 & -0.657864 \\
\hline $\mathrm{H}$ & 0.846095 & -3.407592 & 2.190996 & $\mathrm{H}$ & -6.900486 & -0.049054 & -1.203785 \\
\hline $\mathrm{H}$ & -1.531846 & -3.994085 & 0.385540 & $\mathrm{C}$ & 1.218503 & 0.887114 & -0.986433 \\
\hline $\mathrm{H}$ & -1.542014 & -3.595293 & 2.114822 & $\mathrm{H}$ & 1.768568 & 0.231334 & -1.680700 \\
\hline $\mathrm{N}$ & 0.838362 & -2.217194 & 0.442380 & $\mathrm{C}$ & 1.985724 & 2.052513 & -0.598705 \\
\hline $\mathrm{N}$ & -1.301848 & -1.939710 & 0.811040 & $\mathrm{C}$ & 3.392234 & 1.965946 & -0.602064 \\
\hline $\mathrm{C}$ & 2.206770 & -1.987821 & 0.103497 & $\mathrm{C}$ & 1.381839 & 3.262514 & -0.202507 \\
\hline $\mathrm{C}$ & 3.127889 & -1.631385 & 1.100140 & $\mathrm{C}$ & 4.168175 & 3.028803 & -0.162082 \\
\hline $\mathrm{C}$ & 2.607834 & -2.175380 & -1.229076 & $\mathrm{H}$ & 3.857384 & 1.033429 & -0.918884 \\
\hline $\mathrm{C}$ & 4.449084 & -1.394703 & 0.715440 & $\mathrm{C}$ & 2.168674 & 4.331607 & 0.209429 \\
\hline $\mathrm{C}$ & 3.940220 & -1.933234 & -1.561653 & $\mathrm{H}$ & 0.298514 & 3.354123 & -0.246820 \\
\hline $\mathrm{C}$ & 4.874134 & -1.529343 & -0.605262 & $\mathrm{C}$ & 3.558861 & 4.215409 & 0.247510 \\
\hline $\mathrm{H}$ & 5.163766 & -1.083878 & 1.476362 & $\mathrm{H}$ & 5.251420 & 2.937746 & -0.143619 \\
\hline $\mathrm{H}$ & 4.254074 & -2.058114 & -2.597222 & $\mathrm{H}$ & 1.692056 & 5.263951 & 0.500953 \\
\hline $\mathrm{C}$ & -2.609185 & -1.416120 & 0.533957 & $\mathrm{H}$ & 4.166899 & 5.052430 & 0.580138 \\
\hline $\mathrm{C}$ & -3.316488 & -0.761017 & 1.555933 & $\mathrm{O}$ & -0.617748 & 1.374352 & 1.354709 \\
\hline $\mathrm{C}$ & -3.155216 & -1.560895 & -0.757511 & $\mathrm{H}$ & -0.452413 & -0.528630 & -1.915724 \\
\hline $\mathrm{C}$ & -4.561778 & -0.214150 & 1.247411 & $\mathrm{C}$ & 0.143055 & 0.947365 & 2.408417 \\
\hline $\mathrm{C}$ & -4.388423 & -0.965510 & -1.020763 & $\mathrm{H}$ & -0.220998 & 1.470340 & 3.316608 \\
\hline $\mathrm{C}$ & -5.098783 & -0.276968 & -0.038306 & $\mathrm{H}$ & 0.045683 & -0.142557 & 2.594740 \\
\hline $\mathrm{H}$ & -5.109157 & 0.309417 & 2.030094 & $\mathrm{O}$ & -1.835206 & 1.942037 & -1.156262 \\
\hline $\mathrm{H}$ & -4.786129 & -1.023359 & -2.031895 & $\mathrm{H}$ & -0.963848 & 0.074991 & -2.182642 \\
\hline $\mathrm{C}$ & 1.632395 & -2.594233 & -2.284632 & $\mathrm{C}$ & -2.576664 & 2.678746 & -0.232014 \\
\hline $\mathrm{H}$ & 1.009741 & -1.754658 & -2.618765 & $\mathrm{H}$ & -3.347264 & 3.239293 & -0.784565 \\
\hline $\mathrm{H}$ & 2.150320 & -2.979018 & -3.166363 & $\mathrm{H}$ & -3.087936 & 2.000575 & 0.479310 \\
\hline $\mathrm{H}$ & 0.943394 & -3.367236 & -1.928284 & $\mathrm{O}$ & -2.728785 & 0.644949 & -3.351917 \\
\hline $\mathrm{C}$ & 2.743382 & -1.546308 & 2.542470 & $\mathrm{H}$ & -2.593060 & 1.196017 & -2.545013 \\
\hline $\mathrm{H}$ & 2.937066 & -2.499823 & 3.051327 & $\mathrm{O}$ & -1.828285 & 3.627951 & 0.477428 \\
\hline $\mathrm{H}$ & 3.314568 & -0.772428 & 3.056813 & $\mathrm{H}$ & -1.286852 & 3.076248 & 1.071034 \\
\hline $\mathrm{H}$ & 1.688773 & -1.296779 & 2.670094 & $\mathrm{O}$ & 1.535041 & 1.146011 & 2.258641 \\
\hline $\mathrm{C}$ & 6.298932 & -1.262885 & -0.983909 & $\mathrm{H}$ & 1.638939 & 2.038354 & 1.902546 \\
\hline $\mathrm{H}$ & 6.906551 & -2.173276 & -0.926344 & $\mathrm{H}$ & -2.401025 & 1.206918 & -4.058067 \\
\hline $\mathrm{H}$ & 6.382029 & -0.888653 & -2.008041 & & & & \\
\hline $\mathrm{H}$ & 6.759924 & -0.528925 & -0.317076 & \multicolumn{4}{|c|}{ TSI-1 $_{\text {w3-4 }}$} \\
\hline $\mathrm{C}$ & -2.758321 & -0.593611 & 2.933237 & $\mathrm{Ru}$ & -0.341269 & 0.334212 & -0.596558 \\
\hline $\mathrm{H}$ & -2.358759 & 0.419857 & 3.050734 & $\mathrm{C}$ & -0.053564 & -1.256400 & 0.539397 \\
\hline $\mathrm{H}$ & -3.534507 & -0.733049 & 3.690937 & $\mathrm{C}$ & 0.590946 & -3.149305 & 1.770107 \\
\hline $\mathrm{H}$ & -1.938363 & -1.285422 & 3.141124 & $\mathrm{C}$ & -0.927958 & -3.106622 & 1.699232 \\
\hline $\mathrm{C}$ & -2.479459 & -2.335340 & -1.850207 & $\mathrm{H}$ & 1.017108 & -4.111991 & 1.469227 \\
\hline $\mathrm{H}$ & -1.441686 & -2.589804 & -1.618455 & $\mathrm{H}$ & 0.969830 & -2.914501 & 2.776088 \\
\hline $\mathrm{H}$ & -3.012679 & -3.273506 & -2.044102 & $\mathrm{H}$ & -1.333454 & -3.872171 & 1.019239 \\
\hline $\mathrm{H}$ & -2.488686 & -1.753121 & -2.777138 & $\mathrm{H}$ & -1.411936 & -3.228570 & 2.673100 \\
\hline $\mathrm{C}$ & -6.379714 & 0.423575 & -0.367073 & $\mathrm{~N}$ & 0.975896 & -2.091337 & 0.828549 \\
\hline $\mathrm{H}$ & -7.059562 & 0.454698 & 0.488778 & $\mathrm{~N}$ & -1.170330 & -1.767847 & 1.153869 \\
\hline
\end{tabular}




\begin{tabular}{|c|c|c|c|c|c|c|c|}
\hline $\mathrm{C}$ & 2.332432 & -1.935303 & 0.422425 & $\mathrm{C}$ & 1.094907 & 3.330863 & -0.525900 \\
\hline $\mathrm{C}$ & 3.213387 & -1.137409 & 1.164467 & $\mathrm{C}$ & 3.822849 & 3.608067 & 0.025384 \\
\hline $\mathrm{C}$ & 2.754849 & -2.615779 & -0.730342 & $\mathrm{H}$ & 3.949020 & 1.481915 & -0.314343 \\
\hline $\mathrm{C}$ & 4.533484 & -1.029739 & 0.721111 & $\mathrm{C}$ & 1.626678 & 4.578949 & -0.232993 \\
\hline $\mathrm{C}$ & 4.080032 & -2.471084 & -1.138250 & $\mathrm{H}$ & 0.040569 & 3.222967 & -0.767932 \\
\hline $\mathrm{C}$ & 4.984403 & -1.678209 & -0.428799 & $\mathrm{C}$ & 2.984624 & 4.722194 & 0.054778 \\
\hline $\mathrm{H}$ & 5.224646 & -0.407990 & 1.289932 & $\mathrm{H}$ & 4.883275 & 3.717979 & 0.237601 \\
\hline $\mathrm{H}$ & 4.414372 & -2.990371 & -2.035284 & $\mathrm{H}$ & 0.978066 & 5.450918 & -0.237729 \\
\hline $\mathrm{C}$ & -2.481579 & -1.392492 & 0.703471 & $\mathrm{H}$ & 3.392232 & 5.702610 & 0.287315 \\
\hline $\mathrm{C}$ & -3.313506 & -0.643566 & 1.554276 & $\mathrm{O}$ & -0.946004 & 1.544689 & 0.954723 \\
\hline $\mathrm{C}$ & -2.919660 & -1.805380 & -0.571221 & $\mathrm{H}$ & 0.022659 & -0.769600 & -1.913537 \\
\hline $\mathrm{C}$ & -4.571571 & -0.271130 & 1.081429 & $\mathrm{C}$ & -0.201270 & 1.572050 & 2.109403 \\
\hline $\mathrm{C}$ & -4.175396 & -1.376537 & -1.006602 & $\mathrm{H}$ & -0.108881 & 0.568866 & 2.570961 \\
\hline $\mathrm{C}$ & -5.009642 & -0.601953 & -0.201357 & $\mathrm{H}$ & 0.826170 & 1.946672 & 1.911852 \\
\hline $\mathrm{H}$ & -5.217776 & 0.318778 & 1.729855 & $\mathrm{O}$ & -1.536252 & 1.549486 & -1.893442 \\
\hline $\mathrm{H}$ & -4.508113 & -1.666010 & -2.002663 & $\mathrm{H}$ & -0.586809 & -0.500219 & -2.581473 \\
\hline $\mathrm{C}$ & 1.792911 & -3.446503 & -1.521280 & $\mathrm{C}$ & -2.677696 & 2.173882 & -1.343378 \\
\hline $\mathrm{H}$ & 0.997808 & -2.825499 & -1.951423 & $\mathrm{H}$ & -3.319043 & 2.520969 & -2.166101 \\
\hline $\mathrm{H}$ & 2.297990 & -3.962623 & -2.340939 & $\mathrm{H}$ & -3.243051 & 1.439004 & -0.739308 \\
\hline $\mathrm{H}$ & 1.294192 & -4.204403 & -0.905651 & $\mathrm{O}$ & -1.463553 & -0.165236 & -3.541645 \\
\hline $\mathrm{C}$ & 2.757677 & -0.353511 & 2.352269 & $\mathrm{H}$ & -1.658210 & 0.770577 & -2.853103 \\
\hline $\mathrm{H}$ & 3.549146 & -0.267983 & 3.101465 & $\mathrm{O}$ & -2.338788 & 3.281923 & -0.579509 \\
\hline $\mathrm{H}$ & 2.487325 & 0.666777 & 2.049223 & $\mathrm{H}$ & -1.867672 & 2.893856 & 0.186251 \\
\hline $\mathrm{H}$ & 1.871996 & -0.780974 & 2.829211 & $\mathrm{O}$ & -0.820912 & 2.370456 & 3.093782 \\
\hline $\mathrm{C}$ & 6.395561 & -1.505438 & -0.900770 & $\mathrm{H}$ & -0.922994 & 3.244123 & 2.700454 \\
\hline $\mathrm{H}$ & 7.109351 & -1.560086 & -0.073446 & $\mathrm{H}$ & -0.949025 & 0.140037 & -4.296292 \\
\hline $\mathrm{H}$ & 6.672433 & -2.264272 & -1.636851 & & & & \\
\hline $\mathrm{H}$ & 6.537004 & -0.527198 & -1.374635 & \multicolumn{2}{|c|}{ TSI-2 ${ }_{\mathrm{w} 3-4}$} & & \\
\hline $\mathrm{C}$ & -2.876436 & -0.215342 & 2.916807 & $\mathrm{Ru}$ & -0.584135 & 0.595130 & -0.530269 \\
\hline $\mathrm{H}$ & -2.505013 & 0.816429 & 2.897953 & $\mathrm{C}$ & -0.194805 & -1.127004 & 0.373959 \\
\hline $\mathrm{H}$ & -3.711361 & -0.253273 & 3.622569 & $\mathrm{C}$ & 0.525120 & -2.985586 & 1.604424 \\
\hline $\mathrm{H}$ & -2.064067 & -0.833341 & 3.307252 & $\mathrm{C}$ & -0.941487 & -2.724295 & 1.928499 \\
\hline $\mathrm{C}$ & -2.132550 & -2.735643 & -1.447624 & $\mathrm{H}$ & 0.674092 & -3.908175 & 1.022361 \\
\hline $\mathrm{H}$ & -1.101539 & -2.870805 & -1.115258 & $\mathrm{H}$ & 1.155542 & -3.044179 & 2.496833 \\
\hline $\mathrm{H}$ & -2.607498 & -3.724159 & -1.471473 & $\mathrm{H}$ & -1.575017 & -3.610850 & 1.822134 \\
\hline $\mathrm{H}$ & -2.100334 & -2.365546 & -2.476342 & $\mathrm{H}$ & -1.063317 & -2.325276 & 2.944183 \\
\hline $\mathrm{C}$ & -6.322979 & -0.099918 & -0.715611 & $\mathrm{~N}$ & 0.897499 & -1.818172 & 0.790215 \\
\hline $\mathrm{H}$ & -7.056426 & 0.018255 & 0.086628 & $\mathrm{~N}$ & -1.290646 & -1.709807 & 0.935595 \\
\hline $\mathrm{H}$ & -6.202740 & 0.883975 & -1.185100 & $\mathrm{C}$ & 2.217814 & -1.752080 & 0.248533 \\
\hline $\mathrm{H}$ & -6.746615 & -0.765589 & -1.472325 & $\mathrm{C}$ & 3.298994 & -1.393203 & 1.069496 \\
\hline $\mathrm{C}$ & 1.421288 & 0.851947 & -0.792819 & $\mathrm{C}$ & 2.432064 & -2.170919 & -1.080019 \\
\hline $\mathrm{H}$ & 2.201784 & 0.152330 & -1.132699 & $\mathrm{C}$ & 4.582820 & -1.403517 & 0.515623 \\
\hline $\mathrm{C}$ & 1.925974 & 2.191503 & -0.539444 & $\mathrm{C}$ & 3.729610 & -2.152888 & -1.587086 \\
\hline $\mathrm{C}$ & 3.302891 & 2.359235 & -0.285149 & $\mathrm{C}$ & 4.821232 & -1.762005 & -0.807591 \\
\hline
\end{tabular}




\begin{tabular}{|c|c|c|c|c|c|c|c|}
\hline $\mathrm{H}$ & 5.420203 & -1.106618 & 1.146578 & $\mathrm{H}$ & 5.436071 & 2.237302 & -0.440493 \\
\hline $\mathrm{H}$ & 3.893079 & -2.465578 & -2.617759 & $\mathrm{H}$ & 2.307011 & 5.030954 & 0.513970 \\
\hline $\mathrm{C}$ & -2.624558 & -1.355604 & 0.547366 & $\mathrm{H}$ & 4.716398 & 4.438121 & 0.474962 \\
\hline $\mathrm{C}$ & -3.413729 & -0.495833 & 1.328775 & $\mathrm{O}$ & -0.398792 & 1.625827 & 1.287054 \\
\hline $\mathrm{C}$ & -3.129980 & -1.913179 & -0.646042 & $\mathrm{H}$ & -0.887180 & -0.262407 & -2.027565 \\
\hline $\mathrm{C}$ & -4.676837 & -0.139848 & 0.845922 & $\mathrm{C}$ & 0.591561 & 1.300971 & 2.168243 \\
\hline $\mathrm{C}$ & -4.389771 & -1.521935 & -1.085035 & $\mathrm{H}$ & 1.510048 & 0.946298 & 1.669987 \\
\hline $\mathrm{C}$ & -5.171129 & -0.614156 & -0.365973 & $\mathrm{H}$ & 0.852562 & 2.190289 & 2.773889 \\
\hline $\mathrm{H}$ & -5.283643 & 0.542600 & 1.439801 & $\mathrm{O}$ & -1.976751 & 2.068452 & -1.102761 \\
\hline $\mathrm{H}$ & -4.766774 & -1.927971 & -2.022300 & $\mathrm{H}$ & -1.618264 & 0.205689 & -2.459565 \\
\hline $\mathrm{C}$ & 1.298465 & -2.634780 & -1.937295 & $\mathrm{C}$ & -2.587574 & 2.930397 & -0.172912 \\
\hline $\mathrm{H}$ & 0.642897 & -1.802740 & -2.222682 & $\mathrm{H}$ & -3.350755 & 3.524830 & -0.694129 \\
\hline $\mathrm{H}$ & 1.666080 & -3.107095 & -2.851410 & $\mathrm{H}$ & -3.089318 & 2.321991 & 0.605205 \\
\hline $\mathrm{H}$ & 0.660816 & -3.356463 & -1.414355 & $\mathrm{O}$ & -2.605409 & 0.826713 & -3.021429 \\
\hline $\mathrm{C}$ & 3.136875 & -1.018354 & 2.509910 & $\mathrm{H}$ & -2.505834 & 1.567582 & -2.149987 \\
\hline $\mathrm{H}$ & 3.627727 & -1.754521 & 3.157769 & $\mathrm{O}$ & -1.684610 & 3.815419 & 0.397644 \\
\hline $\mathrm{H}$ & 3.620491 & -0.055640 & 2.709715 & $\mathrm{H}$ & -1.097743 & 3.236952 & 0.928890 \\
\hline $\mathrm{H}$ & 2.094583 & -0.925413 & 2.815387 & $\mathrm{O}$ & 0.246147 & 0.226809 & 3.055121 \\
\hline $\mathrm{C}$ & 6.198143 & -1.685248 & -1.390952 & $\mathrm{H}$ & -0.488261 & 0.548566 & 3.587377 \\
\hline $\mathrm{H}$ & 6.969749 & -1.871247 & -0.638955 & $\mathrm{H}$ & -2.229797 & 1.265959 & -3.792628 \\
\hline $\mathrm{H}$ & 6.337138 & -2.400583 & -2.206140 & & & & \\
\hline $\mathrm{H}$ & 6.391257 & -0.687753 & -1.805607 & \multicolumn{2}{|c|}{ TSI-3 $_{\text {w3-4 }}$} & & \\
\hline $\mathrm{C}$ & -2.931647 & 0.079026 & 2.621439 & $\mathrm{Ru}$ & -0.305667 & 0.414419 & -0.577622 \\
\hline $\mathrm{H}$ & -2.200508 & 0.872200 & 2.423222 & $\mathrm{C}$ & -0.146034 & -1.261326 & 0.475880 \\
\hline $\mathrm{H}$ & -3.764640 & 0.503192 & 3.187980 & $\mathrm{C}$ & 0.417753 & -3.235114 & 1.622034 \\
\hline $\mathrm{H}$ & -2.436134 & -0.668906 & 3.248584 & $\mathrm{C}$ & -1.095855 & -3.145136 & 1.517087 \\
\hline $\mathrm{C}$ & -2.324753 & -2.890846 & -1.441189 & $\mathrm{H}$ & 0.826078 & -4.193621 & 1.285085 \\
\hline $\mathrm{H}$ & -1.499598 & -2.391342 & -1.960777 & $\mathrm{H}$ & 0.777852 & -3.059610 & 2.647230 \\
\hline $\mathrm{H}$ & -1.874048 & -3.664411 & -0.809069 & $\mathrm{H}$ & -1.505874 & -3.861996 & 0.788426 \\
\hline $\mathrm{H}$ & -2.943011 & -3.383924 & -2.194849 & $\mathrm{H}$ & -1.609426 & -3.302660 & 2.470707 \\
\hline $\mathrm{C}$ & -6.498764 & -0.165785 & -0.892260 & $\mathrm{~N}$ & 0.852692 & -2.144113 & 0.742830 \\
\hline $\mathrm{H}$ & -7.080327 & 0.360286 & -0.131097 & $\mathrm{~N}$ & -1.286992 & -1.774773 & 1.034688 \\
\hline $\mathrm{H}$ & -6.371288 & 0.517409 & -1.739551 & $\mathrm{C}$ & 2.227536 & -1.996195 & 0.405420 \\
\hline $\mathrm{H}$ & -7.097055 & -1.007365 & -1.255151 & $\mathrm{C}$ & 3.082027 & -1.233899 & 1.212961 \\
\hline $\mathrm{C}$ & 1.118968 & 0.855103 & -1.187240 & $\mathrm{C}$ & 2.692278 & -2.632128 & -0.755836 \\
\hline $\mathrm{H}$ & 1.544059 & 0.172368 & -1.943122 & $\mathrm{C}$ & 4.419247 & -1.118400 & 0.830085 \\
\hline $\mathrm{C}$ & 2.056035 & 1.896720 & -0.795111 & $\mathrm{C}$ & 4.035674 & -2.487038 & -1.099446 \\
\hline $\mathrm{C}$ & 3.431206 & 1.604387 & -0.877896 & $\mathrm{C}$ & 4.915589 & -1.732957 & -0.319969 \\
\hline $\mathrm{C}$ & 1.663844 & 3.154088 & -0.298451 & $\mathrm{H}$ & 5.086062 & -0.511212 & 1.441652 \\
\hline $\mathrm{C}$ & 4.380484 & 2.497941 & -0.402278 & $\mathrm{H}$ & 4.402449 & -2.966941 & -2.005837 \\
\hline $\mathrm{H}$ & 3.733760 & 0.642749 & -1.288464 & $\mathrm{C}$ & -2.582256 & -1.337247 & 0.597237 \\
\hline $\mathrm{C}$ & 2.620597 & 4.058820 & 0.142723 & $\mathrm{C}$ & -3.410125 & -0.639321 & 1.492938 \\
\hline $\mathrm{H}$ & 0.608113 & 3.410043 & -0.282002 & $\mathrm{C}$ & -3.008667 & -1.640675 & -0.710136 \\
\hline $\mathrm{C}$ & 3.976226 & 3.729189 & 0.113243 & $\mathrm{C}$ & -4.647791 & -0.196199 & 1.031677 \\
\hline
\end{tabular}




\begin{tabular}{|c|c|c|c|}
\hline C & .243819 & 57 & I \\
\hline C & 065528 & .404251 & 282502 \\
\hline & 3 & 56 & .711213 \\
\hline & 562939 & 0 & 152101 \\
\hline & 175 & 7 & 1632 \\
\hline & 0.994284 & 67 & 8145 \\
\hline $\mathrm{H}$ & 2.281351 & 89 & -2.452189 \\
\hline $\mathrm{H}$ & 471 & 4 & 63 \\
\hline $\mathrm{C}$ & 3 & & \\
\hline $\mathrm{H}$ & 3.347760 & -0.401371 & (2) \\
\hline $\mathrm{H}$ & 2.285250 & 962 & 8450 \\
\hline $\mathrm{H}$ & 30 & 7 & 2.850073 \\
\hline $\mathrm{C}$ & 6.357680 & 748 & 48 \\
\hline $\mathrm{H}$ & 13384 & & \\
\hline $\mathrm{H}$ & 4 & 3 & 99 \\
\hline $\mathrm{H}$ & 2 & 8 & 14673 \\
\hline T & 38 & -0.3 & 12 \\
\hline $\mathrm{H}$ & 506 & 4 & 2.983370 \\
\hline $\mathrm{H}$ & -3.81 & 4 & 3.590460 \\
\hline $\mathrm{H}$ & -2.1 & 1 & 39 \\
\hline $\mathrm{C}$ & -2.23 & 2 & 1 \\
\hline $\mathrm{H}$ & -1.221786 & -2.7 & 4589 \\
\hline $\mathrm{H}$ & -2.7 & 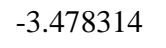 & 0 \\
\hline $\mathrm{H}$ & -2.1 & -2.0 & -2.631503 \\
\hline $\mathrm{C}$ & -6.3 & 2 & 93 \\
\hline $\mathrm{H}$ & 7 & 7 & -0.003270 \\
\hline $\mathrm{H}$ & -6.1 & 733 & 5838 \\
\hline$H$ & -6.744408 & 02408 & 1 \\
\hline $\mathrm{C}$ & 1.4 & 2 & -0 \\
\hline$\Pi$ & 2.196682 & 28 & 668 \\
\hline$C$ & 2.147621 & 050015 & 0466210 \\
\hline $\mathrm{C}$ & 3.554269 & 130937 & -0.550662 \\
\hline $\mathrm{C}$ & $1.4 \mathrm{~J}$ & 3.222826 & 81 \\
\hline $\mathrm{C}$ & 4.228896 & 3.308897 & -0.260800 \\
\hline $\mathrm{H}$ & 4.104757 & 236249 & 39693 \\
\hline$C_{0}+$ & 2.118979 & (1) 207570 & 10415 \\
\hline $\mathrm{H}$ & 0.355872 & 3.170735 & -0.031693 \\
\hline $\mathrm{C}$ & 3.511785 & 725 & 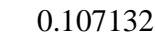 \\
\hline$H$ & 5.313252 & 3.345005 & -0.326113 \\
\hline $\mathrm{H}$ & 1.560143 & 86029 & 0.465118 \\
\hline $\mathrm{H}$ & 290 & 5.372225 & 20104 \\
\hline $\mathrm{O}$ & 372 & 15 & 0 \\
\hline $\mathrm{H}$ & -0.016524 & -0.650403 & -1.939830 \\
\hline $\mathrm{C}$ & -0.310572 & 1.547016 & 2.19752 \\
\hline
\end{tabular}

$\begin{array}{crrr}\mathrm{H} & -0.252304 & 0.509129 & 2.609419 \\ \mathrm{H} & 0.732630 & 1.936657 & 2.140435 \\ \mathrm{O} & -1.212919 & 1.896969 & -1.839659 \\ \mathrm{H} & -0.607418 & -0.274646 & -2.559588 \\ \mathrm{C} & -2.183393 & 2.767391 & -1.320613 \\ \mathrm{H} & -1.661819 & 3.563598 & -0.762797 \\ \mathrm{H} & -2.729222 & 3.221834 & -2.158676 \\ \mathrm{O} & -1.470337 & 0.212521 & -3.503160 \\ \mathrm{H} & -1.500384 & 1.155257 & -2.797073 \\ \mathrm{O} & -3.133333 & 2.139008 & -0.515357 \\ \mathrm{H} & -2.662202 & 1.929623 & 0.311912 \\ \mathrm{O} & -1.071175 & 2.369281 & 3.071998 \\ \mathrm{H} & -0.666421 & 2.308943 & 3.942992 \\ \mathrm{H} & -0.933940 & 0.454789 & -4.265321\end{array}$

\section{TSI-4 ${ }_{\text {w3-4 }}$}

$\begin{array}{llll}\mathrm{Ru} & -0.341415 & 0.328802 & -0.597033\end{array}$

$\begin{array}{llll}\text { C } & -0.056099 & -1.269144 & 0.529735\end{array}$

$\begin{array}{llll}\text { C } & 0.588885 & -3.171824 & 1.745885\end{array}$

$\begin{array}{llll}\text { C } & -0.929730 & -3.132353 & 1.669325\end{array}$

$\mathrm{H} \quad 1.018692-4.131177 \quad 1.439470$

$\mathrm{H} \quad 0.962945 \quad-2.944490 \quad 2.755631$

$\mathrm{H} \quad-1.330511 \quad-3.892415 \quad 0.980310$

$\mathrm{H} \quad-1.417477 \quad-3.265484 \quad 2.639985$

$\begin{array}{llll}\mathrm{N} & 0.974199 & -2.105564 & 0.814481\end{array}$

$\begin{array}{llll}\mathrm{N} & -1.172775 & -1.789242 & 1.136709\end{array}$

$\begin{array}{llll}\text { C } & 2.332849 & -1.936985 & 0.423092\end{array}$

$\begin{array}{llll}\text { C } & 3.196650 & -1.128213 & 1.173405\end{array}$

$\begin{array}{llll}\text { C } & 2.775197 & -2.613826 & -0.723712\end{array}$

$\begin{array}{llll}\text { C } & 4.521640 & -1.011392 & 0.749082\end{array}$

$\begin{array}{llll}\text { C } & 4.105027 & -2.459998 & -1.113531\end{array}$

$\begin{array}{llll}\text { C } & 4.993770 & -1.660305 & -0.392439\end{array}$

$\mathrm{H} \quad 5.199204 \quad-0.380547 \quad 1.324099$

$\mathrm{H} \quad 4.455115 \quad-2.976431 \quad-2.006227$

$\begin{array}{llll}\text { C } & -2.483363 & -1.407515 & 0.691561\end{array}$

$\begin{array}{llll}\text { C } & -3.311405 & -0.662899 & 1.549394\end{array}$

$\begin{array}{llll}\text { C } & -2.921173 & -1.803437 & -0.588295\end{array}$

$\begin{array}{llll}\text { C } & -4.567249 & -0.278984 & 1.079974\end{array}$

$\begin{array}{llll}\text { C } & -4.174273 & -1.363453 & -1.019958\end{array}$

$\begin{array}{llll}\text { C } & -5.005161 & -0.593298 & -0.206981\end{array}$

$\begin{array}{llll}\mathrm{H} & -5.209938 & 0.309767 & 1.732861\end{array}$

$\mathrm{H} \quad-4.506808 \quad-1.638673 \quad-2.020125$

$\begin{array}{llll}\text { C } & 1.827242 & -3.446650 & -1.529447\end{array}$

$\begin{array}{llll}\mathrm{H} & 1.029283 & -2.828439 & -1.958633\end{array}$ 


\begin{tabular}{|c|c|c|c|}
\hline $\mathrm{H}$ & 901 & -3.949774 & - \\
\hline $\mathrm{H}$ & 1.331065 & -4.215401 & -0.925270 \\
\hline & .711 & 8 & 89 \\
\hline & $500 \mathrm{c}-2$ & 7 & 3.078411 \\
\hline & .374394 & 0.653450 & 0150 \\
\hline & 1 & 51 & 49782 \\
\hline & 0 & 6 & 1 \\
\hline & 7.112897 & 503 & 7663 \\
\hline & 8 & & 71 \\
\hline & 7 & 8 & 12 \\
\hline $\mathrm{C}$ & 0 & & 6610 \\
\hline 11 & -2.533210 & 0.792416 & 84 \\
\hline $\mathrm{H}$ & -3.697525 & 3 & 00 \\
\hline $\mathrm{H}$ & 39 & & \\
\hline $\mathrm{C}$ & -2.136326 & 382 & בלנסוד \\
\hline $\mathrm{H}$ & 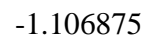 & 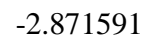 & 1.142 \\
\hline 11 & -2.616912 & -3.710628 & -1.515912 \\
\hline $\mathrm{H}$ & -2.098701 & 04 & (J) \\
\hline $\mathrm{C}$ & -6.314366 & -0.0 & 38 \\
\hline $\mathrm{H}$ & -7.049421 & 25 & \\
\hline $\mathrm{H}$ & 61872 & 0.012466 & -1.173259 \\
\hline $\mathrm{H}$ & -6.739443 & -0.730103 & \\
\hline $\mathrm{C}$ & 1 & 7 & 50 \\
\hline - & 2.197309 & 6095 & 60 \\
\hline 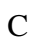 & 1.937820 & 179670 & 719 \\
\hline$C_{1}$ & 2202140 & 2320020 & 0314184 \\
\hline 0 & 514 & 278 & 0501 \\
\hline $\mathrm{C}$ & 3.8 & 509 & 81 \\
\hline $\mathrm{H}$ & 3.96 & 36 & 24 \\
\hline $\mathrm{C}$ & 1.656988 & .566083 & 3587 \\
\hline 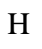 & O5 & (2) & ( \\
\hline$C$ & 10 & 1965 & 448 \\
\hline $\mathrm{H}$ & 4.922227 & 3.689271 & 0.171217 \\
\hline $\mathrm{H}$ & 1.010468 & 0 & -0.181526 \\
\hline $\mathrm{H}$ & 3.440748 & 679508 & 10 \\
\hline U & דינדות & 0 & \\
\hline $\mathrm{H}$ & 0.030029 & -0.763587 & -1.919842 \\
\hline $\mathrm{C}$ & 259173 & 3 & 23 \\
\hline $\mathrm{H}$ & -0.133060 & 0.590797 & $0-5-7100$ \\
\hline $\mathrm{H}$ & 0.762 & 3 & 7680 \\
\hline $\mathrm{O}$ & -1.536677 & 346 & 21 \\
\hline $\mathrm{H}$ & -0.581356 & -0.484428 & -2.579907 \\
\hline $\mathrm{C}$ & -2.669672 & 2.186838 & -1.306858 \\
\hline $\mathbf{T}$ & -3.331576 & 2.500017 & -2.127070 \\
\hline
\end{tabular}

$\begin{array}{rrrr}\mathrm{H} & -3.212758 & 1.464403 & -0.669333 \\ \mathrm{O} & -1.465504 & -0.142052 & -3.538915 \\ \mathrm{H} & -1.662695 & 0.786214 & -2.838964 \\ \mathrm{O} & -2.327738 & 3.320970 & -0.588043 \\ \mathrm{H} & -1.883730 & 2.972973 & 0.211272 \\ \mathrm{O} & -0.983281 & 2.452711 & 2.971309 \\ \mathrm{H} & -0.412971 & 2.648493 & 3.720596 \\ \mathrm{H} & -0.954143 & 0.175297 & -4.290721\end{array}$

\section{TSI-5 ${ }_{\mathrm{w} 3-4}$}

$\begin{array}{llll}\mathrm{Ru} & -0.343223 & 0.815554 & -0.342460\end{array}$

$\begin{array}{llll}\text { C } & -0.448840 & -1.061335 & 0.317648\end{array}$

$\begin{array}{llll}\text { C } & -0.136141 & -3.281286 & 1.065643\end{array}$

$\begin{array}{llll}\text { C } & -1.519048 & -2.819723 & 1.485874\end{array}$

$\begin{array}{llll}\mathrm{H} & -0.161094 & -4.045184 & 0.272608\end{array}$

$\mathrm{H} \quad 0.458703 \quad-3.683503 \quad 1.891654$

$\begin{array}{llll}\mathrm{H} & -2.318896 & -3.499185 & 1.174510\end{array}$

$\begin{array}{llll}\mathrm{H} & -1.600169 & -2.679514 & 2.573937\end{array}$

$\begin{array}{llll}\mathrm{N} & 0.469687 & -2.045138 & 0.552556\end{array}$

$\begin{array}{llll}\mathrm{N} & -1.642792 & -1.530522 & 0.798393\end{array}$

$\begin{array}{llll}\text { C } & 1.838119 & -2.116584 & 0.147589\end{array}$

$\begin{array}{llll}\text { C } & 2.848443 & -1.956820 & 1.107109\end{array}$

$\begin{array}{llll}\text { C } & 2.142830 & -2.454915 & -1.181026\end{array}$

$\begin{array}{llll}\text { C } & 4.175875 & -2.080497 & 0.690467\end{array}$

$\begin{array}{llll}\text { C } & 3.482528 & -2.560736 & -1.551328\end{array}$

$\begin{array}{llll}\text { C } & 4.515200 & -2.364850 & -0.631646\end{array}$

$\begin{array}{llll}\mathrm{H} & 4.966323 & -1.943774 & 1.427188\end{array}$

$\mathrm{H} \quad 3.724457 \quad-2.804743 \quad-2.584904$

$\begin{array}{llll}\text { C } & -2.944227 & -1.069950 & 0.419579\end{array}$

$\begin{array}{llll}\text { C } & -3.730935 & -0.338866 & 1.317654\end{array}$

$\begin{array}{llll}\text { C } & -3.446880 & -1.472194 & -0.830894\end{array}$

$\begin{array}{llll}\text { C } & -5.030779 & -0.003165 & 0.930776\end{array}$

$\begin{array}{llll}\text { C } & -4.741207 & -1.093605 & -1.181589\end{array}$

$\begin{array}{llll}\text { C } & -5.550883 & -0.357694 & -0.311315\end{array}$

$\begin{array}{llll}\mathrm{H} & -5.648027 & 0.568720 & 1.622520\end{array}$

$\begin{array}{llll}\mathrm{H} & -5.135173 & -1.401885 & -2.149437\end{array}$

$\begin{array}{llll}\text { C } & 1.055299 & -2.635598 & -2.191776\end{array}$

$\begin{array}{llll}\mathrm{H} & 0.519693 & -1.693206 & -2.362319\end{array}$

$\begin{array}{llll}\mathrm{H} & 1.456365 & -2.976158 & -3.149092\end{array}$

$\begin{array}{llll}\mathrm{H} & 0.302374 & -3.361496 & -1.864397\end{array}$

$\begin{array}{llll}\text { C } & 2.533488 & -1.625193 & 2.528414\end{array}$

$\mathrm{H} \quad 3.362043 \quad-1.898727 \quad 3.186627$

$\begin{array}{llll}\mathrm{H} & 2.350825 & -0.549689 & 2.640062\end{array}$

$\begin{array}{llll}\mathrm{H} & 1.631707 & -2.132724 & 2.885784\end{array}$ 


\begin{tabular}{|c|c|c|c|c|c|c|c|}
\hline $\mathrm{C}$ & 5.949556 & -2.430924 & -1.058340 & $\mathrm{H}$ & 1.617881 & 2.172284 & 2.373210 \\
\hline $\mathrm{H}$ & 6.577850 & -2.907901 & -0.300560 & $\mathrm{H}$ & -0.271423 & 2.140003 & -3.812916 \\
\hline $\mathrm{H}$ & 6.070156 & -2.981182 & -1.995257 & & & & \\
\hline $\mathrm{H}$ & 6.359307 & -1.426068 & -1.219224 & \multicolumn{4}{|c|}{ TSI-6 ${ }_{w 3-4}$} \\
\hline $\mathrm{C}$ & -3.184905 & 0.146002 & 2.619518 & $\mathrm{Ru}$ & -0.177196 & 0.643725 & -0.489362 \\
\hline $\mathrm{H}$ & -2.577567 & 1.043127 & 2.445354 & $\mathrm{C}$ & -0.484693 & -1.190374 & 0.234704 \\
\hline $\mathrm{H}$ & -3.987634 & 0.399826 & 3.316533 & $\mathrm{C}$ & -0.284870 & -3.384236 & 1.097351 \\
\hline $\mathrm{H}$ & -2.526854 & -0.583948 & 3.100744 & $\mathrm{C}$ & -1.754972 & -3.057235 & 0.937451 \\
\hline $\mathrm{C}$ & -2.616338 & -2.302073 & -1.759047 & $\mathrm{H}$ & 0.008588 & -4.331743 & 0.633269 \\
\hline $\mathrm{H}$ & -1.725537 & -1.757866 & -2.092386 & $\mathrm{H}$ & 0.024849 & -3.419157 & 2.153322 \\
\hline $\mathrm{H}$ & -2.257014 & -3.219701 & -1.275919 & $\mathrm{H}$ & -2.216275 & -3.596729 & 0.094972 \\
\hline $\mathrm{H}$ & -3.185984 & -2.593519 & -2.644463 & $\mathrm{H}$ & -2.351864 & -3.262459 & 1.832094 \\
\hline $\mathrm{C}$ & -6.936047 & 0.048905 & -0.712566 & $\mathrm{~N}$ & 0.352877 & -2.252755 & 0.421428 \\
\hline $\mathrm{H}$ & -7.552695 & 0.291933 & 0.156592 & $\mathrm{~N}$ & -1.724407 & -1.620435 & 0.648147 \\
\hline $\mathrm{H}$ & -6.920641 & 0.937538 & -1.354022 & $\mathrm{C}$ & 1.756692 & -2.284088 & 0.189926 \\
\hline $\mathrm{H}$ & -7.443159 & -0.738437 & -1.278057 & $\mathrm{C}$ & 2.646668 & -1.830443 & 1.173834 \\
\hline $\mathrm{C}$ & 1.462056 & 0.778241 & -0.762958 & $\mathrm{C}$ & 2.209219 & -2.772199 & -1.043340 \\
\hline $\mathrm{H}$ & 1.899241 & -0.040833 & -1.351717 & $\mathrm{C}$ & 4.010624 & -1.855779 & 0.884259 \\
\hline $\mathrm{C}$ & 2.463586 & 1.781985 & -0.446725 & $\mathrm{C}$ & 3.582387 & -2.773769 & -1.290410 \\
\hline $\mathrm{C}$ & 3.815250 & 1.471593 & -0.701309 & $\mathrm{C}$ & 4.497957 & -2.314533 & -0.341550 \\
\hline $\mathrm{C}$ & 2.164684 & 3.027945 & 0.145859 & $\mathrm{H}$ & 4.710426 & -1.480227 & 1.630120 \\
\hline $\mathrm{C}$ & 4.831724 & 2.351527 & -0.355663 & $\mathrm{H}$ & 3.945438 & -3.135008 & -2.251629 \\
\hline $\mathrm{H}$ & 4.047894 & 0.509817 & -1.157837 & $\mathrm{C}$ & -2.980321 & -0.998444 & 0.363377 \\
\hline $\mathrm{C}$ & 3.184656 & 3.909136 & 0.476410 & $\mathrm{C}$ & -3.763507 & -0.534075 & 1.433262 \\
\hline $\mathrm{H}$ & 1.122074 & 3.284224 & 0.311225 & $\mathrm{C}$ & -3.476796 & -0.984693 & -0.953718 \\
\hline $\mathrm{C}$ & 4.518781 & 3.574707 & 0.234345 & $\mathrm{C}$ & -5.017576 & 0.011122 & 1.152638 \\
\hline $\mathrm{H}$ & 5.868518 & 2.087464 & -0.548762 & $\mathrm{C}$ & -4.742555 & -0.447185 & -1.177958 \\
\hline $\mathrm{H}$ & 2.942621 & 4.870231 & 0.923289 & $\mathrm{C}$ & -5.521928 & 0.072469 & -0.145196 \\
\hline $\mathrm{H}$ & 5.311199 & 4.269780 & 0.499640 & $\mathrm{H}$ & -5.621986 & 0.385062 & 1.978559 \\
\hline $\mathrm{O}$ & -0.463946 & 1.621229 & 1.509753 & $\mathrm{H}$ & -5.122420 & -0.420451 & -2.198477 \\
\hline $\mathrm{H}$ & -0.648360 & 0.125891 & -1.950048 & $\mathrm{C}$ & 1.233603 & -3.223257 & -2.086058 \\
\hline $\mathrm{C}$ & 0.067115 & 1.074239 & 2.639000 & $\mathrm{H}$ & 0.616717 & -2.386750 & -2.435799 \\
\hline $\mathrm{H}$ & -0.469016 & 1.499725 & 3.512167 & $\mathrm{H}$ & 1.747058 & -3.649284 & -2.951177 \\
\hline $\mathrm{H}$ & -0.031778 & -0.030652 & 2.690412 & $\mathrm{H}$ & 0.537544 & -3.978439 & -1.702575 \\
\hline $\mathrm{O}$ & -0.690758 & 2.952668 & -1.038929 & $\mathrm{C}$ & 2.150543 & -1.241153 & 2.454658 \\
\hline $\mathrm{H}$ & -0.739003 & 0.902192 & -2.446238 & $\mathrm{H}$ & 2.942337 & -1.218832 & 3.208268 \\
\hline $\mathrm{C}$ & -1.953464 & 3.119403 & -0.519303 & $\mathrm{H}$ & 1.808451 & -0.208687 & 2.296852 \\
\hline $\mathrm{H}$ & -1.904733 & 3.451700 & 0.522319 & $\mathrm{H}$ & 1.301696 & -1.795361 & 2.869349 \\
\hline $\mathrm{H}$ & -2.559624 & 3.817632 & -1.118704 & $\mathrm{C}$ & 5.969630 & -2.318780 & -0.622556 \\
\hline $\mathrm{O}$ & -1.015392 & 2.012024 & -3.216307 & $\mathrm{H}$ & 6.177760 & -2.450998 & -1.687356 \\
\hline $\mathrm{H}$ & -0.810534 & 2.638652 & -2.212127 & $\mathrm{H}$ & 6.442143 & -1.385520 & -0.299707 \\
\hline $\mathrm{O}$ & -2.627048 & 1.839828 & -0.450924 & $\mathrm{H}$ & 6.478121 & -3.129062 & -0.087976 \\
\hline $\mathrm{H}$ & -2.971571 & 1.630212 & -1.329123 & $\mathrm{C}$ & -3.314330 & -0.642509 & 2.858309 \\
\hline $\mathrm{O}$ & 1.461373 & 1.312768 & 2.783947 & $\mathrm{H}$ & -3.324206 & 0.334985 & 3.351138 \\
\hline
\end{tabular}




\begin{tabular}{|c|c|c|c|c|c|c|c|}
\hline $\mathrm{H}$ & -3.987141 & -1.291079 & 3.430850 & $\mathrm{C}$ & -0.017434 & -3.192053 & 1.056273 \\
\hline $\mathrm{H}$ & -2.301961 & -1.042233 & 2.941127 & $\mathrm{C}$ & -1.460836 & -2.821026 & 1.360081 \\
\hline $\mathrm{C}$ & -2.678469 & -1.480169 & -2.118189 & $\mathrm{H}$ & 0.069934 & -4.008667 & 0.323131 \\
\hline $\mathrm{H}$ & -2.152012 & -0.645293 & -2.594615 & $\mathrm{H}$ & 0.545280 & -3.481269 & 1.949513 \\
\hline $\mathrm{H}$ & -1.918816 & -2.215615 & -1.837065 & $\mathrm{H}$ & -2.183644 & -3.559794 & 0.996850 \\
\hline $\mathrm{H}$ & -3.330010 & -1.929676 & -2.872923 & $\mathrm{H}$ & -1.632984 & -2.673660 & 2.434971 \\
\hline $\mathrm{C}$ & -6.855693 & 0.691982 & -0.428060 & $\mathrm{~N}$ & 0.529741 & -1.952828 & 0.490950 \\
\hline $\mathrm{H}$ & -7.481102 & 0.736416 & 0.467469 & $\mathrm{~N}$ & -1.619541 & -1.554510 & 0.642625 \\
\hline $\mathrm{H}$ & -6.743138 & 1.718205 & -0.795985 & $\mathrm{C}$ & 1.890617 & -1.969462 & 0.059343 \\
\hline $\mathrm{H}$ & -7.403636 & 0.140640 & -1.197843 & $\mathrm{C}$ & 2.931226 & -1.916642 & 0.999358 \\
\hline $\mathrm{C}$ & 1.681603 & 0.684610 & -0.436937 & $\mathrm{C}$ & 2.169699 & -2.175969 & -1.307295 \\
\hline $\mathrm{H}$ & 2.290920 & -0.128452 & -0.859452 & $\mathrm{C}$ & 4.248791 & -1.968277 & 0.532222 \\
\hline $\mathrm{C}$ & 2.510303 & 1.811390 & -0.052344 & $\mathrm{C}$ & 3.497398 & -2.226934 & -1.721975 \\
\hline $\mathrm{C}$ & 3.908497 & 1.689983 & -0.206978 & $\mathrm{C}$ & 4.556294 & -2.104986 & -0.817549 \\
\hline $\mathrm{C}$ & 1.995753 & 3.018436 & 0.465755 & $\mathrm{H}$ & 5.057746 & -1.902975 & 1.258927 \\
\hline $\mathrm{C}$ & 4.761554 & 2.729101 & 0.135488 & $\mathrm{H}$ & 3.710324 & -2.371121 & -2.780858 \\
\hline $\mathrm{H}$ & 4.305711 & 0.754677 & -0.600604 & $\mathrm{C}$ & -2.926377 & -1.109625 & 0.267257 \\
\hline $\mathrm{C}$ & 2.852983 & 4.055215 & 0.800649 & $\mathrm{C}$ & -3.741036 & -0.430590 & 1.183670 \\
\hline $\mathrm{H}$ & 0.924647 & 3.109539 & 0.603527 & $\mathrm{C}$ & -3.404320 & -1.450825 & -1.009944 \\
\hline $\mathrm{C}$ & 4.232396 & 3.917990 & 0.637056 & $\mathrm{C}$ & -5.034305 & -0.082065 & 0.790737 \\
\hline $\mathrm{H}$ & 5.835643 & 2.618500 & 0.010232 & $\mathrm{C}$ & -4.695683 & -1.062846 & -1.365820 \\
\hline $\mathrm{H}$ & 2.446094 & 4.981448 & 1.197180 & $\mathrm{C}$ & -5.529702 & -0.380595 & -0.477106 \\
\hline $\mathrm{H}$ & 4.895265 & 4.738157 & 0.902053 & $\mathrm{H}$ & -5.663707 & 0.460307 & 1.495144 \\
\hline $\mathrm{O}$ & -0.920485 & 1.566780 & 1.188210 & $\mathrm{H}$ & -5.064619 & -1.317246 & -2.358781 \\
\hline $\mathrm{H}$ & 0.061598 & -0.226792 & -1.992405 & $\mathrm{C}$ & 1.061589 & -2.316534 & -2.301091 \\
\hline $\mathrm{C}$ & -0.577952 & 1.192281 & 2.440399 & $\mathrm{H}$ & 0.489747 & -1.384989 & -2.395481 \\
\hline $\mathrm{H}$ & -1.333230 & 1.569610 & 3.162520 & $\mathrm{H}$ & 1.447841 & -2.582088 & -3.288043 \\
\hline $\mathrm{H}$ & -0.519643 & 0.084305 & 2.557443 & $\mathrm{H}$ & 0.340204 & -3.084874 & -2.001121 \\
\hline $\mathrm{O}$ & -0.291081 & 2.641243 & -1.467869 & $\mathrm{C}$ & 2.687020 & -1.864573 & 2.475623 \\
\hline $\mathrm{H}$ & 0.146533 & 0.493967 & -2.582358 & $\mathrm{H}$ & 2.926117 & -2.833833 & 2.931663 \\
\hline $\mathrm{C}$ & -1.627230 & 2.946341 & -1.355283 & $\mathrm{H}$ & 3.341696 & -1.129273 & 2.953614 \\
\hline $\mathrm{H}$ & -1.800479 & 3.671485 & -0.546495 & $\mathrm{H}$ & 1.658319 & -1.611289 & 2.732402 \\
\hline $\mathrm{H}$ & -2.029052 & 3.344275 & -2.298918 & $\mathrm{C}$ & 5.975258 & -2.098534 & -1.295384 \\
\hline $\mathrm{O}$ & 0.193735 & 1.489454 & -3.517174 & $\mathrm{H}$ & 6.674380 & -2.360051 & -0.496805 \\
\hline $\mathrm{H}$ & -0.028661 & 2.194463 & -2.618535 & $\mathrm{H}$ & 6.128952 & -2.795818 & -2.124319 \\
\hline $\mathrm{O}$ & -2.383784 & 1.750375 & -1.071668 & $\mathrm{H}$ & 6.263220 & -1.105267 & -1.662074 \\
\hline $\mathrm{H}$ & -2.506149 & 1.723507 & -0.107473 & $\mathrm{C}$ & -3.219556 & -0.008634 & 2.517749 \\
\hline $\mathrm{O}$ & 0.699113 & 1.741968 & 2.780727 & $\mathrm{H}$ & -2.441680 & 0.755514 & 2.382901 \\
\hline $\mathrm{H}$ & 0.900984 & 1.457848 & 3.678950 & $\mathrm{H}$ & -4.016993 & 0.412776 & 3.134814 \\
\hline \multirow[t]{2}{*}{$\mathrm{H}$} & 1.123476 & 1.612645 & -3.736063 & $\mathrm{H}$ & -2.772678 & -0.845200 & 3.066930 \\
\hline & & & & $\mathrm{C}$ & -2.551545 & -2.216940 & -1.969745 \\
\hline \multicolumn{2}{|c|}{ TSI-7w3-4 } & & & $\mathrm{H}$ & -1.701606 & -1.616290 & -2.310245 \\
\hline $\mathrm{Ru}$ & -0.361615 & 0.896256 & -0.307591 & $\mathrm{H}$ & -2.129609 & -3.118697 & -1.509635 \\
\hline $\mathrm{C}$ & -0.432527 & -1.021974 & 0.225589 & $\mathrm{H}$ & -3.123507 & -2.522143 & -2.849009 \\
\hline
\end{tabular}




$\begin{array}{lrrr}\mathrm{C} & -6.928390 & -0.010010 & -0.866324 \\ \mathrm{H} & -7.303069 & 0.829736 & -0.275071 \\ \mathrm{H} & -6.995430 & 0.263844 & -1.923055 \\ \mathrm{H} & -7.620450 & -0.845981 & -0.711058 \\ \mathrm{C} & 1.407900 & 0.891746 & -0.861107 \\ \mathrm{H} & 1.763124 & 0.208304 & -1.647909 \\ \mathrm{C} & 2.475197 & 1.772302 & -0.418240 \\ \mathrm{C} & 3.792670 & 1.474410 & -0.826827 \\ \mathrm{C} & 2.272601 & 2.877782 & 0.435089 \\ \mathrm{C} & 4.869816 & 2.226527 & -0.381085 \\ \mathrm{H} & 3.949390 & 0.622621 & -1.487345 \\ \mathrm{C} & 3.353489 & 3.633889 & 0.866160 \\ \mathrm{H} & 1.258396 & 3.118346 & 0.737180 \\ \mathrm{C} & 4.651568 & 3.310673 & 0.468201 \\ \mathrm{H} & 5.879305 & 1.973691 & -0.696132 \\ \mathrm{H} & 3.186214 & 4.487340 & 1.518236 \\ \mathrm{H} & 5.491568 & 3.908382 & 0.813051 \\ \mathrm{O} & -0.212595 & 1.521986 & 1.627875 \\ \mathrm{H} & -0.795613 & 0.381568 & -1.951406 \\ \mathrm{C} & 0.595918 & 0.889370 & 2.521852 \\ \mathrm{H} & 1.543206 & 0.529267 & 2.080997 \\ \mathrm{H} & 0.844935 & 1.594634 & 3.338929 \\ \mathrm{O} & -0.710361 & 3.104907 & -0.726365 \\ \mathrm{H} & -0.948180 & 1.213237 & -2.342503 \\ \mathrm{C} & -1.902234 & 3.226241 & -0.052123 \\ \mathrm{H} & -1.726965 & 3.438447 & 1.007214 \\ \mathrm{H} & -2.558861 & 3.990262 & -0.497867 \\ \mathrm{O} & -1.305248 & 2.397257 & -2.934561 \\ \mathrm{H} & -0.964892 & 2.921598 & -1.887748 \\ \mathrm{O} & -2.591472 & 1.951098 & -0.046512 \\ \mathrm{H} & -3.028501 & 1.834470 & -0.901031 \\ \mathrm{O} & 0.014229 & -0.289479 & 3.098669 \\ \mathrm{H} & -0.825060 & 0.001482 & 3.470651 \\ & -0.647268 & 2.591574 & -3.609024\end{array}$

\section{II-Br}

$\begin{array}{rrrr}\mathrm{Ru} & 0.087056 & -0.173522 & 0.321254 \\ \mathrm{C} & -2.680131 & 1.058719 & -0.002442 \\ \mathrm{C} & -1.500537 & 2.203060 & 1.637928 \\ \mathrm{C} & -3.810040 & 1.819674 & 0.256427 \\ \mathrm{H} & -2.650116 & 0.296683 & -0.777369 \\ \mathrm{C} & -2.599513 & 3.000118 & 1.926753 \\ \mathrm{H} & -0.548092 & 2.308242 & 2.153779 \\ \mathrm{~N} & -1.543535 & 1.247367 & 0.690732\end{array}$

\begin{tabular}{|c|c|c|c|}
\hline & 86 & & \\
\hline & 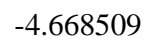 & 10499 & \\
\hline & & 1 & \\
\hline & 24 & 2 & \\
\hline & 1.322253 & -4.1 & 55 \\
\hline & 3.022900 & -3.608 & 1.5411 \\
\hline & 3.486766 & & \\
\hline & 1.00 & -4.8 & -1 \\
\hline & 1.252851 & -4.6 & 68 \\
\hline & 2. & -2.1 & \\
\hline & $0.4 \mathrm{C}$ & -2.9 & \\
\hline & $3.5 \mathrm{~s}$ & -1.2 & \\
\hline & 4.1 & -0.7 & \\
\hline & 3.5 & -0.6 & -1.4216 \\
\hline & & & \\
\hline & 4.959 & 0.3 & -1.43622 \\
\hline & 5.553608 & 0.782940 & -0.2 \\
\hline & & & \\
\hline & 5.256 & 0.739845 & -2.3934 \\
\hline & -0.928462 & -2.922510 & - \\
\hline & -1.48 & -3.00 & \\
\hline & 0 & .78 & -1.2 \\
\hline & -2.855 & -2.837 & \\
\hline & -3.114 & -2.62 & -1.0 \\
\hline & -3.6 & -2.6 & \\
\hline & -3.2958 & & \\
\hline & -3.75 & -2.49 & -1 \\
\hline & 3.391938 & -1.183048 & -2.7 \\
\hline & 84 & -0.35 & \\
\hline & 3.999 & ני. & \\
\hline & 2.376168 & -1.567131 & -2.58 \\
\hline & 3.71 & -1.32 & \\
\hline & & -2.53 & \\
\hline & 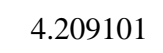 & -0.8 & \\
\hline & 40 & 11 & 400 \\
\hline & 6.553379 & 1.897 & -0. \\
\hline & & 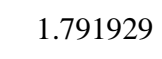 & \\
\hline & 6.055799 & 4 & -0.42 \\
\hline & 57 & 99 & 607 \\
\hline & 0.638 & -3.35 & 2.420 \\
\hline & -1.081921 & -2.968860 & \\
\hline & -0.549864 & -4.440 & 2.5217 \\
\hline & 66679 & -2.930863 & .34 \\
\hline & & 2.8152 & \\
\hline
\end{tabular}




\begin{tabular}{rrrr}
$\mathrm{H}$ & -0.110453 & -3.013407 & -2.603446 \\
$\mathrm{H}$ & -1.683698 & -3.590839 & -3.182699 \\
$\mathrm{H}$ & -1.326765 & -1.857051 & -3.100801 \\
$\mathrm{C}$ & -5.156973 & -2.395478 & 0.449025 \\
$\mathrm{H}$ & -5.692656 & -2.404317 & -0.503453 \\
$\mathrm{H}$ & -5.604666 & -3.154557 & 1.098322 \\
$\mathrm{H}$ & -5.349466 & -1.424531 & 0.919882 \\
$\mathrm{C}$ & 1.428806 & 1.094858 & 0.349843 \\
$\mathrm{H}$ & 2.386733 & 0.816279 & 0.801796 \\
$\mathrm{C}$ & 1.409161 & 2.485304 & -0.072850 \\
$\mathrm{C}$ & 2.445407 & 3.324248 & 0.385254 \\
$\mathrm{C}$ & 0.436179 & 3.033846 & -0.931356 \\
$\mathrm{C}$ & 2.495208 & 4.661960 & 0.020541 \\
$\mathrm{H}$ & 3.196710 & 2.900589 & 1.049380 \\
$\mathrm{C}$ & 0.500194 & 4.367810 & -1.309081 \\
$\mathrm{H}$ & -0.350116 & 2.389539 & -1.314460 \\
$\mathrm{C}$ & 1.523120 & 5.186900 & -0.830723 \\
$\mathrm{H}$ & 3.294165 & 5.297486 & 0.393794 \\
$\mathrm{H}$ & -0.247865 & 4.772828 & -1.985036 \\
$\mathrm{H}$ & 1.565548 & 6.231801 & -1.126314 \\
$\mathrm{O}$ & -0.504846 & -0.054292 & -1.661185 \\
$\mathrm{O}$ & 0.113959 & -0.497780 & 2.402371 \\
$\mathrm{H}$ & 1.304888 & 0.583577 & -2.325778 \\
$\mathrm{C}$ & 0.315589 & 0.176555 & -2.651781 \\
$\mathrm{O}$ & 0.064272 & -0.013709 & -3.830149 \\
$\mathrm{C}$ & 0.655279 & 0.301137 & 3.259502 \\
$\mathrm{H}$ & 0.533670 & -0.077474 & 4.299589 \\
$\mathrm{O}$ & 1.256240 & 1.357347 & 3.059664 \\
$\mathrm{Br}$ & -5.419582 & 1.483504 & -0.766423 \\
$\mathrm{H}$ & -2.523871 & 3.765341 & 2.692194 \\
& & & \\
\hline
\end{tabular}

\section{TSII0-1Br}

$\begin{array}{rrrc}\mathrm{Ru} & -0.292755 & -0.042690 & 0.136533 \\ \mathrm{C} & -0.070059 & 3.087158 & -0.262305 \\ \mathrm{C} & 0.441484 & 2.425615 & 1.899667 \\ \mathrm{C} & 0.133267 & 4.415982 & 0.082123 \\ \mathrm{H} & -0.366228 & 2.787717 & -1.264173 \\ \mathrm{C} & 0.654744 & 3.737376 & 2.296248 \\ \mathrm{H} & 0.548056 & 1.592081 & 2.589060 \\ \mathrm{~N} & 0.091529 & 2.100738 & 0.638960 \\ \mathrm{C} & 0.503621 & 4.766042 & 1.372726 \\ \mathrm{H} & 0.664006 & 5.803456 & 1.646497 \\ \mathrm{C} & -0.763320 & -1.941526 & -0.354320 \\ \mathrm{C} & -0.593382 & -4.174523 & -1.096147\end{array}$

\begin{tabular}{|c|c|c|c|}
\hline & & & \\
\hline & .454685 & 4.265070 & .18 \\
\hline & 18 & & 607 \\
\hline & 75 & & \\
\hline & 2.3 & 66 & 10 \\
\hline & 0.09 & 62 & 0.6048 \\
\hline & 2.0 & & \\
\hline & 1.49 & 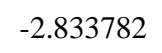 & \\
\hline & 2.44 & & .07472 \\
\hline & 1.8 & & \\
\hline & 3.789062 & -2 & \\
\hline & 3 & & \\
\hline & 4.21 & -2. & 1.37167 \\
\hline & 4.52 & -3 & \\
\hline & & & \\
\hline & 32 & 48 & 0100 \\
\hline & -3.832 & 63 & \\
\hline & -3.85 & & \\
\hline & -5. & & \\
\hline & -5.067 & 92 & \\
\hline & -5.67 & 14 & \\
\hline & 5.5 & & \\
\hline & & & \\
\hline & 0.915 & -2.0 & 3.2 \\
\hline & 1.29 & -1.35 & -3.9 \\
\hline & & & \\
\hline & 0 & 559 & 288 \\
\hline & 2.043 & -3.9 & 350 \\
\hline & 384 & -4 & .35 \\
\hline & & & \\
\hline & 0.96 & 3 & 40 \\
\hline & 5 & -20 & ion \\
\hline & 5 & -1.94 & \\
\hline & 5.879 & & \\
\hline & 221 & 4 & 10 \\
\hline & 185 & 2 & 42 \\
\hline & ו & .0 & $0.15 \pi$ \\
\hline & -3.500 & & \\
\hline & -2.0 & -2.702166 & 80707 \\
\hline & -3.238 & 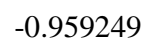 & 2.588660 \\
\hline & -2.999370 & 1.955547 & .980 \\
\hline & -3.917 & 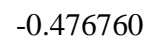 & \\
\hline & 5 & -0.3 & -2.5 \\
\hline & 5.956537 & 21947 & 59 \\
\hline
\end{tabular}




\begin{tabular}{|c|c|c|c|c|c|c|c|}
\hline $\mathrm{H}$ & -7.547762 & 0.368400 & -0.313229 & $\mathrm{H}$ & 0.732188 & -4.902419 & 0.225637 \\
\hline $\mathrm{H}$ & -7.573832 & -0.323469 & 1.315815 & $\mathrm{~N}$ & 1.945934 & -2.318044 & -0.515384 \\
\hline $\mathrm{H}$ & -6.768786 & 1.212106 & 1.019593 & $\mathrm{~N}$ & -0.098366 & -3.085806 & -0.450661 \\
\hline $\mathrm{C}$ & 1.045211 & -0.703068 & 1.236110 & $\mathrm{C}$ & 3.023354 & -1.407655 & -0.741301 \\
\hline $\mathrm{H}$ & 0.881676 & -1.672508 & 1.723602 & $\mathrm{C}$ & 4.010148 & -1.264873 & 0.247569 \\
\hline $\mathrm{C}$ & 2.365358 & -0.166600 & 1.521442 & $\mathrm{C}$ & 3.099825 & -0.700763 & -1.954189 \\
\hline $\mathrm{C}$ & 3.057176 & -0.670901 & 2.642937 & $\mathrm{C}$ & 5.022961 & -0.328918 & 0.040837 \\
\hline $\mathrm{C}$ & 3.028948 & 0.766602 & 0.699814 & $\mathrm{C}$ & 4.121218 & 0.236977 & -2.103892 \\
\hline $\mathrm{C}$ & 4.353936 & -0.269834 & 2.926395 & $\mathrm{C}$ & 5.079781 & 0.453040 & -1.112132 \\
\hline $\mathrm{H}$ & 2.540189 & -1.371168 & 3.296198 & $\mathrm{H}$ & 5.771402 & -0.185284 & 0.819582 \\
\hline $\mathrm{C}$ & 4.336175 & 1.148428 & 0.974901 & $\mathrm{H}$ & 4.167102 & 0.815602 & -3.025718 \\
\hline $\mathrm{H}$ & 2.526456 & 1.170773 & -0.174892 & $\mathrm{C}$ & -1.476020 & -3.246866 & -0.098761 \\
\hline $\mathrm{C}$ & 5.004332 & 0.632586 & 2.084120 & $\mathrm{C}$ & -1.804108 & -3.548025 & 1.233882 \\
\hline $\mathrm{H}$ & 4.864532 & -0.665327 & 3.800641 & $\mathrm{C}$ & -2.451861 & -3.195171 & -1.102624 \\
\hline $\mathrm{H}$ & 4.837585 & 1.851786 & 0.315504 & $\mathrm{C}$ & -3.146077 & -3.722249 & 1.557475 \\
\hline $\mathrm{H}$ & 6.026881 & 0.934557 & 2.294168 & $\mathrm{C}$ & -3.785339 & -3.374171 & -0.724957 \\
\hline $\mathrm{O}$ & -0.496269 & 0.703518 & -1.989954 & $\mathrm{C}$ & -4.154025 & -3.621418 & 0.595184 \\
\hline $\mathrm{O}$ & -1.660363 & 0.019033 & 1.639920 & $\mathrm{H}$ & -3.412909 & -3.935970 & 2.591856 \\
\hline $\mathrm{H}$ & 1.332247 & 0.032123 & -1.432279 & $\mathrm{H}$ & -4.556345 & -3.321444 & -1.492790 \\
\hline $\mathrm{C}$ & 0.777149 & 0.704663 & -2.174675 & $\mathrm{C}$ & 2.164722 & -0.958175 & -3.098315 \\
\hline $\mathrm{O}$ & 1.449837 & 1.309671 & -2.993742 & $\mathrm{H}$ & 1.841907 & -0.024872 & -3.567070 \\
\hline $\mathrm{C}$ & -1.426001 & -0.100758 & 2.910349 & $\mathrm{H}$ & 2.666212 & -1.550280 & -3.873489 \\
\hline $\mathrm{H}$ & -2.375891 & 0.000511 & 3.478910 & $\mathrm{H}$ & 1.264502 & -1.504603 & -2.804006 \\
\hline $\mathrm{O}$ & -0.363779 & -0.302512 & 3.488950 & $\mathrm{C}$ & 3.987608 & -2.079978 & 1.505058 \\
\hline $\mathrm{Br}$ & -0.109503 & 5.781339 & -1.264889 & $\mathrm{H}$ & 4.702819 & -2.909846 & 1.451306 \\
\hline \multirow[t]{2}{*}{$\mathrm{H}$} & 0.938125 & 3.949961 & 3.321683 & $\mathrm{H}$ & 4.271392 & -1.476495 & 2.372626 \\
\hline & & & & $\mathrm{H}$ & 3.001287 & -2.509660 & 1.698151 \\
\hline \multicolumn{2}{|c|}{ 1II-Br } & & & $\mathrm{C}$ & 6.117044 & 1.521437 & -1.265037 \\
\hline $\mathrm{Ru}$ & -0.096475 & -0.129255 & 0.127637 & $\mathrm{H}$ & 6.399597 & 1.669736 & -2.311050 \\
\hline $\mathrm{C}$ & -2.153860 & 2.247423 & -0.236109 & $\mathrm{H}$ & 5.737091 & 2.481474 & -0.894427 \\
\hline $\mathrm{C}$ & -0.825485 & 2.553330 & 1.632733 & $\mathrm{H}$ & 7.022074 & 1.293218 & -0.695422 \\
\hline $\mathrm{C}$ & -2.862246 & 3.403463 & 0.056897 & $\mathrm{C}$ & -0.745173 & -3.710216 & 2.281625 \\
\hline $\mathrm{H}$ & -2.406339 & 1.633579 & -1.094334 & $\mathrm{H}$ & -1.145710 & -3.501745 & 3.276885 \\
\hline $\mathrm{C}$ & -1.500099 & 3.717615 & 1.970935 & $\mathrm{H}$ & -0.357649 & -4.737258 & 2.303297 \\
\hline $\mathrm{H}$ & -0.034407 & 2.152177 & 2.257324 & $\mathrm{H}$ & 0.101963 & -3.037219 & 2.119919 \\
\hline $\mathrm{N}$ & -1.135510 & 1.828927 & 0.538572 & $\mathrm{C}$ & -2.111185 & -3.008310 & -2.548912 \\
\hline $\mathrm{C}$ & -2.541693 & 4.167992 & 1.168748 & $\mathrm{H}$ & -1.048050 & -2.814124 & -2.700319 \\
\hline $\mathrm{H}$ & -3.090485 & 5.073786 & 1.403379 & $\mathrm{H}$ & -2.388379 & -3.898079 & -3.126338 \\
\hline $\mathrm{C}$ & 0.643339 & -1.963236 & -0.284883 & $\mathrm{H}$ & -2.648688 & -2.156943 & -2.972031 \\
\hline $\mathrm{C}$ & 2.073989 & -3.693592 & -1.011589 & $\mathrm{C}$ & -5.595240 & -3.762885 & 0.978462 \\
\hline $\mathrm{C}$ & 0.714898 & -4.283938 & -0.683415 & $\mathrm{H}$ & -6.215232 & -4.046127 & 0.123718 \\
\hline $\mathrm{H}$ & 2.276434 & -3.679923 & -2.092986 & $\mathrm{H}$ & -5.735690 & -4.512270 & 1.762938 \\
\hline $\mathrm{H}$ & 2.906584 & -4.208090 & -0.520986 & $\mathrm{H}$ & -5.994926 & -2.819167 & 1.366769 \\
\hline $\mathrm{H}$ & 0.288558 & -4.885734 & -1.492316 & $\mathrm{C}$ & 1.439692 & 0.280613 & 1.100280 \\
\hline
\end{tabular}




$\begin{array}{cccc}\mathrm{H} & 1.876615 & -0.483393 & 1.759284 \\ \mathrm{C} & 2.228483 & 1.502558 & 1.083775 \\ \mathrm{C} & 3.200438 & 1.704845 & 2.082727 \\ \mathrm{C} & 2.094470 & 2.479369 & 0.077046 \\ \mathrm{C} & 4.002493 & 2.837514 & 2.083098 \\ \mathrm{H} & 3.302632 & 0.953060 & 2.863397 \\ \mathrm{C} & 2.908376 & 3.604284 & 0.070084 \\ \mathrm{H} & 1.365104 & 2.330730 & -0.717524 \\ \mathrm{C} & 3.861188 & 3.789260 & 1.072539 \\ \mathrm{H} & 4.743565 & 2.979004 & 2.865200 \\ \mathrm{H} & 2.802711 & 4.340001 & -0.722492 \\ \mathrm{H} & 4.495739 & 4.671715 & 1.064015 \\ \mathrm{O} & -1.393686 & -0.218417 & -1.872205 \\ \mathrm{O} & -1.150971 & -0.751346 & 1.826262 \\ \mathrm{H} & 0.584766 & 0.367854 & -1.415741 \\ \mathrm{C} & -0.446485 & 0.566427 & -2.108752 \\ \mathrm{O} & -0.202316 & 1.462011 & -2.895648 \\ \mathrm{C} & -0.806092 & -0.450938 & 3.031512 \\ \mathrm{H} & -1.525127 & -0.874799 & 3.769786 \\ \mathrm{O} & 0.162940 & 0.198248 & 3.429351 \\ \mathrm{Br} & -4.312934 & 3.933394 & -1.106795 \\ \mathrm{H} & -1.211058 & 4.264126 & 2.862399 \\ & & & \end{array}$

\section{TSII1-2Br}

$\begin{array}{rrrc}\mathrm{Ru} & -0.022051 & 0.040606 & 0.075075 \\ \mathrm{C} & 2.405144 & 1.858964 & 0.318389 \\ \mathrm{C} & 0.646778 & 3.137683 & -0.472197 \\ \mathrm{C} & 3.276038 & 2.926603 & 0.164936 \\ \mathrm{H} & 2.734367 & 0.892872 & 0.691275 \\ \mathrm{C} & 1.471069 & 4.240557 & -0.644109 \\ \mathrm{H} & -0.403231 & 3.166531 & -0.740286 \\ \mathrm{~N} & 1.102041 & 1.972452 & 0.009713 \\ \mathrm{C} & 2.818955 & 4.144270 & -0.319394 \\ \mathrm{H} & 3.494162 & 4.984284 & -0.443297 \\ \mathrm{C} & -0.694873 & -1.866431 & 0.234830 \\ \mathrm{C} & -2.078116 & -3.752844 & 0.563137 \\ \mathrm{C} & -0.620252 & -4.187636 & 0.646317 \\ \mathrm{H} & -2.659824 & -4.018911 & 1.452078 \\ \mathrm{H} & -2.597132 & -4.167380 & -0.313280 \\ \mathrm{H} & -0.330517 & -4.520697 & 1.652592 \\ \mathrm{H} & -0.365929 & -4.989137 & -0.055174 \\ \mathrm{~N} & -1.968970 & -2.295808 & 0.445305 \\ \mathrm{~N} & 0.102398 & -2.958446 & 0.299560 \\ \mathrm{C} & -3.146086 & -1.491948 & 0.464102\end{array}$

\begin{tabular}{|c|c|c|c|}
\hline & & & \\
\hline & 3.534560 & .846807 & 548 \\
\hline & .059 & 599252 & 695 \\
\hline & 9 & & \\
\hline & 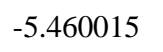 & 30 & .4651 \\
\hline & 5.64 & 31 & 1.6 \\
\hline & & & \\
\hline & 1.4 & & \\
\hline & 1.8 & 314 & 80 \\
\hline & 2. & & \\
\hline & 3.16 & -3 & -1.10 \\
\hline & 3.80 & -3.0 & \\
\hline & 4.17 & -3 & 0.79541 \\
\hline & 3.428065 & 93 & \\
\hline & & & \\
\hline & 76 & -0.97 & 2.92641 \\
\hline & 2.36 & 0.008667 & 20 \\
\hline & 3.40 & & \\
\hline & -1.916 & & \\
\hline & -3.520 & 33 & -1.964 \\
\hline & $-3.95 t$ & -3.1 & -2 \\
\hline & -38 & & \\
\hline & -1 & & \\
\hline & -6.691 & 0.935588 & 0.4627 \\
\hline & -6.658966 & 1.69 & 34 \\
\hline & -0.01 & & \\
\hline & -7.59 & & \\
\hline & 740166 & -3.18 & -2.44936 \\
\hline & 1.165758 & -3.3 & -3.44 \\
\hline & & & \\
\hline & 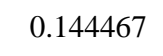 & -2 & -2 \\
\hline & 96 & -3.0 & 4056 \\
\hline & 005 & -2 & 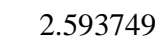 \\
\hline & 2.214478 & & \\
\hline & 78 & -2.42 & \\
\hline & 5.612838 & -3.12 & 301 \\
\hline & 6.2720 & -3.33 & 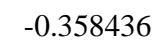 \\
\hline & 0 & & \\
\hline & 5.912037 & -2 & -1.6085 \\
\hline & +4 & 6 & 1.02007 \\
\hline & 637 & -0.039940 & 1.704 \\
\hline & & & \\
\hline & 2020 & $\pi /$ & -1 . \\
\hline & 571573 & 31703 & J. \\
\hline
\end{tabular}




$\begin{array}{rrrr}\mathrm{C} & -4.141310 & 3.019783 & -1.839743 \\ \mathrm{H} & -3.077190 & 1.470417 & -2.894888 \\ \mathrm{C} & -3.514778 & 3.329585 & 0.472790 \\ \mathrm{H} & -1.959583 & 2.040420 & 1.216885 \\ \mathrm{C} & -4.299673 & 3.685553 & -0.624036 \\ \mathrm{H} & -4.749366 & 3.297586 & -2.696517 \\ \mathrm{H} & -3.641882 & 3.841946 & 1.422826 \\ \mathrm{H} & -5.037634 & 4.478227 & -0.529932 \\ \mathrm{O} & 1.170604 & -0.244778 & 2.119178 \\ \mathrm{O} & 1.218808 & -0.278619 & -1.636246 \\ \mathrm{H} & -0.895091 & 0.245395 & 1.494235 \\ \mathrm{H} & 1.061081 & 5.163850 & -1.038757 \\ \mathrm{Br} & 5.138583 & 2.695892 & 0.631311 \\ \mathrm{C} & 0.210225 & 0.533364 & 2.278413 \\ \mathrm{O} & -0.168011 & 1.391667 & 3.047355 \\ \mathrm{C} & 1.075903 & 0.456030 & -2.684616 \\ \mathrm{H} & 1.827154 & 0.204624 & -3.469726 \\ \mathrm{O} & 0.247780 & 1.344015 & -2.901749\end{array}$

\section{II-Br}

$\begin{array}{rrrr}\mathrm{Ru} & 0.036047 & -0.065814 & -0.216495 \\ \mathrm{C} & 2.458611 & -1.943460 & -0.420650 \\ \mathrm{C} & 0.512900 & -3.187217 & -0.538083 \\ \mathrm{C} & 3.218748 & -3.103201 & -0.428176 \\ \mathrm{H} & 2.902333 & -0.956627 & -0.349063 \\ \mathrm{C} & 1.220535 & -4.379700 & -0.542800 \\ \mathrm{H} & -0.572168 & -3.168711 & -0.570710 \\ \mathrm{~N} & 1.118201 & -1.993458 & -0.472878 \\ \mathrm{C} & 2.609439 & -4.348102 & -0.483921 \\ \mathrm{H} & 3.198259 & -5.259113 & -0.480408 \\ \mathrm{C} & -0.742205 & 1.815922 & -0.189766 \\ \mathrm{C} & -2.245551 & 3.638217 & -0.230154 \\ \mathrm{C} & -0.831303 & 4.164253 & -0.412094 \\ \mathrm{H} & -2.926448 & 3.938769 & -1.033326 \\ \mathrm{H} & -2.698777 & 3.945333 & 0.723611 \\ \mathrm{H} & -0.658545 & 4.590494 & -1.409910 \\ \mathrm{H} & -0.550686 & 4.920984 & 0.327276 \\ \mathrm{~N} & -2.048757 & 2.183676 & -0.252393 \\ \mathrm{~N} & -0.010391 & 2.958365 & -0.231297 \\ \mathrm{C} & -3.200073 & 1.348340 & -0.327098 \\ \mathrm{C} & -3.916390 & 1.071136 & 0.848286 \\ \mathrm{C} & -3.656026 & 0.922651 & -1.583707 \\ \mathrm{C} & -5.088660 & 0.324555 & 0.747601 \\ \mathrm{C} & -4.840836 & 0.185429 & -1.632722\end{array}$

\begin{tabular}{|c|c|c|c|}
\hline & 46 & & \\
\hline & .632713 & 77017 & .658 \\
\hline & & & \\
\hline & 8 & & \\
\hline & 1.67 & & 15 \\
\hline & 2.322700 & 3.40 & 0.8219 \\
\hline & 3.00 & & \\
\hline & 3.64 & 28 & -0 . \\
\hline & 4.00 & 3.467655 & .9412 \\
\hline & & & \\
\hline & 4.40 & & \\
\hline & -2.89 & 2 & -2.8 \\
\hline & -2.04 & 2 & 2.9 \\
\hline & -3.53 & 84 & -3.7151 \\
\hline & -2.45 & 235897 & \\
\hline & -3.42 & 1 & 2183 \\
\hline & -3.783097 & 9 & 2.4 \\
\hline & -3.79 & & \\
\hline & -2.33 & 692 & \\
\hline & -6.819 & .944710 & .558 \\
\hline & -7.05 & 9 & 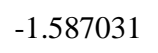 \\
\hline & -6 & 86 & \\
\hline & -7.68 & 55 & .1 \\
\hline & 0.61 & & \\
\hline & 8 & & \\
\hline & -0.222626 & & \\
\hline & 0. & & \\
\hline & 1.968740 & 3.557 & -2 . \\
\hline & 0 & 1 & \\
\hline & & & \\
\hline & 3 & 39 & -2.9 \\
\hline & 5.439 & 3 & .36 \\
\hline & & & \\
\hline & $-\mathrm{cos}$ & & \\
\hline & 5 & 77 & 15274 \\
\hline & -1.243422 & 0.102143 & 0.9924. \\
\hline & & 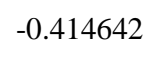 & \\
\hline & -2.3 & 712 & \\
\hline & -2.851 & 52 & 70 \\
\hline & -2.895 & -2.188 & -0.260 \\
\hline & -3.884112 & 3.129038 & 0.780 \\
\hline & -2.4 & 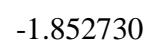 & 3.0861 \\
\hline & -3.931908 & -3.110086 & 0.24222 \\
\hline & .510 & 17 & .203 \\
\hline
\end{tabular}




$\begin{array}{cccc}\mathrm{C} & -4.429401 & -3.582914 & 0.972902 \\ \mathrm{H} & -4.265790 & -3.501215 & 3.120863 \\ \mathrm{H} & -4.367325 & -3.452128 & -1.178088 \\ \mathrm{H} & -5.242818 & -4.304352 & 0.982817 \\ \mathrm{O} & 1.647118 & 0.651685 & -1.601767 \\ \mathrm{O} & 1.496094 & 0.100317 & 1.445110 \\ \mathrm{H} & -1.116471 & -0.445218 & -1.235131 \\ \mathrm{H} & 0.688591 & -5.324181 & -0.585710 \\ \mathrm{Br} & 5.145599 & -2.960157 & -0.348507 \\ \mathrm{C} & 0.691095 & 0.195541 & -2.249459 \\ \mathrm{O} & 0.281713 & -0.023495 & -3.357772 \\ \mathrm{C} & 1.527178 & -0.804240 & 2.354855 \\ \mathrm{H} & 2.336189 & -0.629925 & 3.106214 \\ \mathrm{O} & 0.798397 & -1.795696 & 2.487282\end{array}$

\section{II-Br'}

$\begin{array}{rrrr}\mathrm{Ru} & -0.170465 & -0.500937 & -0.075287 \\ \mathrm{C} & 2.448197 & -1.563204 & 1.138231 \\ \mathrm{C} & 2.869426 & 0.153022 & -0.366506 \\ \mathrm{C} & 3.797843 & -1.698622 & 1.429091 \\ \mathrm{H} & 1.683326 & -2.158375 & 1.630798 \\ \mathrm{C} & 4.226850 & 0.078118 & -0.091411 \\ \mathrm{H} & 2.452750 & 0.874051 & -1.067019 \\ \mathrm{H} & 4.126094 & -2.443753 & 2.146549 \\ \mathrm{~N} & 1.993124 & -0.653015 & 0.258444 \\ \mathrm{C} & 4.717213 & -0.855635 & 0.811571 \\ \mathrm{H} & 5.778058 & -0.917647 & 1.029386 \\ \mathrm{C} & -2.132408 & -0.774922 & -0.394812 \\ \mathrm{C} & -4.463104 & -0.865398 & -0.687058 \\ \mathrm{C} & -3.903573 & -2.275476 & -0.822940 \\ \mathrm{H} & -5.033735 & -0.733142 & 0.243542 \\ \mathrm{H} & -5.104498 & -0.567200 & -1.522008 \\ \mathrm{H} & -4.416815 & -3.010335 & -0.194304 \\ \mathrm{H} & -3.929742 & -2.637912 & -1.861770 \\ \mathrm{~N} & -3.245861 & -0.039369 & -0.649181 \\ \mathrm{~N} & -2.522861 & -2.080586 & -0.385312 \\ \mathrm{C} & -3.345386 & 1.373318 & -0.472358 \\ \mathrm{C} & -3.131257 & 2.204848 & -1.581591 \\ \mathrm{C} & -3.629239 & 1.897274 & 0.798159 \\ \mathrm{C} & -3.162779 & 3.584675 & -1.386303 \\ \mathrm{C} & -3.638141 & 3.285936 & 0.944907 \\ \mathrm{C} & -3.392624 & 4.143799 & -0.127228 \\ \mathrm{H} & -2.977772 & 4.239626 & -2.236778 \\ \mathrm{H} & -3.831435 & 3.706342 & 1.930866\end{array}$

\begin{tabular}{|c|c|c|c|}
\hline C & -1.554048 & -3.123185 & -0.247629 \\
\hline $\mathrm{C}$ & -0.829292 & -3.549281 & -1.378622 \\
\hline $\mathrm{C}$ & -1.335578 & -3.684617 & 1.024404 \\
\hline C & 0.208442 & -4.461411 & -1.184801 \\
\hline $\mathrm{C}$ & -0.296669 & -4.608294 & 1.156278 \\
\hline $\mathrm{C}$ & 0.504578 & -4.981702 & 0.076994 \\
\hline $\mathrm{H}$ & 0.794086 & -4.778807 & -2.046249 \\
\hline $\mathrm{H}$ & -0.105446 & -5.039404 & 2.138226 \\
\hline $\mathrm{C}$ & -3.934747 & 1.014385 & 1.969944 \\
\hline $\mathrm{H}$ & -3.597593 & 1.461956 & 2.908033 \\
\hline $\mathrm{H}$ & -5.015140 & 0.847432 & 2.065696 \\
\hline $\mathrm{H}$ & -3.457422 & 0.033604 & 1.894844 \\
\hline $\mathrm{C}$ & -2.778473 & 1.629451 & -2.916778 \\
\hline $\mathrm{H}$ & -3.356994 & 0.730615 & -3.150080 \\
\hline $\mathrm{H}$ & -2.933739 & 2.360154 & -3.713987 \\
\hline $\mathrm{H}$ & -1.720099 & 1.341899 & -2.940529 \\
\hline $\mathrm{C}$ & -3.335243 & 5.626908 & 0.073622 \\
\hline $\mathrm{H}$ & -3.957135 & 5.948838 & 0.913074 \\
\hline $\mathrm{H}$ & -2.310444 & 5.950304 & 0.292899 \\
\hline $\mathrm{H}$ & -3.657440 & 6.171425 & -0.818202 \\
\hline $\mathrm{C}$ & -1.204013 & -3.113304 & -2.761296 \\
\hline $\mathrm{H}$ & -0.327317 & -3.064360 & -3.409546 \\
\hline $\mathrm{H}$ & -1.907594 & -3.830372 & -3.203578 \\
\hline $\mathrm{H}$ & -1.667904 & -2.125262 & -2.774875 \\
\hline $\mathrm{C}$ & -2.173722 & -3.332416 & 2.211927 \\
\hline $\mathrm{H}$ & -3.050823 & -2.741211 & 1.937835 \\
\hline $\mathrm{H}$ & -2.517174 & -4.240529 & 2.717802 \\
\hline $\mathrm{H}$ & -1.609332 & -2.737037 & 2.937308 \\
\hline $\mathrm{C}$ & 1.666767 & -5.906367 & 0.271330 \\
\hline $\mathrm{H}$ & 1.501910 & -6.597089 & 1.102781 \\
\hline $\mathrm{H}$ & 1.874321 & -6.495505 & -0.626081 \\
\hline $\mathrm{H}$ & 2.579947 & -5.342989 & 0.499892 \\
\hline $\mathrm{C}$ & -0.180662 & 1.344904 & -0.042538 \\
\hline $\mathrm{H}$ & -0.884384 & 1.863504 & -0.701379 \\
\hline $\mathrm{C}$ & 0.694191 & 2.270815 & 0.659637 \\
\hline $\mathrm{C}$ & 0.785669 & 3.588111 & 0.166703 \\
\hline $\mathrm{C}$ & 1.458570 & 1.926823 & 1.791847 \\
\hline $\mathrm{C}$ & 1.633169 & 4.513838 & 0.758704 \\
\hline $\mathrm{H}$ & 0.198639 & 3.852825 & -0.710533 \\
\hline $\mathrm{C}$ & 2.290567 & 2.860751 & 2.392802 \\
\hline $\mathrm{H}$ & 1.374997 & 0.921683 & 2.196869 \\
\hline $\mathrm{C}$ & 2.388709 & 4.152150 & 1.873581 \\
\hline $\mathrm{H}$ & 1.707776 & 5.519108 & 0.352783 \\
\hline $\mathrm{H}$ & 2.866219 & 2.584154 & 3.271876 \\
\hline
\end{tabular}




$\begin{array}{rrrr}\mathrm{H} & 3.050001 & 4.876917 & 2.340885 \\ \mathrm{O} & -0.325573 & -0.919927 & 1.945738 \\ \mathrm{O} & 0.281876 & -0.690607 & -2.113035 \\ \mathrm{H} & -1.187350 & 0.849354 & 2.449665 \\ \mathrm{Br} & 5.419528 & 1.337474 & -0.944848 \\ \mathrm{C} & -0.969349 & -0.185984 & 2.812648 \\ \mathrm{O} & -1.311834 & -0.553780 & 3.924655 \\ \mathrm{C} & 0.685594 & 0.275995 & -2.865539 \\ \mathrm{H} & 0.880096 & -0.071273 & -3.904680 \\ \mathrm{O} & 0.858332 & 1.460192 & -2.571759\end{array}$

\section{TSII' ${ }^{-1 B r}$}

$\begin{array}{rrrr}\mathrm{Ru} & -0.446640 & -0.175862 & 0.374590 \\ \mathrm{C} & 1.234110 & -1.872608 & 2.469299 \\ \mathrm{C} & 1.970470 & -2.122963 & 0.288168 \\ \mathrm{C} & 2.252944 & -2.683174 & 2.948991 \\ \mathrm{H} & 0.496720 & -1.427694 & 3.130367 \\ \mathrm{C} & 3.017126 & -2.924460 & 0.716142 \\ \mathrm{H} & 1.822990 & -1.884616 & -0.761686 \\ \mathrm{H} & 2.323048 & -2.883512 & 4.012905 \\ \mathrm{~N} & 1.099771 & -1.582141 & 1.161357 \\ \mathrm{C} & 3.181172 & -3.218605 & 2.062141 \\ \mathrm{H} & 4.000643 & -3.842122 & 2.403436 \\ \mathrm{C} & -1.899937 & 1.061855 & -0.280545 \\ \mathrm{C} & -3.061203 & 3.018969 & -0.902718 \\ \mathrm{C} & -3.992574 & 1.830063 & -1.048900 \\ \mathrm{H} & -3.328912 & 3.655218 & -0.046732 \\ \mathrm{H} & -3.014460 & 3.654002 & -1.792990 \\ \mathrm{H} & -4.948545 & 1.951472 & -0.529985 \\ \mathrm{H} & -4.207252 & 1.592611 & -2.100577 \\ \mathrm{~N} & -1.765266 & 2.374531 & -0.657254 \\ \mathrm{~N} & -3.209853 & 0.748921 & -0.440472 \\ \mathrm{C} & -0.635214 & 3.204273 & -0.364252 \\ \mathrm{C} & 0.255800 & 3.551154 & -1.392990 \\ \mathrm{C} & -0.455885 & 3.686389 & 0.944751 \\ \mathrm{C} & 1.405833 & 4.265324 & -1.056174 \\ \mathrm{C} & 0.713608 & 4.389949 & 1.233121 \\ \mathrm{C} & 1.673015 & 4.657121 & 0.255314 \\ \mathrm{H} & 2.128213 & 4.493207 & -1.839869 \\ \mathrm{H} & 0.885802 & 4.717224 & 2.257407 \\ \mathrm{C} & -3.781282 & -0.556647 & -0.334592 \\ \mathrm{C} & -3.880040 & -1.358093 & -1.479537 \\ \mathrm{C} & -4.276676 & -0.975009 & 0.907863 \\ \mathrm{C} & -4.458556 & -2.619414 & -1.349160\end{array}$

\begin{tabular}{|c|c|c|c|}
\hline & -4.846056 & -2.246777 & 0.987854 \\
\hline & -4.937170 & -3.085535 & -0.123941 \\
\hline & -4.525641 & -3.260297 & -2.227745 \\
\hline & -5.234296 & -2.587228 & 1.946754 \\
\hline$C$ & -1.493963 & 3.485265 & 2.008272 \\
\hline 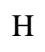 & -1.046759 & 3.523561 & 3.003838 \\
\hline & -2.254287 & 4.275113 & 1.962162 \\
\hline 7 & -2.011115 & 2.525084 & 1.917782 \\
\hline$C$ & 0.009675 & 3.182288 & -2.824545 \\
\hline 1 & -0.147016 & 4.079586 & -3.433945 \\
\hline & 0.872441 & 2.658434 & -3.251061 \\
\hline H & -0.864698 & 2.537733 & -2.942528 \\
\hline$C$ & 2.968789 & 5.319972 & 0.604908 \\
\hline 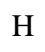 & 2.909515 & 5.854882 & 1.556162 \\
\hline 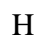 & 3.766903 & 4.573973 & 0.699684 \\
\hline H & 3.286369 & 6.027746 & -0.166500 \\
\hline $\mathrm{C}$ & -3.364035 & -0.890002 & -2.805830 \\
\hline $\mathrm{H}$ & -3.087859 & -1.736087 & -3.440624 \\
\hline I & -4.117960 & -0.315078 & -3.358306 \\
\hline $\mathrm{H}$ & -2.479218 & -0.253269 & -2.698670 \\
\hline $\mathrm{C}$ & -4.206714 & -0.079195 & 2.105130 \\
\hline $\mathrm{H}$ & -4.581100 & 0.925937 & 1.876935 \\
\hline 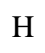 & -4.802224 & -0.481486 & 2.928162 \\
\hline $\mathrm{H}$ & -3.178127 & 0.046570 & 2.461718 \\
\hline $\mathrm{C}$ & -5.516989 & -4.461539 & -0.000326 \\
\hline $\mathrm{H}$ & -6.293494 & -4.506613 & 0.768407 \\
\hline $\mathrm{H}$ & -5.952406 & -4.804842 & -0.942746 \\
\hline H & -4.748852 & -5.191108 & 0.281179 \\
\hline$C$ & 0.670613 & 0.537151 & -0.920124 \\
\hline $\mathrm{H}$ & 0.213072 & 0.875528 & -1.857878 \\
\hline$C$ & 2.107768 & 0.758323 & -0.897680 \\
\hline $\mathrm{C}$ & 2.778969 & 0.842352 & -2.134810 \\
\hline$C$ & 2.860724 & 0.931886 & 0.280350 \\
\hline- & 4.146436 & 1.066757 & -2.192782 \\
\hline $\mathrm{H}$ & 2.204821 & 0.688391 & -3.046087 \\
\hline $\mathrm{C}$ & 4.226412 & 1.178954 & 0.216129 \\
\hline H & 2.364977 & 0.894232 & 1.247549 \\
\hline$C$ & 4.875486 & 1.239475 & -1.016131 \\
\hline $\mathrm{H}$ & 4.647820 & 1.108820 & -3.156064 \\
\hline H & 4.788194 & 1.324355 & 1.135149 \\
\hline $\mathrm{H}$ & 5.945367 & 1.424455 & -1.059905 \\
\hline $\mathrm{O}$ & -0.958687 & 0.183783 & 2.544727 \\
\hline p & -1.030369 & -1.726730 & -0.796966 \\
\hline & 0.404266 & 1.440851 & 1.730990 \\
\hline
\end{tabular}




$\begin{array}{cccc}\mathrm{Br} & 4.266087 & -3.593164 & -0.600053 \\ \mathrm{C} & 0.013669 & 1.008515 & 2.715945 \\ \mathrm{O} & 0.562209 & 1.369106 & 3.745275 \\ \mathrm{C} & -0.503922 & -2.097476 & -1.923809 \\ \mathrm{H} & -1.026013 & -3.001607 & -2.304312 \\ \mathrm{O} & 0.419497 & -1.590058 & -2.550675\end{array}$

\section{II-Br'}

$\mathrm{Ru} \quad-0.435783$

$\begin{array}{llll}\text { C } & 0.726933 & -2.626933 & 1.965982\end{array}$

$\begin{array}{llll}\text { C } & 2.048192 & -2.155090 & 0.128552\end{array}$

$\begin{array}{llll}\text { C } & 1.531628 & -3.697066 & 2.326485\end{array}$

$\begin{array}{llll}\mathrm{H} & -0.155884 & -2.366794 & 2.540919\end{array}$

$\begin{array}{llll}\text { C } & 2.895486 & -3.204943 & 0.451715\end{array}$

$\begin{array}{llll}\mathrm{H} & 2.195715 & -1.555953 & -0.762560\end{array}$

$\begin{array}{llll}\mathrm{H} & 1.281998 & -4.285555 & 3.202757\end{array}$

$\begin{array}{llll}\mathrm{N} & 0.981836 & -1.855077 & 0.892160\end{array}$

$\begin{array}{llll}\text { C } & 2.654308 & -3.998193 & 1.563754\end{array}$

$\begin{array}{llll}\mathrm{H} & 3.313594 & -4.820566 & 1.819835\end{array}$

$\begin{array}{llll}\text { C } & -1.873014 & 1.193246 & 0.002844\end{array}$

$\begin{array}{llll}\text { C } & -2.986853 & 3.273241 & -0.193725\end{array}$

$\begin{array}{llll}\text { C } & -4.026594 & 2.163814 & -0.075254\end{array}$

$\begin{array}{llll}\mathrm{H} & -3.057196 & 4.013980 & 0.611583\end{array}$

$\begin{array}{llll}\mathrm{H} & -3.041194 & 3.815466 & -1.147759\end{array}$

$\begin{array}{llll}\mathrm{H} & -4.648434 & 2.251757 & 0.824449\end{array}$

$\begin{array}{llll}\mathrm{H} & -4.700741 & 2.111455 & -0.938115\end{array}$

$\begin{array}{llll}\mathrm{N} & -1.719152 & 2.543686 & -0.094165\end{array}$

$\begin{array}{llll}\mathrm{N} & -3.205311 & 0.949213 & -0.001895\end{array}$

$\begin{array}{llll}\text { C } & -0.490684 & 3.266590 & -0.050123\end{array}$

$\begin{array}{llll}\text { C } & 0.111958 & 3.654856 & -1.257886\end{array}$

$\begin{array}{llll}\text { C } & 0.077038 & 3.603655 & 1.190743\end{array}$

$\begin{array}{llll}\text { C } & 1.339371 & 4.314311 & -1.201780\end{array}$

$\begin{array}{llll}\text { C } & 1.310536 & 4.255167 & 1.191697\end{array}$

$\begin{array}{llll}\text { C } & 1.966643 & 4.601678 & 0.009249\end{array}$

$\mathrm{H} \quad 1.832441 \quad 4.587373 \quad-2.134285$

$\begin{array}{llll}\mathrm{H} & 1.775649 & 4.490546 & 2.148545\end{array}$

$\begin{array}{llll}\text { C } & -3.811915 & -0.322922 & -0.240632\end{array}$

$\begin{array}{llll}\text { C } & -3.829504 & -0.800150 & -1.562112\end{array}$

$\begin{array}{llll}\text { C } & -4.428621 & -1.018577 & 0.803512\end{array}$

$\begin{array}{llll}\text { C } & -4.427940 & -2.029947 & -1.810641\end{array}$

$\begin{array}{llll}\text { C } & -5.010633 & -2.254887 & 0.504551\end{array}$

$\begin{array}{llll}\text { C } & -5.009107 & -2.781598 & -0.784711\end{array}$

$\begin{array}{llll}\mathrm{H} & -4.433579 & -2.417242 & -2.829103\end{array}$

$\mathrm{H} \quad-5.481777 \quad-2.818185 \quad 1.309118$

\begin{tabular}{|c|c|c|c|}
\hline $\mathrm{C}$ & -0.610487 & 3.313801 & 2.491080 \\
\hline п & 0.059514 & 2.812080 & 3.195097 \\
\hline$H$ & -0.938195 & 4.245023 & 2.967640 \\
\hline H & -1.492116 & 2.678526 & 2.367938 \\
\hline C & -0.527192 & 3.371676 & -2.583528 \\
\hline H & -1.110992 & 4.231242 & -2.935921 \\
\hline $\mathrm{H}$ & 0.227612 & 3.168070 & -3.348533 \\
\hline H & -1.204623 & 2.514216 & -2.542659 \\
\hline $\mathrm{C}$ & 3.318545 & 5.243131 & 0.045740 \\
\hline $\mathrm{H}$ & 3.343617 & 6.102720 & 0.723205 \\
\hline H & 4.074256 & 4.534956 & 0.403072 \\
\hline $\mathrm{H}$ & 3.631271 & 5.583811 & -0.944648 \\
\hline $\mathrm{C}$ & -3.213933 & 0.003430 & -2.665434 \\
\hline $\mathrm{H}$ & -3.321116 & -0.500685 & -3.629055 \\
\hline $\mathrm{H}$ & -3.674498 & 0.996207 & -2.756674 \\
\hline $\mathrm{H}$ & -2.144692 & 0.164646 & -2.489082 \\
\hline C & -4.519908 & -0.455970 & 2.187759 \\
\hline $\mathrm{H}$ & -3.749983 & 0.292784 & 2.377567 \\
\hline $\mathrm{H}$ & -5.502460 & 0.003029 & 2.355633 \\
\hline $\mathrm{H}$ & -4.398501 & -1.239461 & 2.939499 \\
\hline $\mathrm{C}$ & -5.607541 & -4.125092 & -1.070496 \\
\hline $\mathrm{H}$ & -6.225073 & -4.477782 & -0.240357 \\
\hline $\mathrm{H}$ & -6.229359 & -4.109230 & -1.971021 \\
\hline $\mathrm{H}$ & -4.829200 & -4.877527 & -1.239803 \\
\hline $\mathrm{C}$ & 0.735846 & 0.651046 & -0.822246 \\
\hline $\mathrm{H}$ & 0.360937 & 0.995788 & -1.797344 \\
\hline $\mathrm{C}$ & 2.143919 & 0.963450 & -0.637311 \\
\hline $\mathrm{C}$ & 2.924076 & 1.287752 & -1.763786 \\
\hline $\mathrm{C}$ & 2.767425 & 0.951121 & 0.626698 \\
\hline C & 4.278614 & 1.562908 & -1.640252 \\
\hline $\mathrm{H}$ & 2.444719 & 1.293524 & -2.741121 \\
\hline $\mathrm{C}$ & 4.117998 & 1.249047 & 0.750226 \\
\hline $\mathrm{H}$ & 2.176161 & 0.719806 & 1.511719 \\
\hline $\mathrm{C}$ & 4.878946 & 1.546224 & -0.380966 \\
\hline $\mathrm{H}$ & 4.869014 & 1.795533 & -2.522218 \\
\hline $\mathrm{H}$ & 4.582315 & 1.245991 & 1.732752 \\
\hline $\mathrm{H}$ & 5.938030 & 1.769471 & -0.280632 \\
\hline $\mathrm{O}$ & -1.445387 & -0.522152 & 2.512210 \\
\hline $\mathrm{O}$ & -1.073635 & -1.587157 & -1.084558 \\
\hline $\mathrm{H}$ & -0.036281 & 0.839498 & 1.701467 \\
\hline $\mathrm{Br}$ & 4.428897 & -3.534120 & -0.680434 \\
\hline $\mathrm{C}$ & -0.372322 & 0.100826 & 2.678987 \\
\hline $\mathrm{O}$ & 0.429206 & 0.280597 & 3.577404 \\
\hline C & -0.385861 & -1.862303 & -2.140243 \\
\hline
\end{tabular}




\begin{tabular}{|c|c|c|c|c|c|c|c|}
\hline $\mathrm{H}$ & -0.889866 & -2.640358 & -2.757147 & $\mathrm{C}$ & -0.491017 & 3.429845 & -2.493777 \\
\hline \multirow[t]{2}{*}{$\mathrm{O}$} & 0.683347 & -1.380589 & -2.519934 & $\mathrm{H}$ & -1.136736 & 4.271142 & -2.775284 \\
\hline & & & & $\mathrm{H}$ & 0.236934 & 3.307412 & -3.300651 \\
\hline \multicolumn{2}{|c|}{ TSII' $_{1-2 \mathrm{Br}}$} & & & $\mathrm{H}$ & -1.120086 & 2.535078 & -2.468722 \\
\hline $\mathrm{Ru}$ & -0.446866 & -0.201181 & 0.330118 & $\mathrm{C}$ & 3.478191 & 5.151460 & 0.059344 \\
\hline $\mathrm{C}$ & 0.448438 & -2.900737 & 1.628803 & $\mathrm{H}$ & 3.491841 & 6.095216 & 0.615224 \\
\hline $\mathrm{C}$ & 2.061263 & -2.178244 & 0.141316 & $\mathrm{H}$ & 4.203128 & 4.486910 & 0.541570 \\
\hline $\mathrm{C}$ & 1.155879 & -4.060356 & 1.905957 & $\mathrm{H}$ & 3.838683 & 5.353008 & -0.952697 \\
\hline $\mathrm{H}$ & -0.507820 & -2.694367 & 2.100655 & $\mathrm{C}$ & -3.182978 & 0.144729 & -2.631614 \\
\hline $\mathrm{C}$ & 2.819398 & -3.312826 & 0.391889 & $\mathrm{H}$ & -3.303138 & -0.341462 & -3.602834 \\
\hline $\mathrm{H}$ & 2.369605 & -1.441243 & -0.588175 & $\mathrm{H}$ & -3.596448 & 1.159170 & -2.712171 \\
\hline $\mathrm{H}$ & 0.753203 & -4.782557 & 2.607875 & $\mathrm{H}$ & -2.109552 & 0.246848 & -2.440110 \\
\hline $\mathrm{N}$ & 0.894627 & -1.964814 & 0.770442 & $\mathrm{C}$ & -4.619126 & -0.284470 & 2.181685 \\
\hline $\mathrm{C}$ & 2.380381 & -4.279038 & 1.283830 & $\mathrm{H}$ & -3.742166 & 0.313192 & 2.435601 \\
\hline $\mathrm{H}$ & 2.968295 & -5.168746 & 1.481926 & $\mathrm{H}$ & -5.510550 & 0.347843 & 2.285907 \\
\hline $\mathrm{C}$ & -1.840617 & 1.253346 & 0.051772 & $\mathrm{H}$ & -4.701913 & -1.078951 & 2.927178 \\
\hline $\mathrm{C}$ & -2.878012 & 3.371739 & -0.104730 & $\mathrm{C}$ & -5.810022 & -3.876153 & -1.130921 \\
\hline $\mathrm{C}$ & -3.953241 & 2.305385 & 0.068908 & $\mathrm{H}$ & -6.535743 & -4.158268 & -0.363459 \\
\hline $\mathrm{H}$ & -2.910341 & 4.145530 & 0.669987 & $\mathrm{H}$ & -6.328527 & -3.850621 & -2.094279 \\
\hline $\mathrm{H}$ & -2.927833 & 3.875090 & -1.080900 & $\mathrm{H}$ & -5.072199 & -4.684308 & -1.191591 \\
\hline $\mathrm{H}$ & -4.495149 & 2.396180 & 1.019599 & $\mathrm{C}$ & 0.756620 & 0.626959 & -0.834298 \\
\hline $\mathrm{H}$ & -4.693802 & 2.303101 & -0.737800 & $\mathrm{H}$ & 0.397986 & 0.914663 & -1.834917 \\
\hline $\mathrm{N}$ & -1.637718 & 2.598351 & 0.006710 & $\mathrm{C}$ & 2.151057 & 0.978710 & -0.626473 \\
\hline $\mathrm{N}$ & -3.180135 & 1.056741 & 0.049055 & $\mathrm{C}$ & 2.953474 & 1.282706 & -1.742984 \\
\hline $\mathrm{C}$ & -0.381211 & 3.270787 & 0.032279 & $\mathrm{C}$ & 2.742284 & 1.009753 & 0.652423 \\
\hline $\mathrm{C}$ & 0.193967 & 3.674909 & -1.183762 & $\mathrm{C}$ & 4.299115 & 1.586986 & -1.594058 \\
\hline $\mathrm{C}$ & 0.243132 & 3.539883 & 1.261984 & $\mathrm{H}$ & 2.499928 & 1.248247 & -2.731729 \\
\hline $\mathrm{C}$ & 1.439805 & 4.300480 & -1.150283 & $\mathrm{C}$ & 4.084679 & 1.332283 & 0.800030 \\
\hline $\mathrm{C}$ & 1.492980 & 4.158651 & 1.239125 & $\mathrm{H}$ & 2.131351 & 0.778345 & 1.524290 \\
\hline $\mathrm{C}$ & 2.114768 & 4.533370 & 0.046691 & $\mathrm{C}$ & 4.867075 & 1.615597 & -0.320181 \\
\hline $\mathrm{H}$ & 1.910969 & 4.584204 & -2.090848 & $\mathrm{H}$ & 4.907733 & 1.805230 & -2.467255 \\
\hline $\mathrm{H}$ & 2.001124 & 4.342351 & 2.185667 & $\mathrm{H}$ & 4.526288 & 1.360246 & 1.792708 \\
\hline $\mathrm{C}$ & -3.839477 & -0.181422 & -0.221331 & $\mathrm{H}$ & 5.919494 & 1.860258 & -0.199898 \\
\hline $\mathrm{C}$ & -3.849579 & -0.643427 & -1.547245 & $\mathrm{O}$ & -1.553118 & -0.655054 & 2.388034 \\
\hline $\mathrm{C}$ & -4.524388 & -0.849804 & 0.799043 & $\mathrm{O}$ & -1.102945 & -1.571151 & -1.187995 \\
\hline $\mathrm{C}$ & -4.504334 & -1.839818 & -1.821070 & $\mathrm{H}$ & -0.007560 & 0.815284 & 1.554782 \\
\hline $\mathrm{C}$ & -5.164369 & -2.048715 & 0.474911 & $\mathrm{Br}$ & 4.506259 & -3.516578 & -0.533145 \\
\hline $\mathrm{C}$ & -5.152544 & -2.566576 & -0.819182 & $\mathrm{C}$ & -0.414369 & -0.181144 & 2.561098 \\
\hline $\mathrm{H}$ & -4.504216 & -2.217265 & -2.843059 & $\mathrm{O}$ & 0.434747 & -0.087443 & 3.419013 \\
\hline $\mathrm{H}$ & -5.688923 & -2.590149 & 1.261122 & $\mathrm{C}$ & -0.343362 & -1.878061 & -2.177851 \\
\hline $\mathrm{C}$ & -0.385585 & 3.187365 & 2.576531 & $\mathrm{H}$ & -0.820972 & -2.623493 & -2.855833 \\
\hline $\mathrm{H}$ & 0.257059 & 2.519344 & 3.159394 & $\mathrm{O}$ & 0.785156 & -1.454915 & -2.452550 \\
\hline $\mathrm{H}$ & -0.544802 & 4.085617 & 3.183190 & & & & \\
\hline $\mathrm{H}$ & -1.349899 & 2.685781 & 2.457787 & $211-$ & & & \\
\hline
\end{tabular}




\begin{tabular}{|c|c|c|c|c|c|c|c|}
\hline $\mathrm{Ru}$ & -0.430114 & -0.315769 & 0.221486 & $\mathrm{C}$ & 3.956893 & 5.043133 & 0.312937 \\
\hline $\mathrm{C}$ & -0.044141 & -3.380836 & 0.388918 & $\mathrm{H}$ & 3.946082 & 6.014282 & 0.820367 \\
\hline $\mathrm{C}$ & 2.003238 & -2.307130 & 0.430215 & $\mathrm{H}$ & 4.696762 & 4.425144 & 0.832882 \\
\hline $\mathrm{C}$ & 0.563397 & -4.628169 & 0.387749 & $\mathrm{H}$ & 4.317111 & 5.205157 & -0.706411 \\
\hline $\mathrm{H}$ & -1.121696 & -3.258467 & 0.357545 & $\mathrm{C}$ & -3.237982 & 0.805230 & -2.392557 \\
\hline $\mathrm{C}$ & 2.664632 & -3.525103 & 0.422196 & $\mathrm{H}$ & -3.511450 & 0.506840 & -3.407607 \\
\hline $\mathrm{H}$ & 2.543094 & -1.365879 & 0.440559 & $\mathrm{H}$ & -3.433863 & 1.881644 & -2.294968 \\
\hline $\mathrm{H}$ & -0.046089 & -5.525211 & 0.369468 & $\mathrm{H}$ & -2.158536 & 0.656127 & -2.281391 \\
\hline $\mathrm{N}$ & 0.666645 & -2.240892 & 0.407373 & $\mathrm{C}$ & -4.494832 & -0.075291 & 2.414104 \\
\hline $\mathrm{C}$ & 1.950993 & -4.714430 & 0.399529 & $\mathrm{H}$ & -3.540490 & 0.396012 & 2.660318 \\
\hline $\mathrm{H}$ & 2.460962 & -5.671768 & 0.390495 & $\mathrm{H}$ & -5.297008 & 0.612528 & 2.711340 \\
\hline $\mathrm{C}$ & -1.612377 & 1.339168 & 0.287838 & $\mathrm{H}$ & -4.591078 & -0.968277 & 3.036559 \\
\hline $\mathrm{C}$ & -2.355460 & 3.575197 & 0.420021 & $\mathrm{C}$ & -6.519097 & -2.835565 & -1.267839 \\
\hline $\mathrm{C}$ & -3.538740 & 2.649959 & 0.653778 & $\mathrm{H}$ & -7.307513 & -3.028036 & -0.535103 \\
\hline $\mathrm{H}$ & -2.211927 & 4.306988 & 1.221705 & $\mathrm{H}$ & -6.995087 & -2.611078 & -2.227056 \\
\hline $\mathrm{H}$ & -2.423106 & 4.129334 & -0.528188 & $\mathrm{H}$ & -5.968058 & -3.773828 & -1.400742 \\
\hline $\mathrm{H}$ & -3.920525 & 2.698553 & 1.682837 & $\mathrm{C}$ & 0.759627 & 0.412943 & -1.059882 \\
\hline $\mathrm{H}$ & -4.377008 & 2.836035 & -0.024543 & $\mathrm{H}$ & 0.302845 & 0.449590 & -2.058618 \\
\hline $\mathrm{N}$ & -1.226545 & 2.638997 & 0.378528 & $\mathrm{C}$ & 2.142655 & 0.831070 & -1.097216 \\
\hline $\mathrm{N}$ & -2.966063 & 1.321352 & 0.391921 & $\mathrm{C}$ & 2.750363 & 1.012398 & -2.357908 \\
\hline $\mathrm{C}$ & 0.093798 & 3.172965 & 0.350030 & $\mathrm{C}$ & 2.927315 & 1.046853 & 0.053695 \\
\hline $\mathrm{C}$ & 0.630033 & 3.611497 & -0.867713 & $\mathrm{C}$ & 4.079425 & 1.394714 & -2.465334 \\
\hline $\mathrm{C}$ & 0.784297 & 3.346747 & 1.563032 & $\mathrm{H}$ & 2.155853 & 0.820998 & -3.248899 \\
\hline $\mathrm{C}$ & 1.889485 & 4.215597 & -0.856252 & $\mathrm{C}$ & 4.255353 & 1.431582 & -0.055288 \\
\hline $\mathrm{C}$ & 2.034808 & 3.958994 & 1.522393 & $\mathrm{H}$ & 2.462979 & 0.932579 & 1.030762 \\
\hline $\mathrm{C}$ & 2.604810 & 4.400081 & 0.323145 & $\mathrm{C}$ & 4.834168 & 1.611140 & -1.312228 \\
\hline $\mathrm{H}$ & 2.328838 & 4.532468 & -1.801545 & $\mathrm{H}$ & 4.531469 & 1.522948 & -3.444677 \\
\hline $\mathrm{H}$ & 2.582600 & 4.092926 & 2.455012 & $\mathrm{H}$ & 4.841393 & 1.606725 & 0.843534 \\
\hline $\mathrm{C}$ & -3.836159 & 0.258796 & -0.004664 & $\mathrm{H}$ & 5.874058 & 1.917799 & -1.391552 \\
\hline $\mathrm{C}$ & -3.991479 & 0.005984 & -1.376288 & $\mathrm{O}$ & -1.814646 & -1.307933 & 1.683197 \\
\hline $\mathrm{C}$ & -4.588666 & -0.419738 & 0.959946 & $\mathrm{O}$ & -1.456418 & -1.457512 & -1.376772 \\
\hline $\mathrm{C}$ & -4.874466 & -0.997732 & -1.762782 & $\mathrm{H}$ & 0.572281 & 0.453460 & 1.180203 \\
\hline $\mathrm{C}$ & -5.461927 & -1.417384 & 0.523148 & $\mathrm{Br}$ & 4.599631 & -3.534966 & 0.435950 \\
\hline $\mathrm{C}$ & -5.608397 & -1.730268 & -0.827700 & $\mathrm{C}$ & -0.881919 & -0.745877 & 2.280124 \\
\hline $\mathrm{H}$ & -4.989042 & -1.215250 & -2.824110 & $\mathrm{O}$ & -0.412482 & -0.524943 & 3.364147 \\
\hline $\mathrm{H}$ & -6.042802 & -1.966096 & 1.263691 & $\mathrm{C}$ & -0.783667 & -1.940418 & -2.356420 \\
\hline $\mathrm{C}$ & 0.200485 & 2.870681 & 2.855637 & $\mathrm{H}$ & -1.424609 & -2.509970 & -3.074215 \\
\hline $\mathrm{H}$ & 0.251633 & 1.778824 & 2.945438 & $\mathrm{O}$ & 0.428405 & -1.849185 & -2.586273 \\
\hline $\mathrm{H}$ & 0.730042 & 3.301284 & 3.708871 & & & & \\
\hline $\mathrm{H}$ & -0.859994 & 3.130865 & 2.948426 & 5 II & & & \\
\hline $\mathrm{C}$ & -0.102633 & 3.428325 & -2.161408 & $\mathrm{Ru}$ & -0.722482 & 0.794911 & 0.287793 \\
\hline $\mathrm{H}$ & -0.645302 & 4.335851 & -2.453690 & $\mathrm{C}$ & -0.594387 & -1.162779 & 0.028090 \\
\hline $\mathrm{H}$ & 0.596099 & 3.201920 & -2.972977 & $\mathrm{C}$ & -0.143322 & -3.456175 & -0.146363 \\
\hline $\mathrm{H}$ & -0.831147 & 2.614340 & -2.108232 & $\mathrm{C}$ & -1.586392 & -3.210967 & -0.570275 \\
\hline
\end{tabular}




\begin{tabular}{|c|c|c|c|c|c|c|c|}
\hline $\mathrm{H}$ & 0.424549 & -4.056469 & -0.863410 & $\mathrm{H}$ & -7.249610 & 1.082442 & 0.376134 \\
\hline $\mathrm{H}$ & -0.082315 & -3.950847 & 0.835722 & $\mathrm{H}$ & -6.238739 & 2.411435 & -0.177368 \\
\hline $\mathrm{H}$ & -1.711515 & -3.295435 & -1.661699 & $\mathrm{C}$ & 1.007996 & 1.179241 & 0.712917 \\
\hline $\mathrm{H}$ & -2.299709 & -3.889143 & -0.091866 & $\mathrm{H}$ & 1.199491 & 1.097521 & 1.805962 \\
\hline $\mathrm{N}$ & 0.391299 & -2.091953 & -0.067418 & $\mathrm{C}$ & 2.174893 & 1.647343 & -0.015730 \\
\hline $\mathrm{N}$ & -1.778552 & -1.833847 & -0.123609 & $\mathrm{C}$ & 3.382784 & 1.835811 & 0.679732 \\
\hline $\mathrm{C}$ & 1.782293 & -1.827090 & 0.070096 & $\mathrm{C}$ & 2.162482 & 1.901490 & -1.399801 \\
\hline $\mathrm{C}$ & 2.381752 & -1.872748 & 1.335863 & $\mathrm{C}$ & 4.536866 & 2.238647 & 0.018991 \\
\hline $\mathrm{C}$ & 2.513005 & -1.499305 & -1.079562 & $\mathrm{H}$ & 3.401212 & 1.651795 & 1.752429 \\
\hline $\mathrm{C}$ & 3.746451 & -1.599412 & 1.425124 & $\mathrm{C}$ & 3.314664 & 2.299649 & -2.060052 \\
\hline $\mathrm{C}$ & 3.873799 & -1.234594 & -0.943614 & $\mathrm{H}$ & 1.225493 & 1.779810 & -1.937341 \\
\hline $\mathrm{C}$ & 4.508806 & -1.284745 & 0.298476 & $\mathrm{C}$ & 4.508917 & 2.464209 & -1.355162 \\
\hline $\mathrm{H}$ & 4.222614 & -1.611690 & 2.405235 & $\mathrm{H}$ & 5.460814 & 2.375499 & 0.575778 \\
\hline $\mathrm{H}$ & 4.444680 & -0.943857 & -1.824279 & $\mathrm{H}$ & 3.285789 & 2.489867 & -3.130067 \\
\hline $\mathrm{C}$ & -2.988937 & -1.082626 & -0.217560 & $\mathrm{H}$ & 5.410550 & 2.777425 & -1.875601 \\
\hline $\mathrm{C}$ & -3.683574 & -0.797076 & 0.971030 & $\mathrm{O}$ & -1.707839 & 2.582041 & 0.572790 \\
\hline $\mathrm{C}$ & -3.425906 & -0.591051 & -1.458791 & $\mathrm{H}$ & -0.562366 & 0.910986 & -1.264884 \\
\hline $\mathrm{C}$ & -4.834978 & -0.016765 & 0.892843 & $\mathrm{C}$ & -1.130922 & 3.619697 & 1.105139 \\
\hline $\mathrm{C}$ & -4.584456 & 0.186543 & -1.484699 & $\mathrm{H}$ & -1.840236 & 4.470405 & 1.201218 \\
\hline $\mathrm{C}$ & -5.297797 & 0.487876 & -0.324549 & $\mathrm{O}$ & 0.027020 & 3.736105 & 1.484485 \\
\hline $\mathrm{H}$ & -5.378240 & 0.214003 & 1.807836 & & & & \\
\hline $\mathrm{H}$ & -4.929172 & 0.581610 & -2.439117 & \multicolumn{2}{|c|}{ TSII-1 $_{1-2 \mathrm{Br}}$} & & \\
\hline $\mathrm{C}$ & 1.814721 & -1.368179 & -2.395794 & $\mathrm{Ru}$ & -0.122576 & 0.127135 & 0.134487 \\
\hline $\mathrm{H}$ & 1.039677 & -0.592926 & -2.336350 & $\mathrm{C}$ & 2.402562 & 1.710193 & 0.111132 \\
\hline $\mathrm{H}$ & 2.512419 & -1.095127 & -3.191286 & $\mathrm{C}$ & 0.679719 & 3.257543 & 0.228081 \\
\hline $\mathrm{H}$ & 1.306062 & -2.294623 & -2.689364 & $\mathrm{C}$ & 3.343380 & 2.728003 & 0.103096 \\
\hline $\mathrm{C}$ & 1.564850 & -2.165907 & 2.556049 & $\mathrm{H}$ & 2.682557 & 0.661822 & 0.054304 \\
\hline $\mathrm{H}$ & 1.271707 & -3.221282 & 2.612653 & $\mathrm{C}$ & 1.575299 & 4.317604 & 0.211055 \\
\hline $\mathrm{H}$ & 2.119999 & -1.935050 & 3.468271 & $\mathrm{H}$ & -0.394693 & 3.411879 & 0.285842 \\
\hline $\mathrm{H}$ & 0.637928 & -1.580449 & 2.563815 & $\mathrm{~N}$ & 1.087805 & 1.982480 & 0.175032 \\
\hline $\mathrm{C}$ & 5.971631 & -0.989298 & 0.421617 & $\mathrm{C}$ & 2.940249 & 4.055752 & 0.148997 \\
\hline $\mathrm{H}$ & 6.575922 & -1.898707 & 0.322557 & $\mathrm{H}$ & 3.668433 & 4.859406 & 0.135243 \\
\hline $\mathrm{H}$ & 6.304215 & -0.292945 & -0.353643 & $\mathrm{C}$ & -0.728280 & -1.818363 & 0.146687 \\
\hline $\mathrm{H}$ & 6.211899 & -0.549612 & 1.394457 & $\mathrm{C}$ & -2.011284 & -3.778027 & 0.339004 \\
\hline $\mathrm{C}$ & -3.177057 & -1.304944 & 2.286187 & $\mathrm{C}$ & -0.549493 & -4.157657 & 0.163273 \\
\hline $\mathrm{H}$ & -3.881632 & -1.087353 & 3.091825 & $\mathrm{H}$ & -2.379760 & -3.973700 & 1.356793 \\
\hline $\mathrm{H}$ & -2.996528 & -2.385130 & 2.266745 & $\mathrm{H}$ & -2.683739 & -4.282074 & -0.362355 \\
\hline $\mathrm{H}$ & -2.217854 & -0.839424 & 2.546140 & $\mathrm{H}$ & -0.178169 & -4.841785 & 0.932213 \\
\hline $\mathrm{C}$ & -2.673906 & -0.894569 & -2.717499 & $\mathrm{H}$ & -0.343557 & -4.604598 & -0.819856 \\
\hline $\mathrm{H}$ & -1.591607 & -0.874716 & -2.551371 & $\mathrm{~N}$ & -1.985711 & -2.330998 & 0.096314 \\
\hline $\mathrm{H}$ & -2.929381 & -1.885468 & -3.113708 & $\mathrm{~N}$ & 0.127892 & -2.858931 & 0.257968 \\
\hline $\mathrm{H}$ & -2.906716 & -0.165501 & -3.496917 & $\mathrm{C}$ & -3.196611 & -1.581037 & 0.131727 \\
\hline $\mathrm{C}$ & -6.508199 & 1.368043 & -0.375344 & $\mathrm{C}$ & -3.919485 & -1.450977 & -1.067719 \\
\hline $\mathrm{H}$ & -6.989337 & 1.340387 & -1.356559 & $\mathrm{C}$ & -3.649871 & -1.005693 & 1.326828 \\
\hline
\end{tabular}




\begin{tabular}{|c|c|c|c|}
\hline $\mathrm{C}$ & 86 & 783 & -1.053991 \\
\hline $\mathrm{C}$ & 824768 & .251046 & 1282071 \\
\hline & 557968 & 565 & 9136 \\
\hline & $61+2$ & 740 & -1.984144 \\
\hline & -5.169095 & 2 & 50 \\
\hline & 1.553656 & 252 & 129 \\
\hline $\mathrm{C}$ & 2.115923 & -2.744520 & -1.171948 \\
\hline $\mathrm{C}$ & 198 & 440 & 9566 \\
\hline $\mathrm{C}$ & 1 & 9 & 5 \\
\hline $\mathrm{C}$ & 968 & -2.686689 & 853 \\
\hline $\mathrm{C}$ & & & 586 \\
\hline $\mathrm{H}$ & 71 & 2 & 57 \\
\hline $\mathrm{H}$ & 4.357785 & 5460 & 5599 \\
\hline $\mathrm{C}$ & -2.932669 & -1.173822 & 17 \\
\hline $\mathrm{H}$ & 8 & 6 & 84 \\
\hline$U$ & $-35 t r t$ & & 0882 \\
\hline $\mathrm{H}$ & -2.004078 & 55 & 04 \\
\hline $\mathrm{C}$ & 35 & -2.0 & 2 \\
\hline $\mathrm{H}$ & -3.421773 & -3.157271 & 3730 \\
\hline $\mathrm{H}$ & -4.0 & 37 & 30 \\
\hline $\mathrm{H}$ & -2.366543 & 4263 & -2 \\
\hline $\mathrm{C}$ & -6.8 & 30 & \\
\hline $\mathrm{H}$ & -7 & 0.104053 & \\
\hline 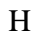 & -6.811786 & 1.480756 & \\
\hline $\mathrm{H}$ & 0 & 2 & 28 \\
\hline $\mathrm{C}$ & 5 & 0 & 8 \\
\hline $\mathrm{H}$ & 790 & 7705 & 2655 \\
\hline $\mathrm{H}$ & 1.404662 & -3.8 & 57 \\
\hline $\mathrm{H}$ & 01 & -2 . & 52 \\
\hline C & 1.767329 & -3.0 & $8 / 5$ \\
\hline$H_{-}$ & 710700 & 2748610 & 060677 \\
\hline $\mathrm{H}$ & 332486 & -4.109191 & 07584 \\
\hline $\mathrm{H}$ & 2.509490 & -2.480 & 91 \\
\hline $\mathrm{C}$ & 7 & -2 . & 9 \\
\hline $\mathrm{H}$ & 6.267994 & 6686 & 76 \\
\hline$-T_{-1}$ & 6.284160 & -3.314973 & 0612007 \\
\hline - & .047856 & -1.622777 & 1.027667 \\
\hline C & 176 & 5179 & 61 \\
\hline $\mathrm{H}$ & -1.707352 & 0.267502 & -1.893099 \\
\hline $\mathrm{C}$ & -2.58 & 163 & 2723 \\
\hline $\mathrm{G}$ & -3.4 & 2113 & 0243 \\
\hline $\mathrm{C}$ & -2.731501 & 2.324257 & 0.657872 \\
\hline $\mathrm{C}$ & -4.421509 & 3.072468 & -1.437886 \\
\hline 1 & -3.345239 & 1.622850 & -2.61267 \\
\hline
\end{tabular}

$\begin{array}{rrrr}\mathrm{C} & -3.712751 & 3.284712 & 0.862057 \\ \mathrm{H} & -2.073059 & 2.032855 & 1.475944 \\ \mathrm{C} & -4.557856 & 3.663477 & -0.182439 \\ \mathrm{H} & -5.078182 & 3.363878 & -2.253379 \\ \mathrm{H} & -3.821871 & 3.741897 & 1.841715 \\ \mathrm{H} & -5.323721 & 4.416590 & -0.016603 \\ \mathrm{O} & 1.104918 & -0.256473 & 2.145241 \\ \mathrm{O} & 1.031241 & -0.194107 & -1.621723 \\ \mathrm{H} & -1.009795 & 0.265405 & 1.525566 \\ \mathrm{H} & 1.208544 & 5.337749 & 0.248073 \\ \mathrm{Br} & 5.214723 & 2.268141 & 0.008249 \\ \mathrm{C} & 0.163287 & 0.531569 & 2.336107 \\ \mathrm{O} & -0.218421 & 1.366146 & 3.126888 \\ \mathrm{C} & 0.918621 & 0.477892 & -2.722215 \\ \mathrm{H} & 0.173845 & 1.321703 & -2.674538 \\ \mathrm{O} & 1.536172 & 0.262060 & -3.755893\end{array}$

\section{TSII-1'1-2Br}

$\begin{array}{rrrr}\mathrm{Ru} & -0.381479 & -0.133815 & 0.086421 \\ \mathrm{C} & -0.434571 & -3.173252 & 0.045585 \\ \mathrm{C} & 1.776783 & -2.499756 & 0.168915 \\ \mathrm{C} & -0.069918 & -4.511637 & 0.045986 \\ \mathrm{H} & -1.471871 & -2.856996 & -0.019860 \\ \mathrm{C} & 2.199854 & -3.820321 & 0.162397 \\ \mathrm{H} & 2.482210 & -1.676970 & 0.223167 \\ \mathrm{H} & -0.834895 & -5.278715 & -0.005554 \\ \mathrm{~N} & 0.476267 & -2.184456 & 0.106966 \\ \mathrm{C} & 1.276882 & -4.854711 & 0.101276 \\ \mathrm{H} & 1.597957 & -5.890646 & 0.096607 \\ \mathrm{C} & -1.544542 & 1.538118 & 0.124473 \\ \mathrm{C} & -2.278789 & 3.769090 & 0.299619 \\ \mathrm{C} & -3.485288 & 2.860947 & 0.126446 \\ \mathrm{H} & -2.213688 & 4.197760 & 1.310489 \\ \mathrm{H} & -2.245833 & 4.595792 & -0.417372 \\ \mathrm{H} & -4.250259 & 2.995745 & 0.897462 \\ \mathrm{H} & -3.967941 & 2.977119 & -0.854290 \\ \mathrm{~N} & -1.158918 & 2.844444 & 0.083953 \\ \mathrm{~N} & -2.891139 & 1.522761 & 0.220734 \\ \mathrm{C} & 0.177689 & 3.326560 & 0.199869 \\ \mathrm{C} & 0.791596 & 3.863975 & -0.943542 \\ \mathrm{C} & 0.841643 & 3.289176 & 1.437923 \\ \mathrm{C} & 2.111356 & 4.303315 & -0.841942 \\ \mathrm{C} & 2.162517 & 3.735161 & 1.483333 \\ \mathrm{C} & 2.820778 & 4.228815 & 0.355351 \\ & & & \end{array}$




\begin{tabular}{|c|c|c|c|}
\hline $\mathrm{H}$ & 607940 & 209 & -1 \\
\hline & 690303 & 678774 & $30^{2}=2+2$ \\
\hline & 3.739144 & 371705 & 0.119514 \\
\hline $\mathrm{C}$ & -4.101201 & .106384 & 1.151135 \\
\hline & -4.241488 & .199675 & 1.296997 \\
\hline $\mathrm{C}$ & -4.9 & & 1089 \\
\hline 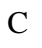 & -5.048625 & 760 & 1.180297 \\
\hline $\mathrm{C}$ & -5.38 & 110 & 32 \\
\hline $\mathrm{H}$ & -5.1 & 742 & 44 \\
\hline $\mathrm{H}$ & -5.4 & 97 & 20 \\
\hline $\mathrm{C}$ & 0.186361 & 268 & 97607 \\
\hline $\mathrm{H}$ & 0.7 & 134 & 40 \\
\hline $\mathrm{H}$ & 0.083044 & 6168 & 361 \\
\hline te $>$ & -0.807418 & .385181 & 27 \\
\hline $\mathrm{C}$ & 4 & 402 & 96 \\
\hline $\mathrm{H}$ & -0.567462 & 576 & -2. \\
\hline $\mathrm{H}$ & 441 & 8983 & -3 \\
\hline $\mathrm{H}$ & -0.60 & 49 & 39 \\
\hline s & 4.254134 & 4.653188 & 81 \\
\hline $\mathrm{H}$ & 4.3 & 11 & 1.149081 \\
\hline 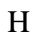 & 4.888636 & 4 & 0.766948 \\
\hline $\mathrm{H}$ & 4.629922 & 986749 & 304 \\
\hline $\mathrm{C}$ & 6 & 9 & 7 \\
\hline $\mathrm{H}$ & -3.377197 & 7138 & -3.1 \\
\hline $\mathrm{H}$ & -4.502007 & 21 & 49 \\
\hline M & -2.821187 & .230432 & -2.272766 \\
\hline $\mathrm{C}$ & -3.98 & 099 & \\
\hline $\mathbf{I}$ & -3.144779 & .095084 & 2.634448 \\
\hline $\mathrm{H}$ & -4.868802 & 29 & 3 \\
\hline $\mathrm{H}$ & -3.746354 & 35 & 188 \\
\hline$C$ & -6.245744 & 3004076 & 0162660 \\
\hline $\mathrm{H}$ & -7.220018 & -2.8 & -0.607051 \\
\hline $\mathrm{H}$ & -5.78807 & -5.0078 & מעוד \\
\hline $\mathrm{H}$ & -6.4302 & 14 & 74 \\
\hline $\mathrm{C}$ & 0.866966 & 688710 & -1.028359 \\
\hline $\mathbf{T}$ & 0.510054 & 115180 & $1+00565$ \\
\hline $\mathrm{C}$ & 2.301288 & 1650 & -0.872991 \\
\hline $\mathrm{C}$ & 3.072723 & & -1.994 \\
\hline $\mathrm{C}$ & 065405 & 0404005 & 026020 \\
\hline $\mathrm{C}$ & 447393 & .391412 & -1.900073 \\
\hline 11 & 2.565831 & 1.379048 & -2.946824 \\
\hline $\mathrm{C}$ & 98 & .866040 & 0.455 \\
\hline $\mathrm{H}$ & 2.382983 & 0.443061 & 1.245933 \\
\hline $\mathrm{C}$ & .085456 & 208608 & 0.073 \\
\hline
\end{tabular}

$\begin{array}{rrrc}\mathrm{H} & 5.024298 & 1.665493 & -2.778852 \\ \mathrm{H} & 4.832588 & 0.738073 & 1.415712 \\ \mathrm{H} & 6.161243 & 1.341589 & -0.594206 \\ \mathrm{O} & -1.341164 & -0.840364 & 2.128452 \\ \mathrm{O} & -1.436558 & -0.920134 & -1.565389 \\ \mathrm{H} & 0.329319 & 0.469277 & 1.481898 \\ \mathrm{Br} & 4.096683 & -4.186286 & 0.242286 \\ \mathrm{C} & -0.145325 & -0.549514 & 2.313438 \\ \mathrm{O} & 0.750312 & -0.738338 & 3.107105 \\ \mathrm{C} & -0.909593 & -1.226918 & -2.708832 \\ \mathrm{H} & 0.194874 & -1.019897 & -2.777291 \\ \mathrm{O} & -1.509535 & -1.694909 & -3.665816\end{array}$

\section{II}

$\begin{array}{llll}\mathrm{Ru} & 0.499019 & 0.291112 & 0.233753\end{array}$

$\begin{array}{llll}\text { C } & 0.024156 & -1.591769 & 0.266113\end{array}$

$\begin{array}{llll}\text { C } & -0.707537 & -3.819676 & 0.194480\end{array}$

$\begin{array}{llll}\text { C } & 0.793113 & -3.791346 & -0.089710\end{array}$

$\mathrm{H} \quad-0.937938 \quad-4.266987 \quad 1.172490$

H $\quad-1.280821 \quad-4.360291 \quad-0.565289$

$\begin{array}{llll}\mathrm{H} & 1.358609 & -4.497601 & 0.526522\end{array}$

$\mathrm{H} \quad 1.017085 \quad-4.003904 \quad-1.145661$

$\begin{array}{llll}\mathrm{N} & -1.064568 & -2.394769 & 0.190146\end{array}$

$\begin{array}{llll}\mathrm{N} & 1.125345 & -2.409901 & 0.247830\end{array}$

$\begin{array}{llll}\text { C } & -2.416890 & -1.952568 & 0.249766\end{array}$

$\begin{array}{llll}\text { C } & -3.144338 & -1.794000 & -0.938857\end{array}$

$\begin{array}{llll}\text { C } & -2.980386 & -1.668089 & 1.503598\end{array}$

$\begin{array}{llll}\text { C } & -4.438669 & -1.275789 & -0.845554\end{array}$

$\begin{array}{llll}\text { C } & -4.278932 & -1.166459 & 1.545905\end{array}$

$\begin{array}{llll}\text { C } & -5.016577 & -0.943964 & 0.379220\end{array}$

$\begin{array}{llll}\mathrm{H} & -5.006640 & -1.121391 & -1.761982\end{array}$

$\mathrm{H} \quad-4.720904 \quad-0.926207 \quad 2.512511$

$\begin{array}{llll}\text { C } & 2.398375 & -1.781033 & 0.036793\end{array}$

$\begin{array}{llll}\text { C } & 2.807399 & -1.427925 & -1.263849\end{array}$

$\begin{array}{llll}\text { C } & 3.189562 & -1.477006 & 1.164547\end{array}$

$\begin{array}{llll}\text { C } & 4.003092 & -0.714684 & -1.400671\end{array}$

$\begin{array}{llll}\text { C } & 4.364735 & -0.755639 & 0.970787\end{array}$

$\begin{array}{llll}\text { C } & 4.786883 & -0.358849 & -0.303215\end{array}$

$\mathrm{H} \quad 4.321161 \quad-0.422898 \quad-2.400747$

$\mathrm{H} \quad 4.971318 \quad-0.497485 \quad 1.837412$

$\begin{array}{llll}\text { C } & -2.168283 & -1.835869 & 2.750781\end{array}$

$\mathrm{H} \quad-1.289813 \quad-1.176729 \quad 2.742863$

$\mathrm{H} \quad-2.758071 \quad-1.606570 \quad 3.641174$

$\mathrm{H} \quad-1.784653 \quad-2.856954 \quad 2.857910$ 


\begin{tabular}{|c|c|c|c|}
\hline & 263 & 186661 & -2.270867 \\
\hline & -2.989592 & .152833 & -2503511 \\
\hline & -2.828844 & -1.457760 & -3.046781 \\
\hline & -1.493589 & .274685 & -2.252036 \\
\hline & 6.379798 & 326815 & 0.441100 \\
\hline & -7.042699 & -0.723630 & -0.332753 \\
\hline & -6.855834 & -0.487713 & 1.412059 \\
\hline & -6.32 & 522 & 361 \\
\hline C & 2.031302 & 5031 & -2 \\
\hline $\mathrm{H}$ & 2.024850 & -1.029033 & -3.227816 \\
\hline $\mathrm{H}$ & 2.463846 & -2.724843 & 36452 \\
\hline $\mathrm{H}$ & 0.982046 & 1673 & -2 \\
\hline$C_{-}+2$ & 2.786071 & -1.942232 & 2.528968 \\
\hline $\mathrm{H}$ & 1.724632 & -1.757959 & 2.717474 \\
\hline 1 & 2.951118 & -3.0 & 1331 \\
\hline $\mathrm{H}$ & 3.369476 & -1.438941 & 3304126 \\
\hline C & 6.029790 & 4648 & -0.479313 \\
\hline $\mathrm{H}$ & 6.530198 & 823 & 84 \\
\hline $\mathrm{H}$ & 5.786520 & 1.523074 & -0.490474 \\
\hline 1 & 740978 & 78 & 56 \\
\hline $\mathrm{C}$ & 1168653 & 0976949 & 0633326 \\
\hline $\mathrm{H}$ & -1.833091 & 0.468350 & 6095 \\
\hline $\mathrm{C}$ & 3 & 2 & 3 \\
\hline $\mathrm{C}$ & -3.124126 & 2.459618 & 0.496853 \\
\hline $\mathrm{C}$ & -1.164589 & 3.068317 & 55 \\
\hline C & 37 & 4 & $77>->$ \\
\hline $\mathrm{H}$ & -3.620200 & 768961 & 8254 \\
\hline $\mathrm{C}$ & -1.837135 & 4.181819 & -1.267230 \\
\hline $\mathrm{H}$ & -0.152463 & 273 & 98 \\
\hline C & -3.151482 & 437690 & -0.873595 \\
\hline $\mathrm{H}$ & 4020260 & 3.767119 & (1) 212000 \\
\hline $\mathrm{H}$ & -1.339815 & 4.854034 & -1.960910 \\
\hline $\mathrm{H}$ & -3.6737 & 9 & -1.258057 \\
\hline $\mathrm{O}$ & .581961 & .640068 & -1.873449 \\
\hline $\mathrm{H}$ & 0.766623 & 2929 & 1.855480 \\
\hline $\mathrm{O}$ & 1005520 & 2.118950 & 0200061 \\
\hline $\mathrm{H}$ & 1.748682 & 1.223706 & 2.644678 \\
\hline $\mathrm{O}$ & 2.191131 & 6 & 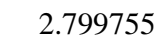 \\
\hline $\mathrm{H}$ & 2.257118 & 2.322764 & 1.115762 \\
\hline $\mathrm{C}$ & 1.193129 & 2.965301 & 3.299661 \\
\hline $\mathrm{H}$ & 0.325132 & 3.024462 & 2.627831 \\
\hline $\mathrm{H}$ & 0.848243 & 2.658375 & 4.29373 \\
\hline $\mathrm{H}$ & 1.644806 & 3.955243 & 3.380243 \\
\hline $\mathrm{C}$ & 0.409906 & .311384 & $2.051 / 51$ \\
\hline
\end{tabular}

$$
\begin{array}{lrrr}
\mathrm{H} & -1.393009 & 0.237299 & -2.116245 \\
\mathrm{O} & -0.339609 & 0.086848 & -3.850211 \\
\mathrm{C} & 2.810572 & 2.396883 & -0.777214 \\
\mathrm{H} & 2.415762 & 2.039146 & -1.740429 \\
\mathrm{O} & 3.857379 & 2.953626 & -0.573510
\end{array}
$$

\section{TSII3-4}

$\begin{array}{llll}\mathrm{Ru} & 0.554285 & 0.382153 & 0.273559\end{array}$

$\begin{array}{llll}\text { C } & 0.047531 & -1.524735 & 0.127545\end{array}$

$\begin{array}{llll}\text { C } & -0.718671 & -3.725487 & -0.118799\end{array}$

$\begin{array}{llll}\text { C } & 0.776616 & -3.695179 & -0.426385\end{array}$

$\mathrm{H} \quad-0.941384 \quad-4.244160 \quad 0.824948$

H $\quad-1.315077 \quad-4.193762 \quad-0.908000$

$\mathrm{H} \quad 1.346443 \quad-4.442180 \quad 0.135105$

H $\quad 0.985702 \quad-3.840106 \quad-1.496298$

$\begin{array}{llll}\mathrm{N} & -1.054905 & -2.298096 & 0.002018\end{array}$

$\begin{array}{llll}\mathrm{N} & 1.131394 & -2.339377 & -0.010501\end{array}$

$\begin{array}{llll}\text { C } & -2.400061 & -1.873011 & 0.198789\end{array}$

$\begin{array}{llll}\text { C } & -3.247894 & -1.730996 & -0.909186\end{array}$

$\begin{array}{llll}\text { C } & -2.852167 & -1.642620 & 1.508927\end{array}$

$\begin{array}{llll}\text { C } & -4.552310 & -1.285486 & -0.677186\end{array}$

$\begin{array}{llll}\text { C } & -4.162507 & -1.204958 & 1.687847\end{array}$

$\begin{array}{llll}\text { C } & -5.022791 & -1.004848 & 0.604186\end{array}$

$\mathrm{H} \quad-5.216313 \quad-1.150871 \quad-1.529905$

$\mathrm{H} \quad-4.521320 \quad-1.012801 \quad 2.698542$

$\begin{array}{llll}\text { C } & 2.427181 & -1.735304 & -0.135238\end{array}$

$\begin{array}{llll}\text { C } & 2.879069 & -1.277899 & -1.387345\end{array}$

$\begin{array}{llll}\text { C } & 3.199627 & -1.572271 & 1.032491\end{array}$

$\begin{array}{llll}\text { C } & 4.093126 & -0.585368 & -1.425891\end{array}$

$\begin{array}{llll}\text { C } & 4.398832 & -0.871503 & 0.935499\end{array}$

$\begin{array}{llll}\text { C } & 4.854716 & -0.353067 & -0.281277\end{array}$

$\begin{array}{llll}\mathrm{H} & 4.439290 & -0.197816 & -2.382877\end{array}$

$\begin{array}{llll}\mathrm{H} & 4.990409 & -0.714287 & 1.836178\end{array}$

$\begin{array}{llll}\text { C } & -1.934008 & -1.842004 & 2.675315\end{array}$

$\begin{array}{llll}\mathrm{H} & -1.075887 & -1.158984 & 2.632897\end{array}$

$\begin{array}{llll}\mathrm{H} & -2.456060 & -1.677618 & 3.620838\end{array}$

$\mathrm{H} \quad-1.515213 \quad-2.855173 \quad 2.697251$

$\begin{array}{llll}\text { C } & -2.808196 & -2.065063 & -2.302481\end{array}$

$\mathrm{H} \quad-3.302100 \quad-2.978249 \quad-2.655463$

$\mathrm{H} \quad-3.062158 \quad-1.268114 \quad-3.006582$

$\begin{array}{llll}\mathrm{H} & -1.728986 & -2.211907 & -2.378330\end{array}$

$\begin{array}{llll}\text { C } & -6.406903 & -0.472343 & 0.814274\end{array}$

$\begin{array}{llll}\mathrm{H} & -7.109581 & -0.859826 & 0.071832\end{array}$

$\begin{array}{llll}\mathrm{H} & -6.791191 & -0.719166 & 1.807718\end{array}$ 


\begin{tabular}{|c|c|c|c|}
\hline $\mathrm{H}$ & -6.425093 & 0.620972 & 0.726226 \\
\hline c & 2.134268 & -1.548563 & -2.656880 \\
\hline & 2.122474 & -0.672622 & -3.308017 \\
\hline $\mathrm{H}$ & 2.605111 & -2.374159 & -3.204895 \\
\hline $\mathrm{H}$ & 1.088487 & -1.810073 & -2.486787 \\
\hline C & 2.751672 & -2.152899 & 2.338338 \\
\hline & 1.692884 & -1.955060 & 2.529879 \\
\hline $\mathrm{H}$ & 2.881567 & -3.241994 & 2.352617 \\
\hline $\mathrm{H}$ & 3.333075 & -1.743278 & 3.168122 \\
\hline $\mathrm{C}$ & 6.096321 & 0.476618 & -0.347683 \\
\hline $\mathrm{H}$ & 6.667480 & 0.279810 & -1.259403 \\
\hline $\mathrm{H}$ & 5.825167 & 1.538824 & -0.355963 \\
\hline $\mathrm{H}$ & 6.748543 & 0.301952 & 0.511884 \\
\hline C & -1.099308 & 1.061249 & 0.738953 \\
\hline $\mathrm{H}$ & -1.667642 & 0.632960 & 1.581212 \\
\hline $\mathrm{C}$ & -1.808814 & 2.172134 & 0.129623 \\
\hline $\mathrm{C}$ & -3.170162 & 2.352563 & 0.452781 \\
\hline $\mathrm{C}$ & -1.206932 & 3.075134 & -0.771020 \\
\hline $\mathrm{C}$ & -3.911037 & 3.373907 & -0.123674 \\
\hline $\mathrm{H}$ & -3.634095 & 1.657295 & 1.151616 \\
\hline $\mathrm{C}$ & -1.948764 & 4.104548 & -1.331154 \\
\hline $\mathrm{H}$ & -0.156793 & 2.948236 & -1.015669 \\
\hline C & -3.300312 & 4.253217 & -1.017380 \\
\hline $\mathrm{H}$ & -4.962482 & 3.492773 & 0.125185 \\
\hline $\mathrm{H}$ & -1.474216 & 4.795838 & -2.021618 \\
\hline $\mathrm{H}$ & -3.875992 & 5.058267 & -1.466186 \\
\hline $\mathrm{O}$ & 0.480483 & 0.729374 & -1.791974 \\
\hline $\mathrm{H}$ & 0.811056 & 0.039119 & 1.944853 \\
\hline $\mathrm{O}$ & 1.957399 & 2.058756 & 0.376539 \\
\hline $\mathrm{H}$ & 1.444982 & 0.826728 & 2.357557 \\
\hline $\mathrm{O}$ & 2.118354 & 1.694422 & 2.753852 \\
\hline $\mathrm{H}$ & 2.292236 & 2.033149 & 1.749461 \\
\hline C & 1.258724 & 2.659285 & 3.377613 \\
\hline $\mathrm{H}$ & 0.374072 & 2.847899 & 2.757789 \\
\hline $\mathrm{H}$ & 0.955280 & 2.271783 & 4.350753 \\
\hline $\mathrm{H}$ & 1.817330 & 3.586224 & 3.510754 \\
\hline $\mathrm{C}$ & -0.546911 & 0.361561 & -2.505820 \\
\hline $\mathrm{H}$ & -1.466604 & 0.163931 & -1.897830 \\
\hline $\mathrm{O}$ & -0.562087 & 0.221680 & -3.718430 \\
\hline C & 2.827157 & 2.373218 & -0.571129 \\
\hline $\mathrm{H}$ & 2.485573 & 2.056922 & -1.576820 \\
\hline 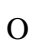 & 3.878917 & 2.948035 & -0.366449 \\
\hline
\end{tabular}

4II

\begin{tabular}{cccc}
$\mathrm{Ru}$ & 0.577559 & 0.413881 & 0.368712 \\
$\mathrm{C}$ & -0.026173 & -1.493445 & 0.037438 \\
$\mathrm{C}$ & -0.883930 & -3.633500 & -0.365887 \\
$\mathrm{C}$ & 0.620580 & -3.645941 & -0.654608 \\
$\mathrm{H}$ & -1.157802 & -4.268467 & 0.486404 \\
$\mathrm{H}$ & -1.490359 & -3.946398 & -1.222304 \\
$\mathrm{H}$ & 1.165787 & -4.381000 & -0.052280 \\
$\mathrm{H}$ & 0.851608 & -3.836118 & -1.710396 \\
$\mathrm{~N}$ & -1.156261 & -2.221241 & -0.039761 \\
$\mathrm{~N}$ & 1.014230 & -2.286356 & -0.282872 \\
$\mathrm{C}$ & -2.476389 & -1.765918 & 0.245867 \\
$\mathrm{C}$ & -3.374839 & -1.545820 & -0.809727 \\
$\mathrm{C}$ & -2.856740 & -1.583488 & 1.586734 \\
$\mathrm{C}$ & -4.651792 & -1.076507 & -0.490842 \\
$\mathrm{C}$ & -4.142381 & -1.116694 & 1.850957 \\
$\mathrm{C}$ & -5.050066 & -0.842577 & 0.823787 \\
$\mathrm{H}$ & -5.352227 & -0.883417 & -1.302058 \\
$\mathrm{H}$ & -4.444114 & -0.965394 & 2.886702 \\
$\mathrm{C}$ & 2.329224 & -1.719442 & -0.380249 \\
$\mathrm{C}$ & 2.794780 & -1.264295 & -1.629705 \\
$\mathrm{C}$ & 3.089578 & -1.564026 & 0.796727 \\
$\mathrm{C}$ & 4.023760 & -0.606525 & -1.661572 \\
$\mathrm{H}$ & 2.508781 & -2.212423 & -3.532937 \\
$\mathrm{C}$ & 4.305944 & -0.888156 & 0.703949 \\
$\mathrm{H}$ & 2.643835 & -2.097632 & 2.125571 \\
$\mathrm{C}$ & 4.782807 & -0.389795 & -0.509146 \\
$\mathrm{H}$ & 4.381938 & -0.219009 & -2.613917 \\
$\mathrm{H}$ & 4.877368 & -0.718453 & 1.614482 \\
$\mathrm{C}$ & -1.907150 & -1.878919 & 2.706171 \\
$\mathrm{H}$ & -1.126045 & -1.113357 & 2.791385 \\
$\mathrm{H}$ & -2.429692 & -1.918829 & 3.664501 \\
$\mathrm{H}$ & -1.386031 & -2.831874 & 2.564866 \\
$\mathrm{C}$ & -3.018687 & -1.819254 & -2.240164 \\
$\mathrm{H}$ & -3.561109 & -2.695718 & -2.613825 \\
$\mathrm{H}$ & -3.283029 & -0.977050 & -2.885387 \\
$\mathrm{H}$ & -1.950544 & -1.991599 & -2.386972 \\
$\mathrm{C}$ & -6.402296 & -0.276306 & 1.127902 \\
$\mathrm{H}$ & -7.135145 & -0.537792 & 0.360319 \\
$\mathrm{H}$ & -6.781761 & -0.621238 & 2.093515 \\
$\mathrm{H}$ & 0.819039 & 1.173372 \\
$\mathrm{H}$ & -1.18861 \\
\hline
\end{tabular}




\begin{tabular}{lrrr}
$\mathrm{H}$ & 3.257362 & -2.955682 & 2.422488 \\
$\mathrm{H}$ & 2.761121 & -1.330014 & 2.896970 \\
$\mathrm{C}$ & 6.036902 & 0.420282 & -0.563329 \\
$\mathrm{H}$ & 6.568954 & 0.284271 & -1.508901 \\
$\mathrm{H}$ & 5.780663 & 1.483031 & -0.479346 \\
$\mathrm{H}$ & 6.716126 & 0.173788 & 0.256706 \\
$\mathrm{C}$ & -1.055918 & 1.150822 & 0.845895 \\
$\mathrm{H}$ & -1.540430 & 0.822650 & 1.781958 \\
$\mathrm{C}$ & -1.836780 & 2.160576 & 0.159594 \\
$\mathrm{C}$ & -3.208819 & 2.261422 & 0.469918 \\
$\mathrm{C}$ & -1.294694 & 3.051243 & -0.789139 \\
$\mathrm{C}$ & -4.019579 & 3.180349 & -0.179089 \\
$\mathrm{H}$ & -3.623989 & 1.584713 & 1.215075 \\
$\mathrm{C}$ & -2.105336 & 3.986128 & -1.415118 \\
$\mathrm{H}$ & -0.234124 & 2.999120 & -1.013337 \\
$\mathrm{C}$ & -3.468343 & 4.046459 & -1.123042 \\
$\mathrm{H}$ & -5.079054 & 3.235014 & 0.057492 \\
$\mathrm{H}$ & -1.674516 & 4.672477 & -2.137854 \\
$\mathrm{H}$ & -4.098615 & 4.776605 & -1.623479 \\
$\mathrm{O}$ & 0.445536 & 0.766015 & -1.664859 \\
$\mathrm{H}$ & 0.586644 & -0.214807 & 1.998216 \\
$\mathrm{O}$ & 2.093255 & 1.863956 & 0.481714 \\
$\mathrm{H}$ & 1.165485 & 0.389384 & 2.058605 \\
$\mathrm{O}$ & 2.976991 & 1.168680 & 2.959693 \\
$\mathrm{H}$ & 2.959322 & 1.499636 & 2.037404 \\
$\mathrm{C}$ & 2.492550 & 2.219469 & 3.762955 \\
$\mathrm{H}$ & 1.472757 & 2.530680 & 3.482571 \\
$\mathrm{H}$ & 2.467032 & 1.868376 & 4.798320 \\
$\mathrm{O}$ & 3.849834 & 2.882905 & -0.446537 \\
$\mathrm{H}$ & 3.135412 & 3.109514 & 3.721705 \\
$\mathrm{C}$ & -0.625819 & 0.447112 & -2.342499 \\
$\mathrm{C}$ & -0.512710 & 0.226287 & -1.699698 \\
& 2.763841 & 2.340065 & -0.549483 \\
\hline
\end{tabular}

\section{TSII-13-4}

$\begin{array}{rrrr}\mathrm{Ru} & 0.575357 & 0.484732 & 0.228526 \\ \mathrm{C} & 0.002623 & -1.333248 & -0.287483 \\ \mathrm{C} & -0.831585 & -3.446075 & -0.833860 \\ \mathrm{C} & 0.616577 & -3.357051 & -1.308701 \\ \mathrm{H} & -0.955922 & -4.137246 & 0.014087 \\ \mathrm{H} & -1.522612 & -3.757513 & -1.623041 \\ \mathrm{H} & 1.231525 & -4.198792 & -0.974627\end{array}$

\begin{tabular}{|c|c|c|c|}
\hline & & & \\
\hline & 12 & 2.069835 & .40 \\
\hline & 047995 & 107573 & \\
\hline & -2.4321 & & \\
\hline & .445 & & \\
\hline & -2.70 & & \\
\hline & -4.72 & & \\
\hline & -3.98 & & \\
\hline & 50 & & \\
\hline & & & \\
\hline & -4.199877 & & \\
\hline & & & \\
\hline & 3.095 & -1 & 1545 \\
\hline & 2.958567 & & \\
\hline & 4.34 & -0.4 & \\
\hline & 4.19 & & \\
\hline & 4.895 & -0.5 & \\
\hline & 4.88 & 24 & \\
\hline & & & \\
\hline & -1.631997 & -2.1 & \\
\hline & -0.87 & -1.3 & \\
\hline & -2.0 & & \\
\hline & -1.0 & & \\
\hline & -3.195 & -1.3 & \\
\hline & -4.096443 & -1.608892 & -2.8 \\
\hline & -2.89 & & \\
\hline & -2.36 & -1 & \\
\hline & -6.371 & -0.78 & \\
\hline & -7.16 & & \\
\hline & -0.31 & & \\
\hline & -6.46 & & \\
\hline & 4 & -0 & \\
\hline & 192510 & -1 & \\
\hline & & & \\
\hline & 1.509 & 9 & 300 \\
\hline & 63 & -2.5 & 3524 \\
\hline & 1.212191 & -2.2 & 1.750 \\
\hline & (5) & & \\
\hline & 5 & - & 28215 \\
\hline & 172 & 0 & \\
\hline & .880117 & 0.153508 & 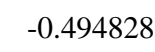 \\
\hline & & & \\
\hline & 6.6576 & -0 & \\
\hline & 116250 & 07227 & 01 \\
\hline
\end{tabular}




\begin{tabular}{|c|c|c|c|c|c|c|c|}
\hline $\mathrm{H}$ & -1.651021 & 0.572945 & 1.502823 & $\mathrm{C}$ & -3.789050 & -1.656209 & 1.999890 \\
\hline $\mathrm{C}$ & -1.929085 & 2.120069 & 0.081535 & $\mathrm{C}$ & -4.838375 & -1.409528 & 1.111823 \\
\hline $\mathrm{C}$ & -3.319658 & 2.073580 & 0.310785 & $\mathrm{H}$ & -5.355490 & -1.191619 & -0.961535 \\
\hline $\mathrm{C}$ & -1.415198 & 3.167266 & -0.708196 & $\mathrm{H}$ & -3.990276 & -1.694386 & 3.069897 \\
\hline $\mathrm{C}$ & -4.172653 & 2.998702 & -0.273194 & $\mathrm{C}$ & 2.605689 & -1.623723 & -0.369588 \\
\hline $\mathrm{H}$ & -3.714962 & 1.275002 & 0.937072 & $\mathrm{C}$ & 3.359513 & -1.093695 & -1.428209 \\
\hline $\mathrm{C}$ & -2.270421 & 4.107112 & -1.266604 & $\mathrm{C}$ & 3.141801 & -1.706294 & 0.935262 \\
\hline $\mathrm{H}$ & -0.343836 & 3.226464 & -0.869728 & $\mathrm{C}$ & 4.615117 & -0.552367 & -1.131787 \\
\hline $\mathrm{C}$ & -3.648456 & 4.020131 & -1.064398 & $\mathrm{C}$ & 4.394666 & -1.153030 & 1.176141 \\
\hline $\mathrm{H}$ & -5.244915 & 2.930191 & -0.105057 & $\mathrm{C}$ & 5.137068 & -0.544928 & 0.157850 \\
\hline $\mathrm{H}$ & -1.862252 & 4.916164 & -1.866469 & $\mathrm{H}$ & 5.196270 & -0.118062 & -1.944110 \\
\hline $\mathrm{H}$ & -4.311193 & 4.755330 & -1.513108 & $\mathrm{H}$ & 4.806251 & -1.201036 & 2.183410 \\
\hline $\mathrm{O}$ & 0.667771 & 1.320604 & -1.717487 & $\mathrm{C}$ & -1.384852 & -2.135942 & 2.530333 \\
\hline $\mathrm{H}$ & 0.656095 & -0.115138 & 1.830384 & $\mathrm{H}$ & -0.687429 & -1.289790 & 2.572261 \\
\hline $\mathrm{O}$ & 2.122835 & 1.947276 & 0.703274 & $\mathrm{H}$ & -1.779276 & -2.302362 & 3.535453 \\
\hline $\mathrm{H}$ & 1.381214 & 0.463626 & 2.405566 & $\mathrm{H}$ & -0.784272 & -3.009416 & 2.252294 \\
\hline $\mathrm{O}$ & 2.136756 & 1.140354 & 2.972619 & $\mathrm{C}$ & -3.012686 & -1.471049 & -2.215939 \\
\hline $\mathrm{H}$ & 2.384487 & 1.641217 & 2.050116 & $\mathrm{H}$ & -3.907178 & -1.742917 & -2.782680 \\
\hline $\mathrm{C}$ & 1.377690 & 2.057014 & 3.773846 & $\mathrm{H}$ & -2.739659 & -0.444457 & -2.483445 \\
\hline $\mathrm{H}$ & 0.501781 & 2.420363 & 3.224026 & $\mathrm{H}$ & -2.168976 & -2.074186 & -2.552471 \\
\hline $\mathrm{H}$ & 1.058867 & 1.539013 & 4.679018 & $\mathrm{C}$ & -6.222445 & -1.130691 & 1.610827 \\
\hline $\mathrm{H}$ & 2.017659 & 2.898578 & 4.040646 & $\mathrm{H}$ & -6.985054 & -1.487136 & 0.913041 \\
\hline $\mathrm{C}$ & 0.051496 & 0.889822 & -2.764394 & $\mathrm{H}$ & -6.407189 & -1.594052 & 2.583756 \\
\hline $\mathrm{H}$ & 0.143099 & 1.605597 & -3.614962 & $\mathrm{H}$ & -6.385542 & -0.052605 & 1.735231 \\
\hline $\mathrm{O}$ & -0.561916 & -0.164793 & -2.917242 & $\mathrm{C}$ & 2.892180 & -1.132790 & -2.847803 \\
\hline $\mathrm{C}$ & 3.048102 & 2.411396 & -0.121237 & $\mathrm{H}$ & 3.019138 & -0.156941 & -3.325929 \\
\hline $\mathrm{H}$ & 2.727515 & 2.361745 & -1.179920 & $\mathrm{H}$ & 3.490894 & -1.849273 & -3.422601 \\
\hline \multirow[t]{2}{*}{$\mathrm{O}$} & 4.123247 & 2.849701 & 0.239439 & $\mathrm{H}$ & 1.841900 & -1.407723 & -2.941429 \\
\hline & & & & $\mathrm{C}$ & 2.387618 & -2.399964 & 2.026537 \\
\hline \multicolumn{2}{|c|}{ TSII-23-4 } & & & $\mathrm{H}$ & 1.465764 & -1.867115 & 2.278497 \\
\hline $\mathrm{Ru}$ & 0.644811 & 0.559979 & 0.191017 & $\mathrm{H}$ & 2.091558 & -3.413507 & 1.730830 \\
\hline $\mathrm{C}$ & 0.198301 & -1.329829 & -0.209875 & $\mathrm{H}$ & 2.992560 & -2.476041 & 2.932932 \\
\hline $\mathrm{C}$ & -0.582979 & -3.484382 & -0.682917 & $\mathrm{C}$ & 6.453577 & 0.102150 & 0.455519 \\
\hline $\mathrm{C}$ & 0.881124 & -3.392435 & -1.106405 & $\mathrm{H}$ & 7.071952 & 0.195870 & -0.440729 \\
\hline $\mathrm{H}$ & -0.737732 & -4.159423 & 0.172689 & $\mathrm{H}$ & 6.309014 & 1.112152 & 0.855217 \\
\hline $\mathrm{H}$ & -1.240467 & -3.816544 & -1.492715 & $\mathrm{H}$ & 7.021178 & -0.456930 & 1.205171 \\
\hline $\mathrm{H}$ & 1.501664 & -4.192503 & -0.688709 & $\mathrm{C}$ & -1.100184 & 1.030087 & 0.577223 \\
\hline $\mathrm{H}$ & 0.995473 & -3.402107 & -2.199236 & $\mathrm{H}$ & -1.562423 & 0.618808 & 1.491964 \\
\hline $\mathrm{N}$ & -0.907834 & -2.103554 & -0.294737 & $\mathrm{C}$ & -2.047644 & 1.863830 & -0.146879 \\
\hline $\mathrm{N}$ & 1.267991 & -2.093107 & -0.563526 & $\mathrm{C}$ & -3.407026 & 1.755530 & 0.212951 \\
\hline $\mathrm{C}$ & -2.225553 & -1.810676 & 0.171114 & $\mathrm{C}$ & -1.704414 & 2.743898 & -1.192527 \\
\hline $\mathrm{C}$ & -3.260347 & -1.575573 & -0.747742 & $\mathrm{C}$ & -4.390691 & 2.445550 & -0.480845 \\
\hline $\mathrm{C}$ & -2.486392 & -1.873165 & 1.552996 & $\mathrm{H}$ & -3.675173 & 1.087049 & 1.029831 \\
\hline $\mathrm{C}$ & -4.552190 & -1.385358 & -0.251327 & $\mathrm{C}$ & -2.689082 & 3.452629 & -1.865978 \\
\hline
\end{tabular}




$\begin{array}{crrc}\mathrm{H} & -0.661228 & 2.850209 & -1.469719 \\ \mathrm{C} & -4.033332 & 3.296952 & -1.524891 \\ \mathrm{H} & -5.435798 & 2.323107 & -0.205919 \\ \mathrm{H} & -2.409458 & 4.130330 & -2.667994 \\ \mathrm{H} & -4.799031 & 3.846538 & -2.065810 \\ \mathrm{O} & 0.792800 & 1.184228 & -1.832137 \\ \mathrm{H} & 0.750072 & 0.120390 & 1.845873 \\ \mathrm{O} & 1.997031 & 2.215008 & 0.573624 \\ \mathrm{H} & 1.166763 & 0.946777 & 2.406229 \\ \mathrm{O} & 1.682488 & 1.854952 & 2.942377 \\ \mathrm{H} & 2.036609 & 2.203996 & 1.996042 \\ \mathrm{C} & 0.668108 & 2.786206 & 3.351783 \\ \mathrm{H} & 1.139587 & 3.754233 & 3.523375 \\ \mathrm{H} & -0.097893 & 2.891383 & 2.573568 \\ \mathrm{H} & 0.217571 & 2.419671 & 4.274447 \\ \mathrm{C} & 0.275043 & 0.594536 & -2.855903 \\ \mathrm{H} & 0.412918 & 1.195702 & -3.785445 \\ \mathrm{O} & -0.292325 & -0.494146 & -2.912849 \\ \mathrm{C} & 2.011044 & 3.389593 & -0.034077 \\ \mathrm{H} & 1.726300 & 3.318446 & -1.101828 \\ \mathrm{O} & 2.307715 & 4.430839 & 0.517492\end{array}$

\section{TSII-33-4}

$\begin{array}{rrrc}\mathrm{Ru} & 0.577394 & 0.351645 & 0.292190 \\ \mathrm{C} & 0.126671 & -1.557559 & 0.085238 \\ \mathrm{C} & -0.590089 & -3.766255 & -0.232086 \\ \mathrm{C} & 0.910631 & -3.698042 & -0.515417 \\ \mathrm{H} & -0.818209 & -4.323424 & 0.688076 \\ \mathrm{H} & -1.164607 & -4.217116 & -1.047422 \\ \mathrm{H} & 1.486538 & -4.444202 & 0.041475 \\ \mathrm{H} & 1.139501 & -3.818890 & -1.584173 \\ \mathrm{~N} & -0.956907 & -2.351661 & -0.066717 \\ \mathrm{~N} & 1.230161 & -2.343656 & -0.069197 \\ \mathrm{C} & -2.311689 & -1.957689 & 0.133765 \\ \mathrm{C} & -3.149989 & -1.776432 & -0.975932 \\ \mathrm{C} & -2.777363 & -1.783157 & 1.447325 \\ \mathrm{C} & -4.458158 & -1.345059 & -0.738794 \\ \mathrm{C} & -4.091181 & -1.357667 & 1.631142 \\ \mathrm{C} & -4.941104 & -1.115052 & 0.548241 \\ \mathrm{H} & -5.113909 & -1.177818 & -1.592111 \\ \mathrm{H} & -4.459918 & -1.205156 & 2.645017 \\ \mathrm{C} & 2.508272 & -1.698445 & -0.163507 \\ \mathrm{C} & 2.946567 & -1.170980 & -1.392017 \\ \mathrm{C} & 3.259841 & -1.541586 & 1.019954\end{array}$

\begin{tabular}{|c|c|c|c|}
\hline & & & \\
\hline & 421415 & 76 & \\
\hline & 856097 & & \\
\hline & & & \\
\hline & 7 & & \\
\hline & .866 & -2. & 143 \\
\hline & 8 & & \\
\hline & 2.40 & & \\
\hline & 1.4 & -3.0 & \\
\hline & -2.69 & & \\
\hline & -3.20 & -2 & \\
\hline & -2.9 & & \\
\hline & -1.61 & -2.2 & -2 \\
\hline & -6.32 & & \\
\hline & -7.0 & & \\
\hline & -6.70 & -1 & \\
\hline & -6.3 & & \\
\hline & 2.2 & & \\
\hline & 2.1 & & \\
\hline & 2.773 & -2 & \\
\hline & 1.210 & -1.7 & -2 \\
\hline & 2.82 & -2 & \\
\hline & 1.75 & & \\
\hline & 3.029 & -3.2 & \\
\hline & 3.36 & -1.76 & 3.15 \\
\hline & 6.10 & & \\
\hline & 6.090 & & \\
\hline & 6.22 & & \\
\hline & 6.995 & & -0 \\
\hline & & & \\
\hline & -1.69 & & \\
\hline & -1.781 & 24 & 2064 \\
\hline & -3.1380 & & \\
\hline & & & \\
\hline & -3.86 & 2321 & 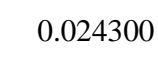 \\
\hline & -3.610 & & 21 \\
\hline & -1.899 & & $n$ \\
\hline & -0.1244 & & \\
\hline & -3.24 & 4.2828 & -0 \\
\hline & -4.91 & & \\
\hline & -1.419663 & 4.821104 & -1.85 \\
\hline & -3.807886 & & \\
\hline & & ( & \\
\hline & 44129 & 05570 & \\
\hline
\end{tabular}




$\begin{array}{cccc}\mathrm{O} & 1.885343 & 2.133552 & 0.549149 \\ \mathrm{H} & 1.424866 & 0.701779 & 2.409820 \\ \mathrm{O} & 2.056968 & 1.597448 & 2.871816 \\ \mathrm{H} & 2.179153 & 1.995800 & 1.843187 \\ \mathrm{C} & 1.128433 & 2.450408 & 3.546461 \\ \mathrm{H} & 0.197560 & 2.545718 & 2.972264 \\ \mathrm{H} & 0.912247 & 2.028072 & 4.529361 \\ \mathrm{H} & 1.576059 & 3.438391 & 3.670688 \\ \mathrm{C} & -0.536701 & 0.359513 & -2.468500 \\ \mathrm{H} & -1.480093 & 0.260131 & -1.871570 \\ \mathrm{O} & -0.543400 & 0.158518 & -3.673713 \\ \mathrm{C} & 2.574252 & 2.881485 & -0.294934 \\ \mathrm{H} & 3.532809 & 3.228255 & 0.158236 \\ \mathrm{O} & 2.248462 & 3.195230 & -1.415996\end{array}$

$\begin{array}{lrrr}\mathrm{H} & 2.698351 & -2.085473 & -3.433102 \\ \mathrm{H} & 1.683195 & -3.161045 & -2.464437 \\ \mathrm{C} & 2.560490 & -1.763704 & 2.498764 \\ \mathrm{H} & 2.885882 & -2.712395 & 2.943453 \\ \mathrm{H} & 2.893286 & -0.965433 & 3.166784 \\ \mathrm{H} & 1.468275 & -1.752066 & 2.505629 \\ \mathrm{C} & 6.409286 & -0.510146 & -0.478022 \\ \mathrm{H} & 6.401396 & 0.584058 & -0.557663 \\ \mathrm{H} & 7.042910 & -0.759790 & 0.377108 \\ \mathrm{H} & 6.890743 & -0.892770 & -1.382204 \\ \mathrm{C} & -2.129057 & -1.322748 & 2.614234 \\ \mathrm{H} & -2.106080 & -0.416748 & 3.222509 \\ \mathrm{H} & -2.598839 & -2.122288 & 3.200755 \\ \mathrm{H} & -1.084880 & -1.596782 & 2.448824 \\ \mathrm{C} & -2.779826 & -2.204997 & -2.331915 \\ \mathrm{H} & -1.719089 & -2.031782 & -2.537035 \\ \mathrm{H} & -2.921991 & -3.290952 & -2.272580 \\ \mathrm{H} & -2.293555 & 2.180888 & -1.597362 \\ \mathrm{H} & -3.362908 & -1.843345 & -3.182686 \\ \mathrm{C} & -6.073057 & 0.631937 & 0.204046 \\ \mathrm{H} & -6.579830 & 0.574342 & 1.171175 \\ \mathrm{H} & -5.825317 & 1.685400 & 0.031022 \\ \mathrm{H} & -6.780138 & 0.329023 & -0.572774 \\ \mathrm{C} & 1.170929 & 0.947246 & -0.846334 \\ \mathrm{H} & 1.818177 & 0.343427 & -1.498333 \\ \mathrm{C} & 1.832619 & 2.187179 & -0.471403 \\ \mathrm{C} & 3.167079 & 2.376126 & -0.886760 \\ \mathrm{C} & 1.225930 & 3.198756 & 0.301642 \\ \mathrm{C} & 3.869635 & 3.522782 & -0.544789 \\ \mathrm{H} & 3.641323 & 1.591445 & -1.475259 \\ \mathrm{C} & 1.929797 & 4.345519 & 0.637590 \\ \mathrm{H} & 0.206975 & 3.052912 & 0.646918 \\ \mathrm{C} & 3.250262 & 4.512434 & 0.217134 \\ \mathrm{H} & 4.899079 & 3.649064 & -0.870486 \\ \mathrm{H} & 1.451249 & 5.115845 & 1.235916 \\ \mathrm{H} & 3.796364 & 5.412486 & 0.486681 \\ \mathrm{H} & -0.649100 & 1.011148 & 1.649998 \\ \mathrm{H} & -0.090403 & -1.963418 \\ \mathrm{H} & 2.158097 & -0.671466 \\ \mathrm{H} & 0.793949 & -2.900270 \\ \mathrm{H} & -1.9984269 \\ \mathrm{H} & 3.708147\end{array}$




\begin{tabular}{|c|c|c|c|c|c|c|c|}
\hline $\mathrm{C}$ & -2.802023 & 2.626167 & 0.249003 & $\mathrm{C}$ & -2.106358 & -1.175381 & 2.693051 \\
\hline $\mathrm{H}$ & -2.394682 & 2.456401 & 1.257150 & $\mathrm{H}$ & -2.034608 & -0.231734 & 3.237399 \\
\hline $\mathrm{O}$ & -3.849221 & 3.140975 & -0.042971 & $\mathrm{H}$ & -2.596796 & -1.909907 & 3.343953 \\
\hline \multirow[t]{2}{*}{$\mathrm{H}$} & -1.959322 & 2.014038 & -3.822260 & $\mathrm{H}$ & -1.078672 & -1.506887 & 2.529127 \\
\hline & & & & $\mathrm{C}$ & -2.873578 & -2.264242 & -2.202817 \\
\hline \multicolumn{2}{|c|}{ TSII $_{w 3-4}$} & & & $\mathrm{H}$ & -1.797685 & -2.198354 & -2.387052 \\
\hline $\mathrm{Ru}$ & -0.574556 & 0.404367 & -0.454159 & $\mathrm{H}$ & -3.125120 & -3.330224 & -2.140410 \\
\hline $\mathrm{C}$ & -0.096595 & -1.478885 & -0.076239 & $\mathrm{H}$ & -3.396924 & -1.853973 & -3.069886 \\
\hline $\mathrm{C}$ & 0.635801 & -3.651535 & 0.407526 & $\mathrm{C}$ & -6.077252 & 0.711157 & 0.281992 \\
\hline $\mathrm{C}$ & -0.868395 & -3.578201 & 0.666694 & $\mathrm{H}$ & -6.579776 & 0.687988 & 1.252936 \\
\hline $\mathrm{H}$ & 0.880957 & -4.270341 & -0.467265 & $\mathrm{H}$ & -5.795630 & 1.752184 & 0.084580 \\
\hline $\mathrm{H}$ & 1.205110 & -4.035594 & 1.259958 & $\mathrm{H}$ & -6.794903 & 0.407814 & -0.484895 \\
\hline $\mathrm{H}$ & -1.430756 & -4.365729 & 0.155124 & $\mathrm{C}$ & 1.097081 & 0.999448 & -0.974456 \\
\hline $\mathrm{H}$ & -1.111392 & -3.627491 & 1.738075 & $\mathrm{H}$ & 1.660363 & 0.459138 & -1.752816 \\
\hline $\mathrm{N}$ & 0.991573 & -2.248742 & 0.144766 & $\mathrm{C}$ & 1.819454 & 2.168799 & -0.507265 \\
\hline $\mathrm{N}$ & -1.194972 & -2.260938 & 0.123146 & $\mathrm{C}$ & 3.176205 & 2.306168 & -0.868199 \\
\hline $\mathrm{C}$ & 2.350128 & -1.854887 & -0.023448 & $\mathrm{C}$ & 1.231963 & 3.176830 & 0.285302 \\
\hline $\mathrm{C}$ & 3.137248 & -1.596405 & 1.107815 & $\mathrm{C}$ & 3.925783 & 3.388510 & -0.431055 \\
\hline $\mathrm{C}$ & 2.875277 & -1.776212 & -1.324110 & $\mathrm{H}$ & 3.630361 & 1.535501 & -1.489785 \\
\hline $\mathrm{C}$ & 4.461944 & -1.199538 & 0.900888 & $\mathrm{C}$ & 1.982270 & 4.263689 & 0.708090 \\
\hline $\mathrm{C}$ & 4.200061 & -1.375710 & -1.477118 & $\mathrm{H}$ & 0.184733 & 3.085030 & 0.555201 \\
\hline $\mathrm{C}$ & 5.005323 & -1.069442 & -0.375377 & $\mathrm{C}$ & 3.329439 & 4.370102 & 0.359628 \\
\hline $\mathrm{H}$ & 5.081334 & -0.977667 & 1.768620 & $\mathrm{H}$ & 4.972647 & 3.474608 & -0.710406 \\
\hline $\mathrm{H}$ & 4.616129 & -1.299005 & -2.481210 & $\mathrm{H}$ & 1.516920 & 5.035127 & 1.314947 \\
\hline $\mathrm{C}$ & -2.472482 & -1.613327 & 0.210163 & $\mathrm{H}$ & 3.911874 & 5.223038 & 0.697467 \\
\hline $\mathrm{C}$ & -2.876778 & -1.015678 & 1.419566 & $\mathrm{O}$ & -0.519436 & 1.005890 & 1.551987 \\
\hline $\mathrm{C}$ & -3.268798 & -1.543989 & -0.950608 & $\mathrm{H}$ & -0.801170 & -0.130692 & -2.086296 \\
\hline $\mathrm{C}$ & -4.068571 & -0.284925 & 1.415139 & $\mathrm{O}$ & -1.973151 & 2.056757 & -0.785405 \\
\hline $\mathrm{C}$ & -4.446028 & -0.802183 & -0.896721 & $\mathrm{H}$ & -1.514169 & 0.511576 & -2.542486 \\
\hline $\mathrm{C}$ & -4.855290 & -0.150205 & 0.271467 & $\mathrm{O}$ & -2.351666 & 1.229513 & -3.004570 \\
\hline $\mathrm{H}$ & -4.377979 & 0.208632 & 2.335373 & $\mathrm{H}$ & -2.387003 & 1.790479 & -2.058489 \\
\hline $\mathrm{H}$ & -5.053490 & -0.716041 & -1.796196 & $\mathrm{C}$ & 0.518193 & 0.751725 & 2.299409 \\
\hline $\mathrm{C}$ & 2.018562 & -2.104917 & -2.508429 & $\mathrm{H}$ & 1.435331 & 0.493380 & 1.712020 \\
\hline $\mathrm{H}$ & 1.108947 & -1.491653 & -2.533316 & $\mathrm{O}$ & 0.542840 & 0.773651 & 3.519961 \\
\hline $\mathrm{H}$ & 2.561983 & -1.950374 & -3.443574 & $\mathrm{C}$ & -2.793370 & 2.579083 & 0.115751 \\
\hline $\mathrm{H}$ & 1.684073 & -3.149132 & -2.489824 & $\mathrm{H}$ & -2.399546 & 2.480430 & 1.146341 \\
\hline $\mathrm{C}$ & 2.611449 & -1.748913 & 2.503123 & $\mathrm{O}$ & -3.849053 & 3.115159 & -0.156759 \\
\hline $\mathrm{H}$ & 3.132046 & -2.558300 & 3.027500 & $\mathrm{H}$ & -1.917728 & 1.797650 & -3.652329 \\
\hline $\mathrm{H}$ & 2.751996 & -0.837806 & 3.091753 & & & & \\
\hline $\mathrm{H}$ & 1.540705 & -1.963000 & 2.524625 & $4 I_{w}$ & & & \\
\hline $\mathrm{C}$ & 6.407807 & -0.580222 & -0.567285 & $\mathrm{Ru}$ & -0.612378 & 0.405471 & -0.571307 \\
\hline $\mathrm{H}$ & 6.432200 & 0.511413 & -0.673377 & $\mathrm{C}$ & -0.070641 & -1.454080 & 0.052704 \\
\hline $\mathrm{H}$ & 7.045647 & -0.829366 & 0.284652 & $\mathrm{C}$ & 0.713500 & -3.529145 & 0.798415 \\
\hline $\mathrm{H}$ & 6.865022 & -0.997137 & -1.469029 & $\mathrm{C}$ & -0.801114 & -3.468287 & 1.021674 \\
\hline
\end{tabular}




\begin{tabular}{|c|c|c|c|c|c|c|c|}
\hline $\mathrm{H}$ & 1.003655 & -4.284926 & 0.057520 & $\mathrm{H}$ & -5.758964 & 1.757014 & -0.015965 \\
\hline $\mathrm{H}$ & 1.277414 & -3.728915 & 1.715459 & $\mathrm{H}$ & -6.738157 & 0.393261 & -0.574519 \\
\hline $\mathrm{H}$ & -1.341702 & -4.274528 & 0.514086 & $\mathrm{C}$ & 1.048313 & 1.045232 & -1.093079 \\
\hline $\mathrm{H}$ & -1.077391 & -3.492431 & 2.083490 & $\mathrm{H}$ & 1.551563 & 0.586888 & -1.962729 \\
\hline $\mathrm{N}$ & 1.035332 & -2.184053 & 0.285301 & $\mathrm{C}$ & 1.828505 & 2.128870 & -0.530372 \\
\hline $\mathrm{N}$ & -1.144272 & -2.172131 & 0.435085 & $\mathrm{C}$ & 3.211484 & 2.162599 & -0.803877 \\
\hline $\mathrm{C}$ & 2.377810 & -1.796519 & 0.003902 & $\mathrm{C}$ & 1.276136 & 3.148703 & 0.270879 \\
\hline $\mathrm{C}$ & 3.232013 & -1.450684 & 1.062707 & $\mathrm{C}$ & 4.022365 & 3.142786 & -0.251595 \\
\hline $\mathrm{C}$ & 2.820865 & -1.801143 & -1.330147 & $\mathrm{H}$ & 3.636213 & 1.384982 & -1.437026 \\
\hline $\mathrm{C}$ & 4.531216 & -1.046352 & 0.744345 & $\mathrm{C}$ & 2.087795 & 4.141848 & 0.797865 \\
\hline $\mathrm{C}$ & 4.125828 & -1.391655 & -1.593092 & $\mathrm{H}$ & 0.207783 & 3.144578 & 0.462232 \\
\hline $\mathrm{C}$ & 4.991696 & -0.994484 & -0.569671 & $\mathrm{C}$ & 3.461200 & 4.135017 & 0.551686 \\
\hline $\mathrm{H}$ & 5.198364 & -0.755607 & 1.554490 & $\mathrm{H}$ & 5.090401 & 3.143648 & -0.453897 \\
\hline $\mathrm{H}$ & 4.477507 & -1.385935 & -2.624189 & $\mathrm{H}$ & 1.649693 & 4.925769 & 1.408612 \\
\hline $\mathrm{C}$ & -2.442777 & -1.559075 & 0.419698 & $\mathrm{H}$ & 4.092470 & 4.910405 & 0.977190 \\
\hline $\mathrm{C}$ & -2.913586 & -0.916858 & 1.582539 & $\mathrm{O}$ & -0.526593 & 1.042716 & 1.397075 \\
\hline $\mathrm{C}$ & -3.174414 & -1.540813 & -0.785839 & $\mathrm{H}$ & -0.621792 & -0.474971 & -2.074631 \\
\hline $\mathrm{C}$ & -4.112656 & -0.213047 & 1.492271 & $\mathrm{O}$ & -2.079050 & 1.865079 & -0.933902 \\
\hline $\mathrm{C}$ & -4.364115 & -0.813942 & -0.816177 & $\mathrm{H}$ & -1.098109 & 0.189821 & -2.271818 \\
\hline $\mathrm{C}$ & -4.841127 & -0.130453 & 0.303084 & $\mathrm{O}$ & -2.918094 & 0.804144 & -3.321447 \\
\hline $\mathrm{H}$ & -4.469966 & 0.319208 & 2.372581 & $\mathrm{H}$ & -2.882096 & 1.313907 & -2.486404 \\
\hline $\mathrm{H}$ & -4.910787 & -0.751852 & -1.754812 & $\mathrm{C}$ & 0.516969 & 0.798013 & 2.144082 \\
\hline $\mathrm{C}$ & 1.916025 & -2.232646 & -2.442768 & $\mathrm{H}$ & 1.413896 & 0.466490 & 1.565907 \\
\hline $\mathrm{H}$ & 1.191926 & -1.451785 & -2.705684 & $\mathrm{O}$ & 0.553505 & 0.900084 & 3.358793 \\
\hline $\mathrm{H}$ & 2.485924 & -2.464440 & -3.345261 & $\mathrm{C}$ & -2.756770 & 2.499208 & 0.002058 \\
\hline $\mathrm{H}$ & 1.327978 & -3.116632 & -2.174930 & $\mathrm{H}$ & -2.290010 & 2.462965 & 1.007168 \\
\hline $\mathrm{C}$ & 2.805118 & -1.521512 & 2.497688 & $\mathrm{O}$ & -3.806752 & 3.080572 & -0.211379 \\
\hline $\mathrm{H}$ & 3.271662 & -2.376603 & 3.001316 & $\mathrm{H}$ & -2.790663 & 1.473352 & -3.997688 \\
\hline $\mathrm{H}$ & 3.100845 & -0.623209 & 3.046020 & & & & \\
\hline $\mathrm{H}$ & 1.723160 & -1.606431 & 2.614466 & \multicolumn{2}{|c|}{ TSII-1 ${ }_{\text {w3-4 }}$} & & \\
\hline $\mathrm{C}$ & 6.368040 & -0.496271 & -0.884663 & $\mathrm{Ru}$ & -0.615200 & 0.531426 & -0.481831 \\
\hline $\mathrm{H}$ & 6.343058 & 0.555855 & -1.193720 & $\mathrm{C}$ & -0.062341 & -1.102697 & 0.476858 \\
\hline $\mathrm{H}$ & 7.031246 & -0.559853 & -0.018527 & $\mathrm{C}$ & 0.789851 & -2.968015 & 1.594601 \\
\hline $\mathrm{H}$ & 6.825811 & -1.054822 & -1.706091 & $\mathrm{C}$ & -0.676291 & -2.793485 & 1.986286 \\
\hline $\mathrm{C}$ & -2.174167 & -0.962787 & 2.882850 & $\mathrm{H}$ & 0.963246 & -3.868482 & 0.985498 \\
\hline $\mathrm{H}$ & -2.070451 & 0.036823 & 3.310665 & $\mathrm{H}$ & 1.458520 & -3.017254 & 2.459667 \\
\hline $\mathrm{H}$ & -2.705059 & -1.589797 & 3.609198 & $\mathrm{H}$ & -1.268736 & -3.705290 & 1.856342 \\
\hline $\mathrm{H}$ & -1.158512 & -1.350253 & 2.781554 & $\mathrm{H}$ & -0.786203 & -2.454116 & 3.025804 \\
\hline $\mathrm{C}$ & -2.723230 & -2.272032 & -2.014906 & $\mathrm{~N}$ & 1.072005 & -1.764391 & 0.796885 \\
\hline $\mathrm{H}$ & -1.692694 & -2.630155 & -1.934899 & $\mathrm{~N}$ & -1.106869 & -1.751530 & 1.059377 \\
\hline $\mathrm{H}$ & -3.360691 & -3.144243 & -2.199493 & $\mathrm{C}$ & 2.373388 & -1.598816 & 0.230378 \\
\hline $\mathrm{H}$ & -2.798602 & -1.619717 & -2.890348 & $\mathrm{C}$ & 3.413580 & -1.059523 & 1.004743 \\
\hline $\mathrm{C}$ & -6.060391 & 0.728298 & 0.214916 & $\mathrm{C}$ & 2.616582 & -2.090199 & -1.064727 \\
\hline $\mathrm{H}$ & -6.611187 & 0.751202 & 1.159307 & $\mathrm{C}$ & 4.691729 & -1.003564 & 0.443510 \\
\hline
\end{tabular}




\begin{tabular}{|c|c|c|c|c|c|c|c|}
\hline $\mathrm{C}$ & 3.905892 & -1.993117 & -1.587715 & $\mathrm{H}$ & 0.584027 & 3.336593 & 0.319610 \\
\hline $\mathrm{C}$ & 4.958582 & -1.449324 & -0.849289 & $\mathrm{C}$ & 3.952375 & 3.828924 & 0.319010 \\
\hline $\mathrm{H}$ & 5.499038 & -0.574898 & 1.036723 & $\mathrm{H}$ & 5.397701 & 2.508881 & -0.590874 \\
\hline $\mathrm{H}$ & 4.093176 & -2.365241 & -2.594277 & $\mathrm{H}$ & 2.291509 & 4.958262 & 1.095126 \\
\hline $\mathrm{C}$ & -2.451202 & -1.388339 & 0.721675 & $\mathrm{H}$ & 4.698068 & 4.534202 & 0.676083 \\
\hline $\mathrm{C}$ & -3.213993 & -0.537018 & 1.539874 & $\mathrm{O}$ & -0.749953 & 1.765302 & 1.234988 \\
\hline $\mathrm{C}$ & -2.970617 & -1.883591 & -0.494166 & $\mathrm{H}$ & -0.657047 & -0.391721 & -1.931609 \\
\hline $\mathrm{C}$ & -4.450178 & -0.098573 & 1.055499 & $\mathrm{O}$ & -2.208562 & 1.796898 & -1.271869 \\
\hline $\mathrm{C}$ & -4.207736 & -1.417196 & -0.928321 & $\mathrm{H}$ & -1.420495 & -0.024714 & -2.565226 \\
\hline $\mathrm{C}$ & -4.948986 & -0.494697 & -0.184021 & $\mathrm{O}$ & -2.313341 & 0.412613 & -3.223623 \\
\hline $\mathrm{H}$ & -5.029894 & 0.594656 & 1.663870 & $\mathrm{H}$ & -2.494990 & 1.174417 & -2.447017 \\
\hline $\mathrm{H}$ & -4.597247 & -1.770637 & -1.881651 & $\mathrm{C}$ & -0.191778 & 1.551914 & 2.378869 \\
\hline $\mathrm{C}$ & 1.514785 & -2.684022 & -1.883100 & $\mathrm{H}$ & -0.326620 & 2.420513 & 3.065726 \\
\hline $\mathrm{H}$ & 0.822077 & -1.907607 & -2.231589 & $\mathrm{O}$ & 0.409712 & 0.553353 & 2.767177 \\
\hline $\mathrm{H}$ & 1.909060 & -3.203683 & -2.759650 & $\mathrm{C}$ & -3.143441 & 2.437313 & -0.583081 \\
\hline $\mathrm{H}$ & 0.909834 & -3.394422 & -1.308718 & $\mathrm{H}$ & -2.810949 & 2.681064 & 0.443886 \\
\hline $\mathrm{C}$ & 3.182435 & -0.507002 & 2.371777 & $\mathrm{O}$ & -4.234625 & 2.730587 & -1.028958 \\
\hline $\mathrm{H}$ & 4.102755 & -0.531207 & 2.961254 & $\mathrm{H}$ & -1.915581 & 0.867108 & -3.975940 \\
\hline $\mathrm{H}$ & 2.842497 & 0.532770 & 2.313929 & & & & \\
\hline $\mathrm{H}$ & 2.390857 & -1.026340 & 2.913328 & \multicolumn{2}{|c|}{ TSII- w $3-4_{4}$} & & \\
\hline $\mathrm{C}$ & 6.325596 & -1.304580 & -1.443722 & $\mathrm{Ru}$ & -0.520638 & 0.327143 & -0.502911 \\
\hline $\mathrm{H}$ & 6.495499 & -2.017042 & -2.255226 & $\mathrm{C}$ & -0.125876 & -1.532601 & 0.001990 \\
\hline $\mathrm{H}$ & 6.465815 & -0.300728 & -1.864262 & $\mathrm{C}$ & 0.532239 & -3.708431 & 0.573718 \\
\hline $\mathrm{H}$ & 7.111091 & -1.445876 & -0.696171 & $\mathrm{C}$ & -0.994304 & -3.642585 & 0.572413 \\
\hline $\mathrm{C}$ & -2.768705 & -0.127956 & 2.907384 & $\mathrm{H}$ & 0.931714 & -4.520275 & -0.042307 \\
\hline $\mathrm{H}$ & -2.912051 & 0.946251 & 3.060794 & $\mathrm{H}$ & 0.941345 & -3.821076 & 1.587188 \\
\hline $\mathrm{H}$ & -3.369941 & -0.640819 & 3.667519 & $\mathrm{H}$ & -1.434330 & -4.183375 & -0.278978 \\
\hline $\mathrm{H}$ & -1.718135 & -0.348844 & 3.094016 & $\mathrm{H}$ & -1.444271 & -4.033827 & 1.490550 \\
\hline $\mathrm{C}$ & -2.213614 & -2.897214 & -1.294012 & $\mathrm{~N}$ & 0.915344 & -2.400817 & 0.016666 \\
\hline $\mathrm{H}$ & -1.261430 & -2.498089 & -1.655682 & $\mathrm{~N}$ & -1.225512 & -2.207192 & 0.439380 \\
\hline $\mathrm{H}$ & -1.974273 & -3.785767 & -0.697490 & $\mathrm{C}$ & 2.282306 & -1.995648 & -0.050488 \\
\hline $\mathrm{H}$ & -2.794073 & -3.220767 & -2.161168 & $\mathrm{C}$ & 2.961380 & -1.622929 & 1.120240 \\
\hline $\mathrm{C}$ & -6.218862 & 0.085290 & -0.717122 & $\mathrm{C}$ & 2.900332 & -1.955003 & -1.307491 \\
\hline $\mathrm{H}$ & -6.966911 & 0.219859 & 0.069346 & $\mathrm{C}$ & 4.275604 & -1.168310 & 0.992109 \\
\hline $\mathrm{H}$ & -6.015850 & 1.075417 & -1.141928 & $\mathrm{C}$ & 4.216894 & -1.501428 & -1.382894 \\
\hline $\mathrm{H}$ & -6.653008 & -0.538058 & -1.503180 & $\mathrm{C}$ & 4.915998 & -1.091705 & -0.245285 \\
\hline $\mathrm{C}$ & 1.104735 & 0.953319 & -1.011496 & $\mathrm{H}$ & 4.809015 & -0.855771 & 1.888796 \\
\hline $\mathrm{H}$ & 1.587667 & 0.303861 & -1.761794 & $\mathrm{H}$ & 4.703286 & -1.451518 & -2.356385 \\
\hline $\mathrm{C}$ & 2.016669 & 1.999657 & -0.575335 & $\mathrm{C}$ & -2.504631 & -1.567247 & 0.340156 \\
\hline $\mathrm{C}$ & 3.385507 & 1.806660 & -0.853683 & $\mathrm{C}$ & -3.081107 & -1.013401 & 1.498735 \\
\hline $\mathrm{C}$ & 1.636475 & 3.162298 & 0.124032 & $\mathrm{C}$ & -3.108896 & -1.430665 & -0.926901 \\
\hline $\mathrm{C}$ & 4.344808 & 2.696098 & -0.392326 & $\mathrm{C}$ & -4.231801 & -0.241558 & 1.342854 \\
\hline $\mathrm{H}$ & 3.680305 & 0.917446 & -1.408973 & $\mathrm{C}$ & -4.248823 & -0.629591 & -1.025518 \\
\hline $\mathrm{C}$ & 2.597705 & 4.063955 & 0.559106 & $\mathrm{C}$ & -4.809685 & -0.011757 & 0.092994 \\
\hline
\end{tabular}




\begin{tabular}{|c|c|c|c|}
\hline & -4.667722 & 0.223113 & 2.225628 \\
\hline & -4.703789 & -0.484686 & -2.004400 \\
\hline & 2.130619 & -2.323009 & -2.537610 \\
\hline & 1.284876 & -1.639489 & -2.687436 \\
\hline & 2.763291 & -2.285475 & -3.427420 \\
\hline & 1.700911 & -3.328359 & -2.467586 \\
\hline & 2.330946 & -1.727490 & 2.476405 \\
\hline & 2.614879 & -2.667943 & 2.965525 \\
\hline 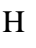 & 2.652406 & -0.915192 & 3.132564 \\
\hline $\mathrm{H}$ & 1.239245 & -1.687004 & 2.445186 \\
\hline $\mathrm{C}$ & 6.307858 & -0.547551 & -0.350908 \\
\hline 11 & 6.964464 & -0.956335 & 0.422831 \\
\hline $\mathrm{H}$ & 6.755636 & -0.765271 & -1.323889 \\
\hline 11 & 6.314802 & 0.541808 & -0.223878 \\
\hline$r$ & -2.481571 & -1.198504 & 2.854203 \\
\hline $\mathrm{H}$ & -1.955091 & -0.291755 & 3.173826 \\
\hline $\mathrm{H}$ & -3.259956 & -1.414716 & 3.592019 \\
\hline$H$ & -1.749433 & -2.009940 & 2.878789 \\
\hline $\mathrm{C}$ & -2.610805 & -2.177040 & -2.129720 \\
\hline U & -1.542721 & -2.403007 & -2.075295 \\
\hline $\mathrm{H}$ & -3.146951 & -3.128748 & -2.234062 \\
\hline$\Pi$ & -2.783245 & -1.606309 & -3.045672 \\
\hline$C$ & -6.010263 & 0.874700 & -0.034335 \\
\hline $\mathrm{H}$ & -5.834327 & 1.846944 & 0.437175 \\
\hline$\Pi$ & -6.274632 & 1.049892 & -1.080396 \\
\hline $\mathrm{H}$ & -6.885328 & 0.439284 & 0.460452 \\
\hline $\mathrm{C}$ & 1.195683 & 0.926825 & -0.838244 \\
\hline $\mathrm{H}$ & 1.894376 & 0.327437 & -1.443705 \\
\hline $\mathrm{C}$ & 1.784185 & 2.176268 & -0.398582 \\
\hline $\mathrm{C}$ & 3.160241 & 2.380902 & -0.636755 \\
\hline 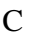 & 1.056833 & 3.181930 & 0.275748 \\
\hline C & 3.795195 & 3.535615 & -0.204244 \\
\hline 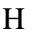 & 3.717944 & 1.601980 & -1.155589 \\
\hline $\mathrm{C}$ & 1.698260 & 4.337710 & 0.695776 \\
\hline $\mathrm{H}$ & -0.004701 & 3.043475 & 0.470147 \\
\hline$C$ & 3.062885 & 4.517110 & 0.463556 \\
\hline$H$ & 4.857898 & 3.674976 & -0.385920 \\
\hline $\mathrm{H}$ & 1.130447 & 5.104501 & 1.214732 \\
\hline $\mathrm{H}$ & 3.556235 & 5.424693 & 0.801985 \\
\hline $\mathrm{O}$ & -0.712935 & 0.904421 & 1.475856 \\
\hline $\mathrm{H}$ & -0.529173 & -0.291022 & -2.133407 \\
\hline $\mathrm{O}$ & -1.744103 & 2.073876 & -1.191186 \\
\hline $\mathrm{H}$ & -1.144275 & 0.300825 & -2.676237 \\
\hline $\mathrm{O}$ & -1.920570 & 1.024261 & -3.321882 \\
\hline
\end{tabular}

$\begin{array}{cccc}\mathrm{H} & -1.986170 & 1.682146 & -2.382901 \\ \mathrm{C} & 0.253501 & 0.779532 & 2.339512 \\ \mathrm{H} & 1.271965 & 0.709021 & 1.879488 \\ \mathrm{O} & 0.117328 & 0.731674 & 3.553233 \\ \mathrm{C} & -2.565171 & 2.902368 & -0.566222 \\ \mathrm{H} & -3.449043 & 3.152578 & -1.198119 \\ \mathrm{O} & -2.411986 & 3.369220 & 0.537618 \\ \mathrm{H} & -1.380391 & 1.493530 & -3.967905\end{array}$

\section{TSII-3 ${ }_{\text {w3-4 }}$}

$\begin{array}{llll}\mathrm{Ru} & 0.452953 & 0.286132 & 0.533150\end{array}$

$\begin{array}{llll}\text { C } & -0.053146 & -1.403601 & -0.341615\end{array}$

$\begin{array}{llll}\text { C } & -0.911753 & -3.203371 & -1.547042\end{array}$

$\begin{array}{llll}\text { C } & 0.583818 & -3.430679 & -1.351792\end{array}$

$\mathrm{H} \quad-1.523014 \quad-4.074039 \quad-1.292662$

$\mathrm{H} \quad-1.141244 \quad-2.897078 \quad-2.577374$

$\mathrm{H} \quad 0.798839 \quad-4.236829 \quad-0.631716$

$\mathrm{H} \quad 1.103271 \quad-3.660553 \quad-2.287113$

$\mathrm{N} \quad-1.184184 \quad-2.088332 \quad-0.629419$

$\mathrm{N} \quad 0.998257 \quad-2.139026 \quad-0.813330$

$\begin{array}{llll}\text { C } & -2.496892 & -1.754319 & -0.184959\end{array}$

$\begin{array}{llll}\text { C } & -3.385492 & -1.063308 & -1.026571\end{array}$

$\begin{array}{llll}\text { C } & -2.868393 & -2.131723 & 1.115420\end{array}$

$\begin{array}{llll}\text { C } & -4.630407 & -0.705249 & -0.509843\end{array}$

$\begin{array}{llll}\text { C } & -4.125255 & -1.747221 & 1.585887\end{array}$

$\begin{array}{llll}\text { C } & -5.013992 & -1.018760 & 0.796076\end{array}$

$\mathrm{H} \quad-5.316143 \quad-0.150104 \quad-1.149510$

$\begin{array}{llll}\mathrm{H} & -4.416759 & -2.028901 & 2.596942\end{array}$

$\begin{array}{llll}\text { C } & 2.322315 & -1.717061 & -0.492227\end{array}$

$\begin{array}{llll}\text { C } & 3.180692 & -1.275132 & -1.514790\end{array}$

$\begin{array}{llll}\text { C } & 2.698511 & -1.626305 & 0.865261\end{array}$

$\begin{array}{llll}\text { C } & 4.348166 & -0.611915 & -1.149670\end{array}$

$\begin{array}{llll}\text { C } & 3.859589 & -0.906634 & 1.175785\end{array}$

$\begin{array}{llll}\text { C } & 4.674866 & -0.360962 & 0.186547\end{array}$

$\begin{array}{llll}\mathrm{H} & 4.996218 & -0.227363 & -1.935752\end{array}$

$\begin{array}{llll}\mathrm{H} & 4.134688 & -0.788422 & 2.224106\end{array}$

$\begin{array}{llll}\text { C } & -1.944008 & -2.922078 & 1.989011\end{array}$

$\mathrm{H} \quad-1.151182 \quad-2.292020 \quad 2.409004$

$\begin{array}{llll}\mathrm{H} & -2.484837 & -3.374704 & 2.823768\end{array}$

$\begin{array}{llll}\mathrm{H} & -1.442415 & -3.721518 & 1.432839\end{array}$

$\begin{array}{llll}\text { C } & -3.007699 & -0.694828 & -2.423335\end{array}$

$\begin{array}{llll}\mathrm{H} & -3.042357 & -1.567204 & -3.088426\end{array}$

$\mathrm{H} \quad-3.700482 \quad 0.045423 \quad-2.831371$

$\mathrm{H} \quad-1.990393 \quad-0.295603 \quad-2.477476$ 


\begin{tabular}{|c|c|c|c|c|c|c|c|}
\hline $\mathrm{C}$ & -6.343834 & -0.579636 & 1.327705 & $\mathrm{P}$ & -0.237634 & -0.071194 & -1.103612 \\
\hline $\mathrm{H}$ & -6.552511 & -1.016798 & 2.307330 & $\mathrm{C}$ & 0.763812 & -1.458906 & -0.344892 \\
\hline $\mathrm{H}$ & -6.387315 & 0.509830 & 1.438924 & $\mathrm{H}$ & 0.067224 & -2.308737 & -0.442534 \\
\hline $\mathrm{H}$ & -7.161134 & -0.857564 & 0.654657 & $\mathrm{C}$ & 1.977706 & -1.825244 & -1.208858 \\
\hline $\mathrm{C}$ & 2.845096 & -1.496079 & -2.956488 & $\mathrm{H}$ & 2.724739 & -1.021109 & -1.162872 \\
\hline $\mathrm{H}$ & 3.408102 & -0.814316 & -3.598743 & $\mathrm{H}$ & 1.677921 & -1.902241 & -2.260655 \\
\hline $\mathrm{H}$ & 3.103543 & -2.516361 & -3.268017 & $\mathrm{C}$ & 2.621483 & -3.123626 & -0.731836 \\
\hline $\mathrm{H}$ & 1.780365 & -1.346393 & -3.154437 & $\mathrm{H}$ & 1.910496 & -3.950813 & -0.876296 \\
\hline $\mathrm{C}$ & 1.994954 & -2.395298 & 1.948467 & $\mathrm{H}$ & 3.498840 & -3.361995 & -1.344341 \\
\hline $\mathrm{H}$ & 0.973386 & -2.670211 & 1.681089 & $\mathrm{C}$ & 3.002938 & -3.044759 & 0.742045 \\
\hline $\mathrm{H}$ & 2.546599 & -3.322931 & 2.147454 & $\mathrm{H}$ & 3.442251 & -3.990685 & 1.078751 \\
\hline $\mathrm{H}$ & 1.958847 & -1.835949 & 2.886784 & $\mathrm{H}$ & 3.783031 & -2.279546 & 0.871037 \\
\hline $\mathrm{C}$ & 5.858188 & 0.486336 & 0.536090 & $\mathrm{C}$ & 1.800858 & -2.676567 & 1.603580 \\
\hline $\mathrm{H}$ & 5.628162 & 1.546149 & 0.375860 & $\mathrm{H}$ & 1.053779 & -3.482500 & 1.547113 \\
\hline $\mathrm{H}$ & 6.147635 & 0.367383 & 1.583840 & $\mathrm{H}$ & 2.090439 & -2.599557 & 2.658247 \\
\hline $\mathrm{H}$ & 6.726708 & 0.251096 & -0.086087 & $\mathrm{C}$ & 1.161500 & -1.372951 & 1.129663 \\
\hline $\mathrm{C}$ & -1.206758 & 1.101483 & 0.464522 & $\mathrm{H}$ & 1.886845 & -0.555872 & 1.259679 \\
\hline $\mathrm{H}$ & -2.107946 & 0.568731 & 0.805774 & $\mathrm{H}$ & 0.294345 & -1.118549 & 1.753086 \\
\hline $\mathrm{C}$ & -1.503300 & 2.436518 & -0.012743 & $\mathrm{C}$ & 0.521522 & 1.544210 & -0.517187 \\
\hline $\mathrm{C}$ & -2.859934 & 2.788467 & -0.186709 & $\mathrm{H}$ & -0.010877 & 2.287139 & -1.136355 \\
\hline $\mathrm{C}$ & -0.511872 & 3.396114 & -0.315770 & $\mathrm{C}$ & 1.995762 & 1.617771 & -0.927670 \\
\hline $\mathrm{C}$ & -3.217244 & 4.039054 & -0.666651 & $\mathrm{H}$ & 2.571592 & 0.914260 & -0.307654 \\
\hline $\mathrm{H}$ & -3.622800 & 2.046344 & 0.049081 & $\mathrm{H}$ & 2.121640 & 1.286524 & -1.966593 \\
\hline $\mathrm{C}$ & -0.880331 & 4.650206 & -0.781188 & $\mathrm{C}$ & 2.565780 & 3.017112 & -0.733557 \\
\hline $\mathrm{H}$ & 0.537400 & 3.149982 & -0.176107 & $\mathrm{H}$ & 2.044732 & 3.714684 & -1.405590 \\
\hline $\mathrm{C}$ & -2.225167 & 4.973788 & -0.963854 & $\mathrm{H}$ & 3.623063 & 3.042226 & -1.021823 \\
\hline $\mathrm{H}$ & -4.265046 & 4.291462 & -0.806369 & $\mathrm{C}$ & 2.390829 & 3.479342 & 0.707571 \\
\hline $\mathrm{H}$ & -0.110739 & 5.384099 & -1.003517 & $\mathrm{H}$ & 2.775190 & 4.497379 & 0.838702 \\
\hline $\mathrm{H}$ & -2.501973 & 5.958374 & -1.332477 & $\mathrm{H}$ & 2.993088 & 2.835505 & 1.366030 \\
\hline $\mathrm{O}$ & 1.265071 & 1.142228 & -1.180659 & $\mathrm{C}$ & 0.929466 & 3.400411 & 1.134887 \\
\hline $\mathrm{H}$ & -0.084539 & -0.466256 & 2.000418 & $\mathrm{H}$ & 0.815437 & 3.712663 & 2.179721 \\
\hline $\mathrm{O}$ & 1.630094 & 1.822276 & 1.708276 & $\mathrm{H}$ & 0.340710 & 4.111170 & 0.535619 \\
\hline $\mathrm{H}$ & 0.458903 & -0.024486 & 2.726782 & $\mathrm{C}$ & 0.357359 & 1.996477 & 0.939015 \\
\hline $\mathrm{O}$ & 1.155238 & 0.495530 & 3.628467 & $\mathrm{H}$ & -0.698469 & 1.979705 & 1.236012 \\
\hline $\mathrm{H}$ & 1.517232 & 1.254346 & 2.823813 & $\mathrm{H}$ & 0.874569 & 1.298542 & 1.610538 \\
\hline $\mathrm{C}$ & 0.862810 & 0.927457 & -2.384925 & $\mathrm{C}$ & -1.800182 & -0.126005 & -0.074772 \\
\hline $\mathrm{H}$ & 1.430746 & 1.548042 & -3.114424 & $\mathrm{H}$ & -1.559699 & 0.018606 & 0.992005 \\
\hline $\mathrm{O}$ & -0.002834 & 0.148894 & -2.786392 & $\mathrm{C}$ & -2.516850 & -1.472488 & -0.214994 \\
\hline $\mathrm{C}$ & 2.758222 & 2.452235 & 1.422569 & $\mathrm{H}$ & -1.883080 & -2.285841 & 0.158490 \\
\hline $\mathrm{H}$ & 3.516078 & 2.330917 & 2.232949 & $\mathrm{H}$ & -2.684741 & -1.678807 & -1.283525 \\
\hline $\mathrm{O}$ & 2.983716 & 3.107920 & 0.432052 & $\mathrm{C}$ & -3.851631 & -1.489136 & 0.522610 \\
\hline \multirow[t]{2}{*}{$\mathrm{H}$} & 0.546880 & 0.955359 & 4.217832 & $\mathrm{H}$ & -4.346701 & -2.457181 & 0.382828 \\
\hline & & & & $\mathrm{H}$ & -3.666037 & -1.391869 & 1.602577 \\
\hline $\mathrm{Cy}_{3}$ & & & & $\mathrm{C}$ & -4.756427 & -0.351065 & 0.068571 \\
\hline
\end{tabular}




$\begin{array}{cccc}\mathrm{H} & -5.015716 & -0.496631 & -0.990533 \\ \mathrm{H} & -5.702204 & -0.365211 & 0.622107 \\ \mathrm{C} & -4.057777 & 0.992610 & 0.230660 \\ \mathrm{H} & -3.875731 & 1.179897 & 1.299721 \\ \mathrm{H} & -4.700735 & 1.808872 & -0.118767 \\ \mathrm{C} & -2.728273 & 1.014029 & -0.515306 \\ \mathrm{H} & -2.914678 & 0.915520 & -1.595693 \\ \mathrm{H} & -2.233995 & 1.985067 & -0.382040\end{array}$

\section{G-I}

$\begin{array}{cccc}\mathrm{Ru} & -0.031120 & 0.005441 & 0.069861 \\ \mathrm{C} & -0.011043 & -1.708198 & 0.759225 \\ \mathrm{Cl} & -0.082346 & -0.273412 & -2.366045 \\ \mathrm{Cl} & -0.032898 & 0.829374 & 2.397908 \\ \mathrm{P} & 2.328145 & 0.504120 & 0.029040 \\ \mathrm{P} & -2.424647 & 0.247058 & -0.022053 \\ \mathrm{H} & -0.268141 & -1.741963 & 1.834828 \\ \mathrm{C} & 3.227704 & 0.228204 & 1.625863 \\ \mathrm{H} & 2.696349 & 0.927736 & 2.289559 \\ \mathrm{C} & 4.710791 & 0.611373 & 1.608565 \\ \mathrm{H} & 4.852163 & 1.608708 & 1.171068 \\ \mathrm{H} & 5.266695 & -0.089067 & 0.967056 \\ \mathrm{C} & 5.288517 & 0.562684 & 3.020982 \\ \mathrm{H} & 6.352901 & 0.823945 & 3.002836 \\ \mathrm{H} & 4.791017 & 1.329174 & 3.633602 \\ \mathrm{C} & 5.078031 & -0.804689 & 3.659230 \\ \mathrm{H} & 5.480894 & -0.816512 & 4.678377 \\ \mathrm{H} & 5.648356 & -1.556483 & 3.093007 \\ \mathrm{C} & 3.603150 & -1.189644 & 3.658378 \\ \mathrm{H} & 3.463385 & -2.183670 & 4.098236 \\ \mathrm{H} & 3.041733 & -0.487696 & 4.291924 \\ \mathrm{C} & 3.016626 & -1.152121 & 2.251560 \\ \mathrm{H} & 1.944452 & -1.370631 & 2.287932 \\ \mathrm{H} & 3.485943 & -1.933525 & 1.638846 \\ \mathrm{C} & 2.510215 & 2.334360 & -0.258376 \\ \mathrm{H} & 3.559826 & 2.506204 & -0.546406 \\ \mathrm{C} & 2.225547 & 3.206317 & 0.967326 \\ \mathrm{H} & 2.886254 & 2.936806 & 1.799808 \\ \mathrm{H} & 1.202417 & 3.020639 & 1.323939 \\ \mathrm{C} & 2.404575 & 4.682735 & 0.627128 \\ \mathrm{H} & 2.196350 & 5.295916 & 1.510900 \\ \mathrm{H} & 3.456424 & 4.870124 & 0.362317 \\ \mathrm{C} & 1.512276 & 5.097580 & -0.534827 \\ \mathrm{H} & 0.460963 & 4.986773 & -0.228008 \\ & & & \end{array}$

\begin{tabular}{|c|c|c|c|}
\hline & & & \\
\hline & 768219 & 4.229602 & -1 . \\
\hline & 789060 & 411727 & 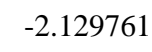 \\
\hline & & & \\
\hline & 2 & 98 & -1. \\
\hline & 79 & 05 & \\
\hline & & & \\
\hline & 264771 & .26 & \\
\hline & 49 & 4 & \\
\hline & 4.45 & & \\
\hline & 4 & 21 & \\
\hline & 5 & 38 & \\
\hline & .995 & -0.22 & -3 . \\
\hline & 4.228632 & .201537 & \\
\hline & 2 & & \\
\hline & 36 & -1.6 & \\
\hline & 5.72 & -2.1 & \\
\hline & .20 & -1.6 & \\
\hline & 18 & -2. & \\
\hline & 4.455 & -3.4 & \\
\hline & 3.370534 & -2.47 & \\
\hline & 3.66 & -1.7 & \\
\hline & 7 & (7) & \\
\hline & 4.46 & -1.6 & \\
\hline & .288 & -0.77 & \\
\hline & .01 & 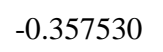 & \\
\hline & 2.884 & 2 & \\
\hline & -3.228348 & -2.745055 & -0.387 \\
\hline & -1.7936 & -2.32 & \\
\hline & & & \\
\hline & -3.03 & -2.5 & -3 \\
\hline & -3.198626 & -4.01 & -2.504 \\
\hline & $-4.99 x$ & 2.80 & \\
\hline & -5.4 & -3.29 & \\
\hline & 541 & 33 & -170799 \\
\hline & -5.395801 & 445 & -2.5 \\
\hline & -5.028589 & -0.839786 & \\
\hline & -6 . & -1.2273 & \\
\hline & -4.8 & 8 & -1 \\
\hline & -5.24 & -1.04 & -0 \\
\hline & -5.099139 & 0.444477 & -1.346 \\
\hline & $-3.254^{\prime}$ & 0 & \\
\hline & -2.50 & 28 & 2.3 \\
\hline & -3.407192 & .44835 & 2.00 \\
\hline
\end{tabular}




\begin{tabular}{llll}
$\mathrm{H}$ & -4.188602 & -1.907932 & 1.382441 \\
$\mathrm{H}$ & -2.481079 & -2.000144 & 1.800193 \\
$\mathrm{C}$ & -3.800813 & -1.584185 & 3.473191 \\
$\mathrm{H}$ & -2.991422 & -1.177193 & 4.096421 \\
$\mathrm{H}$ & -3.902056 & -2.641851 & 3.742841 \\
$\mathrm{C}$ & -5.094929 & -0.831987 & 3.758028 \\
$\mathrm{H}$ & -5.362561 & -0.909551 & 4.817908 \\
$\mathrm{H}$ & -5.915090 & -1.305123 & 3.197228 \\
$\mathrm{C}$ & -4.983617 & 0.629746 & 3.343203 \\
$\mathrm{H}$ & -5.929817 & 1.154732 & 3.518784 \\
$\mathrm{H}$ & -4.230977 & 1.126273 & 3.972523 \\
$\mathrm{C}$ & -4.569123 & 0.776706 & 1.880175 \\
$\mathrm{H}$ & -4.467016 & 1.841468 & 1.641876 \\
$\mathrm{H}$ & -5.366108 & 0.385698 & 1.231546 \\
$\mathrm{C}$ & -2.858264 & 1.990836 & -0.500596 \\
$\mathrm{H}$ & -3.942438 & 2.091661 & -0.330648 \\
$\mathrm{C}$ & -2.596563 & 2.324687 & -1.971472 \\
$\mathrm{H}$ & -3.137252 & 1.635020 & -2.629595 \\
$\mathrm{H}$ & 1.173505 & -4.856810 & -2.495584 \\
$\mathrm{H}$ & -1.530462 & 2.183321 & -2.201417 \\
$\mathrm{C}$ & -3.013366 & 3.759608 & -2.278173 \\
$\mathrm{H}$ & -2.814122 & 3.988931 & -3.330963 \\
$\mathrm{H}$ & -4.101243 & 3.855846 & -2.142055 \\
$\mathrm{C}$ & -2.303033 & 4.752747 & -1.368812 \\
$\mathrm{H}$ & -1.223523 & 4.713046 & -1.578913 \\
$\mathrm{H}$ & -2.621305 & 5.778053 & -1.588980 \\
$\mathrm{C}$ & -2.537425 & 4.421401 & 0.100259 \\
$\mathrm{H}$ & -3.603408 & 4.558511 & 0.338340 \\
$\mathrm{H}$ & -1.987052 & 5.115066 & 0.747091 \\
$\mathrm{C}$ & -2.136549 & 2.985853 & 0.416494 \\
$\mathrm{H}$ & -1.048530 & 2.869198 & 0.281112 \\
$\mathrm{H}$ & -2.310576 & 2.748451 & 1.473682 \\
$\mathrm{C}$ & 0.890526 & -5.674822 & -0.522280 \\
$\mathrm{C}$ & 0.563457 & -5.399107 & 0.803808 \\
\hline & 0.266836 & -4.096959 & 1.183765 \\
$\mathrm{H}$ & 0.629650 & -3.338419 & -1.084486 \\
\hline
\end{tabular}

G-ID

\begin{tabular}{|c|c|c|c|}
\hline $\mathrm{Ru}$ & -1.063079 & -0.993702 & -0.111189 \\
\hline $\mathrm{C}$ & -2.165319 & 0.454759 & 0.127328 \\
\hline $\mathrm{Cl}$ & -1.041178 & -1.960200 & 2.021435 \\
\hline $\mathrm{Cl}$ & -0.972692 & -1.271407 & -2.435481 \\
\hline$P$ & 0.904545 & 0.041170 & -0.002277 \\
\hline $\mathrm{H}$ & -1.825639 & 1.497465 & 0.174821 \\
\hline $\mathrm{C}$ & 1.208755 & 1.253927 & -1.375000 \\
\hline $\mathrm{H}$ & 1.260337 & 0.575806 & -2.240557 \\
\hline $\mathrm{C}$ & 2.538874 & 2.009670 & -1.274736 \\
\hline $\mathrm{H}$ & 3.368919 & 1.320909 & -1.071835 \\
\hline $\mathrm{H}$ & 2.500195 & 2.708599 & -0.425576 \\
\hline $\mathrm{C}$ & 2.809998 & 2.798210 & -2.553291 \\
\hline $\mathrm{H}$ & 3.756858 & 3.342417 & -2.462565 \\
\hline $\mathrm{H}$ & 2.936690 & 2.092228 & -3.387231 \\
\hline $\mathrm{C}$ & 1.664218 & 3.751310 & -2.866964 \\
\hline $\mathrm{H}$ & 1.860806 & 4.294093 & -3.798138 \\
\hline $\mathrm{H}$ & 1.601445 & 4.511852 & -2.074266 \\
\hline $\mathrm{C}$ & 0.340407 & 3.003038 & -2.951376 \\
\hline $\mathrm{H}$ & -0.483757 & 3.695667 & -3.154595 \\
\hline $\mathrm{H}$ & 0.369925 & 2.302646 & -3.798729 \\
\hline $\mathrm{C}$ & 0.052126 & 2.209910 & -1.680581 \\
\hline $\mathrm{H}$ & -0.870548 & 1.635013 & -1.807057 \\
\hline $\mathrm{H}$ & -0.102814 & 2.903386 & -0.840159 \\
\hline $\mathrm{C}$ & 2.309525 & -1.164520 & -0.204905 \\
\hline $\mathrm{H}$ & 3.212101 & -0.575982 & 0.025304 \\
\hline $\mathrm{C}$ & 2.492670 & -1.731978 & -1.615092 \\
\hline $\mathrm{H}$ & 2.577459 & -0.931095 & -2.358046 \\
\hline $\mathrm{H}$ & 1.606634 & -2.314896 & -1.899091 \\
\hline $\mathrm{C}$ & 3.741176 & -2.608221 & -1.672215 \\
\hline $\mathrm{H}$ & 3.860194 & -3.018628 & -2.680921 \\
\hline $\mathrm{H}$ & 4.626657 & -1.980887 & -1.488409 \\
\hline $\mathrm{C}$ & 3.699303 & -3.727345 & -0.638444 \\
\hline $\mathrm{H}$ & 2.877866 & -4.414672 & -0.885687 \\
\hline $\mathrm{H}$ & 4.620020 & -4.320038 & -0.676710 \\
\hline $\mathrm{C}$ & 3.474969 & -3.171617 & 0.762507 \\
\hline $\mathrm{H}$ & 4.344219 & -2.564998 & 1.060067 \\
\hline $\mathrm{H}$ & 3.400482 & -3.982721 & 1.494834 \\
\hline $\mathrm{C}$ & 2.221037 & -2.307889 & 0.813540 \\
\hline $\mathrm{H}$ & 2.042300 & -1.928004 & 1.827065 \\
\hline $\mathrm{H}$ & 1.341277 & -2.924890 & 0.580648 \\
\hline $\mathrm{C}$ & 1.246932 & 0.815699 & 1.662066 \\
\hline $\mathrm{H}$ & 0.775514 & 0.092770 & 2.346303 \\
\hline $\mathrm{C}$ & 2.723156 & 0.942902 & 2.066254 \\
\hline $\mathrm{H}$ & 3.246447 & 1.623487 & 1.380228 \\
\hline
\end{tabular}




$\begin{array}{lrrr}\mathrm{H} & 3.232153 & -0.023956 & 1.997113 \\ \mathrm{C} & 2.847662 & 1.469083 & 3.493603 \\ \mathrm{H} & 2.414273 & 0.729460 & 4.182045 \\ \mathrm{H} & 3.906069 & 1.558199 & 3.763181 \\ \mathrm{C} & 2.130008 & 2.799463 & 3.671007 \\ \mathrm{H} & 2.218892 & 3.150498 & 4.704961 \\ \mathrm{H} & 2.616418 & 3.560841 & 3.042843 \\ \mathrm{C} & 0.666443 & 2.677102 & 3.269582 \\ \mathrm{H} & 0.153606 & 3.640489 & 3.366551 \\ \mathrm{H} & 0.157224 & 1.983623 & 3.953478 \\ \mathrm{C} & 0.525057 & 2.155790 & 1.843120 \\ \mathrm{H} & -0.534880 & 2.052143 & 1.587410 \\ \mathrm{H} & 0.948159 & 2.895561 & 1.146536 \\ \mathrm{C} & -6.392692 & -0.016218 & 0.090999 \\ \mathrm{C} & -5.822272 & 1.245461 & -0.071275 \\ \mathrm{C} & -4.443049 & 1.393385 & -0.038303 \\ \mathrm{C} & -3.602840 & 0.278542 & 0.150735 \\ \mathrm{C} & -4.199198 & -0.988633 & 0.324282 \\ \mathrm{C} & -5.577285 & -1.131153 & 0.291641 \\ \mathrm{H} & -7.472695 & -0.130883 & 0.069422 \\ \mathrm{H} & -6.456797 & 2.114541 & -0.221326 \\ \mathrm{H} & -3.992056 & 2.374820 & -0.168295 \\ \mathrm{H} & -3.566914 & -1.854511 & 0.522104 \\ \mathrm{H} & -6.021889 & -2.111760 & 0.432640\end{array}$

\section{G-II}

$\begin{array}{rrrc}\mathrm{Ru} & -0.069282 & -0.399268 & 0.102556 \\ \mathrm{C} & -1.756173 & 0.317403 & 0.330220 \\ \mathrm{Cl} & 0.293178 & -0.451762 & -2.323256 \\ \mathrm{Cl} & 0.132073 & -0.442695 & 2.561010 \\ \mathrm{P} & 1.133760 & 1.733225 & 0.162417 \\ \mathrm{H} & -2.100471 & 0.279125 & 1.380353 \\ \mathrm{C} & 0.914047 & 2.803146 & -1.348557 \\ \mathrm{C} & -0.363710 & 3.646762 & -1.274417 \\ \mathrm{C} & -0.672038 & 4.279777 & -2.625942 \\ \mathrm{C} & 0.489265 & 5.149221 & -3.090732 \\ \mathrm{C} & 1.786735 & 4.351119 & -3.125101 \\ \mathrm{C} & 2.090880 & 3.685093 & -1.783738 \\ \mathrm{C} & 0.874088 & 2.754520 & 1.688842 \\ \mathrm{C} & -0.588981 & 3.018613 & 2.056223 \\ \mathrm{C} & -0.681079 & 3.650060 & 3.441723 \\ \mathrm{C} & 0.141378 & 4.930418 & 3.529458 \\ \mathrm{C} & 1.592395 & 4.678179 & 3.139623 \\ \mathrm{C} & 1.695195 & 4.046924 & 1.753572\end{array}$

\begin{tabular}{|c|c|c|c|}
\hline & & & \\
\hline & 3.406266 & 0.520748 & \\
\hline & 927342 & .502863 & \\
\hline & & & \\
\hline & 04 & & \\
\hline & 51 & & \\
\hline & & & \\
\hline & 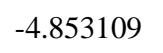 & & \\
\hline & 3.85 & 2 & \\
\hline & -2 & & \\
\hline & -2.684966 & 8 & \\
\hline & -3.6 & 73 & \\
\hline & -0.56 & 2.40 & 22 \\
\hline & -1.76 & -2.9 & \\
\hline & -1.7 & -4.42 & \\
\hline & -0.20 & -4.67 & 06040 \\
\hline & 0.33 & 3 & \\
\hline & 4.42 & -3.2 & \\
\hline & 4.0 & & \\
\hline & 2.68 & -1 & 00 \\
\hline & 1.72 & -3.2 & \\
\hline & 2.08 & & \\
\hline & & & \\
\hline & -5.621 & 12 & \\
\hline & -4.90 & 1.6 & -0.9 \\
\hline & -3.61 & & \\
\hline & -3 & -2.35 & 0 \\
\hline & -3.751516 & -2.02 & 1600 \\
\hline & -5.030 & 1.48 & \\
\hline & & & \\
\hline & 1.04 & -3.7 & 25 \\
\hline & 5.873 & -3.20 & 1.21 \\
\hline & 050 & 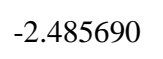 & \\
\hline & & & \\
\hline & -6.99 & -0.6 & 1106 \\
\hline & 60986 & 26 & 362 \\
\hline & -1.211 & 3.04 & 0.9510. \\
\hline & -0.228 & is & \\
\hline & -0.85 & 3. & -3.3606 \\
\hline & -1.59 & & \\
\hline & 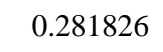 & .585311 & 4.07 \\
\hline & & & 2051 \\
\hline & & 3. & \\
\hline & 626075 & 4.990350 & 3.42 \\
\hline
\end{tabular}




\begin{tabular}{|c|c|c|c|c|c|c|c|}
\hline $\mathrm{H}$ & 2.287450 & 4.455719 & -1.023244 & $\mathrm{H}$ & 6.133378 & -2.239304 & -1.655030 \\
\hline $\mathrm{H}$ & 3.011317 & 3.099193 & -1.879619 & $\mathrm{H}$ & 6.524984 & -3.352309 & -0.347700 \\
\hline $\mathrm{H}$ & 1.245000 & 2.071692 & 2.468082 & $\mathrm{H}$ & -1.958341 & -1.830970 & -2.159515 \\
\hline $\mathrm{H}$ & -1.059745 & 3.685212 & 1.321564 & $\mathrm{H}$ & -2.451871 & -3.513104 & -2.068780 \\
\hline $\mathrm{H}$ & -1.141804 & 2.074238 & 2.040382 & $\mathrm{H}$ & -3.446239 & -2.355410 & -2.966734 \\
\hline $\mathrm{H}$ & -1.729015 & 3.847600 & 3.695378 & $\mathrm{H}$ & -3.775678 & -1.873186 & 3.750307 \\
\hline $\mathrm{H}$ & -0.313918 & 2.925434 & 4.182858 & $\mathrm{H}$ & -2.871979 & -3.264310 & 3.143891 \\
\hline $\mathrm{H}$ & -0.286104 & 5.681936 & 2.848494 & $\mathrm{H}$ & -2.167752 & -1.661392 & 3.016183 \\
\hline $\mathrm{H}$ & 0.084112 & 5.360176 & 4.536112 & $\mathrm{H}$ & -7.216091 & -0.026361 & 0.959940 \\
\hline $\mathrm{H}$ & 2.171173 & 5.608618 & 3.174035 & $\mathrm{H}$ & -7.109079 & -0.116278 & -0.804045 \\
\hline $\mathrm{H}$ & 2.052980 & 3.998928 & 3.872334 & $\mathrm{H}$ & -7.767524 & -1.468730 & 0.114176 \\
\hline $\mathrm{H}$ & 2.748148 & 3.862987 & 1.503922 & & & & \\
\hline $\mathrm{H}$ & 1.319327 & 4.758718 & 1.003237 & G-IID & & & \\
\hline $\mathrm{H}$ & 3.428883 & 2.416226 & 0.041054 & $\mathrm{Ru}$ & 0.239066 & 0.764954 & -0.026234 \\
\hline $\mathrm{H}$ & 3.025775 & -0.498661 & -0.776772 & $\mathrm{C}$ & -1.589812 & 0.944774 & -0.121548 \\
\hline $\mathrm{H}$ & 2.959843 & 0.828585 & -1.911298 & $\mathrm{Cl}$ & 0.804165 & 1.147665 & -2.268560 \\
\hline $\mathrm{H}$ & 5.246123 & -0.152415 & -1.872057 & $\mathrm{Cl}$ & 0.551134 & 1.251060 & 2.249268 \\
\hline $\mathrm{H}$ & 5.278891 & 1.513124 & -1.313600 & $\mathrm{H}$ & -2.283285 & 0.113374 & -0.275834 \\
\hline $\mathrm{H}$ & 5.335861 & -0.994854 & 0.424947 & $\mathrm{C}$ & -3.480384 & 4.751048 & -0.037358 \\
\hline $\mathrm{H}$ & 6.660281 & 0.137411 & 0.199994 & $\mathrm{C}$ & -4.223048 & 3.600078 & -0.297355 \\
\hline $\mathrm{H}$ & 5.455968 & 0.463033 & 2.386075 & $\mathrm{C}$ & -3.598735 & 2.360786 & -0.314678 \\
\hline $\mathrm{H}$ & 5.401314 & 1.902085 & 1.379899 & $\mathrm{C}$ & -2.214301 & 2.248086 & -0.074955 \\
\hline $\mathrm{H}$ & 3.193350 & 1.479984 & 2.367536 & $\mathrm{C}$ & -1.482442 & 3.424737 & 0.192593 \\
\hline $\mathrm{H}$ & 3.120646 & -0.132264 & 1.679670 & $\mathrm{C}$ & -2.109120 & 4.659620 & 0.208271 \\
\hline $\mathrm{H}$ & -5.559422 & 2.781778 & -2.518611 & $\mathrm{C}$ & 0.511565 & -1.141173 & 0.053864 \\
\hline $\mathrm{H}$ & -5.694954 & 2.645315 & -0.041049 & $\mathrm{~N}$ & -0.338629 & -2.189019 & 0.227290 \\
\hline $\mathrm{H}$ & -3.917099 & 1.471683 & 1.228029 & $\mathrm{C}$ & 0.349828 & -3.483363 & 0.189979 \\
\hline $\mathrm{H}$ & -1.828992 & 0.638129 & -2.430994 & $\mathrm{C}$ & 1.817022 & -3.087004 & 0.293530 \\
\hline $\mathrm{H}$ & -3.629423 & 1.766678 & -3.707904 & $\mathrm{~N}$ & 1.775614 & -1.657256 & 0.000096 \\
\hline $\mathrm{H}$ & -2.244686 & -4.786691 & 1.507207 & $\mathrm{C}$ & 5.241603 & 0.752581 & -0.043586 \\
\hline $\mathrm{H}$ & -2.167868 & -4.864821 & -0.263449 & $\mathrm{C}$ & 4.638279 & 0.401214 & 1.163174 \\
\hline $\mathrm{H}$ & 0.143409 & -4.871812 & 1.717810 & $\mathrm{C}$ & 3.509702 & -0.418751 & 1.213786 \\
\hline $\mathrm{H}$ & 0.125318 & -5.505590 & 0.058785 & $\mathrm{C}$ & 2.963609 & -0.860529 & -0.003798 \\
\hline $\mathrm{H}$ & 4.793260 & -3.057503 & 1.273733 & $\mathrm{C}$ & 3.592408 & -0.594143 & -1.233357 \\
\hline $\mathrm{H}$ & 3.724710 & -3.567930 & -2.846415 & $\mathrm{C}$ & 4.719144 & 0.225131 & -1.226479 \\
\hline $\mathrm{H}$ & -5.343292 & -1.464640 & -1.893461 & $\mathrm{C}$ & -4.531079 & -2.013314 & -0.105619 \\
\hline $\mathrm{H}$ & -5.575036 & -1.203917 & 2.381050 & $\mathrm{C}$ & -3.730553 & -2.104960 & -1.246462 \\
\hline $\mathrm{H}$ & 1.331935 & -2.723359 & 2.509283 & $\mathrm{C}$ & -2.338696 & -2.169675 & -1.164563 \\
\hline $\mathrm{H}$ & 2.349307 & -4.159325 & 2.756901 & $\mathrm{C}$ & -1.756522 & -2.147772 & 0.110156 \\
\hline $\mathrm{H}$ & 3.036595 & -2.547168 & 2.909438 & $\mathrm{C}$ & -2.523320 & -2.043564 & 1.278511 \\
\hline $\mathrm{H}$ & 1.512888 & -4.036820 & -3.464364 & $\mathrm{C}$ & -3.909775 & -1.978067 & 1.146153 \\
\hline $\mathrm{H}$ & 0.417803 & -4.633794 & -2.207576 & $\mathrm{C}$ & 2.977258 & -0.873603 & 2.538147 \\
\hline $\mathrm{H}$ & 0.385388 & -2.929248 & -2.667275 & $\mathrm{C}$ & 3.114629 & -1.215218 & -2.508794 \\
\hline $\mathrm{H}$ & 6.125104 & -3.971246 & -1.958658 & $\mathrm{C}$ & 6.419607 & 1.676929 & -0.073715 \\
\hline
\end{tabular}




\begin{tabular}{|c|c|c|c|c|c|c|c|}
\hline $\mathrm{C}$ & -1.486457 & -2.132027 & -2.394889 & $\mathrm{~N}$ & 2.051161 & 0.035637 & -0.203332 \\
\hline $\mathrm{C}$ & -1.854742 & -1.912640 & 2.610462 & $\mathrm{C}$ & 4.822533 & 0.401099 & -0.105500 \\
\hline $\mathrm{C}$ & -6.023867 & -1.955530 & -0.217718 & $\mathrm{H}$ & 5.896994 & 0.544885 & -0.089592 \\
\hline $\mathrm{H}$ & -3.970190 & 5.720845 & -0.020855 & $\mathrm{C}$ & -1.880506 & -1.377027 & -0.291167 \\
\hline $\mathrm{H}$ & -5.290969 & 3.672537 & -0.484139 & $\mathrm{C}$ & -4.117711 & -2.088076 & -0.332174 \\
\hline $\mathrm{H}$ & -4.169177 & 1.455376 & -0.516434 & $\mathrm{C}$ & -3.223311 & -3.302632 & -0.106683 \\
\hline $\mathrm{H}$ & -0.416677 & 3.356416 & 0.411151 & $\mathrm{H}$ & -4.895532 & -1.975239 & 0.428006 \\
\hline $\mathrm{H}$ & -1.533273 & 5.555742 & 0.419394 & $\mathrm{H}$ & -4.604522 & -2.104050 & -1.315256 \\
\hline $\mathrm{H}$ & 0.012740 & -4.119021 & 1.015439 & $\mathrm{H}$ & -3.312727 & -3.702181 & 0.912377 \\
\hline $\mathrm{H}$ & 0.120755 & -4.009516 & -0.748045 & $\mathrm{H}$ & -3.417944 & -4.116742 & -0.809562 \\
\hline $\mathrm{H}$ & 2.226173 & -3.256420 & 1.300024 & $\mathrm{~N}$ & -3.162227 & -0.967502 & -0.259997 \\
\hline $\mathrm{H}$ & 2.458239 & -3.610542 & -0.423428 & $\mathrm{~N}$ & -1.884452 & -2.736313 & -0.316647 \\
\hline $\mathrm{H}$ & 5.058765 & 0.768553 & 2.098157 & $\mathrm{C}$ & -3.610115 & 0.383595 & -0.265650 \\
\hline $\mathrm{H}$ & 5.201664 & 0.458524 & -2.174463 & $\mathrm{C}$ & -3.896866 & 1.008558 & -1.485930 \\
\hline $\mathrm{H}$ & -4.200115 & -2.106636 & -2.229334 & $\mathrm{C}$ & -3.730533 & 1.055948 & 0.959925 \\
\hline $\mathrm{H}$ & -4.519833 & -1.882689 & 2.043315 & $\mathrm{C}$ & -4.343617 & 2.331539 & -1.453452 \\
\hline $\mathrm{H}$ & 3.566179 & -1.722944 & 2.908559 & $\mathrm{C}$ & -4.181522 & 2.374043 & 0.943115 \\
\hline $\mathrm{H}$ & 3.042817 & -0.078821 & 3.284015 & $\mathrm{C}$ & -4.500070 & 3.026695 & -0.252217 \\
\hline $\mathrm{H}$ & 1.929100 & -1.176605 & 2.493174 & $\mathrm{H}$ & -4.562159 & 2.835931 & -2.393689 \\
\hline $\mathrm{H}$ & 3.363155 & -0.588104 & -3.367503 & $\mathrm{H}$ & -4.264289 & 2.913775 & 1.885779 \\
\hline $\mathrm{H}$ & 3.594820 & -2.190351 & -2.664133 & $\mathrm{C}$ & -0.673276 & -3.447366 & -0.039913 \\
\hline $\mathrm{H}$ & 2.033835 & -1.366392 & -2.514027 & $\mathrm{C}$ & 0.086466 & -3.934456 & -1.116765 \\
\hline $\mathrm{H}$ & 7.121061 & 1.414009 & -0.870662 & $\mathrm{C}$ & -0.242875 & -3.592214 & 1.293097 \\
\hline $\mathrm{H}$ & 6.103023 & 2.709755 & -0.258113 & $\mathrm{C}$ & 1.318265 & -4.530548 & -0.833736 \\
\hline $\mathrm{H}$ & 6.963608 & 1.672248 & 0.874646 & $\mathrm{C}$ & 0.993738 & -4.195964 & 1.521887 \\
\hline $\mathrm{H}$ & -0.981184 & -1.160134 & -2.485621 & $\mathrm{C}$ & 1.794869 & -4.656220 & 0.472673 \\
\hline $\mathrm{H}$ & -0.693985 & -2.888426 & -2.384369 & $\mathrm{H}$ & 1.920983 & -4.905827 & -1.659451 \\
\hline $\mathrm{H}$ & -2.083837 & -2.284580 & -3.296422 & $\mathrm{H}$ & 1.345684 & -4.292714 & 2.548372 \\
\hline $\mathrm{H}$ & -2.582450 & -1.942095 & 3.424495 & $\mathrm{C}$ & -3.312118 & 0.388919 & 2.232263 \\
\hline $\mathrm{H}$ & -1.118362 & -2.705021 & 2.783351 & $\mathrm{H}$ & -2.226957 & 0.208570 & 2.229836 \\
\hline $\mathrm{H}$ & -1.306050 & -0.963667 & 2.677089 & $\mathrm{H}$ & -3.549387 & 1.004720 & 3.102397 \\
\hline $\mathrm{H}$ & -6.453869 & -1.236031 & 0.485364 & $\mathrm{H}$ & -3.786313 & -0.590221 & 2.362682 \\
\hline $\mathrm{H}$ & -6.343070 & -1.676833 & -1.225339 & $\mathrm{C}$ & -3.639452 & 0.298035 & -2.777519 \\
\hline \multirow[t]{2}{*}{$\mathrm{H}$} & -6.478278 & -2.926853 & 0.008609 & $\mathrm{H}$ & -4.278797 & -0.583656 & -2.900022 \\
\hline & & & & $\mathrm{H}$ & -3.819024 & 0.955006 & -3.631595 \\
\hline 25 & & & & $\mathrm{H}$ & -2.600362 & -0.057531 & -2.818873 \\
\hline $\mathrm{Ru}$ & -0.070091 & -0.471500 & -0.309870 & $\mathrm{C}$ & -4.965239 & 4.450417 & -0.236531 \\
\hline $\mathrm{C}$ & 2.851216 & -0.876373 & 0.380388 & $\mathrm{H}$ & -5.124503 & 4.835315 & -1.246498 \\
\hline $\mathrm{C}$ & 2.608382 & 1.175075 & -0.673796 & $\mathrm{H}$ & -5.904799 & 4.560013 & 0.315147 \\
\hline $\mathrm{C}$ & 4.238383 & -0.744076 & 0.404833 & $\mathrm{H}$ & -4.236190 & 5.100913 & 0.259844 \\
\hline $\mathrm{H}$ & 2.353067 & -1.761198 & 0.770465 & $\mathrm{C}$ & -0.412189 & -3.807141 & -2.521253 \\
\hline $\mathrm{C}$ & 3.971000 & 1.376519 & -0.627715 & $\mathrm{H}$ & 0.263273 & -4.304680 & -3.221196 \\
\hline $\mathrm{H}$ & 1.932128 & 1.912550 & -1.095025 & $\mathrm{H}$ & -1.406422 & -4.251910 & -2.634463 \\
\hline $\mathrm{H}$ & 4.844291 & -1.523142 & 0.853560 & $\mathrm{H}$ & -0.497407 & -2.752406 & -2.806302 \\
\hline
\end{tabular}




\begin{tabular}{|c|c|c|c|c|c|c|c|}
\hline $\mathrm{C}$ & -1.090537 & -3.140355 & 2.442617 & $\mathrm{H}$ & -4.932356 & 3.186760 & -0.646870 \\
\hline $\mathrm{H}$ & -1.609297 & -2.200534 & 2.226265 & $\mathrm{C}$ & 2.294958 & -0.345060 & -0.147276 \\
\hline $\mathrm{H}$ & -1.855941 & -3.885982 & 2.689520 & $\mathrm{C}$ & 4.412863 & -1.372760 & -0.349370 \\
\hline $\mathrm{H}$ & -0.480603 & -2.988643 & 3.336824 & $\mathrm{C}$ & 4.617652 & 0.117849 & -0.147473 \\
\hline $\mathrm{C}$ & 3.141389 & -5.251045 & 0.748899 & $\mathrm{H}$ & 4.989097 & -1.990036 & 0.345956 \\
\hline $\mathrm{H}$ & 3.844362 & -4.486704 & 1.099819 & $\mathrm{H}$ & 4.661308 & -1.695262 & -1.370110 \\
\hline $\mathrm{H}$ & 3.094417 & -6.012395 & 1.533351 & $\mathrm{H}$ & 5.026392 & 0.348639 & 0.846192 \\
\hline $\mathrm{H}$ & 3.572469 & -5.708698 & -0.144597 & $\mathrm{H}$ & 5.273794 & 0.571386 & -0.895835 \\
\hline $\mathrm{C}$ & -0.669359 & 1.268474 & -0.149936 & $\mathrm{~N}$ & 2.967716 & -1.526980 & -0.114914 \\
\hline $\mathrm{H}$ & -1.394901 & 1.621120 & -0.902313 & $\mathrm{~N}$ & 3.247830 & 0.628537 & -0.265270 \\
\hline $\mathrm{C}$ & -0.297117 & 2.303786 & 0.796366 & $\mathrm{C}$ & 2.388631 & -2.822077 & -0.252991 \\
\hline $\mathrm{C}$ & -0.948645 & 3.554004 & 0.710661 & $\mathrm{C}$ & 1.925158 & -3.248857 & -1.504457 \\
\hline $\mathrm{C}$ & 0.647318 & 2.109925 & 1.828848 & $\mathrm{C}$ & 2.305799 & -3.639113 & 0.884262 \\
\hline $\mathrm{C}$ & -0.686716 & 4.563387 & 1.626672 & $\mathrm{C}$ & 1.343252 & -4.517500 & -1.587373 \\
\hline $\mathrm{H}$ & -1.679167 & 3.705184 & -0.082169 & $\mathrm{C}$ & 1.724358 & -4.898860 & 0.752410 \\
\hline $\mathrm{C}$ & 0.910290 & 3.127197 & 2.735554 & $\mathrm{C}$ & 1.229005 & -5.352376 & -0.474725 \\
\hline $\mathrm{H}$ & 1.198590 & 1.174016 & 1.922935 & $\mathrm{H}$ & 0.970783 & -4.861992 & -2.551292 \\
\hline $\mathrm{C}$ & 0.244228 & 4.351900 & 2.644822 & $\mathrm{H}$ & 1.641984 & -5.536383 & 1.631688 \\
\hline $\mathrm{H}$ & -1.204312 & 5.515759 & 1.548518 & $\mathrm{C}$ & 2.967984 & 2.018846 & -0.094977 \\
\hline $\mathrm{H}$ & 1.641632 & 2.959487 & 3.522753 & $\mathrm{C}$ & 2.837930 & 2.828588 & -1.236048 \\
\hline $\mathrm{H}$ & 0.450407 & 5.139500 & 3.364417 & $\mathrm{C}$ & 2.884782 & 2.560629 & 1.199908 \\
\hline $\mathrm{O}$ & 2.586891 & -0.308781 & 2.907481 & $\mathrm{C}$ & 2.529203 & 4.178983 & -1.053518 \\
\hline $\mathrm{O}$ & -0.220565 & -0.548674 & -2.320811 & $\mathrm{C}$ & 2.580857 & 3.916662 & 1.330093 \\
\hline $\mathrm{C}$ & 1.422167 & -0.853708 & 3.208293 & $\mathrm{C}$ & 2.377971 & 4.737512 & 0.217353 \\
\hline $\mathrm{H}$ & 0.737438 & -0.237268 & 3.859492 & $\mathrm{H}$ & 2.417910 & 4.813916 & -1.931600 \\
\hline $\mathrm{H}$ & 0.737051 & -1.058446 & 2.306237 & $\mathrm{H}$ & 2.504532 & 4.343556 & 2.330029 \\
\hline $\mathrm{H}$ & 1.450624 & -1.872699 & 3.689035 & $\mathrm{C}$ & 2.765267 & -3.127316 & 2.213553 \\
\hline $\mathrm{C}$ & 0.414407 & 0.461033 & -3.032835 & $\mathrm{H}$ & 2.209925 & -2.224087 & 2.493258 \\
\hline $\mathrm{H}$ & 0.057201 & 1.470377 & -2.748284 & $\mathrm{H}$ & 2.620856 & -3.874256 & 2.997201 \\
\hline $\mathrm{H}$ & 1.514010 & 0.466098 & -2.923775 & $\mathrm{H}$ & 3.824077 & -2.846228 & 2.207411 \\
\hline $\mathrm{H}$ & 0.193545 & 0.330659 & -4.103720 & $\mathrm{C}$ & 2.017232 & -2.357184 & -2.703793 \\
\hline \multirow[t]{2}{*}{$\mathrm{Br}$} & 4.700804 & 3.032120 & -1.335124 & $\mathrm{H}$ & 3.043632 & -2.010659 & -2.875544 \\
\hline & & & & $\mathrm{H}$ & 1.690197 & -2.881448 & -3.605239 \\
\hline 26 & & & & $\mathrm{H}$ & 1.405276 & -1.452516 & -2.584014 \\
\hline $\mathrm{Ru}$ & 0.359994 & 0.223810 & -0.100412 & $\mathrm{C}$ & 0.574586 & -6.696166 & -0.581914 \\
\hline $\mathrm{C}$ & -1.680856 & 2.535490 & 0.069338 & $\mathrm{H}$ & 0.382472 & -6.970225 & -1.622071 \\
\hline $\mathrm{C}$ & -2.728875 & 0.596433 & -0.610783 & $\mathrm{H}$ & 1.188840 & -7.482286 & -0.131869 \\
\hline $\mathrm{C}$ & -2.843833 & 3.276143 & -0.082832 & $\mathrm{H}$ & -0.386308 & -6.711789 & -0.055076 \\
\hline $\mathrm{H}$ & -0.753222 & 2.995313 & 0.402333 & $\mathrm{C}$ & 3.035302 & 2.257687 & -2.603416 \\
\hline $\mathrm{C}$ & -3.921090 & 1.281775 & -0.779680 & $\mathrm{H}$ & 2.943107 & 3.033368 & -3.367950 \\
\hline $\mathrm{H}$ & -2.649733 & -0.468043 & -0.810599 & $\mathrm{H}$ & 4.024367 & 1.797248 & -2.706942 \\
\hline $\mathrm{H}$ & -2.843506 & 4.337747 & 0.138210 & $\mathrm{H}$ & 2.288347 & 1.477388 & -2.790130 \\
\hline $\mathrm{N}$ & -1.616477 & 1.215976 & -0.182162 & $\mathrm{C}$ & 3.146521 & 1.717808 & 2.410460 \\
\hline $\mathrm{C}$ & -4.004139 & 2.641961 & -0.517404 & $\mathrm{H}$ & 2.681572 & 0.730253 & 2.324500 \\
\hline
\end{tabular}




\begin{tabular}{|c|c|c|c|c|c|c|c|}
\hline $\mathrm{H}$ & 4.221308 & 1.560360 & 2.559859 & $\mathrm{C}$ & -2.394215 & -2.182689 & 1.548467 \\
\hline $\mathrm{H}$ & 2.771032 & 2.199439 & 3.317323 & $\mathrm{C}$ & -4.248116 & -2.162424 & -0.534722 \\
\hline $\mathrm{C}$ & 1.998918 & 6.176675 & 0.386626 & $\mathrm{C}$ & -3.753001 & -2.004338 & 1.804080 \\
\hline $\mathrm{H}$ & 0.913974 & 6.287052 & 0.499296 & $\mathrm{C}$ & -4.695662 & -1.982189 & 0.774345 \\
\hline $\mathrm{H}$ & 2.454527 & 6.613891 & 1.279479 & $\mathrm{H}$ & -4.968520 & -2.147945 & -1.351754 \\
\hline $\mathrm{H}$ & 2.292639 & 6.777489 & -0.477891 & $\mathrm{H}$ & -4.082162 & -1.864866 & 2.833247 \\
\hline $\mathrm{C}$ & -0.327162 & -1.336275 & 0.645828 & $\mathrm{C}$ & 2.779981 & -1.432949 & -0.165242 \\
\hline $\mathrm{H}$ & 0.217708 & -2.268156 & 0.443317 & $\mathrm{C}$ & 3.593808 & -1.116469 & -1.265122 \\
\hline $\mathrm{C}$ & -1.595390 & -1.622303 & 1.316176 & $\mathrm{C}$ & 3.165320 & -1.065613 & 1.143329 \\
\hline $\mathrm{C}$ & -2.172940 & -2.901584 & 1.171652 & $\mathrm{C}$ & 4.752771 & -0.366714 & -1.041047 \\
\hline $\mathrm{C}$ & -2.277537 & -0.686446 & 2.118036 & $\mathrm{C}$ & 4.320255 & -0.305434 & 1.310497 \\
\hline $\mathrm{C}$ & -3.394067 & -3.213219 & 1.758237 & $\mathrm{C}$ & 5.121699 & 0.068128 & 0.228526 \\
\hline $\mathrm{H}$ & -1.647409 & -3.647112 & 0.574740 & $\mathrm{H}$ & 5.378141 & -0.110066 & -1.895540 \\
\hline $\mathrm{C}$ & -3.489880 & -1.001817 & 2.716901 & $\mathrm{H}$ & 4.599724 & 0.004293 & 2.317068 \\
\hline $\mathrm{H}$ & -1.834800 & 0.294462 & 2.261603 & $\mathrm{C}$ & -1.385477 & -2.098430 & 2.649655 \\
\hline $\mathrm{C}$ & -4.061141 & -2.262329 & 2.531065 & $\mathrm{H}$ & -0.769060 & -1.198463 & 2.522695 \\
\hline $\mathrm{H}$ & -3.825321 & -4.201139 & 1.619480 & $\mathrm{H}$ & -1.866079 & -2.047251 & 3.629994 \\
\hline $\mathrm{H}$ & -3.993099 & -0.262858 & 3.335879 & $\mathrm{H}$ & -0.694159 & -2.949350 & 2.652652 \\
\hline $\mathrm{H}$ & -5.012044 & -2.506219 & 2.996921 & $\mathrm{C}$ & -2.443246 & -2.451337 & -2.254951 \\
\hline $\mathrm{O}$ & -1.402398 & 2.560514 & 3.161689 & $\mathrm{H}$ & -3.275828 & -2.683582 & -2.923251 \\
\hline $\mathrm{O}$ & 0.459755 & 0.569561 & -2.124578 & $\mathrm{H}$ & -2.004016 & -1.502323 & -2.582475 \\
\hline $\mathrm{C}$ & -0.243435 & 2.213042 & 3.211044 & $\mathrm{H}$ & -1.668604 & -3.211609 & -2.394500 \\
\hline $\mathrm{H}$ & 0.077479 & 1.268834 & 3.703933 & $\mathrm{C}$ & -6.145204 & -1.735581 & 1.061023 \\
\hline $\mathrm{H}$ & 0.523609 & 0.861325 & 1.392547 & $\mathrm{H}$ & -6.392522 & -1.937928 & 2.106389 \\
\hline $\mathrm{H}$ & 0.581430 & 2.821303 & 2.779902 & $\mathrm{H}$ & -6.414633 & -0.691860 & 0.860249 \\
\hline $\mathrm{C}$ & -0.553059 & 1.077040 & -2.926005 & $\mathrm{H}$ & -6.794341 & -2.353327 & 0.433764 \\
\hline $\mathrm{H}$ & -1.423216 & 0.400740 & -3.024451 & $\mathrm{C}$ & 3.255075 & -1.541808 & -2.656602 \\
\hline $\mathrm{H}$ & -0.954360 & 2.050173 & -2.582585 & $\mathrm{H}$ & 2.849473 & -0.694603 & -3.217576 \\
\hline $\mathrm{H}$ & -0.171072 & 1.242211 & -3.948442 & $\mathrm{H}$ & 4.146248 & -1.900155 & -3.180967 \\
\hline \multirow[t]{2}{*}{$\mathrm{Br}$} & -5.482765 & 0.296445 & -1.374810 & $\mathrm{H}$ & 2.494943 & -2.325601 & -2.672617 \\
\hline & & & & $\mathrm{C}$ & 2.390423 & -1.496232 & 2.351399 \\
\hline 38-m-r & & & & $\mathrm{H}$ & 1.321134 & -1.580905 & 2.145655 \\
\hline $\mathrm{Ru}$ & 0.363666 & 0.403579 & -0.479782 & $\mathrm{H}$ & 2.739699 & -2.469506 & 2.719797 \\
\hline $\mathrm{C}$ & 0.336701 & -1.522302 & -0.262192 & $\mathrm{H}$ & 2.520437 & -0.779372 & 3.166863 \\
\hline $\mathrm{C}$ & -0.006658 & -3.853506 & -0.092371 & $\mathrm{C}$ & 6.322939 & 0.939065 & 0.429445 \\
\hline $\mathrm{C}$ & 1.488553 & -3.581347 & -0.011099 & $\mathrm{H}$ & 6.043445 & 1.999596 & 0.419482 \\
\hline $\mathrm{H}$ & -0.381169 & -4.472816 & 0.730461 & $\mathrm{H}$ & 6.807565 & 0.749977 & 1.391497 \\
\hline $\mathrm{H}$ & -0.288220 & -4.348173 & -1.034629 & $\mathrm{H}$ & 7.065401 & 0.796621 & -0.360331 \\
\hline $\mathrm{H}$ & 1.883132 & -3.752721 & 1.002520 & $\mathrm{C}$ & -1.420883 & 0.727261 & -0.130114 \\
\hline $\mathrm{H}$ & 2.075556 & -4.192713 & -0.704976 & $\mathrm{H}$ & -2.026796 & 0.068452 & 0.506676 \\
\hline $\mathrm{N}$ & -0.582210 & -2.507964 & -0.032490 & $\mathrm{C}$ & -2.202489 & 1.853056 & -0.626668 \\
\hline $\mathrm{N}$ & 1.561736 & -2.164440 & -0.364495 & $\mathrm{C}$ & -3.442856 & 2.130723 & -0.016496 \\
\hline $\mathrm{C}$ & -1.976521 & -2.345052 & 0.218700 & $\mathrm{C}$ & -1.782735 & 2.676536 & -1.690982 \\
\hline $\mathrm{C}$ & -2.897940 & -2.345360 & -0.836336 & $\mathrm{C}$ & -4.207968 & 3.219884 & -0.409696 \\
\hline
\end{tabular}




\begin{tabular}{|c|c|c|c|c|c|c|c|}
\hline $\mathrm{H}$ & -3.784106 & 1.476220 & 0.785993 & $\mathrm{C}$ & -2.824643 & -1.885635 & -1.146643 \\
\hline $\mathrm{C}$ & -2.561440 & 3.753885 & -2.092873 & $\mathrm{C}$ & -2.239054 & -2.610923 & 1.116328 \\
\hline $\mathrm{H}$ & -0.842871 & 2.444894 & -2.187305 & $\mathrm{C}$ & -4.144973 & -1.729160 & -0.726332 \\
\hline $\mathrm{C}$ & -3.766044 & 4.039472 & -1.448190 & $\mathrm{C}$ & -3.570098 & -2.428202 & 1.493427 \\
\hline $\mathrm{H}$ & -5.153965 & 3.428088 & 0.084280 & $\mathrm{C}$ & -4.536336 & -1.982607 & 0.590050 \\
\hline $\mathrm{H}$ & -2.228974 & 4.379002 & -2.917771 & $\mathrm{H}$ & -4.883078 & -1.374745 & -1.445252 \\
\hline $\mathrm{H}$ & -4.364798 & 4.889982 & -1.763360 & $\mathrm{H}$ & -3.857512 & -2.637345 & 2.522724 \\
\hline $\mathrm{O}$ & 0.822708 & 0.462850 & -2.446561 & $\mathrm{C}$ & 2.819747 & -1.249008 & -0.608833 \\
\hline $\mathrm{H}$ & 0.663244 & 0.456059 & 1.159833 & $\mathrm{C}$ & 3.618019 & -0.709307 & -1.630001 \\
\hline $\mathrm{C}$ & 0.272119 & -0.397680 & -3.379522 & $\mathrm{C}$ & 3.201062 & -1.146066 & 0.745370 \\
\hline $\mathrm{H}$ & 0.285824 & -1.459569 & -3.061104 & $\mathrm{C}$ & 4.769959 & -0.010914 & -1.270321 \\
\hline $\mathrm{H}$ & -0.780732 & -0.146451 & -3.610295 & $\mathrm{C}$ & 4.352259 & -0.415952 & 1.050717 \\
\hline $\mathrm{H}$ & 0.825599 & -0.344699 & -4.333910 & $\mathrm{C}$ & 5.139304 & 0.171823 & 0.062307 \\
\hline $\mathrm{O}$ & 1.043487 & 2.596282 & -0.672907 & $\mathrm{H}$ & 5.385456 & 0.421624 & -2.058362 \\
\hline $\mathrm{H}$ & 1.377217 & 1.176216 & 2.292699 & $\mathrm{H}$ & 4.635432 & -0.308494 & 2.097082 \\
\hline $\mathrm{C}$ & 2.457250 & 2.561139 & -0.828500 & $\mathrm{C}$ & -1.217753 & -3.078640 & 2.106303 \\
\hline $\mathrm{H}$ & 2.951794 & 2.075278 & 0.029066 & $\mathrm{H}$ & -0.476290 & -2.299795 & 2.318895 \\
\hline $\mathrm{H}$ & 2.663443 & 1.979859 & -1.730256 & $\mathrm{H}$ & -1.684777 & -3.352198 & 3.055337 \\
\hline $\mathrm{H}$ & 2.862356 & 3.573038 & -0.945582 & $\mathrm{H}$ & -0.659573 & -3.949613 & 1.742882 \\
\hline $\mathrm{H}$ & 0.846615 & 3.103946 & 0.152511 & $\mathrm{C}$ & -2.425210 & -1.508073 & -2.536213 \\
\hline $\mathrm{C}$ & -0.791613 & 3.884677 & 1.938229 & $\mathrm{H}$ & -3.278259 & -1.544887 & -3.218062 \\
\hline $\mathrm{H}$ & -1.244530 & 2.883942 & 1.885244 & $\mathrm{H}$ & -2.032233 & -0.483966 & -2.552416 \\
\hline $\mathrm{H}$ & -1.008570 & 4.322515 & 2.922136 & $\mathrm{H}$ & -1.634106 & -2.147768 & -2.938256 \\
\hline $\mathrm{H}$ & -1.264879 & 4.504805 & 1.173003 & $\mathrm{C}$ & -5.961106 & -1.793717 & 1.011763 \\
\hline $\mathrm{O}$ & 0.600370 & 3.857137 & 1.676620 & $\mathrm{H}$ & -6.069587 & -1.831224 & 2.098302 \\
\hline $\mathrm{H}$ & 0.999965 & 3.219862 & 2.304984 & $\mathrm{H}$ & -6.358593 & -0.833561 & 0.667220 \\
\hline $\mathrm{O}$ & 1.502392 & 1.675017 & 3.132403 & $\mathrm{H}$ & -6.609307 & -2.570151 & 0.590261 \\
\hline $\mathrm{C}$ & 0.478391 & 1.221926 & 3.997995 & $\mathrm{C}$ & 3.255912 & -0.832436 & -3.074872 \\
\hline $\mathrm{H}$ & -0.506867 & 1.217953 & 3.508725 & $\mathrm{H}$ & 2.922743 & 0.135694 & -3.460346 \\
\hline $\mathrm{H}$ & 0.676594 & 0.205983 & 4.370720 & $\mathrm{H}$ & 4.119447 & -1.144550 & -3.670339 \\
\hline \multirow[t]{2}{*}{$\mathrm{H}$} & 0.439902 & 1.898565 & 4.855068 & $\mathrm{H}$ & 2.440653 & -1.539111 & -3.242531 \\
\hline & & & & $\mathrm{C}$ & 2.456161 & -1.808847 & 1.866725 \\
\hline \multicolumn{2}{|c|}{ TS8-m-r } & & & $\mathrm{H}$ & 1.588780 & -2.378801 & 1.526287 \\
\hline $\mathrm{Ru}$ & 0.361301 & 0.494662 & -0.022577 & $\mathrm{H}$ & 3.118196 & -2.500166 & 2.400041 \\
\hline $\mathrm{C}$ & 0.392223 & -1.413410 & -0.556056 & $\mathrm{H}$ & 2.105403 & -1.064683 & 2.592757 \\
\hline $\mathrm{C}$ & 0.062979 & -3.666334 & -1.118242 & $\mathrm{C}$ & 6.329180 & 1.005579 & 0.424485 \\
\hline $\mathrm{C}$ & 1.559223 & -3.391042 & -1.051370 & $\mathrm{H}$ & 6.033420 & 2.045955 & 0.606980 \\
\hline $\mathrm{H}$ & -0.249231 & -4.534657 & -0.529456 & $\mathrm{H}$ & 6.812965 & 0.649644 & 1.338160 \\
\hline $\mathrm{H}$ & -0.287665 & -3.816873 & -2.150276 & $\mathrm{H}$ & 7.075139 & 1.020151 & -0.374652 \\
\hline $\mathrm{H}$ & 2.025572 & -3.842229 & -0.162035 & $\mathrm{C}$ & -1.467918 & 0.823237 & -0.020152 \\
\hline $\mathrm{H}$ & 2.104790 & -3.740402 & -1.933392 & $\mathrm{H}$ & -2.162587 & 0.165354 & 0.526237 \\
\hline $\mathrm{N}$ & -0.503908 & -2.429339 & -0.570091 & $\mathrm{C}$ & -2.149520 & 1.931366 & -0.657126 \\
\hline $\mathrm{N}$ & 1.599789 & -1.927518 & -0.951178 & $\mathrm{C}$ & -3.559033 & 1.969327 & -0.588375 \\
\hline $\mathrm{C}$ & -1.877952 & -2.316969 & -0.207428 & $\mathrm{C}$ & -1.477640 & 2.972452 & -1.333517 \\
\hline
\end{tabular}




\begin{tabular}{|c|c|c|c|c|c|c|c|}
\hline $\mathrm{C}$ & -4.274262 & 3.001335 & -1.177656 & $\mathrm{C}$ & -1.885211 & -2.053843 & -0.751843 \\
\hline $\mathrm{H}$ & -4.075325 & 1.166095 & -0.063732 & $\mathrm{C}$ & -2.869365 & -1.478511 & -1.569556 \\
\hline $\mathrm{C}$ & -2.201032 & 4.001852 & -1.917336 & $\mathrm{C}$ & -2.206959 & -2.643181 & 0.479855 \\
\hline $\mathrm{H}$ & -0.392313 & 2.942741 & -1.380669 & $\mathrm{C}$ & -4.188774 & -1.490811 & -1.118662 \\
\hline $\mathrm{C}$ & -3.594784 & 4.021711 & -1.842760 & $\mathrm{C}$ & -3.541370 & -2.619359 & 0.892486 \\
\hline $\mathrm{H}$ & -5.359133 & 3.017335 & -1.117042 & $\mathrm{C}$ & -4.544846 & -2.046153 & 0.113046 \\
\hline $\mathrm{H}$ & -1.676964 & 4.801638 & -2.433109 & $\mathrm{H}$ & -4.957799 & -1.036134 & -1.742779 \\
\hline $\mathrm{H}$ & -4.151570 & 4.834976 & -2.300881 & $\mathrm{H}$ & -3.800428 & -3.065641 & 1.851836 \\
\hline $\mathrm{O}$ & 0.999028 & 1.124077 & -1.816594 & $\mathrm{C}$ & 2.869138 & -1.081666 & -0.821663 \\
\hline $\mathrm{H}$ & -0.086207 & -0.277007 & 1.480297 & $\mathrm{C}$ & 3.692023 & -0.302020 & -1.651290 \\
\hline $\mathrm{C}$ & 0.366391 & 0.681835 & -2.967701 & $\mathrm{C}$ & 3.278025 & -1.438678 & 0.478326 \\
\hline $\mathrm{H}$ & 0.318526 & -0.423111 & -3.043524 & $\mathrm{C}$ & 4.910975 & 0.144624 & -1.143024 \\
\hline $\mathrm{H}$ & -0.672184 & 1.055156 & -3.054943 & $\mathrm{C}$ & 4.502959 & -0.956609 & 0.941226 \\
\hline $\mathrm{H}$ & 0.904757 & 1.046433 & -3.858085 & $\mathrm{C}$ & 5.328485 & -0.156738 & 0.152870 \\
\hline $\mathrm{O}$ & 0.975266 & 2.521462 & 0.555717 & $\mathrm{H}$ & 5.548097 & 0.756697 & -1.780054 \\
\hline $\mathrm{H}$ & 0.437601 & 0.267479 & 1.900795 & $\mathrm{H}$ & 4.818110 & -1.218169 & 1.950630 \\
\hline $\mathrm{C}$ & 2.378795 & 2.692420 & 0.461221 & $\mathrm{C}$ & -1.158107 & -3.265073 & 1.347968 \\
\hline $\mathrm{H}$ & 2.909330 & 2.149628 & 1.259055 & $\mathrm{H}$ & -0.578804 & -2.514269 & 1.901533 \\
\hline $\mathrm{H}$ & 2.710484 & 2.314490 & -0.512017 & $\mathrm{H}$ & -1.609584 & -3.923581 & 2.094359 \\
\hline $\mathrm{H}$ & 2.636112 & 3.756033 & 0.532967 & $\mathrm{H}$ & -0.442435 & -3.857862 & 0.767917 \\
\hline $\mathrm{H}$ & 0.649569 & 2.775597 & 1.551798 & $\mathrm{C}$ & -2.511094 & -0.793127 & -2.848283 \\
\hline $\mathrm{C}$ & -1.176345 & 2.984070 & 2.859150 & $\mathrm{H}$ & -3.398006 & -0.607084 & -3.458682 \\
\hline $\mathrm{H}$ & -1.635599 & 2.009928 & 2.595981 & $\mathrm{H}$ & -2.039079 & 0.175232 & -2.641702 \\
\hline $\mathrm{H}$ & -1.574654 & 3.272976 & 3.844549 & $\mathrm{H}$ & -1.796589 & -1.364507 & -3.449140 \\
\hline $\mathrm{H}$ & -1.551314 & 3.716117 & 2.127582 & $\mathrm{C}$ & -5.970882 & -2.035965 & 0.571372 \\
\hline $\mathrm{O}$ & 0.219745 & 2.941722 & 2.858887 & $\mathrm{H}$ & -6.062432 & -2.355604 & 1.612234 \\
\hline $\mathrm{H}$ & 0.587719 & 1.846998 & 3.224857 & $\mathrm{H}$ & -6.412757 & -1.037830 & 0.487302 \\
\hline $\mathrm{O}$ & 0.940980 & 0.708708 & 3.398067 & $\mathrm{H}$ & -6.591632 & -2.705318 & -0.034378 \\
\hline $\mathrm{C}$ & 0.007935 & 0.052659 & 4.191442 & $\mathrm{C}$ & 3.279129 & 0.098565 & -3.029790 \\
\hline $\mathrm{H}$ & -0.874579 & -0.305500 & 3.612155 & $\mathrm{H}$ & 2.852170 & 1.105999 & -2.999203 \\
\hline $\mathrm{H}$ & 0.444902 & -0.836006 & 4.676946 & $\mathrm{H}$ & 4.135515 & 0.112506 & -3.709937 \\
\hline \multirow[t]{2}{*}{$\mathrm{H}$} & -0.391865 & 0.695398 & 4.992772 & $\mathrm{H}$ & 2.508746 & -0.555584 & -3.445259 \\
\hline & & & & $\mathrm{C}$ & 2.462938 & -2.316687 & 1.381137 \\
\hline 48-m-r & & & & $\mathrm{H}$ & 1.553396 & -2.692054 & 0.908752 \\
\hline $\mathrm{Ru}$ & 0.422678 & 0.606798 & 0.172392 & $\mathrm{H}$ & 3.048168 & -3.180954 & 1.713121 \\
\hline $\mathrm{C}$ & 0.413969 & -1.130234 & -0.840402 & $\mathrm{H}$ & 2.153277 & -1.782376 & 2.286922 \\
\hline $\mathrm{C}$ & 0.023366 & -3.133260 & -2.002101 & $\mathrm{C}$ & 6.615543 & 0.386992 & 0.691681 \\
\hline $\mathrm{C}$ & 1.527144 & -2.930139 & -1.863910 & $\mathrm{H}$ & 6.461957 & 1.368677 & 1.154641 \\
\hline $\mathrm{H}$ & -0.321303 & -4.109538 & -1.645909 & $\mathrm{H}$ & 7.042165 & -0.263932 & 1.459497 \\
\hline $\mathrm{H}$ & -0.319913 & -3.013999 & -3.039793 & $\mathrm{H}$ & 7.361916 & 0.519961 & -0.096309 \\
\hline $\mathrm{H}$ & 1.984198 & -3.640462 & -1.158261 & $\mathrm{C}$ & -1.421218 & 0.910118 & 0.137340 \\
\hline $\mathrm{H}$ & 2.064293 & -3.010128 & -2.814199 & $\mathrm{H}$ & -2.139008 & 0.202598 & 0.582966 \\
\hline $\mathrm{N}$ & -0.519630 & -2.054386 & -1.166156 & $\mathrm{C}$ & -2.058335 & 2.126183 & -0.338215 \\
\hline $\mathrm{N}$ & 1.613751 & -1.567346 & -1.326714 & $\mathrm{C}$ & -3.433476 & 2.096577 & -0.651034 \\
\hline
\end{tabular}




\begin{tabular}{|c|c|c|c|c|c|c|c|}
\hline $\mathrm{C}$ & -1.363267 & 3.343019 & -0.497563 & $\mathrm{~N}$ & 1.574533 & -1.558884 & -1.408710 \\
\hline $\mathrm{C}$ & -4.075667 & 3.219112 & -1.154219 & $\mathrm{C}$ & -1.954780 & -1.946104 & -0.942418 \\
\hline $\mathrm{H}$ & -3.979259 & 1.164138 & -0.508178 & $\mathrm{C}$ & -2.872693 & -1.214792 & -1.709594 \\
\hline $\mathrm{C}$ & -2.016184 & 4.466436 & -0.983662 & $\mathrm{C}$ & -2.344094 & -2.636263 & 0.214899 \\
\hline $\mathrm{H}$ & -0.308987 & 3.377636 & -0.236083 & $\mathrm{C}$ & -4.197046 & -1.165036 & -1.276637 \\
\hline $\mathrm{C}$ & -3.368652 & 4.408886 & -1.323093 & $\mathrm{C}$ & -3.681950 & -2.557062 & 0.607212 \\
\hline $\mathrm{H}$ & -5.131960 & 3.172768 & -1.406429 & $\mathrm{C}$ & -4.619874 & -1.822261 & -0.118480 \\
\hline $\mathrm{H}$ & -1.469566 & 5.399036 & -1.096108 & $\mathrm{H}$ & -4.915180 & -0.583882 & -1.854534 \\
\hline $\mathrm{H}$ & -3.872959 & 5.292739 & -1.704833 & $\mathrm{H}$ & -3.994663 & -3.078827 & 1.510858 \\
\hline $\mathrm{O}$ & 0.995065 & 1.655239 & -1.435027 & $\mathrm{C}$ & 2.803731 & -1.094401 & -0.826701 \\
\hline $\mathrm{H}$ & 0.431398 & -0.543950 & 1.508118 & $\mathrm{C}$ & 3.673884 & -0.309576 & -1.598905 \\
\hline $\mathrm{C}$ & 0.339283 & 1.469893 & -2.644773 & $\mathrm{C}$ & 3.119697 & -1.437254 & 0.505963 \\
\hline $\mathrm{H}$ & 0.328183 & 0.412145 & -2.976872 & $\mathrm{C}$ & 4.842393 & 0.166532 & -1.002883 \\
\hline $\mathrm{H}$ & -0.711255 & 1.813453 & -2.620844 & $\mathrm{C}$ & 4.294472 & -0.923954 & 1.055807 \\
\hline $\mathrm{H}$ & 0.843222 & 2.050767 & -3.434588 & $\mathrm{C}$ & 5.163167 & -0.113778 & 0.323968 \\
\hline $\mathrm{O}$ & 1.489393 & 1.866311 & 1.361675 & $\mathrm{H}$ & 5.516528 & 0.783859 & -1.595488 \\
\hline $\mathrm{H}$ & -0.110836 & 0.038565 & 1.763079 & $\mathrm{H}$ & 4.535652 & -1.171812 & 2.088748 \\
\hline $\mathrm{C}$ & 2.409012 & 2.802118 & 0.875188 & $\mathrm{C}$ & -1.348921 & -3.401175 & 1.029907 \\
\hline $\mathrm{H}$ & 3.434071 & 2.519058 & 1.164209 & $\mathrm{H}$ & -0.705399 & -2.736776 & 1.622268 \\
\hline $\mathrm{H}$ & 2.373883 & 2.886493 & -0.219421 & $\mathrm{H}$ & -1.849306 & -4.068287 & 1.736205 \\
\hline $\mathrm{H}$ & 2.207566 & 3.798534 & 1.302223 & $\mathrm{H}$ & -0.686565 & -4.011093 & 0.405566 \\
\hline $\mathrm{H}$ & 0.989531 & 1.698662 & 2.938186 & $\mathrm{C}$ & -2.433411 & -0.444322 & -2.912818 \\
\hline $\mathrm{C}$ & -0.685855 & 2.397960 & 3.593808 & $\mathrm{H}$ & -3.287238 & -0.148722 & -3.526982 \\
\hline $\mathrm{H}$ & -1.357638 & 2.039039 & 2.794740 & $\mathrm{H}$ & -1.904800 & 0.469261 & -2.614442 \\
\hline $\mathrm{H}$ & -1.246889 & 2.409784 & 4.533110 & $\mathrm{H}$ & -1.738465 & -1.010759 & -3.540862 \\
\hline $\mathrm{H}$ & -0.408416 & 3.432433 & 3.348113 & $\mathrm{C}$ & -6.051519 & -1.753369 & 0.317439 \\
\hline $\mathrm{O}$ & 0.446865 & 1.575120 & 3.760711 & $\mathrm{H}$ & -6.177075 & -2.095769 & 1.347614 \\
\hline $\mathrm{H}$ & 0.064639 & -0.193153 & 3.977064 & $\mathrm{H}$ & -6.444399 & -0.733794 & 0.251004 \\
\hline $\mathrm{O}$ & -0.309456 & -1.099204 & 3.945242 & $\mathrm{H}$ & -6.692550 & -2.378204 & -0.314668 \\
\hline $\mathrm{C}$ & -1.698045 & -0.941015 & 3.831402 & $\mathrm{C}$ & 3.374224 & 0.049726 & -3.018602 \\
\hline $\mathrm{H}$ & -2.008929 & -0.506242 & 2.861162 & $\mathrm{H}$ & 3.143197 & 1.116511 & -3.092198 \\
\hline $\mathrm{H}$ & -2.163233 & -1.929733 & 3.907498 & $\mathrm{H}$ & 4.235260 & -0.148734 & -3.664863 \\
\hline \multirow[t]{2}{*}{$\mathrm{H}$} & -2.124521 & -0.310004 & 4.626069 & $\mathrm{H}$ & 2.509863 & -0.491495 & -3.407722 \\
\hline & & & & $\mathrm{C}$ & 2.269137 & -2.345855 & 1.343590 \\
\hline \multicolumn{2}{|c|}{ 4sw8-m-r } & & & $\mathrm{H}$ & 1.382078 & -2.708558 & 0.822399 \\
\hline $\mathrm{Ru}$ & 0.457420 & 0.581448 & 0.066350 & $\mathrm{H}$ & 2.847280 & -3.218774 & 1.665961 \\
\hline $\mathrm{C}$ & 0.368980 & -1.093471 & -0.942610 & $\mathrm{H}$ & 1.913744 & -1.849933 & 2.252402 \\
\hline $\mathrm{C}$ & -0.038012 & -3.057314 & -2.158479 & $\mathrm{C}$ & 6.403848 & 0.441025 & 0.954201 \\
\hline $\mathrm{C}$ & 1.464375 & -2.917975 & -1.944909 & $\mathrm{H}$ & 6.162390 & 1.211291 & 1.695151 \\
\hline $\mathrm{H}$ & -0.434608 & -4.031143 & -1.853416 & $\mathrm{H}$ & 6.969700 & -0.332730 & 1.482371 \\
\hline $\mathrm{H}$ & -0.326757 & -2.890669 & -3.206661 & $\mathrm{H}$ & 7.065754 & 0.895520 & 0.212751 \\
\hline $\mathrm{H}$ & 1.852309 & -3.646084 & -1.215684 & $\mathrm{C}$ & -1.355129 & 0.957344 & 0.161209 \\
\hline $\mathrm{H}$ & 2.043499 & -3.024361 & -2.867724 & $\mathrm{H}$ & -2.084643 & 0.204619 & 0.501570 \\
\hline $\mathrm{N}$ & -0.578887 & -1.986704 & -1.313734 & $\mathrm{C}$ & -1.977796 & 2.248062 & -0.089818 \\
\hline
\end{tabular}




\begin{tabular}{|c|c|c|c|}
\hline E & -3.381964 & 2.348410 & -0.011497 \\
\hline C & -1.240710 & 3.410612 & -0.397678 \\
\hline & -4.025976 & 3.557003 & -0.240554 \\
\hline & -3.954196 & 1.453265 & 0.230652 \\
\hline & -1.889166 & 4.614825 & -0.625361 \\
\hline & -0.158118 & 3.333876 & -0.473686 \\
\hline & -3.280790 & 4.694509 & -0.546755 \\
\hline & -5.109181 & 3.616500 & -0.176589 \\
\hline & -1.309588 & 5.502717 & -0.863324 \\
\hline$U$ & -3.782285 & 5.643120 & -0.719230 \\
\hline $\mathrm{O}$ & 1.159063 & 1.688600 & -1.453266 \\
\hline$\Pi$ & 0.129855 & -0.470552 & 1.457243 \\
\hline $\mathrm{C}$ & 0.530676 & 1.659449 & -2.689531 \\
\hline $\mathrm{H}$ & 0.452388 & 0.637839 & -3.110252 \\
\hline $\mathrm{H}$ & -0.494824 & 2.073504 & -2.656246 \\
\hline $\mathrm{H}$ & 1.097386 & 2.265474 & -3.416009 \\
\hline $\mathrm{O}$ & 1.647752 & 1.886022 & 1.345016 \\
\hline $\mathrm{H}$ & 0.312040 & 0.072137 & 2.066050 \\
\hline $\mathrm{C}$ & 2.412895 & 3.044886 & 1.096144 \\
\hline $\mathrm{H}$ & 3.435052 & 2.928160 & 1.476289 \\
\hline $\mathrm{H}$ & 2.445077 & 3.178333 & 0.011357 \\
\hline $\mathrm{H}$ & 1.955516 & 3.929119 & 1.558049 \\
\hline $\mathrm{H}$ & 1.450038 & 1.633650 & 2.292232 \\
\hline$C$ & -0.508927 & 1.587576 & 3.573841 \\
\hline $\mathrm{H}$ & -1.329581 & 1.433596 & 2.841611 \\
\hline $\mathrm{H}$ & -0.938092 & 1.405900 & 4.573186 \\
\hline $\mathrm{H}$ & -0.263624 & 2.665385 & 3.525134 \\
\hline $\mathrm{O}$ & 0.596021 & 0.776409 & 3.320766 \\
\hline$H$ & 0.151721 & -0.883581 & 3.824532 \\
\hline $\mathrm{O}$ & -0.287104 & -1.764220 & 3.839198 \\
\hline $\mathrm{C}$ & -1.662149 & -1.514485 & 3.749670 \\
\hline $\mathrm{H}$ & -1.946361 & -0.971148 & 2.827697 \\
\hline $\mathrm{H}$ & -2.186113 & -2.477219 & 3.734354 \\
\hline $\mathrm{H}$ & -2.053993 & -0.938878 & 4.603601 \\
\hline
\end{tabular}




\section{Supporting References.}

1 Beach, N. J.; Lummiss, J. A. M.; Bates, J. M.; Fogg, D. E. Reactions of Grubbs Catalysts with Excess Methoxide: Formation of Novel Methoxyhydride Complexes. Organometallics 2012, $31,2349-2356$.

2 Nielsen, M.; Kammer, A.; Cozzula, D.; Junge, H.; Gladiali, S.; Beller, M. Efficient Hydrogen Production from Alcohols under Mild Reaction Conditions. Angew. Chem., Int. Ed. 2011, 50, 9593-9597.

3 Sponholz, P.; Mellmann, D.; Cordes, C.; Alsabeh, P. G.; Li, B.; Li, Y.; Nielsen, M.; Junge, H.; Dixneuf, P.; Beller, M. Efficient and Selective Hydrogen Generation from Bioethanol using Ruthenium Pincer-type Complexes. ChemSusChem 2014, 7, 2419-2422.

4 Wang, Q.; Xia, Y.; Cheng,F.; Chen, Z.; Wang, Y.; Zhu, X.; Qin, L.; Zheng, Z.; Formic Acid Dehydrogenation for Hydrogen Production Promoted by Grubbs and Hoveyda-Grubbs Catalysts. Chin. J. Chem. 2021, 39, 2201-2206.

5 (a) Sinha, V.; Trincado, M.; Grützmacher, H.; d. Bruin, B. DFT Provides Insight into the Additive-Free Conversion of Aqueous Methanol to Dihydrogen Catalyzed by [Ru(trop 2 dad)]: Importance of the (Electronic) Flexibility of the Diazadiene Moiety. J. Am. Chem. Soc. 2018, 140, 13103-13114; (b) Alberico, E.; Sponholz, P.; Cordes, C.; Nielsen, M.; Drexler, H.-J.; Baumann, W.; Junge, H.; Beller, M. Selective Hydrogen Production from Methanol with a Defined Iron Pincer Catalyst under Mild Conditions. Angew. Chem., Int. Ed. 2013, 52, 14162-14166.

6 Frisch, M. J.; Trucks, G. W.; Schlegel, H. B.; Scuseria, G. E.; Robb, M. A.; Cheeseman, J. R.; Scalmani, G.; Barone, V.; Petersson, G. A.; Nakatsuji, H.; Li, X.; Caricato, M.; Marenich, A.; Bloino, J.; Janesko, B. G.; Gomperts, R.; Mennucci, B.; Hratchian, H. P.; Ortiz, J. V.; Izmaylov, A. F.; Sonnenberg, J. L.; Williams-Young, D.; Ding, F.; Lipparini, F.; Egidi, F.; Goings, J.; Peng, B.; Petrone, A.; Henderson, T.; Ranasinghe, D.; Zakrzewski, V. G.; Gao, J.; Rega, N.; Zheng, G.; Liang, W.; Hada, M.; Ehara, M.; Toyota, K.; Fukuda, R.; Hasegawa, J.; Ishida, M.; Nakajima, T.; Honda, Y.; Kitao, O.; Nakai, H.; Vreven, T.; Throssell, K.; Montgomery, J. A.; Jr.; Peralta, J. E.; Ogliaro, F.; Bearpark, M.; Heyd, J. J.; Brothers, E.; Kudin, K. N.; Staroverov, V. N.; Keith, T.; Kobayashi, R.; Normand, J.; Raghavachari, K.; Rendell, A.; Burant, J. C.; Iyengar, S. S.; Tomasi, J.; Cossi, M.; Millam, J. M.; Klene, M.; Adamo, C.; Cammi, R.; Ochterski, J. W.; Martin, R. L.; Morokuma, K.; Farkas, O.; Foresman, J. B.; Fox, D. J. Gaussian 09, Revision A.02, Gaussian, Inc., Wallingford CT, 2016.

7 Frisch, M. J.; Trucks, G. W.; Schlegel, H. B.; Scuseria, G. E.; Robb, M. A.; Cheeseman, J. R.; Scalmani, G.; Barone, V.; Petersson, G. A.; Nakatsuji, H.; Li, X.; Caricato, M.; Marenich, A. V.; Bloino, J.; Janesko, B. G.; Gomperts, R.; Mennucci, B.; Hratchian, H. P.; Ortiz, J. V.; Izmaylov, A. F.; Sonnenberg, J. L.; Williams-Young, D.; Ding, F.; Lipparini, F.; Egidi, F.; Goings, J.; Peng, 
B.; Petrone, A.; Henderson, T.; Ranasinghe, D.; Zakrzewski, V. G.; Gao, J.; Rega, N.; Zheng, G.; Liang, W.; Hada, M.; Ehara, M.; Toyota, K.; Fukuda, R.; Hasegawa, J.; Ishida, M.; Nakajima, T.; Honda, Y.; Kitao, O.; Nakai, H.; Vreven, T.; Throssell, K.; Montgomery, J. A., Jr.; Peralta, J. E.; Ogliaro, F.; Bearpark, M. J.; Heyd, J. J.; Brothers, E. N.; Kudin, K. N.; Staroverov, V. N.; Keith, T. A.; Kobayashi, R.; Normand, J.; Raghavachari, K.; Rendell, A. P.; Burant, J. C.; Iyengar, S. S.; Tomasi, J.; Cossi, M.; Millam, J. M.; Klene, M.; Adamo, C.; Cammi, R.; Ochterski, J. W.; Martin, R. L.; Morokuma, K.; Farkas, O.; Foresman, J. B.; Fox, D. J. Gaussian 16, Revision C.01, Gaussian, Inc., Wallingford CT, 2016.

8 Tomasi, J.; Mennucci, B.; Cammi, R.; Quantum Mechanical Continuum Solvation Models. Chem Rev. 2005, 105, 2999-3093.

9 Barone, V.; Cossi, M. Quantum Calculation of Molecular Energies and Energy Gradients in Solution by a Conductor Solvent Model. J. Phys. Chem. A 1998, 102, 1995-2001.

10 Cossi, M.; Rega, N.; Scalmani, G.; Barone, V. Energies, structures, and electronic properties of molecules in solution with the C-PCM solvation model. J. Comp. Chem. 2003, 24, 669-681.

11 Zhao, Y.; Truhlar, D. G. Density Functionals with Broad Applicability in Chemistry. Acc. Chem. Res. 2008, 41, 157-167.

12 Adamo, C.; Barone, V. Toward reliable density functional methods without adjustable parameters: The PBE0 model. J. Chem. Phys. 1999, 110, 6158-6169.

13 Chai, J.-D.; Head-Gordon, M. Systematic optimization of long-range corrected hybrid density functionals. J. Chem. Phys. 2008, 128, 084106.

14 Lee, C.; Yang, W.; Parr, R. G. Development of the Colle-Salvetti correlation-energy formula into a functional of the electron density. Phys. Rev. B 1988, 37, 785-789.

15 Becke, A. D. Density - functional thermochemistry. III. The role of exact exchange. J. Chem. Phys. 1993, 98, 5648-5652.

16 Grimme, S.; Antony, J.; Ehrlich, S.; Krieg, H. A consistent and accurate $a b$ initio parametrization of density functional dispersion correction (DFT-D) for the 94 elements H-Pu. J. Chem. Phys. 2010, 132, 154104.

17 CYLview, 1.0b; C. Y. Legault, Université de Sherbrooke, 2009 (http://www.cylview.org).

$18 \mathrm{Qu}$, S.; Cramer, C. J. Mechanistic Study of $\mathrm{Cp}^{*} \mathrm{Co}^{\mathrm{III}} / \mathrm{Rh}^{\mathrm{III}}-$ Catalyzed Directed $\mathrm{C}-\mathrm{H}$ Functionalization with Diazo Compounds. J. Org. Chem. 2017, 82, 1195-1204.

19 Willoughby, P. H.; Niu, D.; Wang, T.; Haj, M. K.; Cramer, C. J.; Hoye, T. R. Mechanism of the Reactions of Alcohols with o-Benzynes. J. Am. Chem. Soc. 2014, 136, 13657-13665.

20 Atkins, P.; de Paula, J. Atkins' Physical Chemistry [8th Edition]. Oxford University Press, New York, 2006.

21 Minenkov, Y.; Occhipinti, G.; Jensen, V. R. Metal-Phosphine Bond Strengths of the Transition Metals: A Challenge for DFT. J. Phys. Chem. A 2009, 113, 11833-11844. 